\title{
ATOMIC DATA AND SPECTRAL LINE INTENSITIES FOR CA IX
}

\author{
E. Landi \\ Department of Atmospheric, Oceanic and Space Sciences, University of Michigan \\ Ann Arbor, Michigan 48109 USA
}

A. K. Bhatia

NASA/Goddard Space Flight Center

Greenbelt, Maryland 20771 USA

\begin{abstract}
Electron impact collision strengths, energy levels, oscillator strengths and spontaneous radiative decay rates are calculated for Ca IX. We include in the calculations the 33 lowest configurations in the $n=3,4,5$ complexes, corresponding to 283 fine structure levels in the $3 l 3 l^{\prime}, 3 l 4 l^{\prime \prime}$ and $3 l 4 l^{\prime \prime \prime}$ configurations, where $l, l^{\prime}=s, p, d, l^{\prime \prime}=s, p, d, f$ and $l^{\prime \prime \prime}=s, p, d, f, g$. Collision strengths are calculated at five incident energies for all transitions: 5.8, 13.6, 24.2, 38.6 and 57.9 Ry above the threshold of each transition. An additional energy, very close to the transition threshold, has been added, whose value is between 0.0055 Ry and 0.23 Ry depending on the levels involved. Calculations have been carried out using the Flexible Atomic Code and the distorted wave approximation. Excitation rate coefficients are calculated as a function of electron temperature by assuming a Maxwellian electron velocity distribution. Using the excitation rate coefficients and the radiative transition rates calculated in the present work, statistical equilibrium equations for level populations are solved at electron densities covering the $10^{8}-10^{14} \mathrm{~cm}^{-3}$ range and at an electron temperature of $\log T_{e}(\mathrm{~K})=5.8$, corresponding to the maximum abundance of Ca IX. Spectral line intensities are calculated, and their diagnostic relevance is discussed.
\end{abstract}




\section{CONTENTS}

Introduction $\quad 3$

Atomic Data 3

Assessment of results $\quad 5$

Level Populations and Relative Line Intensities $\quad 7$

Comparison with observations $\quad 8$

Conclusions $\quad 9$

References 10

Explanation of Tables 12

\section{TABLES}

I. Calculated and Experimental Energy Levels for Ca IX 13

II. Ca IX Oscillator Strengths, Radiative Rates, and Collision Strengths 19

III. Ca IX Oscillator Strengths, Radiative Rates 44

IV. Ca IX Level Populations $\quad 112$

V. Intensities of Selected Bright Ca IX Lines 119 


\section{Introduction}

In recent years, we have been pursuing a program to calculate atomic data and transition rates for ions whose lines have been observed in astrophysical spectra, and yet have been neglected in the literature. There are two types of such ions: those for which a large number of calculations are available for low-energy configurations, but whose high-energy configurations have not been considered (i.e. C-like, N-like, O-like systems to name a few); or ions that have never or only seldom been studied. In the present work we focus $\mathrm{Mg}$-like $\mathrm{Ca}$, or Ca IX, which belongs to the second class of ions. This ion emits a number of allowed transitions in the EUV and soft X-ray ranges, some of which have been observed over the years in Extreme Ultraviolet solar spectra from quiet and active solar plasmas (Behring et al. [1], Dere [2], Vernazza \& Reeves [3], Feldman et al. [4], Curdt et al. [5,6,7], Parenti et al. [8]). Laboratory measurements of Ca IX lifetimes were carried out by Träbert et al. [9], while energy levels were determined in several laboratory experiments by Parker \& Phillips [10], Fawcett [11], Ekberg [12], Litzen \& Redfors [13], Redfors [14] and Churilov et al. [15].

Some of the Ca IX can be used to form strongly density and temperature sensitive intensity ratios that can be applied to the measurement of the plasma parameters of solar and stellar plasmas. The strongest line in the Ca IX spectrum can also be used for emission measure and element abundance studies. Configurations with principal quantum number $n=4,5$ emit a number of lines in the soft X-rays between 45 and $80 \AA$ that can also be used for plasma diagnostics.

Calculations of wavefunctions, level energies and radiative decay rates of Ca IX are relatively abundant in the literature, both as part of larger-scope calculations of data along the Mg-like sequence, or specifically dedicated to Ca IX. These calculations have been carried out by a number of authors using different techniques and atomic model (Fawcett [16], Kastner et al. [17], Christensen et al. [18], Tayal [19], Godefroid [20], Froese Fischer et al. [21], Stanek et al. [22], Zou \& Froese Fischer [23], Tayal [24], Safronova et al. [25], Aashamar [26], Karpuskiene et al. [27]). Most of the calculations provided data only for levels in the $n=3$ complex, but a few of them also included some $n=4$ levels (Kastner et al. [17], Christensen et al. [18], Froese Fischer et al. [21], Aashamar [26], Karpuskiene et al. [27] and Tayal [24]). None of these authors provided data for $n=5$ configurations.

Considerable attention has also been paid to the hyperfine-induced transitions from the $3 s 3 p{ }^{3} \mathrm{P}_{0,2}$, and several calculations have been devoted to determine their A values (Marques et al. [28], Brage et al. [29], Kang et al. [30,31] and Andersson et al. [32]). One-photon radiative decays from the metastable ${ }^{3} \mathrm{P}_{0}$ level can have consequences for the overall Ca IX level population and provide excellent density diagnostic intensity ratios.

Contrarily to radiative rates, the available datasets of electron impact excitation rate coefficients for Ca IX are surprisingly scarce. To our knowledge, the only complete dataset of collision strengths for 16 fine structure levels has been published by Christensen et al. [18]. No collision data have ever been published for the entire $n=4$ complex, and none for any of the $n=5$ levels. Furthermore, the available collision rate coefficients for the $n=3$ complex are not complete.

The aim of the present work is to provide a complete dataset of energy levels, A-values and electron collisional excitation rates for Ca IX that allows to predict the spectrum of this ion and use it for plasma diagnostic purposes. We include all levels in the $n=3,4,5$ complexes. While the present results are redundant for most of $n=3,4$ A values, they provide the first complete and up-to-date dataset that allows the calculation of spectral line intensities for all lines within the entire $n=3,4,5$ complexes. We also discuss the diagnostic relevance of the strongest Ca IX lines.

\section{Atomic data}

To calculate a complete set of atomic data and transition rates and to assess its accuracy, we have used two suite of codes: the Flexible Atomic Code (FAC) by Gu [33] and the University College London (UCL) suite of codes (Eissner et al. [34]). 


\begin{tabular}{ll} 
Acronym & Source \\
\hline PP40 & Parker \& Phillips [10] \\
F70 & Fawcett [11] \\
E71 & Ekberg [12] \\
EB76 & Edlen \& Boden [35] \\
F76 & Fawcett [36] \\
LR87 & Litzen \& Redfors [13] \\
R88 & Redfors [14] \\
C89 & Churilov et al. [15] \\
C04 & Curdt et al. [7] \\
& \\
\hline
\end{tabular}

Table A. Literature sources where measured level energies are taken.

\begin{tabular}{rrrrr} 
Lower & \multicolumn{5}{c}{ Upper levels } \\
Level & $\mathrm{E}^{\prime}=0.0055$ & $\mathrm{E}^{\prime}=0.022$ & $\mathrm{E}^{\prime}=0.10$ & $\mathrm{E}^{\prime}=0.23$ \\
\hline & & $2-4$ & $5-45$ & $46-283$ \\
1 & & 5 & $6-64$ & $65-283$ \\
2 & $3-4$ & 5 & $6-64$ & $65-283$ \\
3 & 4 & 5 & $6-64$ & $65-283$ \\
4 & & & & \\
\hline
\end{tabular}

Table B. Values of the energy E' used for the FAC electron-ion scattering calculations (in Ry).

FAC is a relativistic configuration interaction program. The radial wavefunctions for single-electron orbitals are obtained with a self-consistent field method based on Dirac formulation. The Dirac-Coulomb Hamiltonian is diagonalized to obtain energy levels and atomic state wavefunctions. The atomic model we used included all 33 configurations for the $n=3,4,5$ complexes, for a total of 283 fine-structure levels. We did not attempt to calculate hyperfine transitions between the ground and the $3 s 3 p{ }^{3} \mathrm{P}_{0,2}$ levels. We will refer to these results as the "FAC" dataset.

Energy values and radiative decay rates have also been calculated using the SUPERSTRUCTURE program, a part of the UCL suite, with the same atomic model used with FAC. In SUPERSTRUCTURE, wavefunctions are of configuration interaction type and each configuration is expanded in terms of Slater orbitals. The radial functions are calculated in a scaled Thomas-Fermi-Amaldi potential, which depends upon parameters $\lambda$ which are determined variationally by optimizing the weighted sum of the term energies. The relativistic corrections are included by using the Breit-Pauli Hamiltonian as a perturbation to the nonrelativistic Hamiltonian. Energy levels, oscillator strengths, and radiative transition rates are calculated in intermediate coupling. We will refer to this dataset as the "SST" dataset. Both the FAC and SST energies are given in Table I, together with the observed values, where available. Table A lists the literature sources where the observed values are taken from.

The FAC code solves the scattering problem in the distorted wave approximation, as described in $\mathrm{Gu}$ ([33]). FAC calculations were carried out at six incident electron energies. The five highest energies were chosen to be 5.8, 13.6, 24.2,38.6 and 57.9 Ry above the threshold of each transition. The lowest energy was chosen to have a very small value E' above threshold in order to capture the near-threshold behavior of the collision strengths: the selected E' values change from transition to transition and are listed in Table B. Memory limitations prevented us from using the UCL code also to calculate the collision strengths.

The Ca IX collision strengths $\Omega_{i j}$, the transition rates $A_{j i}$, and $g_{i} f_{i j}$, the absorption oscillator strengths multiplied by the statistical weight $g_{i}$ of the lower level, are given in Table II for all transitions involving the 
lowest 4 levels. Transition rates $A_{j i}$ and $g_{i} f_{i j}$ values for all other transitions are given in Table III; collision strengths for these transitions are also available upon request.

The electron impact excitation rate coefficients $\left(\right.$ in $\mathrm{cm}^{3} \mathrm{~s}^{-1}$ ) are obtained by averaging the collision strengths over the Maxwellian electron velocity distribution

$$
C_{i j}^{e}=\frac{8.63 \times 10^{-6}}{g_{i} T_{e}^{1 / 2}} e^{-\frac{\Delta E_{i j}}{k T}} \int_{0}^{\infty} \Omega_{i j}\left(E_{j}\right) e^{-\frac{E_{j}}{k T_{e}}} d\left(\frac{E_{j}}{k T_{e}}\right)
$$

where $T_{e}$ is the electron temperature in $\mathrm{K}, \Delta E_{i j}$ is the transition energy of the $i \rightarrow j$ transition, $E_{j}$ is the incident energy with respect to the level $j$, and $k$ is the Boltzmann constant. The de-excitation rates are given by the principle of detailed balance:

$$
C_{j i}^{d}=\frac{g_{i}}{g_{j}} e^{\frac{\Delta E_{i j}}{k T_{e}}} C_{i j}^{e}
$$

In order to carry out the integration in Eq. (1), it is necessary to know the collision strengths $\Omega$ across the whole energy range, from threshold to infinity. However, the present theoretical calculations only provide collision strengths at a few energy values, so that some interpolation/extrapolation technique is required in order to properly take into account the energy dependence of collision strengths. In the present work we have adopted the method described by Burgess and Tully [37] to extrapolate collision strengths to the highand low-energy limit. This method has been used by Bhatia et al. [38] and details are given there.

\section{Assessment of results}

Table I reports the level energies calculated by both FAC and SST together with the experimental values. Theoretical energies obtained with the two calculations differ by less than $0.3 \%$ for all configurations except a few levels in the $3 s 3 d, 3 p 3 d$ and $3 d^{2}$ where differences rise up to $0.7 \%$, with the FAC calculations being closer to observations than the SST ones. Table A reports the literature sources where the experimental energies have been taken from. A comparison between the two theoretical calculations shows that results are rather similar, except in a few cases where the FAC results are usually closer to the observations. Comparison of the FAC energies with the observed values shows that $n=3$ singlet levels are within $1 \%$ of the measured values, whereas predicted triplet energies reproduce the latter to better than $0.2 \%$. The only exception is the lowest excited configuration $(3 s 3 p)$ where differences rise to $0.75 \%$ and for the ${ }^{3} \mathrm{P}$ energies. FAC energies for the $n=4$ and $n=5$ levels are within $0.2 \%$ of the observed values. The $n=5$ predicted energies reproduce the observed ones to better than $0.15 \%$, with only one exception at $0.25 \%$.

The only other possible direct comparisons of our calculations with observations are level lifetimes, which were measured by Träbert et al. [9]. The comparison with our results is shown in Table C, along with with several other calculations. With the only exception of levels 6 and 15, the present results agree with those of the most recent calculations. The first thing to notice is that the lifetime of level 5 , giving rise to the strong resonance line at $466 \AA$, is within experimental uncertainties for all calculations. Measured lifetimes for all levels of the $3 p^{2}$ and $3 s 3 d$ configurations (levels 6 to 14) are larger than predicted by all calculations, which provide very similar results. The only exception is $3 p^{2}{ }^{1} \mathrm{D}_{2}$ (level 6), for which only our SST calculation, Karpuskiene et al. [27] and Froese Fischer et al. [21] provide results in agreement with observations. The $3 p 3 d{ }^{3} \mathrm{~F}$ triplet (levels 15-17) provides mixed results, with some levels and some calculations giving better agreement. The FAC and SST results are largely the same, with the only exception of level 6 for which SST is superior to FAC.

Table $\mathrm{D}$ compares A values for the $n=3$ levels from different calculations. We have compared results for the forbidden transitions from metastable levels in the $3 s 3 p$ configuration, and allowed transitions giving rise to the strongest lines in the Ca IX spectrum. The forbidden transitions from the $3 s 3 p$ calculation to the ground level show very good agreement (within $20 \%$, mostly down to $5 \%$ ) amongst all calculations, with the only 


\begin{tabular}{lrrrrrrrrr} 
Level & T86 & FAC & SST & F83 & C86 & S00 & FF06 & K04 & T05 \\
\hline 5 & & & & & & & & & \\
6 & $85 \pm 10$ & 87.8 & 91.4 & 89.7 & 87.7 & 101 & 94.9 & 94.0 & 92.1 \\
9 & $134 \pm 8$ & 1013 & 893 & 1332 & 972.9 & 1060 & 848 & 872 & \\
10 & $150 \pm 8$ & 103.1 & 107.2 & 108.2 & 103.8 & 107 & 112.3 & 112.2 & 106.1 \\
12 & $100 \pm 15$ & 74.5 & 75.5 & 74.7 & 71.8 & 78.7 & 76.1 & 76.5 & 74.2 \\
13 & $117 \pm 9$ & 76.3 & 77.3 & 76.9 & 73.0 & 80.6 & 77.9 & 78.1 & 76.0 \\
14 & $72 \pm 3$ & 40.8 & 41.9 & 43.0 & 39.8 & 45.4 & 43.0 & 43.1 & 40.5 \\
15 & $653 \pm 63$ & 425 & 409 & 346 & & 431 & 425 & 505 & 593 \\
16 & $473 \pm 21$ & 460 & 443 & 303 & & 476 & 469 & 468 & 523 \\
17 & $513 \pm 18$ & 444 & 428 & 284 & & 459 & 452 & 452 & 433 \\
& & & & & & & & & \\
\hline
\end{tabular}

Table C. Comparison of observed and calculated lifetimes. T96: Träbert et al. [9] (observed); F83: Fawcett [16]; C86: Christensen et al. [18]; S00: Safronova et al. [25]; FF06: Froese Fischer et al. [21]; K04: Karpuskiene et al. [27]; T05: Tayal [24].

\begin{tabular}{|c|c|c|c|c|c|c|c|c|c|c|c|c|}
\hline Transition & FAC & SST & F 83 & $\mathrm{C} 86$ & $\mathrm{~T} 86$ & T05 & ZFF01 & A 10 & T98 & FF06 & K04 & $\mathrm{K} 10$ \\
\hline $3-4$ & $4.380-1$ & $4.564-1$ & & & & & 4.539-1 & & $4.570-1$ & $4.564-1$ & & $4.539-1$ \\
\hline $5-6$ & $6.475+8$ & $7.360+8$ & $7.315+8$ & $9.890+7$ & $7.160+8$ & & & & & $7.168+8$ & $7.250+8$ & \\
\hline $1-3$ & $2.449+6$ & $2.636+6$ & & $6.050+5$ & & & $2.819+6$ & $2.805+6$ & $2.840+6$ & $2.903+6$ & & \\
\hline $1-4$ & $4.834-1$ & $5.059-1$ & & & & & $5.055-1$ & $5.060-1$ & $5.100-1$ & $4.965-1$ & & $4.902-1$ \\
\hline $4-6$ & $2.287+8$ & $2.600+8$ & & $2.500+8$ & & & & & & $3.139+8$ & $2.880+8$ & \\
\hline $3-6$ & $1.108+8$ & $1.242+8$ & & $9.890+7$ & & & & & & $1.493+8$ & $1.340+8$ & \\
\hline $4-8$ & $3.839+9$ & $3.775+9$ & $3.946+9$ & $3.910+9$ & $4.030+9$ & $3.795+9$ & & & & $3.635+9$ & $3.640+9$ & \\
\hline $3-8$ & $2.431+9$ & $2.389+9$ & $2.420+9$ & $2.430+9$ & $2.520+9$ & $2.400+9$ & & & & $2.302+9$ & $2.300+9$ & \\
\hline $4-9$ & $7.045+9$ & $6.899+9$ & $6.870+9$ & $7.150+9$ & $7.350+9$ & $7.001+9$ & & & & $6.582+9$ & $6.590+9$ & \\
\hline $2-8$ & $3.315+9$ & $3.262+9$ & $3.334+9$ & $3.310+9$ & $3.430+9$ & $3.277+9$ & & & & $3.144+9$ & $3.130+9$ & \\
\hline $3-9$ & $2.434+9$ & $2.386+9$ & $2.366+9$ & $2.450+9$ & $2.530+9$ & $2.428+9$ & & & & $2.268+9$ & $2.270+9$ & \\
\hline $1-5$ & $1.138+10$ & $1.094+10$ & $1.114+10$ & $1.140+10$ & $1.170+10$ & $1.086+10$ & $1.060+10$ & $1.061+10$ & & $1.053+10$ & & \\
\hline 5-14 & $2.448+10$ & $2.382+10$ & $2.324+10$ & $2.510+10$ & $2.690+10$ & $2.468+10$ & & & & $2.325+10$ & $2.320+10$ & \\
\hline $4-13$ & $1.310+10$ & $1.294+10$ & $1.299+10$ & $1.370+10$ & $1.460+10$ & $1.316+10$ & & & & $1.284+10$ & $1.280+10$ & \\
\hline $3-11$ & $5.622+9$ & $5.557+9$ & $5.561+9$ & $5.840+9$ & $6.250+9$ & $5.653+9$ & & & & $5.507+9$ & $5.480+9$ & \\
\hline $3-12$ & $1.015+10$ & $1.002+10$ & $1.012+10$ & $1.012+10$ & $1.050+10$ & $1.130+10$ & $1.019+10$ & & & $9.937+9$ & $9.880+9$ & \\
\hline $2-11$ & $7.639+9$ & $7.534+9$ & $7.552+9$ & $7.890+9$ & $8.460+9$ & $7.665+9$ & & & & $7.481+9$ & $7.420+9$ & \\
\hline
\end{tabular}

Table D. Comparison of A values for transitions within the $n=3$ complex giving rise to observable lines. FAC: this work, FAC calculation; SST: this work, SUPERSTRUCTURE calculation; F83: Fawcett [16]; C86: Christensen et al. [18]; T86: Tayal [19]; T05: Tayal [24]; ZFF01: Zou \& Froese Fischer [23]; A10: Andersson et al. [32]; FF06: Froese Fischer et al. [21]; K04: Karpuskiene et al. [27]; K10: Kang et al. [31]. 


\begin{tabular}{lccccccc} 
Transition & FAC & SST & A88 & C86 & K04 & T05 & FF06 \\
\hline & & & & & & & \\
$4-27$ & $3.840+10$ & $3.607+10$ & $3.720+10$ & $3.490+10$ & $3.720+10$ & $3.673+10$ & $3.801+10$ \\
$5-28$ & $4.400+10$ & $4.258+10$ & $4.139+10$ & $4.100+10$ & $4.180+10$ & $4.233+10$ & $4.245+10$ \\
$1-35$ & $3.701+10$ & $3.394+10$ & $3.433+10$ & & $3.750+10$ & $3.385+10$ & $3.536+10$ \\
$6-35$ & $1.081+10$ & $9.785+9$ & $9.679+9$ & & $9.080+9$ & $1.037+10$ & $8.866+9$ \\
$13-33$ & $1.190+10$ & $1.117+10$ & $1.094+10$ & & $1.090+10$ & $1.072+10$ & $1.103+10$ \\
$4-44$ & $4.759+10$ & $4.304+10$ & & & $4.630+10$ & $4.453+10$ & $4.571+10$ \\
$5-45$ & $2.202+10$ & $2.040+10$ & & & $2.170+10$ & $2.261+10$ & $2.153+10$ \\
$11-49$ & $1.055+11$ & $1.034+11$ & & & $1.020+11$ & & $1.002+11$ \\
$12-50$ & $1.117+11$ & $1.094+11$ & & & $1.080+11$ & & $1.061+11$ \\
$13-51$ & $1.257+11$ & $1.232+11$ & & & $1.210+11$ & & $1.193+11$ \\
$6-53$ & $2.724+10$ & $2.509+10$ & & & $2.350+10$ & $2.885+10$ & $2.459+10$ \\
$14-53$ & $9.092+10$ & $9.180+10$ & & & $8.960+10$ & $9.007+10$ & $8.669+10$ \\
& & & & & & & \\
\hline
\end{tabular}

Table E. Comparison of A values for transitions within the $n=4$ complex giving rise to the strongest lines. FAC: this work, FAC calculation; SST: this work, SUPERSTRUCTURE calculation; A88: Aashamar [26]; C86: Christensen et al. [18]; K04: Karpuskiene et al. [27]; T05: Tayal [24]; FF06: Froese Fischer et al. [21]

exception of Christensen et al. [18] which provide significant differences from all other calculations; such differences are likely to affect level population calculations. Differences in A values for allowed transitions are within $10 \%$ for all calculations, except in a few cases where our FAC calculations are lower than Froese Fischer et al. [21] and Karpuskiene et al. [27] by 20-30\%, while the SST results agree better with the more recent earlier calculations. It is worth noting that Christensen et al. [18] underestimate by a factor 7 the $\mathrm{A}$ value for transition 5-6, giving rise to the line at $821 \AA$ observed by SUMER in the solar spectrum (Curdt et al. [7]); a factor 4 difference also separates the Christensen et al. [18] A value for the important 1-3 transition (giving rise to the observed $691 \AA$ spectral line) from all other results. These differences seem to indicate some problem in this data set. Table E reports the comparison between the A values for $n=4 \rightarrow n=3$ transitions giving rise to the strongest lines, and shows that all calculations agree to within $10 \%$.

The only calculations of collision strengths we are aware of were made by Christensen et al. ([18] - C85), and Maxwellian-averaged collision strengths were provided by Pradhan [39]. They used the Distorted Wave approximation but tried to compensate in an approximate way for resonance excitation, while in the present work no effort has been made to account for resonances. The comparison shows very good agreement for all the allowed transitions within the C85 model, as well as for several intercombination and forbidden transitions. However, most of the forbidden transitions show significant differences from the present results: sometimes the differences consist of larger C85 collision strengths at the lowest incident electron energies, as expected from the neglect of resonances in the present calculation, but in many cases differences consist in constant shifts at all energies. In many other cases C85 collision strengths are lower than ours. Differences are particularly important for the 1-3 intercombination transition, giving rise to the observed $691.4 \AA$ line, and for all transitions between the ground and the $3 p^{2}$ transitions. These differences are likely to have significant effects on the overall level population and line intensity calculations.

\section{Level Populations and Relative Line Intensities}

In the absence of absorption of solar blackbody radiation and proton excitation, the level populations are obtained by solving the equations

$$
\frac{d N_{i}}{d t}=-N_{e} N_{i}\left(\sum_{j>i} C_{i j}^{e}+\sum_{j<i} C_{i j}^{d}\right)+\sum_{j>i} N_{j} A_{j i}-N_{i} \sum_{j<i} A_{i j}+N_{e}\left(\sum_{j>i} N_{j} C_{j i}^{d}+\sum_{j<i} N_{j} C_{j i}^{e}\right)
$$




$$
\frac{d N_{i}}{d t}=0 \text { for steady state }
$$

where $N_{j}$ is the number density of level $j, N_{e}$ is the electron density, and $A_{j i}\left(\mathrm{~s}^{-1}\right)$ is the spontaneous radiative transition rate from level $j$ to level $i$. The equations have been solved at electron densities of $\log N_{e}\left(\mathrm{~cm}^{-3}\right)=8$, $9,10,11,12,13$, and 14 . Level populations and line intensities of various lines at $\log \mathrm{T}_{e}=5.8$, the temperature of maximum abundance of Ca IX (Bryans et al. [40]), are given in Tables IV and V, respectively. The intensity of an optically thin line for a radiative transition from level $j$ to level $i$ is given by $I_{j i}=n_{j} A_{j i}$ in photon units. Line intensities have been calculated and the values for the brightest lines are listed in Table V as a function of density.

Photoexcitation from background black-body radiation has negligible effects on Ca IX level populations at densities larger than $\log N_{e}\left(\mathrm{~cm}^{-3}\right)=8$, for black-body temperatures of $6000 \mathrm{~K}$, typical of the Sun and solartype stars, with a dilution factor of 0.35 , typical of the innermost regions of stellar coronae. Below that density, the $3 s 3 p{ }^{3} \mathrm{P}$ level populations are affected by photoexcitation and this in turn alters the populations of many excited levels that provide lines useful for plasma diagnostics. In Tables IV and V we have omitted photoexcitation at all densities, but recommend the inclusion of photoexcitation in the analysis of Ca IX lines emitted by low density plasmas.

\section{Comparison with observations}

Ca IX lines can be used in several ways to measure the physical properties of the emitting plasma. Electron density measurements at densities typical of the lower corona or upper transition region (e.g. $\log N_{e} \simeq 8.0$ at around $\log T \simeq 5.7-6.0$ ) or more are possible only with the intensity ratio between the forbidden and the intercombination lines from the first excited configuration to the ground configuration: $676.29 \AA / 691.42 \AA$, or ${ }^{3} \mathrm{P}_{2} \rightarrow{ }^{1} \mathrm{~S}_{0} /{ }^{3} \mathrm{P}_{1} \rightarrow{ }^{1} \mathrm{~S}_{0}$. Such dependence is due to the metastable nature of the ${ }^{3} \mathrm{P}_{2}$ level which remains populated at higher densities than the ${ }^{3} \mathrm{P}_{1}$ level, which has a larger $\mathrm{A}$ value to the ground. Comparison of the predicted ratio with the SUMER off-limb observations of Curdt et al. [7] yields a density of $\log N_{e}=$ $8.20 \pm 0.10$ and $7.80 \pm 0.10$ in quiet Sun and coronal hole areas, respectively, after taking into account the effects of photoexcitation. These values are consistent with earlier estimates. Several other intensity ratios are sensitive to the electron densities for $\log N_{e}<8.0$.

Temperature-sensitive line intensity ratios can be formed using lines emitted by configurations with different $n$, because the excitation energies of the levels are different. However, the observable $n=3$ lines are emitted at wavelengths longer than $200 \AA$ so that they are far from $n=4,5$ lines: this makes it difficult for a single instrument to combine one $n=3$ line with one $n=4,5$ line into a temperature-sensitive line intensity ratio. On the contrary, several line pairs emitted by $n=4$ and $n=5$ levels can be found at around $200 \AA$ (e.g. 14-53/5-28: $178.57 \AA / 201.85 \AA$, potentially observable by the Hinode/EIS spectrometer, Culhane et al. [41]),

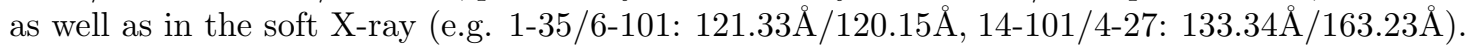

The Ca IX spectrum is dominated by the strong singlet $3 s^{2}{ }^{1} \mathrm{~S}_{0}-3 s 3 p{ }^{1} \mathrm{P}_{1}$ at $466.24 \AA$. This line is very close to the even brighter Ne VII line at $465.22 \AA$, which is formed at similar temperatures (Bryans et al. [40]). Both lines are sensitive to the electron density in a similar way, so that their ratio can be used to determine the relative $\mathrm{Ca} / \mathrm{Ne}$ abundance in solar coronal holes and quiet Sun. Such a ratio is important to invesigate the abundance anomaly in the solar corona known as the FIP effect (Feldman \& Laming [42] and references therein). Also, the $466.24 \AA$ line can be used to estimate the total amount of emitting plasma and in Differential Emission Measure studies. 


\section{Conclusions}

In the present work we have calculated a complete set of energy levels, oscillator strengths, A values and collision strengths for 33 configurations of Ca IX, corresponding to all 283 fine structure levels in the $n=$ 3, 4,5 complexes. Energy levels and radiative data were also calculated using SUPERSTRUCTURE. We compared the radiative rates and level lifetimes we obtained with results from other calculations for a few key transitions, and with laboratory measurements, where available. To our knowledge, this is the first complete dataset that allows to calculate level populations and line intensities for $n=4,5$ and many $n=3$ configurations. Our results allow the calculation of line emissivities of lines in the visible, UV and soft $\mathrm{X}$-ray range, many of which for the first time, and to measure plasma parameters using line intensities and intensity ratios. We discuss the diagnostic applications of our calculations. The main limitation of the present calculations is the neglect of resonant excitation in the collision strengths; this process is expected to provide significant contributions and thus we recommend that future calculations be carried out to include it.

The present results will be distributed in the next release of the CHIANTI database (Dere et al. [43], Landi et al. [44]). We hope that the present results will be useful for the analysis of laboratory and astrophysical spectra.

\section{Acknowledgements}

The work of E. Landi is supported by NASA grants NNX10AQ58G and NNX11AC20G, as well as NSF grant AGS-1154443. Calculations were carried out using the Discover computer of the NASA Center for Computation Science. 


\section{References}

1. W.E. Behring, L. Cohen, G.A. Doschek, U. Feldman, Astrophys. J. 203, 521 (1976)

2. K.P. Dere, Astrophys. J. 221, 1062 (1978)

3. J.E. Vernazza, E.M. Reeves, Astrophys. J. Suppl. Ser. 37, 485 (1978)

4. U. Feldman, J.D. Purcell, B.C. Dohne, An atlas of Ultraviolet Spectroheliograms from 170 to $625 \AA$, Ref 91-4100 (1987), Washington, DC Naval Research Laboratory

5. W. Curdt, U. Feldman, J.M. Laming, et al., Astron. Astrophys. Suppl. Ser. 126, 281 (1997)

6. W. Curdt, P. Brekke, U. Feldman, et al., Astron. Astrophys. 375, 591 (2001)

7. W. Curdt, E. Landi, U. Feldman, Astron. Astrophys. 427, 1045 (2004)

8. S. Parenti, J.-C. Vial, P. Lemaire, Astron. Astrophys. 443, 679 (2005)

9. E. Träbert, E.H. Pinnington, J.A. Kernahan, et al., J. Physics B 29, 2647 (1996)

10. W.L. Parker, L.W. Phillips, Phys. Rev. 57, 140 (1940)

11. B.C. Fawcett, J. Physics B 3, 1732 (1970)

12. J.O. Ekberg, Phys. Scr. 4, 101 (1971)

13. U. Litzen, and A. Redfors, Phys. Scr. 36, 895 (1987)

14. A. Redfors, Phys. Scr. 38, 702 (1988)

15. S.S. Churilov, V.E. Levashov, and J.F. Wyart, Phys. Scr. 40, 625 (1989)

16. B.C. Fawcett, Atomic Data and Nuclear Data Tables 28, 579 (1983)

17. S.O. Kastner, M. Swartz, A.K. Bhatia, and J. Lapides, J. Opt. Soc. Am. 68, 1558 (1978)

18. R.B. Christensen, D.W. Norcross, and A.K. Pradhan, Phys. Rev. A 34, 4704 (1986)

19. S.S. Tayal, J. Phys. B 19, 3421 (1986)

20. M. Godefroid, Phys. Scr. 32, 125 (1985)

21. C. Froese Fischer, G. Tachiev, and A. Irimia, Atomic Data and Nuclear Data Tables 92, 607 (2006)

22. M. Stanek, L. Glowacki, and J. Migdalek, J. Phys. B 29, 2985 (1996)

23. Y. Zou and C. Froese Fischer, J. Phys. B 34, 915 (2001)

24. S.S. Tayal, Phys. Scripta 71, 627 (2005)

25. U.I. Safronova, W.R. Johnson, and H.G. Berry, Phys. Rev. A 61, 052503 (2000)

26. K. Aashamar, Phys. Scr. 37, 13 (1988)

27. R. Karpuskiene, P. Bogdanovich, and A. Udris, J. Phys. B 37, 2067 (2004)

28. J.P. Marques, F. Parente, and P. Indelicato, Atomic Data and Nuclear Data Tables 55, 157 (1993)

29. T. Brage, P.G. Judge, A. Aboussaïd, et al. Astrophys. J. 500, 507 (1998)

30. H. Kang, J. Li, C. Dong, et al., J. Phys. B 42, 195002 (2009) 
31. H. Kang, J. Li, C. Dong, et al., J. Phys. B 43, 095003 (2010)

32. M. Andersson, Y. Zou, R. Hutton, and T. Brage, J. Phys. B 43, 095001 (2010)

33. M.F. Gu, Astrophys. J. 582, 1241 (2003)

34. W. Eissner, M. Jones, H. Nussbaumer, Comput. Phys. Commun. 8, 270 (1974)

35. B. Edlen, and E. Boden, Phys. Scripta 14, 31 (1976)

36. B.C. Fawcett, J. Opt. Soc. Am 66, 632 (1976)

37. A. Burgess, J.A. Tully, Astron. Astrophys. 254, 436 (1992)

38. A.K. Bhatia, E. Landi, H.E. Mason, Atomic Data and Nuclear Data Tables 83, 71 (2003)

39. A.K. Pradhan, Atomic Data and Nuclear Data Tables 40, 335 (1988)

40. P. Bryans, E. Landi, D.W. Savin, Astrophys. J. 691, 1540 (2009)

41. J.L. Culhane, L.K. Harra, A.M. James, et al., Solar Physics 243, 19 (2007)

42. U. Feldman, J.M. Laming, Phys. Scripta 61, 222 (2000)

43. K.P. Dere, E. Landi, H.E. Mason, B.C. Monsignori Fossi, P.R. Young, Astron. Astrophys. Suppl. 125, 149 (1997)

44. E. Landi, G. Del Zanna, P.R. Young, et al., Astroph. J. 744, 99 (2012) 


\section{EXPLANATION OF TABLES}

Table I. Calculated and Experimental Energy Levels for Ca IX

$\begin{array}{ll}\text { Level } & \text { A number assigned to each level } \\ \text { Configuration } & \text { The configuration, with } 1 s^{2} 2 s^{2} 2 p^{6} \text { truncated } \\ \text { Term } & \text { The term designation of the level within the configuration } \\ \mathrm{E}_{\text {obs }} & \text { Measured energies, from laboratory and solar spectra (see Table A). } \\ \mathrm{E}_{\text {calc }} & \text { Calculated energy from FAC and SST, in } \mathrm{cm}^{-1} \\ \text { Source } & \text { Literature sources used to determine the observed energy } \\ & \text { (following Table A). }\end{array}$

Table II. Ca IX Oscillator Strengths, Radiative Decay Rates, and Collision Strengths for transitions involving the lowest four levels
Lower and Upper Level
The lower and upper levels, where the numbers refer to the Level listed in Table I
Oscillator Strength
Radiative Decay Rate
Collision Strength
gf, the (dimensionless) product of the statistical weight $g$ of the lower level and the absorption oscillator strength $\mathrm{f}$
The spontaneous radiative decay rate $\mathrm{A}_{j i}$ in units of $\mathrm{s}^{-1}$
The dimensionless electron impact collision strength $\Omega$
at the energy above threshold (in Ry) given in the table heading.
The lowest energy (E') changes depending on the lower level, as listed in Table B.

Table III. Ca IX Oscillator Strengths and Radiative Decay Rates for transitions from all other levels
Lower and Upper Level
The lower and upper levels, where the numbers refer to the Level listed in Table I
Oscillator Strength
$g f$, the (dimensionless) product of the statistical weight $g$ of the lower level and the absorption oscillator strength $f$
Radiative Decay Rate
The spontaneous radiative decay rate $\mathrm{A}_{j i}$ in units of $\mathrm{s}^{-1}$

Table IV. Ca IX Fractional Level Populations

Den. The electron density in $\mathrm{cm}^{-3}$, in $\log$ values

Key A number assigned to each level as given in Table I

Population The fractional level population $\mathrm{n}_{j}$ as a function of electron density for an electron temperature of $\log \mathrm{T}_{e}(\mathrm{~K})=5.8$ : the sum of all fractional level populations is defined as unity.

\section{Table V. Intensities of selected bright Ca IX lines}

Den. The electron density in $\mathrm{cm}^{-3}$, in log values

$\mathrm{j}$ and $\mathrm{i} \quad$ The upper and lower levels, where numbers refer to the Level listed in Table I

Wavelength The wavelengths in units of $\AA$ calculated from observed energies (when available) or theoretical energies.

Intensity The intensity $\left(\mathrm{n}_{j} \mathrm{~A}_{j i}\right.$ in units of photon/s) for the indicated density given in the table heading and for an electron temperature of $\log \mathrm{T}_{e}(\mathrm{~K})=5.8$. 
Table I. Ca IX experimental and theoretical energy levels.

Level Configuration Term $\quad \mathrm{E}_{o b s} \quad \mathrm{E}_{F A C} \quad \mathrm{E}_{S S T} \quad$ Source

\begin{tabular}{|c|c|c|c|c|c|c|}
\hline 1 & $3 s^{2}$ & ${ }^{1} \mathrm{~S}_{0}$ & 0 & 0 & 0 & \\
\hline 2 & $3 s 3 p$ & ${ }^{3} \mathrm{P}_{0}$ & 143127 & 142044 & 141897 & PP40, E71, LR87 \\
\hline 3 & $3 s 3 p$ & ${ }^{3} \mathrm{P}_{1}$ & 144630 & 143529 & 143420 & $\mathrm{C} 04$ \\
\hline 4 & $3 s 3 p$ & ${ }^{3} \mathrm{P}_{2}$ & 147866 & 146721 & 146692 & $\mathrm{C} 04$ \\
\hline 5 & $3 s 3 p$ & ${ }^{1} \mathrm{P}_{1}$ & 214482 & 217647 & 217812 & E71, LR87 \\
\hline 6 & $3 p^{2}$ & ${ }^{1} \mathrm{D}_{2}$ & 336253 & 335364 & 336180 & C04, E71, LR87 \\
\hline 7 & $3 p^{2}$ & ${ }^{3} \mathrm{P}_{0}$ & 339923 & 340352 & 340761 & PP40, E71, LR87 \\
\hline 8 & $3 p^{2}$ & ${ }^{3} \mathrm{P}_{1}$ & 341827 & 342319 & 342660 & PP40, E71, LR87 \\
\hline 9 & $3 p^{2}$ & ${ }^{3} \mathrm{P}_{2}$ & 345429 & 345772 & 346237 & PP40, E71, LR87 \\
\hline 10 & $3 p^{2}$ & ${ }^{1} \mathrm{~S}_{0}$ & 398895 & 402771 & 403589 & F70, LR87 \\
\hline 11 & $3 s 3 d$ & ${ }^{3} \mathrm{D}_{1}$ & 412020 & 412268 & 414706 & PP40, E71, LR87 \\
\hline 12 & $3 s 3 d$ & ${ }^{3} \mathrm{D}_{2}$ & 412154 & 412382 & 414905 & PP40, E71, LR87 \\
\hline 13 & $3 s 3 d$ & ${ }^{3} \mathrm{D}_{3}$ & 412356 & 412565 & 415219 & PP40, E71, LR87 \\
\hline 14 & $3 s 3 d$ & ${ }^{1} \mathrm{D}_{2}$ & 467633 & 472704 & 475158 & E71, LR87 \\
\hline 15 & $3 p 3 d$ & ${ }^{3} \mathrm{~F}_{2}$ & 563657 & 562509 & 565218 & F70, LR87 \\
\hline 16 & $3 p 3 d$ & ${ }^{3} \mathrm{~F}_{3}$ & 565705 & 564474 & 567309 & F70, LR87 \\
\hline 17 & $3 p 3 d$ & ${ }^{3} \mathrm{~F}_{4}$ & 568146 & 566911 & 569813 & F70, LR87 \\
\hline 18 & $3 p 3 d$ & ${ }^{1} \mathrm{D}_{2}$ & 571899 & 570907 & 573715 & F70, LR87 \\
\hline 19 & $3 p 3 d$ & ${ }^{3} \mathrm{P}_{2}$ & 598281 & 598952 & 601812 & LR87 \\
\hline 20 & $3 p 3 d$ & ${ }^{3} \mathrm{P}_{1}$ & 599315 & 600049 & 602735 & LR87 \\
\hline 21 & $3 p 3 d$ & ${ }^{3} \mathrm{P}_{0}$ & 601096 & 601764 & 604466 & LR87 \\
\hline 22 & $3 p 3 d$ & ${ }^{3} \mathrm{D}_{1}$ & 602058 & 602748 & 605445 & LR87 \\
\hline 23 & $3 p 3 d$ & ${ }^{3} \mathrm{D}_{2}$ & 602613 & 603272 & 606043 & LR87 \\
\hline 24 & $3 p 3 d$ & ${ }^{3} \mathrm{D}_{3}$ & 602657 & 603354 & 606222 & F70, LR87 \\
\hline 25 & $3 p 3 d$ & ${ }^{1} \mathrm{~F}_{3}$ & 644812 & 650100 & 653146 & LR87 \\
\hline 26 & $3 p 3 d$ & ${ }^{1} \mathrm{P}_{1}$ & 654169 & 660181 & 663284 & LR87 \\
\hline 27 & $3 s 4 s$ & ${ }^{3} \mathrm{~S}_{1}$ & 760494 & 758754 & 759002 & PP40, E71, EB76 \\
\hline 28 & $3 s 4 s$ & ${ }^{1} \mathrm{~S}_{0}$ & 774473 & 774337 & 774010 & $\mathrm{E} 71, \mathrm{~EB} 76$ \\
\hline 29 & $3 s 4 p$ & ${ }^{3} \mathrm{P}_{0}$ & & 829142 & 828745 & \\
\hline 30 & $3 s 4 p$ & ${ }^{3} \mathrm{P}_{1}$ & & 829426 & 829011 & \\
\hline 31 & $3 d^{2}$ & ${ }^{3} \mathrm{~F}_{2}$ & 831162 & 830359 & 835666 & LR87, C89 \\
\hline 32 & $3 d^{2}$ & ${ }^{3} \mathrm{~F}_{3}$ & 831385 & 830485 & 835985 & LR87, R88, C89 \\
\hline 33 & $3 s 4 p$ & ${ }^{3} \mathrm{P}_{2}$ & & 830741 & 830263 & \\
\hline 34 & $3 d^{2}$ & ${ }^{3} \mathrm{~F}_{4}$ & 831636 & 830772 & 836389 & LR87, R88, C89 \\
\hline 35 & $3 s 4 p$ & ${ }^{1} \mathrm{P}_{1}$ & 832314 & 831823 & 831256 & E71, EB76 \\
\hline 36 & $3 d^{2}$ & ${ }^{1} \mathrm{G}_{4}$ & 848606 & 850656 & 856244 & R88, C89 \\
\hline 37 & $3 d^{2}$ & ${ }^{1} \mathrm{D}_{2}$ & 849262 & 850815 & 856244 & $\mathrm{R} 88, \mathrm{C} 89$ \\
\hline 38 & $3 d^{2}$ & ${ }^{3} \mathrm{P}_{1}$ & 853097 & 853692 & 859105 & $\mathrm{C} 89$ \\
\hline 39 & $3 d^{2}$ & ${ }^{3} \mathrm{P}_{0}$ & 853028 & 853698 & 858988 & C89 \\
\hline 40 & $3 d^{2}$ & ${ }^{3} \mathrm{P}_{2}$ & 853229 & 853834 & 859105 & $\mathrm{C} 89$ \\
\hline 41 & $3 d^{2}$ & ${ }^{1} \mathrm{~S}_{0}$ & 900061 & 908334 & 913820 & $\mathrm{C} 89$ \\
\hline 42 & $3 s 4 d$ & ${ }^{3} \mathrm{D}_{1}$ & 917157 & 916261 & 916286 & PP40, E71, EB76 \\
\hline 43 & $3 s 4 d$ & ${ }^{3} \mathrm{D}_{2}$ & 917264 & 916388 & 916438 & PP40, E71, EB76 \\
\hline 44 & $3 s 4 d$ & ${ }^{3} \mathrm{D}_{3}$ & 917481 & 916578 & 916666 & E71, EB76 \\
\hline 45 & $3 s 4 d$ & ${ }^{1} \mathrm{D}_{2}$ & 921914 & 918207 & 918272 & E71, EB76 \\
\hline 46 & $3 p 4 s$ & ${ }^{3} \mathrm{P}_{0}$ & 941430 & 941132 & 940500 & E71, EB76 \\
\hline 47 & $3 p 4 s$ & ${ }^{3} \mathrm{P}_{1}$ & 942617 & 942369 & 941714 & E71, EB76 \\
\hline 48 & $3 p 4 s$ & ${ }^{3} \mathrm{P}_{2}$ & 946336 & 945943 & 945235 & E71, EB76 \\
\hline
\end{tabular}


Table I. Ca IX experimental and theoretical energy levels.

\begin{tabular}{|c|c|c|c|c|c|c|}
\hline Level & Configuration & Term & $\mathrm{E}_{o b s}$ & $\mathrm{E}_{\text {calc }}$ & $\mathrm{E}_{S S T}$ & Source \\
\hline 49 & $3 s 4 f$ & ${ }^{3} \mathrm{~F}_{2}$ & 954508 & 954150 & 953687 & PP40, E71, EB76 \\
\hline 50 & $3 s 4 f$ & ${ }^{3} \mathrm{~F}_{3}$ & 954531 & 954175 & 953724 & PP40, E71, EB76 \\
\hline 51 & $3 s 4 f$ & ${ }^{3} \mathrm{~F}_{4}$ & 954563 & 954213 & 953774 & PP40, E71, EB76 \\
\hline 52 & $3 p 4 s$ & ${ }^{1} \mathrm{P}_{1}$ & & 955767 & 954659 & \\
\hline 53 & $3 s 4 f$ & ${ }^{1} \mathrm{~F}_{3}$ & 963058 & 964762 & 964248 & E71, EB76 \\
\hline 54 & $3 p 4 p$ & ${ }^{1} \mathrm{P}_{1}$ & & 997630 & 996913 & \\
\hline 55 & $3 p 4 p$ & ${ }^{3} \mathrm{D}_{1}$ & & 1003558 & 1002857 & \\
\hline 56 & $3 p 4 p$ & ${ }^{3} \mathrm{D}_{2}$ & & 1004842 & 1004154 & \\
\hline 57 & $3 p 4 p$ & ${ }^{3} \mathrm{D}_{3}$ & & 1008148 & 1007366 & \\
\hline 58 & $3 p 4 p$ & ${ }^{3} \mathrm{P}_{0}$ & 1010847 & 1012260 & 1010432 & PP40, E71 \\
\hline 59 & $3 p 4 p$ & ${ }^{3} \mathrm{P}_{1}$ & 1011980 & 1012962 & 1011405 & PP40, E71 \\
\hline 60 & $3 p 4 p$ & ${ }^{3} \mathrm{~S}_{1}$ & 1015597 & 1015457 & 1014617 & PP40, E71 \\
\hline 61 & $3 p 4 p$ & ${ }^{3} \mathrm{P}_{2}$ & 1014350 & 1015620 & 1013775 & PP40, E71 \\
\hline 62 & $3 p 4 p$ & ${ }^{1} \mathrm{D}_{2}$ & & 1027107 & 1025219 & \\
\hline 63 & $3 p 4 p$ & ${ }^{1} \mathrm{~S}_{0}$ & & 1042482 & 1041137 & \\
\hline 64 & $3 s 5 s$ & ${ }^{3} \mathrm{~S}_{1}$ & 1068775 & 1066981 & 1067544 & PP40, E71 \\
\hline 65 & $3 s 5 s$ & ${ }^{1} \mathrm{~S}_{0}$ & 1075141 & 1077817 & 1076503 & PP40 \\
\hline 66 & $3 p 4 d$ & ${ }^{3} \mathrm{D}_{1}$ & & 1090515 & 1090093 & \\
\hline 67 & $3 p 4 d$ & ${ }^{3} \mathrm{D}_{2}$ & & 1090536 & 1090127 & \\
\hline 68 & $3 p 4 d$ & ${ }^{1} \mathrm{D}_{2}$ & & 1091499 & 1090959 & \\
\hline 69 & $3 p 4 d$ & ${ }^{3} \mathrm{D}_{3}$ & & 1091744 & 1091326 & \\
\hline 70 & $3 s 5 p$ & ${ }^{3} \mathrm{P}_{1}$ & & 1095263 & 1095609 & \\
\hline 71 & $3 s 5 p$ & ${ }^{3} \mathrm{P}_{0}$ & & 1095350 & 1095761 & \\
\hline 72 & $3 s 5 p$ & ${ }^{3} \mathrm{P}_{2}$ & & 1095618 & 1096511 & \\
\hline 73 & $3 s 5 p$ & ${ }^{1} \mathrm{P}_{1}$ & 1097580 & 1096249 & 1096402 & E71, EB76 \\
\hline 74 & $3 p 4 d$ & ${ }^{3} \mathrm{~F}_{2}$ & & 1097086 & 1096402 & \\
\hline 75 & $3 p 4 d$ & ${ }^{3} \mathrm{~F}_{3}$ & & 1097505 & 1096935 & \\
\hline 76 & $3 p 4 d$ & ${ }^{3} \mathrm{~F}_{4}$ & & 1100116 & 1099419 & \\
\hline 77 & $3 p 4 d$ & ${ }^{1} \mathrm{~F}_{3}$ & & 1100553 & 1100038 & \\
\hline 78 & $3 p 4 d$ & ${ }^{3} \mathrm{P}_{2}$ & & 1109709 & 1108520 & \\
\hline 79 & $3 p 4 d$ & ${ }^{3} \mathrm{P}_{1}$ & & 1110381 & 1109084 & \\
\hline 80 & $3 p 4 d$ & ${ }^{3} \mathrm{P}_{0}$ & & 1110931 & 1109598 & \\
\hline 81 & $3 p 4 d$ & ${ }^{1} \mathrm{P}_{1}$ & & 1116248 & 1114914 & \\
\hline 82 & $3 p 4 f$ & ${ }^{3} \mathrm{G}_{3}$ & & 1122638 & 1121719 & \\
\hline 83 & $3 p 4 f$ & ${ }^{3} \mathrm{G}_{4}$ & 1124517 & 1124842 & 1123786 & F76 \\
\hline 84 & $3 p 4 f$ & ${ }^{1} \mathrm{~F}_{3}$ & 1125621 & 1125547 & 1124456 & F76 \\
\hline 85 & $3 p 4 f$ & ${ }^{3} \mathrm{G}_{5}$ & 1126958 & 1127168 & 1126205 & F76 \\
\hline 86 & $3 p 4 f$ & ${ }^{3} \mathrm{~F}_{2}$ & & 1128676 & 1127649 & \\
\hline 87 & $3 p 4 f$ & ${ }^{3} \mathrm{~F}_{3}$ & & 1129774 & 1128620 & \\
\hline 88 & $3 p 4 f$ & ${ }^{3} \mathrm{~F}_{4}$ & 1130442 & 1130495 & 1129394 & F76 \\
\hline 89 & $3 p 4 f$ & ${ }^{3} \mathrm{D}_{3}$ & & 1135437 & 1135093 & \\
\hline 90 & $3 p 4 f$ & ${ }^{3} \mathrm{D}_{2}$ & 1135141 & 1135982 & 1135696 & PP40 \\
\hline 91 & $3 p 4 f$ & ${ }^{3} \mathrm{D}_{1}$ & 1136718 & 1136360 & 1136109 & PP40 \\
\hline 92 & $3 s 5 d$ & ${ }^{1} \mathrm{D}_{2}$ & 1139804 & 1138834 & 1139124 & PP40, E71 \\
\hline 93 & $3 p 4 f$ & ${ }^{1} \mathrm{G}_{4}$ & 1139005 & 1141048 & 1140337 & F76 \\
\hline 94 & $3 s 5 d$ & ${ }^{3} \mathrm{D}_{3}$ & 1145711 & 1145367 & 1144806 & E71, EB76 \\
\hline 95 & $3 s 5 d$ & ${ }^{3} \mathrm{D}_{2}$ & 1145220 & 1145924 & 1145263 & E71, EB76 \\
\hline 96 & $3 s 5 d$ & ${ }^{3} \mathrm{D}_{1}$ & 1145181 & 1146491 & 1145711 & E71 \\
\hline
\end{tabular}


Table I. Ca IX experimental and theoretical energy levels.

\begin{tabular}{|c|c|c|c|c|c|c|}
\hline Level & Configuration & Term & $\mathrm{E}_{o b s}$ & $\mathrm{E}_{\text {calc }}$ & $\mathrm{E}_{S S T}$ & Source \\
\hline 97 & $3 p 4 f$ & ${ }^{1} \mathrm{D}_{2}$ & & 1154812 & 1153745 & \\
\hline 98 & $3 s 5 f$ & ${ }^{3} \mathrm{~F}_{2}$ & 1159591 & 1158017 & 1158415 & E71, EB76 \\
\hline 99 & $3 s 5 f$ & ${ }^{3} \mathrm{~F}_{3}$ & 1159633 & 1158031 & 1158433 & E71, EB76 \\
\hline 100 & $3 s 5 f$ & ${ }^{3} \mathrm{~F}_{4}$ & 1159628 & 1158050 & 1158458 & E71, EB76 \\
\hline 101 & $3 s 5 f$ & ${ }^{1} \mathrm{~F}_{3}$ & 1160425 & 1162188 & 1162356 & E71 \\
\hline 102 & $3 s 5 g$ & ${ }^{3} \mathrm{G}_{3}$ & & 1166171 & 1166589 & \\
\hline 103 & $3 s 5 g$ & ${ }^{3} \mathrm{G}_{4}$ & & 1166346 & 1166744 & \\
\hline 104 & $3 s 5 g$ & ${ }^{3} \mathrm{G}_{5}$ & & 1166569 & 1166962 & \\
\hline 105 & $3 s 5 g$ & ${ }^{1} \mathrm{G}_{4}$ & & 1174348 & 1174492 & \\
\hline 106 & $3 d 4 s$ & ${ }^{3} \mathrm{D}_{1}$ & & 1188245 & 1189040 & \\
\hline 107 & $3 d 4 s$ & ${ }^{3} \mathrm{D}_{2}$ & & 1188381 & 1189261 & \\
\hline 108 & $3 d 4 s$ & ${ }^{3} \mathrm{D}_{3}$ & & 1188602 & 1189618 & \\
\hline 109 & $3 d 4 s$ & ${ }^{1} \mathrm{D}_{2}$ & & 1196690 & 1197469 & \\
\hline 110 & $3 p 5 s$ & ${ }^{3} \mathrm{P}_{0}$ & & 1242001 & 1242011 & \\
\hline 111 & $3 p 5 s$ & ${ }^{3} \mathrm{P}_{1}$ & & 1242374 & 1242365 & \\
\hline 112 & $3 d 4 p$ & ${ }^{1} \mathrm{D}_{2}$ & & 1245646 & 1246690 & \\
\hline 113 & $3 p 5 s$ & ${ }^{3} \mathrm{P}_{2}$ & & 1246316 & 1246147 & \\
\hline 114 & $3 p 5 s$ & ${ }^{1} \mathrm{P}_{1}$ & & 1247609 & 1247428 & \\
\hline 115 & $3 d 4 p$ & ${ }^{3} \mathrm{D}_{1}$ & & 1249493 & 1250005 & \\
\hline 116 & $3 d 4 p$ & ${ }^{3} \mathrm{D}_{2}$ & & 1249706 & 1250324 & \\
\hline 117 & $3 d 4 p$ & ${ }^{3} \mathrm{D}_{3}$ & & 1250103 & 1250832 & \\
\hline 118 & $3 d 4 p$ & ${ }^{3} \mathrm{~F}_{2}$ & & 1251557 & 1252275 & \\
\hline 119 & $3 d 4 p$ & ${ }^{3} \mathrm{~F}_{3}$ & & 1252172 & 1252958 & \\
\hline 120 & $3 d 4 p$ & ${ }^{3} \mathrm{~F}_{4}$ & & 1252992 & 1253873 & \\
\hline 121 & $3 d 4 p$ & ${ }^{3} \mathrm{P}_{0}$ & & 1262128 & 1262259 & \\
\hline 122 & $3 d 4 p$ & ${ }^{3} \mathrm{P}_{1}$ & & 1262151 & 1262297 & \\
\hline 123 & $3 d 4 p$ & ${ }^{3} \mathrm{P}_{2}$ & & 1262380 & 1262555 & \\
\hline 124 & $3 d 4 p$ & ${ }^{1} \mathrm{~F}_{3}$ & & 1265416 & 1265937 & \\
\hline 125 & $3 p 5 p$ & ${ }^{1} \mathrm{P}_{1}$ & & 1271833 & 1271658 & \\
\hline 126 & $3 p 5 p$ & ${ }^{3} \mathrm{D}_{1}$ & & 1274626 & 1274077 & \\
\hline 127 & $3 d 4 p$ & ${ }^{1} \mathrm{P}_{1}$ & & 1274833 & 1274748 & \\
\hline 128 & $3 p 5 p$ & ${ }^{3} \mathrm{D}_{2}$ & & 1275269 & 1274735 & \\
\hline 129 & $3 p 5 p$ & ${ }^{3} \mathrm{P}_{0}$ & & 1276692 & 1275334 & \\
\hline 130 & $3 p 5 p$ & ${ }^{3} \mathrm{P}_{1}$ & & 1278295 & 1277286 & \\
\hline 131 & $3 p 5 p$ & ${ }^{3} \mathrm{D}_{3}$ & & 1278756 & 1278246 & \\
\hline 132 & $3 p 5 p$ & ${ }^{3} \mathrm{P}_{2}$ & & 1280161 & 1278882 & \\
\hline 133 & $3 p 5 p$ & ${ }^{3} \mathrm{~S}_{1}$ & & 1281654 & 1280982 & \\
\hline 134 & $3 p 5 p$ & ${ }^{1} \mathrm{D}_{2}$ & & 1286498 & 1284416 & \\
\hline 135 & $3 p 5 p$ & ${ }^{1} \mathrm{~S}_{0}$ & & 1291888 & 1289460 & \\
\hline 136 & $3 p 5 d$ & ${ }^{1} \mathrm{D}_{2}$ & & 1315361 & 1314771 & \\
\hline 137 & $3 p 5 d$ & ${ }^{3} \mathrm{D}_{2}$ & & 1316322 & 1315574 & \\
\hline 138 & $3 p 5 d$ & ${ }^{3} \mathrm{D}_{1}$ & & 1316811 & 1315987 & \\
\hline 139 & $3 p 5 d$ & ${ }^{3} \mathrm{D}_{3}$ & & 1317061 & 1316290 & \\
\hline 140 & $3 p 5 d$ & ${ }^{3} \mathrm{~F}_{2}$ & & 1319896 & 1319123 & \\
\hline 141 & $3 p 5 d$ & ${ }^{3} \mathrm{~F}_{3}$ & & 1320603 & 1319781 & \\
\hline 142 & $3 p 5 d$ & ${ }^{3} \mathrm{~F}_{4}$ & & 1321658 & 1320817 & \\
\hline 143 & $3 p 5 d$ & ${ }^{3} \mathrm{P}_{2}$ & & 1323554 & 1322299 & \\
\hline 144 & $3 d 4 d$ & ${ }^{1} \mathrm{~F}_{3}$ & & 1324414 & 1326293 & \\
\hline
\end{tabular}


Table I. Ca IX experimental and theoretical energy levels.

\begin{tabular}{|c|c|c|c|c|c|c|}
\hline Level & Configuration & Term & $\mathrm{E}_{o b s}$ & $\mathrm{E}_{\text {calc }}$ & $\mathrm{E}_{S S T}$ & Source \\
\hline 145 & $3 p 5 d$ & ${ }^{3} \mathrm{P}_{1}$ & & 1324456 & 1323083 & \\
\hline 146 & $3 p 5 d$ & ${ }^{3} \mathrm{P}_{0}$ & & 1325046 & 1323637 & \\
\hline 147 & $3 p 5 d$ & ${ }^{1} \mathrm{~F}_{3}$ & & 1326871 & 1325158 & \\
\hline 148 & $3 d 4 d$ & ${ }^{3} \mathrm{D}_{1}$ & & 1328477 & 1330214 & \\
\hline 149 & $3 d 4 d$ & ${ }^{3} \mathrm{D}_{2}$ & & 1328538 & 1330408 & \\
\hline 150 & $3 d 4 d$ & ${ }^{3} \mathrm{D}_{3}$ & & 1328745 & 1330663 & \\
\hline 151 & $3 d 4 d$ & ${ }^{3} \mathrm{G}_{3}$ & & 1329606 & 1330259 & \\
\hline 152 & $3 d 4 d$ & ${ }^{3} \mathrm{G}_{4}$ & & 1329982 & 1331128 & \\
\hline 153 & $3 d 4 d$ & ${ }^{3} \mathrm{G}_{5}$ & & 1330488 & 1332172 & \\
\hline 154 & $3 d 4 d$ & ${ }^{1} \mathrm{P}_{1}$ & & 1330929 & 1332764 & \\
\hline 155 & $3 p 5 f$ & ${ }^{1} \mathrm{~F}_{3}$ & & 1331924 & 1332026 & \\
\hline 156 & $3 p 5 d$ & ${ }^{1} \mathrm{P}_{1}$ & & 1332129 & 1329428 & \\
\hline 157 & $3 p 5 g$ & ${ }^{3} \mathrm{H}_{4}$ & & 1332631 & 1332539 & \\
\hline 158 & $3 p 5 f$ & ${ }^{3} \mathrm{~F}_{3}$ & & 1332653 & 1333012 & \\
\hline 159 & $3 p 5 f$ & ${ }^{3} \mathrm{~F}_{2}$ & & 1333075 & 1332800 & \\
\hline 160 & $3 p 5 g$ & ${ }^{3} \mathrm{H}_{5}$ & & 1333102 & 1332933 & \\
\hline 161 & $3 p 5 f$ & ${ }^{3} \mathrm{~F}_{4}$ & & 1333204 & 1333504 & \\
\hline 162 & $3 p 5 g$ & ${ }^{3} \mathrm{G}_{4}$ & & 1334590 & 1334359 & \\
\hline 163 & $3 p 5 g$ & ${ }^{3} \mathrm{G}_{3}$ & & 1334741 & 1334521 & \\
\hline 164 & $3 p 5 f$ & ${ }^{3} \mathrm{G}_{3}$ & & 1336067 & 1335940 & \\
\hline 165 & $3 p 5 f$ & ${ }^{3} \mathrm{G}_{4}$ & & 1336606 & 1336501 & \\
\hline 166 & $3 p 5 g$ & ${ }^{3} \mathrm{H}_{6}$ & & 1336838 & 1336682 & \\
\hline 167 & $3 p 5 g$ & ${ }^{1} \mathrm{G}_{4}$ & & 1337242 & 1336934 & \\
\hline 168 & $3 p 5 g$ & ${ }^{3} \mathrm{G}_{5}$ & & 1337286 & 1336998 & \\
\hline 169 & $3 p 5 f$ & ${ }^{3} \mathrm{G}_{5}$ & & 1337465 & 1337417 & \\
\hline 170 & $3 p 5 g$ & ${ }^{1} \mathrm{H}_{5}$ & & 1338187 & 1337886 & \\
\hline 171 & $3 d 4 d$ & ${ }^{3} \mathrm{~S}_{1}$ & & 1339507 & 1341153 & \\
\hline 172 & $3 p 5 f$ & ${ }^{3} \mathrm{D}_{3}$ & & 1339655 & 1339120 & \\
\hline 173 & $3 p 5 f$ & ${ }^{3} \mathrm{D}_{2}$ & & 1340537 & 1339958 & \\
\hline 174 & $3 p 5 f$ & ${ }^{3} \mathrm{D}_{1}$ & & 1341477 & 1340840 & \\
\hline 175 & $3 p 5 g$ & ${ }^{3} \mathrm{~F}_{4}$ & & 1341551 & 1341382 & \\
\hline 176 & $3 p 5 g$ & ${ }^{3} \mathrm{~F}_{3}$ & & 1341832 & 1341639 & \\
\hline 177 & $3 p 5 g$ & ${ }^{3} \mathrm{~F}_{2}$ & & 1343392 & 1343175 & \\
\hline 178 & $3 p 5 g$ & ${ }^{1} \mathrm{~F}_{3}$ & & 1343700 & 1343481 & \\
\hline 179 & $3 p 5 f$ & ${ }^{1} \mathrm{G}_{4}$ & & 1343815 & 1343308 & \\
\hline 180 & $3 d 4 d$ & ${ }^{3} \mathrm{~F}_{2}$ & & 1344910 & 1345059 & \\
\hline 181 & $3 d 4 d$ & ${ }^{3} \mathrm{~F}_{3}$ & & 1345070 & 1345543 & \\
\hline 182 & $3 d 4 d$ & ${ }^{3} \mathrm{~F}_{4}$ & & 1345298 & 1345912 & \\
\hline 183 & $3 p 5 f$ & ${ }^{1} \mathrm{D}_{2}$ & & 1346113 & 1345607 & \\
\hline 184 & $3 d 4 d$ & ${ }^{3} \mathrm{P}_{1}$ & & 1357254 & 1357370 & \\
\hline 185 & $3 d 4 d$ & ${ }^{3} \mathrm{P}_{0}$ & & 1357276 & 1357275 & \\
\hline 186 & $3 d 4 d$ & ${ }^{3} \mathrm{P}_{2}$ & & 1357329 & 1357426 & \\
\hline 187 & $3 d 4 d$ & ${ }^{1} \mathrm{D}_{2}$ & & 1358147 & 1358015 & \\
\hline 188 & $3 d 4 d$ & ${ }^{1} \mathrm{G}_{4}$ & & 1360352 & 1360215 & \\
\hline 189 & $3 d 4 f$ & ${ }^{1} \mathrm{G}_{4}$ & & 1361724 & 1362240 & \\
\hline 190 & $3 d 4 f$ & ${ }^{3} \mathrm{H}_{4}$ & & 1366667 & 1367097 & \\
\hline 191 & $3 d 4 f$ & ${ }^{3} \mathrm{~F}_{2}$ & & 1366795 & 1367355 & \\
\hline 192 & $3 d 4 f$ & ${ }^{3} \mathrm{~F}_{4}$ & & 1366874 & 1367406 & \\
\hline
\end{tabular}


Table I. Ca IX experimental and theoretical energy levels.

\begin{tabular}{|c|c|c|c|c|c|c|}
\hline Level & Configuration & Term & $\mathrm{E}_{o b s}$ & $\mathrm{E}_{\text {calc }}$ & $\mathrm{E}_{S S T}$ & Source \\
\hline 193 & $3 d 4 f$ & ${ }^{3} \mathrm{~F}_{3}$ & & 1367007 & 1367479 & \\
\hline 194 & $3 d 4 f$ & ${ }^{3} \mathrm{H}_{5}$ & & 1367325 & 1367550 & \\
\hline 195 & $3 d 4 f$ & ${ }^{3} \mathrm{H}_{6}$ & & 1367588 & 1368120 & \\
\hline 196 & $3 d 4 f$ & ${ }^{1} \mathrm{D}_{2}$ & & 1371021 & 1371756 & \\
\hline 197 & $3 d 4 f$ & ${ }^{3} \mathrm{G}_{3}$ & & 1379366 & 1380391 & \\
\hline 198 & $3 d 4 f$ & ${ }^{3} \mathrm{G}_{5}$ & & 1379707 & 1380804 & \\
\hline 199 & $3 d 4 f$ & ${ }^{3} \mathrm{G}_{4}$ & & 1379766 & 1380585 & \\
\hline 200 & $3 d 4 f$ & ${ }^{3} \mathrm{D}_{1}$ & & 1381370 & 1382328 & \\
\hline 201 & $3 d 4 f$ & ${ }^{3} \mathrm{D}_{3}$ & & 1381483 & 1382345 & \\
\hline 202 & $3 d 4 f$ & ${ }^{3} \mathrm{D}_{2}$ & & 1381558 & 1382385 & \\
\hline 203 & $3 d 4 f$ & ${ }^{3} \mathrm{P}_{2}$ & & 1384454 & 1385057 & \\
\hline 204 & $3 d 4 f$ & ${ }^{3} \mathrm{P}_{1}$ & & 1384563 & 1385248 & \\
\hline 205 & $3 d 4 f$ & ${ }^{3} \mathrm{P}_{0}$ & & 1384776 & 1385344 & \\
\hline 206 & $3 d 4 f$ & ${ }^{1} \mathrm{~F}_{3}$ & & 1387675 & 1388873 & \\
\hline 207 & $3 d 4 d$ & ${ }^{1} \mathrm{~S}_{0}$ & & 1388836 & 1387510 & \\
\hline 208 & $3 d 4 f$ & ${ }^{1} \mathrm{P}_{1}$ & & 1399796 & 1401146 & \\
\hline 209 & $3 d 4 f$ & ${ }^{1} \mathrm{H}_{5}$ & & 1403400 & 1404783 & \\
\hline 210 & $3 d 5 s$ & ${ }^{3} \mathrm{D}_{1}$ & & 1487714 & 1488918 & \\
\hline 211 & $3 d 5 s$ & ${ }^{3} \mathrm{D}_{2}$ & & 1487839 & 1489111 & \\
\hline 212 & $3 d 5 s$ & ${ }^{3} \mathrm{D}_{3}$ & & 1488053 & 1489472 & \\
\hline 213 & $3 d 5 s$ & ${ }^{1} \mathrm{D}_{2}$ & & 1490778 & 1491725 & \\
\hline 214 & $3 d 5 p$ & ${ }^{1} \mathrm{D}_{2}$ & & 1515712 & 1516927 & \\
\hline 215 & $3 d 5 p$ & ${ }^{3} \mathrm{D}_{1}$ & & 1516987 & 1517807 & \\
\hline 216 & $3 d 5 p$ & ${ }^{3} \mathrm{D}_{2}$ & & 1517173 & 1518099 & \\
\hline 217 & $3 d 5 p$ & ${ }^{3} \mathrm{D}_{3}$ & & 1517456 & 1518467 & \\
\hline 218 & $3 d 5 p$ & ${ }^{3} \mathrm{~F}_{2}$ & & 1518474 & 1519423 & \\
\hline 219 & $3 d 5 p$ & ${ }^{3} \mathrm{~F}_{3}$ & & 1518825 & 1519842 & \\
\hline 220 & $3 d 5 p$ & ${ }^{3} \mathrm{~F}_{4}$ & & 1519323 & 1520444 & \\
\hline 221 & $3 d 5 p$ & ${ }^{3} \mathrm{P}_{2}$ & & 1522395 & 1523077 & \\
\hline 222 & $3 d 5 p$ & ${ }^{3} \mathrm{P}_{1}$ & & 1522401 & 1522937 & \\
\hline 223 & $3 d 5 p$ & ${ }^{3} \mathrm{P}_{0}$ & & 1522433 & 1522927 & \\
\hline 224 & $3 d 5 p$ & ${ }^{1} \mathrm{~F}_{3}$ & & 1525184 & 1525040 & \\
\hline 225 & $3 d 5 p$ & ${ }^{1} \mathrm{P}_{1}$ & & 1528409 & 1527952 & \\
\hline 226 & $3 d 5 d$ & ${ }^{1} \mathrm{~F}_{3}$ & & 1553867 & 1555960 & \\
\hline 227 & $3 d 5 d$ & ${ }^{3} \mathrm{D}_{1}$ & & 1555529 & 1557356 & \\
\hline 228 & $3 d 5 d$ & ${ }^{3} \mathrm{D}_{2}$ & & 1555607 & 1557521 & \\
\hline 229 & $3 d 5 d$ & ${ }^{3} \mathrm{D}_{3}$ & & 1555751 & 1557735 & \\
\hline 230 & $3 d 5 d$ & ${ }^{3} \mathrm{G}_{3}$ & & 1556540 & 1558290 & \\
\hline 231 & $3 d 5 d$ & ${ }^{3} \mathrm{G}_{4}$ & & 1556678 & 1558534 & \\
\hline 232 & $3 d 5 d$ & ${ }^{3} \mathrm{G}_{5}$ & & 1556905 & 1558864 & \\
\hline 233 & $3 d 5 d$ & ${ }^{1} \mathrm{P}_{1}$ & & 1556975 & 1558830 & \\
\hline 234 & $3 d 5 d$ & ${ }^{3} \mathrm{~S}_{1}$ & & 1560642 & 1562101 & \\
\hline 235 & $3 d 5 d$ & ${ }^{3} \mathrm{~F}_{2}$ & & 1561432 & 1561749 & \\
\hline 236 & $3 d 5 d$ & ${ }^{3} \mathrm{~F}_{3}$ & & 1561527 & 1561963 & \\
\hline 237 & $3 d 5 d$ & ${ }^{3} \mathrm{~F}_{4}$ & & 1561682 & 1562216 & \\
\hline 238 & $3 d 5 d$ & ${ }^{1} \mathrm{D}_{2}$ & & 1567602 & 1566995 & \\
\hline 239 & $3 d 5 d$ & ${ }^{3} \mathrm{P}_{0}$ & & 1567928 & 1567347 & \\
\hline 240 & $3 d 5 d$ & ${ }^{3} \mathrm{P}_{1}$ & & 1567945 & 1567431 & \\
\hline
\end{tabular}


Table I. Ca IX experimental and theoretical energy levels.

\begin{tabular}{|c|c|c|c|c|c|c|}
\hline Level & Configuration & Term & $\mathrm{E}_{o b s}$ & $\mathrm{E}_{\text {calc }}$ & $\mathrm{E}_{S S T}$ & Source \\
\hline 241 & $3 d 5 d$ & ${ }^{3} \mathrm{P}_{2}$ & & 1568042 & 1567630 & \\
\hline 242 & $3 d 5 d$ & ${ }^{1} \mathrm{G}_{4}$ & & 1568999 & 1568386 & \\
\hline 243 & $3 d 5 f$ & ${ }^{1} \mathrm{G}_{4}$ & & 1570694 & 1572511 & \\
\hline 244 & $3 d 5 f$ & ${ }^{3} \mathrm{H}_{4}$ & & 1571608 & 1573419 & \\
\hline 245 & $3 d 5 f$ & ${ }^{3} \mathrm{H}_{5}$ & & 1571764 & 1573627 & \\
\hline 246 & $3 d 5 f$ & ${ }^{3} \mathrm{H}_{6}$ & & 1571920 & 1573911 & \\
\hline 247 & $3 d 5 f$ & ${ }^{3} \mathrm{~F}_{2}$ & & 1572555 & 1574224 & \\
\hline 248 & $3 d 5 f$ & ${ }^{3} \mathrm{~F}_{3}$ & & 1572633 & 1574329 & \\
\hline 249 & $3 d 5 f$ & ${ }^{3} \mathrm{~F}_{4}$ & & 1572705 & 1574473 & \\
\hline 250 & $3 d 5 f$ & ${ }^{1} \mathrm{D}_{2}$ & & 1575112 & 1576725 & \\
\hline 251 & $3 d 5 g$ & ${ }^{1} \mathrm{H}_{5}$ & & 1577239 & 1578892 & \\
\hline 252 & $3 d 5 f$ & ${ }^{3} \mathrm{G}_{3}$ & & 1577762 & 1579012 & \\
\hline 253 & $3 d 5 g$ & ${ }^{3} \mathrm{H}_{4}$ & & 1577853 & 1579536 & \\
\hline 254 & $3 d 5 f$ & ${ }^{3} \mathrm{G}_{4}$ & & 1577893 & 1579195 & \\
\hline 255 & $3 d 5 g$ & ${ }^{3} \mathrm{H}_{5}$ & & 1577986 & 1579723 & \\
\hline 256 & $3 d 5 f$ & ${ }^{3} \mathrm{G}_{5}$ & & 1578001 & 1579391 & \\
\hline 257 & $3 d 5 g$ & ${ }^{3} \mathrm{H}_{6}$ & & 1578102 & 1579934 & \\
\hline 258 & $3 d 5 g$ & ${ }^{3} \mathrm{G}_{3}$ & & 1578149 & 1579838 & \\
\hline 259 & $3 d 5 g$ & ${ }^{3} \mathrm{G}_{4}$ & & 1578251 & 1579988 & \\
\hline 260 & $3 d 5 g$ & ${ }^{3} \mathrm{G}_{5}$ & & 1578319 & 1580142 & \\
\hline 261 & $3 d 5 g$ & ${ }^{1} \mathrm{~F}_{3}$ & & 1579462 & 1581257 & \\
\hline 262 & $3 d 5 f$ & ${ }^{3} \mathrm{D}_{1}$ & & 1579575 & 1580846 & \\
\hline 263 & $3 d 5 f$ & ${ }^{3} \mathrm{D}_{2}$ & & 1579592 & 1580846 & \\
\hline 264 & $3 d 5 f$ & ${ }^{3} \mathrm{D}_{3}$ & & 1579600 & 1580883 & \\
\hline 265 & $3 d 5 g$ & ${ }^{1} \mathrm{G}_{4}$ & & 1579651 & 1581444 & \\
\hline 266 & $3 d 5 g$ & ${ }^{3} \mathrm{I}_{5}$ & & 1579858 & 1581542 & \\
\hline 267 & $3 d 5 g$ & ${ }^{3} \mathrm{~F}_{2}$ & & 1579910 & 1581731 & \\
\hline 268 & $3 d 5 g$ & ${ }^{3} \mathrm{~F}_{3}$ & & 1579915 & 1581733 & \\
\hline 269 & $3 d 5 g$ & ${ }^{3} \mathrm{~F}_{4}$ & & 1579944 & 1581830 & \\
\hline 270 & $3 d 5 g$ & ${ }^{3} \mathrm{I}_{6}$ & & 1580014 & 1581744 & \\
\hline 271 & $3 d 5 g$ & ${ }^{3} \mathrm{I}_{7}$ & & 1580163 & 1582016 & \\
\hline 272 & $3 d 5 f$ & ${ }^{3} \mathrm{P}_{2}$ & & 1580616 & 1581895 & \\
\hline 273 & $3 d 5 f$ & ${ }^{3} \mathrm{P}_{1}$ & & 1580738 & 1582068 & \\
\hline 274 & $3 d 5 f$ & ${ }^{3} \mathrm{P}_{0}$ & & 1580813 & 1582153 & \\
\hline 275 & $3 d 5 f$ & ${ }^{1} \mathrm{~F}_{3}$ & & 1581431 & 1582571 & \\
\hline 276 & $3 d 5 g$ & ${ }^{1} \mathrm{I}_{6}$ & & 1582484 & 1584516 & \\
\hline 277 & $3 d 5 g$ & ${ }^{3} \mathrm{D}_{3}$ & & 1583222 & 1585112 & \\
\hline 278 & $3 d 5 \mathrm{~g}$ & ${ }^{3} \mathrm{D}_{2}$ & & 1583336 & 1585291 & \\
\hline 279 & $3 d 5 g$ & ${ }^{3} \mathrm{D}_{1}$ & & 1583452 & 1585446 & \\
\hline 280 & $3 d 5 g$ & ${ }^{1} \mathrm{D}_{2}$ & & 1584749 & 1586821 & \\
\hline 281 & $3 d 5 d$ & ${ }^{1} \mathrm{~S}_{0}$ & & 1585494 & 1581886 & \\
\hline 282 & $3 d 5 f$ & ${ }^{1} \mathrm{H}_{5}$ & & 1588382 & 1589105 & \\
\hline 283 & $3 d 5 f$ & ${ }^{1} \mathrm{P}_{1}$ & & 1589032 & 1589756 & \\
\hline
\end{tabular}


Table II. Ca IX Oscillator Strengths, Radiative Decay Rates, and Collision Strengths for transitions involving the lowest 4 levels.

Low. Upp. Osc. Rad. Dec. Lev. Lev.

Str. Rate

$$
\text { Str. Rate }
$$

i j $\quad$ E

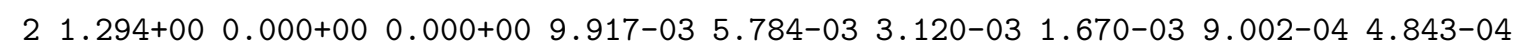
$\begin{array}{llllllllll}3 & 1.308+00 & 5.347-04 & 2.449+06 & 3.429-02 & 2.295-02 & 1.590-02 & 1.237-02 & 1.084-02 & 1.029-02\end{array}$

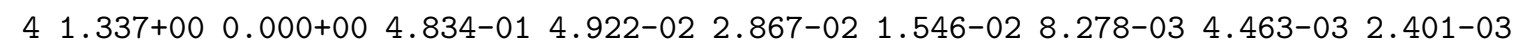

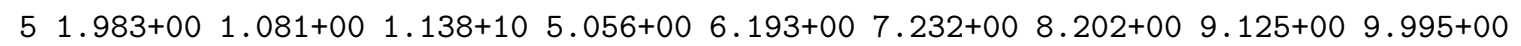
$\begin{array}{lllllllllll}6 & 3.056+00 & 0.000+00 & 8.125+04 & 2.103-01 & 2.447-01 & 2.678-01 & 2.837-01 & 2.947-01 & 3.021-01\end{array}$

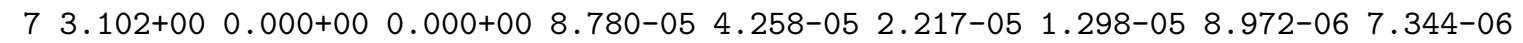
$\begin{array}{llllllllll}8 & 3.119+00 & 0.000+00 & 0.000+00 & 2.494-04 & 1.136-04 & 5.267-05 & 2.500-05 & 1.235-05 & 6.754-06\end{array}$ $\begin{array}{llllllllll}9 & 3.151+00 & 0.000+00 & 3.980+03 & 9.093-03 & 1.034-02 & 1.123-02 & 1.187-02 & 1.232-02 & 1.263-02\end{array}$ $\begin{array}{lllllllllll}10 & 3.670+00 & 0.000+00 & 0.000+00 & 2.046-04 & 1.984-04 & 1.944-04 & 1.901-04 & 1.808-04 & 1.793-04\end{array}$ $\begin{array}{llllllllll}11 & 3.757+00 & 0.000+00 & 0.000+00 & 1.988-02 & 1.164-02 & 6.562-03 & 3.587-03 & 1.929-03 & 1.026-03\end{array}$

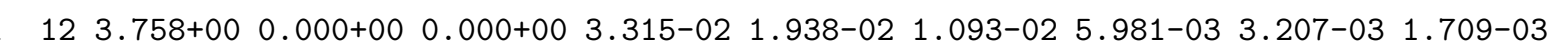
$\begin{array}{lllllllllll}13 & 3.760+00 & 0.000+00 & 0.000+00 & 4.638-02 & 2.717-02 & 1.531-02 & 8.372-03 & 4.501-03 & 2.394-03\end{array}$ $14 \quad 4.308+00 \quad 0.000+00 \quad 8.622+05 \quad 3.336-01 \quad 4.061-01 \quad 4.586-01 \quad 4.969-01 \quad 5.242-01 \quad 5.424-01$ $\begin{array}{llllllllll}15 & 5.126+00 & 0.000+00 & 0.000+00 & 4.600-04 & 2.516-04 & 1.342-04 & 7.082-05 & 3.724-05 & 1.941-05\end{array}$ $\begin{array}{lllllllllll}16 & 5.144+00 & 0.000+00 & 0.000+00 & 6.503-04 & 3.606-04 & 1.947-04 & 1.053-04 & 5.818-05 & 3.348-05\end{array}$ $\begin{array}{llllllllll}17 & 5.166+00 & 0.000+00 & 0.000+00 & 8.156-04 & 4.469-04 & 2.386-04 & 1.260-04 & 6.636-05 & 3.457-05\end{array}$

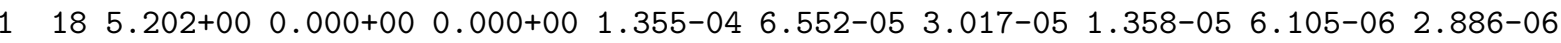
$\begin{array}{llllllllllll}1 & 19 & 5.458+00 & 0.000+00 & 0.000+00 & 2.759-04 & 1.480-04 & 7.656-05 & 3.921-05 & 2.029-05 & 1.091-05\end{array}$ $\begin{array}{lllllllllll}1 & 20 & 5.468+00 & 0.000+00 & 0.000+00 & 1.473-04 & 7.845-05 & 4.021-05 & 2.083-05 & 1.109-05 & 6.452-06\end{array}$ $1215.484+00 \quad 0.000+00 \quad 0.000+00 \quad 5.794-05 \quad 3.145-05 \quad 1.646-05 \quad 8.528-06 \quad 4.460-06 \quad 2.416-06$ $1225.493+00 \quad 0.000+00 \quad 0.000+00 \quad 1.182-04 \quad 6.084-05 \quad 2.989-05 \quad 1.465-05 \quad 7.273-06 \quad 3.914-06$ $12335.497+00 \quad 0.000+00 \quad 0.000+00 \quad 1.715-04 \quad 8.562-05 \quad 4.079-05 \quad 1.908-05 \quad 9.038-06 \quad 4.560-06$

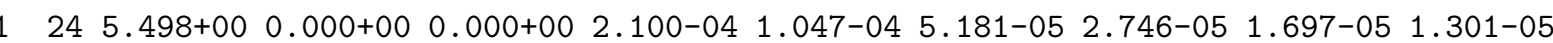
$26 \quad 6.016+00 \quad 2.834-03 \quad 2.747+08 \quad 6.088-04 \quad 1.023-03 \quad 1.505-03 \quad 2.046-03 \quad 2.597-03 \quad 3.175-03$ $276.914+00 \quad 0.000+00 \quad 0.000+00 \quad 7.888-03 \quad 3.994-03 \quad 2.157-03 \quad 1.188-03 \quad 6.340-04 \quad 3.269-04$ $28 \quad 7.056+00 \quad 0.000+00 \quad 0.000+00 \quad 2.169-01 \quad 2.357-012.476-01 \quad 2.547-012.587-01 \quad 2.606-01$ $297.556+00 \quad 0.000+00 \quad 0.000+00 \quad 1.606-03 \quad 9.125-04 \quad 4.710-04 \quad 2.350-04 \quad 1.158-04 \quad 5.856-05$ $307.558+00 \quad 2.943-02 \quad 4.501+09 \quad 6.583-03 \quad 6.606-03 \quad 8.237-03 \quad 1.111-02 \quad 1.467-02 \quad 1.869-02$ $317.567+00 \quad 0.000+00 \quad 0.000+00 \quad 6.188-06 \quad 3.034-06 \quad 1.562-06 \quad 9.364-07 \quad 6.904-07 \quad 6.075-07$ $\begin{array}{lllllllllll}32 & 7.568+00 & 0.000+00 & 0.000+00 & 8.409-06 & 3.848-06 & 1.670-06 & 7.026-07 & 2.899-07 & 1.223-07\end{array}$ $337.570+00 \quad 0.000+00 \quad 0.000+00 \quad 7.958-03 \quad 4.519-03 \quad 2.332-03 \quad 1.165-03 \quad 5.754-04 \quad 2.923-04$ $\begin{array}{llllllllll}34 & 7.571+00 & 0.000+00 & 0.000+00 & 1.088-05 & 4.980-06 & 2.163-06 & 9.107-07 & 3.780-07 & 1.591-07\end{array}$ $357.580+00 \quad 2.405-01 \quad 3.701+10 \quad 2.112-02 \quad 3.543-02 \quad 5.786-02 \quad 8.602-02 \quad 1.175-01 \quad 1.513-01$ $\begin{array}{llllllllll}36 & 7.752+00 & 0.000+00 & 0.000+00 & 5.298-06 & 2.381-06 & 1.777-06 & 2.190-06 & 2.913-06 & 3.495-06\end{array}$ $\begin{array}{llllllllll}37 & 7.753+00 & 0.000+00 & 2.435+05 & 1.552-03 & 2.270-03 & 2.881-03 & 3.385-03 & 3.773-03 & 4.085-03\end{array}$ $38 \quad 7.779+00 \quad 0.000+00 \quad 0.000+00 \quad 1.446-05 \quad 7.753-06 \quad 3.874-06 \quad 1.856-06 \quad 8.812-07 \quad 4.353-07$ $397.779+00 \quad 0.000+00 \quad 0.000+00 \quad 4.948-06 \quad 2.692-06 \quad 1.417-06 \quad 7.625-07 \quad 4.615-07 \quad 3.389-07$ $407.781+00 \quad 0.000+00 \quad 0.000+00 \quad 3.233-05 \quad 2.502-05 \quad 2.185-05 \quad 2.121-05 \quad 2.166-05 \quad 2.260-05$ $418.277+00 \quad 0.000+00 \quad 0.000+00 \quad 2.745-04 \quad 3.135-04 \quad 3.419-04 \quad 3.618-04 \quad 3.760-04 \quad 3.853-04$ $428.350+00 \quad 0.000+00 \quad 0.000+00 \quad 4.595-03 \quad 2.293-03 \quad 1.206-03 \quad 6.312-04 \quad 3.241-04 \quad 1.614-04$ $438.351+00 \quad 0.000+00 \quad 0.000+00 \quad 7.637-03 \quad 3.819-03 \quad 2.025-03 \quad 1.065-03 \quad 5.476-04 \quad 2.836-04$ $448.352+00 \quad 0.000+00 \quad 0.000+00 \quad 1.071-02 \quad 5.346-03 \quad 2.813-03 \quad 1.473-03 \quad 7.564-04 \quad 3.767-04$ $458.367+00 \quad 0.000+00 \quad 1.161+07 \quad 2.512-02 \quad 4.021-02 \quad 5.466-02 \quad 6.685-02 \quad 7.721-02 \quad 8.607-02$ $46 \quad 8.576+00 \quad 0.000+00 \quad 0.000+00 \quad 7.280-06 \quad 3.627-06 \quad 1.700-06 \quad 8.056-07 \quad 3.957-07 \quad 1.849-07$ 
Table II. Ca IX Oscillator Strengths, Radiative Decay Rates, and Collision Strengths for transitions involving the lowest 4 levels.

Low. Upp. Osc. Rad. Dec. Lev. Lev. Rate
Collision Strength

Impact Electron Energy - Eo (Ry)

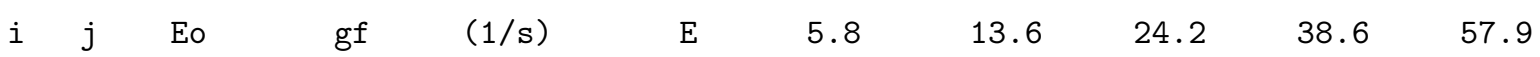

$147 \quad 8.587+00 \quad 4.111-05 \quad 8.118+06 \quad 4.588-05 \quad 3.432-05 \quad 2.970-05 \quad 2.920-05 \quad 3.173-05 \quad 3.552-05$

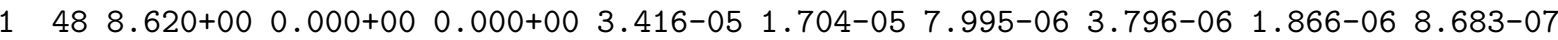

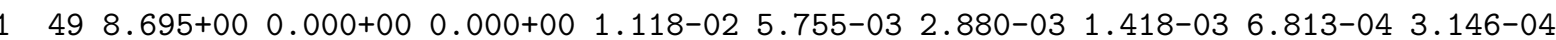

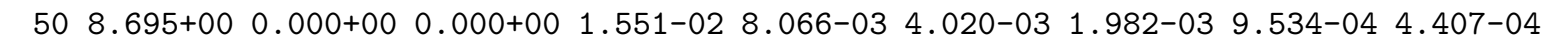
$\begin{array}{llllllllll}51 & 8.695+00 & 0.000+00 & 0.000+00 & 2.012-02 & 1.036-02 & 5.183-03 & 2.553-03 & 1.226-03 & 5.663-04\end{array}$ $\begin{array}{llllllllllll}52 & 8.710+00 & 7.766-04 & 1.577+08 & 6.259-04 & 5.977-04 & 6.076-04 & 6.356-04 & 7.025-04 & 7.808-04\end{array}$ $\begin{array}{lllllllllll}53 & 8.792+00 & 0.000+00 & 0.000+00 & 7.551-02 & 9.977-02 & 1.177-01 & 1.298-01 & 1.374-01 & 1.419-01\end{array}$ $\begin{array}{llllllllll}54 & 9.091+00 & 0.000+00 & 0.000+00 & 3.419-05 & 1.543-05 & 7.374-06 & 3.817-06 & 2.044-06 & 1.020-06\end{array}$ $\begin{array}{llllllllll}55 & 9.145+00 & 0.000+00 & 0.000+00 & 4.472-05 & 2.210-05 & 1.171-05 & 6.590-06 & 3.893-06 & 2.129-06\end{array}$ $\begin{array}{llllllllll}56 & 9.157+00 & 0.000+00 & 0.000+00 & 7.463-05 & 3.766-05 & 2.082-05 & 1.268-05 & 8.120-06 & 5.354-06\end{array}$

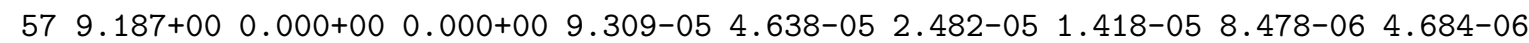

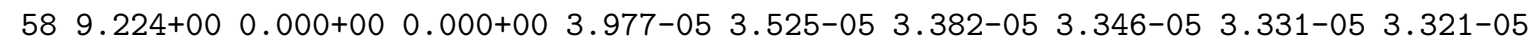
$\begin{array}{lllllllllll}59 & 9.231+00 & 0.000+00 & 0.000+00 & 6.157-05 & 2.959-05 & 1.487-05 & 7.708-06 & 3.966-06 & 2.039-06\end{array}$ $\begin{array}{llllllllll}60 & 9.254+00 & 0.000+00 & 0.000+00 & 9.897-05 & 4.897-05 & 2.543-05 & 1.343-05 & 6.942-06 & 3.663-06\end{array}$

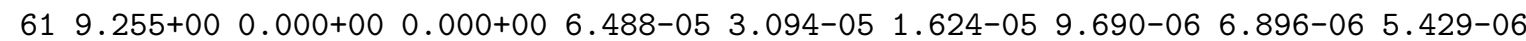
$\begin{array}{llllllllllll}62 & 9.360+00 & 0.000+00 & 1.560+04 & 1.294-04 & 1.129-04 & 1.171-04 & 1.203-04 & 1.373-04 & 1.446-04\end{array}$ $\begin{array}{llllllllllll}63 & 9.500+00 & 0.000+00 & 0.000+00 & 8.002-03 & 8.848-03 & 9.436-03 & 9.826-03 & 1.006-02 & 1.020-02\end{array}$ $\begin{array}{llllllllllll}64 & 9.723+00 & 0.000+00 & 0.000+00 & 2.867-03 & 1.436-03 & 7.734-04 & 4.300-04 & 2.330-04 & 1.197-04\end{array}$ $\begin{array}{lllllllllllll}65 & 9.822+00 & 0.000+00 & 0.000+00 & 3.843-02 & 4.271-02 & 4.575-02 & 4.783-02 & 4.917-02 & 5.003-02\end{array}$ $\begin{array}{lllllllllll}66 & 9.938+00 & 3.625-03 & 9.586+08 & 1.833-04 & 2.983-04 & 4.900-04 & 7.568-04 & 1.071-03 & 1.411-03\end{array}$ $\begin{array}{lllllllllll}67 & 9.938+00 & 0.000+00 & 0.000+00 & 1.427-04 & 8.244-05 & 4.311-05 & 2.167-05 & 1.064-05 & 5.094-06\end{array}$ $\begin{array}{llllllllll}68 & 9.946+00 & 0.000+00 & 0.000+00 & 5.199-05 & 2.815-05 & 1.472-05 & 7.559-06 & 3.901-06 & 2.004-06\end{array}$

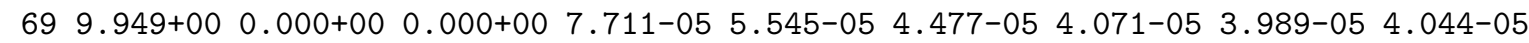
$\begin{array}{llllllllll}70 & 9.981+00 & 1.747-02 & 4.658+09 & 1.924-03 & 2.054-03 & 2.694-03 & 3.824-03 & 5.254-03 & 6.843-03\end{array}$ $\begin{array}{llllllllll}71 & 9.982+00 & 0.000+00 & 0.000+00 & 5.480-04 & 3.196-04 & 1.672-04 & 8.441-05 & 4.149-05 & 1.995-05\end{array}$ $\begin{array}{lllllllllll}72 & 9.984+00 & 0.000+00 & 0.000+00 & 2.376-03 & 1.387-03 & 7.259-04 & 3.669-04 & 1.806-04 & 8.703-05\end{array}$ $\begin{array}{llllllllll}73 & 9.990+00 & 7.554-02 & 2.019+10 & 3.478-03 & 5.997-03 & 1.004-02 & 1.559-02 & 2.212-02 & 2.912-02\end{array}$ $\begin{array}{llllllllll}74 & 9.997+00 & 0.000+00 & 0.000+00 & 1.429-04 & 8.216-05 & 4.566-05 & 2.493-05 & 1.340-05 & 7.144-06\end{array}$ $\begin{array}{lllllllllllll}75 & 1.000+01 & 0.000+00 & 0.000+00 & 2.427-04 & 2.009-04 & 1.811-04 & 1.753-04 & 1.763-04 & 1.810-04\end{array}$ $\begin{array}{llllllllll}76 & 1.002+01 & 0.000+00 & 0.000+00 & 2.885-04 & 1.667-04 & 9.299-05 & 5.092-05 & 2.744-05 & 1.464-05\end{array}$ $\begin{array}{lllllllllllllll}77 & 1.003+01 & 0.000+00 & 0.000+00 & 3.467-04 & 3.905-04 & 4.306-04 & 4.702-04 & 5.049-04 & 5.358-04\end{array}$ $\begin{array}{llllllllll}78 & 1.011+01 & 0.000+00 & 0.000+00 & 9.897-04 & 5.428-04 & 2.720-04 & 1.355-04 & 6.805-05 & 3.448-05\end{array}$ $\begin{array}{llllllllllll}79 & 1.012+01 & 9.922-04 & 2.720+08 & 5.473-04 & 3.544-04 & 2.732-04 & 2.791-04 & 3.311-04 & 4.061-04\end{array}$

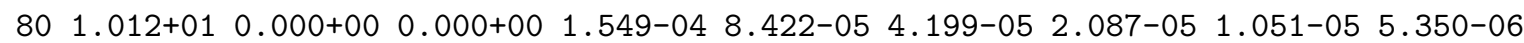

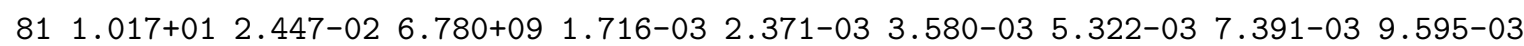
$\begin{array}{lllllllllll}82 & 1.023+01 & 0.000+00 & 0.000+00 & 4.683-04 & 2.075-04 & 9.656-05 & 4.679-05 & 2.208-05 & 1.000-05\end{array}$ $\begin{array}{llllllllllll}83 & 1.025+01 & 0.000+00 & 0.000+00 & 8.298-04 & 3.867-04 & 2.029-04 & 1.221-04 & 8.315-05 & 6.591-05\end{array}$ $\begin{array}{lllllllllll}84 & 1.026+01 & 0.000+00 & 0.000+00 & 2.262-04 & 9.876-05 & 4.497-05 & 2.148-05 & 1.020-05 & 4.837-06\end{array}$ $\begin{array}{llllllllllll}85 & 1.027+01 & 0.000+00 & 0.000+00 & 1.135-03 & 4.994-04 & 2.319-04 & 1.123-04 & 5.251-05 & 2.346-05\end{array}$ $\begin{array}{lllllllllllllll}86 & 1.029+01 & 0.000+00 & 4.410+04 & 1.037-04 & 9.573-05 & 1.047-04 & 1.215-04 & 1.370-04 & 1.513-04\end{array}$ $\begin{array}{lllllllllll}87 & 1.030+01 & 0.000+00 & 0.000+00 & 1.438-04 & 6.226-05 & 2.801-05 & 1.321-05 & 6.435-06 & 3.204-06\end{array}$ $\begin{array}{llllllllll}88 & 1.030+01 & 0.000+00 & 0.000+00 & 1.486-04 & 7.258-05 & 4.182-05 & 3.005-05 & 2.606-05 & 2.477-05\end{array}$ $\begin{array}{llllllllllll}89 & 1.035+01 & 0.000+00 & 0.000+00 & 2.585-03 & 1.207-03 & 6.022-04 & 3.031-04 & 1.502-04 & 7.135-05\end{array}$ $\begin{array}{llllllllllll}90 & 1.035+01 & 0.000+00 & 6.656+04 & 2.142-03 & 1.081-03 & 6.160-04 & 4.103-04 & 3.083-04 & 2.675-04\end{array}$ $\begin{array}{llllllllll}91 & 1.036+01 & 0.000+00 & 0.000+00 & 1.390-03 & 6.534-04 & 3.284-04 & 1.665-04 & 8.308-05 & 3.983-05\end{array}$ 
Table II. Ca IX Oscillator Strengths, Radiative Decay Rates, and Collision Strengths for transitions involving the lowest 4 levels.

Low. Upp. Osc. Rad. Dec.

Lev. Lev.

Str. Rate

Collision Strength

Impact Electron Energy - Eo (Ry)

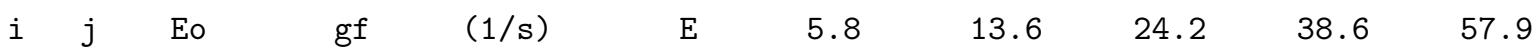

$\begin{array}{lllllllllllllll}1 & 92 & 1.038+01 & 0.000+00 & 1.224+07 & 1.114-02 & 1.719-02 & 2.280-02 & 2.822-02 & 3.272-02 & 3.658-02\end{array}$

$1931.040+01 \quad 0.000+00 \quad 0.000+00 \quad 2.794-03 \quad 4.397-03 \quad 5.210-03 \quad 5.754-03 \quad 6.467-03 \quad 6.953-03$

$\begin{array}{llllllllllll}1 & 94 & 1.044+01 & 0.000+00 & 0.000+00 & 2.750-03 & 1.391-03 & 7.479-04 & 4.017-04 & 2.121-04 & 1.086-04\end{array}$

$\begin{array}{llllllllllllll}1 & 95 & 1.044+01 & 0.000+00 & 2.721+04 & 1.741-03 & 9.120-04 & 5.193-04 & 3.134-04 & 2.007-04 & 1.450-04\end{array}$

$19661.045+01 \quad 0.000+00 \quad 0.000+00 \quad 9.291-04 \quad 4.748-04 \quad 2.571-04 \quad 1.389-04 \quad 7.375-05 \quad 3.800-05$

$197 \quad 1.052+01 \quad 0.000+00 \quad 2.550+05 \quad 3.078-04 \quad 4.011-04 \quad 4.990-04 \quad 6.025-04 \quad 6.944-04 \quad 7.638-04$

$\begin{array}{lllllllllll}1 & 98 & 1.055+01 & 0.000+00 & 0.000+00 & 4.665-03 & 2.560-03 & 1.340-03 & 6.835-04 & 3.430-04 & 1.675-04\end{array}$

$\begin{array}{lllllllllll}1 & 99 & 1.055+01 & 0.000+00 & 0.000+00 & 6.486-03 & 3.529-03 & 1.873-03 & 9.553-04 & 4.760-04 & 2.341-04\end{array}$

$\begin{array}{llllllllllll}1 & 100 & 1.055+01 & 0.000+00 & 0.000+00 & 8.388-03 & 4.603-03 & 2.409-03 & 1.229-03 & 6.168-04 & 3.012-04\end{array}$

$\begin{array}{lllllllllll}1 & 101 & 1.059+01 & 0.000+00 & 0.000+00 & 2.287-02 & 3.033-02 & 3.573-02 & 3.981-02 & 4.285-02 & 4.514-02\end{array}$

$\begin{array}{llllllllllll}1 & 102 & 1.063+01 & 0.000+00 & 0.000+00 & 1.751-03 & 6.636-04 & 2.772-04 & 1.254-04 & 5.388-05 & 2.270-05\end{array}$ $\begin{array}{lllllllllll}1 & 103 & 1.063+01 & 0.000+00 & 0.000+00 & 2.225-03 & 8.471-04 & 3.679-04 & 1.695-04 & 8.477-05 & 4.758-05\end{array}$ $\begin{array}{lllllllllll}1 & 104 & 1.063+01 & 0.000+00 & 0.000+00 & 2.670-03 & 1.010-03 & 4.212-04 & 1.903-04 & 8.169-05 & 3.440-05\end{array}$ $\begin{array}{lllllllllll}1 & 105 & 1.070+01 & 0.000+00 & 0.000+00 & 2.171-03 & 3.419-03 & 3.932-03 & 4.256-03 & 4.745-03 & 5.032-03\end{array}$

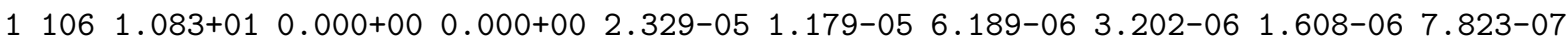
$\begin{array}{llllllllllll}1 & 107 & 1.083+01 & 0.000+00 & 0.000+00 & 3.861-05 & 1.995-05 & 1.079-05 & 6.071-06 & 3.497-06 & 2.301-06\end{array}$ $\begin{array}{lllllllllll}1 & 108 & 1.083+01 & 0.000+00 & 0.000+00 & 5.314-05 & 2.694-05 & 1.415-05 & 7.326-06 & 3.676-06 & 1.789-06\end{array}$ $\begin{array}{lllllllllll}1 & 109 & 1.091+01 & 0.000+00 & 8.143+05 & 4.317-04 & 6.900-04 & 9.541-04 & 1.210-03 & 1.434-03 & 1.632-03\end{array}$ $\begin{array}{lllllllllll}1 & 110 & 1.132+01 & 0.000+00 & 0.000+00 & 3.237-06 & 1.682-06 & 8.184-07 & 4.039-07 & 2.090-07 & 1.042-07\end{array}$ $\begin{array}{lllllllllll}1 & 111 & 1.132+01 & 0.000+00 & 0.000+00 & 1.329-05 & 9.394-06 & 7.352-06 & 6.231-06 & 5.748-06 & 5.636-06\end{array}$ $\begin{array}{lllllllllll}1 & 112 & 1.135+01 & 0.000+00 & 0.000+00 & 2.800-06 & 1.427-06 & 6.873-07 & 3.380-07 & 1.745-07 & 8.627-08\end{array}$

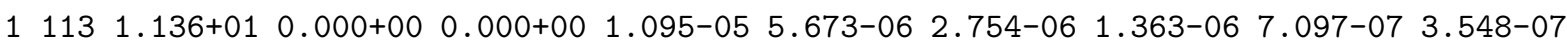
$\begin{array}{lllllllllll}1 & 114 & 1.137+01 & 0.000+00 & 0.000+00 & 1.928-05 & 1.831-05 & 1.839-05 & 1.808-05 & 1.821-05 & 1.871-05\end{array}$ $\begin{array}{llllllllllll}1 & 115 & 1.139+01 & 0.000+00 & 0.000+00 & 1.486-06 & 9.309-07 & 6.483-07 & 5.124-07 & 4.687-07 & 4.970-07\end{array}$ $\begin{array}{llllllllllll}1 & 116 & 1.139+01 & 0.000+00 & 0.000+00 & 1.930-06 & 9.645-07 & 4.623-07 & 2.179-07 & 1.012-07 & 4.662-08\end{array}$ $\begin{array}{llllllllllll}1 & 117 & 1.139+01 & 0.000+00 & 0.000+00 & 2.644-06 & 1.355-06 & 6.999-07 & 3.810-07 & 2.309-07 & 1.646-07\end{array}$ $\begin{array}{lllllllllll}1 & 118 & 1.141+01 & 0.000+00 & 0.000+00 & 6.344-06 & 3.498-06 & 1.865-06 & 9.849-07 & 5.180-07 & 2.646-07\end{array}$ $\begin{array}{lllllllllll}1 & 119 & 1.141+01 & 0.000+00 & 0.000+00 & 8.887-06 & 4.881-06 & 2.626-06 & 1.394-06 & 7.375-07 & 3.903-07\end{array}$ $\begin{array}{lllllllllll}1 & 120 & 1.142+01 & 0.000+00 & 0.000+00 & 1.197-05 & 6.613-06 & 3.531-06 & 1.868-06 & 9.844-07 & 5.031-07\end{array}$ $\begin{array}{lllllllllll}1 & 121 & 1.150+01 & 0.000+00 & 0.000+00 & 1.546-06 & 8.235-07 & 4.102-07 & 1.982-07 & 9.459-08 & 4.425-08\end{array}$ $\begin{array}{lllllllllll}1 & 122 & 1.150+01 & 0.000+00 & 0.000+00 & 5.041-06 & 2.753-06 & 1.485-06 & 8.933-07 & 6.685-07 & 6.239-07\end{array}$ $\begin{array}{lllllllllll}1 & 123 & 1.150+01 & 0.000+00 & 0.000+00 & 9.298-06 & 4.943-06 & 2.455-06 & 1.187-06 & 5.694-07 & 2.667-07\end{array}$

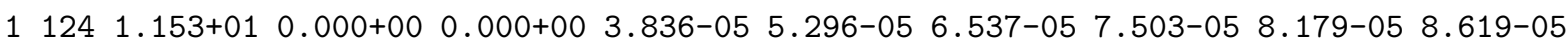

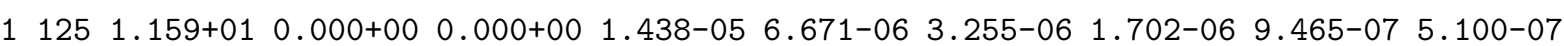

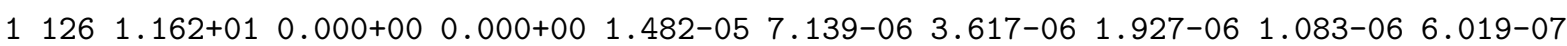
$\begin{array}{lllllllllll}1 & 127 & 1.162+01 & 6.308-04 & 2.280+08 & 6.598-05 & 6.152-05 & 6.962-05 & 9.127-05 & 1.224-04 & 1.612-04\end{array}$ $\begin{array}{lllllllllll}1 & 128 & 1.162+01 & 0.000+00 & 0.000+00 & 2.285-05 & 1.150-05 & 6.135-06 & 3.495-06 & 2.196-06 & 1.475-06\end{array}$

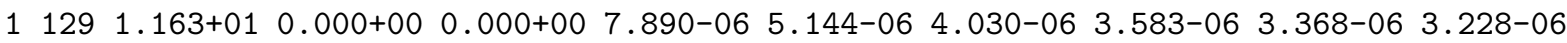
$\begin{array}{lllllllllll}1 & 130 & 1.165+01 & 0.000+00 & 0.000+00 & 1.637-05 & 7.504-06 & 3.600-06 & 1.836-06 & 9.845-07 & 5.019-07\end{array}$ $\begin{array}{lllllllllll}1 & 131 & 1.165+01 & 0.000+00 & 0.000+00 & 2.925-05 & 1.494-05 & 7.983-06 & 4.445-06 & 2.596-06 & 1.537-06\end{array}$ $\begin{array}{lllllllllll}1 & 132 & 1.167+01 & 0.000+00 & 0.000+00 & 2.603-05 & 1.183-05 & 5.647-06 & 2.912-06 & 1.585-06 & 9.138-07\end{array}$

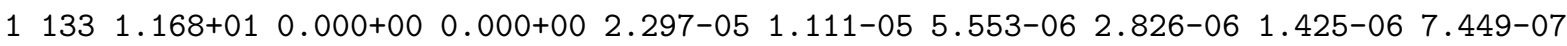
$\begin{array}{lllllllllll}1 & 134 & 1.172+01 & 0.000+00 & 2.614+04 & 2.278-05 & 1.106-05 & 6.887-06 & 5.597-06 & 6.294-06 & 8.220-06\end{array}$ $\begin{array}{lllllllllll}1 & 135 & 1.177+01 & 0.000+00 & 0.000+00 & 5.088-05 & 5.630-05 & 5.970-05 & 6.145-05 & 6.166-05 & 6.107-05\end{array}$

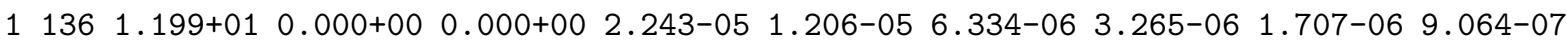


Table II. Ca IX Oscillator Strengths, Radiative Decay Rates, and Collision Strengths for transitions involving the lowest 4 levels.

Low. Upp. Osc. Rad. Dec.

Lev. Lev.

Str. Rate

Collision Strength

Impact Electron Energy - Eo (Ry)

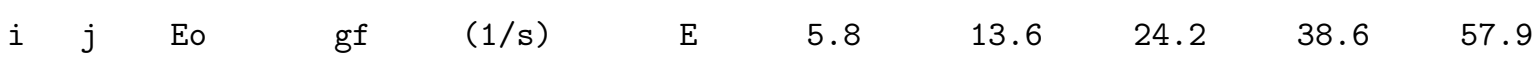

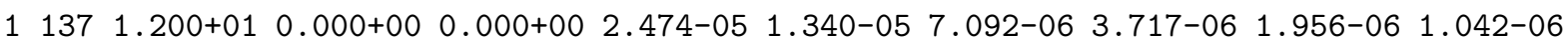

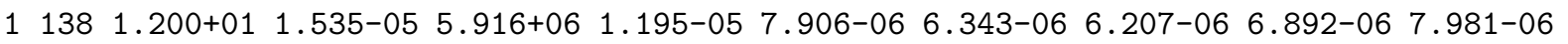

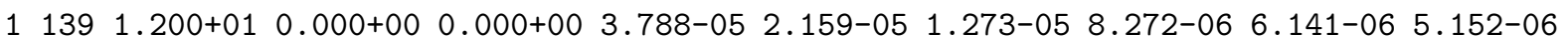

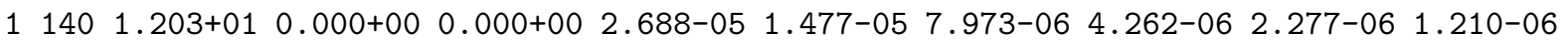
$\begin{array}{lllllllllll}1 & 141 & 1.203+01 & 0.000+00 & 0.000+00 & 4.024-05 & 2.244-05 & 1.229-05 & 6.790-06 & 3.897-06 & 2.320-06\end{array}$ $\begin{array}{llllllllllll}1 & 142 & 1.204+01 & 0.000+00 & 0.000+00 & 7.002-05 & 3.956-05 & 2.217-05 & 1.229-05 & 6.735-06 & 3.610-06\end{array}$

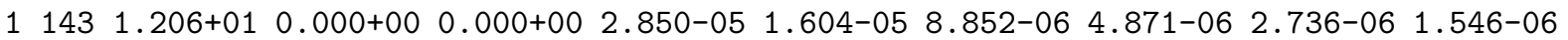
$\begin{array}{llllllllllll}1 & 144 & 1.207+01 & 0.000+00 & 0.000+00 & 1.860-06 & 7.547-07 & 2.981-07 & 1.229-07 & 6.107-08 & 3.409-08\end{array}$ $\begin{array}{lllllllllll}1 & 145 & 1.207+01 & 0.000+00 & 0.000+00 & 1.825-05 & 1.084-05 & 6.851-06 & 4.864-06 & 4.104-06 & 3.815-06\end{array}$ $\begin{array}{lllllllllll}1 & 146 & 1.207+01 & 0.000+00 & 0.000+00 & 5.939-06 & 3.351-06 & 1.859-06 & 1.030-06 & 5.815-07 & 3.304-07\end{array}$

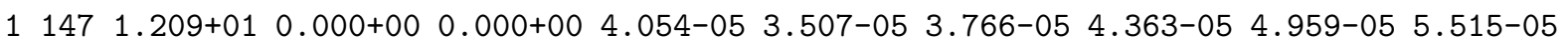
$\begin{array}{lllllllllll}1 & 148 & 1.211+01 & 0.000+00 & 0.000+00 & 1.567-06 & 7.760-07 & 4.026-07 & 2.170-07 & 1.228-07 & 6.763-08\end{array}$ $\begin{array}{llllllllllll}1 & 149 & 1.211+01 & 0.000+00 & 0.000+00 & 2.614-06 & 1.295-06 & 6.763-07 & 3.691-07 & 2.128-07 & 1.227-07\end{array}$ $\begin{array}{lllllllllll}1 & 150 & 1.211+01 & 0.000+00 & 0.000+00 & 3.695-06 & 1.821-06 & 9.422-07 & 5.095-07 & 2.893-07 & 1.600-07\end{array}$

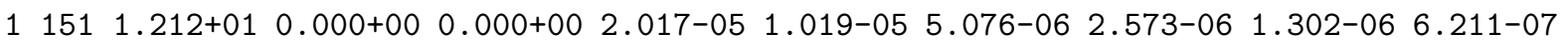
$\begin{array}{lllllllllll}1 & 152 & 1.212+01 & 0.000+00 & 0.000+00 & 1.530-05 & 7.831-06 & 4.187-06 & 2.536-06 & 1.710-06 & 1.363-06\end{array}$ $\begin{array}{llllllllllll}1 & 153 & 1.212+01 & 0.000+00 & 0.000+00 & 9.798-06 & 4.708-06 & 2.259-06 & 1.110-06 & 5.382-07 & 2.456-07\end{array}$ $\begin{array}{llllllllllll}1 & 154 & 1.213+01 & 0.000+00 & 0.000+00 & 2.769-06 & 1.306-06 & 6.013-07 & 2.792-07 & 1.311-07 & 5.946-08\end{array}$

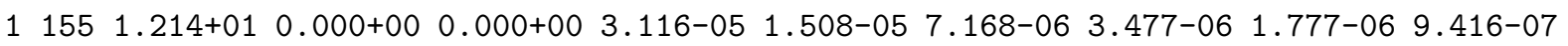
$\begin{array}{llllllllllll}1 & 156 & 1.214+01 & 6.938-04 & 2.738+08 & 1.008-04 & 1.396-04 & 1.937-04 & 2.555-04 & 3.225-04 & 3.930-04\end{array}$

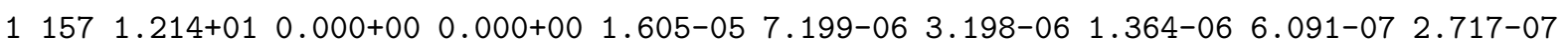
$\begin{array}{lllllllllll}1 & 158 & 1.214+01 & 0.000+00 & 0.000+00 & 3.732-05 & 1.811-05 & 8.651-06 & 4.232-06 & 2.152-06 & 1.130-06\end{array}$

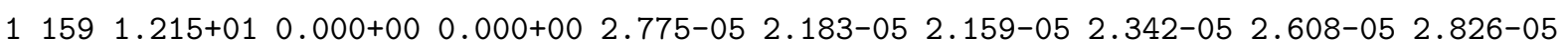

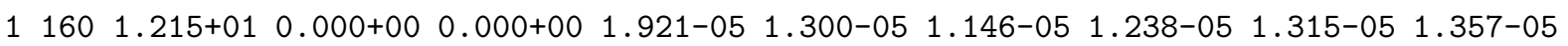
$\begin{array}{lllllllllll}1 & 161 & 1.215+01 & 0.000+00 & 0.000+00 & 4.791-05 & 2.668-05 & 1.678-05 & 1.273-05 & 1.115-05 & 1.100-05\end{array}$

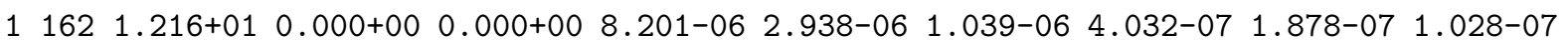

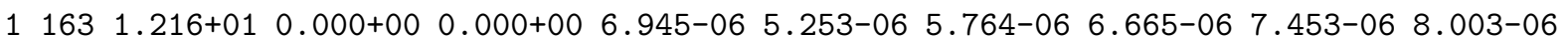
$\begin{array}{lllllllllll}1 & 164 & 1.218+01 & 0.000+00 & 0.000+00 & 4.305-05 & 2.191-05 & 1.093-05 & 5.539-06 & 2.876-06 & 1.475-06\end{array}$ $\begin{array}{lllllllllll}1 & 165 & 1.218+01 & 0.000+00 & 0.000+00 & 6.152-05 & 3.204-05 & 1.658-05 & 9.094-06 & 5.102-06 & 3.173-06\end{array}$

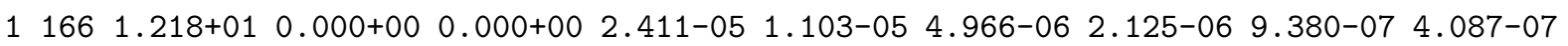
$\begin{array}{lllllllllll}1 & 167 & 1.219+01 & 0.000+00 & 0.000+00 & 8.286-06 & 3.272-06 & 1.300-06 & 5.281-07 & 2.499-07 & 1.296-07\end{array}$

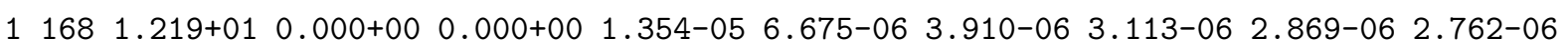
$\begin{array}{lllllllllll}1 & 169 & 1.219+01 & 0.000+00 & 0.000+00 & 1.060-04 & 5.738-05 & 3.010-05 & 1.586-05 & 8.321-06 & 4.108-06\end{array}$ $\begin{array}{lllllllllll}1 & 170 & 1.219+01 & 0.000+00 & 0.000+00 & 1.868-05 & 2.096-05 & 2.606-05 & 3.284-05 & 3.685-05 & 3.894-05\end{array}$

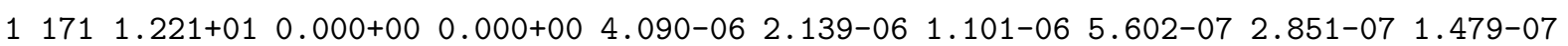

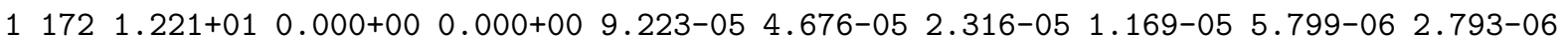
$\begin{array}{lllllllllll}1 & 173 & 1.222+01 & 0.000+00 & 0.000+00 & 7.449-05 & 4.517-05 & 3.256-05 & 2.694-05 & 2.569-05 & 2.559-05\end{array}$

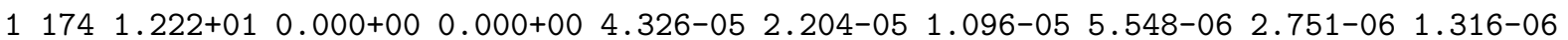
$\begin{array}{llllllllllll}1 & 175 & 1.223+01 & 0.000+00 & 0.000+00 & 1.860-05 & 7.270-06 & 2.776-06 & 1.117-06 & 4.374-07 & 1.605-07\end{array}$ $\begin{array}{llllllllllll}1 & 176 & 1.223+01 & 0.000+00 & 0.000+00 & 1.680-05 & 1.914-05 & 2.497-05 & 3.009-05 & 3.402-05 & 3.669-05\end{array}$ $\begin{array}{llllllllllll}1 & 177 & 1.224+01 & 0.000+00 & 0.000+00 & 1.106-05 & 4.346-06 & 1.664-06 & 6.720-07 & 2.610-07 & 9.102-08\end{array}$

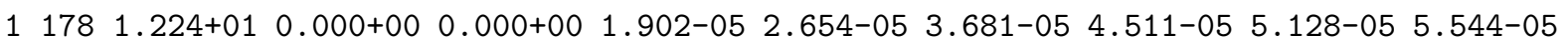
$\begin{array}{llllllllllll}1 & 179 & 1.225+01 & 0.000+00 & 0.000+00 & 6.524-05 & 9.204-05 & 1.244-04 & 1.518-04 & 1.760-04 & 2.004-04\end{array}$ $\begin{array}{llllllllllll}1 & 180 & 1.226+01 & 0.000+00 & 1.057+04 & 1.684-05 & 2.270-05 & 2.868-05 & 3.422-05 & 3.927-05 & 4.293-05\end{array}$ $\begin{array}{lllllllllll}1 & 181 & 1.226+01 & 0.000+00 & 0.000+00 & 3.678-06 & 1.764-06 & 8.042-07 & 3.581-07 & 1.583-07 & 7.270-08\end{array}$ 
Table II. Ca IX Oscillator Strengths, Radiative Decay Rates, and Collision Strengths for transitions involving the lowest 4 levels.

Low. Upp. Osc. Rad. Dec.

Lev. Lev.

Str. Rate

Collision Strength

Impact Electron Energy - Eo (Ry)

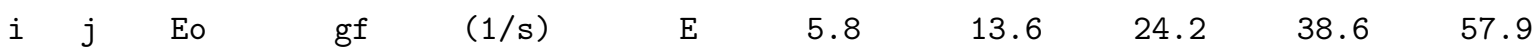

$\begin{array}{lllllllllll}1 & 182 & 1.226+01 & 0.000+00 & 0.000+00 & 5.400-06 & 3.680-06 & 3.187-06 & 3.192-06 & 3.412-06 & 3.774-06\end{array}$ $\begin{array}{llllllllllll}1 & 183 & 1.227+01 & 0.000+00 & 1.597+05 & 2.306-04 & 3.509-04 & 4.604-04 & 5.563-04 & 6.409-04 & 7.010-04\end{array}$

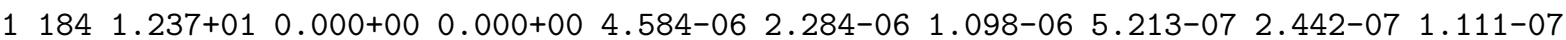
$\begin{array}{lllllllllll}1 & 185 & 1.237+01 & 0.000+00 & 0.000+00 & 1.541-06 & 7.786-07 & 3.895-07 & 2.055-07 & 1.251-07 & 9.435-08\end{array}$ $\begin{array}{lllllllllll}1 & 186 & 1.237+01 & 0.000+00 & 0.000+00 & 8.095-06 & 4.606-06 & 3.005-06 & 2.427-06 & 2.299-06 & 2.426-06\end{array}$ $\begin{array}{lllllllllll}1 & 187 & 1.238+01 & 0.000+00 & 5.509+04 & 1.878-05 & 2.298-05 & 2.999-05 & 3.822-05 & 4.580-05 & 5.409-05\end{array}$ $\begin{array}{lllllllllll}1 & 188 & 1.240+01 & 0.000+00 & 0.000+00 & 8.501-06 & 1.206-05 & 1.669-05 & 2.119-05 & 2.510-05 & 2.916-05\end{array}$ $\begin{array}{llllllllllll}1 & 189 & 1.241+01 & 0.000+00 & 0.000+00 & 2.277-06 & 9.407-07 & 3.791-07 & 1.609-07 & 8.167-08 & 4.536-08\end{array}$ $\begin{array}{lllllllllll}1 & 190 & 1.245+01 & 0.000+00 & 0.000+00 & 1.205-05 & 6.159-06 & 3.025-06 & 1.398-06 & 6.371-07 & 2.867-07\end{array}$ $\begin{array}{lllllllllll}1 & 191 & 1.246+01 & 0.000+00 & 0.000+00 & 1.491-06 & 8.534-07 & 4.687-07 & 2.535-07 & 1.450-07 & 9.043-08\end{array}$ $\begin{array}{lllllllllll}1 & 192 & 1.246+01 & 0.000+00 & 0.000+00 & 3.243-06 & 1.830-06 & 9.919-07 & 5.234-07 & 2.906-07 & 1.728-07\end{array}$ $\begin{array}{lllllllllll}1 & 193 & 1.246+01 & 0.000+00 & 0.000+00 & 2.036-06 & 1.135-06 & 6.634-07 & 3.597-07 & 2.122-07 & 1.458-07\end{array}$ $\begin{array}{lllllllllll}1 & 194 & 1.246+01 & 0.000+00 & 0.000+00 & 1.578-05 & 8.174-06 & 3.935-06 & 1.821-06 & 8.378-07 & 3.805-07\end{array}$ $\begin{array}{lllllllllll}1 & 195 & 1.246+01 & 0.000+00 & 0.000+00 & 1.958-05 & 9.958-06 & 4.882-06 & 2.245-06 & 1.016-06 & 4.510-07\end{array}$ $\begin{array}{llllllllllll}1 & 196 & 1.249+01 & 0.000+00 & 0.000+00 & 1.535-06 & 7.520-07 & 3.441-07 & 1.583-07 & 8.219-08 & 4.917-08\end{array}$ $\begin{array}{lllllllllllll}1 & 197 & 1.257+01 & 0.000+00 & 0.000+00 & 1.439-06 & 7.387-07 & 4.440-07 & 3.500-07 & 3.249-07 & 3.185-07\end{array}$ $\begin{array}{llllllllllll}1 & 198 & 1.257+01 & 0.000+00 & 0.000+00 & 2.080-06 & 8.954-07 & 3.526-07 & 1.540-07 & 8.038-08 & 4.432-08\end{array}$ $\begin{array}{llllllllllll}1 & 199 & 1.257+01 & 0.000+00 & 0.000+00 & 1.701-06 & 7.128-07 & 2.895-07 & 1.245-07 & 6.360-08 & 3.507-08\end{array}$ $\begin{array}{lllllllllll}1200 & 1.259+01 & 0.000+00 & 0.000+00 & 1.037-06 & 5.140-07 & 2.378-07 & 1.123-07 & 6.177-08 & 4.062-08\end{array}$ $\begin{array}{lllllllllll}1201 & 1.259+01 & 0.000+00 & 0.000+00 & 2.352-06 & 1.169-06 & 5.288-07 & 2.443-07 & 1.290-07 & 7.892-08\end{array}$ $\begin{array}{llllllllllll}1 & 202 & 1.259+01 & 0.000+00 & 0.000+00 & 1.681-06 & 8.309-07 & 3.808-07 & 1.747-07 & 8.961-08 & 5.317-08\end{array}$ $\begin{array}{llllllllll}1203 & 1.262+01 & 0.000+00 & 0.000+00 & 5.082-06 & 3.059-06 & 1.719-06 & 9.350-07 & 5.000-07 & 2.705-07\end{array}$ $\begin{array}{lllllllllll}1 & 204 & 1.262+01 & 0.000+00 & 0.000+00 & 3.070-06 & 1.831-06 & 1.036-06 & 5.600-07 & 3.104-07 & 1.738-07\end{array}$ $\begin{array}{lllllllllll}12 & 205 & 1.262+01 & 0.000+00 & 0.000+00 & 1.020-06 & 6.133-07 & 3.443-07 & 1.874-07 & 1.002-07 & 5.415-08\end{array}$ $\begin{array}{lllllllllllll}1206 & 1.265+01 & 0.000+00 & 0.000+00 & 7.916-05 & 1.068-04 & 1.280-04 & 1.445-04 & 1.572-04 & 1.663-04\end{array}$ $12071.266+01 \quad 0.000+00 \quad 0.000+00 \quad 3.691-06 \quad 3.238-06 \quad 3.322-06 \quad 3.738-06 \quad 4.311-06 \quad 4.900-06$ $12081.276+01 \quad 4.034-05 \quad 1.758+07 \quad 4.470-06 \quad 3.521-06 \quad 3.352-06 \quad 4.135-06 \quad 5.978-06 \quad 6.901-06$ $\begin{array}{lllllllllll}1209 & 1.279+01 & 0.000+00 & 0.000+00 & 6.649-06 & 9.118-06 & 1.200-05 & 1.555-05 & 1.805-05 & 1.939-05\end{array}$ $\begin{array}{llllllllllllll}1 & 210 & 1.356+01 & 0.000+00 & 0.000+00 & 1.298-06 & 7.293-07 & 4.032-07 & 2.146-07 & 1.091-07 & 5.388-08\end{array}$ $\begin{array}{llllllllllll}1 & 211 & 1.356+01 & 0.000+00 & 0.000+00 & 2.163-06 & 1.227-06 & 6.939-07 & 3.912-07 & 2.180-07 & 1.390-07\end{array}$ $\begin{array}{llllllllllll}1212 & 1.356+01 & 0.000+00 & 0.000+00 & 3.010-06 & 1.691-06 & 9.344-07 & 4.969-07 & 2.525-07 & 1.245-07\end{array}$ $\begin{array}{lllllllllll}1213 & 1.358+01 & 0.000+00 & 2.109+04 & 4.733-06 & 5.613-06 & 7.751-06 & 1.001-05 & 1.219-05 & 1.466-05\end{array}$ $\begin{array}{lllllllllll}1 & 214 & 1.381+01 & 0.000+00 & 0.000+00 & 2.655-07 & 1.241-07 & 5.668-08 & 2.637-08 & 1.257-08 & 5.488-09\end{array}$

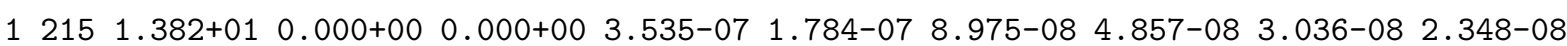
$\begin{array}{lllllllllll}1 & 216 & 1.383+01 & 0.000+00 & 0.000+00 & 5.821-07 & 2.867-07 & 1.354-07 & 6.310-08 & 2.924-08 & 1.256-08\end{array}$ $\begin{array}{lllllllllll}1217 & 1.383+01 & 0.000+00 & 0.000+00 & 8.213-07 & 4.067-07 & 1.950-07 & 9.458-08 & 4.864-08 & 2.667-08\end{array}$ $\begin{array}{llllllllllll}1218 & 1.384+01 & 0.000+00 & 0.000+00 & 8.492-07 & 4.723-07 & 2.585-07 & 1.414-07 & 7.702-08 & 4.180-08\end{array}$ $\begin{array}{lllllllllllll}1 & 219 & 1.384+01 & 0.000+00 & 0.000+00 & 1.196-06 & 6.684-07 & 3.662-07 & 1.987-07 & 1.097-07 & 6.076-08\end{array}$ $\begin{array}{llllllllllll}1220 & 1.385+01 & 0.000+00 & 0.000+00 & 1.555-06 & 8.671-07 & 4.757-07 & 2.604-07 & 1.421-07 & 7.706-08\end{array}$ $\begin{array}{llllllllllll}1221 & 1.387+01 & 0.000+00 & 0.000+00 & 1.336-06 & 7.221-07 & 3.717-07 & 1.843-07 & 8.806-08 & 4.125-08\end{array}$ $\begin{array}{llllllllllll}1 & 222 & 1.387+01 & 0.000+00 & 0.000+00 & 8.151-07 & 4.482-07 & 2.404-07 & 1.299-07 & 7.448-08 & 4.845-08\end{array}$

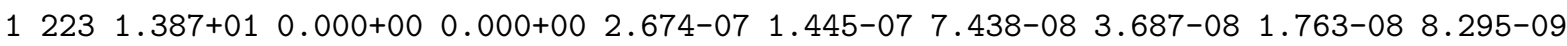
$\begin{array}{llllllllll}1224 & 1.390+01 & 0.000+00 & 0.000+00 & 2.266-06 & 2.651-06 & 3.476-06 & 4.475-06 & 5.332-06 & 6.062-06\end{array}$ $\begin{array}{llllllllll}1225 & 1.393+01 & 0.000+00 & 0.000+00 & 5.402-06 & 5.477-06 & 5.769-06 & 6.157-06 & 6.607-06 & 7.109-06\end{array}$ $\begin{array}{llllllllll}1226 & 1.416+01 & 0.000+00 & 0.000+00 & 7.840-07 & 3.415-07 & 1.434-07 & 5.974-08 & 2.617-08 & 1.216-08\end{array}$ 
Table II. Ca IX Oscillator Strengths, Radiative Decay Rates, and Collision Strengths for transitions involving the lowest 4 levels.

Low. Upp. Osc. Rad. Dec.

Lev. Lev.

Str. Rate

Collision Strength

Impact Electron Energy - Eo (Ry)

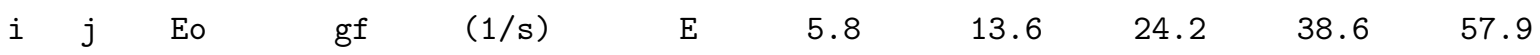

$\begin{array}{llllllllll}1227 & 1.418+01 & 0.000+00 & 0.000+00 & 7.354-07 & 3.571-07 & 1.740-07 & 8.583-08 & 4.303-08 & 2.178-08\end{array}$ $\begin{array}{llllllllllll}1228 & 1.418+01 & 0.000+00 & 0.000+00 & 1.219-06 & 5.919-07 & 2.886-07 & 1.425-07 & 7.148-08 & 3.607-08\end{array}$ $\begin{array}{lllllllllll}1 & 229 & 1.418+01 & 0.000+00 & 0.000+00 & 1.703-06 & 8.261-07 & 4.022-07 & 1.984-07 & 9.932-08 & 5.024-08\end{array}$ $\begin{array}{lllllllllll}1230 & 1.418+01 & 0.000+00 & 0.000+00 & 1.634-06 & 7.878-07 & 3.784-07 & 1.833-07 & 8.733-08 & 4.062-08\end{array}$ $\begin{array}{llllllllllll}1231 & 1.419+01 & 0.000+00 & 0.000+00 & 2.115-06 & 1.020-06 & 4.885-07 & 2.350-07 & 1.129-07 & 5.288-08\end{array}$ $\begin{array}{llllllllllll}1232 & 1.419+01 & 0.000+00 & 0.000+00 & 2.585-06 & 1.247-06 & 5.991-07 & 2.905-07 & 1.383-07 & 6.445-08\end{array}$ $\begin{array}{llllllllllll}1233 & 1.419+01 & 0.000+00 & 0.000+00 & 1.160-06 & 5.544-07 & 2.571-07 & 1.189-07 & 5.567-08 & 2.558-08\end{array}$ $\begin{array}{llllllllllll}1234 & 1.422+01 & 0.000+00 & 0.000+00 & 2.048-06 & 1.095-06 & 5.805-07 & 3.051-07 & 1.593-07 & 8.476-08\end{array}$ $\begin{array}{llllllllllll}1235 & 1.423+01 & 0.000+00 & 0.000+00 & 6.683-07 & 2.953-07 & 1.260-07 & 5.410-08 & 2.518-08 & 1.268-08\end{array}$ $\begin{array}{llllllllllll}1236 & 1.423+01 & 0.000+00 & 0.000+00 & 9.393-07 & 4.138-07 & 1.754-07 & 7.344-08 & 3.216-08 & 1.459-08\end{array}$ $\begin{array}{llllllllll}1237 & 1.423+01 & 0.000+00 & 0.000+00 & 1.211-06 & 5.354-07 & 2.271-07 & 9.515-08 & 4.217-08 & 1.943-08\end{array}$ $\begin{array}{llllllllll}1238 & 1.429+01 & 0.000+00 & 0.000+00 & 3.273-06 & 2.604-06 & 2.439-06 & 2.406-06 & 2.520-06 & 2.682-06\end{array}$ $\begin{array}{lllllllllll}1239 & 1.429+01 & 0.000+00 & 0.000+00 & 5.646-07 & 2.832-07 & 1.432-07 & 7.985-08 & 5.619-08 & 5.151-08\end{array}$ $\begin{array}{lllllllllllll}1240 & 1.429+01 & 0.000+00 & 0.000+00 & 1.667-06 & 8.168-07 & 3.860-07 & 1.807-07 & 8.472-08 & 3.864-08\end{array}$ $\begin{array}{llllllllllll}1 & 241 & 1.429+01 & 0.000+00 & 0.000+00 & 2.809-06 & 1.449-06 & 7.708-07 & 4.521-07 & 3.110-07 & 2.532-07\end{array}$ $\begin{array}{lllllllllll}1242 & 1.430+01 & 0.000+00 & 0.000+00 & 1.452-06 & 1.248-06 & 1.430-06 & 1.602-06 & 1.792-06 & 1.985-06\end{array}$ $\begin{array}{lllllllllll}1243 & 1.431+01 & 0.000+00 & 0.000+00 & 5.442-07 & 2.308-07 & 9.260-08 & 3.742-08 & 1.690-08 & 9.168-09\end{array}$ $\begin{array}{llllllllllll}1244 & 1.432+01 & 0.000+00 & 0.000+00 & 1.320-06 & 6.695-07 & 3.319-07 & 1.613-07 & 7.680-08 & 3.580-08\end{array}$ $\begin{array}{lllllllllll}1245 & 1.432+01 & 0.000+00 & 0.000+00 & 1.633-06 & 8.263-07 & 4.139-07 & 2.016-07 & 9.459-08 & 4.434-08\end{array}$ $\begin{array}{lllllllllllll}1 & 246 & 1.432+01 & 0.000+00 & 0.000+00 & 1.949-06 & 9.885-07 & 4.904-07 & 2.387-07 & 1.137-07 & 5.320-08\end{array}$ $\begin{array}{lllllllllll}1247 & 1.433+01 & 0.000+00 & 0.000+00 & 3.540-07 & 1.515-07 & 6.004-08 & 2.406-08 & 1.057-08 & 5.807-09\end{array}$ $\begin{array}{lllllllllll}1248 & 1.433+01 & 0.000+00 & 0.000+00 & 4.920-07 & 2.108-07 & 8.611-08 & 3.484-08 & 1.579-08 & 9.467-09\end{array}$

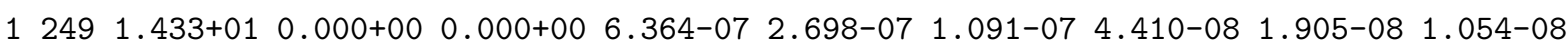
$\begin{array}{lllllllllllll}1250 & 1.435+01 & 0.000+00 & 0.000+00 & 9.042-07 & 4.311-07 & 1.921-07 & 8.374-08 & 3.883-08 & 2.056-08\end{array}$ $\begin{array}{llllllllllll}1251 & 1.437+01 & 0.000+00 & 0.000+00 & 1.279-07 & 4.610-08 & 1.665-08 & 7.120-09 & 3.590-09 & 1.814-09\end{array}$ $\begin{array}{lllllllllll}1252 & 1.438+01 & 0.000+00 & 0.000+00 & 4.857-07 & 2.205-07 & 1.049-07 & 6.046-08 & 4.576-08 & 4.050-08\end{array}$ $\begin{array}{lllllllllll}1253 & 1.438+01 & 0.000+00 & 0.000+00 & 1.149-07 & 4.709-08 & 2.077-08 & 1.225-08 & 9.190-09 & 8.146-09\end{array}$ $\begin{array}{llllllllllll}1254 & 1.438+01 & 0.000+00 & 0.000+00 & 6.139-07 & 2.607-07 & 1.046-07 & 4.233-08 & 1.925-08 & 1.046-08\end{array}$ $\begin{array}{llllllllllll}1255 & 1.438+01 & 0.000+00 & 0.000+00 & 1.429-07 & 5.454-08 & 2.069-08 & 8.952-09 & 4.406-09 & 2.167-09\end{array}$ $\begin{array}{lllllllllllll}1256 & 1.438+01 & 0.000+00 & 0.000+00 & 7.527-07 & 3.178-07 & 1.287-07 & 5.185-08 & 2.389-08 & 1.289-08\end{array}$ $\begin{array}{lllllllllll}1257 & 1.438+01 & 0.000+00 & 0.000+00 & 1.489-07 & 5.454-08 & 1.961-08 & 8.757-09 & 4.831-09 & 2.464-09\end{array}$ $\begin{array}{llllllllll}1258 & 1.438+01 & 0.000+00 & 0.000+00 & 2.119-07 & 9.960-08 & 4.573-08 & 2.020-08 & 8.653-09 & 3.587-09\end{array}$ $\begin{array}{llllllllll}1259 & 1.438+01 & 0.000+00 & 0.000+00 & 2.617-07 & 1.261-07 & 5.859-08 & 2.932-08 & 1.584-08 & 1.067-08\end{array}$

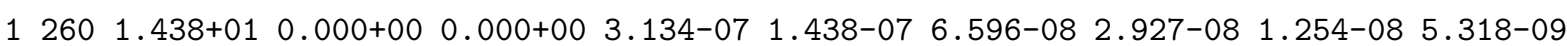
$\begin{array}{llllllllll}1261 & 1.439+01 & 0.000+00 & 0.000+00 & 2.501-07 & 1.086-07 & 4.443-08 & 1.891-08 & 9.481-09 & 4.918-09\end{array}$ $\begin{array}{lllllllllll}1262 & 1.439+01 & 0.000+00 & 0.000+00 & 6.943-07 & 3.360-07 & 1.524-07 & 6.742-08 & 3.181-08 & 1.752-08\end{array}$ $\begin{array}{llllllllllll}1263 & 1.439+01 & 0.000+00 & 0.000+00 & 1.162-06 & 5.635-07 & 2.556-07 & 1.132-07 & 5.278-08 & 2.785-08\end{array}$ $\begin{array}{lllllllllll}1264 & 1.439+01 & 0.000+00 & 0.000+00 & 1.620-06 & 8.170-07 & 4.081-07 & 2.247-07 & 1.519-07 & 1.240-07\end{array}$ $\begin{array}{lllllllllll}1265 & 1.439+01 & 0.000+00 & 0.000+00 & 7.141-07 & 8.744-07 & 1.123-06 & 1.273-06 & 1.394-06 & 1.529-06\end{array}$ $\begin{array}{llllllllllll}1266 & 1.440+01 & 0.000+00 & 0.000+00 & 4.546-07 & 1.781-07 & 7.829-08 & 3.589-08 & 1.594-08 & 6.650-09\end{array}$ $\begin{array}{llllllllllll}1267 & 1.440+01 & 0.000+00 & 0.000+00 & 2.033-07 & 8.805-08 & 3.829-08 & 1.954-08 & 1.334-08 & 1.062-08\end{array}$ $\begin{array}{lllllllllll}1268 & 1.440+01 & 0.000+00 & 0.000+00 & 2.801-07 & 1.211-07 & 4.897-08 & 2.115-08 & 1.071-08 & 5.701-09\end{array}$ $\begin{array}{lllllllllll}1 & 269 & 1.440+01 & 0.000+00 & 0.000+00 & 4.239-07 & 2.936-07 & 2.612-07 & 2.607-07 & 2.727-07 & 2.923-07\end{array}$ $\begin{array}{lllllllllll}1270 & 1.440+01 & 0.000+00 & 0.000+00 & 5.404-07 & 2.101-07 & 9.440-08 & 4.497-08 & 1.993-08 & 9.475-09\end{array}$ $\begin{array}{llllllllll}1271 & 1.440+01 & 0.000+00 & 0.000+00 & 6.301-07 & 2.466-07 & 1.085-07 & 4.970-08 & 2.200-08 & 9.119-09\end{array}$ 
Table II. Ca IX Oscillator Strengths, Radiative Decay Rates, and Collision Strengths for transitions involving the lowest 4 levels.

Low. Upp. Osc. Rad. Dec.

Lev. Lev.

Str. Rate

Collision Strength

Impact Electron Energy - Eo (Ry)

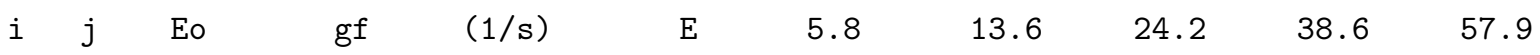

$\begin{array}{lllllllllllll}1 & 272 & 1.440+01 & 0.000+00 & 0.000+00 & 2.770-06 & 1.601-06 & 8.711-07 & 4.559-07 & 2.321-07 & 1.165-07\end{array}$ $\begin{array}{llllllllllll}1 & 273 & 1.440+01 & 0.000+00 & 0.000+00 & 1.676-06 & 9.700-07 & 5.258-07 & 2.786-07 & 1.397-07 & 7.213-08\end{array}$

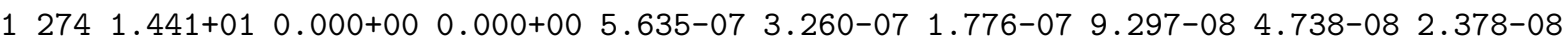
$\begin{array}{lllllllllll}1275 & 1.441+01 & 0.000+00 & 0.000+00 & 9.153-06 & 1.342-05 & 1.727-05 & 2.032-05 & 2.244-05 & 2.389-05\end{array}$ $\begin{array}{lllllllllllll}1276 & 1.442+01 & 0.000+00 & 0.000+00 & 1.501-07 & 2.508-07 & 3.376-07 & 4.047-07 & 4.473-07 & 5.077-07\end{array}$ $\begin{array}{lllllllllllllll}1277 & 1.443+01 & 0.000+00 & 0.000+00 & 8.881-07 & 4.884-07 & 2.574-07 & 1.263-07 & 5.875-08 & 2.723-08\end{array}$ $\begin{array}{lllllllllll}1278 & 1.443+01 & 0.000+00 & 0.000+00 & 6.397-07 & 3.592-07 & 2.052-07 & 1.210-07 & 8.374-08 & 6.409-08\end{array}$ $\begin{array}{llllllllllll}1279 & 1.443+01 & 0.000+00 & 0.000+00 & 3.799-07 & 2.086-07 & 1.100-07 & 5.390-08 & 2.502-08 & 1.159-08\end{array}$ $\begin{array}{llllllllll}1280 & 1.444+01 & 0.000+00 & 0.000+00 & 9.772-07 & 2.055-06 & 3.192-06 & 4.718-06 & 5.638-06 & 6.613-06\end{array}$ $\begin{array}{llllllllll}1281 & 1.445+01 & 0.000+00 & 0.000+00 & 2.842-06 & 3.055-06 & 3.386-06 & 3.802-06 & 4.284-06 & 4.788-06\end{array}$ $\begin{array}{llllllllll}1282 & 1.447+01 & 0.000+00 & 0.000+00 & 6.157-07 & 7.894-07 & 1.060-06 & 1.399-06 & 1.776-06 & 2.064-06\end{array}$ $\begin{array}{llllllllll}1283 & 1.448+01 & 2.781-05 & 1.562+07 & 4.418-06 & 3.602-06 & 3.117-06 & 2.706-06 & 2.820-06 & 2.653-06\end{array}$ $\begin{array}{llllllllll}3 & 1.353-02 & 0.000+00 & 0.000+00 & 8.447-02 & 4.400-02 & 2.368-02 & 1.291-02 & 7.024-03 & 3.785-03\end{array}$ $\begin{array}{llllllllll}4 & 4.262-02 & 0.000+00 & 3.336-05 & 2.820-01 & 2.508-01 & 2.433-01 & 2.415-01 & 2.414-01 & 2.422-01\end{array}$

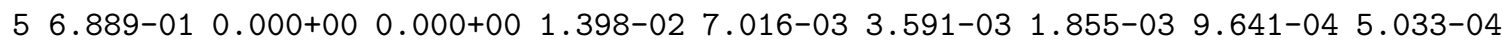
$\begin{array}{llllllllll}6 & 1.762+00 & 0.000+00 & 0.000+00 & 2.873-02 & 1.650-02 & 8.887-03 & 4.743-03 & 2.535-03 & 1.351-03\end{array}$ $\begin{array}{llllllllll}7 & 1.807+00 & 0.000+00 & 0.000+00 & 5.572-03 & 3.220-03 & 1.733-03 & 9.269-04 & 4.997-04 & 2.698-04\end{array}$ $\begin{array}{lllllllllll}8 & 1.825+00 & 3.717-01 & 3.315+09 & 1.992+00 & 2.429+00 & 2.826+00 & 3.196+00 & 3.547+00 & 3.876+00\end{array}$ $\begin{array}{lllllllllll}9 & 1.857+00 & 0.000+00 & 0.000+00 & 2.732-03 & 1.558-03 & 8.307-04 & 4.428-04 & 2.394-04 & 1.303-04\end{array}$ $\begin{array}{lllllllllll}10 & 2.376+00 & 0.000+00 & 0.000+00 & 1.405-03 & 8.081-04 & 4.344-04 & 2.314-04 & 1.237-04 & 6.602-05\end{array}$

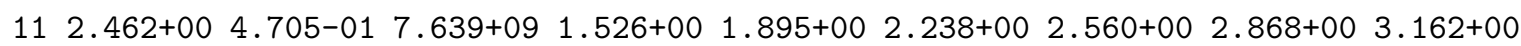

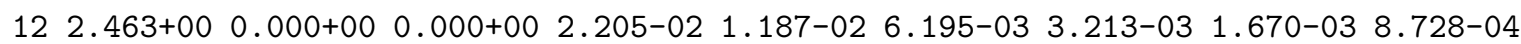
$\begin{array}{lllllllllll}13 & 2.465+00 & 0.000+00 & 0.000+00 & 3.662-02 & 3.463-02 & 3.535-02 & 3.718-02 & 3.949-02 & 4.166-02\end{array}$ $\begin{array}{llllllllll}14 & 3.013+00 & 0.000+00 & 0.000+00 & 1.031-02 & 5.359-03 & 2.747-03 & 1.411-03 & 7.312-04 & 3.839-04\end{array}$ $\begin{array}{llllllllll}15 & 3.832+00 & 0.000+00 & 2.177+05 & 1.690-01 & 1.974-01 & 2.177-01 & 2.325-01 & 2.431-01 & 2.502-01\end{array}$

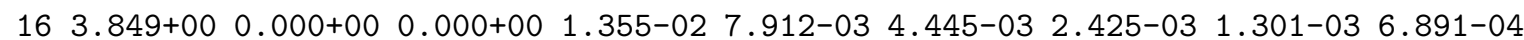
$\begin{array}{lllllllllll}17 & 3.872+00 & 0.000+00 & 0.000+00 & 1.699-04 & 1.214-04 & 1.114-04 & 1.154-04 & 1.238-04 & 1.324-04\end{array}$ $\begin{array}{lllllllllll}18 & 3.908+00 & 0.000+00 & 1.391+04 & 1.295-02 & 1.330-02 & 1.368-02 & 1.408-02 & 1.443-02 & 1.471-02\end{array}$ $\begin{array}{lllllllllllll}19 & 4.164+00 & 0.000+00 & 1.953+05 & 1.001-01 & 1.180-01 & 1.310-01 & 1.407-01 & 1.477-01 & 1.524-01\end{array}$ $\begin{array}{llllllllll}20 & 4.174+00 & 0.000+00 & 0.000+00 & 5.172-03 & 3.046-03 & 1.724-03 & 9.453-04 & 5.096-04 & 2.724-04\end{array}$ $\begin{array}{llllllllllll}21 & 4.189+00 & 0.000+00 & 0.000+00 & 1.925-04 & 1.969-04 & 1.981-04 & 1.975-04 & 1.959-04 & 1.948-04\end{array}$ $\begin{array}{llllllllll}22 & 4.198+00 & 0.000+00 & 0.000+00 & 7.989-04 & 4.742-04 & 2.701-04 & 1.487-04 & 8.039-05 & 4.304-05\end{array}$ $\begin{array}{llllllllllllllll}23 & 4.203+00 & 0.000+00 & 4.580+04 & 2.084-02 & 2.505-02 & 2.810-02 & 3.032-02 & 3.191-02 & 3.298-02\end{array}$ $\begin{array}{llllllllll}24 & 4.204+00 & 0.000+00 & 0.000+00 & 4.094-03 & 2.416-03 & 1.370-03 & 7.515-04 & 4.053-04 & 2.166-04\end{array}$

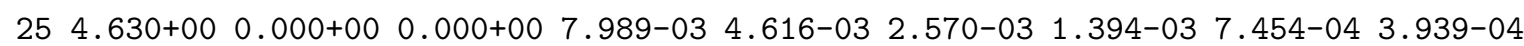
$\begin{array}{lllllllllll}26 & 4.722+00 & 0.000+00 & 0.000+00 & 1.960-03 & 1.151-03 & 6.506-04 & 3.571-04 & 1.933-04 & 1.048-04\end{array}$ $\begin{array}{llllllllllll}27 & 5.620+00 & 8.978-02 & 7.592+09 & 1.708-02 & 3.164-02 & 4.865-02 & 6.734-02 & 8.635-02 & 1.059-01\end{array}$ $\begin{array}{llllllllll}28 & 5.762+00 & 0.000+00 & 0.000+00 & 1.180-03 & 6.531-04 & 3.270-04 & 1.596-04 & 7.635-05 & 3.625-05\end{array}$ $\begin{array}{llllllllllll}29 & 6.261+00 & 0.000+00 & 0.000+00 & 1.568-01 & 1.677-01 & 1.739-01 & 1.770-01 & 1.783-01 & 1.787-01\end{array}$ $\begin{array}{llllllllll}30 & 6.264+00 & 0.000+00 & 0.000+00 & 4.425-03 & 2.135-03 & 1.090-03 & 5.613-04 & 2.848-04 & 1.467-04\end{array}$ $\begin{array}{lllllllllll}31 & 6.272+00 & 0.000+00 & 0.000+00 & 6.584-05 & 3.452-05 & 1.756-05 & 8.824-06 & 4.420-06 & 2.213-06\end{array}$ $\begin{array}{llllllllllll}32 & 6.274+00 & 0.000+00 & 0.000+00 & 2.440-04 & 2.796-04 & 3.077-04 & 3.292-04 & 3.467-04 & 3.608-04\end{array}$ $\begin{array}{lllllllllll}33 & 6.276+00 & 0.000+00 & 9.995+05 & 1.664-02 & 2.009-02 & 2.445-02 & 2.810-02 & 3.097-02 & 3.308-02\end{array}$ $\begin{array}{llllllllll}34 & 6.276+00 & 0.000+00 & 0.000+00 & 4.932-05 & 2.676-05 & 1.412-05 & 7.348-06 & 3.792-06 & 1.923-06\end{array}$

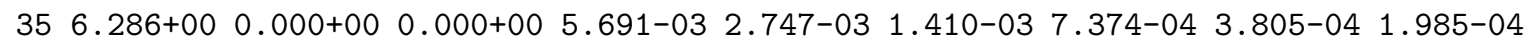


Table II. Ca IX Oscillator Strengths, Radiative Decay Rates, and Collision Strengths for transitions involving the lowest 4 levels.

Low. Upp. Osc. Rad. Dec. Lev. Lev. Rate
Collision Strength

Impact Electron Energy - Eo (Ry)

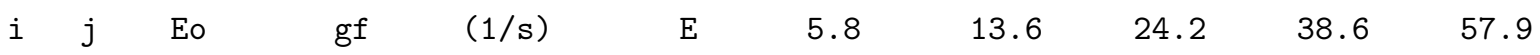

$236 \quad 6.457+00 \quad 0.000+00 \quad 0.000+00 \quad 2.622-04 \quad 1.401-04 \quad 7.294-05 \quad 3.748-05 \quad 1.911-05 \quad 9.565-06$

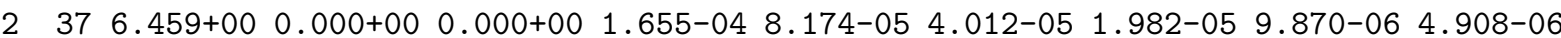

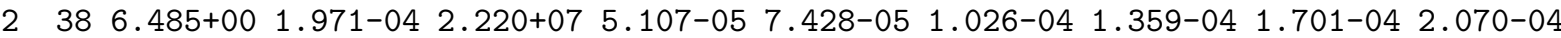
$2396.485+00 \quad 0.000+00 \quad 0.000+00 \quad 3.456-06 \quad 1.891-06 \quad 1.031-06 \quad 5.724-07 \quad 3.251-07 \quad 1.945-07$ $2406.486+00 \quad 0.000+00 \quad 0.000+00 \quad 7.604-06 \quad 3.906-06 \quad 1.971-06 \quad 1.003-06 \quad 5.171-07 \quad 2.796-07$ $2416.983+00 \quad 0.000+00 \quad 0.000+00 \quad 1.205-05 \quad 6.741-06 \quad 3.519-06 \quad 1.753-06 \quad 8.264-07 \quad 4.125-07$ $2427.055+001.987-01 \quad 2.648+10 \quad 1.377-02 \quad 3.083-02 \quad 5.364-02 \quad 8.052-02 \quad 1.096-01 \quad 1.406-01$ $243 \quad 7.056+00 \quad 0.000+00 \quad 0.000+00 \quad 5.249-03 \quad 2.848-03 \quad 1.474-03 \quad 7.424-04 \quad 3.673-04 \quad 1.794-04$

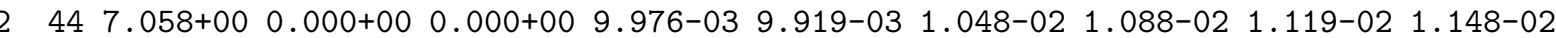
$\begin{array}{lllllllllll}45 & 7.073+00 & 0.000+00 & 0.000+00 & 4.899-03 & 2.609-03 & 1.348-03 & 6.817-04 & 3.383-04 & 1.657-04\end{array}$

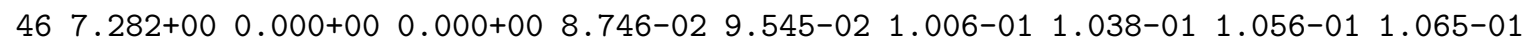
$47 \quad 7.293+00 \quad 0.000+00 \quad 0.000+00 \quad 2.573-03 \quad 1.304-03 \quad 7.041-04 \quad 3.885-04 \quad 2.077-04 \quad 1.065-04$ $48 \quad 7.326+00 \quad 0.000+00 \quad 0.000+00 \quad 4.211-05 \quad 4.176-05 \quad 4.788-05 \quad 5.409-05 \quad 5.994-05 \quad 6.465-05$

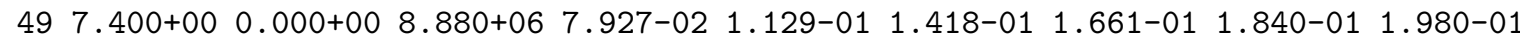
$\begin{array}{llllllllll}50 & 7.401+00 & 0.000+00 & 0.000+00 & 1.121-02 & 5.119-03 & 2.387-03 & 1.143-03 & 5.435-04 & 2.498-04\end{array}$ $\begin{array}{llllllllllll}51 & 7.401+00 & 0.000+00 & 0.000+00 & 1.108-02 & 1.068-02 & 1.137-02 & 1.246-02 & 1.377-02 & 1.475-02\end{array}$ $\begin{array}{lllllllllllll}52 & 7.415+00 & 0.000+00 & 0.000+00 & 5.183-04 & 2.595-04 & 1.384-04 & 7.548-05 & 3.994-05 & 2.018-05\end{array}$ $\begin{array}{lllllllllll}53 & 7.497+00 & 0.000+00 & 0.000+00 & 9.199-03 & 4.171-03 & 1.934-03 & 9.237-04 & 4.363-04 & 1.991-04\end{array}$ $\begin{array}{llllllllllll}54 & 7.797+00 & 2.806-03 & 4.568+08 & 1.532-03 & 1.133-03 & 1.027-03 & 1.160-03 & 1.419-03 & 1.754-03\end{array}$ $\begin{array}{llllllllllllll}55 & 7.851+00 & 5.194-02 & 8.571+09 & 4.913-03 & 7.560-03 & 1.197-02 & 1.769-02 & 2.412-02 & 3.108-02\end{array}$ $\begin{array}{llllllllll}56 & 7.862+00 & 0.000+00 & 0.000+00 & 1.794-03 & 9.988-04 & 5.086-04 & 2.540-04 & 1.274-04 & 6.612-05\end{array}$ $\begin{array}{lllllllllll}57 & 7.893+00 & 0.000+00 & 0.000+00 & 2.128-04 & 2.249-04 & 2.439-04 & 2.572-04 & 2.670-04 & 2.737-04\end{array}$ $\begin{array}{lllllllllll}58 & 7.930+00 & 0.000+00 & 0.000+00 & 5.449-04 & 3.105-04 & 1.608-04 & 8.076-05 & 4.019-05 & 2.048-05\end{array}$ $\begin{array}{lllllllllll}59 & 7.936+00 & 4.431-02 & 7.473+09 & 4.513-03 & 6.355-03 & 9.770-03 & 1.436-02 & 1.965-02 & 2.544-02\end{array}$ $\begin{array}{lllllllllll}60 & 7.959+00 & 0.000+00 & 0.000+00 & 7.231-05 & 5.359-05 & 4.151-05 & 3.434-05 & 3.182-05 & 3.054-05\end{array}$ $\begin{array}{llllllllllll}61 & 7.961+00 & 0.000+00 & 0.000+00 & 5.741-04 & 3.255-04 & 1.682-04 & 8.453-05 & 4.220-05 & 2.160-05\end{array}$ $\begin{array}{lllllllllll}62 & 8.065+00 & 0.000+00 & 0.000+00 & 6.771-04 & 3.736-04 & 1.903-04 & 9.533-05 & 4.808-05 & 2.503-05\end{array}$ $\begin{array}{llllllllllll}63 & 8.205+00 & 0.000+00 & 0.000+00 & 2.688-04 & 1.583-04 & 8.288-05 & 4.174-05 & 2.068-05 & 1.040-05\end{array}$ $\begin{array}{lllllllllllll}64 & 8.429+00 & 1.590-02 & 3.024+09 & 2.284-03 & 3.400-03 & 4.893-03 & 6.729-03 & 8.754-03 & 1.081-02\end{array}$ $\begin{array}{lllllllllllll}65 & 8.527+00 & 0.000+00 & 0.000+00 & 3.205-04 & 1.720-04 & 8.436-05 & 4.123-05 & 2.033-05 & 9.454-06\end{array}$ $\begin{array}{llllllllllll}66 & 8.643+00 & 0.000+00 & 0.000+00 & 1.854-03 & 9.397-04 & 5.004-04 & 2.637-04 & 1.353-04 & 6.727-05\end{array}$ $\begin{array}{lllllllllll}67 & 8.643+00 & 0.000+00 & 1.915+06 & 5.683-03 & 6.827-03 & 8.381-03 & 9.894-03 & 1.124-02 & 1.245-02\end{array}$ $\begin{array}{lllllllllll}68 & 8.652+00 & 0.000+00 & 2.175+06 & 4.678-03 & 6.636-03 & 8.724-03 & 1.056-02 & 1.217-02 & 1.358-02\end{array}$ $\begin{array}{lllllllllll}69 & 8.654+00 & 0.000+00 & 0.000+00 & 2.826-03 & 1.464-03 & 7.896-04 & 4.219-04 & 2.209-04 & 1.122-04\end{array}$ $\begin{array}{lllllllllll}70 & 8.686+00 & 0.000+00 & 0.000+00 & 1.501-03 & 7.332-04 & 3.789-04 & 1.973-04 & 9.997-05 & 4.995-05\end{array}$ $\begin{array}{llllllllllll}71 & 8.687+00 & 0.000+00 & 0.000+00 & 2.386-02 & 2.603-02 & 2.750-02 & 2.845-02 & 2.900-02 & 2.933-02\end{array}$ $\begin{array}{lllllllllll}72 & 8.690+00 & 0.000+00 & 1.049+06 & 4.187-03 & 4.727-03 & 5.537-03 & 6.475-03 & 7.300-03 & 7.943-03\end{array}$ $\begin{array}{llllllllllll}73 & 8.695+00 & 0.000+00 & 0.000+00 & 1.657-03 & 7.933-04 & 4.050-04 & 2.129-04 & 1.099-04 & 5.613-05\end{array}$ $\begin{array}{llllllllll}74 & 8.703+00 & 0.000+00 & 9.162+05 & 2.394-03 & 3.085-03 & 3.918-03 & 4.643-03 & 5.329-03 & 5.907-03\end{array}$ $\begin{array}{llllllllllll}75 & 8.707+00 & 0.000+00 & 0.000+00 & 2.142-03 & 1.152-03 & 6.315-04 & 3.413-04 & 1.806-04 & 9.336-05\end{array}$ $\begin{array}{llllllllll}76 & 8.731+00 & 0.000+00 & 0.000+00 & 1.056-04 & 8.016-05 & 7.553-05 & 7.910-05 & 8.712-05 & 9.550-05\end{array}$ $\begin{array}{llllllllll}77 & 8.735+00 & 0.000+00 & 0.000+00 & 1.685-04 & 8.992-05 & 4.768-05 & 2.515-05 & 1.314-05 & 6.714-06\end{array}$ $\begin{array}{llllllllllll}78 & 8.818+00 & 0.000+00 & 5.126+04 & 5.844-04 & 3.645-04 & 3.032-04 & 2.876-04 & 3.219-04 & 3.360-04\end{array}$ $\begin{array}{llllllllllll}79 & 8.824+00 & 0.000+00 & 0.000+00 & 4.556-04 & 2.164-04 & 1.112-04 & 6.018-05 & 3.296-05 & 1.768-05\end{array}$ $808.829+00 \quad 0.000+00 \quad 0.000+00 \quad 6.672-03 \quad 7.285-03 \quad 7.708-03 \quad 7.985-03 \quad 8.146-03 \quad 8.241-03$ 
Table II. Ca IX Oscillator Strengths, Radiative Decay Rates, and Collision Strengths for transitions involving the lowest 4 levels.

Low. Upp. Osc. Rad. Dec.

Lev. Lev.

Str. Rate

Collision Strength

Impact Electron Energy - Eo (Ry)

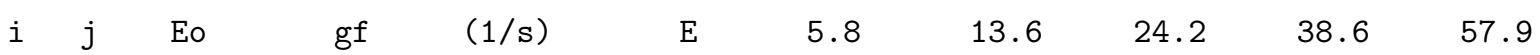

$2818.878+00 \quad 0.000+00 \quad 0.000+00 \quad 4.962-04 \quad 2.352-04 \quad 1.202-04 \quad 6.503-05 \quad 3.575-05 \quad 1.917-05$

$\begin{array}{llllllllllll}2 & 82 & 8.936+00 & 0.000+00 & 0.000+00 & 2.334-02 & 2.667-02 & 2.971-02 & 3.196-02 & 3.345-02 & 3.434-02\end{array}$

$2838.956+00 \quad 0.000+00 \quad 0.000+00 \quad 8.342-03 \quad 4.211-03 \quad 2.080-03 \quad 1.015-03 \quad 4.830-04 \quad 2.201-04$

$2 \quad 84 \quad 8.962+00 \quad 0.000+00 \quad 0.000+00 \quad 1.627-02 \quad 2.131-02 \quad 2.509-02 \quad 2.764-02 \quad 2.926-02 \quad 3.021-02$

$\begin{array}{llllllllllll}2 & 85 & 8.977+00 & 0.000+00 & 0.000+00 & 3.474-04 & 2.532-04 & 2.526-04 & 2.774-04 & 2.952-04 & 3.055-04\end{array}$

$2868.991+00 \quad 0.000+00 \quad 0.000+00 \quad 3.330-03 \quad 1.716-03 \quad 8.585-04 \quad 4.225-04 \quad 2.029-04 \quad 9.321-05$

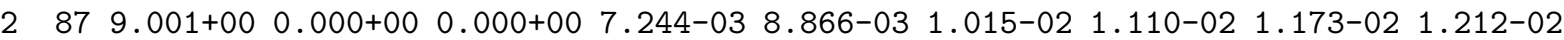

$2888.007+00 \quad 0.000+00 \quad 0.000+00 \quad 8.260-04 \quad 4.333-04 \quad 2.198-04 \quad 1.097-04 \quad 5.369-05 \quad 2.539-05$

$2899.052+00 \quad 0.000+00 \quad 0.000+00 \quad 7.323-03 \quad 7.996-03 \quad 8.792-03 \quad 9.482-03 \quad 9.982-03 \quad 1.025-02$

$290 \quad 9.057+00 \quad 0.000+00 \quad 0.000+00 \quad 1.743-03 \quad 8.849-04 \quad 4.378-04 \quad 2.124-04 \quad 1.008-04 \quad 4.560-05$

$\begin{array}{lllllllllll}91 & 9.061+00 & 5.225-02 & 1.149+10 & 3.011-03 & 6.056-03 & 1.003-02 & 1.486-02 & 2.017-02 & 2.581-02\end{array}$

$2929.083+00 \quad 0.000+00 \quad 0.000+00 \quad 2.257-03 \quad 1.170-03 \quad 5.910-04 \quad 2.936-04 \quad 1.434-04 \quad 6.765-05$

$\begin{array}{lllllllllll}93 & 9.104+00 & 0.000+00 & 0.000+00 & 3.223-03 & 1.522-03 & 7.158-04 & 3.361-04 & 1.547-04 & 6.785-05\end{array}$

$\begin{array}{llllllllll}94 & 9.143+00 & 0.000+00 & 0.000+00 & 1.977-03 & 1.516-03 & 1.340-03 & 1.291-03 & 1.283-03 & 1.323-03\end{array}$

$\begin{array}{lllllllllll}95 & 9.148+00 & 0.000+00 & 0.000+00 & 1.237-03 & 6.962-04 & 3.756-04 & 1.979-04 & 1.031-04 & 5.327-05\end{array}$

$\begin{array}{llllllllll}96 & 9.153+00 & 3.198-02 & 7.175+09 & 1.728-03 & 3.402-03 & 5.667-03 & 8.471-03 & 1.159-02 & 1.497-02\end{array}$

$297 \quad 9.229+00 \quad 0.000+00 \quad 0.000+00 \quad 8.416-04 \quad 4.570-04 \quad 2.384-04 \quad 1.220-04 \quad 6.115-05 \quad 3.031-05$

$298 \quad 9.258+00 \quad 0.000+00 \quad 4.193+06 \quad 2.131-02 \quad 2.809-02 \quad 3.414-02 \quad 3.946-02 \quad 4.394-02 \quad 4.732-02$

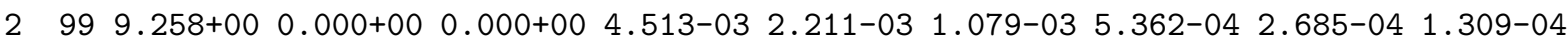

$\begin{array}{lllllllllll}2 & 100 & 9.259+00 & 0.000+00 & 0.000+00 & 3.391-03 & 2.678-03 & 2.674-03 & 2.892-03 & 3.189-03 & 3.531-03\end{array}$

$\begin{array}{lllllllllllll}2 & 101 & 9.296+00 & 0.000+00 & 0.000+00 & 4.366-03 & 2.137-03 & 1.042-03 & 5.177-04 & 2.585-04 & 1.256-04\end{array}$

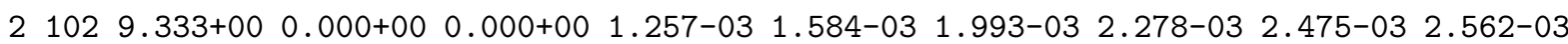

$2103 \quad 9.334+00 \quad 0.000+00 \quad 0.000+00 \quad 1.080-03 \quad 3.945-04 \quad 1.514-04 \quad 6.094-05 \quad 2.557-05 \quad 1.112-05$

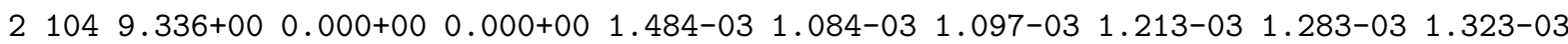

$\begin{array}{llllllllllllll}2 & 105 & 9.407+00 & 0.000+00 & 0.000+00 & 7.610-04 & 3.177-04 & 1.386-04 & 6.274-05 & 2.943-05 & 1.420-05\end{array}$

$2106 \quad 9.534+00 \quad 4.223-04 \quad 1.028+08 \quad 3.269-05 \quad 3.461-05 \quad 4.777-05 \quad 7.141-05 \quad 1.020-04 \quad 1.392-04$

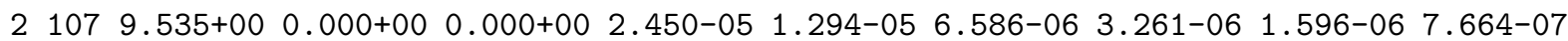

$\begin{array}{llllllllllll}2 & 108 & 9.537+00 & 0.000+00 & 0.000+00 & 8.779-05 & 9.832-05 & 1.113-04 & 1.217-04 & 1.286-04 & 1.326-04\end{array}$

$\begin{array}{lllllllllllll}2 & 109 & 9.611+00 & 0.000+00 & 0.000+00 & 1.493-04 & 8.308-05 & 4.458-05 & 2.344-05 & 1.211-05 & 6.068-06\end{array}$

$\begin{array}{lllllllllllllll}2 & 110 & 1.002+01 & 0.000+00 & 0.000+00 & 1.729-02 & 1.925-02 & 2.066-02 & 2.162-02 & 2.223-02 & 2.262-02\end{array}$

$\begin{array}{llllllllllll}2 & 111 & 1.003+01 & 0.000+00 & 0.000+00 & 1.194-03 & 5.998-04 & 3.227-04 & 1.790-04 & 9.701-05 & 4.993-05\end{array}$

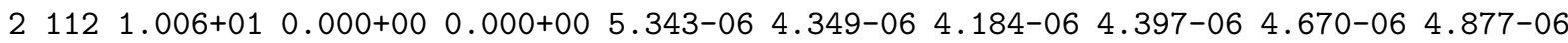

$21131.006+01 \quad 0.000+00 \quad 0.000+00 \quad 4.092-06 \quad 3.697-06 \quad 3.824-06 \quad 4.111-06 \quad 4.355-06 \quad 4.571-06$

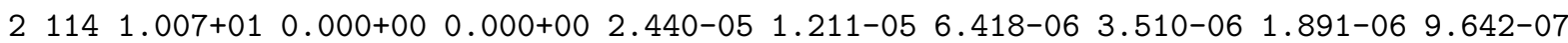

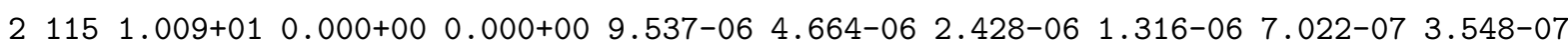

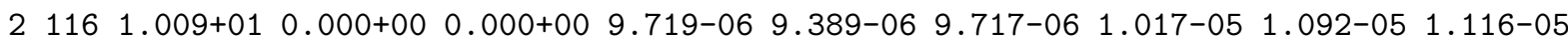

$\begin{array}{llllllllllll}2 & 117 & 1.010+01 & 0.000+00 & 0.000+00 & 1.137-06 & 5.740-07 & 2.932-07 & 1.523-07 & 7.630-08 & 4.019-08\end{array}$

$\begin{array}{lllllllllll}2 & 118 & 1.011+01 & 0.000+00 & 1.842+04 & 4.172-05 & 5.485-05 & 6.754-05 & 8.047-05 & 9.128-05 & 9.848-05\end{array}$

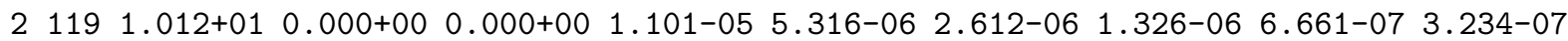

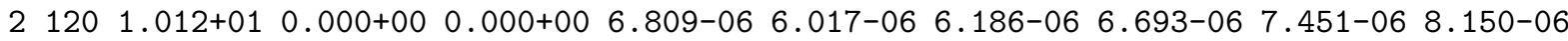

$\begin{array}{lllllllllllll}2 & 121 & 1.021+01 & 0.000+00 & 0.000+00 & 1.777-03 & 1.986-03 & 2.136-03 & 2.242-03 & 2.311-03 & 2.356-03\end{array}$

$\begin{array}{llllllllllll}2 & 122 & 1.021+01 & 0.000+00 & 0.000+00 & 1.065-04 & 5.355-05 & 2.891-05 & 1.614-05 & 8.818-06 & 4.460-06\end{array}$

$\begin{array}{lllllllllll}2 & 123 & 1.021+01 & 0.000+00 & 0.000+00 & 6.438-06 & 6.566-06 & 7.080-06 & 7.624-06 & 8.133-06 & 8.605-06\end{array}$

$\begin{array}{lllllllllll}2 & 124 & 1.024+01 & 0.000+00 & 0.000+00 & 9.420-06 & 4.447-06 & 2.250-06 & 1.185-06 & 6.103-07 & 3.178-07\end{array}$

$\begin{array}{lllllllllll}2 & 125 & 1.030+01 & 4.854-03 & 1.378+09 & 9.149-04 & 7.843-04 & 8.561-04 & 1.112-03 & 1.477-03 & 1.892-03\end{array}$ 
Table II. Ca IX Oscillator Strengths, Radiative Decay Rates, and Collision Strengths for transitions involving the lowest 4 levels.

Low. Upp. Osc. Rad. Dec.

Lev. Lev.

Str. Rate

Collision Strength

Impact Electron Energy - Eo (Ry)

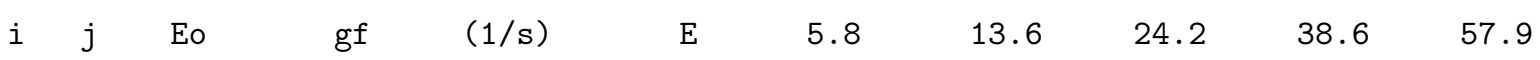

$\begin{array}{llllllllllll}2 & 126 & 1.032+01 & 3.673-02 & 1.047+10 & 1.796-03 & 2.901-03 & 4.752-03 & 7.337-03 & 1.041-02 & 1.367-02\end{array}$ $\begin{array}{llllllllllll}2 & 127 & 1.032+01 & 0.000+00 & 0.000+00 & 7.926-05 & 3.840-05 & 1.990-05 & 1.056-05 & 5.476-06 & 2.659-06\end{array}$ $\begin{array}{llllllllllll}2 & 128 & 1.033+01 & 0.000+00 & 0.000+00 & 1.165-03 & 6.627-04 & 3.409-04 & 1.715-04 & 8.538-05 & 4.227-05\end{array}$ $\begin{array}{lllllllllllll}2 & 129 & 1.034+01 & 0.000+00 & 0.000+00 & 2.695-04 & 1.549-04 & 8.024-05 & 4.047-05 & 2.010-05 & 9.817-06\end{array}$

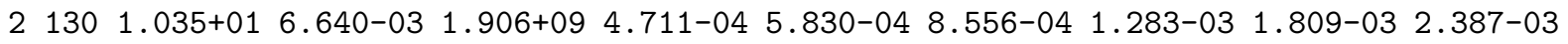
$\begin{array}{llllllllllll}2 & 131 & 1.036+01 & 0.000+00 & 0.000+00 & 1.560-05 & 1.263-05 & 1.171-05 & 1.143-05 & 1.154-05 & 1.188-05\end{array}$ $\begin{array}{lllllllllll}2 & 132 & 1.037+01 & 0.000+00 & 0.000+00 & 1.017-04 & 5.817-05 & 3.010-05 & 1.521-05 & 7.595-06 & 3.708-06\end{array}$

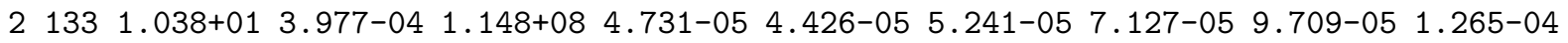
$\begin{array}{llllllllllll}2 & 134 & 1.043+01 & 0.000+00 & 0.000+00 & 2.581-04 & 1.444-04 & 7.389-05 & 3.719-05 & 1.867-05 & 9.419-06\end{array}$ $\begin{array}{lllllllllll}2 & 135 & 1.048+01 & 0.000+00 & 0.000+00 & 5.322-05 & 3.064-05 & 1.599-05 & 8.034-06 & 3.945-06 & 1.949-06\end{array}$ $\begin{array}{lllllllllll}2 & 136 & 1.069+01 & 0.000+00 & 1.648+05 & 1.515-03 & 8.706-04 & 5.998-04 & 4.916-04 & 4.492-04 & 4.454-04\end{array}$ $\begin{array}{llllllllllll}2 & 137 & 1.070+01 & 0.000+00 & 5.551+06 & 4.220-03 & 6.514-03 & 8.692-03 & 1.081-02 & 1.260-02 & 1.414-02\end{array}$ $\begin{array}{llllllllllll}2 & 138 & 1.071+01 & 0.000+00 & 0.000+00 & 7.271-04 & 3.555-04 & 1.860-04 & 9.777-05 & 5.078-05 & 2.548-05\end{array}$ $\begin{array}{llllllllllllll}2 & 139 & 1.071+01 & 0.000+00 & 0.000+00 & 2.035-03 & 1.008-03 & 5.319-04 & 2.806-04 & 1.458-04 & 7.327-05\end{array}$ $\begin{array}{llllllllllll}2 & 140 & 1.073+01 & 0.000+00 & 1.097+05 & 2.084-04 & 1.749-04 & 1.782-04 & 1.973-04 & 2.193-04 & 2.414-04\end{array}$ $\begin{array}{llllllllllll}2 & 141 & 1.074+01 & 0.000+00 & 0.000+00 & 1.711-04 & 8.679-05 & 4.605-05 & 2.432-05 & 1.264-05 & 6.411-06\end{array}$ $\begin{array}{lllllllllll}2 & 142 & 1.075+01 & 0.000+00 & 0.000+00 & 1.689-05 & 8.939-06 & 5.595-06 & 4.652-06 & 4.537-06 & 4.930-06\end{array}$ $\begin{array}{lllllllllllll}2 & 143 & 1.077+01 & 0.000+00 & 4.955+05 & 5.392-04 & 6.187-04 & 7.449-04 & 8.919-04 & 1.023-03 & 1.144-03\end{array}$ $\begin{array}{lllllllllll}2 & 144 & 1.077+01 & 0.000+00 & 0.000+00 & 4.037-05 & 2.277-05 & 1.267-05 & 6.974-06 & 3.937-06 & 2.381-06\end{array}$ $\begin{array}{llllllllllll}2 & 145 & 1.077+01 & 0.000+00 & 0.000+00 & 1.017-04 & 4.964-05 & 2.572-05 & 1.346-05 & 7.045-06 & 3.584-06\end{array}$ $\begin{array}{lllllllllll}2 & 146 & 1.078+01 & 0.000+00 & 0.000+00 & 2.061-05 & 2.304-05 & 2.538-05 & 2.760-05 & 2.957-05 & 3.121-05\end{array}$ $\begin{array}{llllllllllll}2 & 147 & 1.080+01 & 0.000+00 & 0.000+00 & 3.998-04 & 2.020-04 & 1.075-04 & 5.704-05 & 2.982-05 & 1.511-05\end{array}$ $\begin{array}{lllllllllll}2 & 148 & 1.081+01 & 0.000+00 & 0.000+00 & 1.091-05 & 8.357-06 & 7.650-06 & 8.089-06 & 8.983-06 & 9.818-06\end{array}$ $\begin{array}{lllllllllll}2 & 149 & 1.081+01 & 0.000+00 & 0.000+00 & 7.087-06 & 3.494-06 & 1.711-06 & 8.981-07 & 5.167-07 & 3.090-07\end{array}$ $\begin{array}{lllllllllll}2 & 150 & 1.081+01 & 0.000+00 & 0.000+00 & 5.767-06 & 4.902-06 & 4.701-06 & 4.810-06 & 4.979-06 & 5.154-06\end{array}$ $\begin{array}{lllllllllllll}2 & 151 & 1.082+01 & 0.000+00 & 0.000+00 & 2.349-03 & 2.708-03 & 3.005-03 & 3.256-03 & 3.461-03 & 3.630-03\end{array}$ $\begin{array}{lllllllllllll}2 & 152 & 1.083+01 & 0.000+00 & 0.000+00 & 6.047-04 & 3.375-04 & 1.797-04 & 9.313-05 & 4.743-05 & 2.362-05\end{array}$ $\begin{array}{lllllllllllll}2 & 153 & 1.083+01 & 0.000+00 & 0.000+00 & 8.542-07 & 3.374-07 & 1.747-07 & 1.368-07 & 1.415-07 & 1.485-07\end{array}$ $\begin{array}{llllllllllllll}2 & 154 & 1.083+01 & 0.000+00 & 0.000+00 & 2.132-06 & 1.073-06 & 5.514-07 & 3.303-07 & 2.400-07 & 1.916-07\end{array}$ $\begin{array}{llllllllllll}2 & 155 & 1.084+01 & 0.000+00 & 0.000+00 & 2.058-03 & 1.208-03 & 7.350-04 & 4.753-04 & 3.434-04 & 2.802-04\end{array}$ $\begin{array}{lllllllllllll}2 & 156 & 1.084+01 & 0.000+00 & 0.000+00 & 2.040-04 & 1.007-04 & 5.278-05 & 2.802-05 & 1.492-05 & 7.667-06\end{array}$ $\begin{array}{lllllllllll}2 & 157 & 1.085+01 & 0.000+00 & 0.000+00 & 1.777-03 & 2.055-03 & 2.227-03 & 2.359-03 & 2.608-03 & 2.777-03\end{array}$ $\begin{array}{lllllllllll}2 & 158 & 1.085+01 & 0.000+00 & 0.000+00 & 7.500-03 & 9.896-03 & 1.164-02 & 1.296-02 & 1.395-02 & 1.471-02\end{array}$ $\begin{array}{llllllllllllll}2 & 159 & 1.085+01 & 0.000+00 & 0.000+00 & 1.549-03 & 8.569-04 & 4.513-04 & 2.313-04 & 1.167-04 & 5.733-05\end{array}$ $\begin{array}{lllllllllll}2 & 160 & 1.085+01 & 0.000+00 & 0.000+00 & 1.680-03 & 6.668-04 & 2.870-04 & 1.327-04 & 5.850-05 & 2.497-05\end{array}$ $\begin{array}{lllllllllllll}2 & 161 & 1.085+01 & 0.000+00 & 0.000+00 & 2.830-03 & 1.560-03 & 8.200-04 & 4.194-04 & 2.110-04 & 1.032-04\end{array}$ $\begin{array}{llllllllllll}2 & 162 & 1.087+01 & 0.000+00 & 0.000+00 & 1.277-03 & 1.432-03 & 1.534-03 & 1.617-03 & 1.785-03 & 1.899-03\end{array}$ $\begin{array}{lllllllllllll}2 & 163 & 1.087+01 & 0.000+00 & 0.000+00 & 7.748-04 & 3.030-04 & 1.288-04 & 5.936-05 & 2.608-05 & 1.112-05\end{array}$ $\begin{array}{lllllllllllllll}2 & 164 & 1.088+01 & 0.000+00 & 0.000+00 & 3.187-04 & 2.911-04 & 2.858-04 & 2.905-04 & 2.991-04 & 3.083-04\end{array}$ $\begin{array}{llllllllllll}2 & 165 & 1.089+01 & 0.000+00 & 0.000+00 & 2.080-04 & 1.118-04 & 5.783-05 & 2.922-05 & 1.449-05 & 6.955-06\end{array}$ $\begin{array}{lllllllllll}2 & 166 & 1.089+01 & 0.000+00 & 0.000+00 & 2.696-06 & 1.433-06 & 1.163-06 & 1.144-06 & 1.244-06 & 1.402-06\end{array}$ $\begin{array}{lllllllllll}2 & 167 & 1.089+01 & 0.000+00 & 0.000+00 & 1.021-05 & 1.185-05 & 1.258-05 & 1.322-05 & 1.458-05 & 1.553-05\end{array}$ $\begin{array}{llllllllllll}2 & 168 & 1.089+01 & 0.000+00 & 0.000+00 & 1.089-05 & 3.932-06 & 1.575-06 & 7.298-07 & 3.307-07 & 1.472-07\end{array}$ $\begin{array}{lllllllllll}2 & 169 & 1.089+01 & 0.000+00 & 0.000+00 & 1.241-05 & 6.767-06 & 4.931-06 & 4.685-06 & 5.108-06 & 5.604-06\end{array}$

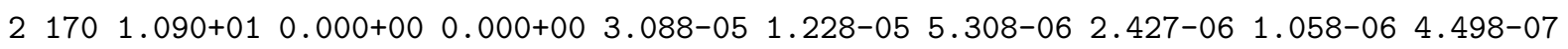


Table II. Ca IX Oscillator Strengths, Radiative Decay Rates, and Collision Strengths for transitions involving the lowest 4 levels.

Low. Upp. Osc. Rad. Dec.

Lev. Lev.

Str. Rate

Collision Strength

Impact Electron Energy - Eo (Ry)

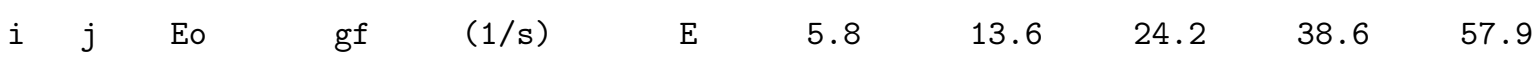

$\begin{array}{lllllllllll}2 & 171 & 1.091+01 & 4.273-05 & 1.362+07 & 9.242-06 & 7.050-06 & 7.179-06 & 9.039-06 & 1.187-05 & 1.510-05\end{array}$ $\begin{array}{lllllllllll}2 & 172 & 1.091+01 & 0.000+00 & 0.000+00 & 1.458-03 & 1.595-03 & 1.733-03 & 1.860-03 & 1.968-03 & 2.060-03\end{array}$ $\begin{array}{llllllllllll}2 & 173 & 1.092+01 & 0.000+00 & 0.000+00 & 3.868-04 & 2.112-04 & 1.103-04 & 5.610-05 & 2.798-05 & 1.357-05\end{array}$

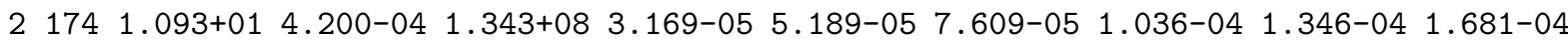
$\begin{array}{lllllllllllll}2 & 175 & 1.093+01 & 0.000+00 & 0.000+00 & 3.660-04 & 3.799-04 & 3.965-04 & 4.132-04 & 4.544-04 & 4.810-04\end{array}$ $\begin{array}{lllllllllll}2 & 176 & 1.093+01 & 0.000+00 & 0.000+00 & 2.276-04 & 8.717-05 & 3.641-05 & 1.644-05 & 7.033-06 & 2.939-06\end{array}$ $\begin{array}{lllllllllll}2 & 177 & 1.095+01 & 0.000+00 & 0.000+00 & 1.431-06 & 2.438-06 & 4.043-06 & 5.314-06 & 6.698-06 & 7.477-06\end{array}$ $\begin{array}{llllllllllll}2 & 178 & 1.095+01 & 0.000+00 & 0.000+00 & 1.201-05 & 5.303-06 & 2.460-06 & 1.189-06 & 5.685-07 & 2.640-07\end{array}$ $\begin{array}{lllllllllllll}2 & 179 & 1.095+01 & 0.000+00 & 0.000+00 & 7.985-04 & 4.394-04 & 2.310-04 & 1.182-04 & 5.939-05 & 2.900-05\end{array}$ $\begin{array}{lllllllllll}2 & 180 & 1.096+01 & 0.000+00 & 0.000+00 & 7.313-05 & 3.967-05 & 2.048-05 & 1.027-05 & 5.064-06 & 2.413-06\end{array}$ $\begin{array}{llllllllllll}2 & 181 & 1.096+01 & 0.000+00 & 0.000+00 & 5.055-05 & 6.469-05 & 7.546-05 & 8.360-05 & 8.961-05 & 9.416-05\end{array}$ $\begin{array}{lllllllllll}2 & 182 & 1.096+01 & 0.000+00 & 0.000+00 & 2.223-06 & 1.081-06 & 5.187-07 & 2.514-07 & 1.247-07 & 6.037-08\end{array}$ $\begin{array}{llllllllllll}2 & 183 & 1.097+01 & 0.000+00 & 0.000+00 & 3.312-04 & 1.812-04 & 9.478-05 & 4.829-05 & 2.411-05 & 1.171-05\end{array}$ $\begin{array}{llllllllllll}2 & 184 & 1.107+01 & 2.127-05 & 6.984+06 & 2.707-05 & 2.424-05 & 2.333-05 & 2.383-05 & 2.500-05 & 2.637-05\end{array}$ $\begin{array}{lllllllllll}2 & 185 & 1.107+01 & 0.000+00 & 0.000+00 & 3.359-06 & 1.729-06 & 8.492-07 & 4.366-07 & 2.403-07 & 1.371-07\end{array}$ $\begin{array}{lllllllllll}2 & 186 & 1.107+01 & 0.000+00 & 0.000+00 & 8.871-06 & 4.515-06 & 2.212-06 & 1.130-06 & 6.168-07 & 3.460-07\end{array}$ $\begin{array}{llllllllllll}2 & 187 & 1.108+01 & 0.000+00 & 0.000+00 & 4.011-05 & 2.034-05 & 1.004-05 & 4.977-06 & 2.516-06 & 1.245-06\end{array}$ $\begin{array}{lllllllllllll}2 & 188 & 1.110+01 & 0.000+00 & 0.000+00 & 1.009-04 & 5.378-05 & 2.743-05 & 1.362-05 & 6.634-06 & 3.083-06\end{array}$ $\begin{array}{llllllllllll}2 & 189 & 1.111+01 & 0.000+00 & 0.000+00 & 1.908-05 & 7.339-06 & 3.585-06 & 2.121-06 & 1.720-06 & 1.568-06\end{array}$ $\begin{array}{lllllllllllll}2 & 190 & 1.116+01 & 0.000+00 & 0.000+00 & 1.139-04 & 1.237-04 & 1.306-04 & 1.362-04 & 1.500-04 & 1.581-04\end{array}$ $21911.116+01 \quad 0.000+00 \quad 0.000+00 \quad 3.766-05 \quad 4.254-05 \quad 4.864-05 \quad 5.431-05 \quad 6.031-05 \quad 6.266-05$ $\begin{array}{lllllllllll}2 & 192 & 1.116+01 & 0.000+00 & 0.000+00 & 3.331-05 & 4.902-05 & 5.460-05 & 5.791-05 & 6.409-05 & 6.700-05\end{array}$

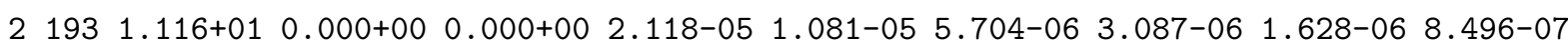

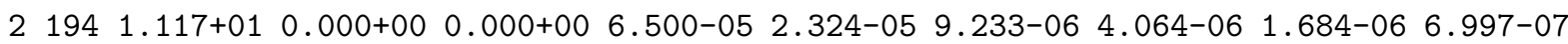
$\begin{array}{lllllllllllll}2 & 195 & 1.117+01 & 0.000+00 & 0.000+00 & 1.265-06 & 8.751-07 & 8.460-07 & 8.699-07 & 9.517-07 & 1.074-06\end{array}$ $\begin{array}{lllllllllll}2 & 196 & 1.120+01 & 0.000+00 & 0.000+00 & 3.816-06 & 2.164-06 & 1.339-06 & 8.247-07 & 5.781-07 & 4.194-07\end{array}$ $\begin{array}{lllllllllll}2 & 197 & 1.128+01 & 0.000+00 & 0.000+00 & 8.518-06 & 3.042-06 & 1.166-06 & 5.106-07 & 2.317-07 & 1.206-07\end{array}$ $\begin{array}{lllllllllllllll}2 & 198 & 1.128+01 & 0.000+00 & 0.000+00 & 5.949-06 & 1.972-06 & 7.296-07 & 3.186-07 & 1.302-07 & 5.518-08\end{array}$ $\begin{array}{llllllllllll}2 & 199 & 1.128+01 & 0.000+00 & 0.000+00 & 8.512-06 & 1.066-05 & 1.127-05 & 1.189-05 & 1.317-05 & 1.373-05\end{array}$ $\begin{array}{lllllllllllll}2 & 200 & 1.129+01 & 0.000+00 & 0.000+00 & 1.904-06 & 1.055-06 & 5.773-07 & 3.174-07 & 1.697-07 & 9.278-08\end{array}$ $\begin{array}{llllllllllll}2 & 201 & 1.129+01 & 0.000+00 & 0.000+00 & 2.282-06 & 1.157-06 & 5.828-07 & 3.031-07 & 1.607-07 & 9.255-08\end{array}$ $22021.130+01 \quad 0.000+00 \quad 0.000+00 \quad 3.219-06 \quad 3.998-06 \quad 4.581-06 \quad 6.005-06 \quad 6.355-06 \quad 7.058-06$ $22031.132+01 \quad 0.000+00 \quad 0.000+00 \quad 8.718-06 \quad 7.066-06 \quad 6.716-06 \quad 6.935-06 \quad 7.456-06 \quad 7.462-06$ $22041.132+01 \quad 0.000+00 \quad 0.000+00 \quad 1.667-06 \quad 9.217-07 \quad 5.152-07 \quad 2.930-07 \quad 1.655-07 \quad 9.184-08$ $22051.132+01 \quad 0.000+00 \quad 0.000+00 \quad 3.223-06 \quad 3.995-06 \quad 4.534-06 \quad 4.905-06 \quad 5.016-06 \quad 5.188-06$ $22061.135+01 \quad 0.000+00 \quad 0.000+00 \quad 1.875-05 \quad 9.285-06 \quad 4.636-06 \quad 2.418-06 \quad 1.234-06 \quad 6.264-07$ $\begin{array}{llllllllllll}2 & 207 & 1.136+01 & 0.000+00 & 0.000+00 & 7.060-06 & 3.709-06 & 1.838-06 & 9.106-07 & 4.674-07 & 2.489-07\end{array}$ $22081.146+01 \quad 0.000+00 \quad 0.000+00 \quad 2.073-06 \quad 1.162-06 \quad 6.522-07 \quad 3.813-07 \quad 2.296-07 \quad 1.503-07$ $22091.149+01 \quad 0.000+00 \quad 0.000+00 \quad 2.106-05 \quad 7.284-06 \quad 2.796-06 \quad 1.203-06 \quad 4.843-07 \quad 1.994-07$ $\begin{array}{llllllllllll}2 & 210 & 1.226+01 & 0.000+00 & 0.000+00 & 4.762-06 & 4.289-06 & 4.023-06 & 3.819-06 & 3.772-06 & 3.942-06\end{array}$ $\begin{array}{llllllllllllll}2 & 211 & 1.226+01 & 0.000+00 & 0.000+00 & 1.854-06 & 9.534-07 & 4.667-07 & 2.260-07 & 1.130-07 & 5.573-08\end{array}$ $\begin{array}{lllllllllll}2 & 212 & 1.227+01 & 0.000+00 & 0.000+00 & 1.527-06 & 1.761-06 & 2.168-06 & 2.634-06 & 3.037-06 & 3.342-06\end{array}$ $\begin{array}{lllllllllllll}2 & 213 & 1.229+01 & 0.000+00 & 0.000+00 & 4.181-06 & 2.190-06 & 1.148-06 & 6.131-07 & 3.353-07 & 1.801-07\end{array}$ $\begin{array}{llllllllllllll}2 & 214 & 1.252+01 & 0.000+00 & 0.000+00 & 1.746-06 & 9.124-07 & 5.181-07 & 3.353-07 & 2.483-07 & 2.093-07\end{array}$ $\begin{array}{lllllllllll}2 & 215 & 1.253+01 & 0.000+00 & 0.000+00 & 2.199-06 & 1.087-06 & 5.665-07 & 3.080-07 & 1.742-07 & 9.296-08\end{array}$ 
Table II. Ca IX Oscillator Strengths, Radiative Decay Rates, and Collision Strengths for transitions involving the lowest 4 levels.

Low. Upp. Osc. Rad. Dec.

Lev. Lev.

Str. Rate

Collision Strength

Impact Electron Energy - Eo (Ry)

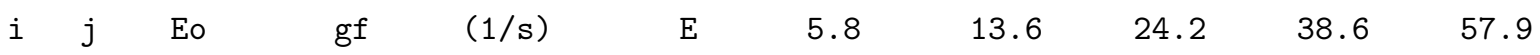

$2216 \quad 1.253+01 \quad 0.000+00 \quad 0.000+00 \quad 2.630-06 \quad 2.644-06 \quad 3.228-06 \quad 3.918-06 \quad 4.518-06 \quad 5.157-06$ $\begin{array}{lllllllllllll}2 & 217 & 1.253+01 & 0.000+00 & 0.000+00 & 1.267-06 & 6.739-07 & 3.723-07 & 2.067-07 & 1.153-07 & 6.158-08\end{array}$ $22181.254+01 \quad 0.000+00 \quad 0.000+00 \quad 3.976-06 \quad 4.549-06 \quad 5.513-06 \quad 6.769-06 \quad 7.943-06 \quad 8.602-06$ $\begin{array}{llllllllllll}2 & 219 & 1.255+01 & 0.000+00 & 0.000+00 & 2.470-06 & 1.268-06 & 6.683-07 & 3.590-07 & 1.961-07 & 1.077-07\end{array}$ $\begin{array}{lllllllllllll}2 & 220 & 1.255+01 & 0.000+00 & 0.000+00 & 2.069-07 & 1.427-07 & 1.440-07 & 1.842-07 & 2.329-07 & 2.823-07\end{array}$

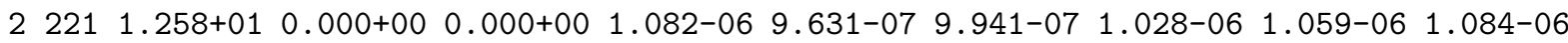
$\begin{array}{llllllllllll}2 & 222 & 1.258+01 & 0.000+00 & 0.000+00 & 1.249-06 & 6.113-07 & 3.023-07 & 1.519-07 & 7.762-08 & 3.801-08\end{array}$ $\begin{array}{lllllllllll}2 & 223 & 1.258+01 & 0.000+00 & 0.000+00 & 2.119-06 & 2.212-06 & 2.312-06 & 2.381-06 & 2.406-06 & 2.397-06\end{array}$

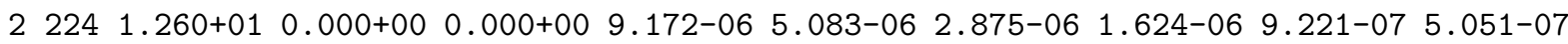
$\begin{array}{llllllllllll}2 & 225 & 1.263+01 & 0.000+00 & 0.000+00 & 3.191-06 & 1.688-06 & 9.202-07 & 5.140-07 & 2.976-07 & 1.655-07\end{array}$ $\begin{array}{lllllllllll}2 & 226 & 1.287+01 & 0.000+00 & 0.000+00 & 1.635-06 & 9.117-07 & 4.900-07 & 2.609-07 & 1.408-07 & 7.608-08\end{array}$ $22271.288+01 \quad 0.000+00 \quad 0.000+00 \quad 1.583-06 \quad 1.850-06 \quad 2.740-06 \quad 4.061-06 \quad 5.521-06 \quad 7.103-06$ $\begin{array}{llllllllllll}2 & 228 & 1.288+01 & 0.000+00 & 0.000+00 & 1.443-06 & 7.772-07 & 4.195-07 & 2.311-07 & 1.323-07 & 7.751-08\end{array}$ $\begin{array}{llllllllllll}2 & 229 & 1.288+01 & 0.000+00 & 0.000+00 & 1.252-06 & 1.006-06 & 9.268-07 & 9.751-07 & 1.081-06 & 1.209-06\end{array}$ $22301.289+01 \quad 0.000+00 \quad 0.000+00 \quad 6.270-06 \quad 5.416-06 \quad 5.213-06 \quad 5.334-06 \quad 5.607-06 \quad 5.982-06$ $\begin{array}{llllllllllll}2 & 231 & 1.289+01 & 0.000+00 & 0.000+00 & 4.912-06 & 2.847-06 & 1.615-06 & 9.038-07 & 4.983-07 & 2.699-07\end{array}$ $\begin{array}{llllllllllllll}2 & 232 & 1.289+01 & 0.000+00 & 0.000+00 & 8.822-08 & 1.005-07 & 1.325-07 & 1.674-07 & 2.155-07 & 2.303-07\end{array}$ $\begin{array}{lllllllllllll}2 & 233 & 1.289+01 & 0.000+00 & 0.000+00 & 5.361-07 & 3.117-07 & 2.108-07 & 1.753-07 & 1.768-07 & 1.902-07\end{array}$ $\begin{array}{lllllllllll}2 & 234 & 1.293+01 & 0.000+00 & 0.000+00 & 9.587-07 & 9.961-07 & 1.368-06 & 1.864-06 & 2.275-06 & 2.566-06\end{array}$ $\begin{array}{lllllllllll}2 & 235 & 1.293+01 & 0.000+00 & 0.000+00 & 2.026-06 & 1.112-06 & 5.902-07 & 3.088-07 & 1.623-07 & 8.650-08\end{array}$

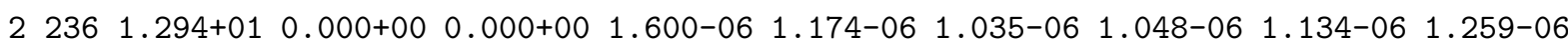
$\begin{array}{lllllllllllll}2 & 237 & 1.294+01 & 0.000+00 & 0.000+00 & 1.002-06 & 5.772-07 & 3.262-07 & 1.825-07 & 1.009-07 & 5.482-08\end{array}$ $\begin{array}{lllllllllll}2 & 238 & 1.299+01 & 0.000+00 & 0.000+00 & 3.763-06 & 2.011-06 & 1.091-06 & 6.113-07 & 3.548-07 & 2.067-07\end{array}$ $\begin{array}{lllllllllll}2 & 239 & 1.299+01 & 0.000+00 & 0.000+00 & 4.610-07 & 2.336-07 & 1.219-07 & 6.797-08 & 4.115-08 & 2.600-08\end{array}$ $22401.299+01 \quad 9.515-05 \quad 4.302+07 \quad 4.512-06 \quad 5.645-06 \quad 7.302-06 \quad 9.435-06 \quad 1.213-05 \quad 1.552-05$ $\begin{array}{llllllllllllllll}2 & 241 & 1.299+01 & 0.000+00 & 0.000+00 & 5.030-07 & 2.636-07 & 1.367-07 & 7.159-08 & 3.848-08 & 2.159-08\end{array}$ $22421.300+01 \quad 0.000+00 \quad 0.000+00 \quad 4.738-06 \quad 2.750-06 \quad 1.560-06 \quad 8.734-07 \quad 4.814-07 \quad 2.608-07$ $\begin{array}{lllllllllllll}2 & 243 & 1.302+01 & 0.000+00 & 0.000+00 & 2.854-06 & 1.458-06 & 7.452-07 & 4.113-07 & 2.396-07 & 1.596-07\end{array}$ $22441.303+01 \quad 0.000+00 \quad 0.000+00 \quad 6.698-06 \quad 7.212-06 \quad 8.375-06 \quad 9.466-06 \quad 1.058-051.185-05$ $\begin{array}{llllllllllll}2 & 245 & 1.303+01 & 0.000+00 & 0.000+00 & 5.538-06 & 2.926-06 & 1.501-06 & 7.807-07 & 4.044-07 & 1.952-07\end{array}$ $\begin{array}{lllllllllll}2 & 246 & 1.303+01 & 0.000+00 & 0.000+00 & 3.200-08 & 3.768-08 & 5.339-08 & 6.527-08 & 7.704-08 & 7.435-08\end{array}$ $\begin{array}{lllllllllll}2 & 247 & 1.304+01 & 0.000+00 & 0.000+00 & 1.016-05 & 1.399-05 & 1.798-05 & 2.153-05 & 2.474-05 & 2.700-05\end{array}$ $22481.304+01 \quad 0.000+00 \quad 0.000+00 \quad 3.408-06 \quad 1.723-06 \quad 8.456-07 \quad 4.241-07 \quad 2.116-07 \quad 1.037-07$ $\begin{array}{lllllllllll}2 & 249 & 1.304+01 & 0.000+00 & 0.000+00 & 1.702-06 & 1.500-06 & 1.524-06 & 1.632-06 & 1.805-06 & 2.024-06\end{array}$ $\begin{array}{lllllllllll}2 & 250 & 1.306+01 & 0.000+00 & 0.000+00 & 1.683-06 & 8.767-07 & 4.957-07 & 2.950-07 & 2.129-07 & 1.744-07\end{array}$ $2251 \quad 1.308+01 \quad 0.000+00 \quad 0.000+00 \quad 1.042-06 \quad 4.891-07 \quad 2.256-07 \quad 1.124-07 \quad 6.553-08$ 4.364-08 $\begin{array}{llllllllllll}2 & 252 & 1.308+01 & 0.000+00 & 0.000+00 & 3.303-06 & 1.643-06 & 8.020-07 & 3.964-07 & 2.040-07 & 1.086-07\end{array}$ $\begin{array}{llllllllllll}2 & 253 & 1.308+01 & 0.000+00 & 0.000+00 & 1.152-06 & 5.137-07 & 2.237-07 & 9.535-08 & 4.411-08 & 2.149-08\end{array}$ $22541.308+01 \quad 0.000+00 \quad 0.000+00 \quad 2.495-06 \quad 2.534-06 \quad 3.048-06 \quad 3.577-06 \quad 4.071-06 \quad 4.596-06$ $\begin{array}{lllllllllll}2 & 255 & 1.309+01 & 0.000+00 & 0.000+00 & 1.013-06 & 1.096-06 & 1.327-06 & 1.665-06 & 1.888-06 & 2.000-06\end{array}$ $\begin{array}{llllllllllll}2 & 256 & 1.309+01 & 0.000+00 & 0.000+00 & 1.721-06 & 9.403-07 & 4.994-07 & 2.655-07 & 1.396-07 & 6.918-08\end{array}$ $\begin{array}{llllllllllll}2 & 257 & 1.309+01 & 0.000+00 & 0.000+00 & 7.572-07 & 3.709-07 & 1.744-07 & 7.652-08 & 3.404-08 & 1.496-08\end{array}$ $\begin{array}{lllllllllllll}2 & 258 & 1.309+01 & 0.000+00 & 0.000+00 & 4.936-07 & 2.688-07 & 2.309-07 & 2.713-07 & 3.527-07 & 3.972-07\end{array}$ $\begin{array}{llllllllllll}2 & 259 & 1.309+01 & 0.000+00 & 0.000+00 & 4.154-07 & 1.801-07 & 7.995-08 & 3.860-08 & 1.901-08 & 1.034-08\end{array}$ $22601.309+01 \quad 0.000+00 \quad 0.000+00 \quad 1.640-07 \quad 7.801-08 \quad 4.795-08 \quad 3.691-08 \quad 4.327-08$ 4.181-08 
Table II. Ca IX Oscillator Strengths, Radiative Decay Rates, and Collision Strengths for transitions involving the lowest 4 levels.

Low. Upp. Osc. Rad. Dec.

Lev. Lev.

Str. Rate

Collision Strength

Impact Electron Energy - Eo (Ry)

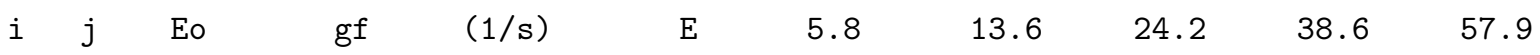

$\begin{array}{lllllllllll}2 & 261 & 1.310+01 & 0.000+00 & 0.000+00 & 2.869-07 & 1.149-07 & 4.992-08 & 2.263-08 & 1.299-08 & 1.019-08\end{array}$ $\begin{array}{llllllllllll}2 & 262 & 1.310+01 & 0.000+00 & 0.000+00 & 1.115-06 & 5.429-07 & 2.562-07 & 1.245-07 & 5.718-08 & 2.510-08\end{array}$

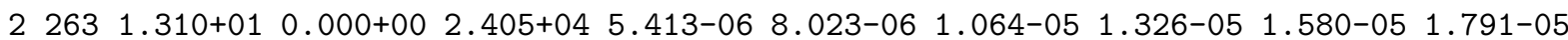
$22641.310+01 \quad 0.000+00 \quad 0.000+00 \quad 1.576-06 \quad 7.304-07 \quad 3.279-07 \quad 1.514-07 \quad 6.913-08 \quad 3.298-08$ $\begin{array}{lllllllllllll}2 & 265 & 1.310+01 & 0.000+00 & 0.000+00 & 1.398-06 & 7.714-07 & 4.193-07 & 2.248-07 & 1.193-07 & 6.399-08\end{array}$ $\begin{array}{lllllllllll}2 & 266 & 1.310+01 & 0.000+00 & 0.000+00 & 2.289-06 & 2.045-06 & 2.156-06 & 2.539-06 & 2.825-06 & 2.973-06\end{array}$ $\begin{array}{lllllllllll}2 & 267 & 1.310+01 & 0.000+00 & 0.000+00 & 2.778-07 & 1.264-07 & 5.784-08 & 2.840-08 & 1.363-08 & 7.356-09\end{array}$ $\begin{array}{lllllllllllll}2 & 268 & 1.310+01 & 0.000+00 & 0.000+00 & 3.086-07 & 5.075-07 & 7.083-07 & 8.789-07 & 1.033-06 & 1.070-06\end{array}$ $\begin{array}{lllllllllll}2 & 269 & 1.310+01 & 0.000+00 & 0.000+00 & 2.364-07 & 1.062-07 & 4.686-08 & 2.079-08 & 9.007-09 & 4.111-09\end{array}$ $22701.310+01 \quad 0.000+00 \quad 0.000+00 \quad 1.853-06 \quad 8.987-07 \quad 4.295-07 \quad 1.899-07 \quad 8.521-08$ 3.747-08 $2271 \quad 1.311+01 \quad 0.000+00 \quad 0.000+00 \quad 4.505-08 \quad 4.045-08 \quad 4.844-08 \quad 5.811-08 \quad 6.191-08$ 6.793-08 $22721.311+01 \quad 0.000+00 \quad 0.000+00 \quad 4.556-06 \quad 5.738-06 \quad 7.093-06 \quad 8.272-06 \quad 9.347-06 \quad 9.995-06$ $\begin{array}{lllllllllll}2 & 273 & 1.311+01 & 0.000+00 & 0.000+00 & 4.481-07 & 2.310-07 & 1.163-07 & 6.092-08 & 3.103-08 & 1.545-08\end{array}$ $22741.311+010.000+00 \quad 0.000+00 \quad 9.734-07 \quad 1.276-06 \quad 1.845-06 \quad 2.871-06 \quad 4.394-06 \quad 6.284-06$ $\begin{array}{lllllllllllll}2 & 275 & 1.312+01 & 0.000+00 & 0.000+00 & 5.685-06 & 2.838-06 & 1.390-06 & 6.965-07 & 3.389-07 & 1.554-07\end{array}$ $22761.313+010.000+00 \quad 0.000+00 \quad 1.319-06 \quad 6.388-07 \quad 3.056-07 \quad 1.351-07 \quad 6.054-08$ 2.661-08 $2277 \begin{array}{llllllllllll}2 & 1.313+01 & 0.000+00 & 0.000+00 & 8.284-07 & 6.157-07 & 5.680-07 & 6.091-07 & 7.054-07 & 7.493-07\end{array}$ $2278 \begin{array}{llllllllll}2 & 1.313+01 & 0.000+00 & 0.000+00 & 3.196-07 & 1.695-07 & 9.110-08 & 4.931-08 & 2.588-08 & 1.427-08\end{array}$ $\begin{array}{llllllllllllll}2 & 279 & 1.314+01 & 0.000+00 & 0.000+00 & 1.815-07 & 1.354-07 & 1.807-07 & 3.320-07 & 5.187-07 & 6.404-07\end{array}$ $\begin{array}{llllllllllllll}2 & 280 & 1.315+01 & 0.000+00 & 0.000+00 & 6.315-07 & 3.637-07 & 2.038-07 & 1.119-07 & 6.004-08 & 3.329-08\end{array}$ $\begin{array}{llllllllllll}2 & 281 & 1.315+01 & 0.000+00 & 0.000+00 & 9.251-07 & 4.550-07 & 2.234-07 & 1.154-07 & 6.460-08 & 3.717-08\end{array}$ $\begin{array}{lllllllllll}2 & 282 & 1.318+01 & 0.000+00 & 0.000+00 & 5.260-06 & 2.934-06 & 1.583-06 & 8.517-07 & 4.549-07 & 2.292-07\end{array}$ $22831.319+01 \quad 0.000+00 \quad 0.000+00 \quad 1.150-06 \quad 6.321-07 \quad 3.468-07 \quad 1.998-07 \quad 1.114-07 \quad 6.426-08$ $42.909-02 \quad 0.000+00 \quad 4.380-01 \quad 7.413-01 \quad 6.189-01 \quad 5.769-01 \quad 5.595-01 \quad 5.520-01 \quad 5.497-01$ $\begin{array}{llllllllll}5 & 6.754-01 & 0.000+00 & 0.000+00 & 4.307-02 & 2.209-02 & 1.177-02 & 6.550-03 & 3.818-03 & 2.425-03\end{array}$ $\begin{array}{lllllllllll}6 & 1.748+00 & 2.257-02 & 1.108+08 & 2.086-01 & 2.027-01 & 2.071-01 & 2.193-01 & 2.356-01 & 2.533-01\end{array}$

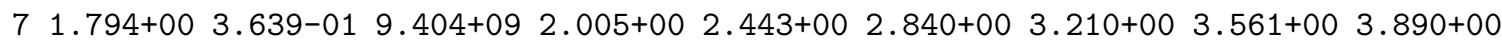
$\begin{array}{llllllllll}8 & 1.812+00 & 2.767-01 & 2.431+09 & 1.521+00 & 1.841+00 & 2.133+00 & 2.408+00 & 2.671+00 & 2.917+00\end{array}$ $\begin{array}{llllllllll}9 & 1.843+00 & 4.461-01 & 2.434+09 & 2.365+00 & 2.878+00 & 3.346+00 & 3.783+00 & 4.199+00 & 4.589+00\end{array}$ $10 \quad 2.362+00 \quad 6.625-04 \quad 2.970+07 \quad 7.178-03 \quad 5.670-03 \quad 4.898-03 \quad 4.688-03 \quad 4.779-03 \quad 5.018-03$ $\begin{array}{llllllllll}11 & 2.449+00 & 3.501-01 & 5.622+09 & 1.174+00 & 1.438+00 & 1.688+00 & 1.925+00 & 2.154+00 & 2.372+00\end{array}$

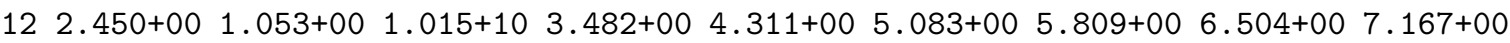
$\begin{array}{lllllllllll}13 & 2.452+00 & 0.000+00 & 0.000+00 & 1.048-01 & 8.629-02 & 7.958-02 & 7.899-02 & 8.138-02 & 8.458-02\end{array}$ $\begin{array}{lllllllllll}14 & 3.000+00 & 2.271-03 & 3.283+07 & 3.626-02 & 2.280-02 & 1.625-02 & 1.345-02 & 1.259-02 & 1.269-02\end{array}$ $\begin{array}{llllllllll}15 & 3.818+00 & 0.000+00 & 2.095+05 & 1.864-01 & 2.056-01 & 2.201-01 & 2.316-01 & 2.402-01 & 2.462-01\end{array}$ $\begin{array}{llllllllllllllll}16 & 3.836+00 & 0.000+00 & 3.292+05 & 3.534-01 & 4.141-01 & 4.573-01 & 4.890-01 & 5.116-01 & 5.267-01\end{array}$ $\begin{array}{lllllllllll}17 & 3.858+00 & 0.000+00 & 0.000+00 & 1.712-02 & 1.003-02 & 5.715-03 & 3.219-03 & 1.843-03 & 1.102-03\end{array}$

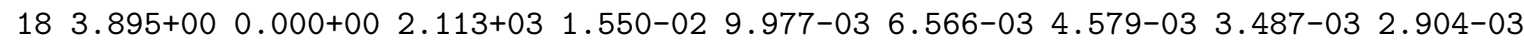
$\begin{array}{llllllllllll}19 & 4.150+00 & 0.000+00 & 2.590+05 & 1.454-01 & 1.656-01 & 1.807-01 & 1.923-01 & 2.010-01 & 2.069-01\end{array}$ $\begin{array}{lllllllllll}20 & 4.160+00 & 0.000+00 & 4.061+05 & 1.244-01 & 1.470-01 & 1.634-01 & 1.756-01 & 1.843-01 & 1.902-01\end{array}$ $\begin{array}{lllllllllllll}21 & 4.176+00 & 0.000+00 & 0.000+00 & 1.435-03 & 8.391-04 & 4.726-04 & 2.584-04 & 1.391-04 & 7.436-05\end{array}$ $\begin{array}{lllllllllllllll}22 & 4.185+00 & 0.000+00 & 7.195+04 & 2.433-02 & 2.667-02 & 2.853-02 & 3.003-02 & 3.120-02 & 3.203-02\end{array}$ $\begin{array}{llllllllll}23 & 4.189+00 & 0.000+00 & 0.000+00 & 8.503-03 & 5.052-03 & 2.901-03 & 1.631-03 & 9.193-04 & 5.323-04\end{array}$ $\begin{array}{llllllllll}24 & 4.190+00 & 0.000+00 & 1.428+05 & 1.032-01 & 1.184-01 & 1.298-01 & 1.384-01 & 1.448-01 & 1.491-01\end{array}$

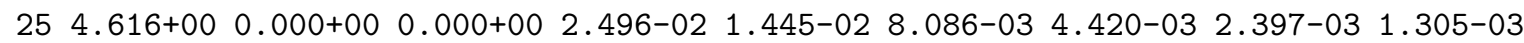


Table II. Ca IX Oscillator Strengths, Radiative Decay Rates, and Collision Strengths for transitions involving the lowest 4 levels.

Low. Upp. Osc. Rad. Dec. Lev. Lev. Rate
Collision Strength

Impact Electron Energy - Eo (Ry)

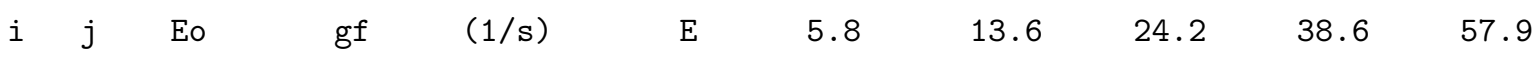

$\begin{array}{lllllllllll}3 & 26 & 4.708+00 & 0.000+00 & 0.000+00 & 6.240-03 & 3.662-03 & 2.076-03 & 1.140-03 & 6.180-04 & 3.374-04\end{array}$ $\begin{array}{lllllllllllll}3 & 27 & 5.606+00 & 2.714-01 & 2.284+10 & 5.207-02 & 9.639-02 & 1.481-01 & 2.048-01 & 2.625-01 & 3.218-01\end{array}$ $\begin{array}{lllllllllll}3 & 28 & 5.748+00 & 1.021-04 & 2.710+07 & 3.538-03 & 1.984-03 & 1.020-03 & 5.487-04 & 3.131-04 & 2.180-04\end{array}$ $\begin{array}{llllllllllll}3 & 29 & 6.248+00 & 0.000+00 & 0.000+00 & 5.204-03 & 2.499-03 & 1.277-03 & 6.639-04 & 3.404-04 & 1.770-04\end{array}$ $\begin{array}{llllllllllllll}3 & 30 & 6.250+00 & 0.000+00 & 1.089+06 & 4.446-01 & 4.750-01 & 4.940-01 & 5.049-01 & 5.106-01 & 5.132-01\end{array}$ $\begin{array}{llllllllllll}3 & 31 & 6.259+00 & 0.000+00 & 0.000+00 & 4.192-04 & 4.349-04 & 4.556-04 & 4.764-04 & 4.964-04 & 5.139-04\end{array}$ $\begin{array}{lllllllllll}3 & 32 & 6.260+00 & 0.000+00 & 0.000+00 & 2.155-04 & 1.331-04 & 8.923-05 & 6.724-05 & 5.685-05 & 5.226-05\end{array}$ $\begin{array}{lllllllllll}3 & 33 & 6.262+00 & 0.000+00 & 2.241+06 & 4.438-02 & 4.874-02 & 5.704-02 & 6.453-02 & 7.061-02 & 7.517-02\end{array}$ $\begin{array}{lllllllllll}34 & 6.263+00 & 0.000+00 & 0.000+00 & 4.374-04 & 4.507-04 & 4.692-04 & 4.886-04 & 5.077-04 & 5.249-04\end{array}$

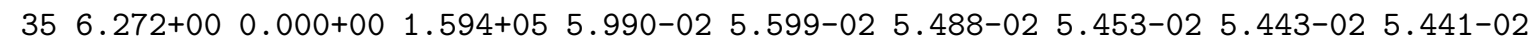
$\begin{array}{llllllllll}36 & 6.444+00 & 0.000+00 & 0.000+00 & 7.933-04 & 4.311-04 & 2.301-04 & 1.249-04 & 7.046-05 & 4.247-05\end{array}$

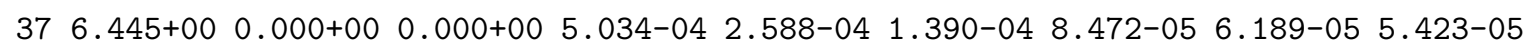
$\begin{array}{llllllllll}38 & 6.471+00 & 1.379-04 & 1.547+07 & 5.403-05 & 6.236-05 & 7.790-05 & 9.905-05 & 1.218-04 & 1.471-04\end{array}$ $\begin{array}{lllllllllll}39 & 6.472+00 & 1.772-04 & 5.961+07 & 4.653-05 & 6.667-05 & 9.171-05 & 1.215-04 & 1.521-04 & 1.853-04\end{array}$ $\begin{array}{llllllllll}40 & 6.473+00 & 2.403-04 & 1.618+07 & 8.005-05 & 9.962-05 & 1.296-04 & 1.680-04 & 2.086-04 & 2.530-04\end{array}$ $\begin{array}{llllllllll}41 & 6.969+00 & 0.000+00 & 0.000+00 & 3.539-05 & 2.001-05 & 1.065-05 & 5.626-06 & 3.076-06 & 1.938-06\end{array}$ $\begin{array}{lllllllllll}42 & 7.042+00 & 1.504-01 & 1.997+10 & 1.758-02 & 2.735-02 & 4.285-02 & 6.230-02 & 8.384-02 & 1.072-01\end{array}$ $\begin{array}{llllllllllll}43 & 7.043+00 & 4.473-01 & 3.564+10 & 4.236-02 & 8.033-02 & 1.321-01 & 1.931-01 & 2.588-01 & 3.291-01\end{array}$ $\begin{array}{lllllllllllllll}44 & 7.045+00 & 0.000+00 & 0.000+00 & 2.740-02 & 2.394-02 & 2.315-02 & 2.292-02 & 2.301-02 & 2.332-02\end{array}$ $\begin{array}{llllllllll}45 & 7.059+00 & 0.000+00 & 0.000+00 & 1.465-02 & 7.826-03 & 4.043-03 & 2.065-03 & 1.044-03 & 5.342-04\end{array}$ $\begin{array}{llllllllll}46 & 7.268+00 & 0.000+00 & 0.000+00 & 2.308-03 & 1.169-03 & 6.313-04 & 3.481-04 & 1.861-04 & 9.555-05\end{array}$ $\begin{array}{llllllllllll}47 & 7.280+00 & 0.000+00 & 5.485+03 & 2.608-01 & 2.831-01 & 2.977-01 & 3.067-01 & 3.118-01 & 3.144-01\end{array}$ $\begin{array}{llllllllll}48 & 7.312+00 & 0.000+00 & 1.010+04 & 2.594-03 & 1.361-03 & 7.923-04 & 5.000-04 & 3.380-04 & 2.507-04\end{array}$ $\begin{array}{llllllllll}49 & 7.387+00 & 0.000+00 & 8.827+06 & 9.528-02 & 1.204-01 & 1.455-01 & 1.682-01 & 1.853-01 & 1.989-01\end{array}$ $\begin{array}{llllllllllll}50 & 7.387+00 & 0.000+00 & 1.262+07 & 1.729-01 & 2.391-01 & 2.975-01 & 3.474-01 & 3.847-01 & 4.138-01\end{array}$ $\begin{array}{lllllllllll}51 & 7.387+00 & 0.000+00 & 0.000+00 & 3.752-02 & 2.771-02 & 2.493-02 & 2.513-02 & 2.670-02 & 2.811-02\end{array}$ $\begin{array}{llllllllll}52 & 7.402+00 & 0.000+00 & 0.000+00 & 6.527-03 & 5.939-03 & 5.748-03 & 5.681-03 & 5.652-03 & 5.638-03\end{array}$ $\begin{array}{lllllllllll}53 & 7.484+00 & 0.000+00 & 1.285+04 & 2.778-02 & 1.278-02 & 6.158-03 & 3.168-03 & 1.747-03 & 1.067-03\end{array}$ $\begin{array}{llllllllll}54 & 7.783+00 & 1.030-02 & 1.670+09 & 3.896-03 & 3.160-03 & 3.241-03 & 3.967-03 & 5.043-03 & 6.336-03\end{array}$ $\begin{array}{lllllllllll}55 & 7.837+00 & 2.028-02 & 3.335+09 & 4.224-03 & 4.263-03 & 5.376-03 & 7.306-03 & 9.667-03 & 1.232-02\end{array}$ $\begin{array}{llllllllll}56 & 7.849+00 & 1.158-01 & 1.146+10 & 1.309-02 & 1.824-02 & 2.763-02 & 4.016-02 & 5.438-02 & 6.987-02\end{array}$ $\begin{array}{llllllllll}57 & 7.879+00 & 0.000+00 & 0.000+00 & 2.327-03 & 1.505-03 & 1.023-03 & 7.798-04 & 6.654-04 & 6.141-04\end{array}$ $\begin{array}{llllllllll}58 & 7.916+00 & 3.994-02 & 2.011+10 & 3.909-03 & 5.619-03 & 8.739-03 & 1.291-02 & 1.769-02 & 2.293-02\end{array}$ $\begin{array}{llllllllll}59 & 7.923+00 & 7.187-02 & 1.208+10 & 8.137-03 & 1.083-02 & 1.620-02 & 2.361-02 & 3.218-02 & 4.157-02\end{array}$ $\begin{array}{llllllllll}60 & 7.946+00 & 6.778-03 & 1.146+09 & 1.494-03 & 1.482-03 & 1.816-03 & 2.437-03 & 3.207-03 & 4.073-03\end{array}$ $\begin{array}{llllllllll}61 & 7.947+00 & 3.390-02 & 3.439+09 & 5.113-03 & 5.800-03 & 7.944-03 & 1.118-02 & 1.508-02 & 1.943-02\end{array}$ $\begin{array}{llllllllllll}62 & 8.052+00 & 1.044-03 & 1.088+08 & 2.489-03 & 1.463-03 & 8.916-04 & 6.617-04 & 6.156-04 & 6.669-04\end{array}$ $\begin{array}{lllllllllll}63 & 8.192+00 & 1.711-04 & 9.224+07 & 9.134-04 & 5.527-04 & 3.129-04 & 1.938-04 & 1.418-04 & 1.284-04\end{array}$ $\begin{array}{llllllllllll}64 & 8.415+00 & 4.744-02 & 8.996+09 & 6.902-03 & 1.029-02 & 1.479-02 & 2.031-02 & 2.640-02 & 3.257-02\end{array}$ $\begin{array}{lllllllllll}65 & 8.514+00 & 2.829-05 & 1.647+07 & 9.512-04 & 5.104-04 & 2.553-04 & 1.305-04 & 7.072-05 & 4.164-05\end{array}$ $\begin{array}{llllllllll}66 & 8.630+00 & 0.000+00 & 5.456+06 & 9.731-03 & 1.236-02 & 1.539-02 & 1.815-02 & 2.057-02 & 2.270-02\end{array}$ $\begin{array}{llllllllll}67 & 8.630+00 & 0.000+00 & 1.469+06 & 8.785-03 & 7.585-03 & 7.786-03 & 8.440-03 & 9.208-03 & 9.986-03\end{array}$ $\begin{array}{llllllllll}68 & 8.639+00 & 0.000+00 & 1.768+04 & 5.080-03 & 2.678-03 & 1.521-03 & 8.982-04 & 5.692-04 & 3.957-04\end{array}$ $\begin{array}{lllllllllll}69 & 8.641+00 & 0.000+00 & 3.391+06 & 1.260-02 & 1.576-02 & 1.979-02 & 2.351-02 & 2.687-02 & 2.988-02\end{array}$

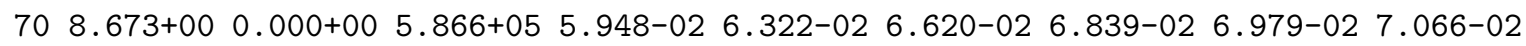


Table II. Ca IX Oscillator Strengths, Radiative Decay Rates, and Collision Strengths for transitions involving the lowest 4 levels.

Low. Upp. Osc. Rad. Dec.

Lev. Lev. Str. Rate

Collision Strength

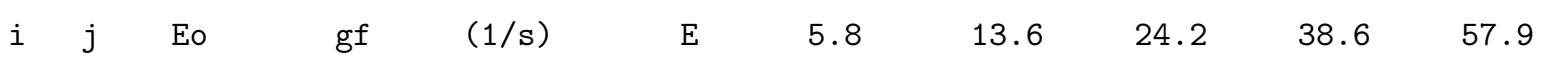

$\begin{array}{llllllllllll}3 & 71 & 8.674+00 & 0.000+00 & 0.000+00 & 1.903-03 & 9.196-04 & 4.732-04 & 2.480-04 & 1.270-04 & 6.420-05\end{array}$

$\begin{array}{llllllllllll}3 & 72 & 8.676+00 & 0.000+00 & 3.155+06 & 1.371-02 & 1.458-02 & 1.669-02 & 1.928-02 & 2.163-02 & 2.353-02\end{array}$

$\begin{array}{llllllllllll}3 & 73 & 8.682+00 & 0.000+00 & 9.396+05 & 1.938-02 & 1.886-02 & 1.920-02 & 1.975-02 & 2.023-02 & 2.064-02\end{array}$

$\begin{array}{llllllllllll}3 & 74 & 8.689+00 & 0.000+00 & 2.229+06 & 6.449-03 & 7.669-03 & 9.410-03 & 1.103-02 & 1.257-02 & 1.393-02\end{array}$

$\begin{array}{lllllllllll}3 & 75 & 8.693+00 & 0.000+00 & 7.746+05 & 6.897-03 & 5.890-03 & 5.947-03 & 6.302-03 & 6.848-03 & 7.389-03\end{array}$

$\begin{array}{llllllllllll}3 & 76 & 8.717+00 & 0.000+00 & 0.000+00 & 3.285-03 & 1.797-03 & 1.041-03 & 6.330-04 & 4.192-04 & 3.108-04\end{array}$

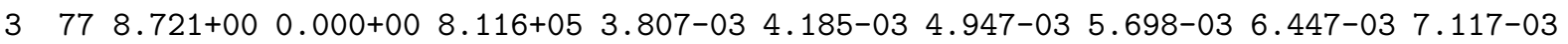

$\begin{array}{lllllllllll}3 & 78 & 8.804+00 & 0.000+00 & 2.108+05 & 2.227-03 & 1.368-03 & 1.122-03 & 1.053-03 & 1.139-03 & 1.202-03\end{array}$

$\begin{array}{lllllllllllllllllll}3 & 79 & 8.811+00 & 0.000+00 & 1.093+05 & 2.237-02 & 2.401-02 & 2.525-02 & 2.609-02 & 2.661-02 & 2.691-02\end{array}$

$\begin{array}{llllllllllll}3 & 80 & 8.816+00 & 0.000+00 & 0.000+00 & 4.850-04 & 2.301-04 & 1.182-04 & 6.375-05 & 3.456-05 & 1.836-05\end{array}$

$\begin{array}{lllllllllllll}3 & 81 & 8.864+00 & 0.000+00 & 1.435+04 & 1.652-03 & 9.158-04 & 5.988-04 & 4.508-04 & 3.748-04 & 3.335-04\end{array}$

$\begin{array}{lllllllllll}82 & 8.922+00 & 0.000+00 & 0.000+00 & 3.448-02 & 3.358-02 & 3.453-02 & 3.579-02 & 3.681-02 & 3.749-02\end{array}$

$\begin{array}{lllllllllllll}83 & 8.942+00 & 0.000+00 & 0.000+00 & 6.468-02 & 7.949-02 & 9.148-02 & 9.983-02 & 1.052-01 & 1.083-01\end{array}$

$\begin{array}{lllllllllll}84 & 8.949+00 & 0.000+00 & 0.000+00 & 1.090-02 & 6.949-03 & 4.985-03 & 4.050-03 & 3.608-03 & 3.399-03\end{array}$

$\begin{array}{llllllllll}85 & 8.964+00 & 0.000+00 & 0.000+00 & 8.493-03 & 4.386-03 & 2.384-03 & 1.436-03 & 9.766-04 & 7.533-04\end{array}$

$\begin{array}{lllllllllll}86 & 8.977+00 & 1.287-03 & 1.666+08 & 2.569-02 & 3.116-02 & 3.560-02 & 3.886-02 & 4.108-02 & 4.249-02\end{array}$

$\begin{array}{lllllllllll}87 & 8.987+00 & 0.000+00 & 0.000+00 & 1.571-02 & 1.723-02 & 1.882-02 & 2.009-02 & 2.097-02 & 2.150-02\end{array}$

$\begin{array}{lllllllllll}88 & 8.994+00 & 0.000+00 & 0.000+00 & 9.326-03 & 8.282-03 & 7.974-03 & 8.024-03 & 8.163-03 & 8.286-03\end{array}$

$\begin{array}{lllllllllllllll}89 & 9.039+00 & 0.000+00 & 0.000+00 & 2.184-02 & 2.322-02 & 2.514-02 & 2.688-02 & 2.818-02 & 2.890-02\end{array}$

$\begin{array}{lllllllllll}90 & 9.044+00 & 1.062-01 & 1.396+10 & 1.479-02 & 2.082-02 & 2.934-02 & 3.967-02 & 5.091-02 & 6.265-02\end{array}$

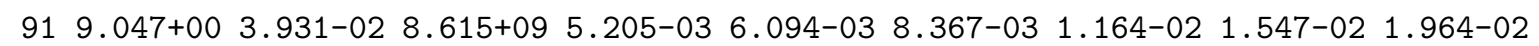

$\begin{array}{llllllllll}92 & 9.070+00 & 5.854-04 & 7.737+07 & 7.203-03 & 3.967-03 & 2.277-03 & 1.463-03 & 1.099-03 & 9.487-04\end{array}$

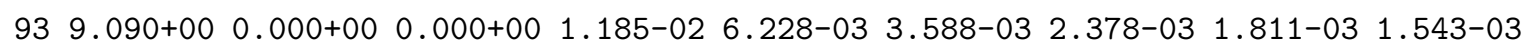

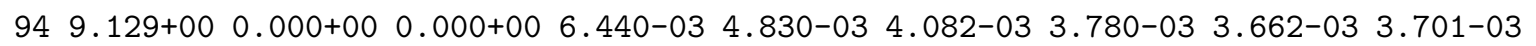

$\begin{array}{lllllllllll}95 & 9.134+00 & 8.151-02 & 1.093+10 & 7.087-03 & 1.086-02 & 1.645-02 & 2.357-02 & 3.155-02 & 4.023-02\end{array}$

$\begin{array}{llllllllll}96 & 9.140+00 & 2.398-02 & 5.363+09 & 2.956-03 & 3.511-03 & 4.799-03 & 6.681-03 & 8.907-03 & 1.139-02\end{array}$

$\begin{array}{llllllllll}97 & 9.215+00 & 1.206-04 & 1.645+07 & 3.101-03 & 1.781-03 & 1.047-03 & 6.692-04 & 4.805-04 & 3.944-04\end{array}$

$\begin{array}{llllllllllll}98 & 9.245+00 & 0.000+00 & 4.149+06 & 2.771-02 & 3.125-02 & 3.573-02 & 4.027-02 & 4.437-02 & 4.756-02\end{array}$

$\begin{array}{llllllllll}99 & 9.245+00 & 0.000+00 & 5.930+06 & 4.701-02 & 5.957-02 & 7.153-02 & 8.234-02 & 9.161-02 & 9.872-02\end{array}$

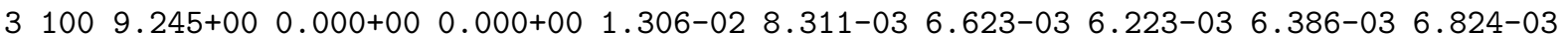

$\begin{array}{lllllllllll}3 & 101 & 9.283+00 & 0.000+00 & 9.079+03 & 1.318-02 & 6.464-03 & 3.215-03 & 1.660-03 & 8.916-04 & 5.075-04\end{array}$

$\begin{array}{lllllllllll}3 & 102 & 9.319+00 & 0.000+00 & 0.000+00 & 2.953-03 & 2.266-03 & 2.327-03 & 2.490-03 & 2.646-03 & 2.717-03\end{array}$

$\begin{array}{lllllllllll}3 & 103 & 9.321+00 & 0.000+00 & 0.000+00 & 4.039-03 & 4.067-03 & 4.772-03 & 5.404-03 & 5.839-03 & 6.041-03\end{array}$

$\begin{array}{lllllllllll}3 & 104 & 9.323+00 & 0.000+00 & 0.000+00 & 4.270-03 & 2.532-03 & 2.207-03 & 2.284-03 & 2.358-03 & 2.410-03\end{array}$

$\begin{array}{lllllllllll}3 & 105 & 9.394+00 & 0.000+00 & 0.000+00 & 2.316-03 & 9.813-04 & 4.356-04 & 2.061-04 & 1.037-04 & 5.620-05\end{array}$

$\begin{array}{llllllllllll}3 & 106 & 9.520+00 & 3.321-04 & 8.060+07 & 5.891-05 & 4.475-05 & 4.655-05 & 6.078-05 & 8.274-05 & 1.111-04\end{array}$ $\begin{array}{llllllllllll}3 & 107 & 9.521+00 & 9.543-04 & 1.390+08 & 1.726-04 & 1.842-04 & 2.260-04 & 2.897-04 & 3.660-04 & 4.543-04\end{array}$ $\begin{array}{lllllllllllll}3 & 108 & 9.523+00 & 0.000+00 & 0.000+00 & 2.145-04 & 2.200-04 & 2.376-04 & 2.541-04 & 2.657-04 & 2.726-04\end{array}$ $\begin{array}{lllllllllllll}3 & 109 & 9.597+00 & 0.000+00 & 0.000+00 & 4.428-04 & 2.472-04 & 1.328-04 & 7.024-05 & 3.684-05 & 1.896-05\end{array}$ $\begin{array}{lllllllllllll}3 & 110 & 1.001+01 & 0.000+00 & 0.000+00 & 8.904-04 & 4.472-04 & 2.406-04 & 1.334-04 & 7.226-05 & 3.722-05\end{array}$ $\begin{array}{llllllllllll}3 & 111 & 1.001+01 & 0.000+00 & 0.000+00 & 4.380-02 & 4.787-02 & 5.093-02 & 5.308-02 & 5.444-02 & 5.531-02\end{array}$ $\begin{array}{llllllllllll}3 & 112 & 1.004+01 & 0.000+00 & 0.000+00 & 1.698-04 & 8.541-05 & 4.615-05 & 2.596-05 & 1.451-05 & 8.021-06\end{array}$ $\begin{array}{llllllllllll}3 & 113 & 1.005+01 & 0.000+00 & 0.000+00 & 7.394-04 & 3.772-04 & 2.094-04 & 1.233-04 & 7.451-05 & 4.684-05\end{array}$ $\begin{array}{llllllllllll}3 & 114 & 1.006+01 & 0.000+00 & 0.000+00 & 8.627-03 & 9.402-03 & 9.987-03 & 1.039-02 & 1.064-02 & 1.080-02\end{array}$ $\begin{array}{lllllllllll}3 & 115 & 1.008+01 & 0.000+00 & 0.000+00 & 4.376-05 & 3.569-05 & 3.341-05 & 3.346-05 & 3.475-05 & 3.499-05\end{array}$ 
Table II. Ca IX Oscillator Strengths, Radiative Decay Rates, and Collision Strengths for transitions involving the lowest 4 levels.

Low. Upp. Osc. Rad. Dec.

Lev. Lev.

Str. Rate

Collision Strength

Impact Electron Energy - Eo (Ry)

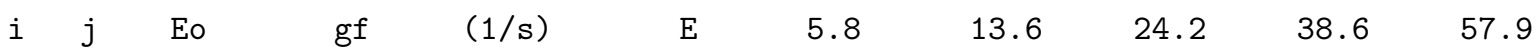

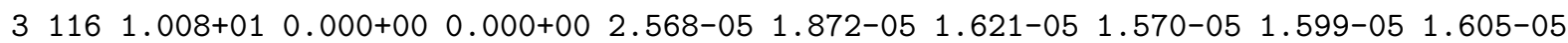
$\begin{array}{llllllllllll}3 & 117 & 1.008+01 & 0.000+00 & 0.000+00 & 1.464-05 & 1.249-05 & 1.196-05 & 1.200-05 & 1.266-05 & 1.287-05\end{array}$

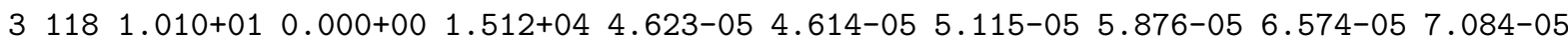
$\begin{array}{llllllllllll}3 & 119 & 1.010+01 & 0.000+00 & 2.537+04 & 8.923-05 & 1.121-04 & 1.359-04 & 1.609-04 & 1.823-04 & 1.968-04\end{array}$ $\begin{array}{lllllllllll}3 & 120 & 1.011+01 & 0.000+00 & 0.000+00 & 2.652-05 & 1.787-05 & 1.477-05 & 1.408-05 & 1.467-05 & 1.554-05\end{array}$ $\begin{array}{llllllllllll}3 & 121 & 1.019+01 & 0.000+00 & 0.000+00 & 9.791-05 & 4.925-05 & 2.658-05 & 1.485-05 & 8.117-06 & 4.106-06\end{array}$ $\begin{array}{lllllllllll}3 & 122 & 1.019+01 & 0.000+00 & 0.000+00 & 6.353-03 & 7.041-03 & 7.546-03 & 7.897-03 & 8.123-03 & 8.267-03\end{array}$ $\begin{array}{llllllllllll}3 & 123 & 1.020+01 & 0.000+00 & 0.000+00 & 1.982-04 & 1.079-04 & 6.686-05 & 4.626-05 & 3.491-05 & 2.863-05\end{array}$ $\begin{array}{llllllllllll}3 & 124 & 1.022+01 & 0.000+00 & 0.000+00 & 3.003-05 & 1.893-05 & 1.471-05 & 1.377-05 & 1.378-05 & 1.436-05\end{array}$ $\begin{array}{lllllllllll}3 & 125 & 1.028+01 & 1.328-02 & 3.760+09 & 2.054-03 & 1.860-03 & 2.150-03 & 2.887-03 & 3.896-03 & 5.030-03\end{array}$ $\begin{array}{llllllllllll}3 & 126 & 1.031+01 & 3.829-04 & 1.089+08 & 1.610-03 & 9.457-04 & 5.315-04 & 3.310-04 & 2.487-04 & 2.263-04\end{array}$ $\begin{array}{lllllllllll}3 & 127 & 1.031+01 & 0.000+00 & 0.000+00 & 2.801-04 & 1.566-04 & 1.013-04 & 7.459-05 & 6.011-05 & 5.259-05\end{array}$ $\begin{array}{lllllllllll}3 & 128 & 1.031+01 & 7.084-02 & 1.210+10 & 4.717-03 & 6.364-03 & 9.639-03 & 1.449-02 & 2.035-02 & 2.663-02\end{array}$ $\begin{array}{lllllllllll}3 & 129 & 1.033+01 & 1.837-02 & 1.573+10 & 1.098-03 & 1.542-03 & 2.400-03 & 3.658-03 & 5.174-03 & 6.810-03\end{array}$ $\begin{array}{lllllllllll}3 & 130 & 1.034+01 & 3.081-02 & 8.821+09 & 1.774-03 & 2.544-03 & 3.985-03 & 6.079-03 & 8.600-03 & 1.131-02\end{array}$ $\begin{array}{llllllllllllll}3 & 131 & 1.034+01 & 0.000+00 & 0.000+00 & 9.575-04 & 5.509-04 & 2.930-04 & 1.577-04 & 8.953-05 & 5.598-05\end{array}$ $\begin{array}{llllllllllll}3 & 132 & 1.036+01 & 4.765-03 & 8.212+08 & 1.056-03 & 8.269-04 & 8.243-04 & 1.027-03 & 1.351-03 & 1.742-03\end{array}$ $\begin{array}{llllllllllllll}3 & 133 & 1.037+01 & 5.079-03 & 1.463+09 & 5.998-04 & 5.783-04 & 7.063-04 & 9.771-04 & 1.339-03 & 1.746-03\end{array}$ $\begin{array}{llllllllllll}3 & 134 & 1.042+01 & 2.187-03 & 3.812+08 & 1.193-03 & 7.852-04 & 6.011-04 & 6.033-04 & 7.105-04 & 8.681-04\end{array}$ $\begin{array}{lllllllllllll}3 & 135 & 1.046+01 & 3.113-04 & 2.738+08 & 2.323-04 & 1.491-04 & 1.045-04 & 9.353-05 & 1.018-04 & 1.209-04\end{array}$ $\begin{array}{lllllllllll}3 & 136 & 1.068+01 & 0.000+00 & 1.811+06 & 3.981-03 & 3.365-03 & 3.442-03 & 3.799-03 & 4.198-03 & 4.601-03\end{array}$ $\begin{array}{lllllllllll}3 & 137 & 1.069+01 & 0.000+00 & 1.619+04 & 3.398-03 & 1.697-03 & 9.161-04 & 5.134-04 & 2.994-04 & 1.881-04\end{array}$

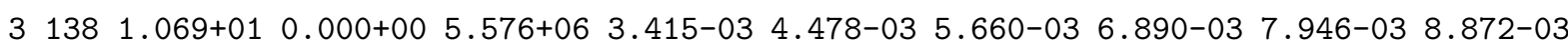
$\begin{array}{lllllllllll}3 & 139 & 1.069+01 & 0.000+00 & 5.601+06 & 8.127-03 & 1.026-02 & 1.283-02 & 1.556-02 & 1.794-02 & 2.003-02\end{array}$ $\begin{array}{llllllllllll}3 & 140 & 1.072+01 & 0.000+00 & 2.704+06 & 2.306-03 & 3.231-03 & 4.190-03 & 5.162-03 & 5.992-03 & 6.716-03\end{array}$ $\begin{array}{llllllllllll}3 & 141 & 1.073+01 & 0.000+00 & 3.259+05 & 1.525-03 & 1.056-03 & 9.206-04 & 9.224-04 & 9.724-04 & 1.043-03\end{array}$ $\begin{array}{llllllllllll}3 & 142 & 1.074+01 & 0.000+00 & 0.000+00 & 1.566-03 & 7.825-04 & 4.156-04 & 2.226-04 & 1.196-04 & 6.508-05\end{array}$ $\begin{array}{llllllllllll}3 & 143 & 1.075+01 & 0.000+00 & 1.964+06 & 2.234-03 & 2.564-03 & 3.085-03 & 3.689-03 & 4.226-03 & 4.719-03\end{array}$ $\begin{array}{lllllllllllll}3 & 144 & 1.076+01 & 0.000+00 & 0.000+00 & 1.096-04 & 6.933-05 & 4.585-05 & 3.334-05 & 2.706-05 & 2.410-05\end{array}$ $\begin{array}{llllllllllll}3 & 145 & 1.076+01 & 0.000+00 & 6.749+05 & 8.852-04 & 7.627-04 & 7.847-04 & 8.655-04 & 9.544-04 & 1.045-03\end{array}$ $\begin{array}{lllllllllll}3 & 146 & 1.077+01 & 0.000+00 & 0.000+00 & 1.693-04 & 8.265-05 & 4.292-05 & 2.247-05 & 1.171-05 & 5.914-06\end{array}$ $\begin{array}{lllllllllll}3 & 147 & 1.078+01 & 0.000+00 & 2.605+05 & 2.056-03 & 1.316-03 & 1.031-03 & 9.421-04 & 9.338-04 & 9.672-04\end{array}$

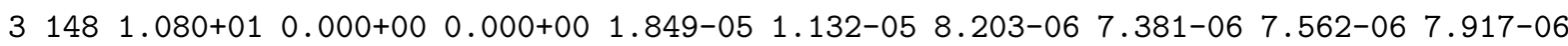
$\begin{array}{lllllllllll}3 & 149 & 1.080+01 & 0.000+00 & 0.000+00 & 3.223-05 & 2.431-05 & 2.179-05 & 2.261-05 & 2.475-05 & 2.689-05\end{array}$

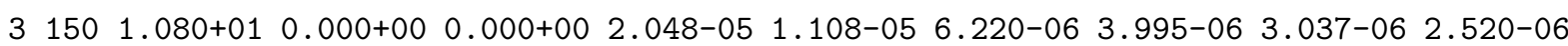
$\begin{array}{lllllllllll}3 & 151 & 1.081+01 & 0.000+00 & 0.000+00 & 3.303-03 & 2.935-03 & 2.789-03 & 2.778-03 & 2.830-03 & 2.905-03\end{array}$ $\begin{array}{llllllllllll}3 & 152 & 1.081+01 & 0.000+00 & 0.000+00 & 2.932-03 & 3.392-03 & 3.765-03 & 4.083-03 & 4.343-03 & 4.555-03\end{array}$ $\begin{array}{llllllllllll}3 & 153 & 1.082+01 & 0.000+00 & 0.000+00 & 2.493-04 & 1.399-04 & 7.515-05 & 3.939-05 & 2.036-05 & 1.040-05\end{array}$ $\begin{array}{lllllllllll}3 & 154 & 1.082+01 & 0.000+00 & 0.000+00 & 6.753-06 & 3.338-06 & 1.652-06 & 9.336-07 & 6.352-07 & 4.931-07\end{array}$ $\begin{array}{lllllllllll}3 & 155 & 1.083+01 & 0.000+00 & 0.000+00 & 5.824-03 & 4.871-03 & 4.418-03 & 4.280-03 & 4.296-03 & 4.376-03\end{array}$ $\begin{array}{lllllllllllll}3 & 156 & 1.083+01 & 0.000+00 & 1.027+05 & 8.338-04 & 4.636-04 & 3.014-04 & 2.307-04 & 1.992-04 & 1.888-04\end{array}$

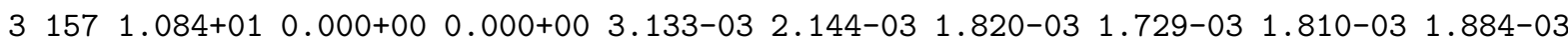
$\begin{array}{llllllllllll}3 & 158 & 1.084+01 & 0.000+00 & 0.000+00 & 5.165-03 & 2.884-03 & 1.561-03 & 8.444-04 & 4.731-04 & 2.837-04\end{array}$ $\begin{array}{llllllllllll}3 & 159 & 1.084+01 & 6.200-05 & 1.170+07 & 7.535-03 & 8.666-03 & 9.604-03 & 1.040-02 & 1.106-02 & 1.160-02\end{array}$

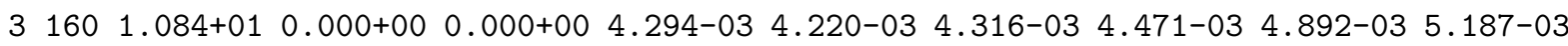


Table II. Ca IX Oscillator Strengths, Radiative Decay Rates, and Collision Strengths for transitions involving the lowest 4 levels.

Low. Upp. Osc. Rad. Dec.

Lev. Lev.

Str. Rate

Collision Strength

Impact Electron Energy - Eo (Ry)

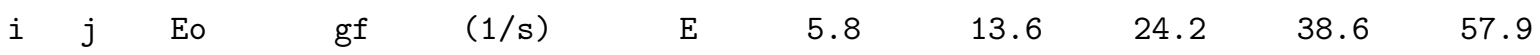

$\begin{array}{lllllllllllllll}3 & 161 & 1.084+01 & 0.000+00 & 0.000+00 & 1.322-02 & 1.515-02 & 1.678-02 & 1.815-02 & 1.928-02 & 2.021-02\end{array}$ $\begin{array}{lllllllllllll}3 & 162 & 1.085+01 & 0.000+00 & 0.000+00 & 2.303-03 & 1.164-03 & 7.531-04 & 6.008-04 & 5.632-04 & 5.559-04\end{array}$

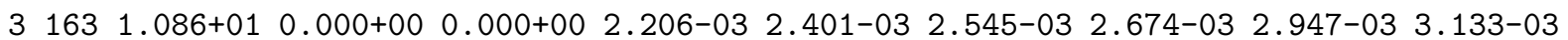

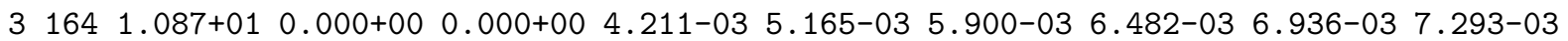
$\begin{array}{llllllllllll}3 & 165 & 1.087+01 & 0.000+00 & 0.000+00 & 2.516-03 & 1.900-03 & 1.582-03 & 1.443-03 & 1.398-03 & 1.396-03\end{array}$ $\begin{array}{llllllllllllll}3 & 166 & 1.087+01 & 0.000+00 & 0.000+00 & 8.930-04 & 3.571-04 & 1.552-04 & 7.276-05 & 3.336-05 & 1.575-05\end{array}$ $\begin{array}{lllllllllllll}3 & 167 & 1.088+01 & 0.000+00 & 0.000+00 & 7.555-04 & 8.017-04 & 8.449-04 & 8.864-04 & 9.755-04 & 1.037-03\end{array}$ $\begin{array}{llllllllllll}3 & 168 & 1.088+01 & 0.000+00 & 0.000+00 & 7.626-04 & 3.147-04 & 1.510-04 & 8.385-05 & 5.476-05 & 4.219-05\end{array}$ $\begin{array}{llllllllllll}3 & 169 & 1.088+01 & 0.000+00 & 0.000+00 & 2.294-03 & 1.260-03 & 6.636-04 & 3.429-04 & 1.771-04 & 9.198-05\end{array}$ $\begin{array}{lllllllllll}3 & 170 & 1.089+01 & 0.000+00 & 0.000+00 & 1.031-03 & 1.016-03 & 1.038-03 & 1.075-03 & 1.176-03 & 1.247-03\end{array}$ $\begin{array}{lllllllllll}3 & 171 & 1.090+01 & 1.411-04 & 4.488+07 & 2.857-05 & 2.195-05 & 2.269-05 & 2.894-05 & 3.833-05 & 4.902-05\end{array}$

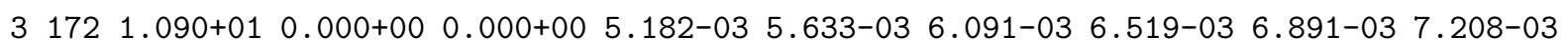
$\begin{array}{lllllllllll}3 & 173 & 1.091+01 & 8.935-04 & 1.708+08 & 2.462-03 & 2.240-03 & 2.193-03 & 2.254-03 & 2.365-03 & 2.495-03\end{array}$ $\begin{array}{lllllllllllll}3 & 174 & 1.092+01 & 3.239-04 & 1.033+08 & 7.069-04 & 4.145-04 & 2.548-04 & 1.798-04 & 1.536-04 & 1.537-04\end{array}$

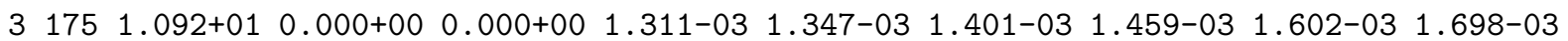
$\begin{array}{llllllllllllll}3 & 176 & 1.092+01 & 0.000+00 & 0.000+00 & 8.443-04 & 3.856-04 & 2.226-04 & 1.611-04 & 1.409-04 & 1.339-04\end{array}$ $\begin{array}{lllllllllllll}3 & 177 & 1.093+01 & 0.000+00 & 0.000+00 & 3.248-04 & 1.275-04 & 5.662-05 & 2.918-05 & 1.690-05 & 1.163-05\end{array}$ $\begin{array}{llllllllllllll}3 & 178 & 1.094+01 & 0.000+00 & 0.000+00 & 5.648-04 & 5.322-04 & 5.360-04 & 5.504-04 & 6.013-04 & 6.359-04\end{array}$ $\begin{array}{lllllllllll}3 & 179 & 1.094+01 & 0.000+00 & 0.000+00 & 3.644-03 & 2.285-03 & 1.524-03 & 1.124-03 & 9.302-04 & 8.433-04\end{array}$ $\begin{array}{llllllllllll}3 & 180 & 1.095+01 & 0.000+00 & 0.000+00 & 2.456-04 & 1.571-04 & 1.100-04 & 8.578-05 & 7.483-05 & 7.081-05\end{array}$

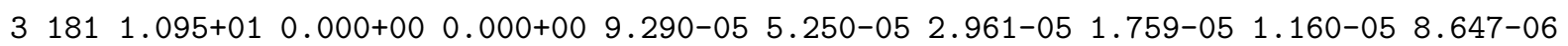
$\begin{array}{lllllllllllll}3 & 182 & 1.095+01 & 0.000+00 & 0.000+00 & 2.539-04 & 2.784-04 & 3.022-04 & 3.237-04 & 3.419-04 & 3.570-04\end{array}$

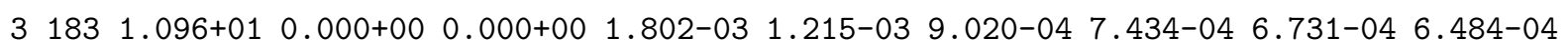

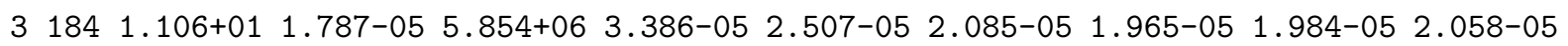
$\begin{array}{lllllllllll}3 & 185 & 1.106+01 & 2.579-05 & 2.534+07 & 2.647-05 & 2.348-05 & 2.257-05 & 2.321-05 & 2.459-05 & 2.622-05\end{array}$ $\begin{array}{lllllllllll}3 & 186 & 1.106+01 & 2.654-05 & 5.216+06 & 5.541-05 & 4.202-05 & 3.565-05 & 3.377-05 & 3.401-05 & 3.509-05\end{array}$ $\begin{array}{llllllllllll}3 & 187 & 1.107+01 & 0.000+00 & 0.000+00 & 1.372-04 & 7.121-05 & 3.696-05 & 2.000-05 & 1.198-05 & 8.011-06\end{array}$ $\begin{array}{llllllllllllll}3 & 188 & 1.109+01 & 0.000+00 & 0.000+00 & 3.461-04 & 1.882-04 & 1.007-04 & 5.436-05 & 3.108-05 & 1.952-05\end{array}$ $\begin{array}{llllllllllll}3 & 189 & 1.110+01 & 0.000+00 & 0.000+00 & 5.732-05 & 2.013-05 & 7.831-06 & 3.486-06 & 1.545-06 & 7.385-07\end{array}$ $\begin{array}{lllllllllllll}3 & 190 & 1.115+01 & 0.000+00 & 0.000+00 & 2.603-04 & 2.618-04 & 2.677-04 & 2.757-04 & 3.016-04 & 3.164-04\end{array}$ $\begin{array}{lllllllllll}3 & 191 & 1.115+01 & 0.000+00 & 0.000+00 & 7.314-05 & 6.015-05 & 5.802-05 & 5.935-05 & 6.311-05 & 6.420-05\end{array}$ $\begin{array}{lllllllllll}3 & 192 & 1.115+01 & 0.000+00 & 0.000+00 & 7.830-05 & 3.740-05 & 2.188-05 & 1.524-05 & 1.290-05 & 1.164-05\end{array}$ $\begin{array}{llllllllllll}3 & 193 & 1.115+01 & 0.000+00 & 0.000+00 & 1.062-04 & 1.146-04 & 1.260-04 & 1.378-04 & 1.522-04 & 1.580-04\end{array}$ $\begin{array}{llllllllllll}3 & 194 & 1.115+01 & 0.000+00 & 0.000+00 & 2.569-04 & 3.021-04 & 3.248-04 & 3.416-04 & 3.766-04 & 3.968-04\end{array}$ $\begin{array}{lllllllllll}3 & 195 & 1.115+01 & 0.000+00 & 0.000+00 & 1.289-04 & 4.795-05 & 2.027-05 & 9.888-06 & 5.183-06 & 3.353-06\end{array}$ $\begin{array}{lllllllllll}3 & 196 & 1.119+01 & 0.000+00 & 0.000+00 & 1.250-05 & 7.211-06 & 4.259-06 & 2.653-06 & 1.776-06 & 1.325-06\end{array}$ $\begin{array}{lllllllllll}3 & 197 & 1.126+01 & 0.000+00 & 0.000+00 & 2.596-05 & 2.347-05 & 2.253-05 & 2.278-05 & 2.491-05 & 2.589-05\end{array}$

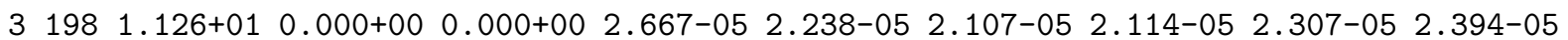
$\begin{array}{lllllllllll}3 & 199 & 1.127+01 & 0.000+00 & 0.000+00 & 2.525-05 & 9.714-06 & 4.500-06 & 2.747-06 & 2.092-06 & 1.843-06\end{array}$ $\begin{array}{lllllllllll}3 & 200 & 1.128+01 & 0.000+00 & 0.000+00 & 6.366-06 & 6.890-06 & 7.466-06 & 9.392-06 & 1.000-05 & 1.106-05\end{array}$ $\begin{array}{lllllllllll}3 & 201 & 1.128+01 & 0.000+00 & 0.000+00 & 9.245-06 & 7.820-06 & 7.505-06 & 8.872-06 & 9.088-06 & 9.885-06\end{array}$

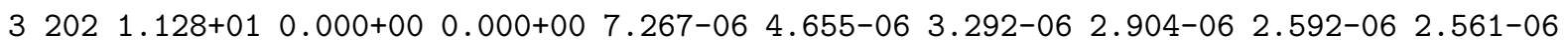
$\begin{array}{lllllllllll}3 & 203 & 1.131+01 & 0.000+00 & 0.000+00 & 2.316-05 & 1.809-05 & 1.641-05 & 1.657-05 & 1.751-05 & 1.751-05\end{array}$ $\begin{array}{lllllllllll}3 & 204 & 1.131+01 & 0.000+00 & 0.000+00 & 1.842-05 & 1.899-05 & 2.009-05 & 2.131-05 & 2.209-05 & 2.277-05\end{array}$ $\begin{array}{lllllllllll}3 & 205 & 1.131+01 & 0.000+00 & 0.000+00 & 1.883-06 & 1.030-06 & 5.736-07 & 3.252-07 & 1.840-07 & 1.023-07\end{array}$ 
Table II. Ca IX Oscillator Strengths, Radiative Decay Rates, and Collision Strengths for transitions involving the lowest 4 levels.

Low. Upp. Osc. Rad. Dec.

Lev. Lev.

Str. Rate

Collision Strength

Impact Electron Energy - Eo (Ry)

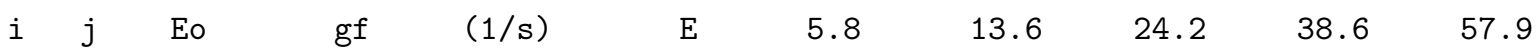

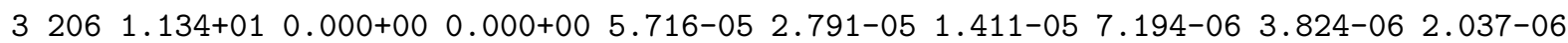
$\begin{array}{lllllllllll}3 & 207 & 1.135+01 & 0.000+00 & 0.000+00 & 2.167-05 & 1.143-05 & 5.684-06 & 2.850-06 & 1.489-06 & 8.193-07\end{array}$

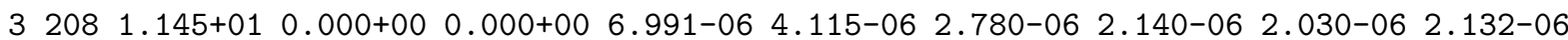

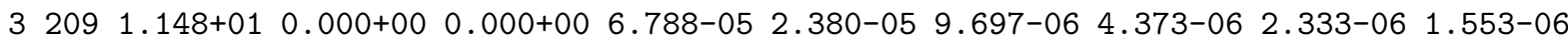
$\begin{array}{lllllllllll}3 & 210 & 1.225+01 & 0.000+00 & 0.000+00 & 6.058-06 & 4.473-06 & 3.609-06 & 3.132-06 & 2.950-06 & 3.017-06\end{array}$ $\begin{array}{lllllllllllll}3 & 211 & 1.225+01 & 0.000+00 & 0.000+00 & 1.307-05 & 1.184-05 & 1.142-05 & 1.132-05 & 1.157-05 & 1.225-05\end{array}$

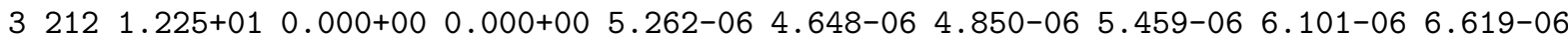
$\begin{array}{lllllllllll}3 & 213 & 1.228+01 & 0.000+00 & 0.000+00 & 1.274-05 & 6.690-06 & 3.511-06 & 1.895-06 & 1.073-06 & 6.045-07\end{array}$ $\begin{array}{lllllllllll}3 & 214 & 1.250+01 & 0.000+00 & 0.000+00 & 4.888-06 & 2.533-06 & 1.422-06 & 9.000-07 & 6.462-07 & 5.104-07\end{array}$

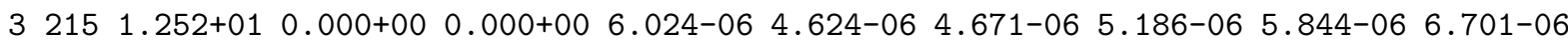

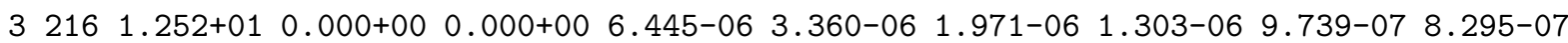

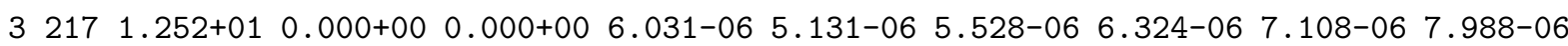

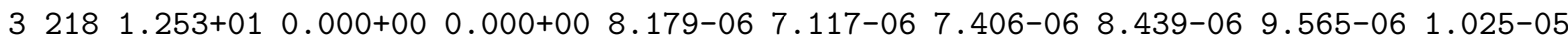
$\begin{array}{lllllllllll}3 & 219 & 1.253+01 & 0.000+00 & 4.244+03 & 8.916-06 & 9.548-06 & 1.122-05 & 1.362-05 & 1.591-05 & 1.721-05\end{array}$ $\begin{array}{llllllllllll}3 & 220 & 1.254+01 & 0.000+00 & 0.000+00 & 3.215-06 & 1.714-06 & 1.028-06 & 7.480-07 & 6.529-07 & 6.443-07\end{array}$

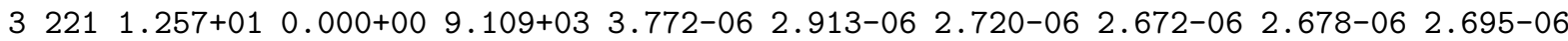
$\begin{array}{lllllllllll}3 & 222 & 1.257+01 & 0.000+00 & 5.046+03 & 8.573-06 & 8.098-06 & 8.093-06 & 8.162-06 & 8.167-06 & 8.101-06\end{array}$ $\begin{array}{llllllllllll}3 & 223 & 1.257+01 & 0.000+00 & 0.000+00 & 1.250-06 & 6.088-07 & 2.999-07 & 1.503-07 & 7.652-08 & 3.769-08\end{array}$

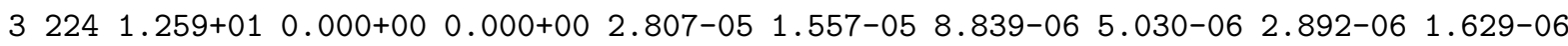

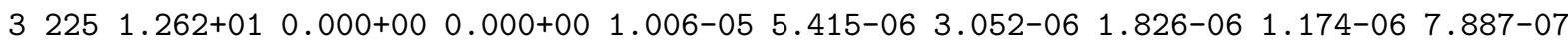
$\begin{array}{lllllllllll}3 & 226 & 1.285+01 & 0.000+00 & 0.000+00 & 4.645-06 & 2.590-06 & 1.409-06 & 7.691-07 & 4.349-07 & 2.607-07\end{array}$ $\begin{array}{llllllllll}3227 & 1.287+01 & 0.000+00 & 0.000+00 & 3.252-06 & 2.419-06 & 2.497-06 & 3.140-06 & 4.015-06 & 5.035-06\end{array}$

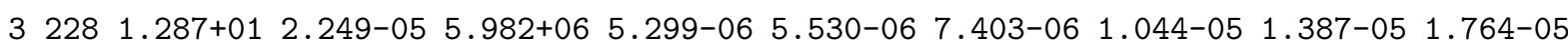

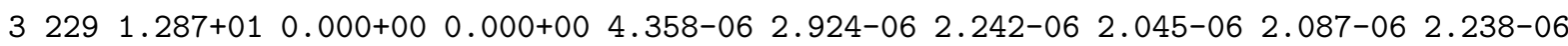
$\begin{array}{lllllllllll}3 & 230 & 1.288+01 & 0.000+00 & 0.000+00 & 1.500-05 & 1.074-05 & 8.555-06 & 7.577-06 & 7.256-06 & 7.326-06\end{array}$ $\begin{array}{lllllllllllll}3 & 231 & 1.288+01 & 0.000+00 & 0.000+00 & 1.299-05 & 1.100-05 & 1.046-05 & 1.062-05 & 1.113-05 & 1.183-05\end{array}$ $\begin{array}{llllllllllll}3 & 232 & 1.288+01 & 0.000+00 & 0.000+00 & 6.251-06 & 3.720-06 & 2.245-06 & 1.423-06 & 1.004-06 & 7.460-07\end{array}$ $\begin{array}{llllllllllll}3 & 233 & 1.288+01 & 0.000+00 & 0.000+00 & 1.661-06 & 9.014-07 & 5.064-07 & 3.103-07 & 2.183-07 & 1.694-07\end{array}$ $\begin{array}{lllllllllll}3 & 234 & 1.291+01 & 0.000+00 & 0.000+00 & 2.921-06 & 2.997-06 & 4.074-06 & 5.513-06 & 6.691-06 & 7.525-06\end{array}$ $\begin{array}{lllllllllll}3 & 235 & 1.292+01 & 0.000+00 & 0.000+00 & 4.761-06 & 3.065-06 & 2.254-06 & 1.947-06 & 1.907-06 & 2.008-06\end{array}$ $\begin{array}{lllllllllll}3 & 236 & 1.292+01 & 0.000+00 & 0.000+00 & 5.307-06 & 2.979-06 & 1.660-06 & 9.532-07 & 5.917-07 & 4.136-07\end{array}$

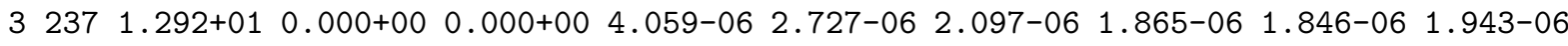

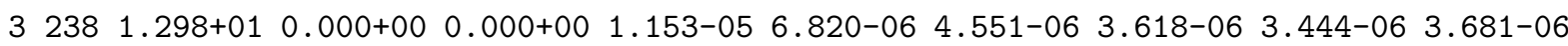
$\begin{array}{lllllllllll}3 & 239 & 1.298+01 & 9.229-05 & 1.249+08 & 4.579-06 & 5.658-06 & 7.292-06 & 9.400-06 & 1.206-05 & 1.541-05\end{array}$

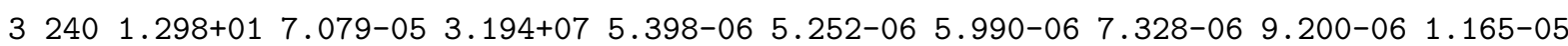

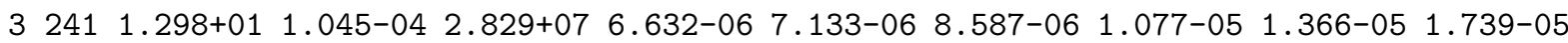
$\begin{array}{lllllllllll}3 & 242 & 1.299+01 & 0.000+00 & 0.000+00 & 1.461-05 & 8.503-06 & 4.843-06 & 2.739-06 & 1.548-06 & 8.756-07\end{array}$ $\begin{array}{lllllllllll}3 & 243 & 1.301+01 & 0.000+00 & 0.000+00 & 7.757-06 & 4.036-06 & 2.170-06 & 1.298-06 & 8.946-07 & 7.159-07\end{array}$ $\begin{array}{lllllllllll}3 & 244 & 1.301+01 & 0.000+00 & 0.000+00 & 1.711-05 & 1.328-05 & 1.220-05 & 1.224-05 & 1.288-05 & 1.399-05\end{array}$ $\begin{array}{llllllllllll}3 & 245 & 1.302+01 & 0.000+00 & 0.000+00 & 1.392-05 & 1.409-05 & 1.581-05 & 1.759-05 & 1.955-05 & 2.180-05\end{array}$ $\begin{array}{llllllllllll}3 & 246 & 1.302+01 & 0.000+00 & 0.000+00 & 6.633-06 & 3.534-06 & 1.872-06 & 1.040-06 & 6.147-07 & 3.624-07\end{array}$ $\begin{array}{lllllllllll}3 & 247 & 1.302+01 & 0.000+00 & 0.000+00 & 1.545-05 & 1.663-05 & 1.926-05 & 2.211-05 & 2.496-05 & 2.703-05\end{array}$ $\begin{array}{lllllllllll}3 & 248 & 1.302+01 & 0.000+00 & 0.000+00 & 2.284-05 & 3.011-05 & 3.798-05 & 4.518-05 & 5.175-05 & 5.647-05\end{array}$ $\begin{array}{llllllllllll}3 & 249 & 1.302+01 & 0.000+00 & 0.000+00 & 7.557-06 & 4.992-06 & 3.877-06 & 3.511-06 & 3.543-06 & 3.792-06\end{array}$

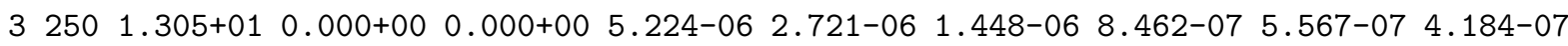


Table II. Ca IX Oscillator Strengths, Radiative Decay Rates, and Collision Strengths for transitions involving the lowest 4 levels.

Low. Upp. Osc. Rad. Dec.

Lev. Lev.

Str. Rate

Collision Strength

Impact Electron Energy - Eo (Ry)

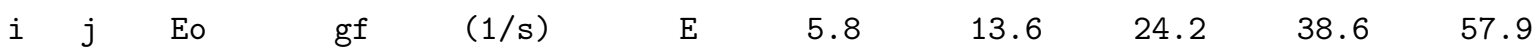

$\begin{array}{lllllllllll}3 & 251 & 1.306+01 & 0.000+00 & 0.000+00 & 2.867-06 & 1.348-06 & 6.297-07 & 3.127-07 & 1.887-07 & 1.325-07\end{array}$

$\begin{array}{lllllllllll}3 & 252 & 1.307+01 & 0.000+00 & 0.000+00 & 8.093-06 & 6.012-06 & 5.715-06 & 6.007-06 & 6.513-06 & 7.197-06\end{array}$

$\begin{array}{lllllllllll}3 & 253 & 1.307+01 & 0.000+00 & 0.000+00 & 2.795-06 & 2.093-06 & 2.032-06 & 2.324-06 & 2.560-06 & 2.685-06\end{array}$

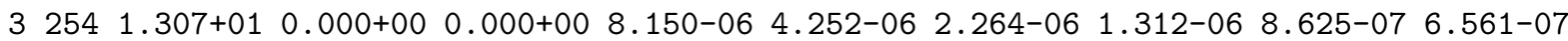

$\begin{array}{lllllllllll}3 & 255 & 1.307+01 & 0.000+00 & 0.000+00 & 2.904-06 & 1.389-06 & 6.735-07 & 3.619-07 & 2.376-07 & 1.827-07\end{array}$

$\begin{array}{lllllllllll}3 & 256 & 1.307+01 & 0.000+00 & 0.000+00 & 6.601-06 & 5.389-06 & 5.421-06 & 5.830-06 & 6.344-06 & 7.011-06\end{array}$

$\begin{array}{lllllllllll}3 & 257 & 1.307+01 & 0.000+00 & 0.000+00 & 2.492-06 & 2.032-06 & 2.049-06 & 2.353-06 & 2.584-06 & 2.702-06\end{array}$

$\begin{array}{llllllllllll}3 & 258 & 1.307+01 & 0.000+00 & 0.000+00 & 1.393-06 & 6.280-07 & 3.699-07 & 3.177-07 & 3.577-07 & 3.899-07\end{array}$

$\begin{array}{lllllllllll}3 & 259 & 1.307+01 & 0.000+00 & 0.000+00 & 1.254-06 & 6.733-07 & 5.212-07 & 5.615-07 & 7.076-07 & 7.810-07\end{array}$

$\begin{array}{lllllllllll}3 & 260 & 1.307+01 & 0.000+00 & 0.000+00 & 9.742-07 & 5.323-07 & 3.868-07 & 3.678-07 & 3.896-07 & 3.963-07\end{array}$

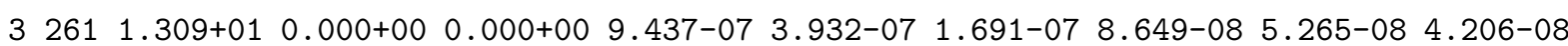

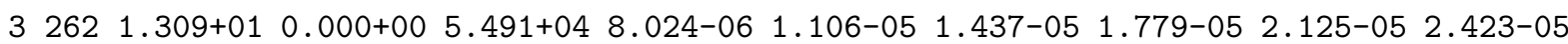

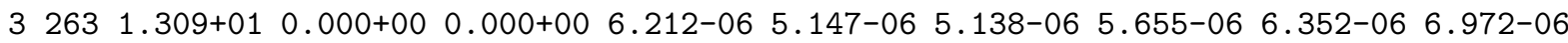

$\begin{array}{llllllllllll}3 & 264 & 1.309+01 & 0.000+00 & 2.443+04 & 9.167-06 & 1.041-05 & 1.261-05 & 1.526-05 & 1.808-05 & 2.057-05\end{array}$

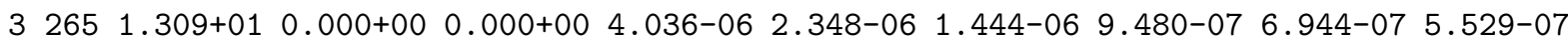

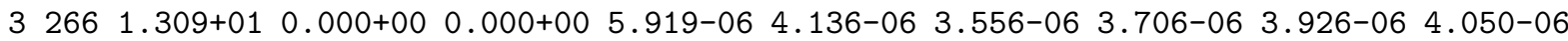

$\begin{array}{llllllllllllll}3 & 267 & 1.309+01 & 0.000+00 & 0.000+00 & 7.378-07 & 8.816-07 & 1.130-06 & 1.367-06 & 1.597-06 & 1.652-06\end{array}$

$\begin{array}{llllllllllllll}3 & 268 & 1.309+01 & 0.000+00 & 0.000+00 & 9.392-07 & 4.652-07 & 2.802-07 & 2.238-07 & 2.141-07 & 2.067-07\end{array}$

$\begin{array}{lllllllllll}3 & 269 & 1.309+01 & 0.000+00 & 0.000+00 & 1.145-06 & 9.412-07 & 9.410-07 & 1.014-06 & 1.123-06 & 1.132-06\end{array}$

$\begin{array}{lllllllllll}3 & 270 & 1.309+01 & 0.000+00 & 0.000+00 & 4.703-06 & 3.957-06 & 4.052-06 & 4.676-06 & 5.165-06 & 5.426-06\end{array}$

$\begin{array}{lllllllllll}3 & 271 & 1.309+01 & 0.000+00 & 0.000+00 & 2.193-06 & 1.095-06 & 5.728-07 & 3.163-07 & 2.035-07 & 1.594-07\end{array}$

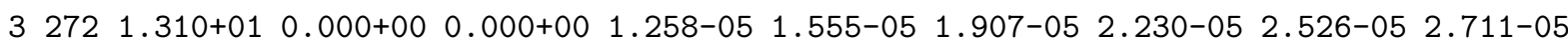

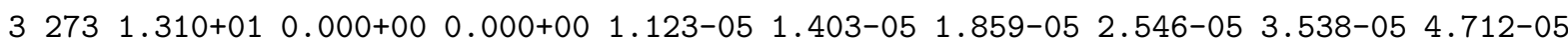

$\begin{array}{lllllllllll}3 & 274 & 1.310+01 & 0.000+00 & 0.000+00 & 4.216-07 & 2.163-07 & 1.083-07 & 5.665-08 & 2.872-08 & 1.428-08\end{array}$

$\begin{array}{lllllllllll}3 & 275 & 1.310+01 & 0.000+00 & 0.000+00 & 1.761-05 & 8.769-06 & 4.314-06 & 2.162-06 & 1.052-06 & 4.898-07\end{array}$

$\begin{array}{lllllllllll}3 & 276 & 1.311+01 & 0.000+00 & 0.000+00 & 4.232-06 & 2.064-06 & 9.824-07 & 4.436-07 & 2.030-07 & 9.268-08\end{array}$

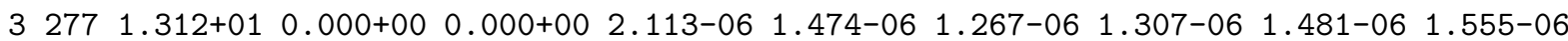

$\begin{array}{lllllllllll}3 & 278 & 1.312+01 & 0.000+00 & 0.000+00 & 1.450-06 & 1.046-06 & 1.037-06 & 1.388-06 & 1.878-06 & 2.175-06\end{array}$

$\begin{array}{lllllllllllllll}3 & 279 & 1.312+01 & 0.000+00 & 0.000+00 & 4.779-07 & 2.843-07 & 2.350-07 & 3.050-07 & 4.236-07 & 5.076-07\end{array}$

$\begin{array}{llllllllllll}3 & 280 & 1.313+01 & 0.000+00 & 0.000+00 & 2.004-06 & 1.155-06 & 6.823-07 & 3.925-07 & 2.322-07 & 1.538-07\end{array}$

$\begin{array}{lllllllllllll}3 & 281 & 1.314+01 & 0.000+00 & 0.000+00 & 2.836-06 & 1.430-06 & 7.504-07 & 4.492-07 & 3.327-07 & 2.881-07\end{array}$

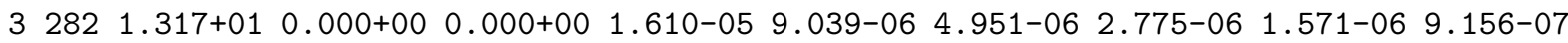

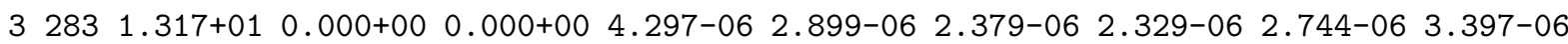

$4556.463-01 \quad 0.000+00 \quad 0.000+00 \quad 7.243-02 \quad 3.678-02 \quad 1.915-02 \quad 1.017-02 \quad 5.530-03 \quad 3.123-03$

$466 \quad 1.719+00 \quad 4.816-02 \quad 2.287+08 \quad 3.942-01 \quad 4.083-01 \quad 4.346-01 \quad 4.703-01 \quad 5.108-01 \quad 5.519-01$

$47 \begin{array}{llllllllll}4 & 1.764+00 & 0.000+00 & 0.000+00 & 5.508-03 & 3.178-03 & 1.708-03 & 9.141-04 & 4.934-04 & 2.668-04\end{array}$

$\begin{array}{llllllllllll}4 & 8 & 1.782+00 & 4.513-01 & 3.839+09 & 2.525+00 & 3.067+00 & 3.560+00 & 4.021+00 & 4.458+00 & 4.869+00\end{array}$

$\begin{array}{llllllllllll}4 & 9 & 1.814+00 & 1.333+00 & 7.045+09 & 7.265+00 & 8.823+00 & 1.024+01 & 1.157+01 & 1.284+01 & 1.402+01\end{array}$

$\begin{array}{lllllllllll}4 & 10 & 2.333+00 & 0.000+00 & 0.000+00 & 1.007-02 & 5.799-03 & 3.119-03 & 1.664-03 & 8.914-04 & 4.764-04\end{array}$

$\begin{array}{llllllllllll}4 & 11 & 2.420+00 & 2.304-02 & 3.612+08 & 1.525-01 & 1.630-01 & 1.789-01 & 1.971-01 & 2.160-01 & 2.342-01\end{array}$

$\begin{array}{lllllllllllll}4 & 12 & 2.421+00 & 3.464-01 & 3.262+09 & 1.264+00 & 1.521+00 & 1.771+00 & 2.012+00 & 2.245+00 & 2.469+00\end{array}$

$\begin{array}{llllllllllll}4 & 13 & 2.423+00 & 1.946+00 & 1.310+10 & 6.570+00 & 8.104+00 & 9.539+00 & 1.089+01 & 1.219+01 & 1.343+01\end{array}$

$\begin{array}{llllllllllll}4 & 14 & 2.971+00 & 0.000+00 & 0.000+00 & 5.154-02 & 2.695-02 & 1.402-02 & 7.430-03 & 4.120-03 & 2.458-03\end{array}$

$\begin{array}{llllllllllll}4 & 15 & 3.789+00 & 0.000+00 & 2.428+04 & 2.416-02 & 2.577-02 & 2.714-02 & 2.832-02 & 2.926-02 & 2.994-02\end{array}$

$\begin{array}{lllllllllllllll}4 & 16 & 3.807+00 & 0.000+00 & 1.390+05 & 1.772-01 & 1.946-01 & 2.079-01 & 2.185-01 & 2.265-01 & 2.321-01\end{array}$ 
Table II. Ca IX Oscillator Strengths, Radiative Decay Rates, and Collision Strengths for transitions involving the lowest 4 levels.

$\begin{array}{lll}\text { Low. Upp. } & \text { Osc. Rad. Dec }\end{array}$ Lev. Lev. Rate
Collision Strength

Impact Electron Energy - Eo (Ry)

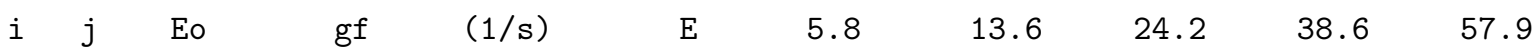

$4 \begin{array}{llllllllll}4 & 3.829+00 & 0.000+00 & 4.708+05 & 6.817-01 & 7.831-01 & 8.565-01 & 9.112-01 & 9.508-01 & 9.776-01\end{array}$ $\begin{array}{llllllllllll}4 & 18 & 3.865+00 & 0.000+00 & 1.762+03 & 3.070-02 & 1.886-02 & 1.153-02 & 7.237-03 & 4.858-03 & 3.581-03\end{array}$ $4 \quad 194.121+00 \quad 0.000+00 \quad 1.796+04 \quad 2.011-02 \quad 1.813-02 \quad 1.712-02 \quad 1.673-02 \quad 1.667-02 \quad 1.672-02$ $4204.131+00 \quad 0.000+00 \quad 6.953+04 \quad 2.955-02 \quad 3.088-02 \quad 3.216-02 \quad 3.338-02 \quad 3.443-02 \quad 3.520-02$ $4214.147+00 \quad 0.000+00 \quad 4.719+05 \quad 5.201-02 \quad 6.003-02 \quad 6.600-02 \quad 7.051-02 \quad 7.381-02 \quad 7.606-02$ $4224.156+00 \quad 0.000+00 \quad 4.040+05 \quad 1.316-01 \quad 1.514-01 \quad 1.662-01 \quad 1.773-01 \quad 1.856-011.912-01$ $423 \quad 4.160+00 \quad 0.000+00 \quad 4.317+05 \quad 2.297-01 \quad 2.652-01 \quad 2.915-01 \quad 3.113-013.258-01 \quad 3.358-01$ $424 \quad 4.161+00 \quad 0.000+00 \quad 3.368+05 \quad 2.528-01 \quad 2.895-01 \quad 3.170-01 \quad 3.378-01 \quad 3.532-01 \quad 3.638-01$ $4 \quad 25 \quad 4.587+00 \quad 0.000+00 \quad 0.000+00 \quad 4.518-02 \quad 2.614-02 \quad 1.460-02 \quad 7.939-03 \quad 4.262-03 \quad 2.276-03$ $4 \quad 26 \quad 4.679+00 \quad 0.000+00 \quad 0.000+00 \quad 1.175-02 \quad 6.907-03 \quad 3.910-03 \quad 2.146-03 \quad 1.162-03 \quad 6.295-04$ $275.577+00 \quad 4.610-01 \quad 3.840+10 \quad 8.997-02 \quad 1.664-01 \quad 2.551-01 \quad 3.524-01 \quad 4.511-01 \quad 5.526-01$ $\begin{array}{lllllllllll}28 & 5.719+00 & 0.000+00 & 0.000+00 & 5.881-03 & 3.253-03 & 1.627-03 & 7.930-04 & 3.790-04 & 1.795-04\end{array}$ $\begin{array}{lllllllllll}29 & 6.219+00 & 0.000+00 & 4.967+06 & 1.737-02 & 2.105-02 & 2.560-02 & 2.940-02 & 3.238-02 & 3.456-02\end{array}$ $306.221+00 \quad 0.000+00 \quad 3.344+06 \quad 4.525-02 \quad 4.728-02 \quad 5.405-02 \quad 6.051-02 \quad 6.587-02 \quad 6.994-02$ $\begin{array}{lllllllllll}31 & 6.230+00 & 0.000+00 & 0.000+00 & 2.780-04 & 2.540-04 & 2.458-04 & 2.462-04 & 2.508-04 & 2.567-04\end{array}$ $\begin{array}{lllllllllll}32 & 6.231+00 & 0.000+00 & 0.000+00 & 6.052-04 & 5.989-04 & 6.108-04 & 6.296-04 & 6.513-04 & 6.719-04\end{array}$ $\begin{array}{llllllllllll}33 & 6.233+00 & 0.000+00 & 1.732+06 & 8.396-01 & 8.914-01 & 9.263-01 & 9.472-01 & 9.589-01 & 9.647-01\end{array}$ $\begin{array}{llllllllllll}34 & 6.234+00 & 0.000+00 & 0.000+00 & 8.753-04 & 8.217-04 & 8.133-04 & 8.258-04 & 8.481-04 & 8.721-04\end{array}$ $\begin{array}{lllllllllllll}35 & 6.243+00 & 0.000+00 & 3.767+05 & 2.389-02 & 1.439-02 & 1.065-02 & 9.074-03 & 8.469-03 & 8.320-03\end{array}$ $\begin{array}{llllllllll}36 & 6.415+00 & 0.000+00 & 0.000+00 & 1.310-03 & 7.027-04 & 3.652-04 & 1.879-04 & 9.589-05 & 4.821-05\end{array}$ $\begin{array}{llllllllll}37 & 6.416+00 & 0.000+00 & 0.000+00 & 8.328-04 & 4.113-04 & 2.016-04 & 9.947-05 & 4.978-05 & 2.494-05\end{array}$ $\begin{array}{llllllllllll}38 & 6.442+00 & 1.983-04 & 2.204+07 & 7.042-05 & 8.484-05 & 1.088-04 & 1.405-04 & 1.741-04 & 2.111-04\end{array}$ $\begin{array}{llllllllll}39 & 6.442+00 & 0.000+00 & 0.000+00 & 7.531-06 & 3.874-06 & 1.958-06 & 1.003-06 & 5.298-07 & 2.986-07\end{array}$ $406.444+00 \quad 6.520-04 \quad 4.349+07 \quad 2.145-04 \quad 2.725-04 \quad 3.566-04 \quad 4.626-04 \quad 5.737-04 \quad 6.956-04$ $416.940+00 \quad 0.000+00 \quad 0.000+00 \quad 5.839-05 \quad 3.253-05 \quad 1.698-05 \quad 8.468-06 \quad 3.998-06 \quad 2.002-06$ $427.013+00 \quad 1.024-02 \quad 1.348+092.127-02 \quad 2.105-02 \quad 2.267-02 \quad 2.448-02 \quad 2.639-02 \quad 2.843-02$

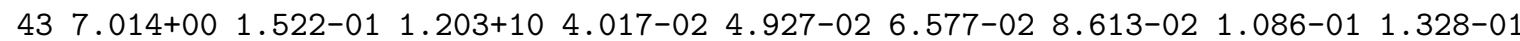
$447.015+00 \quad 8.426-01 \quad 4.759+10 \quad 8.571-02 \quad 1.528-01 \quad 2.478-01 \quad 3.614-01 \quad 4.849-01 \quad 6.174-01$ $\begin{array}{llllllllllllll}45 & 7.030+00 & 0.000+00 & 0.000+00 & 2.421-02 & 1.288-02 & 6.619-03 & 3.357-03 & 1.661-03 & 8.177-04\end{array}$ $46 \quad 7.239+00 \quad 0.000+00 \quad 2.303+04 \quad 4.476-05 \quad 4.498-05 \quad 5.192-05 \quad 5.884-05 \quad 6.523-05 \quad 7.039-05$ $\begin{array}{lllllllllll}47 & 7.250+00 & 0.000+00 & 1.643+04 & 1.873-03 & 9.952-04 & 5.954-04 & 3.921-04 & 2.816-04 & 2.237-04\end{array}$ $48 \quad 7.283+00 \quad 0.000+00 \quad 7.482+03 \quad 4.478-01 \quad 4.838-01 \quad 5.077-01 \quad 5.224-01 \quad 5.308-01 \quad 5.351-01$ $497.358+00 \quad 0.000+00 \quad 1.249+06 \quad 4.354-02 \quad 4.317-02 \quad 4.689-02 \quad 5.187-02 \quad 5.687-02 \quad 6.079-02$ $\begin{array}{llllllllllll}50 & 7.358+00 & 0.000+00 & 6.246+06 & 1.226-01 & 1.443-01 & 1.696-01 & 1.939-01 & 2.133-01 & 2.287-01\end{array}$ $\begin{array}{llllllllllllll}51 & 7.358+00 & 0.000+00 & 1.874+07 & 3.478-01 & 4.638-01 & 5.706-01 & 6.633-01 & 7.329-01 & 7.874-01\end{array}$ $\begin{array}{lllllllllll}52 & 7.373+00 & 0.000+00 & 0.000+00 & 5.651-03 & 2.851-03 & 1.534-03 & 8.447-04 & 4.514-04 & 2.312-04\end{array}$ $\begin{array}{llllllllllll}53 & 7.455+00 & 0.000+00 & 0.000+00 & 4.626-02 & 2.097-02 & 9.722-03 & 4.636-03 & 2.194-03 & 1.000-03\end{array}$ $\begin{array}{lllllllllll}54 & 7.754+00 & 0.000+00 & 0.000+00 & 2.210-03 & 1.288-03 & 7.034-04 & 3.957-04 & 2.413-04 & 1.683-04\end{array}$ $\begin{array}{lllllllllll}55 & 7.808+00 & 1.816-03 & 2.965+08 & 2.133-03 & 1.553-03 & 1.307-03 & 1.307-03 & 1.437-03 & 1.640-03\end{array}$ $\begin{array}{lllllllllllll}56 & 7.820+00 & 2.314-02 & 2.273+09 & 5.088-03 & 5.255-03 & 6.635-03 & 8.913-03 & 1.166-02 & 1.472-02\end{array}$ $\begin{array}{llllllllllllllll}57 & 7.850+00 & 1.944-01 & 1.374+10 & 2.554-02 & 3.269-02 & 4.752-02 & 6.808-02 & 9.173-02 & 1.176-01\end{array}$ $\begin{array}{llllllllllll}58 & 7.887+00 & 0.000+00 & 0.000+00 & 4.560-04 & 2.576-04 & 1.328-04 & 6.670-05 & 3.326-05 & 1.699-05\end{array}$ $\begin{array}{lllllllllll}59 & 7.894+00 & 2.531-03 & 4.223+08 & 2.035-03 & 1.375-03 & 1.080-03 & 1.070-03 & 1.235-03 & 1.500-03\end{array}$ $607.916+00 \quad 1.259-01 \quad 2.113+10 \quad 1.310-02 \quad 1.836-02 \quad 2.812-02 \quad 4.137-02 \quad 5.652-02 \quad 7.307-02$ $\begin{array}{llllllllll}61 & 7.918+00 & 1.600-01 & 1.612+10 & 1.861-02 & 2.427-02 & 3.601-02 & 5.230-02 & 7.127-02 & 9.215-02\end{array}$ 
Table II. Ca IX Oscillator Strengths, Radiative Decay Rates, and Collision Strengths for transitions involving the lowest 4 levels.

Low. Upp. Osc. Rad. Dec. Lev. Lev. Rate
Collision Strength

Impact Electron Energy - Eo (Ry)

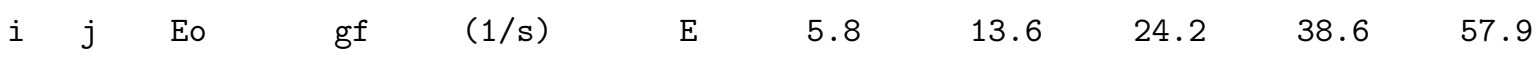

$462 \quad 8.023+00 \quad 1.112-03 \quad 1.150+08 \quad 5.752-03 \quad 3.312-03 \quad 1.865-03 \quad 1.179-03 \quad 9.104-04 \quad 8.564-04$

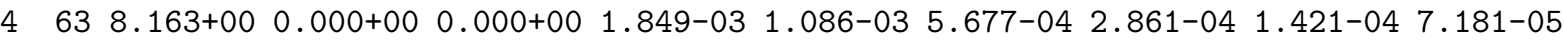
$\begin{array}{lllllllllll}4 & 64 & 8.386+00 & 7.864-02 & 1.481+10 & 1.172-02 & 1.754-02 & 2.517-02 & 3.446-02 & 4.467-02 & 5.500-02\end{array}$ $465 \quad 8.485+00 \quad 0.000+00 \quad 0.000+00 \quad 1.544-03 \quad 8.249-04 \quad 4.026-04 \quad 1.963-04 \quad 9.674-05 \quad 4.471-05$

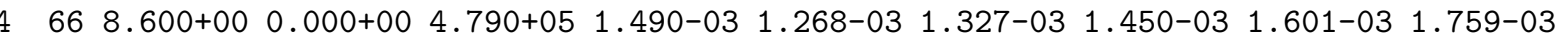
$\begin{array}{lllllllllll}67 & 8.601+00 & 0.000+00 & 6.429+05 & 7.364-03 & 7.738-03 & 8.360-03 & 8.928-03 & 9.429-03 & 9.872-03\end{array}$ $\begin{array}{llllllllll}68 & 8.609+00 & 0.000+00 & 8.956+05 & 4.685-03 & 4.198-03 & 4.465-03 & 4.928-03 & 5.430-03 & 5.941-03\end{array}$ $\begin{array}{llllllllll}69 & 8.612+00 & 0.000+00 & 2.341+06 & 9.695-03 & 1.160-02 & 1.428-02 & 1.688-02 & 1.919-02 & 2.130-02\end{array}$ $\begin{array}{llllllllll}70 & 8.644+00 & 0.000+00 & 6.620+06 & 1.760-02 & 1.846-02 & 2.104-02 & 2.423-02 & 2.712-02 & 2.956-02\end{array}$ $\begin{array}{llllllllll}71 & 8.645+00 & 0.000+00 & 8.818+06 & 6.750-03 & 7.843-03 & 9.313-03 & 1.092-02 & 1.232-02 & 1.345-02\end{array}$ $\begin{array}{lllllllllll}72 & 8.647+00 & 0.000+00 & 4.551+06 & 1.279-01 & 1.368-01 & 1.454-01 & 1.528-01 & 1.584-01 & 1.625-01\end{array}$ $\begin{array}{llllllllll}73 & 8.653+00 & 0.000+00 & 1.037+06 & 1.188-02 & 7.533-03 & 5.816-03 & 5.213-03 & 5.079-03 & 5.148-03\end{array}$ $\begin{array}{llllllllll}74 & 8.660+00 & 0.000+00 & 8.830+05 & 6.555-03 & 5.154-03 & 4.931-03 & 5.087-03 & 5.435-03 & 5.849-03\end{array}$ $\begin{array}{lllllllllll}75 & 8.664+00 & 0.000+00 & 2.911+06 & 1.223-02 & 1.422-02 & 1.730-02 & 2.027-02 & 2.306-02 & 2.556-02\end{array}$ $\begin{array}{llllllllll}76 & 8.688+00 & 0.000+00 & 4.427+06 & 2.483-02 & 2.847-02 & 3.438-02 & 4.001-02 & 4.546-02 & 5.029-02\end{array}$ $\begin{array}{llllllllll}77 & 8.692+00 & 0.000+00 & 3.185+05 & 1.317-02 & 7.877-03 & 5.395-03 & 4.132-03 & 3.575-03 & 3.382-03\end{array}$ $\begin{array}{lllllllllllll}78 & 8.775+00 & 0.000+00 & 3.883+05 & 4.655-02 & 4.888-02 & 5.103-02 & 5.257-02 & 5.358-02 & 5.420-02\end{array}$ $\begin{array}{llllllllllllll}79 & 8.782+00 & 0.000+00 & 8.313+05 & 2.760-03 & 2.038-03 & 2.002-03 & 2.106-03 & 2.359-03 & 2.593-03\end{array}$ $\begin{array}{lllllllllllll}80 & 8.787+00 & 0.000+00 & 1.099+06 & 8.332-04 & 7.188-04 & 7.904-04 & 8.791-04 & 1.012-03 & 1.128-03\end{array}$ $\begin{array}{lllllllllll}81 & 8.835+00 & 0.000+00 & 7.424+03 & 2.446-03 & 1.176-03 & 6.129-04 & 3.433-04 & 2.030-04 & 1.221-04\end{array}$ $\begin{array}{llllllllll}82 & 8.893+00 & 0.000+00 & 0.000+00 & 2.248-03 & 2.287-03 & 2.485-03 & 2.673-03 & 2.804-03 & 2.885-03\end{array}$ $\begin{array}{lllllllllll}83 & 8.913+00 & 0.000+00 & 0.000+00 & 2.125-02 & 2.010-02 & 2.048-02 & 2.116-02 & 2.172-02 & 2.209-02\end{array}$ $\begin{array}{llllllllll}84 & 8.920+00 & 1.254-03 & 1.144+08 & 1.166-02 & 6.224-03 & 3.399-03 & 2.049-03 & 1.441-03 & 1.210-03\end{array}$ $\begin{array}{lllllllllllllll}85 & 8.934+00 & 0.000+00 & 0.000+00 & 1.100-01 & 1.272-01 & 1.428-01 & 1.542-01 & 1.616-01 & 1.660-01\end{array}$ $\begin{array}{lllllllllll}86 & 8.948+00 & 4.230-04 & 5.441+07 & 8.225-03 & 7.798-03 & 7.849-03 & 8.070-03 & 8.298-03 & 8.488-03\end{array}$ $\begin{array}{lllllllllll}87 & 8.958+00 & 3.024-03 & 2.785+08 & 2.907-02 & 3.032-02 & 3.236-02 & 3.433-02 & 3.590-02 & 3.706-02\end{array}$ $\begin{array}{llllllllll}88 & 8.965+00 & 0.000+00 & 0.000+00 & 5.851-02 & 6.667-02 & 7.409-02 & 7.970-02 & 8.351-02 & 8.586-02\end{array}$ $\begin{array}{lllllllllllll}89 & 9.010+00 & 1.756-01 & 1.636+10 & 4.126-02 & 5.208-02 & 6.741-02 & 8.568-02 & 1.052-01 & 1.252-01\end{array}$ $90 \quad 9.015+00 \quad 3.541-02 \quad 4.623+09 \quad 3.407-02 \quad 3.847-02 \quad 4.419-02 \quad 5.011-02 \quad 5.567-02 \quad 6.062-02$ $\begin{array}{lllllllllll}91 & 9.018+00 & 2.653-03 & 5.777+08 & 2.224-02 & 2.439-02 & 2.691-02 & 2.916-02 & 3.088-02 & 3.197-02\end{array}$ $\begin{array}{llllllllll}92 & 9.041+00 & 4.628-04 & 6.077+07 & 1.280-02 & 6.547-03 & 3.336-03 & 1.747-03 & 9.872-04 & 6.293-04\end{array}$ $\begin{array}{lllllllllll}93 & 9.061+00 & 0.000+00 & 0.000+00 & 2.500-02 & 1.205-02 & 5.747-03 & 2.744-03 & 1.287-03 & 5.802-04\end{array}$ $\begin{array}{llllllllll}94 & 9.100+00 & 1.755-01 & 1.668+10 & 1.804-02 & 2.563-02 & 3.742-02 & 5.272-02 & 6.997-02 & 8.875-02\end{array}$ $\begin{array}{llllllllll}95 & 9.105+00 & 2.718-02 & 3.620+09 & 1.041-02 & 1.060-02 & 1.209-02 & 1.441-02 & 1.711-02 & 2.015-02\end{array}$

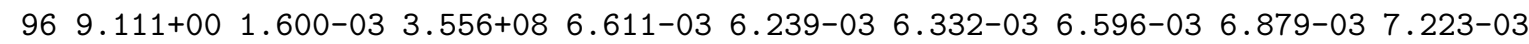

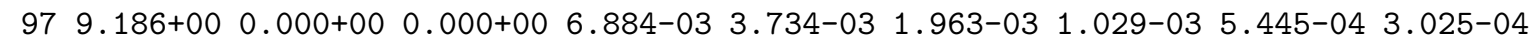
$\begin{array}{lllllllllll}98 & 9.216+00 & 0.000+00 & 5.815+05 & 1.364-02 & 1.141-02 & 1.147-02 & 1.231-02 & 1.340-02 & 1.453-02\end{array}$ $\begin{array}{lllllllllll}99 & 9.216+00 & 0.000+00 & 2.904+06 & 3.641-02 & 3.756-02 & 4.150-02 & 4.621-02 & 5.076-02 & 5.452-02\end{array}$ $\begin{array}{lllllllllll}4 & 100 & 9.216+00 & 0.000+00 & 8.720+06 & 9.715-02 & 1.172-01 & 1.380-01 & 1.574-01 & 1.744-01 & 1.874-01\end{array}$ $\begin{array}{lllllllllll}4 & 101 & 9.254+00 & 0.000+00 & 0.000+00 & 2.194-02 & 1.069-02 & 5.224-03 & 2.588-03 & 1.292-03 & 6.287-04\end{array}$ $\begin{array}{lllllllllll}4 & 102 & 9.290+00 & 0.000+00 & 0.000+00 & 4.775-03 & 3.247-03 & 3.141-03 & 3.391-03 & 3.563-03 & 3.664-03\end{array}$ $\begin{array}{lllllllllll}4 & 103 & 9.292+00 & 0.000+00 & 0.000+00 & 6.046-03 & 4.227-03 & 4.130-03 & 4.420-03 & 4.668-03 & 4.796-03\end{array}$ $\begin{array}{lllllllllll}4 & 104 & 9.294+00 & 0.000+00 & 0.000+00 & 7.416-03 & 6.419-03 & 7.078-03 & 7.842-03 & 8.452-03 & 8.728-03\end{array}$ $\begin{array}{llllllllllll}4 & 105 & 9.364+00 & 0.000+00 & 0.000+00 & 4.017-03 & 1.737-03 & 7.836-04 & 3.687-04 & 1.795-04 & 9.160-05\end{array}$ $\begin{array}{llllllllllll}4 & 106 & 9.491+00 & 0.000+00 & 0.000+00 & 1.878-04 & 2.041-04 & 2.278-04 & 2.481-04 & 2.626-04 & 2.722-04\end{array}$ 
Table II. Ca IX Oscillator Strengths, Radiative Decay Rates, and Collision Strengths for transitions involving the lowest 4 levels.

Low. Upp. Osc. Rad. Dec.

Lev. Lev.

Str. Rate

Collision Strength

Impact Electron Energy - Eo (Ry)

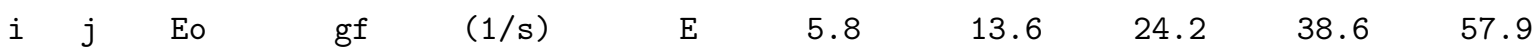

$4 \begin{array}{lllllllllll}4 & 107 & 9.492+00 & 3.520-04 & 5.096+07 & 2.637-04 & 2.682-04 & 2.965-04 & 3.332-04 & 3.711-04 & 4.098-04\end{array}$ $4 \begin{array}{lllllllllll}4 & 108 & 9.494+00 & 1.874-03 & 1.938+08 & 3.307-04 & 3.215-04 & 3.803-04 & 4.913-04 & 6.336-04 & 8.032-04\end{array}$ $\begin{array}{llllllllllllll}4 & 109 & 9.568+00 & 0.000+00 & 0.000+00 & 7.259-04 & 4.039-04 & 2.168-04 & 1.142-04 & 5.910-05 & 2.969-05\end{array}$

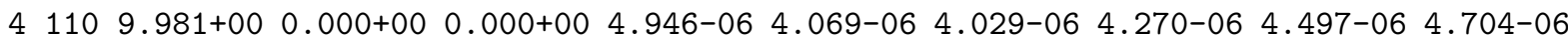
$\begin{array}{lllllllllll}4 & 111 & 9.984+00 & 0.000+00 & 0.000+00 & 1.138-04 & 5.946-05 & 3.483-05 & 2.254-05 & 1.578-05 & 1.215-05\end{array}$ $\begin{array}{lllllllllllll}4 & 112 & 1.001+01 & 0.000+00 & 0.000+00 & 1.494-02 & 1.630-02 & 1.734-02 & 1.806-02 & 1.852-02 & 1.881-02\end{array}$ $\begin{array}{llllllllllllll}4 & 113 & 1.002+01 & 0.000+00 & 0.000+00 & 6.740-02 & 7.357-02 & 7.824-02 & 8.151-02 & 8.359-02 & 8.490-02\end{array}$ $\begin{array}{llllllllllll}4 & 114 & 1.003+01 & 0.000+00 & 0.000+00 & 2.719-03 & 1.367-03 & 7.367-04 & 4.106-04 & 2.245-04 & 1.176-04\end{array}$ $\begin{array}{llllllllllll}4 & 115 & 1.005+01 & 0.000+00 & 0.000+00 & 4.132-05 & 2.426-05 & 1.651-05 & 1.273-05 & 1.094-05 & 9.726-06\end{array}$ $\begin{array}{lllllllllll}4 & 116 & 1.005+01 & 0.000+00 & 0.000+00 & 7.480-04 & 8.147-04 & 8.689-04 & 9.100-04 & 9.402-04 & 9.597-04\end{array}$ $4117 \quad 1.005+01 \quad 0.000+00 \quad 0.000+00 \quad 7.557-05 \quad 7.260-05 \quad 7.480-05 \quad 7.931-05 \quad 8.520-05 \quad 8.720-05$

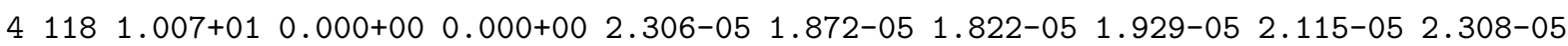

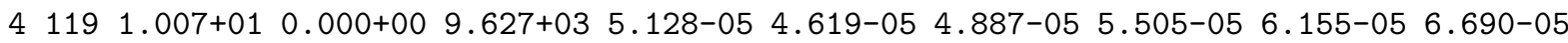
$\begin{array}{llllllllllll}4 & 120 & 1.008+01 & 0.000+00 & 3.441+04 & 1.601-04 & 1.849-04 & 2.185-04 & 2.572-04 & 2.907-04 & 3.141-04\end{array}$

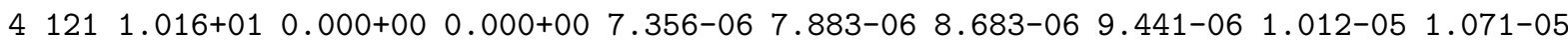
$\begin{array}{llllllllllll}4 & 122 & 1.016+01 & 0.000+00 & 0.000+00 & 1.718-04 & 9.587-05 & 6.175-05 & 4.483-05 & 3.566-05 & 3.068-05\end{array}$ $\begin{array}{llllllllllll}4 & 123 & 1.017+01 & 0.000+00 & 0.000+00 & 1.553-02 & 1.696-02 & 1.805-02 & 1.883-02 & 1.932-02 & 1.964-02\end{array}$ $\begin{array}{lllllllllll}4 & 124 & 1.019+01 & 0.000+00 & 0.000+00 & 3.854-05 & 1.923-05 & 9.747-06 & 5.317-06 & 2.831-06 & 1.597-06\end{array}$ $\begin{array}{lllllllllll}4 & 125 & 1.025+01 & 4.463-05 & 1.256+07 & 3.274-04 & 1.921-04 & 1.066-04 & 6.397-05 & 4.462-05 & 3.727-05\end{array}$ $\begin{array}{llllllllllllll}4 & 126 & 1.028+01 & 0.000+00 & 0.000+00 & 4.690-04 & 2.696-04 & 1.429-04 & 7.649-05 & 4.271-05 & 2.616-05\end{array}$ $\begin{array}{lllllllllll}4 & 127 & 1.028+01 & 0.000+00 & 0.000+00 & 4.691-04 & 2.297-04 & 1.203-04 & 6.493-05 & 3.416-05 & 1.680-05\end{array}$ $\begin{array}{llllllllllll}4 & 128 & 1.028+01 & 2.785-03 & 4.731+08 & 8.934-04 & 6.700-04 & 6.181-04 & 7.156-04 & 9.004-04 & 1.124-03\end{array}$

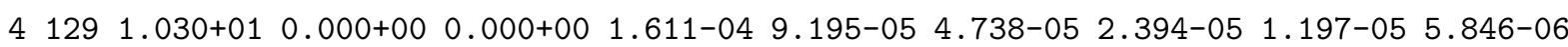

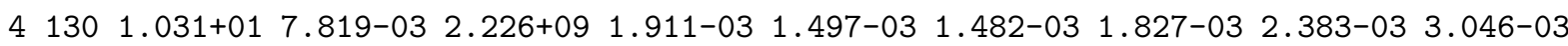
$\begin{array}{llllllllllllll}4 & 131 & 1.032+01 & 1.023-01 & 1.249+10 & 8.255-03 & 1.006-02 & 1.441-02 & 2.122-02 & 2.959-02 & 3.859-02\end{array}$ $\begin{array}{llllllllllll}4 & 132 & 1.033+01 & 8.468-02 & 1.451+10 & 6.244-03 & 7.813-03 & 1.146-02 & 1.709-02 & 2.400-02 & 3.149-02\end{array}$ $\begin{array}{llllllllllll}4 & 133 & 1.034+01 & 4.579-02 & 1.311+10 & 3.320-03 & 4.108-03 & 5.977-03 & 8.896-03 & 1.249-02 & 1.642-02\end{array}$ $4 \begin{array}{lllllllllll}4 & 134 & 1.039+01 & 5.896-04 & 1.022+08 & 3.298-03 & 1.893-03 & 1.022-03 & 5.896-04 & 3.937-04 & 3.259-04\end{array}$ $\begin{array}{lllllllllllll}4 & 135 & 1.044+01 & 0.000+00 & 0.000+00 & 6.080-04 & 3.502-04 & 1.824-04 & 9.180-05 & 4.531-05 & 2.237-05\end{array}$ $\begin{array}{lllllllllllll}4 & 136 & 1.065+01 & 0.000+00 & 4.690+04 & 7.085-04 & 3.870-04 & 2.533-04 & 1.948-04 & 1.699-04 & 1.623-04\end{array}$

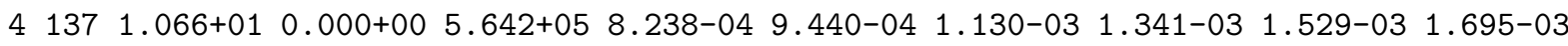
$\begin{array}{lllllllllll}4 & 138 & 1.066+01 & 0.000+00 & 5.650+05 & 9.416-04 & 7.694-04 & 7.658-04 & 8.314-04 & 9.085-04 & 9.881-04\end{array}$

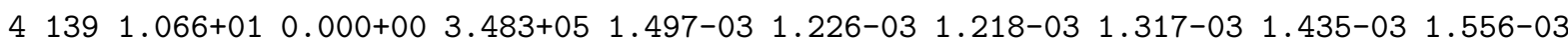
$\begin{array}{lllllllllll}4 & 140 & 1.069+01 & 0.000+00 & 1.751+06 & 4.851-03 & 3.843-03 & 3.733-03 & 4.000-03 & 4.348-03 & 4.725-03\end{array}$ $\begin{array}{lllllllllll}4 & 141 & 1.070+01 & 0.000+00 & 5.834+06 & 9.792-03 & 1.142-02 & 1.383-02 & 1.655-02 & 1.896-02 & 2.112-02\end{array}$ $\begin{array}{lllllllllll}4 & 142 & 1.071+01 & 0.000+00 & 6.220+06 & 1.300-02 & 1.495-02 & 1.802-02 & 2.155-02 & 2.472-02 & 2.756-02\end{array}$ $\begin{array}{llllllllllll}4 & 143 & 1.072+01 & 0.000+00 & 4.064+06 & 5.694-03 & 6.046-03 & 7.019-03 & 8.239-03 & 9.359-03 & 1.039-02\end{array}$ $4144 \quad 1.073+01 \quad 0.000+00 \quad 0.000+00 \quad 1.029-04 \quad 5.798-05 \quad 3.155-05 \quad 1.685-05 \quad 9.036-06 \quad 4.975-06$

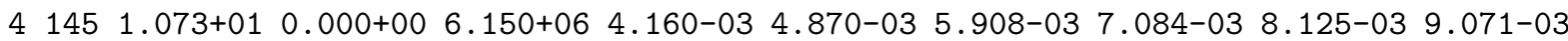
$\begin{array}{llllllllllll}4 & 146 & 1.074+01 & 0.000+00 & 6.900+06 & 1.528-03 & 1.798-03 & 2.184-03 & 2.622-03 & 3.009-03 & 3.361-03\end{array}$ $\begin{array}{lllllllllllll}4 & 147 & 1.075+01 & 0.000+00 & 0.000+00 & 5.835-03 & 2.934-03 & 1.557-03 & 8.325-04 & 4.370-04 & 2.294-04\end{array}$ $\begin{array}{lllllllllll}4 & 148 & 1.077+01 & 0.000+00 & 0.000+00 & 1.320-05 & 9.626-06 & 8.031-06 & 7.527-06 & 7.449-06 & 7.535-06\end{array}$ $\begin{array}{lllllllllll}4 & 149 & 1.077+01 & 0.000+00 & 0.000+00 & 3.371-05 & 2.257-05 & 1.780-05 & 1.665-05 & 1.706-05 & 1.787-05\end{array}$ $41501.077+01 \quad 5.658-05 \quad 7.533+06 \quad 8.809-05 \quad 6.379-05 \quad 5.479-05 \quad 5.507-05 \quad 5.923-05 \quad 6.385-05$ $\begin{array}{llllllllllll}4 & 151 & 1.078+01 & 0.000+00 & 0.000+00 & 1.288-04 & 1.088-04 & 1.011-04 & 9.995-05 & 1.016-04 & 1.043-04\end{array}$ 
Table II. Ca IX Oscillator Strengths, Radiative Decay Rates, and Collision Strengths for transitions involving the lowest 4 levels.

Low. Upp. Osc. Rad. Dec.

Lev. Lev.

Str. Rate

Collision Strength

Impact Electron Energy - Eo (Ry)

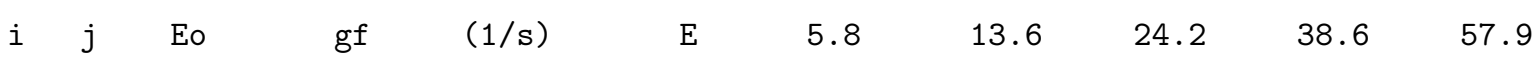

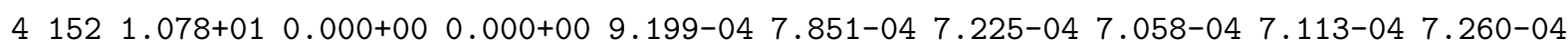
$\begin{array}{lllllllllll}4 & 153 & 1.079+01 & 0.000+00 & 0.000+00 & 2.406-03 & 2.562-03 & 2.727-03 & 2.896-03 & 3.049-03 & 3.182-03\end{array}$

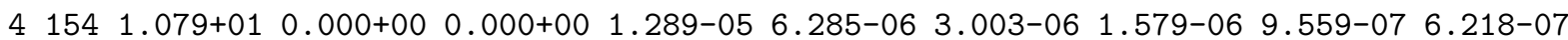
$41551.080+01 \quad 9.396-051.258+07 \quad 1.161-03 \quad 6.831-04 \quad 4.099-04 \quad 2.647-04 \quad 1.945-04 \quad 1.637-04$ $\begin{array}{lllllllllll}4 & 156 & 1.080+01 & 0.000+00 & 1.431+04 & 2.078-03 & 1.029-03 & 5.452-04 & 2.970-04 & 1.658-04 & 9.511-05\end{array}$ $\begin{array}{lllllllllll}4 & 157 & 1.081+01 & 0.000+00 & 0.000+00 & 1.871-05 & 1.299-05 & 1.178-05 & 1.178-05 & 1.275-05 & 1.363-05\end{array}$ $\begin{array}{lllllllllll}4 & 158 & 1.081+01 & 1.713-04 & 2.295+07 & 1.637-03 & 1.695-03 & 1.777-03 & 1.876-03 & 1.977-03 & 2.071-03\end{array}$

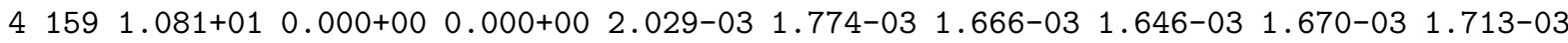
$\begin{array}{lllllllllll}4 & 160 & 1.081+01 & 0.000+00 & 0.000+00 & 7.361-05 & 4.051-05 & 2.966-05 & 2.605-05 & 2.611-05 & 2.669-05\end{array}$

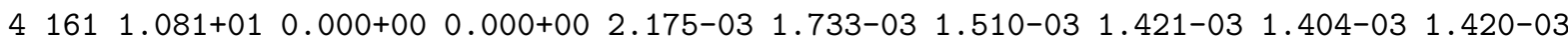
$\begin{array}{lllllllllllll}4 & 162 & 1.082+01 & 0.000+00 & 0.000+00 & 8.709-04 & 6.354-04 & 5.612-04 & 5.455-04 & 5.777-04 & 6.051-04\end{array}$ $\begin{array}{lllllllllll}4 & 163 & 1.083+01 & 0.000+00 & 0.000+00 & 7.240-04 & 5.761-04 & 5.335-04 & 5.292-04 & 5.664-04 & 5.959-04\end{array}$

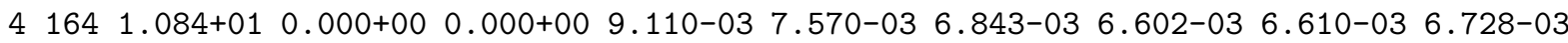
$\begin{array}{llllllllllll}4 & 165 & 1.084+01 & 0.000+00 & 0.000+00 & 1.718-02 & 1.854-02 & 1.987-02 & 2.118-02 & 2.235-02 & 2.334-02\end{array}$

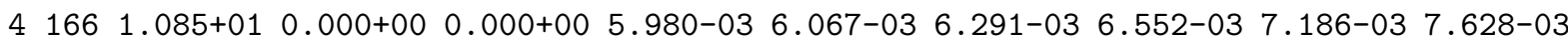
$\begin{array}{lllllllllll}4 & 167 & 1.085+01 & 0.000+00 & 0.000+00 & 3.555-03 & 2.397-03 & 2.015-03 & 1.904-03 & 1.988-03 & 2.068-03\end{array}$ $\begin{array}{lllllllllll}4 & 168 & 1.085+01 & 0.000+00 & 0.000+00 & 4.945-03 & 4.863-03 & 4.969-03 & 5.150-03 & 5.633-03 & 5.974-03\end{array}$ $\begin{array}{llllllllllllll}4 & 169 & 1.085+01 & 0.000+00 & 0.000+00 & 2.312-02 & 2.506-02 & 2.699-02 & 2.883-02 & 3.044-02 & 3.180-02\end{array}$ $\begin{array}{lllllllllll}4 & 170 & 1.086+01 & 0.000+00 & 0.000+00 & 4.352-03 & 1.871-03 & 9.529-04 & 5.814-04 & 4.253-04 & 3.611-04\end{array}$ $\begin{array}{lllllllllll}4 & 171 & 1.087+01 & 2.806-04 & 8.878+07 & 4.992-05 & 3.888-05 & 4.156-05 & 5.461-05 & 7.362-05 & 9.517-05\end{array}$

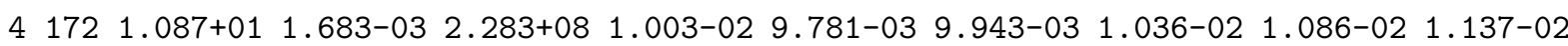
$\begin{array}{llllllllllll}4 & 173 & 1.088+01 & 3.118-04 & 5.929+07 & 9.243-03 & 9.975-03 & 1.073-02 & 1.146-02 & 1.212-02 & 1.269-02\end{array}$

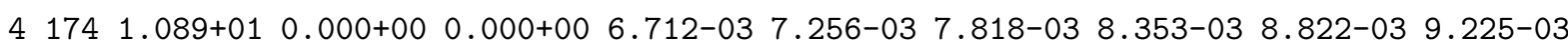

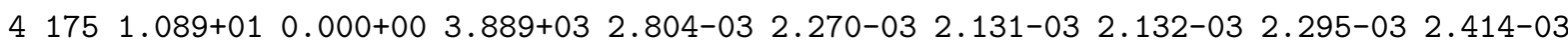
$\begin{array}{lllllllllll}4 & 176 & 1.089+01 & 0.000+00 & 0.000+00 & 2.281-03 & 2.030-03 & 1.990-03 & 2.024-03 & 2.198-03 & 2.320-03\end{array}$ $\begin{array}{lllllllllll}4 & 177 & 1.090+01 & 0.000+00 & 0.000+00 & 2.144-03 & 2.149-03 & 2.214-03 & 2.297-03 & 2.519-03 & 2.667-03\end{array}$ $\begin{array}{lllllllllll}4 & 178 & 1.091+01 & 0.000+00 & 0.000+00 & 2.608-03 & 1.462-03 & 1.065-03 & 9.278-04 & 9.247-04 & 9.406-04\end{array}$ $\begin{array}{llllllllllll}4 & 179 & 1.091+01 & 0.000+00 & 0.000+00 & 9.493-03 & 5.247-03 & 2.806-03 & 1.471-03 & 7.733-04 & 4.163-04\end{array}$ $\begin{array}{llllllllllll}4 & 180 & 1.092+01 & 0.000+00 & 0.000+00 & 5.900-04 & 5.524-04 & 5.458-04 & 5.572-04 & 5.750-04 & 5.940-04\end{array}$ $\begin{array}{llllllllllll}4 & 181 & 1.092+01 & 0.000+00 & 0.000+00 & 6.427-04 & 6.971-04 & 7.518-04 & 8.038-04 & 8.487-04 & 8.865-04\end{array}$ $\begin{array}{lllllllllllll}4 & 182 & 1.092+01 & 0.000+00 & 0.000+00 & 9.646-04 & 8.614-04 & 8.253-04 & 8.266-04 & 8.443-04 & 8.674-04\end{array}$ $\begin{array}{llllllllllll}4 & 183 & 1.093+01 & 0.000+00 & 0.000+00 & 5.015-03 & 2.765-03 & 1.471-03 & 7.699-04 & 4.064-04 & 2.213-04\end{array}$

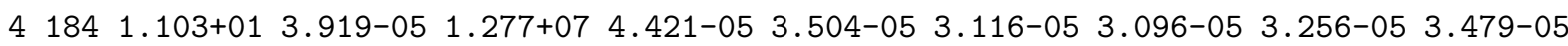
$\begin{array}{llllllllllll}4 & 185 & 1.103+01 & 0.000+00 & 0.000+00 & 4.680-06 & 2.399-06 & 1.175-06 & 5.987-07 & 3.260-07 & 1.834-07\end{array}$

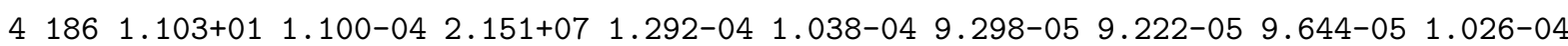
$\begin{array}{llllllllllll}4 & 187 & 1.104+01 & 0.000+00 & 0.000+00 & 2.957-04 & 1.536-04 & 7.856-05 & 4.144-05 & 2.352-05 & 1.438-05\end{array}$ $\begin{array}{lllllllllllllll}4 & 188 & 1.106+01 & 0.000+00 & 0.000+00 & 7.288-04 & 3.902-04 & 2.012-04 & 1.003-04 & 4.893-05 & 2.300-05\end{array}$ $\begin{array}{lllllllllll}4 & 189 & 1.107+01 & 0.000+00 & 0.000+00 & 9.769-05 & 3.507-05 & 1.425-05 & 6.529-06 & 3.332-06 & 1.998-06\end{array}$ $\begin{array}{llllllllllll}4 & 190 & 1.112+01 & 0.000+00 & 0.000+00 & 1.553-04 & 8.731-05 & 6.661-05 & 6.020-05 & 6.159-05 & 6.308-05\end{array}$ $\begin{array}{lllllllllll}4 & 191 & 1.112+01 & 0.000+00 & 0.000+00 & 9.434-05 & 8.700-05 & 8.486-05 & 8.537-05 & 9.226-05 & 9.542-05\end{array}$ $\begin{array}{llllllllllllll}4 & 192 & 1.112+01 & 0.000+00 & 0.000+00 & 2.314-04 & 2.263-04 & 2.366-04 & 2.514-04 & 2.737-04 & 2.821-04\end{array}$ $\begin{array}{lllllllllllllll}4 & 193 & 1.112+01 & 0.000+00 & 0.000+00 & 1.448-04 & 1.250-04 & 1.199-04 & 1.211-04 & 1.293-04 & 1.325-04\end{array}$ $\begin{array}{llllllllllllll}4 & 194 & 1.112+01 & 0.000+00 & 0.000+00 & 3.881-04 & 3.149-04 & 2.964-04 & 2.963-04 & 3.192-04 & 3.338-04\end{array}$ $\begin{array}{llllllllllll}4 & 195 & 1.113+01 & 0.000+00 & 0.000+00 & 8.150-04 & 8.106-04 & 8.292-04 & 8.550-04 & 9.359-04 & 9.844-04\end{array}$

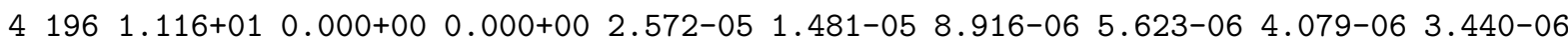


Table II. Ca IX Oscillator Strengths, Radiative Decay Rates, and Collision Strengths for transitions involving the lowest 4 levels.

Low. Upp. Osc. Rad. Dec.

Lev. Lev.

Str. Rate

Collision Strength

Impact Electron Energy - Eo (Ry)

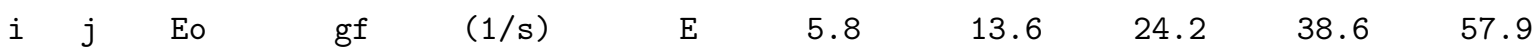

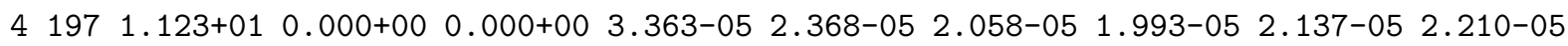

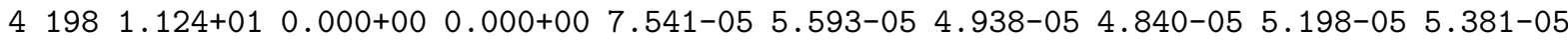
$41991.124+01 \quad 0.000+00 \quad 0.000+00 \quad 5.366-05 \quad 4.436-05 \quad 4.144-05 \quad 4.162-05 \quad 4.515-05 \quad 4.689-05$

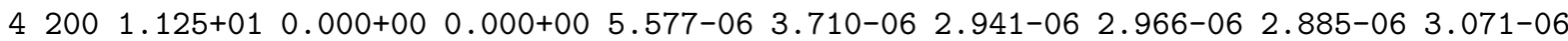
$\begin{array}{lllllllllll}4 & 201 & 1.125+01 & 0.000+00 & 0.000+00 & 1.990-05 & 1.709-05 & 1.596-05 & 1.842-05 & 1.861-05 & 2.003-05\end{array}$ $\begin{array}{llllllllllll}4 & 202 & 1.125+01 & 0.000+00 & 0.000+00 & 1.237-05 & 1.068-05 & 1.024-05 & 1.198-05 & 1.240-05 & 1.353-05\end{array}$ $42031.128+01 \quad 0.000+00 \quad 0.000+00 \quad 4.659-05 \quad 4.465-05 \quad 4.556-05 \quad 4.793-05 \quad 4.990-05 \quad 5.154-05$ $\begin{array}{lllllllllll}4 & 204 & 1.128+01 & 0.000+00 & 0.000+00 & 2.578-05 & 2.038-05 & 1.871-05 & 1.906-05 & 2.024-05 & 2.039-05\end{array}$

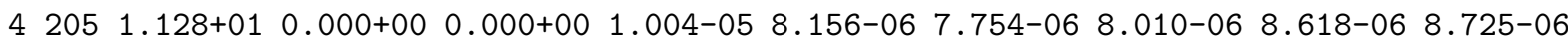

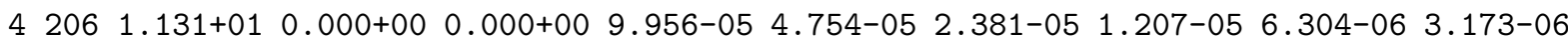

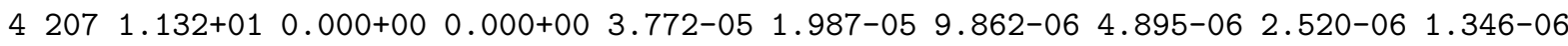

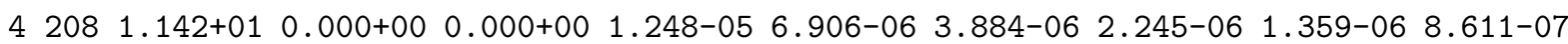

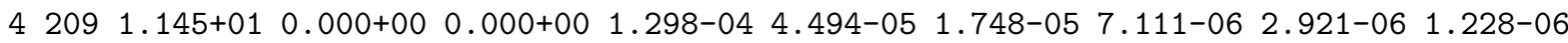
$\begin{array}{lllllllllll}4 & 210 & 1.222+01 & 0.000+00 & 0.000+00 & 3.812-06 & 3.818-06 & 4.314-06 & 5.022-06 & 5.682-06 & 6.204-06\end{array}$ $42111.222+01 \quad 0.000+00 \quad 0.000+00 \quad 9.577-06 \quad 8.306-06 \quad 8.154-06 \quad 8.546-06 \quad 9.133-06 \quad 9.786-06$ $\begin{array}{llllllllllll}4 & 212 & 1.222+01 & 0.000+00 & 0.000+00 & 2.724-05 & 2.308-05 & 2.111-05 & 2.015-05 & 2.017-05 & 2.116-05\end{array}$

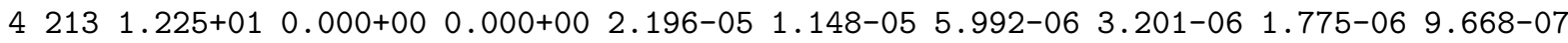
$\begin{array}{lllllllllll}4 & 214 & 1.248+01 & 0.000+00 & 0.000+00 & 7.030-06 & 3.850-06 & 2.490-06 & 2.051-06 & 2.240-06 & 2.758-06\end{array}$

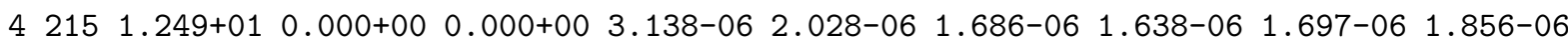

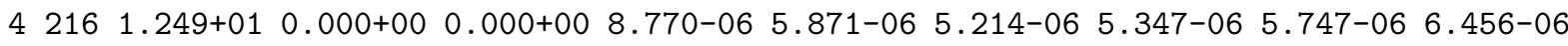

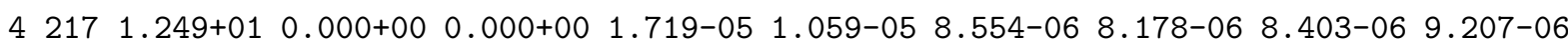

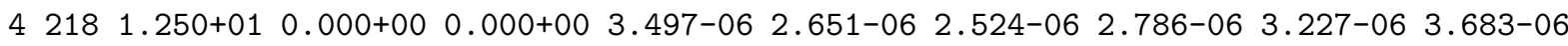

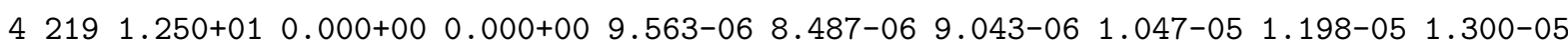

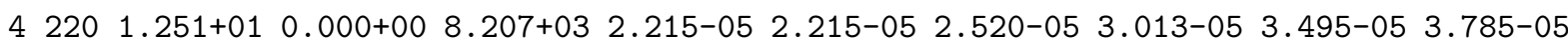
$\begin{array}{lllllllllll}4 & 221 & 1.254+01 & 0.000+00 & 5.898+03 & 1.537-05 & 1.293-05 & 1.206-05 & 1.168-05 & 1.135-05 & 1.104-05\end{array}$ $\begin{array}{lllllllllll}4 & 222 & 1.254+01 & 0.000+00 & 1.381+04 & 3.948-06 & 3.086-06 & 2.919-06 & 2.895-06 & 2.907-06 & 2.922-06\end{array}$

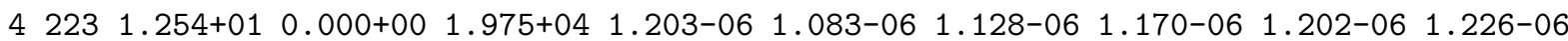
$\begin{array}{lllllllllll}4224 & 1.256+01 & 0.000+00 & 0.000+00 & 4.887-05 & 2.705-05 & 1.530-05 & 8.638-06 & 4.891-06 & 2.691-06\end{array}$

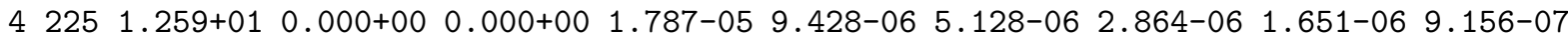
$\begin{array}{lllllllllll}4 & 226 & 1.282+01 & 0.000+00 & 0.000+00 & 6.549-06 & 3.633-06 & 1.973-06 & 1.086-06 & 6.354-07 & 4.135-07\end{array}$

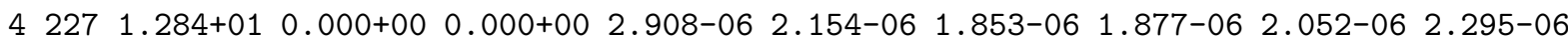

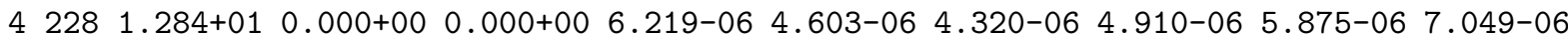

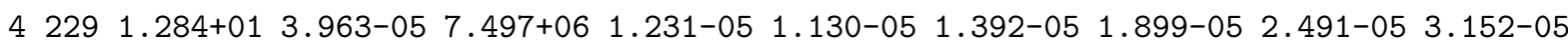

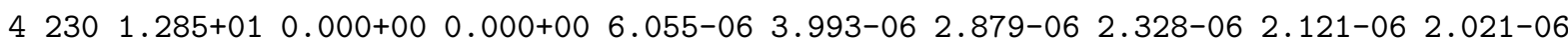

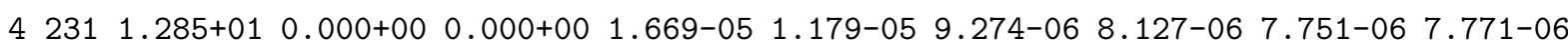

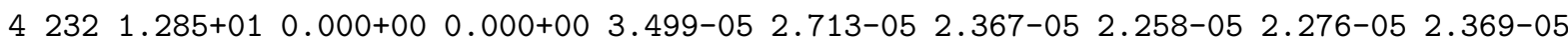
$\begin{array}{lllllllllll}4233 & 1.285+01 & 0.000+00 & 0.000+00 & 3.146-06 & 1.691-06 & 9.199-07 & 5.184-07 & 3.202-07 & 2.098-07\end{array}$ $42341.288+01 \quad 2.512-05 \quad 1.117+07 \quad 4.739-06 \quad 4.617-06 \quad 6.126-06 \quad 8.207-06 \quad 9.880-06 \quad 1.102-05$

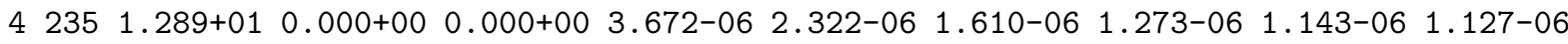
$\begin{array}{lllllllllll}4 & 236 & 1.289+01 & 0.000+00 & 0.000+00 & 7.496-06 & 4.821-06 & 3.495-06 & 2.934-06 & 2.787-06 & 2.866-06\end{array}$ $\begin{array}{lllllllllll}4237 & 1.289+01 & 0.000+00 & 0.000+00 & 1.306-05 & 8.043-06 & 5.452-06 & 4.271-06 & 3.863-06 & 3.866-06\end{array}$ $42381.295+01 \quad 2.382-05 \quad 6.414+06 \quad 1.691-05 \quad 9.889-06 \quad 6.398-06 \quad 4.845-06 \quad 4.396-06 \quad 4.565-06$ $\begin{array}{llllllllllll}4 & 239 & 1.295+01 & 0.000+00 & 0.000+00 & 7.913-07 & 4.012-07 & 2.054-07 & 1.094-07 & 6.267-08 & 3.806-08\end{array}$

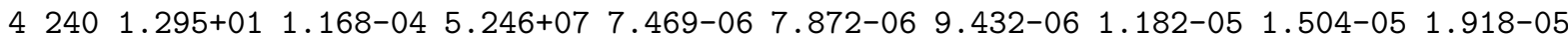

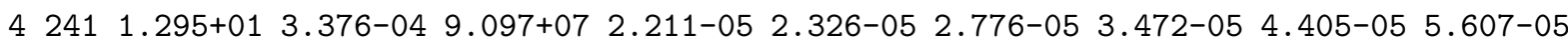


Table II. Ca IX Oscillator Strengths, Radiative Decay Rates, and Collision Strengths for transitions involving the lowest 4 levels.

Low. Upp. Osc. Rad. Dec.

Low. Upp.
Lev. Lev.
Str.

Rate

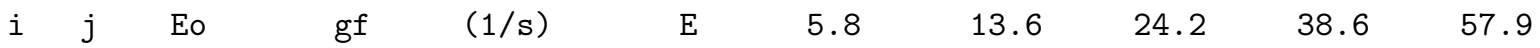

Collision Strength

Impact Electron Energy - Eo (Ry)

$42421.296+01 \quad 0.000+00 \quad 0.000+00 \quad 2.576-05 \quad 1.495-05 \quad 8.466-06 \quad 4.735-06 \quad 2.614-06 \quad 1.416-06$ $\begin{array}{lllllllllll}4 & 243 & 1.298+01 & 0.000+00 & 0.000+00 & 9.307-06 & 4.674-06 & 2.384-06 & 1.321-06 & 8.178-07 & 5.764-07\end{array}$ $42441.298+01 \quad 0.000+00 \quad 0.000+00 \quad 8.468-06 \quad 5.416-06 \quad 3.996-06 \quad 3.416-06 \quad 3.250-06 \quad 3.301-06$ $\begin{array}{lllllllllll}4 & 245 & 1.299+01 & 0.000+00 & 0.000+00 & 1.925-05 & 1.456-05 & 1.306-05 & 1.291-05 & 1.349-05 & 1.456-05\end{array}$ $42461.299+01 \quad 0.000+00 \quad 0.000+00 \quad 3.752-05 \quad 3.304-05 \quad 3.381-05 \quad 3.601-05 \quad 3.912-05 \quad 4.318-05$ $42471.299+01 \quad 0.000+00 \quad 0.000+00 \quad 7.207-06 \quad 6.193-06 \quad 6.215-06 \quad 6.648-06 \quad 7.342-06 \quad 8.079-06$ $42481.299+01 \quad 0.000+00 \quad 0.000+00 \quad 1.929-05 \quad 1.954-05 \quad 2.182-05 \quad 2.467-05 \quad 2.770-05 \quad 3.008-05$

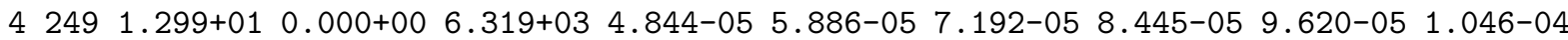
$42501.302+01 \quad 0.000+00 \quad 0.000+00 \quad 9.763-06 \quad 5.222-06 \quad 3.031-06 \quad 2.067-06 \quad 1.840-06 \quad 1.969-06$ $4251 \quad 1.304+01 \quad 0.000+00 \quad 0.000+00 \quad 3.471-06 \quad 1.637-06 \quad 7.583-07 \quad 3.632-07 \quad 2.019-07 \quad 1.306-07$ $42521.304+01 \quad 0.000+00 \quad 0.000+00 \quad 6.906-06 \quad 4.891-06 \quad 4.239-06 \quad 4.155-06 \quad 4.308-06 \quad 4.644-06$

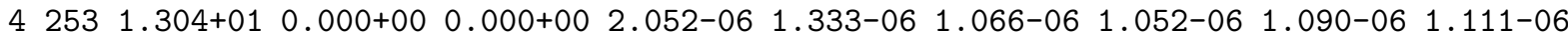
$\begin{array}{lllllllllll}4 & 254 & 1.304+01 & 0.000+00 & 0.000+00 & 1.246-05 & 9.162-06 & 8.466-06 & 8.685-06 & 9.288-06 & 1.018-05\end{array}$ $\begin{array}{lllllllllll}4 & 255 & 1.304+01 & 0.000+00 & 0.000+00 & 3.283-06 & 2.226-06 & 1.953-06 & 2.095-06 & 2.264-06 & 2.355-06\end{array}$

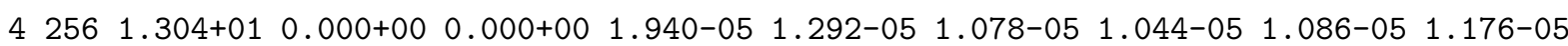
$4257 \quad 1.304+01 \quad 0.000+00 \quad 0.000+00 \quad 5.349-06 \quad 3.395-06 \quad 2.771-06 \quad 2.851-06 \quad 3.015-06 \quad 3.105-06$ $\begin{array}{lllllllllllllll}4 & 258 & 1.304+01 & 0.000+00 & 0.000+00 & 1.333-06 & 7.775-07 & 6.074-07 & 6.167-07 & 6.741-07 & 6.992-07\end{array}$

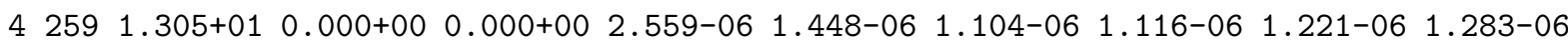
$\begin{array}{lllllllllll}4 & 260 & 1.305+01 & 0.000+00 & 0.000+00 & 3.994-06 & 2.207-06 & 1.674-06 & 1.722-06 & 1.975-06 & 2.124-06\end{array}$ $\begin{array}{llllllllllll}4 & 261 & 1.306+01 & 0.000+00 & 0.000+00 & 1.819-06 & 7.345-07 & 2.995-07 & 1.327-07 & 6.747-08 & 4.304-08\end{array}$ $42621.306+01 \quad 0.000+00 \quad 1.883+04 \quad 3.887-06 \quad 3.165-06 \quad 3.200-06 \quad 3.604-06 \quad 4.195-06 \quad 4.792-06$ $42631.306+01 \quad 0.000+00 \quad 4.395+04 \quad 9.702-06 \quad 1.097-05 \quad 1.323-05 \quad 1.606-05 \quad 1.912-05 \quad 2.190-05$ $\begin{array}{lllllllllll}4 & 264 & 1.306+01 & 0.000+00 & 5.006+04 & 1.883-05 & 2.250-05 & 2.762-05 & 3.358-05 & 3.974-05 & 4.512-05\end{array}$

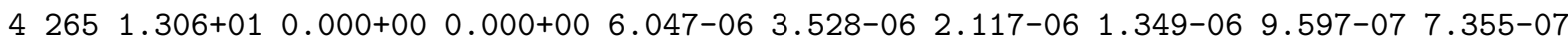
$\begin{array}{lllllllllll}4 & 266 & 1.306+01 & 0.000+00 & 0.000+00 & 3.098-06 & 1.901-06 & 1.411-06 & 1.302-06 & 1.293-06 & 1.307-06\end{array}$ $42671.306+01 \quad 0.000+00 \quad 0.000+00 \quad 9.853-07 \quad 6.757-07 \quad 6.515-07 \quad 7.042-07 \quad 7.846-07 \quad 8.013-07$ $\begin{array}{lllllllllll}4 & 268 & 1.306+01 & 0.000+00 & 0.000+00 & 1.540-06 & 1.385-06 & 1.555-06 & 1.803-06 & 2.065-06 & 2.123-06\end{array}$

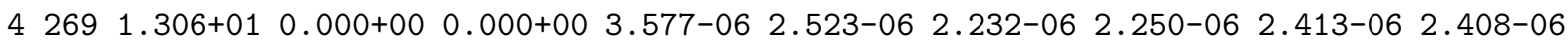
$42701.306+01 \quad 0.000+00 \quad 0.000+00 \quad 6.530-06 \quad 4.557-06 \quad 3.961-06 \quad 4.135-06 \quad 4.383-06 \quad 4.531-06$ $\begin{array}{lllllllllll}4 & 271 & 1.306+01 & 0.000+00 & 0.000+00 & 1.226-05 & 9.460-06 & 9.021-06 & 1.003-05 & 1.091-05 & 1.138-05\end{array}$ $42721.307+01 \quad 0.000+00 \quad 0.000+00 \quad 2.755-05 \quad 3.373-05 \quad 4.349-05 \quad 5.729-05 \quad 7.618-05 \quad 9.758-05$ $\begin{array}{llllllllllll}4 & 273 & 1.307+01 & 0.000+00 & 0.000+00 & 1.481-05 & 1.868-05 & 2.310-05 & 2.715-05 & 3.086-05 & 3.325-05\end{array}$ $42741.307+01 \quad 0.000+00 \quad 0.000+00 \quad 5.883-06 \quad 7.570-06 \quad 9.442-06 \quad 1.109-05 \quad 1.260-05 \quad 1.356-05$ $\begin{array}{llllllllllll}4 & 275 & 1.307+01 & 0.000+00 & 0.000+00 & 3.166-05 & 1.575-05 & 7.785-06 & 3.897-06 & 1.947-06 & 9.328-07\end{array}$ $\begin{array}{llllllllllll}4 & 276 & 1.308+01 & 0.000+00 & 0.000+00 & 8.282-06 & 4.040-06 & 1.918-06 & 8.605-07 & 3.901-07 & 1.746-07\end{array}$ $\begin{array}{llllllllllll}4 & 277 & 1.309+01 & 0.000+00 & 0.000+00 & 2.747-06 & 1.864-06 & 1.766-06 & 2.342-06 & 3.188-06 & 3.706-06\end{array}$ $\begin{array}{lllllllllll}4 & 278 & 1.309+01 & 0.000+00 & 0.000+00 & 2.266-06 & 1.598-06 & 1.436-06 & 1.613-06 & 1.957-06 & 2.137-06\end{array}$

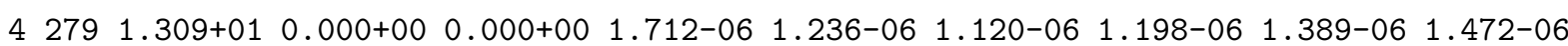
$\begin{array}{llllllllllll}4 & 280 & 1.310+01 & 0.000+00 & 0.000+00 & 3.845-06 & 2.201-06 & 1.244-06 & 6.874-07 & 3.762-07 & 2.173-07\end{array}$ $\begin{array}{llllllllllll}4 & 281 & 1.311+01 & 0.000+00 & 0.000+00 & 4.645-06 & 2.280-06 & 1.120-06 & 5.814-07 & 3.275-07 & 1.890-07\end{array}$ $42821.314+01 \quad 0.000+00 \quad 0.000+00 \quad 2.776-05 \quad 1.547-05 \quad 8.330-06 \quad 4.508-06 \quad 2.383-06 \quad 1.203-06$ $\begin{array}{lllllllllll}4 & 283 & 1.314+01 & 0.000+00 & 0.000+00 & 6.124-06 & 3.341-06 & 1.832-06 & 1.038-06 & 5.826-07 & 3.327-07\end{array}$ 
Table III. Ca IX Oscillator Strengths and Radiative Decay Rates for transitions involving levels higher than 4.

\begin{tabular}{|c|c|c|c|c|c|c|c|c|c|c|c|}
\hline $\begin{array}{l}\text { Low. } \\
\text { Lev. }\end{array}$ & $\begin{array}{l}\text { Upp. } \\
\text { Lev. }\end{array}$ & $\begin{array}{l}\text { Osc. } \\
\text { Str. }\end{array}$ & $\begin{array}{l}\text { Rad. } \\
\text { Rate }\end{array}$ & $\begin{array}{l}\text { Low. } \\
\text { Lev. }\end{array}$ & $\begin{array}{l}\text { - Upp } \\
\text {. Lev }\end{array}$ & $\begin{array}{l}\text { Osc. } \\
\text { Str. }\end{array}$ & $\begin{array}{l}\text { Rad. } \\
\text { Rate }\end{array}$ & $\begin{array}{l}\text { Low. } \\
\text { Lev. }\end{array}$ & $\begin{array}{l}\text { Upp } \\
\text { Lev }\end{array}$ & $\begin{array}{l}\text { Osc. } \\
\text { Str. }\end{array}$ & $\begin{array}{l}\text { Rad. } \\
\text { Rate }\end{array}$ \\
\hline i & $j$ & $g f$ & $(1 / s)$ & $i$ & $j$ & $g f$ & $(1 / s)$ & $i$ & $j$ & $g f$ & $(1 / s)$ \\
\hline 5 & 6 & $3.503 e-01$ & $6.475 \mathrm{e}+08$ & 5 & 7 & $5.584 e-04$ & $5.608 e+06$ & 5 & 9 & $1.653 e-02$ & $3.620 \mathrm{e}+07$ \\
\hline 5 & 10 & $4.228 e-01$ & $9.666 \mathrm{e}+09$ & 5 & 11 & $2.207 e-04$ & $1.859 e+06$ & 5 & 12 & $5.256 e-04$ & $2.659 e+06$ \\
\hline 5 & 14 & $2.820 \mathrm{e}+00$ & $2.448 e+10$ & 5 & 15 & $0.000 e+00$ & $5.888 e+03$ & 5 & 18 & $0.000 e+00$ & $2.127 e+05$ \\
\hline 5 & 19 & $0.000 e+00$ & $1.831 \mathrm{e}+03$ & 5 & 25 & $0.000 e+00$ & $1.608 e+05$ & 5 & 26 & $0.000 e+00$ & $6.197 e+05$ \\
\hline 5 & 27 & $3.268 e-04$ & $2.128 e+07$ & 5 & 28 & $2.129 \mathrm{e}-01$ & $4.400 e+10$ & 5 & 30 & $0.000 e+00$ & $2.654 \mathrm{e}+05$ \\
\hline 5 & 33 & $0.000 e+00$ & $1.710 \mathrm{e}+03$ & 5 & 35 & $0.000 e+00$ & $2.031 e+06$ & 5 & 37 & $1.960 \mathrm{e}-02$ & $1.048 e+09$ \\
\hline 5 & 40 & $1.239 \mathrm{e}-04$ & $6.689 e+06$ & 5 & 41 & $3.460 e-04$ & $1.101 e+08$ & 5 & 42 & $1.337 e-04$ & $1.451 \mathrm{e}+07$ \\
\hline 5 & 43 & $2.890 \mathrm{e}-04$ & $1.882 \mathrm{e}+07$ & 5 & 45 & $3.362 \mathrm{e}-01$ & $2.202 \mathrm{e}+10$ & 5 & 53 & $0.000 e+00$ & $1.384 \mathrm{e}+07$ \\
\hline 5 & 54 & $1.627 e-01$ & $2.201 \mathrm{e}+10$ & 5 & 55 & $1.997 e-02$ & $2.742 \mathrm{e}+09$ & 5 & 56 & $4.860 e-04$ & $4.018 e+07$ \\
\hline 5 & 58 & $1.164 \mathrm{e}-04$ & $4.901 \mathrm{e}+07$ & 5 & 59 & $3.497 e-03$ & $4.918 e+08$ & 5 & 60 & $6.654 \mathrm{e}-04$ & $9.417 e+07$ \\
\hline 5 & 61 & $4.156 \mathrm{e}-03$ & $3.531 \mathrm{e}+08$ & 5 & 62 & $2.948 \mathrm{e}-01$ & $2.577 e+10$ & 5 & 63 & $1.023 e-02$ & $4.643 e+09$ \\
\hline 5 & 64 & $3.966 \mathrm{e}-05$ & $6.361 \mathrm{e}+06$ & 5 & 65 & $8.612 \mathrm{e}-02$ & $4.250 e+10$ & 5 & 66 & $0.000 e+00$ & $8.317 e+03$ \\
\hline 5 & 67 & $0.000 e+00$ & $2.655 e+06$ & 5 & 68 & $0.000 e+00$ & $3.020 e+06$ & 5 & 69 & $0.000 e+00$ & $3.079 e+05$ \\
\hline 5 & 70 & $0.000 e+00$ & $6.719 e+03$ & 5 & 72 & $0.000 e+00$ & $2.402 \mathrm{e}+05$ & 5 & 73 & $0.000 e+00$ & $1.468 \mathrm{e}+04$ \\
\hline 5 & 74 & $0.000 e+00$ & $7.028 e+05$ & 5 & 75 & $0.000 e+00$ & $1.616 \mathrm{e}+06$ & 5 & 77 & $0.000 e+00$ & $5.330 e+06$ \\
\hline 5 & 78 & $0.000 e+00$ & $3.906 e+04$ & 5 & 79 & $0.000 e+00$ & $1.548 e+05$ & 5 & 81 & $0.000 e+00$ & $7.852 e+06$ \\
\hline 5 & 86 & $1.010 e-03$ & $1.119 \mathrm{e}+08$ & 5 & 90 & $8.057 e-04$ & $9.065 e+07$ & 5 & 92 & $2.695 \mathrm{e}-01$ & $3.051 e+10$ \\
\hline 5 & 95 & $1.870 \mathrm{e}-04$ & $2.150 e+07$ & 5 & 97 & $2.084 \mathrm{e}-03$ & $2.442 \mathrm{e}+08$ & 5 & 101 & $0.000 e+00$ & $6.578 e+06$ \\
\hline 5 & 109 & $5.045 e-03$ & $6.451 \mathrm{e}+08$ & 5 & 112 & $0.000 e+00$ & $1.395 e+04$ & 5 & 124 & $0.000 e+00$ & $3.069 e+05$ \\
\hline 5 & 125 & $4.597 e-02$ & $1.136 e+10$ & 5 & 126 & $1.530 \mathrm{e}-02$ & $3.800 e+09$ & 5 & 127 & $0.000 e+00$ & $6.081 e+04$ \\
\hline 5 & 128 & $3.569 e-03$ & $5.326 e+08$ & 5 & 129 & $5.769 e-04$ & $4.316 e+08$ & 5 & 130 & $1.134 \mathrm{e}-02$ & $2.837 e+09$ \\
\hline 5 & 132 & $2.126 \mathrm{e}-03$ & $3.202 \mathrm{e}+08$ & 5 & 133 & $1.192 \mathrm{e}-03$ & $3.002 e+08$ & 5 & 134 & $1.417 \mathrm{e}-01$ & $2.160 \mathrm{e}+10$ \\
\hline 5 & 135 & $2.364 \mathrm{e}-02$ & $1.820 \mathrm{e}+10$ & 5 & 136 & $0.000 e+00$ & $5.370 e+06$ & 5 & 137 & $0.000 e+00$ & $1.300 e+05$ \\
\hline 5 & 138 & $0.000 e+00$ & $7.593 e+04$ & 5 & 139 & $0.000 e+00$ & $2.448 e+05$ & 5 & 140 & $0.000 e+00$ & $2.043 e+06$ \\
\hline 5 & 141 & $0.000 e+00$ & $3.608 e+04$ & 5 & 143 & $0.000 e+00$ & $3.122 \mathrm{e}+05$ & 5 & 145 & $0.000 e+00$ & $4.192 \mathrm{e}+04$ \\
\hline 5 & 147 & $0.000 e+00$ & $6.208 e+06$ & 5 & 154 & $3.061 \mathrm{e}-04$ & $8.434 \mathrm{e}+07$ & 5 & 156 & $0.000 e+00$ & $6.390 \mathrm{e}+06$ \\
\hline 5 & 159 & $1.988 e-04$ & $3.299 e+07$ & 5 & 163 & $0.000 e+00$ & $4.527 e+03$ & 5 & 173 & $1.563 e-04$ & $2.629 \mathrm{e}+07$ \\
\hline 5 & 180 & $3.742 \mathrm{e}-04$ & $6.344 \mathrm{e}+07$ & 5 & 183 & $5.892 \mathrm{e}-03$ & $1.001 \mathrm{e}+09$ & 5 & 186 & $8.673 e-05$ & $1.503 e+07$ \\
\hline 5 & 187 & $2.328 e-03$ & $4.040 e+08$ & 5 & 196 & $0.000 e+00$ & $2.260 e+05$ & 5 & 206 & $0.000 e+00$ & $2.942 e+04$ \\
\hline 5 & 207 & $1.223 e-04$ & $1.119 \mathrm{e}+08$ & 5 & 208 & $0.000 e+00$ & $1.923 e+05$ & 5 & 213 & $5.319 e-05$ & $1.150 \mathrm{e}+07$ \\
\hline 5 & 225 & $0.000 e+00$ & $1.297 \mathrm{e}+05$ & 5 & 233 & $4.396 \mathrm{e}-05$ & $1.753 e+07$ & 5 & 238 & $1.576 \mathrm{e}-03$ & $3.832 \mathrm{e}+08$ \\
\hline 5 & 241 & $1.208 e-04$ & $2.939 e+07$ & 5 & 275 & $0.000 e+00$ & $7.829 e+04$ & 5 & 281 & $2.707 e-03$ & $3.379 e+09$ \\
\hline 5 & 283 & $0.000 e+00$ & $9.525 e+05$ & 6 & 15 & $5.103 e-02$ & $3.512 \mathrm{e}+08$ & 6 & 16 & $1.409 \mathrm{e}-03$ & $7.046 e+06$ \\
\hline 6 & 18 & $1.323 e+00$ & $9.791 e+09$ & 6 & 19 & $1.300 e-03$ & $1.205 e+07$ & 6 & 20 & $3.732 \mathrm{e}-03$ & $5.813 e+07$ \\
\hline 6 & 22 & $9.164 \mathrm{e}-03$ & $1.457 \mathrm{e}+08$ & 6 & 23 & $4.155 \mathrm{e}-02$ & $3.979 e+08$ & 6 & 24 & $1.346 \mathrm{e}-01$ & $9.210 e+08$ \\
\hline 6 & 25 & $1.811 \mathrm{e}+00$ & $1.709 \mathrm{e}+10$ & 6 & 26 & $1.200 \mathrm{e}-02$ & $2.815 e+08$ & 6 & 28 & $0.000 e+00$ & $6.147 e+05$ \\
\hline 6 & 30 & $2.214 \mathrm{e}-02$ & $1.202 \mathrm{e}+09$ & 6 & 35 & $1.973 e-01$ & $1.081 e+10$ & 6 & 36 & $0.000 e+00$ & $8.810 e+05$ \\
\hline 6 & 37 & $0.000 e+00$ & $3.492 \mathrm{e}+05$ & 6 & 41 & $0.000 e+00$ & $5.144 e+04$ & 6 & 45 & $0.000 e+00$ & $5.995 e+05$ \\
\hline 6 & 47 & $4.320 \mathrm{e}-02$ & $3.539 \mathrm{e}+09$ & 6 & 48 & $2.316 \mathrm{e}-02$ & $1.152 \mathrm{e}+09$ & 6 & 52 & $4.710 e-01$ & $4.031 e+10$ \\
\hline 6 & 53 & $7.215 e-01$ & $2.724 e+10$ & 6 & 54 & $0.000 e+00$ & $4.266 e+06$ & 6 & 55 & $0.000 e+00$ & $2.879 \mathrm{e}+05$ \\
\hline 6 & 56 & $0.000 e+00$ & $1.283 e+05$ & 6 & 57 & $0.000 e+00$ & $9.811 e+04$ & 6 & 58 & $0.000 e+00$ & $2.620 \mathrm{e}+05$ \\
\hline 6 & 60 & $0.000 e+00$ & $6.899 \mathrm{e}+04$ & 6 & 62 & $0.000 e+00$ & $2.038 e+06$ & 6 & 63 & $0.000 e+00$ & $6.425 e+06$ \\
\hline 6 & 65 & $0.000 e+00$ & $1.160 \mathrm{e}+05$ & 6 & 66 & $1.202 \mathrm{e}-03$ & $1.524 e+08$ & 6 & 67 & $6.180 e-02$ & $4.702 \mathrm{e}+09$ \\
\hline 6 & 68 & $1.179 \mathrm{e}-01$ & $8.990 e+09$ & 6 & 69 & $1.574 \mathrm{e}-01$ & $8.583 e+09$ & 6 & 70 & $1.092 \mathrm{e}-02$ & $1.402 \mathrm{e}+09$ \\
\hline
\end{tabular}


Table III. Ca IX Oscillator Strengths and Radiative Decay Rates for transitions involving levels higher than 4.

\begin{tabular}{|c|c|c|c|c|c|c|c|c|c|c|c|}
\hline $\begin{array}{l}\text { Low. } \\
\text { Lev. }\end{array}$ & $\begin{array}{l}\text { Upp } \\
\text {. Lev }\end{array}$ & $\begin{array}{l}\text { Osc. } \\
\text { Str. }\end{array}$ & $\begin{array}{l}\text { Rad. } \\
\text { Rate }\end{array}$ & $\begin{array}{l}\text { Low. } \\
\text { Lev. }\end{array}$ & $\begin{array}{l}\text { Upp } \\
\text { Lev }\end{array}$ & $\begin{array}{l}\text { Osc. } \\
\text { Str. }\end{array}$ & $\begin{array}{l}\text { Rad. } \\
\text { Rate }\end{array}$ & $\begin{array}{l}\text { Low } \\
\text { Lev }\end{array}$ & $\begin{array}{l}\text { Upp } \\
\text { Lev }\end{array}$ & $\begin{array}{l}\text { Osc. } \\
\text { Str. }\end{array}$ & $\begin{array}{l}\text { Rad. } \\
\text { Rate }\end{array}$ \\
\hline$i$ & $j$ & $g f$ & $(1 / s)$ & $i$ & $j$ & $g f$ & $(1 / s)$ & $i$ & j & $g f$ & $(1 / s)$ \\
\hline 6 & 73 & $2.097 e-02$ & $2.700 e+09$ & 6 & 74 & $2.241 \mathrm{e}-02$ & $1.735 e+09$ & 6 & 75 & $1.514 \mathrm{e}-01$ & $8.379 e+09$ \\
\hline 6 & 77 & $6.214 \mathrm{e}-01$ & $3.467 e+10$ & 6 & 78 & $3.037 e-03$ & $2.429 e+08$ & 6 & 79 & $2.869 \mathrm{e}-03$ & $3.832 \mathrm{e}+08$ \\
\hline 6 & 81 & $3.627 e-03$ & $4.918 e+08$ & 6 & 82 & $0.000 e+00$ & $2.935 e+06$ & 6 & 83 & $0.000 e+00$ & $5.877 e+05$ \\
\hline 6 & 84 & $0.000 e+00$ & $5.274 \mathrm{e}+06$ & 6 & 87 & $0.000 e+00$ & $1.133 e+06$ & 6 & 88 & $0.000 e+00$ & $1.421 \mathrm{e}+06$ \\
\hline 6 & 89 & $0.000 e+00$ & $7.721 \mathrm{e}+04$ & 6 & 90 & $0.000 e+00$ & $3.064 e+05$ & 6 & 91 & $0.000 e+00$ & $6.756 e+04$ \\
\hline 6 & 92 & $0.000 e+00$ & $9.402 \mathrm{e}+05$ & 6 & 93 & $0.000 e+00$ & $2.098 e+07$ & 6 & 94 & $0.000 e+00$ & $8.740 e+04$ \\
\hline 6 & 95 & $0.000 e+00$ & $2.258 \mathrm{e}+05$ & 6 & 96 & $0.000 e+00$ & $8.091 e+04$ & 6 & 97 & $0.000 e+00$ & $8.899 e+05$ \\
\hline 6 & 101 & $2.740 \mathrm{e}-01$ & $1.785 e+10$ & 6 & 105 & $0.000 e+00$ & $4.350 e+06$ & 6 & 111 & $4.501 \mathrm{e}-02$ & $8.234 e+09$ \\
\hline 6 & 112 & $1.816 \mathrm{e}-02$ & $2.008 e+09$ & 6 & 113 & $1.963 e-02$ & $2.173 e+09$ & 6 & 114 & $1.063 \mathrm{e}-01$ & $1.968 \mathrm{e}+10$ \\
\hline 6 & 115 & $1.957 \mathrm{e}-03$ & $3.635 e+08$ & 6 & 116 & $5.255 e-05$ & $5.861 e+06$ & 6 & 117 & $2.039 e-04$ & $1.626 e+07$ \\
\hline 6 & 118 & $2.578 e-04$ & $2.887 e+07$ & 6 & 123 & $5.501 e-04$ & $6.307 e+07$ & 6 & 124 & $6.177 \mathrm{e}-02$ & $5.092 e+09$ \\
\hline 6 & 125 & $0.000 e+00$ & $3.182 \mathrm{e}+06$ & 6 & 126 & $0.000 e+00$ & $8.730 e+05$ & 6 & 127 & $4.705 e-04$ & $9.233 e+07$ \\
\hline 6 & 128 & $0.000 e+00$ & $2.398 e+05$ & 6 & 129 & $0.000 e+00$ & $5.421 e+05$ & 6 & 130 & $0.000 e+00$ & $2.906 e+05$ \\
\hline 6 & 131 & $0.000 e+00$ & $8.761 e+04$ & 6 & 133 & $0.000 e+00$ & $1.313 e+05$ & 6 & 134 & $0.000 e+00$ & $2.531 e+06$ \\
\hline 6 & 135 & $0.000 e+00$ & $7.277 e+06$ & 6 & 136 & $7.684 \mathrm{e}-02$ & $9.845 e+09$ & 6 & 137 & $2.022 \mathrm{e}-04$ & $2.595 e+07$ \\
\hline 6 & 138 & $6.989 e-05$ & $1.497 e+07$ & 6 & 139 & $6.383 e-02$ & $5.862 e+09$ & 6 & 140 & $3.279 \mathrm{e}-02$ & $4.240 e+09$ \\
\hline 6 & 141 & $1.364 \mathrm{e}-03$ & $1.262 \mathrm{e}+08$ & 6 & 143 & $7.879 e-04$ & $1.026 e+08$ & 6 & 144 & $0.000 e+00$ & $6.829 e+05$ \\
\hline 6 & 145 & $2.938 e-03$ & $6.390 e+08$ & 6 & 147 & $4.046 e-01$ & $3.790 e+10$ & 6 & 148 & $0.000 e+00$ & $5.889 e+03$ \\
\hline 6 & 151 & $0.000 e+00$ & $2.061 \mathrm{e}+05$ & 6 & 152 & $0.000 e+00$ & $7.647 e+04$ & 6 & 154 & $0.000 e+00$ & $1.031 \mathrm{e}+06$ \\
\hline 6 & 155 & $0.000 e+00$ & $2.833 e+06$ & 6 & 156 & $8.952 e-03$ & $1.978 e+09$ & 6 & 158 & $0.000 e+00$ & $6.485 e+04$ \\
\hline 6 & 161 & $0.000 e+00$ & $1.278 \mathrm{e}+06$ & 6 & 163 & $6.936 \mathrm{e}-04$ & $6.601 \mathrm{e}+07$ & 6 & 164 & $0.000 e+00$ & $1.335 e+06$ \\
\hline 6 & 165 & $0.000 e+00$ & $2.735 \mathrm{e}+04$ & 6 & 173 & $0.000 e+00$ & $3.216 e+05$ & 6 & 174 & $0.000 e+00$ & $7.342 \mathrm{e}+04$ \\
\hline 6 & 176 & $8.597 e-04$ & $8.299 e+07$ & 6 & 178 & $1.063 e-03$ & $1.029 e+08$ & 6 & 179 & $0.000 e+00$ & $1.026 \mathrm{e}+07$ \\
\hline 6 & 180 & $0.000 e+00$ & $1.154 \mathrm{e}+04$ & 6 & 181 & $0.000 e+00$ & $1.455 e+04$ & 6 & 182 & $0.000 e+00$ & $8.145 e+04$ \\
\hline 6 & 183 & $0.000 e+00$ & $4.654 \mathrm{e}+05$ & 6 & 186 & $0.000 e+00$ & $4.661 e+04$ & 6 & 187 & $0.000 e+00$ & $9.042 e+05$ \\
\hline 6 & 188 & $0.000 e+00$ & $4.730 e+06$ & 6 & 207 & $0.000 e+00$ & $1.216 \mathrm{e}+04$ & 6 & 208 & $8.202 e-05$ & $2.066 \mathrm{e}+07$ \\
\hline 6 & 214 & $1.626 \mathrm{e}-02$ & $3.023 e+09$ & 6 & 215 & $2.005 e-05$ & $6.226 e+06$ & 6 & 218 & $1.824 \mathrm{e}-04$ & $3.406 \mathrm{e}+07$ \\
\hline 6 & 222 & $2.717 e-05$ & $8.513 e+06$ & 6 & 224 & $1.047 \mathrm{e}-02$ & $1.413 e+09$ & 6 & 225 & $7.897 e-03$ & $2.499 \mathrm{e}+09$ \\
\hline 6 & 226 & $0.000 e+00$ & $1.082 \mathrm{e}+06$ & 6 & 227 & $0.000 e+00$ & $2.284 e+04$ & 6 & 229 & $0.000 e+00$ & $6.993 e+03$ \\
\hline 6 & 233 & $0.000 e+00$ & $1.629 \mathrm{e}+06$ & 6 & 238 & $0.000 e+00$ & $1.263 e+06$ & 6 & 241 & $0.000 e+00$ & $9.780 e+04$ \\
\hline 6 & 242 & $0.000 e+00$ & $1.455 \mathrm{e}+06$ & 6 & 250 & $3.479 \mathrm{e}-04$ & $7.132 e+07$ & 6 & 264 & $9.811 \mathrm{e}-05$ & $1.447 \mathrm{e}+07$ \\
\hline 6 & 265 & $0.000 e+00$ & $5.596 e+03$ & 6 & 275 & $1.045 e-03$ & $1.546 e+08$ & 6 & 280 & $0.000 e+00$ & $1.179 e+04$ \\
\hline 6 & 281 & $0.000 e+00$ & $1.433 e+06$ & 7 & 20 & $8.239 e-01$ & $1.235 \mathrm{e}+10$ & 7 & 22 & $1.321 \mathrm{e}-01$ & $2.022 \mathrm{e}+09$ \\
\hline 7 & 26 & $1.499 \mathrm{e}-03$ & $3.410 \mathrm{e}+07$ & 7 & 31 & $0.000 e+00$ & $1.165 e+04$ & 7 & 43 & $0.000 e+00$ & $9.615 e+03$ \\
\hline 7 & 47 & $1.724 \mathrm{e}-01$ & $1.389 \mathrm{e}+10$ & 7 & 52 & $2.408 e-03$ & $2.028 e+08$ & 7 & 56 & $0.000 e+00$ & $1.420 \mathrm{e}+06$ \\
\hline 7 & 61 & $0.000 e+00$ & $2.735 e+05$ & 7 & 66 & $2.660 \mathrm{e}-01$ & $3.328 e+10$ & 7 & 73 & $1.189 \mathrm{e}-02$ & $1.510 \mathrm{e}+09$ \\
\hline 7 & 79 & $3.553 e-02$ & $4.684 \mathrm{e}+09$ & 7 & 81 & $1.017 \mathrm{e}-03$ & $1.361 e+08$ & 7 & 86 & $0.000 e+00$ & $1.264 \mathrm{e}+07$ \\
\hline 7 & 90 & $0.000 e+00$ & $1.063 e+06$ & 7 & 92 & $0.000 e+00$ & $4.837 e+04$ & 7 & 95 & $0.000 e+00$ & $1.840 \mathrm{e}+06$ \\
\hline 7 & 97 & $0.000 e+00$ & $3.048 e+04$ & 7 & 107 & $0.000 e+00$ & $1.603 e+04$ & 7 & 111 & $3.028 e-02$ & $5.477 e+09$ \\
\hline 7 & 114 & $5.084 e-03$ & $9.305 e+08$ & 7 & 115 & $3.071 \mathrm{e}-05$ & $5.644 \mathrm{e}+06$ & 7 & 122 & $3.898 e-03$ & $7.364 e+08$ \\
\hline 7 & 127 & $2.347 e-04$ & $4.557 e+07$ & 7 & 128 & $0.000 e+00$ & $1.412 \mathrm{e}+06$ & 7 & 132 & $0.000 \mathrm{e}+00$ & $8.212 \mathrm{e}+04$ \\
\hline 7 & 134 & $0.000 e+00$ & $2.530 \mathrm{e}+04$ & 7 & 138 & $1.518 \mathrm{e}-01$ & $3.218 e+10$ & 7 & 145 & $1.485 \mathrm{e}-02$ & $3.198 e+09$ \\
\hline 7 & 156 & $1.206 \mathrm{e}-03$ & $2.637 e+08$ & 7 & 159 & $0.000 e+00$ & $5.893 e+06$ & 7 & 173 & $0.000 e+00$ & $1.050 \mathrm{e}+06$ \\
\hline 7 & 180 & $0.000 e+00$ & $5.494 e+04$ & 7 & 183 & $0.000 e+00$ & $1.862 \mathrm{e}+05$ & 7 & 186 & $0.000 e+00$ & $4.761 \mathrm{e}+03$ \\
\hline
\end{tabular}


Table III. Ca IX Oscillator Strengths and Radiative Decay Rates for transitions involving levels higher than 4.

\begin{tabular}{|c|c|c|c|c|c|c|c|c|c|c|c|}
\hline $\begin{array}{l}\text { Low. } \\
\text { Lev. }\end{array}$ & $\begin{array}{l}\text { Upp } \\
\text { Lev }\end{array}$ & $\begin{array}{l}\text { Osc. } \\
\text { Str. }\end{array}$ & $\begin{array}{l}\text { Rad. } \\
\text { Rate }\end{array}$ & $\begin{array}{l}\text { Low. } \\
\text { Lev. }\end{array}$ & $\begin{array}{l}\text { Upp } \\
\text { Lev }\end{array}$ & $\begin{array}{l}\text { Osc. } \\
\text { Str. }\end{array}$ & $\begin{array}{l}\text { Rad. } \\
\text { Rate }\end{array}$ & $\begin{array}{l}\text { Low } \\
\text { Lev }\end{array}$ & $\begin{array}{l}\text { Upp } \\
\text { Lev }\end{array}$ & $\begin{array}{l}\text { Osc. } \\
\text { Str. }\end{array}$ & $\begin{array}{l}\text { Rad. } \\
\text { Rate }\end{array}$ \\
\hline$i$ & $j$ & $g f$ & $(1 / s)$ & $i$ & $j$ & $g f$ & $(1 / s)$ & $i$ & $j$ & $g f$ & $(1 / s)$ \\
\hline 7 & 215 & $1.708 e-04$ & $5.258 e+07$ & 7 & 228 & $0.000 e+00$ & $6.335 e+03$ & 7 & 262 & $4.595 e-04$ & $1.569 \mathrm{e}+08$ \\
\hline 7 & 273 & $1.048 e-04$ & $3.587 e+07$ & 8 & 15 & $7.920 \mathrm{e}-04$ & $5.123 e+06$ & 8 & 18 & $3.684 \mathrm{e}-03$ & $2.568 e+07$ \\
\hline 8 & 19 & $1.091 \mathrm{e}+00$ & $9.589 e+09$ & 8 & 20 & $8.877 e-03$ & $1.311 \mathrm{e}+08$ & 8 & 21 & $2.513 e-01$ & $1.128 \mathrm{e}+10$ \\
\hline 8 & 22 & $7.087 e-01$ & $1.069 e+10$ & 8 & 23 & $7.930 e-01$ & $7.204 e+09$ & 8 & 26 & $2.479 \mathrm{e}-04$ & $5.568 e+06$ \\
\hline 8 & 29 & $1.419 \mathrm{e}-05$ & $2.243 e+06$ & 8 & 31 & $0.000 e+00$ & $1.127 e+04$ & 8 & 32 & $0.000 e+00$ & $1.649 \mathrm{e}+04$ \\
\hline 8 & 42 & $0.000 e+00$ & $2.431 \mathrm{e}+04$ & 8 & 44 & $0.000 e+00$ & $7.934 e+03$ & 8 & 46 & $1.780 \mathrm{e}-01$ & $4.258 e+10$ \\
\hline 8 & 47 & $1.289 \mathrm{e}-01$ & $1.032 e+10$ & 8 & 48 & $2.185 e-01$ & $1.062 \mathrm{e}+10$ & 8 & 52 & $3.327 e-03$ & $2.784 e+08$ \\
\hline 8 & 54 & $0.000 e+00$ & $2.912 \mathrm{e}+05$ & 8 & 55 & $0.000 e+00$ & $2.766 e+06$ & 8 & 56 & $0.000 e+00$ & $4.199 \mathrm{e}+05$ \\
\hline 8 & 57 & $0.000 e+00$ & $1.306 e+06$ & 8 & 59 & $0.000 e+00$ & $2.089 e+05$ & 8 & 60 & $0.000 e+00$ & $1.202 e+05$ \\
\hline 8 & 61 & $0.000 e+00$ & $7.848 e+05$ & 8 & 62 & $0.000 e+00$ & $2.215 e+04$ & 8 & 66 & $1.430 \mathrm{e}-01$ & $1.780 e+10$ \\
\hline 8 & 67 & $3.600 e-01$ & $2.689 e+10$ & 8 & 68 & $2.206 \mathrm{e}-01$ & $1.652 e+10$ & 8 & 70 & $4.477 \mathrm{e}-02$ & $5.644 e+09$ \\
\hline 8 & 71 & $1.789 \mathrm{e}-02$ & $6.767 e+09$ & 8 & 74 & $3.199 \mathrm{e}-02$ & $2.431 e+09$ & 8 & 78 & $3.272 \mathrm{e}-02$ & $2.571 \mathrm{e}+09$ \\
\hline 8 & 79 & $4.778 e-02$ & $6.268 e+09$ & 8 & 80 & $5.103 e-02$ & $2.011 e+10$ & 8 & 81 & $5.466 e-05$ & $7.280 e+06$ \\
\hline 8 & 82 & $0.000 e+00$ & $1.117 e+05$ & 8 & 84 & $0.000 e+00$ & $2.334 \mathrm{e}+06$ & 8 & 86 & $0.000 e+00$ & $8.792 e+06$ \\
\hline 8 & 87 & $0.000 e+00$ & $1.546 \mathrm{e}+07$ & 8 & 89 & $0.000 e+00$ & $6.688 e+05$ & 8 & 90 & $0.000 e+00$ & $1.846 e+06$ \\
\hline 8 & 91 & $0.000 e+00$ & $5.370 e+06$ & 8 & 92 & $0.000 e+00$ & $1.910 e+04$ & 8 & 94 & $0.000 e+00$ & $1.396 e+06$ \\
\hline 8 & 95 & $0.000 e+00$ & $1.079 \mathrm{e}+06$ & 8 & 96 & $0.000 e+00$ & $6.401 e+06$ & 8 & 97 & $0.000 e+00$ & $1.113 e+04$ \\
\hline 8 & 106 & $0.000 e+00$ & $4.028 e+04$ & 8 & 108 & $0.000 e+00$ & $1.400 \mathrm{e}+04$ & 8 & 110 & $3.611 \mathrm{e}-02$ & $1.950 \mathrm{e}+10$ \\
\hline 8 & 111 & $2.129 \mathrm{e}-02$ & $3.835 e+09$ & 8 & 112 & $7.694 \mathrm{e}-03$ & $8.376 e+08$ & 8 & 113 & $3.449 e-02$ & $3.760 \mathrm{e}+09$ \\
\hline 8 & 114 & $5.133 e-03$ & $9.353 e+08$ & 8 & 116 & $1.074 \mathrm{e}-04$ & $1.179 e+07$ & 8 & 121 & $3.327 e-03$ & $1.877 e+09$ \\
\hline 8 & 122 & $3.037 e-03$ & $5.714 e+08$ & 8 & 123 & $7.268 e-03$ & $8.207 e+08$ & 8 & 125 & $0.000 e+00$ & $6.943 e+05$ \\
\hline 8 & 126 & $0.000 e+00$ & $2.152 \mathrm{e}+06$ & 8 & 128 & $0.000 e+00$ & $5.839 e+05$ & 8 & 130 & $0.000 e+00$ & $5.486 e+03$ \\
\hline 8 & 131 & $0.000 e+00$ & $1.130 \mathrm{e}+06$ & 8 & 132 & $0.000 e+00$ & $5.577 e+05$ & 8 & 133 & $0.000 e+00$ & $6.158 e+04$ \\
\hline 8 & 134 & $0.000 e+00$ & $5.610 e+04$ & 8 & 136 & $1.254 \mathrm{e}-02$ & $1.584 \mathrm{e}+09$ & 8 & 137 & $2.345 e-01$ & $2.967 e+10$ \\
\hline 8 & 138 & $7.085 e-02$ & $1.496 e+10$ & 8 & 140 & $8.860 e-02$ & $1.130 \mathrm{e}+10$ & 8 & 143 & $3.131 \mathrm{e}-03$ & $4.022 e+08$ \\
\hline 8 & 145 & $5.479 e-02$ & $1.175 e+10$ & 8 & 146 & $4.026 e-02$ & $2.594 e+10$ & 8 & 151 & $0.000 e+00$ & $7.826 e+04$ \\
\hline 8 & 155 & $0.000 e+00$ & $2.785 e+05$ & 8 & 156 & $2.033 e-04$ & $4.428 e+07$ & 8 & 158 & $0.000 e+00$ & $5.818 e+06$ \\
\hline 8 & 159 & $0.000 e+00$ & $3.424 \mathrm{e}+06$ & 8 & 164 & $0.000 e+00$ & $2.050 e+06$ & 8 & 172 & $0.000 e+00$ & $5.663 e+05$ \\
\hline 8 & 173 & $0.000 e+00$ & $1.605 \mathrm{e}+06$ & 8 & 174 & $0.000 e+00$ & $5.652 e+06$ & 8 & 180 & $0.000 e+00$ & $2.378 e+05$ \\
\hline 8 & 181 & $0.000 e+00$ & $2.343 e+05$ & 8 & 183 & $0.000 e+00$ & $5.365 e+04$ & 8 & 184 & $0.000 e+00$ & $4.939 e+03$ \\
\hline 8 & 186 & $0.000 e+00$ & $8.554 \mathrm{e}+03$ & 8 & 205 & $4.550 \mathrm{e}-05$ & $3.298 e+07$ & 8 & 210 & $0.000 e+00$ & $7.003 e+03$ \\
\hline 8 & 215 & $1.262 \mathrm{e}-04$ & $3.872 \mathrm{e}+07$ & 8 & 216 & $3.824 \mathrm{e}-04$ & $7.041 \mathrm{e}+07$ & 8 & 227 & $0.000 e+00$ & $1.459 \mathrm{e}+04$ \\
\hline 8 & 229 & $0.000 e+00$ & $5.671 \mathrm{e}+03$ & 8 & 241 & $0.000 e+00$ & $5.364 e+03$ & 8 & 262 & $2.755 e-04$ & $9.376 e+07$ \\
\hline 8 & 263 & $1.029 \mathrm{e}-03$ & $2.102 \mathrm{e}+08$ & 8 & 272 & $1.005 e-04$ & $2.056 e+07$ & 8 & 273 & $1.412 \mathrm{e}-04$ & $4.814 e+07$ \\
\hline 8 & 274 & $1.428 e-04$ & $1.461 \mathrm{e}+08$ & 9 & 15 & $3.839 e-04$ & $2.406 e+06$ & 9 & 16 & $3.012 \mathrm{e}-03$ & $1.373 e+07$ \\
\hline 9 & 18 & $3.682 \mathrm{e}-02$ & $2.490 e+08$ & 9 & 19 & $3.115 e-01$ & $2.663 e+09$ & 9 & 20 & $1.047 e-01$ & $1.505 e+09$ \\
\hline 9 & 22 & $2.279 \mathrm{e}-01$ & $3.347 e+09$ & 9 & 23 & $1.108 e+00$ & $9.802 e+09$ & 9 & 24 & $2.796 \mathrm{e}+00$ & $1.768 e+10$ \\
\hline 9 & 25 & $8.658 e-02$ & $7.641 \mathrm{e}+08$ & 9 & 26 & $5.922 e-04$ & $1.302 \mathrm{e}+07$ & 9 & 28 & $0.000 e+00$ & $2.354 e+04$ \\
\hline 9 & 30 & $1.079 \mathrm{e}-03$ & $5.610 e+07$ & 9 & 32 & $0.000 e+00$ & $7.544 e+03$ & 9 & 34 & $0.000 e+00$ & $2.260 \mathrm{e}+04$ \\
\hline 9 & 35 & $7.563 e-03$ & $3.973 e+08$ & 9 & 36 & $0.000 e+00$ & $3.354 e+04$ & 9 & 37 & $0.000 e+00$ & $1.436 \mathrm{e}+04$ \\
\hline 9 & 42 & $0.000 e+00$ & $7.197 e+03$ & 9 & 43 & $0.000 e+00$ & $1.630 \mathrm{e}+04$ & 9 & 44 & $0.000 e+00$ & $1.595 e+04$ \\
\hline 9 & 45 & $0.000 e+00$ & $2.278 \mathrm{e}+04$ & 9 & 47 & $1.900 \mathrm{e}-01$ & $1.503 e+10$ & 9 & 48 & $6.408 \mathrm{e}-01$ & $3.079 e+10$ \\
\hline 9 & 52 & $4.558 e-02$ & $3.771 \mathrm{e}+09$ & 9 & 53 & $3.273 e-02$ & $1.195 e+09$ & 9 & 55 & $0.000 e+00$ & $8.465 e+05$ \\
\hline 9 & 56 & $0.000 e+00$ & $1.876 \mathrm{e}+06$ & 9 & 57 & $0.000 e+00$ & $2.485 e+06$ & 9 & 58 & $0.000 e+00$ & $1.798 e+06$ \\
\hline
\end{tabular}


Table III. Ca IX Oscillator Strengths and Radiative Decay Rates for transitions involving levels higher than 4.

\begin{tabular}{|c|c|c|c|c|c|c|c|c|c|c|c|}
\hline $\begin{array}{l}\text { Low } \\
\text { Lev }\end{array}$ & $\begin{array}{l}\text { Upp } \\
\text { Lev }\end{array}$ & $\begin{array}{l}\text { Osc. } \\
\text { Str. }\end{array}$ & $\begin{array}{l}\text { Rad. } \\
\text { Rate }\end{array}$ & $\begin{array}{l}\text { Low } \\
\text { Lev }\end{array}$ & $\begin{array}{l}\text { Upp } \\
\text { Lev }\end{array}$ & $\begin{array}{l}\text { Osc. } \\
\text { Str. }\end{array}$ & $\begin{array}{l}\text { Rad. } \\
\text { Rate }\end{array}$ & $\begin{array}{l}\text { Low. } \\
\text { Lev. }\end{array}$ & $\begin{array}{l}\text { Upp } \\
\text { Lev }\end{array}$ & $\begin{array}{l}\text { Osc. } \\
\text { Str. }\end{array}$ & $\begin{array}{l}\text { Rad. } \\
\text { Rate }\end{array}$ \\
\hline$i$ & $j$ & $g f$ & $(1 / s)$ & $i$ & $j$ & $g f$ & $(1 / s)$ & $i$ & j & $g f$ & $(1 / s)$ \\
\hline 9 & 59 & $0.000 e+00$ & $.300 e+06$ & 9 & 60 & $0.000 e+00$ & $4.073 e+05$ & 9 & 61 & $0.000 e+00$ & $.913 e+$ \\
\hline 9 & 62 & $0.000 e+00$ & $6.169 e+04$ & 9 & 63 & $0.000 e+00$ & $3.873 e+05$ & 9 & 65 & $0.000 e+00$ & $7.734 e+03$ \\
\hline 9 & 66 & $5.147 e-03$ & $6.347 e+08$ & 9 & 67 & $5.305 e-02$ & $3.925 e+09$ & 9 & 68 & $4.113 e-02$ & $3.052 \mathrm{e}+09$ \\
\hline 9 & 69 & $7.996 e-01$ & $4.240 e+10$ & 9 & 70 & $2.154 \mathrm{e}-02$ & $2.690 \mathrm{e}+09$ & 9 & 72 & $1.404 \mathrm{e}-01$ & $1.053 e+10$ \\
\hline 9 & 73 & $7.323 e-03$ & $9.170 e+08$ & 9 & 74 & $5.386 e-03$ & $4.056 e+08$ & 9 & 75 & $2.056 \mathrm{e}-01$ & $1.107 e+10$ \\
\hline 9 & 77 & $3.949 e-02$ & $2.144 \mathrm{e}+09$ & 9 & 78 & $2.015 \mathrm{e}-01$ & $1.569 \mathrm{e}+10$ & 9 & 79 & $6.241 \mathrm{e}-02$ & $8.113 e+09$ \\
\hline 9 & 81 & $2.106 \mathrm{e}-03$ & $2.779 e+08$ & 9 & 82 & $0.000 e+00$ & $1.591 e+04$ & 9 & 83 & $0.000 e+00$ & $1.470 e+06$ \\
\hline 9 & 84 & $0.000 e+00$ & $6.716 \mathrm{e}+05$ & 9 & 86 & $0.000 e+00$ & $4.987 e+05$ & 9 & 87 & $0.000 e+00$ & $3.090 e+06$ \\
\hline 9 & 88 & $0.000 e+00$ & $1.891 \mathrm{e}+07$ & 9 & 89 & $0.000 e+00$ & $8.531 e+06$ & 9 & 90 & $0.000 e+00$ & $5.009 e+06$ \\
\hline 9 & 91 & $0.000 e+00$ & $1.711 \mathrm{e}+06$ & 9 & 92 & $0.000 e+00$ & $6.118 e+04$ & 9 & 93 & $0.000 e+00$ & $1.737 e+06$ \\
\hline 9 & 94 & $0.000 e+00$ & $5.092 e+06$ & 9 & 95 & $0.000 e+00$ & $4.503 e+06$ & 9 & 96 & $0.000 e+00$ & $2.031 e+06$ \\
\hline 9 & 97 & $0.000 e+00$ & $1.510 e+05$ & 9 & 101 & $1.227 e-02$ & $7.791 e+08$ & 9 & 105 & $0.000 e+00$ & $2.153 e+05$ \\
\hline 9 & 106 & $0.000 e+00$ & $1.201 \mathrm{e}+04$ & 9 & 107 & $0.000 e+00$ & $2.750 e+04$ & 9 & 108 & $0.000 e+00$ & $3.035 e+04$ \\
\hline 9 & 111 & $2.294 \mathrm{e}-02$ & $4.101 e+09$ & 9 & 112 & $3.361 \mathrm{e}-02$ & $3.630 e+09$ & 9 & 113 & $8.819 e-02$ & $9.542 e+09$ \\
\hline 9 & 114 & $2.515 e-02$ & $4.548 e+09$ & 9 & 115 & $8.372 e-05$ & $1.520 \mathrm{e}+07$ & 9 & 116 & $1.165 e-03$ & $1.270 \mathrm{e}+08$ \\
\hline 9 & 118 & $4.951 e-05$ & $5.420 e+06$ & 9 & 122 & $5.203 e-03$ & $9.714 e+08$ & 9 & 123 & $2.131 \mathrm{e}-02$ & $2.388 e+09$ \\
\hline 9 & 124 & $2.116 \mathrm{e}-03$ & $1.705 e+08$ & 9 & 125 & $0.000 e+00$ & $8.541 e+04$ & 9 & 126 & $0.000 e+00$ & $1.018 \mathrm{e}+05$ \\
\hline 9 & 127 & $1.142 \mathrm{e}-04$ & $2.192 \mathrm{e}+07$ & 9 & 128 & $0.000 e+00$ & $9.351 e+05$ & 9 & 129 & $0.000 e+00$ & $1.628 e+06$ \\
\hline 9 & 130 & $0.000 e+00$ & $2.009 e+06$ & 9 & 131 & $0.000 e+00$ & $2.099 e+06$ & 9 & 132 & $0.000 e+00$ & $1.537 e+06$ \\
\hline 9 & 133 & $0.000 e+00$ & $1.808 e+05$ & 9 & 134 & $0.000 e+00$ & $1.190 \mathrm{e}+05$ & 9 & 135 & $0.000 e+00$ & $5.928 e+05$ \\
\hline 9 & 136 & $6.301 \mathrm{e}-05$ & $7.902 e+06$ & 9 & 137 & $2.210 \mathrm{e}-02$ & $2.778 e+09$ & 9 & 138 & $1.252 \mathrm{e}-03$ & $2.624 e+08$ \\
\hline 9 & 139 & $2.564 \mathrm{e}-01$ & $2.305 e+10$ & 9 & 140 & $1.012 \mathrm{e}-02$ & $1.281 e+09$ & 9 & 141 & $2.369 \mathrm{e}-01$ & $2.145 \mathrm{e}+10$ \\
\hline 9 & 143 & $2.101 \mathrm{e}-01$ & $2.680 e+10$ & 9 & 144 & $0.000 e+00$ & $3.881 e+04$ & 9 & 145 & $5.284 \mathrm{e}-02$ & $1.125 \mathrm{e}+10$ \\
\hline 9 & 147 & $4.303 e-02$ & $3.947 e+09$ & 9 & 151 & $0.000 e+00$ & $2.098 e+04$ & 9 & 152 & $0.000 e+00$ & $1.203 e+05$ \\
\hline 9 & 154 & $0.000 e+00$ & $4.490 e+04$ & 9 & 156 & $1.345 e-03$ & $2.909 e+08$ & 9 & 158 & $0.000 e+00$ & $6.982 e+05$ \\
\hline 9 & 159 & $0.000 e+00$ & $1.021 e+05$ & 9 & 161 & $0.000 e+00$ & $4.474 e+06$ & 9 & 163 & $4.998 e-05$ & $4.658 e+06$ \\
\hline 9 & 164 & $0.000 e+00$ & $3.673 e+05$ & 9 & 165 & $0.000 e+00$ & $3.854 e+06$ & 9 & 172 & $0.000 e+00$ & $6.602 e+06$ \\
\hline 9 & 173 & $0.000 \mathrm{e}+00$ & $4.341 \mathrm{e}+06$ & 9 & 174 & $0.000 \mathrm{e}+00$ & $1.766 e+06$ & 9 & 176 & $5.259 \mathrm{e}-05$ & $4.972 e+06$ \\
\hline 9 & 178 & $5.179 \mathrm{e}-05$ & $4.914 \mathrm{e}+06$ & 9 & 179 & $0.000 e+00$ & $7.119 e+05$ & 9 & 180 & $0.000 e+00$ & $1.941 \mathrm{e}+05$ \\
\hline 9 & 181 & $0.000 e+00$ & $2.948 e+05$ & 9 & 182 & $0.000 e+00$ & $6.518 e+05$ & 9 & 183 & $0.000 e+00$ & $1.263 e+05$ \\
\hline 9 & 184 & $0.000 e+00$ & $1.548 \mathrm{e}+04$ & 9 & 185 & $0.000 e+00$ & $2.021 e+04$ & 9 & 187 & $0.000 e+00$ & $5.510 e+04$ \\
\hline 9 & 188 & $0.000 e+00$ & $2.356 \mathrm{e}+05$ & 9 & 203 & $1.390 e-04$ & $2.001 e+07$ & 9 & 214 & $7.172 \mathrm{e}-04$ & $1.310 \mathrm{e}+08$ \\
\hline 9 & 216 & $1.496 \mathrm{e}-04$ & $2.739 e+07$ & 9 & 217 & $7.405 e-04$ & $9.687 e+07$ & 9 & 224 & $4.785 e-04$ & $6.342 e+07$ \\
\hline 9 & 225 & $3.655 e-04$ & $1.136 \mathrm{e}+08$ & 9 & 226 & $0.000 e+00$ & $5.302 e+04$ & 9 & 228 & $0.000 e+00$ & $1.043 e+0$ \\
\hline 9 & 229 & $0.000 e+00$ & $9.121 \mathrm{e}+03$ & 9 & 233 & $0.000 e+00$ & $7.099 e+04$ & 9 & 238 & $0.000 e+00$ & $6.831 \mathrm{e}+0$ \\
\hline 9 & 239 & $0.000 e+00$ & $1.365 e+04$ & 9 & 240 & $0.000 e+00$ & $1.007 e+04$ & 9 & 242 & $0.000 e+00$ & $6.609 \mathrm{e}+0$ \\
\hline 9 & 263 & $2.133 e-04$ & $4.333 e+07$ & 9 & 264 & $1.692 \mathrm{e}-03$ & $2.455 e+08$ & 9 & 272 & $6.315 e-04$ & $1.285 e+0$ \\
\hline 9 & 273 & $1.808 \mathrm{e}-04$ & $6.130 e+07$ & 9 & 281 & $0.000 e+00$ & $7.018 e+04$ & 10 & 20 & $5.422 \mathrm{e}-04$ & $4.692 \mathrm{e}+0$ \\
\hline 10 & 26 & $1.041 \mathrm{e}+00$ & $1.534 \mathrm{e}+10$ & 10 & 30 & $8.325 e-04$ & $3.370 e+07$ & 10 & 35 & $8.464 \mathrm{e}-03$ & $3.464 \mathrm{e}+0$ \\
\hline 10 & 37 & $0.000 e+00$ & $1.132 \mathrm{e}+04$ & 10 & 47 & $5.659 \mathrm{e}-04$ & $3.663 e+07$ & 10 & 52 & $1.133 \mathrm{e}-01$ & $7.707 e+0$ \\
\hline 10 & 61 & $0.000 e+00$ & $9.134 e+03$ & 10 & 62 & $0.000 e+00$ & $1.197 e+06$ & 10 & 66 & $3.375 e-03$ & $3.550 e+0$ \\
\hline 10 & 70 & $1.360 \mathrm{e}-02$ & $1.450 e+09$ & 10 & 73 & $6.474 \mathrm{e}-02$ & $6.922 \mathrm{e}+09$ & 10 & 79 & $2.616 \mathrm{e}-03$ & $2.912 \mathrm{e}+08$ \\
\hline 10 & 81 & $2.486 e-01$ & $2.814 \mathrm{e}+10$ & 10 & 90 & $0.000 e+00$ & $1.105 e+04$ & 10 & 92 & $0.000 e+00$ & $7.581 \mathrm{e}+05$ \\
\hline 10 & 95 & $.000 e+00$ & $.544 e+04$ & 10 & 97 & $.000 e+00$ & $1.233 e+07$ & 10 & 109 & $0.000 e+00$ & $0<0$ \\
\hline
\end{tabular}


Table III. Ca IX Oscillator Strengths and Radiative Decay Rates for transitions involving levels higher than 4.

\begin{tabular}{|c|c|c|c|c|c|c|c|c|c|c|c|}
\hline $\begin{array}{l}\text { Low. } \\
\text { Lev. }\end{array}$ & $\begin{array}{l}\text { Upp } \\
\text { Lev }\end{array}$ & $\begin{array}{l}\text { Osc. } \\
\text { Str. }\end{array}$ & $\begin{array}{l}\text { Rad. } \\
\text { Rate }\end{array}$ & $\begin{array}{l}\text { Low. } \\
\text { Lev. }\end{array}$ & $\begin{array}{l}\text { Upp. } \\
\text { Lev }\end{array}$ & $\begin{array}{l}\text { Osc. } \\
\text { Str. }\end{array}$ & $\begin{array}{l}\text { Rad. } \\
\text { Rate }\end{array}$ & $\begin{array}{l}\text { Low. } \\
\text { Lev. }\end{array}$ & $\begin{array}{l}\text {. Upp } \\
\text {. Lev }\end{array}$ & $\begin{array}{l}\text { Osc. } \\
\text { Str. }\end{array}$ & $\begin{array}{l}\text { Rad. } \\
\text { Rate }\end{array}$ \\
\hline$i$ & $j$ & $g f$ & $(1 / s)$ & $i$ & $j$ & $g f$ & $(1 / s)$ & $i$ & j & $g f$ & $(1 / s)$ \\
\hline 10 & 111 & $4.969 \mathrm{e}-03$ & $7.788 e+08$ & 10 & 114 & $2.746 \mathrm{e}-02$ & $4.357 e+09$ & 10 & 115 & $4.322 \mathrm{e}-04$ & $6.889 e+07$ \\
\hline 10 & 122 & $9.386 e-05$ & $1.541 \mathrm{e}+07$ & 10 & 127 & $3.442 \mathrm{e}-05$ & $5.819 e+06$ & 10 & 128 & $0.000 e+00$ & $2.374 \mathrm{e}+04$ \\
\hline 10 & 132 & $0.000 e+00$ & $1.709 e+04$ & 10 & 134 & $0.000 e+00$ & $1.486 e+06$ & 10 & 138 & $1.205 e-03$ & $2.238 e+08$ \\
\hline 10 & 145 & $1.090 e-03$ & $2.059 e+08$ & 10 & 156 & $2.094 \mathrm{e}-01$ & $4.021 e+10$ & 10 & 159 & $0.000 e+00$ & $9.764 e+04$ \\
\hline 10 & 173 & $0.000 e+00$ & $1.523 e+05$ & 10 & 180 & $0.000 e+00$ & $3.829 e+05$ & 10 & 183 & $0.000 e+00$ & $6.920 \mathrm{e}+06$ \\
\hline 10 & 186 & $0.000 e+00$ & $9.369 e+03$ & 10 & 187 & $0.000 e+00$ & $1.782 \mathrm{e}+05$ & 10 & 208 & $8.877 e-04$ & $1.962 \mathrm{e}+08$ \\
\hline 10 & 225 & $3.087 e-04$ & $8.698 e+07$ & 10 & 238 & $0.000 e+00$ & $3.147 e+04$ & 10 & 280 & $0.000 e+00$ & $2.591 e+04$ \\
\hline 10 & 283 & $4.511 \mathrm{e}-03$ & $1.411 \mathrm{e}+09$ & 11 & 15 & $5.427 e-01$ & $1.634 \mathrm{e}+09$ & 11 & 18 & $2.147 \mathrm{e}-02$ & $7.209 e+07$ \\
\hline 11 & 19 & $7.053 e-03$ & $3.279 e+07$ & 11 & 20 & $5.675 e-03$ & $4.449 e+07$ & 11 & 21 & $2.135 \mathrm{e}-01$ & $5.115 e+09$ \\
\hline 11 & 22 & $5.791 \mathrm{e}-01$ & $4.672 \mathrm{e}+09$ & 11 & 23 & $1.661 \mathrm{e}-01$ & $8.085 e+08$ & 11 & 26 & $2.163 e-04$ & $2.956 e+06$ \\
\hline 11 & 27 & $0.000 e+00$ & $1.260 \mathrm{e}+05$ & 11 & 29 & $1.235 \mathrm{e}-01$ & $1.431 e+10$ & 11 & 30 & $8.277 e-02$ & $3.203 e+09$ \\
\hline 11 & 31 & $0.000 e+00$ & $4.094 e+05$ & 11 & 32 & $0.000 e+00$ & $1.463 e+05$ & 11 & 33 & $6.037 \mathrm{e}-03$ & $1.410 \mathrm{e}+08$ \\
\hline 11 & 35 & $9.149 e-03$ & $3.581 \mathrm{e}+08$ & 11 & 38 & $0.000 e+00$ & $4.241 e+05$ & 11 & 40 & $0.000 e+00$ & $8.394 e+04$ \\
\hline 11 & 42 & $0.000 e+00$ & $3.240 e+05$ & 11 & 43 & $0.000 e+00$ & $3.248 e+05$ & 11 & 44 & $0.000 e+00$ & $2.668 e+04$ \\
\hline 11 & 46 & $2.100 e-03$ & $3.917 e+08$ & 11 & 47 & $1.527 e-03$ & $9.542 \mathrm{e}+07$ & 11 & 49 & $2.694 e+00$ & $1.055 \mathrm{e}+11$ \\
\hline 11 & 55 & $0.000 e+00$ & $2.629 \mathrm{e}+04$ & 11 & 56 & $0.000 e+00$ & $2.456 e+04$ & 11 & 59 & $0.000 e+00$ & $2.472 \mathrm{e}+04$ \\
\hline 11 & 64 & $0.000 e+00$ & $1.485 e+05$ & 11 & 66 & $1.361 \mathrm{e}-03$ & $1.392 \mathrm{e}+08$ & 11 & 67 & $1.023 e-04$ & $6.281 e+06$ \\
\hline 11 & 68 & $9.271 \mathrm{e}-03$ & $5.706 e+08$ & 11 & 70 & $1.538 e-02$ & $1.595 e+09$ & 11 & 71 & $2.135 \mathrm{e}-02$ & $6.644 \mathrm{e}+09$ \\
\hline 11 & 72 & $1.227 e-03$ & $7.642 \mathrm{e}+07$ & 11 & 73 & $2.548 e-03$ & $2.651 e+08$ & 11 & 74 & $2.979 \mathrm{e}-02$ & $1.864 \mathrm{e}+09$ \\
\hline 11 & 78 & $1.914 \mathrm{e}-04$ & $1.242 \mathrm{e}+07$ & 11 & 79 & $2.950 e-03$ & $3.197 e+08$ & 11 & 80 & $4.173 e-03$ & $1.359 e+09$ \\
\hline 11 & 81 & $9.285 e-05$ & $1.023 e+07$ & 11 & 82 & $0.000 e+00$ & $2.409 e+05$ & 11 & 84 & $0.000 e+00$ & $1.067 \mathrm{e}+05$ \\
\hline 11 & 87 & $0.000 e+00$ & $3.093 e+04$ & 11 & 89 & $0.000 e+00$ & $1.437 e+04$ & 11 & 90 & $0.000 \mathrm{e}+00$ & $1.696 \mathrm{e}+05$ \\
\hline 11 & 91 & $0.000 e+00$ & $1.939 e+05$ & 11 & 94 & $0.000 e+00$ & $2.218 e+04$ & 11 & 95 & $0.000 e+00$ & $2.529 e+05$ \\
\hline 11 & 96 & $0.000 e+00$ & $2.342 e+05$ & 11 & 98 & $6.237 e-01$ & $4.628 e+10$ & 11 & 102 & $0.000 e+00$ & $4.452 \mathrm{e}+06$ \\
\hline 11 & 110 & $5.995 e-03$ & $2.753 e+09$ & 11 & 111 & $2.393 e-03$ & $3.666 e+08$ & 11 & 112 & $1.297 e-03$ & $1.202 \mathrm{e}+08$ \\
\hline 11 & 113 & $9.338 e-04$ & $8.666 e+07$ & 11 & 114 & $5.255 e-03$ & $8.153 e+08$ & 11 & 115 & $9.652 \mathrm{e}-02$ & $1.504 \mathrm{e}+10$ \\
\hline 11 & 116 & $9.299 \mathrm{e}-03$ & $8.700 e+08$ & 11 & 118 & $1.850 \mathrm{e}-01$ & $1.739 e+10$ & 11 & 121 & $3.468 \mathrm{e}-02$ & $1.671 \mathrm{e}+10$ \\
\hline 11 & 122 & $2.815 \mathrm{e}-02$ & $4.521 \mathrm{e}+09$ & 11 & 123 & $2.076 \mathrm{e}-03$ & $2.002 e+08$ & 11 & 126 & $0.000 e+00$ & $9.438 e+04$ \\
\hline 11 & 128 & $0.000 e+00$ & $4.363 e+04$ & 11 & 130 & $0.000 e+00$ & $1.097 e+05$ & 11 & 132 & $0.000 e+00$ & $2.454 e+04$ \\
\hline 11 & 133 & $0.000 e+00$ & $5.031 e+04$ & 11 & 136 & $8.163 e-04$ & $8.882 e+07$ & 11 & 137 & $6.515 \mathrm{e}-04$ & $7.104 e+07$ \\
\hline 11 & 138 & $8.551 \mathrm{e}-05$ & $1.556 \mathrm{e}+07$ & 11 & 140 & $1.620 \mathrm{e}-03$ & $1.780 e+08$ & 11 & 145 & $4.043 e-04$ & $7.480 e+07$ \\
\hline 11 & 146 & $5.745 e-04$ & $3.193 e+08$ & 11 & 148 & $0.000 e+00$ & $2.057 e+06$ & 11 & 149 & $0.000 e+00$ & $2.068 e+06$ \\
\hline 11 & 150 & $0.000 e+00$ & $2.136 \mathrm{e}+05$ & 11 & 151 & $0.000 e+00$ & $3.372 e+06$ & 11 & 154 & $0.000 e+00$ & $1.560 e+04$ \\
\hline 11 & 155 & $0.000 e+00$ & $7.003 e+05$ & 11 & 158 & $0.000 e+00$ & $2.323 e+05$ & 11 & 159 & $0.000 e+00$ & $6.982 e+04$ \\
\hline 11 & 164 & $0.000 e+00$ & $2.119 \mathrm{e}+05$ & 11 & 171 & $0.000 e+00$ & $1.247 e+06$ & 11 & 174 & $0.000 e+00$ & $4.150 e+04$ \\
\hline 11 & 177 & $3.679 e-04$ & $4.256 \mathrm{e}+07$ & 11 & 180 & $0.000 e+00$ & $3.363 e+06$ & 11 & 181 & $0.000 e+00$ & $1.214 \mathrm{e}+06$ \\
\hline 11 & 183 & $0.000 e+00$ & $1.620 \mathrm{e}+05$ & 11 & 184 & $0.000 e+00$ & $2.360 e+06$ & 11 & 186 & $0.000 e+00$ & $4.529 e+05$ \\
\hline 11 & 187 & $0.000 e+00$ & $1.390 \mathrm{e}+04$ & 11 & 191 & $4.010 e-04$ & $4.874 e+07$ & 11 & 214 & $8.693 e-04$ & $1.412 \mathrm{e}+08$ \\
\hline 11 & 215 & $4.468 e-02$ & $1.212 \mathrm{e}+10$ & 11 & 216 & $8.285 e-03$ & $1.349 e+09$ & 11 & 218 & $8.614 \mathrm{e}-02$ & $1.406 e+10$ \\
\hline 11 & 221 & $1.246 \mathrm{e}-03$ & $2.048 e+08$ & 11 & 222 & $1.626 \mathrm{e}-02$ & $4.455 e+09$ & 11 & 223 & $1.969 \mathrm{e}-02$ & $1.618 \mathrm{e}+10$ \\
\hline 11 & 227 & $0.000 e+00$ & $2.054 \mathrm{e}+06$ & 11 & 228 & $0.000 e+00$ & $2.132 \mathrm{e}+06$ & 11 & 229 & $0.000 e+00$ & $2.067 e+05$ \\
\hline 11 & 230 & $0.000 e+00$ & $4.893 e+06$ & 11 & 233 & $0.000 e+00$ & $3.692 \mathrm{e}+04$ & 11 & 234 & $0.000 e+00$ & $1.086 e+06$ \\
\hline 11 & 235 & $0.000 e+00$ & $4.239 e+06$ & 11 & 236 & $0.000 e+00$ & $1.463 e+06$ & 11 & 238 & $0.000 e+00$ & $6.516 e+04$ \\
\hline 11 & 240 & $0.000 e+00$ & $3.792 \mathrm{e}+06$ & 11 & 241 & $0.000 e+00$ & $6.905 e+05$ & 11 & 258 & $0.000 e+00$ & $6.507 e+03$ \\
\hline
\end{tabular}


Table III. Ca IX Oscillator Strengths and Radiative Decay Rates for transitions involving levels higher than 4.

\begin{tabular}{|c|c|c|c|c|c|c|c|c|c|c|c|}
\hline $\begin{array}{l}\text { Low. } \\
\text { Lev. }\end{array}$ & $\begin{array}{l}\text { Upp } \\
\text { Lev }\end{array}$ & $\begin{array}{l}\text { Osc. } \\
\text { Str. }\end{array}$ & $\begin{array}{l}\text { Rad. } \\
\text { Rate }\end{array}$ & $\begin{array}{l}\text { Low. } \\
\text { Lev. }\end{array}$ & $\begin{array}{l}\text { - Upp } \\
\text {. Lev }\end{array}$ & $\begin{array}{l}\text { Osc. } \\
\text { Str. }\end{array}$ & $\begin{array}{l}\text { Rad. } \\
\text { Rate }\end{array}$ & $\begin{array}{l}\text { Low. } \\
\text { Lev. }\end{array}$ & $\begin{array}{l}\text { Upp } \\
\text { Lev }\end{array}$ & $\begin{array}{l}\text { Osc. } \\
\text { Str. }\end{array}$ & $\begin{array}{l}\text { Rad. } \\
\text { Rate }\end{array}$ \\
\hline$i$ & $j$ & $g f$ & $(1 / s)$ & $i$ & $j$ & $g f$ & $(1 / s)$ & $i$ & j & $g f$ & $(1 / s)$ \\
\hline 11 & 273 & $3.532 \mathrm{e}-05$ & $1.072 \mathrm{e}+07$ & 11 & 274 & $3.830 e-05$ & $3.489 e+07$ & 11 & 278 & $0.000 e+00$ & $6.747 e+03$ \\
\hline 11 & 279 & $0.000 e+00$ & $7.109 e+03$ & 12 & 15 & $1.170 \mathrm{e}-01$ & $3.516 e+08$ & 12 & 16 & $8.433 e-01$ & $1.859 e+09$ \\
\hline 12 & 18 & $4.062 \mathrm{e}-03$ & $1.362 \mathrm{e}+07$ & 12 & 20 & $5.988 e-01$ & $4.689 e+09$ & 12 & 22 & $1.508 e-02$ & $1.215 e+08$ \\
\hline 12 & 23 & $7.992 \mathrm{e}-01$ & $3.885 e+09$ & 12 & 24 & $1.801 \mathrm{e}-01$ & $6.259 e+08$ & 12 & 26 & $8.271 \mathrm{e}-04$ & $1.129 e+07$ \\
\hline 12 & 27 & $0.000 e+00$ & $2.096 \mathrm{e}+05$ & 12 & 30 & $2.492 \mathrm{e}-01$ & $9.635 e+09$ & 12 & 31 & $0.000 e+00$ & $2.911 \mathrm{e}+05$ \\
\hline 12 & 32 & $0.000 e+00$ & $3.653 e+05$ & 12 & 33 & $9.083 e-02$ & $2.121 e+09$ & 12 & 34 & $0.000 e+00$ & $1.222 \mathrm{e}+05$ \\
\hline 12 & 35 & $2.721 \mathrm{e}-02$ & $1.064 \mathrm{e}+09$ & 12 & 38 & $0.000 e+00$ & $7.832 e+04$ & 12 & 39 & $0.000 e+00$ & $9.409 e+05$ \\
\hline 12 & 40 & $0.000 e+00$ & $3.295 e+05$ & 12 & 42 & $0.000 e+00$ & $5.405 e+05$ & 12 & 43 & $0.000 e+00$ & $2.316 e+05$ \\
\hline 12 & 44 & $0.000 e+00$ & $2.663 e+05$ & 12 & 47 & $4.545 e-03$ & $2.838 e+08$ & 12 & 48 & $1.528 \mathrm{e}-03$ & $5.802 e+07$ \\
\hline 12 & 49 & $4.987 e-01$ & $1.953 e+10$ & 12 & 50 & $3.994 e+00$ & $1.117 e+11$ & 12 & 52 & $1.532 \mathrm{e}-04$ & $1.006 e+07$ \\
\hline 12 & 54 & $0.000 e+00$ & $5.902 e+03$ & 12 & 55 & $0.000 e+00$ & $2.993 e+04$ & 12 & 56 & $0.000 e+00$ & $1.928 e+04$ \\
\hline 12 & 57 & $0.000 e+00$ & $1.760 e+04$ & 12 & 58 & $0.000 e+00$ & $5.593 e+04$ & 12 & 59 & $0.000 e+00$ & $1.358 e+04$ \\
\hline 12 & 61 & $0.000 e+00$ & $1.659 e+04$ & 12 & 64 & $0.000 e+00$ & $2.463 e+05$ & 12 & 66 & $3.811 \mathrm{e}-03$ & $3.896 e+08$ \\
\hline 12 & 67 & $5.910 e-04$ & $3.626 \mathrm{e}+07$ & 12 & 68 & $1.075 e-04$ & $6.613 e+06$ & 12 & 69 & $9.582 \mathrm{e}-03$ & $4.214 e+08$ \\
\hline 12 & 70 & $3.291 \mathrm{e}-02$ & $3.413 e+09$ & 12 & 72 & $1.811 \mathrm{e}-02$ & $1.128 e+09$ & 12 & 73 & $1.076 \mathrm{e}-02$ & $1.119 \mathrm{e}+09$ \\
\hline 12 & 74 & $8.819 e-03$ & $5.515 e+08$ & 12 & 75 & $3.072 \mathrm{e}-02$ & $1.374 \mathrm{e}+09$ & 12 & 77 & $1.711 \mathrm{e}-02$ & $7.722 \mathrm{e}+08$ \\
\hline 12 & 78 & $3.517 e-03$ & $2.281 \mathrm{e}+08$ & 12 & 79 & $1.078 e-02$ & $1.168 \mathrm{e}+09$ & 12 & 81 & $1.065 e-04$ & $1.173 e+07$ \\
\hline 12 & 82 & $0.000 e+00$ & $7.058 e+04$ & 12 & 83 & $0.000 e+00$ & $3.935 e+05$ & 12 & 84 & $0.000 e+00$ & $1.940 e+04$ \\
\hline 12 & 87 & $0.000 e+00$ & $2.096 \mathrm{e}+04$ & 12 & 88 & $0.000 e+00$ & $4.775 e+04$ & 12 & 89 & $0.000 e+00$ & $1.127 e+05$ \\
\hline 12 & 90 & $0.000 e+00$ & $1.214 \mathrm{e}+05$ & 12 & 91 & $0.000 e+00$ & $3.229 e+05$ & 12 & 94 & $0.000 e+00$ & $2.301 \mathrm{e}+05$ \\
\hline 12 & 95 & $0.000 e+00$ & $1.819 \mathrm{e}+05$ & 12 & 96 & $0.000 e+00$ & $3.881 e+05$ & 12 & 98 & $1.153 \mathrm{e}-01$ & $8.554 e+09$ \\
\hline 12 & 99 & $9.235 e-01$ & $4.893 e+10$ & 12 & 102 & $0.000 e+00$ & $1.239 \mathrm{e}+06$ & 12 & 103 & $0.000 e+00$ & $4.768 e+06$ \\
\hline 12 & 105 & $0.000 e+00$ & $7.478 e+03$ & 12 & 111 & $1.349 \mathrm{e}-02$ & $2.066 e+09$ & 12 & 112 & $1.652 \mathrm{e}-03$ & $1.530 \mathrm{e}+08$ \\
\hline 12 & 113 & $1.374 \mathrm{e}-03$ & $1.274 \mathrm{e}+08$ & 12 & 114 & $1.774 \mathrm{e}-03$ & $2.751 e+08$ & 12 & 115 & $3.912 \mathrm{e}-02$ & $6.095 e+09$ \\
\hline 12 & 116 & $1.795 \mathrm{e}-01$ & $1.679 e+10$ & 12 & 117 & $5.089 e-03$ & $3.403 e+08$ & 12 & 118 & $8.572 \mathrm{e}-03$ & $8.054 e+08$ \\
\hline 12 & 119 & $2.671 \mathrm{e}-01$ & $1.795 e+10$ & 12 & 122 & $7.364 \mathrm{e}-02$ & $1.182 \mathrm{e}+10$ & 12 & 123 & $2.812 \mathrm{e}-02$ & $2.711 e+09$ \\
\hline 12 & 125 & $0.000 e+00$ & $1.238 \mathrm{e}+04$ & 12 & 126 & $0.000 e+00$ & $6.335 e+03$ & 12 & 127 & $1.987 e-04$ & $3.286 e+07$ \\
\hline 12 & 128 & $0.000 \mathrm{e}+00$ & $6.036 \mathrm{e}+04$ & 12 & 129 & $0.000 e+00$ & $4.989 e+05$ & 12 & 130 & $0.000 e+00$ & $4.771 e+04$ \\
\hline 12 & 131 & $0.000 e+00$ & $2.149 \mathrm{e}+04$ & 12 & 132 & $0.000 e+00$ & $1.338 e+05$ & 12 & 133 & $0.000 e+00$ & $1.838 e+04$ \\
\hline 12 & 134 & $0.000 e+00$ & $9.949 e+03$ & 12 & 135 & $0.000 e+00$ & $1.930 \mathrm{e}+04$ & 12 & 136 & $3.429 \mathrm{e}-05$ & $3.730 e+06$ \\
\hline 12 & 137 & $5.730 e-04$ & $6.247 e+07$ & 12 & 139 & $1.561 \mathrm{e}-03$ & $1.217 e+08$ & 12 & 140 & $1.637 \mathrm{e}-04$ & $1.798 e+07$ \\
\hline 12 & 141 & $2.681 \mathrm{e}-03$ & $2.107 e+08$ & 12 & 143 & $3.627 e-04$ & $4.017 e+07$ & 12 & 144 & $0.000 e+00$ & $6.168 e+03$ \\
\hline 12 & 145 & $1.224 \mathrm{e}-03$ & $2.263 e+08$ & 12 & 147 & $2.413 e-04$ & $1.923 e+07$ & 12 & 148 & $0.000 e+00$ & $3.390 e+06$ \\
\hline 12 & 149 & $0.000 e+00$ & $1.463 e+06$ & 12 & 150 & $0.000 e+00$ & $1.605 e+06$ & 12 & 151 & $0.000 e+00$ & $9.787 e+05$ \\
\hline 12 & 152 & $0.000 e+00$ & $4.329 \mathrm{e}+06$ & 12 & 154 & $0.000 e+00$ & $9.642 e+03$ & 12 & 155 & $0.000 e+00$ & $1.239 \mathrm{e}+05$ \\
\hline 12 & 158 & $0.000 e+00$ & $2.820 \mathrm{e}+05$ & 12 & 159 & $0.000 e+00$ & $1.812 \mathrm{e}+04$ & 12 & 161 & $0.000 e+00$ & $3.427 e+05$ \\
\hline 12 & 165 & $0.000 e+00$ & $2.495 \mathrm{e}+05$ & 12 & 171 & $0.000 e+00$ & $2.088 e+06$ & 12 & 173 & $0.000 e+00$ & $1.217 e+05$ \\
\hline 12 & 174 & $0.000 e+00$ & $6.826 e+04$ & 12 & 176 & $2.986 e-04$ & $2.458 e+07$ & 12 & 177 & $6.496 \mathrm{e}-05$ & $7.512 \mathrm{e}+06$ \\
\hline 12 & 178 & $2.159 \mathrm{e}-04$ & $1.785 e+07$ & 12 & 179 & $0.000 e+00$ & $1.232 \mathrm{e}+04$ & 12 & 180 & $0.000 e+00$ & $2.275 e+06$ \\
\hline 12 & 181 & $0.000 e+00$ & $3.133 e+06$ & 12 & 182 & $0.000 e+00$ & $9.879 e+05$ & 12 & 183 & $0.000 e+00$ & $1.631 \mathrm{e}+05$ \\
\hline 12 & 184 & $0.000 e+00$ & $4.386 e+05$ & 12 & 185 & $0.000 e+00$ & $5.322 \mathrm{e}+06$ & 12 & 186 & $0.000 e+00$ & $1.778 e+06$ \\
\hline 12 & 187 & $0.000 e+00$ & $8.057 e+04$ & 12 & 191 & $7.187 e-05$ & $8.734 e+06$ & 12 & 193 & $5.922 \mathrm{e}-04$ & $5.142 \mathrm{e}+07$ \\
\hline 12 & 214 & $7.659 \mathrm{e}-04$ & $1.244 \mathrm{e}+08$ & 12 & 215 & $1.745 e-02$ & $4.734 e+09$ & 12 & 216 & $7.469 \mathrm{e}-02$ & $1.216 \mathrm{e}+10$ \\
\hline 12 & 217 & $6.140 e-03$ & $7.145 e+08$ & 12 & 218 & $8.503 e-03$ & $1.388 e+09$ & 12 & 219 & $1.274 \mathrm{e}-01$ & $1.486 e+10$ \\
\hline
\end{tabular}


Table III. Ca IX Oscillator Strengths and Radiative Decay Rates for transitions involving levels higher than 4.

\begin{tabular}{|c|c|c|c|c|c|c|c|c|c|c|c|}
\hline $\begin{array}{l}\text { Low. } \\
\text { Lev. }\end{array}$ & $\begin{array}{l}\text { Upp } \\
\text { Lev }\end{array}$ & $\begin{array}{l}\text { Osc. } \\
\text { Str. }\end{array}$ & $\begin{array}{l}\text { Rad. } \\
\text { Rate }\end{array}$ & $\begin{array}{l}\text { Low. } \\
\text { Lev. }\end{array}$ & $\begin{array}{l}\text { - Upp } \\
\text {. Lev }\end{array}$ & $\begin{array}{l}\text { Osc. } \\
\text { Str. }\end{array}$ & $\begin{array}{l}\text { Rad. } \\
\text { Rate }\end{array}$ & $\begin{array}{l}\text { Low. } \\
\text { Lev. }\end{array}$ & $\begin{array}{l}\text { Upp } \\
\text { Lev }\end{array}$ & $\begin{array}{l}\text { Osc. } \\
\text { Str. }\end{array}$ & $\begin{array}{l}\text { Rad. } \\
\text { Rate }\end{array}$ \\
\hline$i$ & $j$ & $g f$ & $(1 / s)$ & $i$ & $j$ & $g f$ & $(1 / s)$ & $i$ & j & $g f$ & $(1 / s)$ \\
\hline 12 & 221 & $1.734 \mathrm{e}-02$ & $2.850 e+09$ & 12 & 222 & $4.300 e-02$ & $1.178 \mathrm{e}+10$ & 12 & 225 & $1.685 \mathrm{e}-04$ & $4.667 e+07$ \\
\hline 12 & 226 & $0.000 e+00$ & $2.107 e+04$ & 12 & 227 & $0.000 e+00$ & $3.352 \mathrm{e}+06$ & 12 & 228 & $0.000 e+00$ & $1.446 e+06$ \\
\hline 12 & 229 & $0.000 e+00$ & $1.684 \mathrm{e}+06$ & 12 & 230 & $0.000 e+00$ & $1.335 e+06$ & 12 & 231 & $0.000 e+00$ & $5.307 e+06$ \\
\hline 12 & 233 & $0.000 e+00$ & $1.965 \mathrm{e}+04$ & 12 & 234 & $0.000 e+00$ & $1.768 e+06$ & 12 & 235 & $0.000 e+00$ & $3.120 \mathrm{e}+06$ \\
\hline 12 & 236 & $0.000 e+00$ & $3.818 e+06$ & 12 & 237 & $0.000 e+00$ & $1.214 \mathrm{e}+06$ & 12 & 238 & $0.000 e+00$ & $1.815 e+05$ \\
\hline 12 & 239 & $0.000 e+00$ & $8.654 \mathrm{e}+06$ & 12 & 240 & $0.000 e+00$ & $7.024 e+05$ & 12 & 241 & $0.000 e+00$ & $2.810 \mathrm{e}+06$ \\
\hline 12 & 259 & $0.000 e+00$ & $6.572 \mathrm{e}+03$ & 12 & 273 & $8.000 e-05$ & $2.428 e+07$ & 12 & 277 & $0.000 e+00$ & $5.772 e+03$ \\
\hline 12 & 278 & $0.000 e+00$ & $5.319 \mathrm{e}+03$ & 12 & 279 & $0.000 e+00$ & $1.177 e+04$ & 13 & 15 & $3.311 \mathrm{e}-03$ & $9.932 e+06$ \\
\hline 13 & 16 & $1.330 e-01$ & $2.925 e+08$ & 13 & 17 & $1.276 \mathrm{e}+00$ & $2.253 e+09$ & 13 & 18 & $4.505 e-03$ & $1.507 e+07$ \\
\hline 13 & 19 & $1.013 e+00$ & $4.695 e+09$ & 13 & 23 & $4.736 e-03$ & $2.298 e+07$ & 13 & 24 & $1.143 e+00$ & $3.966 e+09$ \\
\hline 13 & 25 & $1.686 \mathrm{e}-03$ & $9.066 e+06$ & 13 & 27 & $0.000 e+00$ & $2.930 e+05$ & 13 & 31 & $0.000 e+00$ & $2.934 e+04$ \\
\hline 13 & 32 & $0.000 e+00$ & $2.187 e+05$ & 13 & 33 & $5.103 e-01$ & $1.190 e+10$ & 13 & 34 & $0.000 e+00$ & $6.096 e+05$ \\
\hline 13 & 37 & $0.000 e+00$ & $2.769 \mathrm{e}+03$ & 13 & 38 & $0.000 e+00$ & $4.382 e+05$ & 13 & 40 & $0.000 e+00$ & $5.235 e+05$ \\
\hline 13 & 42 & $0.000 e+00$ & $6.187 e+04$ & 13 & 43 & $0.000 e+00$ & $3.719 e+05$ & 13 & 44 & $0.000 e+00$ & $6.379 e+05$ \\
\hline 13 & 48 & $8.601 \mathrm{e}-03$ & $3.264 \mathrm{e}+08$ & 13 & 49 & $1.424 \mathrm{e}-02$ & $5.573 e+08$ & 13 & 50 & $4.992 \mathrm{e}-01$ & $1.395 e+10$ \\
\hline 13 & 51 & $5.780 e+00$ & $1.257 \mathrm{e}+11$ & 13 & 56 & $0.000 e+00$ & $2.045 e+04$ & 13 & 57 & $0.000 e+00$ & $4.174 \mathrm{e}+04$ \\
\hline 13 & 59 & $0.000 e+00$ & $8.269 e+03$ & 13 & 60 & $0.000 e+00$ & $2.853 e+04$ & 13 & 61 & $0.000 e+00$ & $3.694 \mathrm{e}+04$ \\
\hline 13 & 64 & $0.000 e+00$ & $3.435 e+05$ & 13 & 67 & $7.598 e-03$ & $4.659 e+08$ & 13 & 68 & $3.591 \mathrm{e}-04$ & $2.208 e+07$ \\
\hline 13 & 69 & $3.985 e-03$ & $1.752 \mathrm{e}+08$ & 13 & 72 & $7.432 \mathrm{e}-02$ & $4.626 e+09$ & 13 & 74 & $6.341 e-04$ & $3.963 e+07$ \\
\hline 13 & 75 & $1.026 \mathrm{e}-02$ & $4.586 e+08$ & 13 & 76 & $8.101 \mathrm{e}-02$ & $2.838 e+09$ & 13 & 77 & $1.796 \mathrm{e}-03$ & $8.100 e+07$ \\
\hline 13 & 78 & $2.486 \mathrm{e}-02$ & $1.612 \mathrm{e}+09$ & 13 & 83 & $0.000 e+00$ & $7.535 e+04$ & 13 & 85 & $0.000 e+00$ & $5.854 e+05$ \\
\hline 13 & 89 & $0.000 e+00$ & $2.829 \mathrm{e}+05$ & 13 & 90 & $0.000 e+00$ & $1.934 \mathrm{e}+05$ & 13 & 91 & $0.000 \mathrm{e}+00$ & $3.687 e+04$ \\
\hline 13 & 94 & $0.000 e+00$ & $5.474 e+05$ & 13 & 95 & $0.000 e+00$ & $2.870 e+05$ & 13 & 96 & $0.000 e+00$ & $4.422 \mathrm{e}+04$ \\
\hline 13 & 98 & $3.286 \mathrm{e}-03$ & $2.436 \mathrm{e}+08$ & 13 & 99 & $1.152 \mathrm{e}-01$ & $6.103 e+09$ & 13 & 100 & $1.334 \mathrm{e}+00$ & $5.494 e+10$ \\
\hline 13 & 102 & $0.000 e+00$ & $8.257 e+04$ & 13 & 103 & $0.000 e+00$ & $9.549 e+05$ & 13 & 104 & $0.000 e+00$ & $5.687 e+06$ \\
\hline 13 & 112 & $1.135 \mathrm{e}-02$ & $1.050 \mathrm{e}+09$ & 13 & 113 & $3.447 e-02$ & $3.197 e+09$ & 13 & 116 & $3.736 e-02$ & $3.493 e+09$ \\
\hline 13 & 117 & $3.110 \mathrm{e}-01$ & $2.079 e+10$ & 13 & 118 & $8.323 e-05$ & $7.816 e+06$ & 13 & 119 & $2.941 \mathrm{e}-03$ & $1.976 \mathrm{e}+08$ \\
\hline 13 & 120 & $3.390 e-01$ & $1.775 \mathrm{e}+10$ & 13 & 123 & $1.312 \mathrm{e}-01$ & $1.264 \mathrm{e}+10$ & 13 & 124 & $3.744 \mathrm{e}-04$ & $2.595 e+07$ \\
\hline 13 & 125 & $0.000 e+00$ & $8.072 \mathrm{e}+03$ & 13 & 126 & $0.000 e+00$ & $3.235 e+04$ & 13 & 130 & $0.000 e+00$ & $2.055 e+05$ \\
\hline 13 & 131 & $0.000 e+00$ & $5.067 e+04$ & 13 & 132 & $0.000 e+00$ & $3.257 e+05$ & 13 & 133 & $0.000 e+00$ & $1.565 e+04$ \\
\hline 13 & 134 & $0.000 e+00$ & $5.160 e+03$ & 13 & 136 & $1.195 \mathrm{e}-04$ & $1.300 \mathrm{e}+07$ & 13 & 137 & $4.989 \mathrm{e}-05$ & $5.436 e+06$ \\
\hline 13 & 139 & $4.955 e-04$ & $3.863 e+07$ & 13 & 140 & $2.614 \mathrm{e}-05$ & $2.871 \mathrm{e}+06$ & 13 & 141 & $1.794 \mathrm{e}-04$ & $1.410 \mathrm{e}+07$ \\
\hline 13 & 142 & $6.471 \mathrm{e}-03$ & $3.964 \mathrm{e}+08$ & 13 & 143 & $2.148 \mathrm{e}-03$ & $2.378 e+08$ & 13 & 148 & $0.000 e+00$ & $3.885 e+05$ \\
\hline 13 & 149 & $0.000 e+00$ & $2.323 e+06$ & 13 & 150 & $0.000 e+00$ & $3.997 e+06$ & 13 & 151 & $0.000 e+00$ & $3.042 e+04$ \\
\hline 13 & 152 & $0.000 e+00$ & $8.011 \mathrm{e}+05$ & 13 & 153 & $0.000 e+00$ & $5.593 e+06$ & 13 & 154 & $0.000 e+00$ & $5.599 e+03$ \\
\hline 13 & 155 & $0.000 e+00$ & $3.304 e+04$ & 13 & 158 & $0.000 e+00$ & $9.733 e+03$ & 13 & 161 & $0.000 e+00$ & $2.477 e+05$ \\
\hline 13 & 162 & $1.163 e-04$ & $7.330 e+06$ & 13 & 165 & $0.000 e+00$ & $1.476 e+04$ & 13 & 169 & $0.000 e+00$ & $2.434 e+05$ \\
\hline 13 & 171 & $0.000 e+00$ & $2.842 \mathrm{e}+06$ & 13 & 172 & $0.000 e+00$ & $1.716 e+05$ & 13 & 173 & $0.000 e+00$ & $7.197 e+04$ \\
\hline 13 & 174 & $0.000 e+00$ & $7.985 e+03$ & 13 & 175 & $7.002 \mathrm{e}-04$ & $4.479 e+07$ & 13 & 179 & $0.000 e+00$ & $1.245 e+05$ \\
\hline 13 & 180 & $0.000 e+00$ & $2.164 \mathrm{e}+05$ & 13 & 181 & $0.000 e+00$ & $1.826 \mathrm{e}+06$ & 13 & 182 & $0.000 e+00$ & $5.079 e+06$ \\
\hline 13 & 183 & $0.000 e+00$ & $2.153 e+04$ & 13 & 184 & $0.000 e+00$ & $2.519 e+06$ & 13 & 186 & $0.000 e+00$ & $2.882 \mathrm{e}+06$ \\
\hline 13 & 187 & $0.000 e+00$ & $1.037 e+05$ & 13 & 190 & $4.860 e-05$ & $3.279 e+06$ & 13 & 192 & $7.905 e-04$ & $5.336 e+07$ \\
\hline 13 & 214 & $7.411 \mathrm{e}-05$ & $1.203 e+07$ & 13 & 216 & $2.028 \mathrm{e}-02$ & $3.301 e+09$ & 13 & 217 & $1.375 e-01$ & $1.599 \mathrm{e}+10$ \\
\hline 13 & 218 & $6.511 \mathrm{e}-05$ & $1.062 \mathrm{e}+07$ & 13 & 219 & $5.669 e-03$ & $6.611 e+08$ & 13 & 220 & $1.686 \mathrm{e}-01$ & $1.530 \mathrm{e}+10$ \\
\hline
\end{tabular}


Table III. Ca IX Oscillator Strengths and Radiative Decay Rates for transitions involving levels higher than 4.

\begin{tabular}{|c|c|c|c|c|c|c|c|c|c|c|c|}
\hline $\begin{array}{l}\text { Low. } \\
\text { Lev. }\end{array}$ & $\begin{array}{l}\text { Upp } \\
\text { Lev }\end{array}$ & $\begin{array}{l}\text { Osc. } \\
\text { Str. }\end{array}$ & $\begin{array}{l}\text { Rad. } \\
\text { Rate }\end{array}$ & $\begin{array}{l}\text { Low. } \\
\text { Lev. }\end{array}$ & $\begin{array}{l}\text { Upp. } \\
\text { Lev }\end{array}$ & $\begin{array}{l}\text { Osc. } \\
\text { Str. }\end{array}$ & $\begin{array}{l}\text { Rad. } \\
\text { Rate }\end{array}$ & $\begin{array}{l}\text { Low. } \\
\text { Lev. }\end{array}$ & $\begin{array}{l}\text { Upp } \\
\text { Lev }\end{array}$ & $\begin{array}{l}\text { Osc. } \\
\text { Str. }\end{array}$ & $\begin{array}{l}\text { Rad. } \\
\text { Rate }\end{array}$ \\
\hline$i$ & $j$ & $g f$ & $(1 / s)$ & $i$ & $j$ & $g f$ & $(1 / s)$ & $i$ & j & $g f$ & $(1 / s)$ \\
\hline 13 & 221 & $8.117 e-02$ & $1.334 \mathrm{e}+10$ & 13 & 224 & $1.461 \mathrm{e}-04$ & $1.723 e+07$ & 13 & 226 & $0.000 e+00$ & $9.379 e+03$ \\
\hline 13 & 227 & $0.000 e+00$ & $3.688 e+05$ & 13 & 228 & $0.000 e+00$ & $2.257 e+06$ & 13 & 229 & $0.000 e+00$ & $3.916 e+06$ \\
\hline 13 & 230 & $0.000 e+00$ & $6.656 \mathrm{e}+04$ & 13 & 231 & $0.000 e+00$ & $9.863 e+05$ & 13 & 232 & $0.000 e+00$ & $6.284 e+06$ \\
\hline 13 & 233 & $0.000 e+00$ & $1.191 \mathrm{e}+04$ & 13 & 234 & $0.000 e+00$ & $2.301 e+06$ & 13 & 235 & $0.000 e+00$ & $3.310 e+05$ \\
\hline 13 & 236 & $0.000 e+00$ & $2.398 e+06$ & 13 & 237 & $0.000 e+00$ & $6.463 e+06$ & 13 & 238 & $0.000 e+00$ & $3.613 e+05$ \\
\hline 13 & 240 & $0.000 e+00$ & $4.150 e+06$ & 13 & 241 & $0.000 e+00$ & $4.527 e+06$ & 13 & 260 & $0.000 e+00$ & $7.331 \mathrm{e}+03$ \\
\hline 13 & 264 & $9.936 \mathrm{e}-05$ & $1.290 \mathrm{e}+07$ & 13 & 272 & $1.455 \mathrm{e}-04$ & $2.649 e+07$ & 13 & 277 & $0.000 e+00$ & $1.422 \mathrm{e}+04$ \\
\hline 13 & 278 & $0.000 e+00$ & $8.334 e+03$ & 14 & 15 & $6.448 e-03$ & $6.938 e+06$ & 14 & 16 & $5.791 \mathrm{e}-04$ & $4.648 e+05$ \\
\hline 14 & 18 & $1.878 \mathrm{e}-01$ & $2.417 e+08$ & 14 & 20 & $5.409 e-04$ & $1.950 e+06$ & 14 & 25 & $2.567 e+00$ & $7.699 e+09$ \\
\hline 14 & 26 & $8.071 \mathrm{e}-01$ & $6.308 e+09$ & 14 & 28 & $0.000 e+00$ & $2.524 e+05$ & 14 & 30 & $1.453 \mathrm{e}-02$ & $4.111 \mathrm{e}+08$ \\
\hline 14 & 35 & $1.207 e-01$ & $3.462 \mathrm{e}+09$ & 14 & 36 & $0.000 e+00$ & $2.338 e+05$ & 14 & 37 & $0.000 e+00$ & $5.098 e+05$ \\
\hline 14 & 40 & $0.000 e+00$ & $2.859 \mathrm{e}+03$ & 14 & 41 & $0.000 e+00$ & $8.240 e+05$ & 14 & 45 & $0.000 e+00$ & $2.651 e+05$ \\
\hline 14 & 47 & $6.386 e-03$ & $3.132 \mathrm{e}+08$ & 14 & 52 & $2.299 \mathrm{e}-01$ & $1.193 e+10$ & 14 & 53 & $3.941 \mathrm{e}+00$ & $9.092 e+10$ \\
\hline 14 & 54 & $0.000 e+00$ & $5.970 e+05$ & 14 & 55 & $0.000 e+00$ & $7.550 e+04$ & 14 & 59 & $0.000 e+00$ & $1.135 e+04$ \\
\hline 14 & 61 & $0.000 e+00$ & $5.546 e+03$ & 14 & 62 & $0.000 e+00$ & $2.988 e+05$ & 14 & 63 & $0.000 e+00$ & $2.393 e+05$ \\
\hline 14 & 65 & $0.000 e+00$ & $5.838 e+05$ & 14 & 66 & $1.410 \mathrm{e}-03$ & $1.197 e+08$ & 14 & 67 & $3.721 \mathrm{e}-02$ & $1.895 e+09$ \\
\hline 14 & 68 & $3.815 e-02$ & $1.949 \mathrm{e}+09$ & 14 & 69 & $2.015 e-02$ & $7.357 e+08$ & 14 & 70 & $7.332 \mathrm{e}-03$ & $6.319 e+08$ \\
\hline 14 & 72 & $2.929 e-03$ & $1.516 \mathrm{e}+08$ & 14 & 73 & $3.119 \mathrm{e}-02$ & $2.696 e+09$ & 14 & 74 & $7.648 e-03$ & $3.978 e+08$ \\
\hline 14 & 75 & $1.042 \mathrm{e}-01$ & $3.874 \mathrm{e}+09$ & 14 & 77 & $3.023 e-01$ & $1.136 e+10$ & 14 & 78 & $5.890 e-04$ & $3.188 e+07$ \\
\hline 14 & 79 & $2.335 e-04$ & $2.111 e+07$ & 14 & 81 & $7.240 e-03$ & $6.667 e+08$ & 14 & 82 & $0.000 e+00$ & $4.570 e+05$ \\
\hline 14 & 83 & $0.000 e+00$ & $1.251 \mathrm{e}+04$ & 14 & 84 & $0.000 e+00$ & $9.864 e+05$ & 14 & 87 & $0.000 e+00$ & $7.602 e+04$ \\
\hline 14 & 89 & $0.000 e+00$ & $1.945 \mathrm{e}+04$ & 14 & 92 & $0.000 e+00$ & $1.883 e+05$ & 14 & 93 & $0.000 \mathrm{e}+00$ & $2.761 \mathrm{e}+05$ \\
\hline 14 & 97 & $0.000 e+00$ & $7.409 e+05$ & 14 & 101 & $9.662 \mathrm{e}-01$ & $4.377 e+10$ & 14 & 103 & $0.000 e+00$ & $1.371 \mathrm{e}+04$ \\
\hline 14 & 105 & $0.000 e+00$ & $8.667 e+06$ & 14 & 111 & $1.720 \mathrm{e}-03$ & $2.266 \mathrm{e}+08$ & 14 & 112 & $2.051 \mathrm{e}-01$ & $1.635 \mathrm{e}+10$ \\
\hline 14 & 113 & $4.928 \mathrm{e}-02$ & $3.935 e+09$ & 14 & 114 & $4.694 \mathrm{e}-03$ & $6.267 e+08$ & 14 & 115 & $5.663 e-04$ & $7.597 e+07$ \\
\hline 14 & 117 & $4.470 \mathrm{e}-04$ & $2.574 \mathrm{e}+07$ & 14 & 118 & $2.389 e-03$ & $1.933 e+08$ & 14 & 119 & $2.333 e-04$ & $1.351 \mathrm{e}+07$ \\
\hline 14 & 122 & $3.608 e-04$ & $5.000 e+07$ & 14 & 123 & $1.246 \mathrm{e}-04$ & $1.037 e+07$ & 14 & 124 & $1.774 \mathrm{e}-01$ & $1.062 \mathrm{e}+10$ \\
\hline 14 & 125 & $0.000 \mathrm{e}+00$ & $1.054 \mathrm{e}+06$ & 14 & 126 & $0.000 e+00$ & $3.525 e+05$ & 14 & 127 & $1.490 \mathrm{e}-01$ & $2.132 \mathrm{e}+10$ \\
\hline 14 & 130 & $0.000 e+00$ & $2.418 \mathrm{e}+05$ & 14 & 133 & $0.000 e+00$ & $2.603 e+04$ & 14 & 134 & $0.000 e+00$ & $3.516 e+04$ \\
\hline 14 & 135 & $0.000 e+00$ & $1.185 \mathrm{e}+05$ & 14 & 136 & $3.581 \mathrm{e}-02$ & $3.392 e+09$ & 14 & 137 & $9.337 e-04$ & $8.865 e+07$ \\
\hline 14 & 138 & $9.645 e-05$ & $1.528 \mathrm{e}+07$ & 14 & 139 & $1.702 \mathrm{e}-02$ & $1.156 \mathrm{e}+09$ & 14 & 140 & $1.196 \mathrm{e}-02$ & $1.146 \mathrm{e}+09$ \\
\hline 14 & 141 & $2.859 \mathrm{e}-03$ & $1.959 \mathrm{e}+08$ & 14 & 143 & $2.117 \mathrm{e}-03$ & $2.045 e+08$ & 14 & 144 & $0.000 e+00$ & $5.504 e+06$ \\
\hline 14 & 147 & $3.068 e-01$ & $2.133 e+10$ & 14 & 148 & $0.000 e+00$ & $1.856 e+04$ & 14 & 150 & $0.000 e+00$ & $9.120 \mathrm{e}+03$ \\
\hline 14 & 151 & $0.000 e+00$ & $1.067 e+04$ & 14 & 152 & $0.000 e+00$ & $1.189 e+04$ & 14 & 154 & $0.000 e+00$ & $4.111 \mathrm{e}+06$ \\
\hline 14 & 155 & $0.000 e+00$ & $4.937 e+05$ & 14 & 156 & $3.717 e-04$ & $6.104 e+07$ & 14 & 158 & $0.000 e+00$ & $2.875 e+04$ \\
\hline 14 & 161 & $0.000 e+00$ & $1.281 \mathrm{e}+05$ & 14 & 163 & $1.823 e-03$ & $1.291 \mathrm{e}+08$ & 14 & 164 & $0.000 e+00$ & $2.258 \mathrm{e}+05$ \\
\hline 14 & 165 & $0.000 e+00$ & $1.780 \mathrm{e}+04$ & 14 & 172 & $0.000 e+00$ & $5.239 e+04$ & 14 & 176 & $4.908 e-03$ & $3.533 e+08$ \\
\hline 14 & 178 & $6.929 e-03$ & $5.009 e+08$ & 14 & 179 & $0.000 e+00$ & $4.361 e+06$ & 14 & 180 & $0.000 e+00$ & $4.658 e+03$ \\
\hline 14 & 182 & $0.000 e+00$ & $9.982 e+04$ & 14 & 183 & $0.000 e+00$ & $1.474 \mathrm{e}+05$ & 14 & 186 & $0.000 e+00$ & $1.825 e+05$ \\
\hline 14 & 187 & $0.000 \mathrm{e}+00$ & $4.746 e+06$ & 14 & 188 & $0.000 e+00$ & $2.236 \mathrm{e}+06$ & 14 & 196 & $4.196 \mathrm{e}-03$ & $4.518 \mathrm{e}+08$ \\
\hline 14 & 206 & $6.368 \mathrm{e}-04$ & $5.080 e+07$ & 14 & 207 & $0.000 e+00$ & $2.590 e+06$ & 14 & 208 & $1.346 \mathrm{e}-04$ & $2.573 e+07$ \\
\hline 14 & 213 & $0.000 e+00$ & $2.059 \mathrm{e}+04$ & 14 & 214 & $1.030 \mathrm{e}-01$ & $1.495 e+10$ & 14 & 215 & $7.410 \mathrm{e}-05$ & $1.797 \mathrm{e}+07$ \\
\hline 14 & 216 & $1.652 \mathrm{e}-04$ & $2.405 e+07$ & 14 & 217 & $2.544 \mathrm{e}-04$ & $2.647 e+07$ & 14 & 218 & $1.174 \mathrm{e}-03$ & $1.713 e+08$ \\
\hline 14 & 219 & $7.221 \mathrm{e}-05$ & $7.530 e+06$ & 14 & 221 & $1.899 \mathrm{e}-04$ & $2.792 \mathrm{e}+07$ & 14 & 222 & $1.349 \mathrm{e}-04$ & $3.304 \mathrm{e}+07$ \\
\hline
\end{tabular}


Table III. Ca IX Oscillator Strengths and Radiative Decay Rates for transitions involving levels higher than 4.

\begin{tabular}{|c|c|c|c|c|c|c|c|c|c|c|c|}
\hline $\begin{array}{l}\text { Low. } \\
\text { Lev. }\end{array}$ & $\begin{array}{l}\text { Upp } \\
\text { Lev }\end{array}$ & $\begin{array}{l}\text { Osc. } \\
\text { Str. }\end{array}$ & $\begin{array}{l}\text { Rad. } \\
\text { Rate }\end{array}$ & $\begin{array}{l}\text { Low. } \\
\text { Lev. }\end{array}$ & $\begin{array}{l}\text { Upp } \\
\text { Lev }\end{array}$ & $\begin{array}{l}\text { Osc. } \\
\text { Str. }\end{array}$ & $\begin{array}{l}\text { Rad. } \\
\text { Rate }\end{array}$ & $\begin{array}{l}\text { Low. } \\
\text { Lev. }\end{array}$ & $\begin{array}{l}\text { Upp } \\
\text { Lev }\end{array}$ & $\begin{array}{l}\text { Osc. } \\
\text { Str. }\end{array}$ & $\begin{array}{l}\text { Rad. } \\
\text { Rate }\end{array}$ \\
\hline$i$ & $j$ & $g f$ & $(1 / s)$ & $i$ & $j$ & $g f$ & $(1 / s)$ & $i$ & $j$ & $g f$ & $(1 / s)$ \\
\hline 14 & 224 & $1.655 \mathrm{e}-01$ & $1.746 \mathrm{e}+10$ & 14 & 225 & $4.770 e-02$ & $1.182 \mathrm{e}+10$ & 14 & 226 & $0.000 e+00$ & $5.165 e+06$ \\
\hline 14 & 227 & $0.000 e+00$ & $2.636 \mathrm{e}+04$ & 14 & 229 & $0.000 e+00$ & $2.068 e+04$ & 14 & 230 & $0.000 e+00$ & $6.044 e+03$ \\
\hline 14 & 233 & $0.000 e+00$ & $2.534 \mathrm{e}+06$ & 14 & 238 & $0.000 e+00$ & $6.908 e+06$ & 14 & 241 & $0.000 e+00$ & $5.237 e+05$ \\
\hline 14 & 242 & $0.000 e+00$ & $5.688 e+06$ & 14 & 250 & $4.156 e-03$ & $6.738 e+08$ & 14 & 261 & $0.000 e+00$ & $1.947 e+04$ \\
\hline 14 & 265 & $0.000 e+00$ & $4.062 \mathrm{e}+03$ & 14 & 275 & $5.204 \mathrm{e}-03$ & $6.096 e+08$ & 14 & 281 & $0.000 e+00$ & $7.987 e+06$ \\
\hline 14 & 283 & $8.416 \mathrm{e}-04$ & $2.332 \mathrm{e}+08$ & 15 & 29 & $0.000 e+00$ & $4.049 e+03$ & 15 & 31 & $9.398 e-01$ & $8.995 e+09$ \\
\hline 15 & 32 & $8.874 e-02$ & $6.072 \mathrm{e}+08$ & 15 & 37 & $6.720 \mathrm{e}-02$ & $7.452 e+08$ & 15 & 38 & $1.637 e-04$ & $3.086 e+06$ \\
\hline 15 & 42 & $3.543 e-02$ & $9.859 e+08$ & 15 & 43 & $6.222 \mathrm{e}-03$ & $1.039 e+08$ & 15 & 45 & $5.547 e-04$ & $9.363 e+06$ \\
\hline 15 & 46 & $0.000 e+00$ & $4.711 e+05$ & 15 & 47 & $0.000 e+00$ & $1.637 e+05$ & 15 & 48 & $0.000 e+00$ & $1.133 e+04$ \\
\hline 15 & 54 & $8.613 e-02$ & $3.626 e+09$ & 15 & 55 & $2.664 \mathrm{e}-01$ & $1.152 e+10$ & 15 & 56 & $6.013 e-02$ & $1.570 e+09$ \\
\hline 15 & 57 & $1.510 e-03$ & $2.857 e+07$ & 15 & 59 & $8.525 e-04$ & $3.846 e+07$ & 15 & 60 & $9.663 e-05$ & $4.408 e+06$ \\
\hline 15 & 62 & $1.911 \mathrm{e}-03$ & $5.504 e+07$ & 15 & 66 & $0.000 e+00$ & $4.262 e+05$ & 15 & 67 & $0.000 e+00$ & $4.890 e+04$ \\
\hline 15 & 68 & $0.000 e+00$ & $4.034 e+05$ & 15 & 69 & $0.000 e+00$ & $6.936 e+04$ & 15 & 71 & $0.000 e+00$ & $8.168 e+04$ \\
\hline 15 & 73 & $0.000 e+00$ & $7.080 e+03$ & 15 & 74 & $0.000 e+00$ & $3.357 e+05$ & 15 & 75 & $0.000 e+00$ & $1.259 \mathrm{e}+05$ \\
\hline 15 & 76 & $0.000 e+00$ & $5.832 e+03$ & 15 & 77 & $0.000 e+00$ & $1.326 e+04$ & 15 & 80 & $0.000 e+00$ & $1.391 \mathrm{e}+04$ \\
\hline 15 & 82 & $3.236 e+00$ & $9.673 e+10$ & 15 & 84 & $4.868 e-01$ & $1.471 \mathrm{e}+10$ & 15 & 86 & $2.818 \mathrm{e}-01$ & $1.205 e+10$ \\
\hline 15 & 87 & $3.474 \mathrm{e}-02$ & $1.065 e+09$ & 15 & 91 & $2.034 \mathrm{e}-02$ & $1.489 e+09$ & 15 & 92 & $4.050 e-04$ & $1.795 e+07$ \\
\hline 15 & 96 & $8.391 e-04$ & $6.362 e+07$ & 15 & 97 & $2.882 \mathrm{e}-03$ & $1.349 e+08$ & 15 & 98 & $0.000 e+00$ & $1.857 e+04$ \\
\hline 15 & 102 & $3.418 \mathrm{e}-01$ & $1.187 e+10$ & 15 & 106 & $2.586 e-01$ & $2.251 \mathrm{e}+10$ & 15 & 107 & $5.726 e-02$ & $2.992 \mathrm{e}+09$ \\
\hline 15 & 108 & $1.641 \mathrm{e}-03$ & $6.128 e+07$ & 15 & 109 & $1.548 \mathrm{e}-02$ & $8.307 e+08$ & 15 & 110 & $0.000 e+00$ & $1.365 e+06$ \\
\hline 15 & 111 & $0.000 e+00$ & $4.094 e+05$ & 15 & 112 & $0.000 e+00$ & $4.655 e+04$ & 15 & 113 & $0.000 e+00$ & $6.596 \mathrm{e}+03$ \\
\hline 15 & 114 & $0.000 e+00$ & $1.012 \mathrm{e}+05$ & 15 & 115 & $0.000 e+00$ & $1.198 \mathrm{e}+06$ & 15 & 116 & $0.000 e+00$ & $3.779 \mathrm{e}+05$ \\
\hline 15 & 117 & $0.000 e+00$ & $1.460 \mathrm{e}+04$ & 15 & 118 & $0.000 e+00$ & $7.897 e+05$ & 15 & 119 & $0.000 \mathrm{e}+00$ & $2.324 \mathrm{e}+05$ \\
\hline 15 & 120 & $0.000 e+00$ & $7.778 e+03$ & 15 & 121 & $0.000 e+00$ & $1.998 e+06$ & 15 & 122 & $0.000 e+00$ & $7.387 e+05$ \\
\hline 15 & 123 & $0.000 e+00$ & $6.713 e+04$ & 15 & 124 & $0.000 e+00$ & $5.612 e+04$ & 15 & 125 & $3.021 \mathrm{e}-02$ & $3.380 e+09$ \\
\hline 15 & 126 & $2.420 \mathrm{e}-02$ & $2.728 e+09$ & 15 & 128 & $8.197 e-03$ & $5.555 e+08$ & 15 & 130 & $3.147 e-03$ & $3.585 e+08$ \\
\hline 15 & 131 & $2.179 \mathrm{e}-04$ & $1.065 \mathrm{e}+07$ & 15 & 132 & $7.447 e-04$ & $5.117 e+07$ & 15 & 133 & $4.310 e-05$ & $4.956 e+06$ \\
\hline 15 & 134 & $2.304 \mathrm{e}-04$ & $1.611 \mathrm{e}+07$ & 15 & 136 & $0.000 e+00$ & $2.351 e+05$ & 15 & 137 & $0.000 e+00$ & $5.222 \mathrm{e}+05$ \\
\hline 15 & 138 & $0.000 e+00$ & $2.742 \mathrm{e}+05$ & 15 & 139 & $0.000 e+00$ & $1.421 \mathrm{e}+05$ & 15 & 140 & $0.000 e+00$ & $1.656 \mathrm{e}+05$ \\
\hline 15 & 141 & $0.000 e+00$ & $9.816 e+04$ & 15 & 142 & $0.000 e+00$ & $7.495 e+03$ & 15 & 144 & $8.090 e-03$ & $4.475 e+08$ \\
\hline 15 & 145 & $0.000 e+00$ & $6.254 \mathrm{e}+04$ & 15 & 146 & $0.000 e+00$ & $2.940 e+05$ & 15 & 148 & $1.980 e-02$ & $2.583 e+09$ \\
\hline 15 & 149 & $2.421 \mathrm{e}-03$ & $1.896 \mathrm{e}+08$ & 15 & 151 & $3.483 e-03$ & $1.953 e+08$ & 15 & 154 & $1.575 e-03$ & $2.068 e+08$ \\
\hline 15 & 155 & $5.911 \mathrm{e}-01$ & $3.334 \mathrm{e}+10$ & 15 & 156 & $0.000 e+00$ & $1.447 e+04$ & 15 & 157 & $0.000 e+00$ & $1.233 e+07$ \\
\hline 15 & 158 & $3.862 \mathrm{e}-01$ & $2.183 e+10$ & 15 & 159 & $3.394 \mathrm{e}-02$ & $2.688 e+09$ & 15 & 162 & $0.000 e+00$ & $7.115 e+04$ \\
\hline 15 & 163 & $0.000 e+00$ & $1.848 \mathrm{e}+06$ & 15 & 164 & $3.590 e-01$ & $2.047 e+10$ & 15 & 167 & $0.000 e+00$ & $1.314 \mathrm{e}+06$ \\
\hline 15 & 172 & $8.033 e-04$ & $4.623 e+07$ & 15 & 173 & $9.016 \mathrm{e}-04$ & $7.281 \mathrm{e}+07$ & 15 & 174 & $2.984 e-03$ & $4.026 e+08$ \\
\hline 15 & 177 & $0.000 e+00$ & $3.055 e+05$ & 15 & 180 & $1.395 \mathrm{e}-01$ & $1.139 e+10$ & 15 & 181 & $9.355 e-03$ & $5.460 \mathrm{e}+08$ \\
\hline 15 & 183 & $6.552 \mathrm{e}-03$ & $5.367 e+08$ & 15 & 186 & $5.124 \mathrm{e}-04$ & $4.318 e+07$ & 15 & 187 & $6.671 \mathrm{e}-03$ & $5.634 e+08$ \\
\hline 15 & 189 & $0.000 e+00$ & $4.899 e+05$ & 15 & 190 & $0.000 e+00$ & $6.766 e+06$ & 15 & 191 & $0.000 e+00$ & $1.827 e+06$ \\
\hline 15 & 192 & $0.000 e+00$ & $5.965 e+05$ & 15 & 193 & $0.000 e+00$ & $5.049 e+05$ & 15 & 196 & $0.000 e+00$ & $1.786 e+05$ \\
\hline 15 & 197 & $0.000 e+00$ & $4.872 \mathrm{e}+06$ & 15 & 199 & $0.000 e+00$ & $5.517 e+05$ & 15 & 200 & $0.000 \mathrm{e}+00$ & $4.533 e+05$ \\
\hline 15 & 201 & $0.000 e+00$ & $2.233 e+04$ & 15 & 202 & $0.000 e+00$ & $1.326 \mathrm{e}+05$ & 15 & 204 & $0.000 e+00$ & $1.449 \mathrm{e}+04$ \\
\hline 15 & 205 & $0.000 e+00$ & $9.016 \mathrm{e}+04$ & 15 & 206 & $0.000 e+00$ & $2.547 e+05$ & 15 & 208 & $0.000 e+00$ & $7.977 \mathrm{e}+04$ \\
\hline 15 & 210 & $6.469 e-02$ & $1.231 \mathrm{e}+10$ & 15 & 211 & $1.487 e-02$ & $1.699 e+09$ & 15 & 212 & $4.094 e-04$ & $3.342 e+07$ \\
\hline
\end{tabular}


Table III. Ca IX Oscillator Strengths and Radiative Decay Rates for transitions involving levels higher than 4.

\begin{tabular}{|c|c|c|c|c|c|c|c|c|c|c|c|}
\hline $\begin{array}{l}\text { Low. } \\
\text { Lev. }\end{array}$ & $\begin{array}{l}\text { Upp } \\
\text { Lev }\end{array}$ & $\begin{array}{l}\text { Osc. } \\
\text { Str. }\end{array}$ & $\begin{array}{l}\text { Rad. } \\
\text { Rate }\end{array}$ & $\begin{array}{l}\text { Low. } \\
\text { Lev. }\end{array}$ & $\begin{array}{l}\text { Upp } \\
\text {. Lev }\end{array}$ & $\begin{array}{l}\text { Osc. } \\
\text { Str. }\end{array}$ & $\begin{array}{l}\text { Rad. } \\
\text { Rate }\end{array}$ & $\begin{array}{l}\text { Low. } \\
\text { Lev. }\end{array}$ & $\begin{array}{l}\text { Upp } \\
\text { Lev }\end{array}$ & $\begin{array}{l}\text { Osc. } \\
\text { Str. }\end{array}$ & $\begin{array}{l}\text { Rad. } \\
\text { Rate }\end{array}$ \\
\hline$i$ & $j$ & $g f$ & $(1 / s)$ & $i$ & $j$ & $g f$ & $(1 / s)$ & $i$ & $j$ & $g f$ & $(1 / s)$ \\
\hline 15 & 213 & $2.999 \mathrm{e}-03$ & $3.448 e+08$ & 15 & 214 & $0.000 e+00$ & $1.765 e+04$ & 15 & 215 & $0.000 e+00$ & $8.904 e+05$ \\
\hline 15 & 216 & $0.000 e+00$ & $3.381 \mathrm{e}+05$ & 15 & 217 & $0.000 e+00$ & $2.008 e+04$ & 15 & 218 & $0.000 e+00$ & $8.022 e+05$ \\
\hline 15 & 219 & $0.000 e+00$ & $2.634 \mathrm{e}+05$ & 15 & 220 & $0.000 e+00$ & $9.158 e+03$ & 15 & 221 & $0.000 e+00$ & $1.170 \mathrm{e}+05$ \\
\hline 15 & 222 & $0.000 e+00$ & $1.326 \mathrm{e}+06$ & 15 & 223 & $0.000 e+00$ & $3.594 e+06$ & 15 & 224 & $0.000 e+00$ & $6.276 e+04$ \\
\hline 15 & 225 & $0.000 e+00$ & $3.792 \mathrm{e}+04$ & 15 & 226 & $1.032 \mathrm{e}-02$ & $9.662 e+08$ & 15 & 227 & $1.655 \mathrm{e}-02$ & $3.628 \mathrm{e}+09$ \\
\hline 15 & 228 & $2.181 \mathrm{e}-03$ & $2.869 \mathrm{e}+08$ & 15 & 229 & $2.363 e-04$ & $2.222 \mathrm{e}+07$ & 15 & 230 & $2.451 \mathrm{e}-01$ & $2.308 e+10$ \\
\hline 15 & 233 & $8.288 e-04$ & $1.823 e+08$ & 15 & 235 & $6.801 \mathrm{e}-02$ & $9.053 e+09$ & 15 & 236 & $5.437 e-03$ & $5.171 e+08$ \\
\hline 15 & 238 & $3.391 \mathrm{e}-03$ & $4.570 e+08$ & 15 & 241 & $1.240 \mathrm{e}-04$ & $1.673 e+07$ & 15 & 243 & $0.000 e+00$ & $4.372 e+05$ \\
\hline 15 & 244 & $0.000 e+00$ & $6.315 e+06$ & 15 & 247 & $0.000 e+00$ & $1.098 e+06$ & 15 & 248 & $0.000 e+00$ & $2.875 e+05$ \\
\hline 15 & 249 & $0.000 e+00$ & $8.820 e+03$ & 15 & 250 & $0.000 e+00$ & $8.371 e+04$ & 15 & 252 & $0.000 e+00$ & $2.685 e+06$ \\
\hline 15 & 254 & $0.000 e+00$ & $3.065 e+05$ & 15 & 262 & $0.000 e+00$ & $3.589 e+05$ & 15 & 263 & $0.000 e+00$ & $1.050 e+05$ \\
\hline 15 & 273 & $0.000 e+00$ & $2.794 e+04$ & 15 & 274 & $0.000 e+00$ & $1.285 e+05$ & 15 & 275 & $0.000 e+00$ & $1.533 e+05$ \\
\hline 15 & 283 & $0.000 e+00$ & $8.639 e+04$ & 16 & 30 & $0.000 e+00$ & $2.277 e+03$ & 16 & 31 & $1.192 \mathrm{e}-01$ & $1.124 \mathrm{e}+09$ \\
\hline 16 & 32 & $1.284 e+00$ & $8.656 e+09$ & 16 & 34 & $7.715 e-02$ & $4.055 e+08$ & 16 & 36 & $1.728 \mathrm{e}-03$ & $1.049 e+07$ \\
\hline 16 & 37 & $3.721 \mathrm{e}-04$ & $4.070 e+06$ & 16 & 40 & $1.118 \mathrm{e}-03$ & $1.249 \mathrm{e}+07$ & 16 & 43 & $5.250 e-02$ & $8.674 e+08$ \\
\hline 16 & 44 & $6.212 \mathrm{e}-03$ & $7.339 e+07$ & 16 & 45 & $1.643 e-04$ & $2.743 e+06$ & 16 & 47 & $0.000 e+00$ & $3.216 e+05$ \\
\hline 16 & 48 & $0.000 e+00$ & $8.797 e+04$ & 16 & 52 & $0.000 e+00$ & $8.345 e+03$ & 16 & 56 & $5.233 e-01$ & $1.354 \mathrm{e}+10$ \\
\hline 16 & 57 & $5.832 e-02$ & $1.094 \mathrm{e}+09$ & 16 & 61 & $2.689 \mathrm{e}-03$ & $7.302 \mathrm{e}+07$ & 16 & 62 & $9.931 e-04$ & $2.835 e+07$ \\
\hline 16 & 66 & $0.000 e+00$ & $1.462 \mathrm{e}+05$ & 16 & 67 & $0.000 e+00$ & $2.024 \mathrm{e}+05$ & 16 & 68 & $0.000 e+00$ & $1.764 \mathrm{e}+04$ \\
\hline 16 & 69 & $0.000 e+00$ & $3.151 \mathrm{e}+05$ & 16 & 70 & $0.000 e+00$ & $1.014 \mathrm{e}+05$ & 16 & 74 & $0.000 e+00$ & $3.743 e+05$ \\
\hline 16 & 75 & $0.000 e+00$ & $1.910 \mathrm{e}+05$ & 16 & 76 & $0.000 e+00$ & $1.420 \mathrm{e}+05$ & 16 & 77 & $0.000 e+00$ & $1.529 \mathrm{e}+05$ \\
\hline 16 & 79 & $0.000 e+00$ & $1.599 \mathrm{e}+04$ & 16 & 82 & $1.728 \mathrm{e}-01$ & $5.129 e+09$ & 16 & 83 & $4.726 \mathrm{e}+00$ & $1.100 \mathrm{e}+11$ \\
\hline 16 & 84 & $4.293 e-03$ & $1.288 e+08$ & 16 & 86 & $2.337 e-02$ & $9.923 e+08$ & 16 & 87 & $5.096 \mathrm{e}-01$ & $1.552 \mathrm{e}+10$ \\
\hline 16 & 88 & $1.298 \mathrm{e}-01$ & $3.081 \mathrm{e}+09$ & 16 & 89 & $2.649 \mathrm{e}-03$ & $8.229 e+07$ & 16 & 90 & $4.279 \mathrm{e}-02$ & $1.864 \mathrm{e}+09$ \\
\hline 16 & 93 & $2.041 \mathrm{e}-02$ & $5.028 e+08$ & 16 & 94 & $4.200 e-04$ & $1.350 \mathrm{e}+07$ & 16 & 95 & $1.679 \mathrm{e}-03$ & $7.573 e+07$ \\
\hline 16 & 98 & $0.000 e+00$ & $8.404 e+03$ & 16 & 99 & $0.000 e+00$ & $1.576 e+04$ & 16 & 102 & $3.457 \mathrm{e}-02$ & $1.192 \mathrm{e}+09$ \\
\hline 16 & 103 & $5.091 \mathrm{e}-01$ & $1.367 e+10$ & 16 & 105 & $2.874 \mathrm{e}-03$ & $7.922 \mathrm{e}+07$ & 16 & 107 & $3.992 \mathrm{e}-01$ & $2.073 e+10$ \\
\hline 16 & 108 & $6.342 \mathrm{e}-02$ & $2.354 \mathrm{e}+09$ & 16 & 109 & $5.159 e-04$ & $2.751 e+07$ & 16 & 111 & $0.000 e+00$ & $8.354 \mathrm{e}+05$ \\
\hline 16 & 112 & $0.000 e+00$ & $6.320 \mathrm{e}+04$ & 16 & 113 & $0.000 e+00$ & $1.993 e+05$ & 16 & 114 & $0.000 e+00$ & $1.603 e+05$ \\
\hline 16 & 115 & $0.000 e+00$ & $7.507 e+05$ & 16 & 116 & $0.000 e+00$ & $1.172 \mathrm{e}+06$ & 16 & 117 & $0.000 e+00$ & $2.879 e+05$ \\
\hline 16 & 118 & $0.000 e+00$ & $8.238 e+04$ & 16 & 119 & $0.000 e+00$ & $6.442 e+05$ & 16 & 120 & $0.000 e+00$ & $1.858 \mathrm{e}+05$ \\
\hline 16 & 122 & $0.000 e+00$ & $1.231 \mathrm{e}+06$ & 16 & 123 & $0.000 e+00$ & $4.399 e+05$ & 16 & 127 & $0.000 e+00$ & $8.020 e+03$ \\
\hline 16 & 128 & $7.767 e-02$ & $5.235 e+09$ & 16 & 131 & $8.830 e-03$ & $4.293 e+08$ & 16 & 132 & $6.959 \mathrm{e}-03$ & $4.756 e+08$ \\
\hline 16 & 134 & $1.272 \mathrm{e}-03$ & $8.847 e+07$ & 16 & 136 & $0.000 e+00$ & $1.599 e+05$ & 16 & 138 & $0.000 e+00$ & $4.774 e+04$ \\
\hline 16 & 139 & $0.000 e+00$ & $5.291 e+05$ & 16 & 140 & $0.000 e+00$ & $4.168 e+05$ & 16 & 141 & $0.000 e+00$ & $2.073 e+05$ \\
\hline 16 & 142 & $0.000 e+00$ & $1.901 \mathrm{e}+05$ & 16 & 143 & $0.000 e+00$ & $1.779 e+04$ & 16 & 144 & $1.059 e-04$ & $5.829 e+06$ \\
\hline 16 & 145 & $0.000 e+00$ & $2.595 e+05$ & 16 & 147 & $0.000 e+00$ & $4.273 e+04$ & 16 & 149 & $2.936 \mathrm{e}-02$ & $2.287 e+09$ \\
\hline 16 & 150 & $1.584 \mathrm{e}-03$ & $8.819 e+07$ & 16 & 151 & $3.529 e-03$ & $1.969 e+08$ & 16 & 152 & $9.503 e-02$ & $4.127 e+09$ \\
\hline 16 & 155 & $4.750 e-02$ & $2.666 \mathrm{e}+09$ & 16 & 157 & $0.000 e+00$ & $6.853 e+05$ & 16 & 159 & $2.159 \mathrm{e}-03$ & $1.701 \mathrm{e}+08$ \\
\hline 16 & 160 & $0.000 e+00$ & $8.572 e+06$ & 16 & 161 & $8.708 e-01$ & $3.814 \mathrm{e}+10$ & 16 & 162 & $0.000 e+00$ & $7.070 e+05$ \\
\hline 16 & 163 & $0.000 e+00$ & $3.130 \mathrm{e}+05$ & 16 & 164 & $9.762 \mathrm{e}-02$ & $5.538 e+09$ & 16 & 165 & $7.776 e-01$ & $3.436 e+10$ \\
\hline 16 & 167 & $0.000 e+00$ & $2.236 \mathrm{e}+06$ & 16 & 168 & $0.000 e+00$ & $4.827 e+06$ & 16 & 170 & $0.000 e+00$ & $1.275 \mathrm{e}+06$ \\
\hline 16 & 173 & $4.697 e-03$ & $3.774 \mathrm{e}+08$ & 16 & 175 & $0.000 e+00$ & $1.274 \mathrm{e}+04$ & 16 & 176 & $0.000 e+00$ & $3.779 e+05$ \\
\hline 16 & 177 & $0.000 e+00$ & $1.390 \mathrm{e}+05$ & 16 & 178 & $0.000 e+00$ & $8.974 e+04$ & 16 & 179 & $3.212 \mathrm{e}-02$ & $1.446 \mathrm{e}+09$ \\
\hline
\end{tabular}


Table III. Ca IX Oscillator Strengths and Radiative Decay Rates for transitions involving levels higher than 4.

\begin{tabular}{|c|c|c|c|c|c|c|c|c|c|c|c|}
\hline $\begin{array}{l}\text { Low. } \\
\text { Lev. }\end{array}$ & $\begin{array}{l}\text { Upp } \\
\text { Lev }\end{array}$ & $\begin{array}{l}\text { Osc. } \\
\text { Str. }\end{array}$ & $\begin{array}{l}\text { Rad. } \\
\text { Rate }\end{array}$ & $\begin{array}{l}\text { Low. } \\
\text { Lev. }\end{array}$ & $\begin{array}{l}\text { Upp. } \\
\text { Lev. }\end{array}$ & $\begin{array}{l}\text { Osc. } \\
\text { Str. }\end{array}$ & $\begin{array}{l}\text { Rad. } \\
\text { Rate }\end{array}$ & $\begin{array}{l}\text { Low. } \\
\text { Lev. }\end{array}$ & $\begin{array}{l}\text { - Upp } \\
\text {. Lev }\end{array}$ & $\begin{array}{l}\text { Osc. } \\
\text { Str. }\end{array}$ & $\begin{array}{l}\text { Rad. } \\
\text { Rate }\end{array}$ \\
\hline$i$ & $j$ & $g f$ & $(1 / s)$ & $i$ & $j$ & $g f$ & $(1 / s)$ & $i$ & j & $g f$ & $(1 / s)$ \\
\hline 16 & 180 & $2.034 \mathrm{e}-02$ & $1.653 e+09$ & 16 & 181 & $2.145 e-01$ & $1.246 e+10$ & 16 & 182 & $9.917 e-03$ & $4.481 e+08$ \\
\hline 16 & 183 & $7.900 \mathrm{e}-04$ & $6.439 e+07$ & 16 & 186 & $9.373 e-05$ & $7.861 e+06$ & 16 & 188 & $1.264 \mathrm{e}-03$ & $5.934 e+07$ \\
\hline 16 & 190 & $0.000 e+00$ & $1.176 \mathrm{e}+06$ & 16 & 191 & $0.000 e+00$ & $7.495 e+05$ & 16 & 192 & $0.000 e+00$ & $1.023 e+05$ \\
\hline 16 & 193 & $0.000 e+00$ & $1.476 \mathrm{e}+06$ & 16 & 194 & $0.000 e+00$ & $7.694 e+06$ & 16 & 197 & $0.000 e+00$ & $1.498 e+06$ \\
\hline 16 & 198 & $0.000 e+00$ & $4.145 e+05$ & 16 & 199 & $0.000 e+00$ & $4.670 e+06$ & 16 & 200 & $0.000 e+00$ & $1.942 \mathrm{e}+05$ \\
\hline 16 & 201 & $0.000 e+00$ & $4.188 e+04$ & 16 & 202 & $0.000 e+00$ & $3.782 \mathrm{e}+05$ & 16 & 204 & $0.000 e+00$ & $9.801 e+04$ \\
\hline 16 & 211 & $9.898 e-02$ & $1.126 \mathrm{e}+10$ & 16 & 212 & $1.591 \mathrm{e}-02$ & $1.294 e+09$ & 16 & 213 & $6.243 e-04$ & $7.147 e+07$ \\
\hline 16 & 214 & $0.000 e+00$ & $5.456 e+03$ & 16 & 215 & $0.000 e+00$ & $5.728 e+05$ & 16 & 216 & $0.000 e+00$ & $7.566 e+05$ \\
\hline 16 & 217 & $0.000 e+00$ & $2.832 \mathrm{e}+05$ & 16 & 218 & $0.000 e+00$ & $2.009 e+05$ & 16 & 219 & $0.000 e+00$ & $6.595 e+05$ \\
\hline 16 & 220 & $0.000 e+00$ & $2.202 \mathrm{e}+05$ & 16 & 221 & $0.000 e+00$ & $8.326 e+05$ & 16 & 222 & $0.000 e+00$ & $2.371 e+06$ \\
\hline 16 & 225 & $0.000 e+00$ & $2.064 \mathrm{e}+04$ & 16 & 228 & $2.418 \mathrm{e}-02$ & $3.169 e+09$ & 16 & 229 & $1.454 \mathrm{e}-03$ & $1.361 e+08$ \\
\hline 16 & 230 & $2.171 \mathrm{e}-02$ & $2.036 \mathrm{e}+09$ & 16 & 231 & $3.386 e-01$ & $2.470 e+10$ & 16 & 235 & $9.065 e-03$ & $1.202 \mathrm{e}+09$ \\
\hline 16 & 236 & $9.477 e-02$ & $8.977 e+09$ & 16 & 237 & $4.162 \mathrm{e}-03$ & $3.068 e+08$ & 16 & 241 & $3.555 e-05$ & $4.777 e+06$ \\
\hline 16 & 242 & $4.004 e-04$ & $2.994 \mathrm{e}+07$ & 16 & 243 & $0.000 e+00$ & $7.797 e+03$ & 16 & 244 & $0.000 e+00$ & $8.939 e+05$ \\
\hline 16 & 245 & $0.000 e+00$ & $6.951 \mathrm{e}+06$ & 16 & 247 & $0.000 e+00$ & $4.453 e+05$ & 16 & 248 & $0.000 e+00$ & $8.870 e+05$ \\
\hline 16 & 249 & $0.000 e+00$ & $1.983 e+05$ & 16 & 252 & $0.000 e+00$ & $8.180 e+05$ & 16 & 254 & $0.000 e+00$ & $2.548 e+06$ \\
\hline 16 & 256 & $0.000 e+00$ & $2.232 \mathrm{e}+05$ & 16 & 262 & $0.000 e+00$ & $1.415 e+05$ & 16 & 263 & $0.000 e+00$ & $2.975 e+05$ \\
\hline 16 & 264 & $0.000 e+00$ & $4.965 e+04$ & 16 & 273 & $0.000 e+00$ & $1.239 e+05$ & 17 & 32 & $1.177 \mathrm{e}-01$ & $7.790 e+08$ \\
\hline 17 & 33 & $0.000 e+00$ & $3.027 e+03$ & 17 & 34 & $1.769 e+00$ & $9.128 e+09$ & 17 & 44 & $7.279 e-02$ & $8.481 e+08$ \\
\hline 17 & 48 & $0.000 e+00$ & $3.681 \mathrm{e}+05$ & 17 & 57 & $7.532 \mathrm{e}-01$ & $1.397 e+10$ & 17 & 67 & $0.000 e+00$ & $2.954 e+04$ \\
\hline 17 & 68 & $0.000 e+00$ & $3.218 e+04$ & 17 & 69 & $0.000 e+00$ & $2.592 \mathrm{e}+05$ & 17 & 72 & $0.000 e+00$ & $1.151 \mathrm{e}+05$ \\
\hline 17 & 74 & $0.000 e+00$ & $3.628 e+04$ & 17 & 75 & $0.000 e+00$ & $3.690 e+05$ & 17 & 76 & $0.000 e+00$ & $7.331 e+05$ \\
\hline 17 & 77 & $0.000 e+00$ & $4.433 e+04$ & 17 & 78 & $0.000 e+00$ & $1.780 e+04$ & 17 & 82 & $7.615 \mathrm{e}-04$ & $2.241 \mathrm{e}+07$ \\
\hline 17 & 83 & $1.064 \mathrm{e}-01$ & $2.454 e+09$ & 17 & 85 & $6.198 e+00$ & $1.180 e+11$ & 17 & 87 & $2.344 e-02$ & $7.078 e+08$ \\
\hline 17 & 88 & $7.750 e-01$ & $1.824 \mathrm{e}+10$ & 17 & 89 & $6.863 e-02$ & $2.114 \mathrm{e}+09$ & 17 & 93 & $7.476 e-04$ & $1.826 e+07$ \\
\hline 17 & 94 & $1.697 \mathrm{e}-03$ & $5.412 e+07$ & 17 & 100 & $0.000 e+00$ & $2.294 e+04$ & 17 & 102 & $6.277 e-04$ & $2.148 e+07$ \\
\hline 17 & 103 & $3.857 e-02$ & $1.027 e+09$ & 17 & 104 & $7.359 e-01$ & $1.605 e+10$ & 17 & 108 & $6.013 e-01$ & $2.215 \mathrm{e}+10$ \\
\hline 17 & 112 & $0.000 e+00$ & $3.167 e+05$ & 17 & 113 & $0.000 e+00$ & $1.155 e+06$ & 17 & 116 & $0.000 e+00$ & $3.930 e+05$ \\
\hline 17 & 117 & $0.000 e+00$ & $1.730 e+06$ & 17 & 119 & $0.000 e+00$ & $2.133 e+04$ & 17 & 120 & $0.000 e+00$ & $6.901 \mathrm{e}+05$ \\
\hline 17 & 123 & $0.000 e+00$ & $1.323 e+06$ & 17 & 131 & $1.226 \mathrm{e}-01$ & $5.919 e+09$ & 17 & 140 & $0.000 e+00$ & $3.540 e+04$ \\
\hline 17 & 141 & $0.000 e+00$ & $5.084 e+05$ & 17 & 142 & $0.000 e+00$ & $1.008 e+06$ & 17 & 143 & $0.000 e+00$ & $3.045 e+05$ \\
\hline 17 & 150 & $3.936 \mathrm{e}-02$ & $2.177 e+09$ & 17 & 151 & $6.225 \mathrm{e}-05$ & $3.451 e+06$ & 17 & 152 & $1.451 \mathrm{e}-02$ & $6.260 \mathrm{e}+08$ \\
\hline 17 & 153 & $3.114 \mathrm{e}-01$ & $1.101 \mathrm{e}+10$ & 17 & 155 & $1.754 \mathrm{e}-04$ & $9.779 e+06$ & 17 & 160 & $0.000 e+00$ & $1.928 e+04$ \\
\hline 17 & 161 & $6.463 e-04$ & $2.813 e+07$ & 17 & 162 & $0.000 e+00$ & $5.100 e+04$ & 17 & 163 & $0.000 e+00$ & $7.255 e+03$ \\
\hline 17 & 164 & $2.774 \mathrm{e}-03$ & $1.564 \mathrm{e}+08$ & 17 & 165 & $1.470 \mathrm{e}-01$ & $6.453 e+09$ & 17 & 166 & $0.000 e+00$ & $1.658 e+07$ \\
\hline 17 & 167 & $0.000 e+00$ & $2.656 \mathrm{e}+05$ & 17 & 168 & $0.000 e+00$ & $3.635 e+06$ & 17 & 169 & $1.994 \mathrm{e}+00$ & $7.180 \mathrm{e}+10$ \\
\hline 17 & 170 & $0.000 e+00$ & $1.624 \mathrm{e}+05$ & 17 & 172 & $8.375 e-03$ & $4.765 e+08$ & 17 & 175 & $0.000 e+00$ & $5.486 e+05$ \\
\hline 17 & 176 & $0.000 e+00$ & $8.000 e+04$ & 17 & 177 & $0.000 e+00$ & $6.552 \mathrm{e}+03$ & 17 & 178 & $0.000 e+00$ & $4.174 e+04$ \\
\hline 17 & 179 & $9.989 e-03$ & $4.469 e+08$ & 17 & 181 & $2.204 \mathrm{e}-02$ & $1.272 \mathrm{e}+09$ & 17 & 182 & $3.021 \mathrm{e}-01$ & $1.356 e+10$ \\
\hline 17 & 190 & $0.000 e+00$ & $2.909 e+04$ & 17 & 191 & $0.000 e+00$ & $3.172 \mathrm{e}+04$ & 17 & 192 & $0.000 e+00$ & $1.941 \mathrm{e}+06$ \\
\hline 17 & 193 & $0.000 \mathrm{e}+00$ & $5.245 \mathrm{e}+05$ & 17 & 194 & $0.000 e+00$ & $7.246 e+05$ & 17 & 195 & $0.000 e+00$ & $7.803 e+06$ \\
\hline 17 & 197 & $0.000 \mathrm{e}+00$ & $6.038 \mathrm{e}+04$ & 17 & 198 & $0.000 e+00$ & $5.621 e+06$ & 17 & 199 & $0.000 e+00$ & $1.129 \mathrm{e}+06$ \\
\hline 17 & 201 & $0.000 e+00$ & $4.591 \mathrm{e}+05$ & 17 & 202 & $0.000 e+00$ & $1.195 e+05$ & 17 & 203 & $0.000 e+00$ & $6.794 e+04$ \\
\hline 17 & 212 & $1.492 \mathrm{e}-01$ & $1.206 \mathrm{e}+10$ & 17 & 216 & $0.000 e+00$ & $4.328 e+05$ & 17 & 217 & $0.000 e+00$ & $1.319 e+06$ \\
\hline
\end{tabular}


Table III. Ca IX Oscillator Strengths and Radiative Decay Rates for transitions involving levels higher than 4.

\begin{tabular}{|c|c|c|c|c|c|c|c|c|c|c|c|}
\hline $\begin{array}{l}\text { Low. } \\
\text { Lev. }\end{array}$ & $\begin{array}{l}\text { Upp } \\
\text { Lev }\end{array}$ & $\begin{array}{l}\text { Osc. } \\
\text { Str. }\end{array}$ & $\begin{array}{l}\text { Rad. } \\
\text { Rate }\end{array}$ & $\begin{array}{l}\text { Low. } \\
\text { Lev. }\end{array}$ & $\begin{array}{l}\text { Upp. } \\
\text { Lev. }\end{array}$ & $\begin{array}{l}\text { Osc. } \\
\text { Str. }\end{array}$ & $\begin{array}{l}\text { Rad. } \\
\text { Rate }\end{array}$ & $\begin{array}{l}\text { Low. } \\
\text { Lev. }\end{array}$ & $\begin{array}{l}\text { Upp } \\
\text { Lev }\end{array}$ & $\begin{array}{l}\text { Osc. } \\
\text { Str. }\end{array}$ & $\begin{array}{l}\text { Rad. } \\
\text { Rate }\end{array}$ \\
\hline$i$ & $j$ & $g f$ & $(1 / s)$ & $i$ & $j$ & $g f$ & $(1 / s)$ & $i$ & j & $g f$ & $(1 / s)$ \\
\hline 17 & 219 & $0.000 e+00$ & $1.074 e+05$ & 17 & 220 & $0.000 e+00$ & $8.349 e+05$ & 17 & 221 & $0.000 e+00$ & $2.757 e+06$ \\
\hline 17 & 226 & $9.167 e-05$ & $8.509 e+06$ & 17 & 229 & $3.250 e-02$ & $3.028 e+09$ & 17 & 230 & $2.443 e-04$ & $2.280 e+07$ \\
\hline 17 & 231 & $2.137 e-02$ & $1.552 \mathrm{e}+09$ & 17 & 232 & $4.434 \mathrm{e}-01$ & $2.635 e+10$ & 17 & 236 & $9.278 e-03$ & $8.746 e+08$ \\
\hline 17 & 237 & $1.318 e-01$ & $9.669 \mathrm{e}+09$ & 17 & 244 & $0.000 e+00$ & $3.406 e+04$ & 17 & 245 & $0.000 e+00$ & $7.204 e+05$ \\
\hline 17 & 246 & $0.000 e+00$ & $7.580 e+06$ & 17 & 247 & $0.000 e+00$ & $1.868 e+04$ & 17 & 248 & $0.000 e+00$ & $3.023 e+05$ \\
\hline 17 & 249 & $0.000 e+00$ & $1.131 \mathrm{e}+06$ & 17 & 252 & $0.000 e+00$ & $4.119 e+04$ & 17 & 254 & $0.000 e+00$ & $6.365 e+05$ \\
\hline 17 & 256 & $0.000 e+00$ & $3.099 e+06$ & 17 & 263 & $0.000 e+00$ & $7.528 e+04$ & 17 & 264 & $0.000 e+00$ & $3.527 e+05$ \\
\hline 17 & 272 & $0.000 e+00$ & $1.230 e+05$ & 18 & 31 & $3.431 \mathrm{e}-02$ & $3.081 e+08$ & 18 & 32 & $7.492 \mathrm{e}-04$ & $4.810 e+06$ \\
\hline 18 & 37 & $1.816 \mathrm{e}+00$ & $1.898 \mathrm{e}+10$ & 18 & 38 & $3.853 e-03$ & $6.851 e+07$ & 18 & 40 & $9.683 e-04$ & $1.034 \mathrm{e}+07$ \\
\hline 18 & 42 & $1.169 \mathrm{e}-03$ & $3.100 e+07$ & 18 & 45 & $1.240 \mathrm{e}-02$ & $1.996 e+08$ & 18 & 46 & $0.000 e+00$ & $2.625 e+04$ \\
\hline 18 & 52 & $0.000 e+00$ & $3.863 e+05$ & 18 & 54 & $2.129 \mathrm{e}-01$ & $8.622 e+09$ & 18 & 55 & $8.099 e-02$ & $3.371 \mathrm{e}+09$ \\
\hline 18 & 56 & $6.469 \mathrm{e}-04$ & $1.625 e+07$ & 18 & 59 & $1.737 \mathrm{e}-03$ & $7.548 e+07$ & 18 & 60 & $3.459 e-04$ & $1.520 \mathrm{e}+07$ \\
\hline 18 & 61 & $2.572 \mathrm{e}-03$ & $6.786 e+07$ & 18 & 62 & $8.910 e-02$ & $2.474 \mathrm{e}+09$ & 18 & 67 & $0.000 e+00$ & $1.883 e+05$ \\
\hline 18 & 68 & $0.000 e+00$ & $5.533 e+04$ & 18 & 69 & $0.000 e+00$ & $1.686 e+04$ & 18 & 70 & $0.000 e+00$ & $3.469 e+04$ \\
\hline 18 & 71 & $0.000 e+00$ & $3.912 \mathrm{e}+03$ & 18 & 72 & $0.000 e+00$ & $8.401 e+03$ & 18 & 73 & $0.000 e+00$ & $1.644 \mathrm{e}+05$ \\
\hline 18 & 74 & $0.000 e+00$ & $8.307 e+04$ & 18 & 75 & $0.000 e+00$ & $7.434 e+04$ & 18 & 77 & $0.000 e+00$ & $3.780 e+05$ \\
\hline 18 & 79 & $0.000 e+00$ & $4.851 \mathrm{e}+03$ & 18 & 81 & $0.000 e+00$ & $2.126 e+05$ & 18 & 82 & $5.827 e-01$ & $1.690 \mathrm{e}+10$ \\
\hline 18 & 84 & $3.177 e+00$ & $9.313 e+10$ & 18 & 86 & $6.014 \mathrm{e}-04$ & $2.496 e+07$ & 18 & 87 & $1.993 e-01$ & $5.931 e+09$ \\
\hline 18 & 89 & $1.365 e-02$ & $4.146 e+08$ & 18 & 90 & $3.610 e-03$ & $1.538 e+08$ & 18 & 91 & $1.002 e-03$ & $7.123 e+07$ \\
\hline 18 & 92 & $8.495 e-02$ & $3.656 \mathrm{e}+09$ & 18 & 94 & $6.137 e-04$ & $1.930 \mathrm{e}+07$ & 18 & 95 & $3.329 e-03$ & $1.469 e+08$ \\
\hline 18 & 96 & $1.204 \mathrm{e}-04$ & $8.867 e+06$ & 18 & 97 & $2.308 e-01$ & $1.050 e+10$ & 18 & 101 & $0.000 e+00$ & $3.253 e+04$ \\
\hline 18 & 102 & $1.957 \mathrm{e}-02$ & $6.606 e+08$ & 18 & 106 & $9.881 \mathrm{e}-03$ & $8.373 e+08$ & 18 & 107 & $9.264 \mathrm{e}-04$ & $4.712 \mathrm{e}+07$ \\
\hline 18 & 108 & $1.593 e-03$ & $5.791 e+07$ & 18 & 109 & $4.584 \mathrm{e}-01$ & $2.395 e+10$ & 18 & 110 & $0.000 e+00$ & $6.312 \mathrm{e}+04$ \\
\hline 18 & 111 & $0.000 e+00$ & $6.889 e+04$ & 18 & 112 & $0.000 e+00$ & $1.130 \mathrm{e}+06$ & 18 & 113 & $0.000 e+00$ & $3.219 e+05$ \\
\hline 18 & 114 & $0.000 e+00$ & $6.429 e+05$ & 18 & 115 & $0.000 e+00$ & $1.469 e+04$ & 18 & 116 & $0.000 e+00$ & $1.386 e+04$ \\
\hline 18 & 119 & $0.000 e+00$ & $1.271 \mathrm{e}+04$ & 18 & 120 & $0.000 e+00$ & $8.619 e+03$ & 18 & 121 & $0.000 \mathrm{e}+00$ & $6.419 e+04$ \\
\hline 18 & 122 & $0.000 e+00$ & $1.683 e+04$ & 18 & 123 & $0.000 e+00$ & $9.097 e+03$ & 18 & 124 & $0.000 \mathrm{e}+00$ & $1.326 \mathrm{e}+06$ \\
\hline 18 & 125 & $2.866 \mathrm{e}-02$ & $3.131 \mathrm{e}+09$ & 18 & 126 & $2.367 e-02$ & $2.606 e+09$ & 18 & 127 & $0.000 e+00$ & $6.887 e+04$ \\
\hline 18 & 128 & $2.934 \mathrm{e}-04$ & $1.942 \mathrm{e}+07$ & 18 & 130 & $9.276 \mathrm{e}-03$ & $1.032 \mathrm{e}+09$ & 18 & 133 & $3.787 e-04$ & $4.254 e+07$ \\
\hline 18 & 134 & $1.258 \mathrm{e}-04$ & $8.593 e+06$ & 18 & 136 & $0.000 e+00$ & $2.102 e+05$ & 18 & 137 & $0.000 e+00$ & $7.004 e+04$ \\
\hline 18 & 138 & $0.000 e+00$ & $6.543 e+03$ & 18 & 140 & $0.000 e+00$ & $1.577 e+05$ & 18 & 143 & $0.000 e+00$ & $7.436 e+03$ \\
\hline 18 & 144 & $2.634 \mathrm{e}-01$ & $1.425 \mathrm{e}+10$ & 18 & 145 & $0.000 e+00$ & $8.404 e+03$ & 18 & 146 & $0.000 e+00$ & $2.241 \mathrm{e}+04$ \\
\hline 18 & 147 & $0.000 e+00$ & $5.278 e+04$ & 18 & 148 & $2.350 \mathrm{e}-04$ & $2.999 e+07$ & 18 & 149 & $8.315 e-05$ & $6.367 e+06$ \\
\hline 18 & 150 & $5.664 \mathrm{e}-04$ & $3.100 e+07$ & 18 & 151 & $4.929 e-02$ & $2.703 e+09$ & 18 & 154 & $6.721 \mathrm{e}-02$ & $8.632 e+09$ \\
\hline 18 & 155 & $4.509 e-01$ & $2.489 e+10$ & 18 & 156 & $0.000 e+00$ & $1.184 \mathrm{e}+06$ & 18 & 157 & $0.000 e+00$ & $2.568 e+05$ \\
\hline 18 & 158 & $1.286 \mathrm{e}-01$ & $7.108 e+09$ & 18 & 159 & $4.775 e-04$ & $3.701 \mathrm{e}+07$ & 18 & 162 & $0.000 e+00$ & $4.335 e+06$ \\
\hline 18 & 163 & $0.000 e+00$ & $4.798 e+04$ & 18 & 164 & $3.488 e-01$ & $1.946 e+10$ & 18 & 167 & $0.000 e+00$ & $4.433 e+06$ \\
\hline 18 & 171 & $2.238 e-04$ & $2.940 e+07$ & 18 & 172 & $2.430 \mathrm{e}-02$ & $1.369 e+09$ & 18 & 173 & $2.635 \mathrm{e}-03$ & $2.082 e+08$ \\
\hline 18 & 174 & $1.909 \mathrm{e}-04$ & $2.520 e+07$ & 18 & 175 & $0.000 e+00$ & $3.515 e+05$ & 18 & 176 & $0.000 e+00$ & $1.355 e+06$ \\
\hline 18 & 177 & $0.000 \mathrm{e}+00$ & $1.830 \mathrm{e}+04$ & 18 & 178 & $0.000 e+00$ & $1.811 \mathrm{e}+06$ & 18 & 180 & $4.528 e-04$ & $3.619 \mathrm{e}+07$ \\
\hline 18 & 183 & $5.198 e-02$ & $4.167 e+09$ & 18 & 184 & $3.522 \mathrm{e}-04$ & $4.842 \mathrm{e}+07$ & 18 & 186 & $1.344 \mathrm{e}-02$ & $1.109 e+09$ \\
\hline 18 & 187 & $2.235 e-01$ & $1.848 \mathrm{e}+10$ & 18 & 189 & $0.000 e+00$ & $5.323 e+06$ & 18 & 190 & $0.000 e+00$ & $3.637 e+05$ \\
\hline 18 & 191 & $0.000 e+00$ & $2.917 e+04$ & 18 & 192 & $0.000 e+00$ & $4.895 e+04$ & 18 & 196 & $0.000 e+00$ & $6.430 e+06$ \\
\hline 18 & 197 & $0.000 e+00$ & $1.065 e+05$ & 18 & 200 & $0.000 e+00$ & $4.920 e+04$ & 18 & 201 & $0.000 e+00$ & $2.558 e+04$ \\
\hline
\end{tabular}


Table III. Ca IX Oscillator Strengths and Radiative Decay Rates for transitions involving levels higher than 4.

\begin{tabular}{|c|c|c|c|c|c|c|c|c|c|c|c|}
\hline $\begin{array}{l}\text { Low. } \\
\text { Lev. }\end{array}$ & $\begin{array}{l}\text { Upp } \\
\text { Lev }\end{array}$ & $\begin{array}{l}\text { Osc. } \\
\text { Str. }\end{array}$ & $\begin{array}{l}\text { Rad. } \\
\text { Rate }\end{array}$ & $\begin{array}{l}\text { Low. } \\
\text { Lev. }\end{array}$ & $\begin{array}{l}\text { - Upp } \\
\text {. Lev }\end{array}$ & $\begin{array}{l}\text { Osc. } \\
\text { Str. }\end{array}$ & $\begin{array}{l}\text { Rad. } \\
\text { Rate }\end{array}$ & $\begin{array}{l}\text { Low. } \\
\text { Lev. }\end{array}$ & $\begin{array}{l}\text { Upp } \\
\text { Lev }\end{array}$ & $\begin{array}{l}\text { Osc. } \\
\text { Str. }\end{array}$ & $\begin{array}{l}\text { Rad. } \\
\text { Rate }\end{array}$ \\
\hline$i$ & $j$ & $g f$ & $(1 / s)$ & $i$ & $j$ & $g f$ & $(1 / s)$ & $i$ & j & $g f$ & $(1 / s)$ \\
\hline 18 & 204 & $0.000 e+00$ & $3.807 e+04$ & 18 & 205 & $0.000 e+00$ & $9.390 e+04$ & 18 & 206 & $0.000 e+00$ & $8.586 e+06$ \\
\hline 18 & 208 & $0.000 e+00$ & $2.162 \mathrm{e}+06$ & 18 & 210 & $2.424 \mathrm{e}-03$ & $4.530 e+08$ & 18 & 212 & $4.353 e-04$ & $3.489 e+07$ \\
\hline 18 & 213 & $1.010 e-01$ & $1.140 \mathrm{e}+10$ & 18 & 214 & $0.000 e+00$ & $9.082 e+05$ & 18 & 215 & $0.000 e+00$ & $4.367 e+04$ \\
\hline 18 & 216 & $0.000 e+00$ & $1.930 \mathrm{e}+04$ & 18 & 219 & $0.000 e+00$ & $9.515 e+03$ & 18 & 220 & $0.000 e+00$ & $1.005 e+04$ \\
\hline 18 & 221 & $0.000 e+00$ & $1.924 e+04$ & 18 & 222 & $0.000 e+00$ & $1.634 \mathrm{e}+04$ & 18 & 223 & $0.000 e+00$ & $1.547 e+05$ \\
\hline 18 & 224 & $0.000 e+00$ & $1.507 e+06$ & 18 & 225 & $0.000 e+00$ & $1.237 e+06$ & 18 & 226 & $1.973 e-01$ & $1.816 e+10$ \\
\hline 18 & 227 & $5.655 e-05$ & $1.219 \mathrm{e}+07$ & 18 & 229 & $8.689 e-05$ & $8.031 e+06$ & 18 & 230 & $1.261 \mathrm{e}-02$ & $1.168 \mathrm{e}+09$ \\
\hline 18 & 233 & $4.714 \mathrm{e}-02$ & $1.019 \mathrm{e}+10$ & 18 & 234 & $1.249 \mathrm{e}-04$ & $2.721 \mathrm{e}+07$ & 18 & 235 & $2.387 e-03$ & $3.124 e+08$ \\
\hline 18 & 238 & $1.025 e-01$ & $1.358 \mathrm{e}+10$ & 18 & 240 & $2.205 e-04$ & $4.874 e+07$ & 18 & 241 & $4.889 e-03$ & $6.485 e+08$ \\
\hline 18 & 243 & $0.000 e+00$ & $3.869 e+06$ & 18 & 244 & $0.000 e+00$ & $4.821 e+05$ & 18 & 247 & $0.000 e+00$ & $2.345 e+04$ \\
\hline 18 & 250 & $0.000 e+00$ & $3.048 e+06$ & 18 & 252 & $0.000 e+00$ & $6.431 e+04$ & 18 & 261 & $2.232 \mathrm{e}-04$ & $2.163 e+07$ \\
\hline 18 & 262 & $0.000 e+00$ & $2.547 e+04$ & 18 & 264 & $0.000 e+00$ & $4.514 e+04$ & 18 & 273 & $0.000 e+00$ & $2.442 e+04$ \\
\hline 18 & 274 & $0.000 e+00$ & $7.232 e+04$ & 18 & 275 & $0.000 e+00$ & $4.821 e+06$ & 18 & 280 & $2.031 \mathrm{e}-04$ & $2.785 e+07$ \\
\hline 18 & 283 & $0.000 e+00$ & $2.279 \mathrm{e}+06$ & 19 & 31 & $4.004 e-02$ & $2.860 e+08$ & 19 & 32 & $3.732 \mathrm{e}-01$ & $1.907 e+09$ \\
\hline 19 & 38 & $9.379 e-01$ & $1.353 e+10$ & 19 & 40 & $1.083 e+00$ & $9.390 e+09$ & 19 & 46 & $0.000 e+00$ & $2.421 \mathrm{e}+05$ \\
\hline 19 & 47 & $0.000 e+00$ & $1.039 \mathrm{e}+05$ & 19 & 48 & $0.000 e+00$ & $4.962 e+03$ & 19 & 52 & $0.000 e+00$ & $8.105 e+03$ \\
\hline 19 & 55 & $8.528 e-03$ & $3.104 \mathrm{e}+08$ & 19 & 56 & $2.237 e-02$ & $4.917 e+08$ & 19 & 59 & $2.631 \mathrm{e}-01$ & $1.003 e+10$ \\
\hline 19 & 60 & $3.245 e-02$ & $1.252 \mathrm{e}+09$ & 19 & 61 & $2.563 e-02$ & $5.935 e+08$ & 19 & 62 & $2.223 e-03$ & $5.436 e+07$ \\
\hline 19 & 64 & $3.658 e-03$ & $1.782 \mathrm{e}+08$ & 19 & 66 & $0.000 e+00$ & $1.999 e+05$ & 19 & 67 & $0.000 e+00$ & $1.786 e+05$ \\
\hline 19 & 68 & $0.000 e+00$ & $1.499 \mathrm{e}+05$ & 19 & 69 & $0.000 e+00$ & $1.064 e+05$ & 19 & 71 & $0.000 e+00$ & $8.245 e+03$ \\
\hline 19 & 72 & $0.000 e+00$ & $1.851 \mathrm{e}+04$ & 19 & 73 & $0.000 e+00$ & $2.275 e+04$ & 19 & 75 & $0.000 e+00$ & $5.292 e+03$ \\
\hline 19 & 76 & $0.000 e+00$ & $3.090 \mathrm{e}+03$ & 19 & 77 & $0.000 e+00$ & $3.137 e+04$ & 19 & 78 & $0.000 e+00$ & $1.515 \mathrm{e}+05$ \\
\hline 19 & 79 & $0.000 e+00$ & $1.107 e+05$ & 19 & 80 & $0.000 e+00$ & $7.054 \mathrm{e}+04$ & 19 & 81 & $0.000 e+00$ & $1.160 \mathrm{e}+04$ \\
\hline 19 & 82 & $2.320 \mathrm{e}-03$ & $6.062 e+07$ & 19 & 84 & $1.825 \mathrm{e}-01$ & $4.823 e+09$ & 19 & 86 & $1.395 \mathrm{e}-01$ & $5.221 \mathrm{e}+09$ \\
\hline 19 & 87 & $1.271 \mathrm{e}+00$ & $3.412 \mathrm{e}+10$ & 19 & 89 & $1.424 \mathrm{e}+00$ & $3.904 e+10$ & 19 & 90 & $7.004 e-02$ & $2.695 e+09$ \\
\hline 19 & 91 & $1.445 \mathrm{e}-03$ & $9.280 e+07$ & 19 & 92 & $2.750 e-03$ & $1.069 e+08$ & 19 & 94 & $1.378 \mathrm{e}+00$ & $3.922 \mathrm{e}+10$ \\
\hline 19 & 95 & $1.135 \mathrm{e}-01$ & $4.530 \mathrm{e}+09$ & 19 & 96 & $3.822 \mathrm{e}-03$ & $2.548 e+08$ & 19 & 97 & $9.133 e-03$ & $3.765 e+08$ \\
\hline 19 & 102 & $4.522 \mathrm{e}-04$ & $1.386 \mathrm{e}+07$ & 19 & 106 & $5.191 \mathrm{e}-03$ & $4.008 e+08$ & 19 & 107 & $2.407 e-03$ & $1.116 \mathrm{e}+08$ \\
\hline 19 & 108 & $2.961 \mathrm{e}-01$ & $9.810 \mathrm{e}+09$ & 19 & 109 & $1.808 \mathrm{e}-03$ & $8.620 e+07$ & 19 & 110 & $0.000 e+00$ & $1.661 \mathrm{e}+05$ \\
\hline 19 & 111 & $0.000 e+00$ & $7.458 e+04$ & 19 & 112 & $0.000 e+00$ & $2.685 e+04$ & 19 & 113 & $0.000 e+00$ & $1.639 \mathrm{e}+04$ \\
\hline 19 & 114 & $0.000 e+00$ & $4.023 e+04$ & 19 & 115 & $0.000 e+00$ & $1.435 \mathrm{e}+04$ & 19 & 116 & $0.000 e+00$ & $2.088 e+04$ \\
\hline 19 & 117 & $0.000 e+00$ & $5.278 e+05$ & 19 & 118 & $0.000 e+00$ & $2.327 e+04$ & 19 & 120 & $0.000 e+00$ & $1.477 \mathrm{e}+06$ \\
\hline 19 & 121 & $0.000 e+00$ & $2.255 \mathrm{e}+05$ & 19 & 122 & $0.000 e+00$ & $2.431 e+04$ & 19 & 123 & $0.000 e+00$ & $2.973 e+04$ \\
\hline 19 & 125 & $2.017 e-04$ & $2.030 e+07$ & 19 & 126 & $1.689 \mathrm{e}-02$ & $1.714 \mathrm{e}+09$ & 19 & 128 & $1.130 \mathrm{e}-02$ & $6.893 e+08$ \\
\hline 19 & 130 & $3.034 \mathrm{e}-02$ & $3.113 e+09$ & 19 & 132 & $6.663 e-04$ & $4.125 e+07$ & 19 & 133 & $2.872 \mathrm{e}-02$ & $2.976 \mathrm{e}+09$ \\
\hline 19 & 134 & $4.184 \mathrm{e}-04$ & $2.639 \mathrm{e}+07$ & 19 & 137 & $0.000 e+00$ & $4.250 e+05$ & 19 & 138 & $0.000 e+00$ & $2.038 e+05$ \\
\hline 19 & 139 & $0.000 e+00$ & $2.657 e+05$ & 19 & 140 & $0.000 e+00$ & $1.682 \mathrm{e}+04$ & 19 & 142 & $0.000 e+00$ & $8.490 e+03$ \\
\hline 19 & 143 & $0.000 e+00$ & $2.722 e+05$ & 19 & 144 & $1.399 e-03$ & $7.016 e+07$ & 19 & 145 & $0.000 e+00$ & $3.456 \mathrm{e}+05$ \\
\hline 19 & 146 & $0.000 e+00$ & $3.451 e+05$ & 19 & 147 & $0.000 e+00$ & $1.422 \mathrm{e}+04$ & 19 & 148 & $1.913 e-02$ & $2.264 e+09$ \\
\hline 19 & 149 & $1.466 \mathrm{e}-01$ & $1.041 \mathrm{e}+10$ & 19 & 150 & $1.178 \mathrm{e}-01$ & $5.979 e+09$ & 19 & 151 & $4.624 \mathrm{e}-03$ & $2.353 e+08$ \\
\hline 19 & 155 & $3.492 \mathrm{e}-02$ & $1.788 e+09$ & 19 & 156 & $0.000 e+00$ & $3.338 \mathrm{e}+04$ & 19 & 157 & $0.000 \mathrm{e}+00$ & $3.314 \mathrm{e}+04$ \\
\hline 19 & 158 & $3.323 e-01$ & $1.704 \mathrm{e}+10$ & 19 & 159 & $4.142 \mathrm{e}-02$ & $2.978 e+09$ & 19 & 162 & $0.000 e+00$ & $1.285 e+06$ \\
\hline 19 & 163 & $0.000 e+00$ & $3.305 e+05$ & 19 & 164 & $1.148 \mathrm{e}-01$ & $5.943 e+09$ & 19 & 167 & $0.000 \mathrm{e}+00$ & $3.887 e+05$ \\
\hline 19 & 171 & $1.190 \mathrm{e}-01$ & $1.451 \mathrm{e}+10$ & 19 & 172 & $5.152 \mathrm{e}-01$ & $2.693 e+10$ & 19 & 173 & $3.174 \mathrm{e}-02$ & $2.329 \mathrm{e}+09$ \\
\hline
\end{tabular}


Table III. Ca IX Oscillator Strengths and Radiative Decay Rates for transitions involving levels higher than 4.

\begin{tabular}{|c|c|c|c|c|c|c|c|c|c|c|c|}
\hline $\begin{array}{l}\text { Low. } \\
\text { Lev . }\end{array}$ & $\begin{array}{l}\text { Upp } \\
\text { Lev }\end{array}$ & $\begin{array}{l}\text { Osc. } \\
\text { Str. }\end{array}$ & $\begin{array}{l}\text { Rad. } \\
\text { Rate }\end{array}$ & $\begin{array}{l}\text { Low. } \\
\text { Lev. }\end{array}$ & $\begin{array}{l}\text { Upp. } \\
\text { Lev. }\end{array}$ & $\begin{array}{l}\text { Osc. } \\
\text { Str. }\end{array}$ & $\begin{array}{l}\text { Rad. } \\
\text { Rate }\end{array}$ & $\begin{array}{l}\text { Low. } \\
\text { Lev. }\end{array}$ & $\begin{array}{l}\text { - Upp } \\
\text {. Lev }\end{array}$ & $\begin{array}{l}\text { Osc. } \\
\text { Str. }\end{array}$ & $\begin{array}{l}\text { Rad. } \\
\text { Rate }\end{array}$ \\
\hline$i$ & $j$ & $g f$ & $(1 / s)$ & $i$ & $j$ & $g f$ & $(1 / s)$ & i & j & $g f$ & $(1 / s)$ \\
\hline 19 & 174 & $1.279 \mathrm{e}-03$ & $1.568 e+08$ & 19 & 175 & $0.000 e+00$ & $4.207 e+06$ & 19 & 176 & $0.000 e+00$ & $4.611 e+05$ \\
\hline 19 & 177 & $0.000 e+00$ & $2.434 \mathrm{e}+05$ & 19 & 178 & $0.000 e+00$ & $8.172 \mathrm{e}+05$ & 19 & 180 & $9.927 e-03$ & $7.369 e+08$ \\
\hline 19 & 181 & $6.147 \mathrm{e}-02$ & $3.261 \mathrm{e}+09$ & 19 & 183 & $3.362 \mathrm{e}-03$ & $2.504 e+08$ & 19 & 184 & $1.177 \mathrm{e}-01$ & $1.505 e+10$ \\
\hline 19 & 186 & $1.340 \mathrm{e}-01$ & $1.028 e+10$ & 19 & 187 & $1.191 \mathrm{e}-02$ & $9.155 e+08$ & 19 & 190 & $0.000 e+00$ & $5.550 e+04$ \\
\hline 19 & 191 & $0.000 e+00$ & $1.313 e+06$ & 19 & 192 & $0.000 e+00$ & $5.085 e+05$ & 19 & 193 & $0.000 e+00$ & $2.956 e+06$ \\
\hline 19 & 197 & $0.000 e+00$ & $3.350 \mathrm{e}+05$ & 19 & 199 & $0.000 e+00$ & $1.722 \mathrm{e}+06$ & 19 & 200 & $0.000 e+00$ & $3.854 e+06$ \\
\hline 19 & 201 & $0.000 e+00$ & $2.783 e+06$ & 19 & 202 & $0.000 e+00$ & $6.959 e+06$ & 19 & 203 & $0.000 e+00$ & $2.464 e+06$ \\
\hline 19 & 204 & $0.000 e+00$ & $1.024 \mathrm{e}+07$ & 19 & 205 & $0.000 e+00$ & $1.574 e+07$ & 19 & 206 & $0.000 e+00$ & $2.169 e+04$ \\
\hline 19 & 210 & $1.136 \mathrm{e}-03$ & $1.995 e+08$ & 19 & 211 & $2.991 \mathrm{e}-04$ & $3.152 \mathrm{e}+07$ & 19 & 212 & $8.477 e-02$ & $6.385 e+09$ \\
\hline 19 & 213 & $3.465 e-04$ & $3.676 \mathrm{e}+07$ & 19 & 214 & $0.000 e+00$ & $6.088 e+03$ & 19 & 215 & $0.000 e+00$ & $6.331 e+04$ \\
\hline 19 & 217 & $0.000 e+00$ & $5.352 e+05$ & 19 & 218 & $0.000 e+00$ & $4.479 e+03$ & 19 & 219 & $0.000 e+00$ & $2.164 \mathrm{e}+04$ \\
\hline 19 & 220 & $0.000 e+00$ & $1.537 e+06$ & 19 & 221 & $0.000 e+00$ & $1.861 e+05$ & 19 & 222 & $0.000 e+00$ & $2.430 e+05$ \\
\hline 19 & 223 & $0.000 e+00$ & $1.707 e+05$ & 19 & 225 & $0.000 e+00$ & $6.064 e+03$ & 19 & 226 & $2.036 e-04$ & $1.769 e+07$ \\
\hline 19 & 227 & $1.087 e-02$ & $2.211 \mathrm{e}+09$ & 19 & 228 & $8.382 e-02$ & $1.023 e+10$ & 19 & 229 & $6.323 e-02$ & $5.516 e+09$ \\
\hline 19 & 230 & $2.361 \mathrm{e}-04$ & $2.063 e+07$ & 19 & 233 & $4.945 e-05$ & $1.009 e+07$ & 19 & 234 & $9.481 \mathrm{e}-02$ & $1.950 \mathrm{e}+10$ \\
\hline 19 & 235 & $5.485 e-03$ & $6.778 e+08$ & 19 & 236 & $4.671 \mathrm{e}-02$ & $4.124 e+09$ & 19 & 238 & $3.563 e-03$ & $4.460 e+08$ \\
\hline 19 & 240 & $6.016 \mathrm{e}-02$ & $1.256 \mathrm{e}+10$ & 19 & 241 & $8.089 e-02$ & $1.013 e+10$ & 19 & 243 & $0.000 e+00$ & $4.836 e+03$ \\
\hline 19 & 244 & $0.000 e+00$ & $3.056 e+03$ & 19 & 247 & $0.000 e+00$ & $8.658 e+05$ & 19 & 248 & $0.000 e+00$ & $2.130 \mathrm{e}+06$ \\
\hline 19 & 249 & $0.000 e+00$ & $8.769 e+05$ & 19 & 252 & $0.000 e+00$ & $1.480 e+05$ & 19 & 254 & $0.000 e+00$ & $6.153 e+05$ \\
\hline 19 & 262 & $0.000 e+00$ & $1.337 e+06$ & 19 & 263 & $0.000 e+00$ & $2.138 e+06$ & 19 & 264 & $0.000 e+00$ & $8.700 e+05$ \\
\hline 19 & 267 & $4.177 e-05$ & $5.363 e+06$ & 19 & 268 & $3.609 e-04$ & $3.310 e+07$ & 19 & 272 & $0.000 e+00$ & $2.459 \mathrm{e}+06$ \\
\hline 19 & 273 & $0.000 e+00$ & $6.269 e+06$ & 19 & 274 & $0.000 e+00$ & $8.719 e+06$ & 19 & 275 & $0.000 e+00$ & $2.402 \mathrm{e}+04$ \\
\hline 19 & 277 & $1.066 \mathrm{e}-03$ & $9.845 e+07$ & 20 & 31 & $4.821 \mathrm{e}-01$ & $3.412 \mathrm{e}+09$ & 20 & 37 & $4.468 e-03$ & $3.749 e+07$ \\
\hline 20 & 38 & $6.207 e-02$ & $8.879 e+08$ & 20 & 39 & $6.454 \mathrm{e}-01$ & $2.770 e+10$ & 20 & 40 & $3.024 e-01$ & $2.599 e+09$ \\
\hline 20 & 41 & $8.718 e-04$ & $5.527 e+07$ & 20 & 47 & $0.000 e+00$ & $9.876 e+04$ & 20 & 48 & $0.000 e+00$ & $1.116 \mathrm{e}+04$ \\
\hline 20 & 54 & $7.815 e-03$ & $2.747 e+08$ & 20 & 55 & $1.196 \mathrm{e}-02$ & $4.330 e+08$ & 20 & 56 & $1.781 \mathrm{e}-03$ & $3.894 \mathrm{e}+07$ \\
\hline 20 & 58 & $8.952 e-02$ & $1.015 \mathrm{e}+10$ & 20 & 59 & $3.729 e-02$ & $1.414 \mathrm{e}+09$ & 20 & 60 & $5.138 \mathrm{e}-02$ & $1.972 \mathrm{e}+09$ \\
\hline 20 & 61 & $1.042 \mathrm{e}-02$ & $2.401 \mathrm{e}+08$ & 20 & 63 & $2.731 \mathrm{e}-04$ & $3.566 e+07$ & 20 & 64 & $1.771 \mathrm{e}-03$ & $8.587 e+07$ \\
\hline 20 & 66 & $0.000 e+00$ & $2.882 \mathrm{e}+05$ & 20 & 67 & $0.000 e+00$ & $2.418 e+04$ & 20 & 68 & $0.000 e+00$ & $8.135 e+03$ \\
\hline 20 & 69 & $0.000 e+00$ & $3.824 \mathrm{e}+04$ & 20 & 70 & $0.000 e+00$ & $1.289 e+04$ & 20 & 72 & $0.000 e+00$ & $3.577 e+04$ \\
\hline 20 & 73 & $0.000 e+00$ & $5.358 e+03$ & 20 & 74 & $0.000 e+00$ & $9.210 e+04$ & 20 & 78 & $0.000 e+00$ & $1.457 e+05$ \\
\hline 20 & 86 & $1.345 \mathrm{e}+00$ & $5.014 e+10$ & 20 & 90 & $5.545 e-01$ & $2.125 e+10$ & 20 & 91 & $5.133 e-02$ & $3.283 e+09$ \\
\hline 20 & 92 & $7.784 \mathrm{e}-03$ & $3.015 e+08$ & 20 & 95 & $7.036 \mathrm{e}-01$ & $2.797 e+10$ & 20 & 96 & $8.697 e-02$ & $5.774 e+09$ \\
\hline 20 & 97 & $1.777 e-02$ & $7.294 e+08$ & 20 & 106 & $1.082 \mathrm{e}-02$ & $8.327 e+08$ & 20 & 107 & $1.887 e-01$ & $8.715 e+09$ \\
\hline 20 & 111 & $0.000 e+00$ & $4.050 e+04$ & 20 & 112 & $0.000 e+00$ & $5.387 e+03$ & 20 & 113 & $0.000 e+00$ & $1.595 e+04$ \\
\hline 20 & 114 & $0.000 e+00$ & $6.831 \mathrm{e}+03$ & 20 & 116 & $0.000 e+00$ & $2.923 e+05$ & 20 & 117 & $0.000 e+00$ & $2.206 e+04$ \\
\hline 20 & 118 & $0.000 e+00$ & $1.658 e+04$ & 20 & 119 & $0.000 \mathrm{e}+00$ & $1.275 \mathrm{e}+06$ & 20 & 122 & $0.000 e+00$ & $1.610 \mathrm{e}+05$ \\
\hline 20 & 123 & $0.000 e+00$ & $1.848 e+04$ & 20 & 125 & $7.412 e-03$ & $7.438 e+08$ & 20 & 126 & $8.133 e-04$ & $8.229 e+07$ \\
\hline 20 & 128 & $9.455 e-04$ & $5.751 e+07$ & 20 & 129 & $1.400 \mathrm{e}-02$ & $4.277 e+09$ & 20 & 130 & $9.108 \mathrm{e}-03$ & $9.316 e+08$ \\
\hline 20 & 132 & $7.857 e-04$ & $4.849 \mathrm{e}+07$ & 20 & 133 & $1.947 e-02$ & $2.011 e+09$ & 20 & 135 & $2.036 \mathrm{e}-04$ & $6.501 \mathrm{e}+07$ \\
\hline 20 & 136 & $0.000 e+00$ & $1.268 \mathrm{e}+05$ & 20 & 137 & $0.000 e+00$ & $1.832 \mathrm{e}+04$ & 20 & 138 & $0.000 e+00$ & $2.029 e+05$ \\
\hline 20 & 139 & $0.000 e+00$ & $4.721 \mathrm{e}+04$ & 20 & 140 & $0.000 e+00$ & $1.104 e+05$ & 20 & 141 & $0.000 e+00$ & $1.781 \mathrm{e}+04$ \\
\hline 20 & 143 & $0.000 e+00$ & $3.638 \mathrm{e}+05$ & 20 & 145 & $0.000 e+00$ & $4.755 e+04$ & 20 & 148 & $1.497 \mathrm{e}-01$ & $1.766 e+10$ \\
\hline 20 & 49 & $2.289 \mathrm{e}-02$ & $1.621 \mathrm{e}+09$ & 20 & 159 & $4.060 e-01$ & $2.910 e+10$ & 20 & 163 & $0.000 e+00$ & $1.567 e+06$ \\
\hline
\end{tabular}


Table III. Ca IX Oscillator Strengths and Radiative Decay Rates for transitions involving levels higher than 4.

\begin{tabular}{|c|c|c|c|c|c|c|c|c|c|c|c|}
\hline $\begin{array}{l}\text { Low. } \\
\text { Lev. }\end{array}$ & $\begin{array}{l}\text { - Upp } \\
\text {. Lev }\end{array}$ & $\begin{array}{l}\text { Osc. } \\
\text { Str. }\end{array}$ & $\begin{array}{l}\text { Rad. } \\
\text { Rate }\end{array}$ & $\begin{array}{l}\text { Low. } \\
\text { Lev. }\end{array}$ & $\begin{array}{l}\text { - Upp } \\
\text { Lev }\end{array}$ & $\begin{array}{l}\text { Osc. } \\
\text { Str. }\end{array}$ & $\begin{array}{l}\text { Rad. } \\
\text { Rate }\end{array}$ & $\begin{array}{l}\text { Low. } \\
\text { Lev. }\end{array}$ & $\begin{array}{l}\text {. Upp } \\
\text {. Lev }\end{array}$ & $\begin{array}{l}\text { Osc. } \\
\text { Str. }\end{array}$ & $\begin{array}{l}\text { Rad. } \\
\text { Rate }\end{array}$ \\
\hline$i$ & $j$ & $g f$ & $(1 / s)$ & i & $j$ & $g f$ & $(1 / s)$ & $i$ & j & $g f$ & $(1 / s)$ \\
\hline 20 & 171 & $5.736 e-02$ & $6.973 e+09$ & 20 & 173 & $2.064 \mathrm{e}-01$ & $1.510 \mathrm{e}+10$ & 20 & 174 & $3.335 e-02$ & $4.076 e+09$ \\
\hline 20 & 176 & $0.000 e+00$ & $9.719 e+05$ & 20 & 177 & $0.000 \mathrm{e}+00$ & $1.547 e+06$ & 20 & 178 & $0.000 e+00$ & $1.291 \mathrm{e}+06$ \\
\hline 20 & 180 & $9.162 \mathrm{e}-02$ & $6.782 \mathrm{e}+09$ & 20 & 183 & $6.650 \mathrm{e}-03$ & $4.938 e+08$ & 20 & 184 & $9.392 \mathrm{e}-03$ & $1.197 \mathrm{e}+09$ \\
\hline 20 & 185 & $8.060 e-02$ & $3.083 e+10$ & 20 & 186 & $4.121 \mathrm{e}-02$ & $3.153 e+09$ & 20 & 187 & $3.896 \mathrm{e}-04$ & $2.987 e+07$ \\
\hline 20 & 191 & $0.000 e+00$ & $4.928 \mathrm{e}+06$ & 20 & 197 & $0.000 e+00$ & $3.237 e+06$ & 20 & 200 & $0.000 e+00$ & $1.010 e+07$ \\
\hline 20 & 201 & $0.000 e+00$ & $1.092 \mathrm{e}+06$ & 20 & 202 & $0.000 e+00$ & $2.661 \mathrm{e}+04$ & 20 & 203 & $0.000 e+00$ & $2.612 \mathrm{e}+06$ \\
\hline 20 & 204 & $0.000 e+00$ & $3.438 e+06$ & 20 & 207 & $2.384 \mathrm{e}-05$ & $9.893 e+06$ & 20 & 210 & $1.994 \mathrm{e}-03$ & $3.494 e+08$ \\
\hline 20 & 211 & $5.278 \mathrm{e}-02$ & $5.549 e+09$ & 20 & 214 & $0.000 e+00$ & $6.080 e+03$ & 20 & 215 & $0.000 e+00$ & $2.791 e+04$ \\
\hline 20 & 216 & $0.000 e+00$ & $3.802 \mathrm{e}+05$ & 20 & 217 & $0.000 \mathrm{e}+00$ & $5.701 e+04$ & 20 & 219 & $0.000 e+00$ & $1.210 e+06$ \\
\hline 20 & 221 & $0.000 e+00$ & $1.923 e+05$ & 20 & 222 & $0.000 e+00$ & $1.285 e+04$ & 20 & 227 & $8.708 e-02$ & $1.768 e+10$ \\
\hline 20 & 228 & $1.201 \mathrm{e}-02$ & $1.463 e+09$ & 20 & 233 & $7.066 e-05$ & $1.439 e+07$ & 20 & 234 & $4.625 e-02$ & $9.489 e+09$ \\
\hline 20 & 235 & $6.003 e-02$ & $7.402 \mathrm{e}+09$ & 20 & 238 & $2.092 \mathrm{e}-03$ & $2.613 e+08$ & 20 & 239 & $3.920 e-02$ & $2.449 e+10$ \\
\hline 20 & 240 & $6.419 e-03$ & $1.337 e+09$ & 20 & 241 & $2.079 \mathrm{e}-02$ & $2.599 e+09$ & 20 & 247 & $0.000 e+00$ & $3.398 e+06$ \\
\hline 20 & 248 & $0.000 e+00$ & $1.231 \mathrm{e}+05$ & 20 & 252 & $0.000 \mathrm{e}+00$ & $1.058 e+06$ & 20 & 262 & $0.000 e+00$ & $3.944 e+06$ \\
\hline 20 & 264 & $0.000 e+00$ & $3.940 e+05$ & 20 & 267 & $4.591 \mathrm{e}-04$ & $5.881 e+07$ & 20 & 272 & $0.000 e+00$ & $2.090 e+06$ \\
\hline 20 & 273 & $0.000 e+00$ & $1.633 e+06$ & 20 & 278 & $5.837 e-04$ & $7.529 e+07$ & 20 & 281 & $1.142 \mathrm{e}-05$ & $7.394 e+06$ \\
\hline 21 & 38 & $4.685 e-01$ & $6.612 \mathrm{e}+09$ & 21 & 48 & $0.000 e+00$ & $2.430 \mathrm{e}+04$ & 21 & 55 & $8.819 e-04$ & $3.166 e+07$ \\
\hline 21 & 59 & $7.557 e-04$ & $2.841 e+07$ & 21 & 60 & $7.236 e-02$ & $2.754 e+09$ & 21 & 64 & $9.845 e-04$ & $4.737 e+07$ \\
\hline 21 & 67 & $0.000 e+00$ & $2.611 \mathrm{e}+04$ & 21 & 68 & $0.000 e+00$ & $2.264 e+04$ & 21 & 72 & $0.000 e+00$ & $3.674 e+04$ \\
\hline 21 & 74 & $0.000 e+00$ & $3.122 \mathrm{e}+04$ & 21 & 78 & $0.000 e+00$ & $7.200 e+04$ & 21 & 91 & $4.082 e-01$ & $2.594 e+10$ \\
\hline 21 & 96 & $5.097 e-01$ & $3.363 e+10$ & 21 & 106 & $5.529 e-02$ & $4.229 e+09$ & 21 & 113 & $0.000 e+00$ & $2.985 e+04$ \\
\hline 21 & 116 & $0.000 e+00$ & $4.290 e+04$ & 21 & 118 & $0.000 e+00$ & $6.300 e+05$ & 21 & 126 & $3.544 \mathrm{e}-04$ & $3.567 e+07$ \\
\hline 21 & 130 & $4.951 \mathrm{e}-04$ & $5.039 e+07$ & 21 & 133 & $1.733 \mathrm{e}-02$ & $1.782 \mathrm{e}+09$ & 21 & 137 & $0.000 e+00$ & $5.797 e+03$ \\
\hline 21 & 140 & $0.000 e+00$ & $6.956 \mathrm{e}+04$ & 21 & 143 & $0.000 e+00$ & $2.545 e+05$ & 21 & 148 & $5.524 e-02$ & $6.486 e+09$ \\
\hline 21 & 154 & $4.173 e-04$ & $4.933 e+07$ & 21 & 171 & $3.046 \mathrm{e}-02$ & $3.686 e+09$ & 21 & 174 & $2.148 \mathrm{e}-01$ & $2.613 e+10$ \\
\hline 21 & 177 & $0.000 e+00$ & $3.032 \mathrm{e}+06$ & 21 & 184 & $6.236 \mathrm{e}-02$ & $7.914 \mathrm{e}+09$ & 21 & 191 & $0.000 e+00$ & $9.188 e+05$ \\
\hline 21 & 202 & $0.000 e+00$ & $3.139 \mathrm{e}+06$ & 21 & 203 & $0.000 e+00$ & $2.498 e+06$ & 21 & 210 & $1.651 \mathrm{e}-02$ & $2.881 e+09$ \\
\hline 21 & 214 & $0.000 e+00$ & $4.543 e+03$ & 21 & 216 & $0.000 e+00$ & $3.852 e+04$ & 21 & 218 & $0.000 e+00$ & $6.371 \mathrm{e}+05$ \\
\hline 21 & 221 & $0.000 e+00$ & $9.106 \mathrm{e}+04$ & 21 & 227 & $3.153 e-02$ & $6.378 \mathrm{e}+09$ & 21 & 233 & $5.917 e-04$ & $1.200 \mathrm{e}+08$ \\
\hline 21 & 234 & $2.483 e-02$ & $5.075 e+09$ & 21 & 240 & $3.276 \mathrm{e}-02$ & $6.799 e+09$ & 21 & 247 & $0.000 e+00$ & $1.052 \mathrm{e}+06$ \\
\hline 21 & 263 & $0.000 e+00$ & $1.338 \mathrm{e}+06$ & 21 & 272 & $0.000 e+00$ & $1.388 e+06$ & 21 & 279 & $2.569 \mathrm{e}-04$ & $5.506 e+07$ \\
\hline 22 & 31 & $8.237 e-01$ & $5.693 e+09$ & 22 & 37 & $1.170 \mathrm{e}-03$ & $9.606 e+06$ & 22 & 38 & $4.229 \mathrm{e}-01$ & $5.922 \mathrm{e}+09$ \\
\hline 22 & 39 & $6.120 \mathrm{e}-03$ & $2.571 \mathrm{e}+08$ & 22 & 40 & $2.883 e-01$ & $2.425 e+09$ & 22 & 41 & $1.936 \mathrm{e}-04$ & $1.206 \mathrm{e}+07$ \\
\hline 22 & 47 & $0.000 e+00$ & $1.623 e+04$ & 22 & 48 & $0.000 e+00$ & $6.102 \mathrm{e}+04$ & 22 & 54 & $1.429 \mathrm{e}-03$ & $4.955 e+07$ \\
\hline 22 & 55 & $1.460 e-02$ & $5.215 e+08$ & 22 & 56 & $1.153 e-02$ & $2.487 e+08$ & 22 & 58 & $1.534 \mathrm{e}-02$ & $1.716 e+09$ \\
\hline 22 & 59 & $1.616 \mathrm{e}-02$ & $6.046 e+08$ & 22 & 60 & $1.173 e-01$ & $4.444 e+09$ & 22 & 61 & $2.886 \mathrm{e}-02$ & $6.564 e+08$ \\
\hline 22 & 62 & $2.205 \mathrm{e}-04$ & $5.296 \mathrm{e}+06$ & 22 & 63 & $5.160 e-05$ & $6.655 e+06$ & 22 & 64 & $1.120 \mathrm{e}-03$ & $5.366 e+07$ \\
\hline 22 & 67 & $0.000 e+00$ & $4.907 e+04$ & 22 & 68 & $0.000 e+00$ & $1.273 e+04$ & 22 & 69 & $0.000 e+00$ & $2.689 e+04$ \\
\hline 22 & 70 & $0.000 e+00$ & $5.600 e+04$ & 22 & 72 & $0.000 e+00$ & $1.173 e+04$ & 22 & 73 & $0.000 e+00$ & $1.053 e+04$ \\
\hline 22 & 74 & $0.000 e+00$ & $8.509 e+04$ & 22 & 75 & $0.000 e+00$ & $9.107 e+04$ & 22 & 77 & $0.000 e+00$ & $1.571 \mathrm{e}+04$ \\
\hline 22 & 78 & $0.000 e+00$ & $1.488 \mathrm{e}+04$ & 22 & 79 & $0.000 \mathrm{e}+00$ & $2.155 e+05$ & 22 & 86 & $1.256 \mathrm{e}+00$ & $4.634 e+10$ \\
\hline 22 & 90 & $4.225 e-01$ & $1.603 e+10$ & 22 & 91 & $3.785 e-01$ & $2.396 e+10$ & 22 & 92 & $1.542 \mathrm{e}-03$ & $5.912 \mathrm{e}+07$ \\
\hline 22 & 95 & $3.191 \mathrm{e}-01$ & $1.256 \mathrm{e}+10$ & 22 & 96 & $3.994 \mathrm{e}-01$ & $2.626 e+10$ & 22 & 106 & $2.254 \mathrm{e}-01$ & $1.718 e+10$ \\
\hline 22 & 112 & $0.000 e+00$ & $3.714 \mathrm{e}+04$ & 22 & 113 & $0.000 e+00$ & $1.505 e+04$ & 22 & 114 & $0.000 e+00$ & $1.530 e+04$ \\
\hline
\end{tabular}


Table III. Ca IX Oscillator Strengths and Radiative Decay Rates for transitions involving levels higher than 4.

\begin{tabular}{|c|c|c|c|c|c|c|c|c|c|c|c|}
\hline $\begin{array}{l}\text { Low. } \\
\text { Lev }\end{array}$ & $\begin{array}{l}\text { Upp } \\
\text {. Lev }\end{array}$ & $\begin{array}{l}\text { Osc. } \\
\text { Str. }\end{array}$ & $\begin{array}{l}\text { Rad. } \\
\text { Rate }\end{array}$ & $\begin{array}{l}\text { Low. } \\
\text { Lev. }\end{array}$ & $\begin{array}{l}\text { - Upp } \\
\text {. Lev }\end{array}$ & $\begin{array}{l}\text { Osc. } \\
\text { Str. }\end{array}$ & $\begin{array}{l}\text { Rad. } \\
\text { Rate }\end{array}$ & $\begin{array}{l}\text { Low } \\
\text { Lev }\end{array}$ & $\begin{array}{l}\text { - Upp } \\
\text {. Lev }\end{array}$ & $\begin{array}{l}\text { Osc. } \\
\text { Str. }\end{array}$ & $\begin{array}{l}\text { Rad. } \\
\text { Rate }\end{array}$ \\
\hline$i$ & $\mathrm{j}$ & $g f$ & $(1 / s)$ & $i$ & $j$ & $g f$ & $(1 / s)$ & $i$ & j & $g f$ & $(1 / s)$ \\
\hline 22 & 115 & $0.000 e+00$ & $6.885 e+05$ & 22 & 116 & $0.000 \mathrm{e}+00$ & $2.044 e+04$ & 22 & 118 & $0.000 e+00$ & $1.685 e+06$ \\
\hline 22 & 119 & $0.000 e+00$ & $5.379 e+03$ & 22 & 122 & $0.000 e+00$ & $2.449 e+05$ & 22 & 123 & $0.000 e+00$ & $7.691 \mathrm{e}+04$ \\
\hline 22 & 125 & $2.270 \mathrm{e}-03$ & $2.259 \mathrm{e}+08$ & 22 & 126 & $2.661 \mathrm{e}-03$ & $2.671 \mathrm{e}+08$ & 22 & 128 & $4.081 \mathrm{e}-03$ & $2.462 \mathrm{e}+08$ \\
\hline 22 & 129 & $3.247 e-03$ & $9.836 e+08$ & 22 & 130 & $7.545 e-03$ & $7.656 e+08$ & 22 & 132 & $7.116 \mathrm{e}-03$ & $4.357 e+08$ \\
\hline 22 & 133 & $2.518 \mathrm{e}-02$ & $2.580 e+09$ & 22 & 135 & $6.279 \mathrm{e}-05$ & $1.989 e+07$ & 22 & 136 & $0.000 e+00$ & $2.115 \mathrm{e}+04$ \\
\hline 22 & 137 & $0.000 e+00$ & $6.322 \mathrm{e}+03$ & 22 & 138 & $0.000 \mathrm{e}+00$ & $6.925 e+03$ & 22 & 139 & $0.000 e+00$ & $4.521 \mathrm{e}+03$ \\
\hline 22 & 140 & $0.000 e+00$ & $1.003 e+05$ & 22 & 141 & $0.000 \mathrm{e}+00$ & $1.975 e+05$ & 22 & 143 & $0.000 e+00$ & $8.201 e+04$ \\
\hline 22 & 145 & $0.000 e+00$ & $4.434 e+05$ & 22 & 148 & $3.125 \mathrm{e}-02$ & $3.660 e+09$ & 22 & 149 & $1.497 \mathrm{e}-01$ & $1.052 \mathrm{e}+10$ \\
\hline 22 & 154 & $1.029 \mathrm{e}-04$ & $1.213 e+07$ & 22 & 159 & $3.260 e-01$ & $2.320 e+10$ & 22 & 163 & $0.000 e+00$ & $7.075 e+05$ \\
\hline 22 & 171 & $3.381 \mathrm{e}-02$ & $4.080 e+09$ & 22 & 173 & $2.464 \mathrm{e}-01$ & $1.790 e+10$ & 22 & 174 & $1.782 \mathrm{e}-01$ & $2.163 e+10$ \\
\hline 22 & 176 & $0.000 e+00$ & $1.353 e+06$ & 22 & 177 & $0.000 e+00$ & $1.545 e+06$ & 22 & 178 & $0.000 e+00$ & $5.315 e+05$ \\
\hline 22 & 180 & $6.720 \mathrm{e}-02$ & $4.938 e+09$ & 22 & 183 & $3.820 e-03$ & $2.816 e+08$ & 22 & 184 & $5.272 \mathrm{e}-02$ & $6.673 e+09$ \\
\hline 22 & 185 & $1.544 \mathrm{e}-03$ & $5.864 e+08$ & 22 & 186 & $3.616 \mathrm{e}-02$ & $2.747 e+09$ & 22 & 187 & $1.760 e-03$ & $1.340 e+08$ \\
\hline 22 & 191 & $0.000 e+00$ & $1.761 \mathrm{e}+06$ & 22 & 193 & $0.000 e+00$ & $3.475 e+06$ & 22 & 197 & $0.000 e+00$ & $4.885 e+06$ \\
\hline 22 & 200 & $0.000 e+00$ & $1.553 e+05$ & 22 & 201 & $0.000 e+00$ & $2.258 e+06$ & 22 & 202 & $0.000 e+00$ & $3.127 e+06$ \\
\hline 22 & 203 & $0.000 e+00$ & $3.342 e+06$ & 22 & 204 & $0.000 e+00$ & $3.224 e+04$ & 22 & 210 & $5.879 e-02$ & $1.024 \mathrm{e}+10$ \\
\hline 22 & 211 & $2.574 \mathrm{e}-04$ & $2.690 \mathrm{e}+07$ & 22 & 214 & $0.000 e+00$ & $1.208 e+04$ & 22 & 215 & $0.000 e+00$ & $8.431 \mathrm{e}+05$ \\
\hline 22 & 216 & $0.000 e+00$ & $2.113 e+05$ & 22 & 218 & $0.000 e+00$ & $1.472 \mathrm{e}+06$ & 22 & 219 & $0.000 e+00$ & $2.022 \mathrm{e}+04$ \\
\hline 22 & 221 & $0.000 e+00$ & $2.563 e+04$ & 22 & 222 & $0.000 e+00$ & $1.719 e+05$ & 22 & 227 & $1.831 \mathrm{e}-02$ & $3.695 e+09$ \\
\hline 22 & 228 & $8.906 e-02$ & $1.079 \mathrm{e}+10$ & 22 & 233 & $2.125 e-04$ & $4.302 \mathrm{e}+07$ & 22 & 234 & $2.740 e-02$ & $5.590 e+09$ \\
\hline 22 & 235 & $1.010 \mathrm{e}-01$ & $1.239 \mathrm{e}+10$ & 22 & 238 & $1.307 \mathrm{e}-03$ & $1.623 e+08$ & 22 & 239 & $1.918 \mathrm{e}-03$ & $1.192 \mathrm{e}+09$ \\
\hline 22 & 240 & $2.341 \mathrm{e}-02$ & $4.849 \mathrm{e}+09$ & 22 & 241 & $1.768 e-02$ & $2.198 e+09$ & 22 & 247 & $0.000 e+00$ & $5.359 e+05$ \\
\hline 22 & 248 & $0.000 e+00$ & $2.445 \mathrm{e}+06$ & 22 & 252 & $0.000 e+00$ & $1.655 \mathrm{e}+06$ & 22 & 262 & $0.000 e+00$ & $2.583 e+04$ \\
\hline 22 & 263 & $0.000 e+00$ & $1.598 \mathrm{e}+06$ & 22 & 264 & $0.000 e+00$ & $6.994 e+05$ & 22 & 267 & $7.139 \mathrm{e}-04$ & $9.094 \mathrm{e}+07$ \\
\hline 22 & 272 & $0.000 e+00$ & $1.468 \mathrm{e}+06$ & 22 & 273 & $0.000 e+00$ & $2.466 e+05$ & 22 & 278 & $6.609 \mathrm{e}-05$ & $8.478 e+06$ \\
\hline 22 & 279 & $4.658 \mathrm{e}-04$ & $9.961 \mathrm{e}+07$ & 23 & 31 & $1.740 \mathrm{e}-01$ & $1.197 e+09$ & 23 & 32 & $1.584 \mathrm{e}+00$ & $7.792 \mathrm{e}+09$ \\
\hline 23 & 37 & $2.787 e-03$ & $2.278 e+07$ & 23 & 38 & $5.757 e-02$ & $8.027 e+08$ & 23 & 40 & $8.150 e-01$ & $6.826 e+09$ \\
\hline 23 & 46 & $0.000 e+00$ & $5.124 \mathrm{e}+04$ & 23 & 48 & $0.000 e+00$ & $1.069 e+05$ & 23 & 54 & $1.325 e-03$ & $4.583 e+07$ \\
\hline 23 & 55 & $9.069 e-03$ & $3.231 \mathrm{e}+08$ & 23 & 56 & $3.419 \mathrm{e}-02$ & $7.356 e+08$ & 23 & 57 & $1.850 e-02$ & $2.890 e+08$ \\
\hline 23 & 59 & $1.836 \mathrm{e}-02$ & $6.851 \mathrm{e}+08$ & 23 & 60 & $1.119 \mathrm{e}-01$ & $4.226 e+09$ & 23 & 61 & $1.382 \mathrm{e}-01$ & $3.136 e+09$ \\
\hline 23 & 62 & $1.101 \mathrm{e}-03$ & $2.639 \mathrm{e}+07$ & 23 & 64 & $9.596 \mathrm{e}-04$ & $4.588 e+07$ & 23 & 66 & $0.000 e+00$ & $3.234 \mathrm{e}+04$ \\
\hline 23 & 68 & $0.000 e+00$ & $1.415 \mathrm{e}+04$ & 23 & 69 & $0.000 e+00$ & $9.892 \mathrm{e}+04$ & 23 & 70 & $0.000 e+00$ & $4.837 e+04$ \\
\hline 23 & 71 & $0.000 e+00$ & $1.619 \mathrm{e}+05$ & 23 & 72 & $0.000 e+00$ & $9.685 e+03$ & 23 & 74 & $0.000 e+00$ & $4.766 e+04$ \\
\hline 23 & 75 & $0.000 e+00$ & $1.528 \mathrm{e}+05$ & 23 & 76 & $0.000 e+00$ & $6.663 e+04$ & 23 & 77 & $0.000 e+00$ & $1.769 \mathrm{e}+04$ \\
\hline 23 & 78 & $0.000 e+00$ & $1.946 \mathrm{e}+04$ & 23 & 79 & $0.000 e+00$ & $1.342 \mathrm{e}+05$ & 23 & 80 & $0.000 e+00$ & $5.750 e+05$ \\
\hline 23 & 82 & $3.007 e-02$ & $7.728 e+08$ & 23 & 84 & $1.901 \mathrm{e}-01$ & $4.942 e+09$ & 23 & 86 & $2.127 e-01$ & $7.834 e+09$ \\
\hline 23 & 87 & $2.203 e+00$ & $5.820 e+10$ & 23 & 89 & $6.201 \mathrm{e}-01$ & $1.674 \mathrm{e}+10$ & 23 & 90 & $5.691 \mathrm{e}-01$ & $2.154 e+10$ \\
\hline 23 & 91 & $6.191 \mathrm{e}-02$ & $3.912 \mathrm{e}+09$ & 23 & 94 & $2.895 \mathrm{e}-01$ & $8.108 e+09$ & 23 & 95 & $4.022 e-01$ & $1.580 \mathrm{e}+10$ \\
\hline 23 & 96 & $5.694 \mathrm{e}-02$ & $3.736 \mathrm{e}+09$ & 23 & 97 & $2.154 \mathrm{e}-03$ & $8.740 e+07$ & 23 & 102 & $1.380 e-03$ & $4.168 e+07$ \\
\hline 23 & 106 & $7.166 \mathrm{e}-02$ & $5.452 e+09$ & 23 & 107 & $3.325 e-01$ & $1.518 e+10$ & 23 & 108 & $5.705 e-04$ & $1.863 e+07$ \\
\hline 23 & 109 & $3.036 \mathrm{e}-04$ & $1.426 \mathrm{e}+07$ & 23 & 111 & $0.000 e+00$ & $1.240 \mathrm{e}+04$ & 23 & 113 & $0.000 e+00$ & $5.350 e+04$ \\
\hline 23 & 115 & $0.000 e+00$ & $8.879 e+05$ & 23 & 116 & $0.000 e+00$ & $6.865 e+05$ & 23 & 118 & $0.000 e+00$ & $5.530 e+05$ \\
\hline 23 & 119 & $0.000 e+00$ & $1.339 \mathrm{e}+06$ & 23 & 121 & $0.000 e+00$ & $6.931 e+05$ & 23 & 122 & $0.000 e+00$ & $2.547 e+04$ \\
\hline 23 & 123 & $0.000 e+00$ & $2.928 \mathrm{e}+05$ & 23 & 125 & $1.742 \mathrm{e}-03$ & $1.731 \mathrm{e}+08$ & 23 & 126 & $6.056 \mathrm{e}-03$ & $6.069 \mathrm{e}+08$ \\
\hline
\end{tabular}


Table III. Ca IX Oscillator Strengths and Radiative Decay Rates for transitions involving levels higher than 4.

\begin{tabular}{|c|c|c|c|c|c|c|c|c|c|c|c|}
\hline $\begin{array}{l}\text { Low. } \\
\text { Lev. }\end{array}$ & $\begin{array}{l}\text { Upp } \\
\text { Lev }\end{array}$ & $\begin{array}{l}\text { Osc. } \\
\text { Str. }\end{array}$ & $\begin{array}{l}\text { Rad. } \\
\text { Rate }\end{array}$ & $\begin{array}{l}\text { Low. } \\
\text { Lev. }\end{array}$ & $\begin{array}{l}\text { Upp } \\
\text { Lev }\end{array}$ & $\begin{array}{l}\text { Osc. } \\
\text { Str. }\end{array}$ & $\begin{array}{l}\text { Rad. } \\
\text { Rate }\end{array}$ & $\begin{array}{l}\text { Low. } \\
\text { Lev. }\end{array}$ & $\begin{array}{l}\text {. Upp } \\
\text {. Lev }\end{array}$ & $\begin{array}{l}\text { Osc. } \\
\text { Str. }\end{array}$ & $\begin{array}{l}\text { Rad. } \\
\text { Rate }\end{array}$ \\
\hline$i$ & $j$ & $g f$ & $(1 / s)$ & $i$ & $j$ & $g f$ & $(1 / s)$ & $i$ & j & $g f$ & $(1 / s)$ \\
\hline 23 & 128 & $9.770 e-03$ & $5.886 e+08$ & 23 & 130 & $2.701 \mathrm{e}-03$ & $2.737 e+08$ & 23 & 131 & $9.526 \mathrm{e}-03$ & $4.142 e+08$ \\
\hline 23 & 132 & $3.220 e-02$ & $1.968 \mathrm{e}+09$ & 23 & 133 & $2.373 e-02$ & $2.429 e+09$ & 23 & 136 & $0.000 e+00$ & $2.678 e+04$ \\
\hline 23 & 137 & $0.000 e+00$ & $1.814 \mathrm{e}+04$ & 23 & 138 & $0.000 e+00$ & $6.642 \mathrm{e}+03$ & 23 & 140 & $0.000 e+00$ & $9.357 e+04$ \\
\hline 23 & 141 & $0.000 e+00$ & $2.635 \mathrm{e}+05$ & 23 & 142 & $0.000 e+00$ & $1.528 e+05$ & 23 & 143 & $0.000 e+00$ & $1.551 \mathrm{e}+04$ \\
\hline 23 & 144 & $1.853 e-04$ & $9.185 e+06$ & 23 & 145 & $0.000 e+00$ & $2.638 \mathrm{e}+05$ & 23 & 146 & $0.000 e+00$ & $1.029 \mathrm{e}+06$ \\
\hline 23 & 148 & $2.858 e-02$ & $3.342 \mathrm{e}+09$ & 23 & 149 & $1.128 \mathrm{e}-01$ & $7.917 e+09$ & 23 & 150 & $1.678 \mathrm{e}-01$ & $8.413 e+09$ \\
\hline 23 & 151 & $1.041 \mathrm{e}-02$ & $5.233 e+08$ & 23 & 154 & $9.331 e-05$ & $1.099 e+07$ & 23 & 155 & $1.824 \mathrm{e}-03$ & $9.228 e+07$ \\
\hline 23 & 157 & $0.000 e+00$ & $1.099 \mathrm{e}+05$ & 23 & 158 & $3.863 e-01$ & $1.958 e+10$ & 23 & 159 & $4.767 e-02$ & $3.387 e+09$ \\
\hline 23 & 162 & $0.000 e+00$ & $3.749 e+05$ & 23 & 163 & $0.000 e+00$ & $2.070 e+05$ & 23 & 164 & $2.530 \mathrm{e}-01$ & $1.295 e+10$ \\
\hline 23 & 167 & $0.000 e+00$ & $6.656 e+05$ & 23 & 171 & $2.898 \mathrm{e}-02$ & $3.493 e+09$ & 23 & 172 & $3.227 e-01$ & $1.668 \mathrm{e}+10$ \\
\hline 23 & 173 & $2.495 \mathrm{e}-01$ & $1.809 \mathrm{e}+10$ & 23 & 174 & $2.673 e-02$ & $3.239 e+09$ & 23 & 175 & $0.000 e+00$ & $1.551 e+06$ \\
\hline 23 & 176 & $0.000 e+00$ & $7.190 e+05$ & 23 & 177 & $0.000 e+00$ & $2.290 e+05$ & 23 & 178 & $0.000 e+00$ & $2.779 e+05$ \\
\hline 23 & 180 & $6.850 \mathrm{e}-03$ & $5.026 e+08$ & 23 & 181 & $1.424 \mathrm{e}-01$ & $7.467 e+09$ & 23 & 183 & $4.793 e-03$ & $3.529 e+08$ \\
\hline 23 & 184 & $4.984 \mathrm{e}-03$ & $6.300 e+08$ & 23 & 186 & $9.983 e-02$ & $7.572 e+09$ & 23 & 187 & $4.818 e-03$ & $3.663 e+08$ \\
\hline 23 & 190 & $0.000 e+00$ & $1.268 e+05$ & 23 & 191 & $0.000 e+00$ & $2.650 e+06$ & 23 & 192 & $0.000 e+00$ & $3.156 e+06$ \\
\hline 23 & 193 & $0.000 e+00$ & $2.816 \mathrm{e}+06$ & 23 & 197 & $0.000 e+00$ & $2.122 \mathrm{e}+06$ & 23 & 199 & $0.000 e+00$ & $7.392 e+06$ \\
\hline 23 & 200 & $0.000 e+00$ & $1.370 \mathrm{e}+06$ & 23 & 201 & $0.000 e+00$ & $5.021 e+06$ & 23 & 202 & $0.000 e+00$ & $5.171 e+04$ \\
\hline 23 & 203 & $0.000 e+00$ & $3.199 \mathrm{e}+06$ & 23 & 204 & $0.000 e+00$ & $6.816 e+05$ & 23 & 210 & $1.795 \mathrm{e}-02$ & $3.123 e+09$ \\
\hline 23 & 211 & $8.458 e-02$ & $8.829 e+09$ & 23 & 213 & $3.544 e-04$ & $3.724 e+07$ & 23 & 214 & $0.000 e+00$ & $1.210 e+04$ \\
\hline 23 & 215 & $0.000 e+00$ & $1.241 \mathrm{e}+06$ & 23 & 216 & $0.000 e+00$ & $7.509 e+05$ & 23 & 217 & $0.000 e+00$ & $1.335 e+05$ \\
\hline 23 & 218 & $0.000 e+00$ & $5.064 e+05$ & 23 & 219 & $0.000 e+00$ & $1.149 e+06$ & 23 & 220 & $0.000 e+00$ & $3.971 \mathrm{e}+03$ \\
\hline 23 & 222 & $0.000 e+00$ & $7.450 \mathrm{e}+04$ & 23 & 223 & $0.000 e+00$ & $4.130 e+05$ & 23 & 226 & $4.710 e-04$ & $4.055 e+07$ \\
\hline 23 & 227 & $1.640 \mathrm{e}-02$ & $3.306 e+09$ & 23 & 228 & $6.545 e-02$ & $7.918 e+09$ & 23 & 229 & $9.976 e-02$ & $8.624 \mathrm{e}+09$ \\
\hline 23 & 230 & $2.257 e-04$ & $1.954 \mathrm{e}+07$ & 23 & 233 & $8.654 \mathrm{e}-05$ & $1.750 e+07$ & 23 & 234 & $2.297 e-02$ & $4.682 \mathrm{e}+09$ \\
\hline 23 & 235 & $2.316 \mathrm{e}-02$ & $2.837 e+09$ & 23 & 236 & $1.945 \mathrm{e}-01$ & $1.702 \mathrm{e}+10$ & 23 & 238 & $2.769 e-03$ & $3.435 \mathrm{e}+08$ \\
\hline 23 & 240 & $6.292 \mathrm{e}-04$ & $1.302 \mathrm{e}+08$ & 23 & 241 & $4.606 \mathrm{e}-02$ & $5.719 e+09$ & 23 & 243 & $0.000 e+00$ & $5.255 e+03$ \\
\hline 23 & 244 & $0.000 e+00$ & $7.464 \mathrm{e}+03$ & 23 & 247 & $0.000 e+00$ & $1.167 \mathrm{e}+06$ & 23 & 248 & $0.000 \mathrm{e}+00$ & $1.151 \mathrm{e}+06$ \\
\hline 23 & 249 & $0.000 e+00$ & $2.221 \mathrm{e}+06$ & 23 & 252 & $0.000 e+00$ & $6.064 e+05$ & 23 & 254 & $0.000 e+00$ & $2.367 e+06$ \\
\hline 23 & 262 & $0.000 e+00$ & $7.670 \mathrm{e}+05$ & 23 & 263 & $0.000 e+00$ & $1.210 \mathrm{e}+05$ & 23 & 264 & $0.000 e+00$ & $2.057 \mathrm{e}+06$ \\
\hline 23 & 267 & $1.537 \mathrm{e}-04$ & $1.956 \mathrm{e}+07$ & 23 & 268 & $1.363 e-03$ & $1.239 e+08$ & 23 & 272 & $0.000 e+00$ & $1.176 \mathrm{e}+06$ \\
\hline 23 & 273 & $0.000 e+00$ & $6.829 \mathrm{e}+05$ & 23 & 274 & $0.000 e+00$ & $4.097 e+05$ & 23 & 275 & $0.000 e+00$ & $1.734 \mathrm{e}+04$ \\
\hline 23 & 277 & $4.637 e-05$ & $4.243 e+06$ & 23 & 278 & $6.097 e-04$ & $7.812 \mathrm{e}+07$ & 23 & 279 & $1.024 \mathrm{e}-04$ & $2.187 e+07$ \\
\hline 24 & 31 & $5.088 e-03$ & $3.498 e+07$ & 24 & 32 & $2.030 e-01$ & $9.978 e+08$ & 24 & 34 & $2.826 e+00$ & $1.083 e+10$ \\
\hline 24 & 36 & $1.299 \mathrm{e}-03$ & $5.888 e+06$ & 24 & 37 & $6.138 \mathrm{e}-03$ & $5.015 e+07$ & 24 & 40 & $7.436 e-01$ & $6.224 e+09$ \\
\hline 24 & 47 & $0.000 e+00$ & $7.601 \mathrm{e}+04$ & 24 & 48 & $0.000 e+00$ & $1.155 e+05$ & 24 & 56 & $1.963 e-02$ & $4.221 \mathrm{e}+08$ \\
\hline 24 & 57 & $1.153 e-01$ & $1.801 \mathrm{e}+09$ & 24 & 61 & $3.108 e-01$ & $7.048 e+09$ & 24 & 62 & $3.722 \mathrm{e}-03$ & $8.916 e+07$ \\
\hline 24 & 67 & $0.000 e+00$ & $1.292 \mathrm{e}+04$ & 24 & 68 & $0.000 e+00$ & $6.043 e+04$ & 24 & 69 & $0.000 e+00$ & $2.007 e+05$ \\
\hline 24 & 70 & $0.000 e+00$ & $5.858 e+04$ & 24 & 72 & $0.000 e+00$ & $9.686 e+04$ & 24 & 73 & $0.000 e+00$ & $5.439 e+03$ \\
\hline 24 & 75 & $0.000 \mathrm{e}+00$ & $3.211 \mathrm{e}+03$ & 24 & 76 & $0.000 \mathrm{e}+00$ & $2.239 e+05$ & 24 & 77 & $0.000 e+00$ & $1.841 \mathrm{e}+04$ \\
\hline 24 & 78 & $0.000 e+00$ & $1.785 e+05$ & 24 & 79 & $0.000 e+00$ & $1.500 e+05$ & 24 & 82 & $1.174 \mathrm{e}-02$ & $3.016 e+08$ \\
\hline 24 & 83 & $3.412 \mathrm{e}-01$ & $6.877 e+09$ & 24 & 84 & $5.390 e-03$ & $1.401 \mathrm{e}+08$ & 24 & 86 & $4.779 e-03$ & $1.760 \mathrm{e}+08$ \\
\hline 24 & 87 & $2.591 \mathrm{e}-01$ & $6.841 \mathrm{e}+09$ & 24 & 88 & $4.929 e+00$ & $1.015 e+11$ & 24 & 89 & $5.825 e-01$ & $1.571 \mathrm{e}+10$ \\
\hline 24 & 90 & $5.725 e-02$ & $2.167 e+09$ & 24 & 93 & $2.163 e-02$ & $4.634 e+08$ & 24 & 94 & $2.592 \mathrm{e}-01$ & $7.255 e+09$ \\
\hline 24 & 95 & $3.491 \mathrm{e}-02$ & $1.371 \mathrm{e}+09$ & 24 & 97 & $3.951 \mathrm{e}-04$ & $1.603 e+07$ & 24 & 102 & $2.578 e-04$ & $7.782 e+06$ \\
\hline
\end{tabular}


Table III. Ca IX Oscillator Strengths and Radiative Decay Rates for transitions involving levels higher than 4.

\begin{tabular}{|c|c|c|c|c|c|c|c|c|c|c|c|}
\hline $\begin{array}{l}\text { Low. } \\
\text { Lev . }\end{array}$ & $\begin{array}{l}\text { Upp } \\
\text { Lev }\end{array}$ & $\begin{array}{l}\text { Osc. } \\
\text { Str. }\end{array}$ & $\begin{array}{l}\text { Rad. } \\
\text { Rate }\end{array}$ & $\begin{array}{l}\text { Low. } \\
\text { Lev. }\end{array}$ & $\begin{array}{l}\text { Upp. } \\
\text { Lev. }\end{array}$ & $\begin{array}{l}\text { Osc. } \\
\text { Str. }\end{array}$ & $\begin{array}{l}\text { Rad. } \\
\text { Rate }\end{array}$ & $\begin{array}{l}\text { Low. } \\
\text { Lev. }\end{array}$ & $\begin{array}{l}\text { Upp } \\
\text { Lev }\end{array}$ & $\begin{array}{l}\text { Osc. } \\
\text { Str. }\end{array}$ & $\begin{array}{l}\text { Rad. } \\
\text { Rate }\end{array}$ \\
\hline$i$ & $j$ & $g f$ & $(1 / s)$ & $i$ & $j$ & $g f$ & $(1 / s)$ & $i$ & j & $g f$ & $(1 / s)$ \\
\hline 24 & 103 & $3.502 \mathrm{e}-03$ & $8.226 e+07$ & 24 & 107 & $8.218 e-02$ & $3.752 \mathrm{e}+09$ & 24 & 108 & $5.264 \mathrm{e}-01$ & $1.718 e+10$ \\
\hline 24 & 115 & $0.000 e+00$ & $1.176 e+05$ & 24 & 116 & $0.000 e+00$ & $6.812 e+05$ & 24 & 117 & $0.000 e+00$ & $1.123 e+06$ \\
\hline 24 & 118 & $0.000 e+00$ & $1.442 \mathrm{e}+04$ & 24 & 119 & $0.000 e+00$ & $2.859 e+05$ & 24 & 120 & $0.000 e+00$ & $1.457 e+06$ \\
\hline 24 & 122 & $0.000 e+00$ & $4.355 e+05$ & 24 & 123 & $0.000 e+00$ & $4.135 e+05$ & 24 & 128 & $1.876 e-02$ & $1.130 \mathrm{e}+09$ \\
\hline 24 & 131 & $5.599 e-02$ & $2.434 \mathrm{e}+09$ & 24 & 132 & $4.173 e-02$ & $2.550 e+09$ & 24 & 134 & $1.681 \mathrm{e}-03$ & $1.047 e+08$ \\
\hline 24 & 136 & $0.000 e+00$ & $2.267 e+04$ & 24 & 139 & $0.000 e+00$ & $1.250 \mathrm{e}+05$ & 24 & 141 & $0.000 e+00$ & $3.235 e+04$ \\
\hline 24 & 142 & $0.000 e+00$ & $4.882 e+05$ & 24 & 143 & $0.000 e+00$ & $2.308 e+05$ & 24 & 144 & $1.404 \mathrm{e}-03$ & $6.958 e+07$ \\
\hline 24 & 145 & $0.000 e+00$ & $2.102 \mathrm{e}+05$ & 24 & 149 & $4.597 e-02$ & $3.225 e+09$ & 24 & 150 & $3.904 e-01$ & $1.958 e+10$ \\
\hline 24 & 151 & $4.661 \mathrm{e}-03$ & $2.343 e+08$ & 24 & 152 & $3.100 e-02$ & $1.213 e+09$ & 24 & 155 & $2.815 e-03$ & $1.424 \mathrm{e}+08$ \\
\hline 24 & 157 & $0.000 e+00$ & $3.334 e+04$ & 24 & 158 & $2.803 e-02$ & $1.421 e+09$ & 24 & 159 & $8.088 e-04$ & $5.746 e+07$ \\
\hline 24 & 160 & $0.000 e+00$ & $6.136 e+05$ & 24 & 161 & $7.844 \mathrm{e}-01$ & $3.097 e+10$ & 24 & 162 & $0.000 e+00$ & $9.636 e+04$ \\
\hline 24 & 163 & $0.000 e+00$ & $1.952 \mathrm{e}+04$ & 24 & 164 & $3.704 \mathrm{e}-02$ & $1.895 e+09$ & 24 & 165 & $7.248 e-01$ & $2.888 e+10$ \\
\hline 24 & 167 & $0.000 e+00$ & $2.040 e+05$ & 24 & 168 & $0.000 e+00$ & $1.554 e+06$ & 24 & 170 & $0.000 e+00$ & $4.762 e+05$ \\
\hline 24 & 172 & $2.148 e-01$ & $1.110 \mathrm{e}+10$ & 24 & 173 & $2.161 \mathrm{e}-02$ & $1.567 e+09$ & 24 & 175 & $0.000 e+00$ & $2.585 e+05$ \\
\hline 24 & 176 & $0.000 e+00$ & $5.526 e+04$ & 24 & 177 & $0.000 e+00$ & $6.410 e+03$ & 24 & 178 & $0.000 e+00$ & $1.437 e+04$ \\
\hline 24 & 179 & $2.672 \mathrm{e}-02$ & $1.086 e+09$ & 24 & 181 & $1.286 \mathrm{e}-02$ & $6.744 e+08$ & 24 & 182 & $2.694 \mathrm{e}-01$ & $1.099 \mathrm{e}+10$ \\
\hline 24 & 183 & $2.761 \mathrm{e}-04$ & $2.032 \mathrm{e}+07$ & 24 & 186 & $8.391 \mathrm{e}-02$ & $6.364 e+09$ & 24 & 187 & $2.700 \mathrm{e}-03$ & $2.052 e+08$ \\
\hline 24 & 188 & $4.339 e-04$ & $1.843 e+07$ & 24 & 189 & $0.000 e+00$ & $1.603 e+04$ & 24 & 190 & $0.000 e+00$ & $5.515 e+05$ \\
\hline 24 & 191 & $0.000 e+00$ & $3.314 e+05$ & 24 & 192 & $0.000 e+00$ & $7.866 e+06$ & 24 & 193 & $0.000 e+00$ & $2.828 e+06$ \\
\hline 24 & 194 & $0.000 e+00$ & $1.224 e+04$ & 24 & 197 & $0.000 e+00$ & $7.864 e+04$ & 24 & 198 & $0.000 e+00$ & $1.105 e+07$ \\
\hline 24 & 199 & $0.000 e+00$ & $1.700 e+06$ & 24 & 200 & $0.000 e+00$ & $3.097 e+05$ & 24 & 201 & $0.000 e+00$ & $4.687 e+06$ \\
\hline 24 & 202 & $0.000 e+00$ & $2.521 \mathrm{e}+06$ & 24 & 203 & $0.000 e+00$ & $1.628 \mathrm{e}+06$ & 24 & 204 & $0.000 e+00$ & $1.359 \mathrm{e}+06$ \\
\hline 24 & 211 & $2.023 e-02$ & $2.111 \mathrm{e}+09$ & 24 & 212 & $1.304 \mathrm{e}-01$ & $9.723 e+09$ & 24 & 214 & $0.000 e+00$ & $9.611 \mathrm{e}+03$ \\
\hline 24 & 215 & $0.000 e+00$ & $1.602 \mathrm{e}+05$ & 24 & 216 & $0.000 e+00$ & $9.095 e+05$ & 24 & 217 & $0.000 e+00$ & $1.512 \mathrm{e}+06$ \\
\hline 24 & 218 & $0.000 e+00$ & $1.942 \mathrm{e}+04$ & 24 & 219 & $0.000 e+00$ & $2.513 e+05$ & 24 & 220 & $0.000 e+00$ & $1.100 \mathrm{e}+06$ \\
\hline 24 & 221 & $0.000 e+00$ & $3.744 e+04$ & 24 & 222 & $0.000 e+00$ & $7.715 e+04$ & 24 & 224 & $0.000 e+00$ & $6.487 e+03$ \\
\hline 24 & 226 & $1.497 e-03$ & $1.289 \mathrm{e}+08$ & 24 & 228 & $2.596 \mathrm{e}-02$ & $3.141 e+09$ & 24 & 229 & $2.259 \mathrm{e}-01$ & $1.953 e+10$ \\
\hline 24 & 230 & $1.824 \mathrm{e}-04$ & $1.579 \mathrm{e}+07$ & 24 & 231 & $9.447 e-04$ & $6.363 e+07$ & 24 & 235 & $7.643 e-04$ & $9.359 e+07$ \\
\hline 24 & 236 & $2.836 \mathrm{e}-02$ & $2.481 \mathrm{e}+09$ & 24 & 237 & $3.529 e-01$ & $2.402 \mathrm{e}+10$ & 24 & 238 & $2.513 e-03$ & $3.117 e+08$ \\
\hline 24 & 241 & $2.899 e-02$ & $3.600 e+09$ & 24 & 242 & $1.552 \mathrm{e}-04$ & $1.073 e+07$ & 24 & 243 & $0.000 e+00$ & $2.342 e+04$ \\
\hline 24 & 245 & $0.000 e+00$ & $1.224 \mathrm{e}+04$ & 24 & 247 & $0.000 e+00$ & $1.640 \mathrm{e}+05$ & 24 & 248 & $0.000 e+00$ & $1.352 \mathrm{e}+06$ \\
\hline 24 & 249 & $0.000 e+00$ & $4.128 e+06$ & 24 & 252 & $0.000 e+00$ & $4.285 e+04$ & 24 & 254 & $0.000 e+00$ & $5.748 e+05$ \\
\hline 24 & 256 & $0.000 e+00$ & $3.632 \mathrm{e}+06$ & 24 & 262 & $0.000 e+00$ & $1.497 e+05$ & 24 & 263 & $0.000 e+00$ & $1.030 \mathrm{e}+06$ \\
\hline 24 & 264 & $0.000 e+00$ & $2.140 \mathrm{e}+06$ & 24 & 265 & $5.182 \mathrm{e}-04$ & $3.661 e+07$ & 24 & 268 & $1.830 \mathrm{e}-04$ & $1.663 e+07$ \\
\hline 24 & 269 & $1.951 \mathrm{e}-03$ & $1.379 \mathrm{e}+08$ & 24 & 272 & $0.000 e+00$ & $4.223 e+05$ & 24 & 273 & $0.000 e+00$ & $2.543 e+05$ \\
\hline 24 & 277 & $7.645 e-04$ & $6.994 \mathrm{e}+07$ & 24 & 278 & $9.770 e-05$ & $1.252 \mathrm{e}+07$ & 25 & 32 & $8.627 e-04$ & $2.675 e+06$ \\
\hline 25 & 36 & $3.335 e+00$ & $9.942 e+09$ & 25 & 37 & $3.083 e-01$ & $1.657 e+09$ & 25 & 40 & $3.821 \mathrm{e}-03$ & $2.116 \mathrm{e}+07$ \\
\hline 25 & 45 & $2.007 e-01$ & $1.925 \mathrm{e}+09$ & 25 & 52 & $0.000 e+00$ & $1.081 e+05$ & 25 & 61 & $2.242 \mathrm{e}-03$ & $3.996 \mathrm{e}+07$ \\
\hline 25 & 62 & $2.492 \mathrm{e}-01$ & $4.725 e+09$ & 25 & 67 & $0.000 e+00$ & $9.234 e+04$ & 25 & 68 & $0.000 e+00$ & $1.021 \mathrm{e}+05$ \\
\hline 25 & 69 & $0.000 e+00$ & $9.249 e+03$ & 25 & 70 & $0.000 e+00$ & $1.588 e+04$ & 25 & 72 & $0.000 e+00$ & $8.118 e+03$ \\
\hline 25 & 73 & $0.000 e+00$ & $7.512 \mathrm{e}+04$ & 25 & 74 & $0.000 e+00$ & $2.523 e+04$ & 25 & 75 & $0.000 e+00$ & $5.741 e+04$ \\
\hline 25 & 77 & $0.000 e+00$ & $1.973 e+05$ & 25 & 81 & $0.000 e+00$ & $2.800 \mathrm{e}+04$ & 25 & 82 & $1.932 \mathrm{e}-01$ & $4.111 \mathrm{e}+09$ \\
\hline 25 & 83 & $3.266 \mathrm{e}-02$ & $5.456 e+08$ & 25 & 84 & $4.314 e-01$ & $9.293 e+09$ & 25 & 86 & $4.727 e-04$ & $1.444 e+07$ \\
\hline 25 & 87 & $3.607 e-02$ & $7.908 e+08$ & 25 & 88 & $8.872 \mathrm{e}-03$ & $1.518 \mathrm{e}+08$ & 25 & 89 & $9.666 \mathrm{e}-03$ & $2.170 \mathrm{e}+08$ \\
\hline
\end{tabular}


Table III. Ca IX Oscillator Strengths and Radiative Decay Rates for transitions involving levels higher than 4.

\begin{tabular}{|c|c|c|c|c|c|c|c|c|c|c|c|}
\hline $\begin{array}{l}\text { Low. } \\
\text { Lev. }\end{array}$ & $\begin{array}{l}\text { Upp } \\
\text { Lev }\end{array}$ & $\begin{array}{l}\text { Osc. } \\
\text { Str. }\end{array}$ & $\begin{array}{l}\text { Rad. } \\
\text { Rate }\end{array}$ & $\begin{array}{l}\text { Low. } \\
\text { Lev. }\end{array}$ & $\begin{array}{l}\text { - Upp } \\
\text {. Lev }\end{array}$ & $\begin{array}{l}\text { Osc. } \\
\text { Str. }\end{array}$ & $\begin{array}{l}\text { Rad. } \\
\text { Rate }\end{array}$ & $\begin{array}{l}\text { Low. } \\
\text { Lev. }\end{array}$ & $\begin{array}{l}\text { Upp } \\
\text { Lev }\end{array}$ & $\begin{array}{l}\text { Osc. } \\
\text { Str. }\end{array}$ & $\begin{array}{l}\text { Rad. } \\
\text { Rate }\end{array}$ \\
\hline$i$ & $j$ & $g f$ & $(1 / s)$ & $i$ & $j$ & $g f$ & $(1 / s)$ & $i$ & $j$ & $g f$ & $(1 / s)$ \\
\hline 25 & 90 & $6.601 \mathrm{e}-04$ & $2.079 e+07$ & 25 & 92 & $1.376 \mathrm{e}-01$ & $4.384 e+09$ & 25 & 93 & $4.128 e+00$ & $7.375 e+10$ \\
\hline 25 & 94 & $2.726 \mathrm{e}-03$ & $6.372 \mathrm{e}+07$ & 25 & 95 & $4.857 e-04$ & $1.593 e+07$ & 25 & 97 & $4.842 \mathrm{e}-03$ & $1.646 \mathrm{e}+08$ \\
\hline 25 & 101 & $0.000 e+00$ & $7.896 \mathrm{e}+04$ & 25 & 105 & $1.456 \mathrm{e}+00$ & $2.967 e+10$ & 25 & 107 & $5.335 e-04$ & $2.062 e+07$ \\
\hline 25 & 108 & $7.436 \mathrm{e}-04$ & $2.055 e+07$ & 25 & 109 & $5.275 e-01$ & $2.103 e+10$ & 25 & 111 & $0.000 e+00$ & $2.115 e+04$ \\
\hline 25 & 112 & $0.000 e+00$ & $1.422 \mathrm{e}+06$ & 25 & 113 & $0.000 e+00$ & $3.240 e+05$ & 25 & 114 & $0.000 e+00$ & $9.542 e+04$ \\
\hline 25 & 118 & $0.000 e+00$ & $1.818 e+04$ & 25 & 122 & $0.000 e+00$ & $8.989 e+03$ & 25 & 124 & $0.000 e+00$ & $7.538 e+05$ \\
\hline 25 & 127 & $0.000 e+00$ & $2.070 e+06$ & 25 & 128 & $2.839 e-03$ & $1.480 e+08$ & 25 & 132 & $1.436 \mathrm{e}-03$ & $7.607 e+07$ \\
\hline 25 & 134 & $1.062 \mathrm{e}-01$ & $5.736 e+09$ & 25 & 136 & $0.000 e+00$ & $5.204 e+04$ & 25 & 138 & $0.000 e+00$ & $2.067 e+04$ \\
\hline 25 & 139 & $0.000 e+00$ & $3.275 e+04$ & 25 & 140 & $0.000 e+00$ & $2.324 e+04$ & 25 & 141 & $0.000 e+00$ & $3.690 e+03$ \\
\hline 25 & 143 & $0.000 e+00$ & $4.224 e+03$ & 25 & 144 & $3.775 e-01$ & $1.636 e+10$ & 25 & 145 & $0.000 e+00$ & $7.901 e+03$ \\
\hline 25 & 147 & $0.000 e+00$ & $6.959 e+05$ & 25 & 149 & $1.262 \mathrm{e}-04$ & $7.752 e+06$ & 25 & 150 & $1.863 e-03$ & $8.175 e+07$ \\
\hline 25 & 151 & $1.301 \mathrm{e}-03$ & $5.726 e+07$ & 25 & 152 & $9.053 e-03$ & $3.102 \mathrm{e}+08$ & 25 & 155 & $4.207 e-02$ & $1.864 e+09$ \\
\hline 25 & 156 & $0.000 e+00$ & $8.399 e+05$ & 25 & 157 & $0.000 e+00$ & $1.382 e+04$ & 25 & 158 & $3.094 \mathrm{e}-03$ & $1.374 e+08$ \\
\hline 25 & 159 & $4.671 \mathrm{e}-04$ & $2.906 \mathrm{e}+07$ & 25 & 160 & $0.000 e+00$ & $3.301 e+05$ & 25 & 161 & $5.671 \mathrm{e}-02$ & $1.961 \mathrm{e}+09$ \\
\hline 25 & 162 & $0.000 e+00$ & $3.663 e+04$ & 25 & 164 & $2.013 e-02$ & $9.026 e+08$ & 25 & 165 & $9.369 e-03$ & $3.273 e+08$ \\
\hline 25 & 167 & $0.000 e+00$ & $2.657 e+04$ & 25 & 168 & $0.000 e+00$ & $8.379 e+04$ & 25 & 170 & $0.000 e+00$ & $1.273 e+06$ \\
\hline 25 & 172 & $5.660 e-03$ & $2.565 e+08$ & 25 & 173 & $3.930 e-04$ & $2.499 e+07$ & 25 & 179 & $2.245 e+00$ & $8.006 e+10$ \\
\hline 25 & 180 & $7.661 \mathrm{e}-04$ & $4.934 \mathrm{e}+07$ & 25 & 181 & $2.170 \mathrm{e}-04$ & $9.985 e+06$ & 25 & 182 & $4.162 \mathrm{e}-02$ & $1.491 \mathrm{e}+09$ \\
\hline 25 & 183 & $2.039 e-02$ & $1.318 \mathrm{e}+09$ & 25 & 186 & $6.304 e-04$ & $4.207 e+07$ & 25 & 187 & $3.146 e-02$ & $2.104 \mathrm{e}+09$ \\
\hline 25 & 188 & $3.324 e-01$ & $1.243 e+10$ & 25 & 189 & $0.000 e+00$ & $7.730 e+06$ & 25 & 190 & $0.000 e+00$ & $4.723 e+04$ \\
\hline 25 & 196 & $0.000 e+00$ & $8.136 e+05$ & 25 & 206 & $0.000 e+00$ & $1.954 \mathrm{e}+06$ & 25 & 208 & $0.000 e+00$ & $5.060 e+04$ \\
\hline 25 & 209 & $0.000 e+00$ & $1.564 \mathrm{e}+07$ & 25 & 211 & $6.791 \mathrm{e}-04$ & $6.358 e+07$ & 25 & 212 & $1.574 \mathrm{e}-04$ & $1.053 e+07$ \\
\hline 25 & 213 & $1.593 e-01$ & $1.502 \mathrm{e}+10$ & 25 & 214 & $0.000 e+00$ & $3.435 e+06$ & 25 & 216 & $0.000 e+00$ & $6.029 e+03$ \\
\hline 25 & 218 & $0.000 e+00$ & $4.340 e+04$ & 25 & 221 & $0.000 e+00$ & $5.114 e+03$ & 25 & 222 & $0.000 e+00$ & $8.084 e+03$ \\
\hline 25 & 224 & $0.000 e+00$ & $8.120 e+05$ & 25 & 225 & $0.000 e+00$ & $2.148 e+06$ & 25 & 226 & $1.859 \mathrm{e}-01$ & $1.447 \mathrm{e}+10$ \\
\hline 25 & 228 & $6.445 e-05$ & $7.050 e+06$ & 25 & 229 & $1.521 \mathrm{e}-03$ & $1.189 e+08$ & 25 & 230 & $1.215 \mathrm{e}-04$ & $9.515 e+06$ \\
\hline 25 & 231 & $4.037 e-04$ & $2.459 e+07$ & 25 & 238 & $5.906 e-03$ & $6.633 e+08$ & 25 & 241 & $6.964 \mathrm{e}-04$ & $7.829 e+07$ \\
\hline 25 & 242 & $6.405 e-01$ & $4.008 e+10$ & 25 & 243 & $0.000 e+00$ & $3.827 e+06$ & 25 & 244 & $0.000 e+00$ & $3.692 \mathrm{e}+04$ \\
\hline 25 & 245 & $0.000 e+00$ & $3.238 e+03$ & 25 & 249 & $0.000 e+00$ & $1.845 e+04$ & 25 & 250 & $0.000 e+00$ & $4.631 \mathrm{e}+04$ \\
\hline 25 & 253 & $1.264 \mathrm{e}-04$ & $8.061 \mathrm{e}+06$ & 25 & 259 & $9.271 \mathrm{e}-05$ & $5.920 e+06$ & 25 & 261 & $1.438 \mathrm{e}-04$ & $1.183 e+07$ \\
\hline 25 & 265 & $2.179 \mathrm{e}-02$ & $1.395 \mathrm{e}+09$ & 25 & 269 & $4.114 \mathrm{e}-03$ & $2.636 e+08$ & 25 & 275 & $0.000 e+00$ & $6.271 \mathrm{e}+05$ \\
\hline 25 & 280 & $9.194 \mathrm{e}-04$ & $1.071 \mathrm{e}+08$ & 25 & 282 & $0.000 e+00$ & $6.924 e+06$ & 25 & 283 & $0.000 e+00$ & $2.825 e+04$ \\
\hline 26 & 28 & $9.179 \mathrm{e}-03$ & $7.979 \mathrm{e}+07$ & 26 & 31 & $6.203 e-04$ & $2.397 e+06$ & 26 & 37 & $7.696 \mathrm{e}-01$ & $3.731 \mathrm{e}+09$ \\
\hline 26 & 39 & $3.755 e-04$ & $9.380 e+06$ & 26 & 40 & $5.564 \mathrm{e}-03$ & $2.784 e+07$ & 26 & 41 & $8.135 \mathrm{e}-01$ & $3.341 \mathrm{e}+10$ \\
\hline 26 & 45 & $1.859 e-03$ & $1.651 \mathrm{e}+07$ & 26 & 52 & $0.000 e+00$ & $5.789 e+04$ & 26 & 53 & $0.000 e+00$ & $2.086 e+04$ \\
\hline 26 & 54 & $7.045 e-02$ & $1.784 \mathrm{e}+09$ & 26 & 55 & $9.861 \mathrm{e}-03$ & $2.585 e+08$ & 26 & 58 & $1.748 \mathrm{e}-04$ & $1.445 e+07$ \\
\hline 26 & 59 & $1.885 e-03$ & $5.215 e+07$ & 26 & 60 & $4.685 e-04$ & $1.315 e+07$ & 26 & 62 & $1.169 \mathrm{e}-02$ & $2.099 \mathrm{e}+08$ \\
\hline 26 & 63 & $7.229 e-02$ & $7.048 e+09$ & 26 & 65 & $1.055 \mathrm{e}-02$ & $1.227 e+09$ & 26 & 66 & $0.000 e+00$ & $6.856 e+03$ \\
\hline 26 & 67 & $0.000 e+00$ & $4.261 e+04$ & 26 & 68 & $0.000 e+00$ & $4.981 e+04$ & 26 & 70 & $0.000 e+00$ & $1.437 e+04$ \\
\hline 26 & 72 & $0.000 e+00$ & $3.875 \mathrm{e}+03$ & 26 & 73 & $0.000 e+00$ & $6.352 e+04$ & 26 & 74 & $0.000 e+00$ & $1.359 \mathrm{e}+04$ \\
\hline 26 & 75 & $0.000 e+00$ & $4.502 \mathrm{e}+03$ & 26 & 77 & $0.000 e+00$ & $1.459 e+04$ & 26 & 79 & $0.000 e+00$ & $4.123 e+03$ \\
\hline 26 & 81 & $0.000 e+00$ & $2.787 e+05$ & 26 & 86 & $1.122 \mathrm{e}-02$ & $3.285 e+08$ & 26 & 90 & $5.587 e-03$ & $1.687 \mathrm{e}+08$ \\
\hline 26 & 92 & $3.274 \mathrm{e}-01$ & $1.001 \mathrm{e}+10$ & 26 & 95 & $8.457 e-03$ & $2.662 \mathrm{e}+08$ & 26 & 97 & $2.329 e+00$ & $7.602 \mathrm{e}+10$ \\
\hline 26 & 106 & $1.539 \mathrm{e}-04$ & $9.542 e+06$ & 26 & 109 & $3.426 \mathrm{e}-01$ & $1.316 \mathrm{e}+10$ & 26 & 112 & $0.000 e+00$ & $1.384 \mathrm{e}+05$ \\
\hline
\end{tabular}


Table III. Ca IX Oscillator Strengths and Radiative Decay Rates for transitions involving levels higher than 4.

\begin{tabular}{|c|c|c|c|c|c|c|c|c|c|c|c|}
\hline $\begin{array}{l}\text { Low. } \\
\text { Lev. }\end{array}$ & $\begin{array}{l}\text { Upp } \\
\text { Lev }\end{array}$ & $\begin{array}{l}\text { Osc. } \\
\text { Str. }\end{array}$ & $\begin{array}{l}\text { Rad. } \\
\text { Rate }\end{array}$ & $\begin{array}{l}\text { Low. } \\
\text { Lev. }\end{array}$ & $\begin{array}{l}\text { - Upp } \\
\text {. Lev }\end{array}$ & $\begin{array}{l}\text { Osc. } \\
\text { Str. }\end{array}$ & $\begin{array}{l}\text { Rad. } \\
\text { Rate }\end{array}$ & $\begin{array}{l}\text { Low. } \\
\text { Lev. }\end{array}$ & $\begin{array}{l}\text {. Upp } \\
\text {. Lev }\end{array}$ & $\begin{array}{l}\text { Osc. } \\
\text { Str. }\end{array}$ & $\begin{array}{l}\text { Rad. } \\
\text { Rate }\end{array}$ \\
\hline$i$ & $j$ & $g f$ & $(1 / s)$ & $i$ & $j$ & $g f$ & $(1 / s)$ & $i$ & j & $g f$ & $(1 / s)$ \\
\hline 26 & 113 & $0.000 e+00$ & $3.372 \mathrm{e}+04$ & 26 & 114 & $0.000 e+00$ & $1.123 e+04$ & 26 & 124 & $0.000 e+00$ & $9.928 e+05$ \\
\hline 26 & 125 & $9.155 e-03$ & $7.615 e+08$ & 26 & 126 & $3.609 \mathrm{e}-03$ & $3.030 e+08$ & 26 & 127 & $0.000 e+00$ & $1.107 e+05$ \\
\hline 26 & 128 & $3.663 e-04$ & $1.849 \mathrm{e}+07$ & 26 & 129 & $2.965 e-04$ & $7.518 e+07$ & 26 & 130 & $2.801 \mathrm{e}-03$ & $2.380 e+08$ \\
\hline 26 & 132 & $1.484 \mathrm{e}-04$ & $7.610 \mathrm{e}+06$ & 26 & 133 & $3.718 e-04$ & $3.193 e+07$ & 26 & 134 & $6.851 \mathrm{e}-03$ & $3.585 e+08$ \\
\hline 26 & 135 & $1.343 e-02$ & $3.574 \mathrm{e}+09$ & 26 & 136 & $0.000 e+00$ & $1.434 \mathrm{e}+05$ & 26 & 137 & $0.000 e+00$ & $4.376 e+03$ \\
\hline 26 & 138 & $0.000 e+00$ & $6.405 e+03$ & 26 & 139 & $0.000 e+00$ & $9.202 \mathrm{e}+03$ & 26 & 140 & $0.000 e+00$ & $6.267 e+04$ \\
\hline 26 & 143 & $0.000 e+00$ & $1.131 \mathrm{e}+04$ & 26 & 147 & $0.000 e+00$ & $1.093 e+05$ & 26 & 148 & $3.636 e-04$ & $3.611 \mathrm{e}+07$ \\
\hline 26 & 154 & $2.905 e-01$ & $2.906 e+10$ & 26 & 156 & $0.000 e+00$ & $3.370 e+05$ & 26 & 159 & $1.506 \mathrm{e}-02$ & $9.096 e+08$ \\
\hline 26 & 163 & $0.000 e+00$ & $1.631 \mathrm{e}+05$ & 26 & 171 & $2.894 \mathrm{e}-04$ & $2.970 e+07$ & 26 & 173 & $2.042 \mathrm{e}-02$ & $1.261 \mathrm{e}+09$ \\
\hline 26 & 176 & $0.000 e+00$ & $9.968 e+05$ & 26 & 178 & $0.000 e+00$ & $1.625 e+06$ & 26 & 180 & $5.353 e-02$ & $3.348 e+09$ \\
\hline 26 & 183 & $8.178 e-01$ & $5.134 e+10$ & 26 & 185 & $8.902 e-05$ & $2.885 e+07$ & 26 & 186 & $2.219 \mathrm{e}-03$ & $1.439 e+08$ \\
\hline 26 & 187 & $7.720 e-02$ & $5.017 e+09$ & 26 & 196 & $0.000 e+00$ & $7.454 e+06$ & 26 & 206 & $0.000 e+00$ & $4.664 e+06$ \\
\hline 26 & 207 & $7.100 e-02$ & $2.515 e+10$ & 26 & 208 & $0.000 e+00$ & $1.167 e+07$ & 26 & 213 & $6.947 e-02$ & $6.393 e+09$ \\
\hline 26 & 214 & $0.000 e+00$ & $5.981 e+05$ & 26 & 218 & $0.000 e+00$ & $5.600 e+03$ & 26 & 224 & $0.000 e+00$ & $1.460 e+06$ \\
\hline 26 & 225 & $0.000 e+00$ & $1.702 \mathrm{e}+05$ & 26 & 227 & $1.073 e-03$ & $1.912 \mathrm{e}+08$ & 26 & 233 & $1.947 e-01$ & $3.482 e+10$ \\
\hline 26 & 234 & $4.169 e-04$ & $7.517 e+07$ & 26 & 235 & $6.666 e-05$ & $7.223 e+06$ & 26 & 238 & $1.119 \mathrm{e}-01$ & $1.229 \mathrm{e}+10$ \\
\hline 26 & 239 & $2.872 \mathrm{e}-05$ & $1.579 \mathrm{e}+07$ & 26 & 241 & $9.034 \mathrm{e}-03$ & $9.934 e+08$ & 26 & 247 & $0.000 e+00$ & $6.182 e+03$ \\
\hline 26 & 250 & $0.000 e+00$ & $6.039 e+06$ & 26 & 275 & $0.000 e+00$ & $9.778 e+05$ & 26 & 278 & $4.687 e-05$ & $5.329 e+06$ \\
\hline 26 & 280 & $7.748 e-03$ & $8.836 e+08$ & 26 & 281 & $2.981 \mathrm{e}-02$ & $1.702 \mathrm{e}+10$ & 26 & 283 & $0.000 e+00$ & $4.674 e+06$ \\
\hline 27 & 29 & $2.443 e-01$ & $8.073 e+08$ & 27 & 30 & $6.621 \mathrm{e}-01$ & $7.353 e+08$ & 27 & 33 & $1.254 \mathrm{e}+00$ & $8.666 e+08$ \\
\hline 27 & 35 & $7.691 \mathrm{e}-02$ & $9.130 e+07$ & 27 & 42 & $0.000 e+00$ & $4.625 e+04$ & 27 & 43 & $0.000 e+00$ & $4.649 e+04$ \\
\hline 27 & 44 & $0.000 e+00$ & $4.685 e+04$ & 27 & 46 & $1.957 \mathrm{e}-01$ & $4.341 e+09$ & 27 & 47 & $5.748 e-01$ & $4.309 \mathrm{e}+09$ \\
\hline 27 & 48 & $1.002 \mathrm{e}+00$ & $4.683 e+09$ & 27 & 52 & $1.693 e-02$ & $1.461 \mathrm{e}+08$ & 27 & 55 & $0.000 e+00$ & $4.435 e+03$ \\
\hline 27 & 56 & $0.000 e+00$ & $4.823 e+03$ & 27 & 57 & $0.000 e+00$ & $4.583 e+03$ & 27 & 66 & $1.820 \mathrm{e}-03$ & $4.454 \mathrm{e}+07$ \\
\hline 27 & 67 & $6.717 e-03$ & $9.864 \mathrm{e}+07$ & 27 & 70 & $7.316 \mathrm{e}-02$ & $1.842 \mathrm{e}+09$ & 27 & 71 & $3.285 e-02$ & $2.482 \mathrm{e}+09$ \\
\hline 27 & 72 & $1.371 \mathrm{e}-01$ & $2.076 \mathrm{e}+09$ & 27 & 73 & $1.955 \mathrm{e}-02$ & $4.950 e+08$ & 27 & 78 & $5.060 e-02$ & $8.314 e+08$ \\
\hline 27 & 79 & $2.633 e-02$ & $7.238 e+08$ & 27 & 80 & $8.311 \mathrm{e}-03$ & $6.875 e+08$ & 27 & 81 & $3.399 e-04$ & $9.658 e+06$ \\
\hline 27 & 89 & $0.000 \mathrm{e}+00$ & $1.424 \mathrm{e}+05$ & 27 & 90 & $0.000 e+00$ & $1.670 \mathrm{e}+05$ & 27 & 91 & $0.000 e+00$ & $1.907 \mathrm{e}+05$ \\
\hline 27 & 94 & $0.000 e+00$ & $2.644 \mathrm{e}+05$ & 27 & 95 & $0.000 e+00$ & $2.381 e+05$ & 27 & 96 & $0.000 e+00$ & $2.194 \mathrm{e}+05$ \\
\hline 27 & 106 & $0.000 e+00$ & $3.710 \mathrm{e}+05$ & 27 & 107 & $0.000 e+00$ & $3.711 e+05$ & 27 & 108 & $0.000 e+00$ & $3.722 \mathrm{e}+05$ \\
\hline 27 & 110 & $1.324 \mathrm{e}-04$ & $2.063 e+07$ & 27 & 111 & $3.208 e-04$ & $1.668 \mathrm{e}+07$ & 27 & 113 & $4.887 e-04$ & $1.550 \mathrm{e}+07$ \\
\hline 27 & 121 & $3.225 e-05$ & $5.452 e+06$ & 27 & 122 & $7.288 e-05$ & $4.106 e+06$ & 28 & 30 & $4.816 e-02$ & $3.250 e+07$ \\
\hline 28 & 35 & $4.392 e-01$ & $3.228 e+08$ & 28 & 45 & $0.000 e+00$ & $2.944 e+04$ & 28 & 47 & $2.331 e-02$ & $1.463 e+08$ \\
\hline 28 & 52 & $8.454 \mathrm{e}-01$ & $6.188 e+09$ & 28 & 66 & $3.459 e-03$ & $7.687 e+07$ & 28 & 70 & $1.729 \mathrm{e}-02$ & $3.959 \mathrm{e}+08$ \\
\hline 28 & 73 & $7.249 e-02$ & $1.670 \mathrm{e}+09$ & 28 & 79 & $1.081 \mathrm{e}-03$ & $2.714 e+07$ & 28 & 81 & $2.763 e-02$ & $7.181 \mathrm{e}+08$ \\
\hline 28 & 92 & $0.000 e+00$ & $5.477 e+05$ & 28 & 97 & $0.000 e+00$ & $1.345 e+05$ & 28 & 109 & $0.000 e+00$ & $2.374 \mathrm{e}+05$ \\
\hline 28 & 114 & $2.410 e-04$ & $1.200 \mathrm{e}+07$ & 28 & 127 & $1.252 \mathrm{e}-04$ & $6.971 \mathrm{e}+06$ & 28 & 187 & $0.000 e+00$ & $1.696 \mathrm{e}+04$ \\
\hline 28 & 208 & $2.278 e-03$ & $1.982 e+08$ & 28 & 225 & $1.332 \mathrm{e}-04$ & $1.684 \mathrm{e}+07$ & 28 & 280 & $0.000 e+00$ & $7.257 e+03$ \\
\hline 28 & 283 & $7.576 \mathrm{e}-04$ & $1.118 \mathrm{e}+08$ & 29 & 42 & $5.767 e-01$ & $9.732 \mathrm{e}+08$ & 29 & 54 & $4.576 e-02$ & $2.889 \mathrm{e}+08$ \\
\hline 29 & 55 & $3.597 e-01$ & $2.433 e+09$ & 29 & 59 & $8.310 e-02$ & $6.244 \mathrm{e}+08$ & 29 & 60 & $2.312 \mathrm{e}-01$ & $1.785 e+09$ \\
\hline 29 & 64 & $1.260 e-01$ & $1.585 \mathrm{e}+09$ & 29 & 72 & $0.000 e+00$ & $6.305 e+04$ & 29 & 74 & $0.000 e+00$ & $2.300 e+03$ \\
\hline 29 & 78 & $0.000 e+00$ & $4.192 \mathrm{e}+04$ & 29 & 91 & $5.961 \mathrm{e}-02$ & $1.251 \mathrm{e}+09$ & 29 & 96 & $4.414 \mathrm{e}-02$ & $9.884 e+08$ \\
\hline 29 & 98 & $0.000 e+00$ & $6.258 e+05$ & 29 & 113 & $0.000 e+00$ & $1.825 e+04$ & 29 & 116 & $0.000 e+00$ & $1.658 \mathrm{e}+05$ \\
\hline 29 & 118 & $0.000 e+00$ & $9.987 e+04$ & 29 & 123 & $0.000 e+00$ & $5.488 e+04$ & 29 & 126 & $1.798 e-04$ & $7.933 e+06$ \\
\hline
\end{tabular}


Table III. Ca IX Oscillator Strengths and Radiative Decay Rates for transitions involving levels higher than 4.

\begin{tabular}{|c|c|c|c|c|c|c|c|c|c|c|c|}
\hline $\begin{array}{l}\text { Low. } \\
\text { Lev. }\end{array}$ & $\begin{array}{l}\text { Upp } \\
\text { Lev }\end{array}$ & $\begin{array}{l}\text { Osc. } \\
\text { Str. }\end{array}$ & $\begin{array}{l}\text { Rad. } \\
\text { Rate }\end{array}$ & $\begin{array}{l}\text { Low. } \\
\text { Lev. }\end{array}$ & $\begin{array}{l}\text { Upp. } \\
\text { Lev }\end{array}$ & $\begin{array}{l}\text { Osc. } \\
\text { Str. }\end{array}$ & $\begin{array}{l}\text { Rad. } \\
\text { Rate }\end{array}$ & $\begin{array}{l}\text { Low. } \\
\text { Lev. }\end{array}$ & $\begin{array}{l}\text { Upp } \\
\text { Lev }\end{array}$ & $\begin{array}{l}\text { Osc. } \\
\text { Str. }\end{array}$ & $\begin{array}{l}\text { Rad. } \\
\text { Rate }\end{array}$ \\
\hline$i$ & $j$ & $g f$ & $(1 / s)$ & $i$ & $j$ & $g f$ & $(1 / s)$ & $i$ & j & $g f$ & $(1 / s)$ \\
\hline 29 & 174 & $1.849 \mathrm{e}-04$ & $1.079 \mathrm{e}+07$ & 29 & 177 & $0.000 e+00$ & $1.096 \mathrm{e}+04$ & 30 & 42 & $3.854 \mathrm{e}-01$ & $6.462 \mathrm{e}+08$ \\
\hline 30 & 43 & $1.181 \mathrm{e}+00$ & $1.191 \mathrm{e}+09$ & 30 & 45 & $1.694 \mathrm{e}-01$ & $1.781 e+08$ & 30 & 54 & $2.348 e-01$ & $1.477 e+09$ \\
\hline 30 & 55 & $1.803 e-01$ & $1.215 \mathrm{e}+09$ & 30 & 56 & $7.766 \mathrm{e}-01$ & $3.188 e+09$ & 30 & 58 & $1.842 \mathrm{e}-01$ & $4.107 e+09$ \\
\hline 30 & 59 & $4.508 e-03$ & $3.376 \mathrm{e}+07$ & 30 & 60 & $2.952 \mathrm{e}-01$ & $2.271 e+09$ & 30 & 61 & $3.585 e-01$ & $1.658 \mathrm{e}+09$ \\
\hline 30 & 62 & $8.282 \mathrm{e}-02$ & $4.318 e+08$ & 30 & 63 & $1.629 \mathrm{e}-02$ & $4.934 e+08$ & 30 & 64 & $3.433 e-01$ & $4.307 e+09$ \\
\hline 30 & 65 & $2.475 e-02$ & $1.019 \mathrm{e}+09$ & 30 & 67 & $0.000 e+00$ & $8.755 e+03$ & 30 & 70 & $0.000 e+00$ & $3.239 e+04$ \\
\hline 30 & 72 & $0.000 e+00$ & $1.251 \mathrm{e}+05$ & 30 & 73 & $0.000 e+00$ & $7.577 e+04$ & 30 & 74 & $0.000 e+00$ & $1.975 e+03$ \\
\hline 30 & 77 & $0.000 e+00$ & $7.635 e+03$ & 30 & 78 & $0.000 e+00$ & $8.530 e+04$ & 30 & 79 & $0.000 e+00$ & $2.867 e+04$ \\
\hline 30 & 81 & $0.000 e+00$ & $4.599 e+04$ & 30 & 86 & $1.452 \mathrm{e}-03$ & $1.735 e+07$ & 30 & 90 & $1.137 \mathrm{e}-01$ & $1.426 e+09$ \\
\hline 30 & 91 & $4.047 e-02$ & $8.478 e+08$ & 30 & 92 & $1.159 \mathrm{e}-02$ & $1.480 e+08$ & 30 & 95 & $1.070 e-01$ & $1.430 e+09$ \\
\hline 30 & 96 & $3.014 e-02$ & $6.736 e+08$ & 30 & 97 & $4.414 e-03$ & $6.235 e+07$ & 30 & 98 & $0.000 e+00$ & $5.615 e+05$ \\
\hline 30 & 99 & $0.000 e+00$ & $8.031 e+05$ & 30 & 101 & $0.000 e+00$ & $1.228 e+05$ & 30 & 109 & $3.112 \mathrm{e}-03$ & $5.600 e+07$ \\
\hline 30 & 111 & $0.000 e+00$ & $1.758 e+04$ & 30 & 112 & $0.000 e+00$ & $7.740 e+04$ & 30 & 115 & $0.000 e+00$ & $2.269 e+05$ \\
\hline 30 & 116 & $0.000 e+00$ & $6.886 e+03$ & 30 & 117 & $0.000 e+00$ & $1.722 \mathrm{e}+05$ & 30 & 118 & $0.000 e+00$ & $1.425 e+05$ \\
\hline 30 & 119 & $0.000 e+00$ & $1.359 \mathrm{e}+05$ & 30 & 122 & $0.000 e+00$ & $6.884 e+04$ & 30 & 123 & $0.000 e+00$ & $1.155 \mathrm{e}+05$ \\
\hline 30 & 124 & $0.000 e+00$ & $2.138 e+04$ & 30 & 127 & $0.000 e+00$ & $1.110 e+04$ & 30 & 128 & $3.296 e-04$ & $8.740 e+06$ \\
\hline 30 & 130 & $1.411 \mathrm{e}-04$ & $6.323 e+06$ & 30 & 173 & $3.184 \mathrm{e}-04$ & $1.110 \mathrm{e}+07$ & 30 & 176 & $0.000 e+00$ & $1.422 \mathrm{e}+04$ \\
\hline 30 & 177 & $0.000 e+00$ & $9.930 e+03$ & 30 & 185 & $6.365 e-05$ & $1.183 e+07$ & 30 & 207 & $2.312 \mathrm{e}-05$ & $4.826 e+06$ \\
\hline 30 & 233 & $1.582 e-04$ & $1.862 \mathrm{e}+07$ & 30 & 234 & $8.876 e-05$ & $1.055 e+07$ & 31 & 66 & $2.125 \mathrm{e}-02$ & $3.198 e+08$ \\
\hline 31 & 67 & $2.236 e-03$ & $2.019 e+07$ & 31 & 68 & $9.707 e-04$ & $8.831 e+06$ & 31 & 70 & $1.480 e-03$ & $2.309 e+07$ \\
\hline 31 & 73 & $3.685 e-04$ & $5.792 e+06$ & 31 & 74 & $1.597 \mathrm{e}-03$ & $1.515 e+07$ & 31 & 106 & $0.000 e+00$ & $2.819 e+05$ \\
\hline 31 & 107 & $0.000 e+00$ & $1.206 \mathrm{e}+05$ & 31 & 108 & $0.000 e+00$ & $8.716 e+03$ & 31 & 111 & $7.067 e-04$ & $2.667 e+07$ \\
\hline 31 & 112 & $1.663 e-03$ & $3.827 e+07$ & 31 & 113 & $2.434 \mathrm{e}-03$ & $5.619 e+07$ & 31 & 114 & $5.363 e-03$ & $2.076 e+08$ \\
\hline 31 & 115 & $3.962 \mathrm{e}-01$ & $1.548 \mathrm{e}+10$ & 31 & 116 & $1.397 e-01$ & $3.278 e+09$ & 31 & 117 & $7.586 e-03$ & $1.274 \mathrm{e}+08$ \\
\hline 31 & 118 & $2.129 \mathrm{e}-01$ & $5.039 e+09$ & 31 & 119 & $2.954 \mathrm{e}-02$ & $5.009 e+08$ & 31 & 122 & $3.723 e-04$ & $1.543 e+07$ \\
\hline 31 & 126 & $0.000 e+00$ & $5.467 e+03$ & 31 & 129 & $0.000 e+00$ & $3.640 \mathrm{e}+04$ & 31 & 130 & $0.000 \mathrm{e}+00$ & $6.470 e+03$ \\
\hline 31 & 136 & $1.203 e-03$ & $3.777 e+07$ & 31 & 137 & $2.281 \mathrm{e}-03$ & $7.187 e+07$ & 31 & 138 & $5.524 e-04$ & $2.906 e+07$ \\
\hline 31 & 139 & $3.488 e-04$ & $7.873 e+06$ & 31 & 140 & $2.388 e-03$ & $7.635 e+07$ & 31 & 141 & $4.170 \mathrm{e}-04$ & $9.551 e+06$ \\
\hline 31 & 148 & $0.000 e+00$ & $5.021 \mathrm{e}+05$ & 31 & 149 & $0.000 e+00$ & $2.171 e+05$ & 31 & 150 & $0.000 e+00$ & $1.155 e+04$ \\
\hline 31 & 151 & $0.000 e+00$ & $3.521 \mathrm{e}+05$ & 31 & 152 & $0.000 e+00$ & $6.581 e+04$ & 31 & 155 & $0.000 e+00$ & $9.152 e+04$ \\
\hline 31 & 158 & $0.000 e+00$ & $5.156 e+04$ & 31 & 161 & $0.000 e+00$ & $1.023 e+04$ & 31 & 163 & $3.106 \mathrm{e}-01$ & $7.529 e+09$ \\
\hline 31 & 164 & $0.000 e+00$ & $2.552 e+04$ & 31 & 176 & $1.786 \mathrm{e}-02$ & $4.451 e+08$ & 31 & 177 & $1.435 \mathrm{e}-01$ & $5.039 e+09$ \\
\hline 31 & 178 & $1.225 e-02$ & $3.076 \mathrm{e}+08$ & 31 & 180 & $0.000 e+00$ & $5.129 e+04$ & 31 & 181 & $0.000 e+00$ & $1.909 e+04$ \\
\hline 31 & 184 & $0.000 e+00$ & $2.471 \mathrm{e}+05$ & 31 & 185 & $0.000 e+00$ & $7.429 e+05$ & 31 & 186 & $0.000 e+00$ & $1.995 e+04$ \\
\hline 31 & 191 & $1.718 e+00$ & $6.596 \mathrm{e}+10$ & 31 & 193 & $2.609 e-01$ & $7.161 e+09$ & 31 & 196 & $3.804 \mathrm{e}-03$ & $1.484 \mathrm{e}+08$ \\
\hline 31 & 197 & $5.835 e+00$ & $1.676 \mathrm{e}+11$ & 31 & 200 & $1.508 e-01$ & $1.018 e+10$ & 31 & 201 & $2.012 \mathrm{e}-02$ & $5.824 e+08$ \\
\hline 31 & 202 & $2.259 \mathrm{e}-02$ & $9.156 \mathrm{e}+08$ & 31 & 204 & $1.071 \mathrm{e}-03$ & $7.315 e+07$ & 31 & 206 & $4.587 e-03$ & $1.358 e+08$ \\
\hline 31 & 210 & $0.000 e+00$ & $2.819 e+05$ & 31 & 211 & $0.000 e+00$ & $1.209 e+05$ & 31 & 212 & $0.000 e+00$ & $8.760 e+03$ \\
\hline 31 & 214 & $9.237 e-04$ & $5.788 e+07$ & 31 & 215 & $7.974 \mathrm{e}-02$ & $8.358 e+09$ & 31 & 216 & $2.058 \mathrm{e}-02$ & $1.295 \mathrm{e}+09$ \\
\hline 31 & 217 & $9.354 e-04$ & $4.208 e+07$ & 31 & 218 & $3.965 e-02$ & $2.504 e+09$ & 31 & 219 & $5.295 e-03$ & $2.392 \mathrm{e}+08$ \\
\hline 31 & 222 & $7.991 \mathrm{e}-05$ & $8.510 e+06$ & 31 & 227 & $0.000 e+00$ & $4.543 e+05$ & 31 & 228 & $0.000 e+00$ & $1.986 \mathrm{e}+05$ \\
\hline 31 & 229 & $0.000 e+00$ & $1.175 \mathrm{e}+04$ & 31 & 230 & $0.000 e+00$ & $5.692 e+05$ & 31 & 231 & $0.000 e+00$ & $8.878 e+04$ \\
\hline 31 & 235 & $0.000 e+00$ & $6.719 \mathrm{e}+04$ & 31 & 236 & $0.000 e+00$ & $2.383 e+04$ & 31 & 239 & $0.000 e+00$ & $1.235 \mathrm{e}+06$ \\
\hline 31 & 240 & $0.000 e+00$ & $4.071 \mathrm{e}+05$ & 31 & 241 & $0.000 e+00$ & $3.084 e+04$ & 31 & 247 & $3.744 \mathrm{e}-01$ & $2.751 e+10$ \\
\hline
\end{tabular}


Table III. Ca IX Oscillator Strengths and Radiative Decay Rates for transitions involving levels higher than 4.

\begin{tabular}{|c|c|c|c|c|c|c|c|c|c|c|c|}
\hline $\begin{array}{l}\text { Low. } \\
\text { Lev . }\end{array}$ & $\begin{array}{l}\text { Upp } \\
\text { Lev }\end{array}$ & $\begin{array}{l}\text { Osc. } \\
\text { Str. }\end{array}$ & $\begin{array}{l}\text { Rad. } \\
\text { Rate }\end{array}$ & $\begin{array}{l}\text { Low. } \\
\text { Lev. }\end{array}$ & $\begin{array}{l}\text { Upp. } \\
\text { Lev. }\end{array}$ & $\begin{array}{l}\text { Osc. } \\
\text { Str. }\end{array}$ & $\begin{array}{l}\text { Rad. } \\
\text { Rate }\end{array}$ & $\begin{array}{l}\text { Low. } \\
\text { Lev. }\end{array}$ & $\begin{array}{l}\text { - Upp } \\
\text {. Lev }\end{array}$ & $\begin{array}{l}\text { Osc. } \\
\text { Str. }\end{array}$ & $\begin{array}{l}\text { Rad. } \\
\text { Rate }\end{array}$ \\
\hline$i$ & $j$ & $g f$ & $(1 / s)$ & $i$ & $j$ & $g f$ & $(1 / s)$ & i & j & $g f$ & $(1 / s)$ \\
\hline 31 & 248 & $5.724 \mathrm{e}-02$ & $3.005 e+09$ & 31 & 250 & $7.438 e-04$ & $5.504 e+07$ & 31 & 252 & $1.621 \mathrm{e}+00$ & $8.629 e+10$ \\
\hline 31 & 253 & $0.000 e+00$ & $8.399 e+06$ & 31 & 258 & $0.000 e+00$ & $3.738 e+06$ & 31 & 261 & $0.000 e+00$ & $3.407 e+04$ \\
\hline 31 & 262 & $4.887 e-02$ & $6.099 e+09$ & 31 & 263 & $7.239 e-03$ & $5.421 e+08$ & 31 & 264 & $5.921 e-04$ & $3.168 e+07$ \\
\hline 31 & 265 & $0.000 e+00$ & $2.972 \mathrm{e}+04$ & 31 & 267 & $0.000 e+00$ & $8.839 e+05$ & 31 & 268 & $0.000 e+00$ & $1.554 \mathrm{e}+05$ \\
\hline 31 & 273 & $4.384 e-04$ & $5.489 e+07$ & 31 & 275 & $9.357 e-04$ & $5.030 e+07$ & 31 & 278 & $0.000 e+00$ & $2.789 e+04$ \\
\hline 31 & 279 & $0.000 e+00$ & $9.713 e+04$ & 32 & 67 & $1.695 \mathrm{e}-02$ & $1.530 \mathrm{e}+08$ & 32 & 68 & $1.430 \mathrm{e}-02$ & $1.300 \mathrm{e}+08$ \\
\hline 32 & 69 & $2.821 \mathrm{e}-03$ & $1.835 \mathrm{e}+07$ & 32 & 72 & $1.586 \mathrm{e}-03$ & $1.487 e+07$ & 32 & 74 & $1.395 e-03$ & $1.323 e+07$ \\
\hline 32 & 75 & $2.230 \mathrm{e}-03$ & $1.515 e+07$ & 32 & 78 & $2.501 \mathrm{e}-04$ & $2.602 \mathrm{e}+06$ & 32 & 106 & $0.000 e+00$ & $1.409 \mathrm{e}+05$ \\
\hline 32 & 107 & $0.000 e+00$ & $2.113 e+05$ & 32 & 108 & $0.000 e+00$ & $9.078 e+04$ & 32 & 112 & $2.659 \mathrm{e}-03$ & $6.113 e+07$ \\
\hline 32 & 113 & $1.431 \mathrm{e}-03$ & $3.302 \mathrm{e}+07$ & 32 & 116 & $5.119 e-01$ & $1.200 e+10$ & 32 & 117 & $1.614 \mathrm{e}-01$ & $2.708 e+09$ \\
\hline 32 & 118 & $1.129 \mathrm{e}-01$ & $2.671 \mathrm{e}+09$ & 32 & 119 & $2.842 \mathrm{e}-01$ & $4.816 e+09$ & 32 & 120 & $3.516 e-02$ & $4.652 e+08$ \\
\hline 32 & 123 & $7.421 \mathrm{e}-04$ & $1.847 \mathrm{e}+07$ & 32 & 124 & $6.287 e-04$ & $1.133 e+07$ & 32 & 130 & $0.000 e+00$ & $1.934 \mathrm{e}+04$ \\
\hline 32 & 132 & $0.000 e+00$ & $5.457 e+03$ & 32 & 136 & $2.453 e-04$ & $7.692 e+06$ & 32 & 139 & $3.935 e-03$ & $8.877 e+07$ \\
\hline 32 & 140 & $1.415 \mathrm{e}-03$ & $4.520 e+07$ & 32 & 141 & $3.763 e-03$ & $8.613 e+07$ & 32 & 142 & $8.449 e-04$ & $1.511 \mathrm{e}+07$ \\
\hline 32 & 143 & $1.429 \mathrm{e}-04$ & $4.636 e+06$ & 32 & 147 & $6.246 e-04$ & $1.467 e+07$ & 32 & 148 & $0.000 e+00$ & $2.485 e+05$ \\
\hline 32 & 149 & $0.000 e+00$ & $3.776 e+05$ & 32 & 150 & $0.000 e+00$ & $1.693 e+05$ & 32 & 151 & $0.000 e+00$ & $9.354 e+04$ \\
\hline 32 & 152 & $0.000 e+00$ & $3.890 e+05$ & 32 & 153 & $0.000 e+00$ & $6.183 e+04$ & 32 & 155 & $0.000 e+00$ & $2.586 e+04$ \\
\hline 32 & 157 & $3.415 \mathrm{e}-02$ & $6.382 e+08$ & 32 & 158 & $0.000 e+00$ & $1.805 e+04$ & 32 & 161 & $0.000 e+00$ & $6.024 e+04$ \\
\hline 32 & 162 & $2.222 \mathrm{e}-01$ & $4.185 e+09$ & 32 & 163 & $5.941 e-03$ & $1.440 e+08$ & 32 & 165 & $0.000 e+00$ & $2.577 e+04$ \\
\hline 32 & 167 & $1.748 \mathrm{e}-01$ & $3.328 e+09$ & 32 & 175 & $7.862 \mathrm{e}-03$ & $1.522 \mathrm{e}+08$ & 32 & 176 & $1.277 e-01$ & $3.183 e+09$ \\
\hline 32 & 177 & $1.780 e-02$ & $6.246 e+08$ & 32 & 178 & $7.767 e-02$ & $1.949 e+09$ & 32 & 180 & $0.000 e+00$ & $2.586 e+04$ \\
\hline 32 & 181 & $0.000 e+00$ & $4.427 e+04$ & 32 & 182 & $0.000 e+00$ & $1.543 e+04$ & 32 & 184 & $0.000 e+00$ & $4.973 e+05$ \\
\hline 32 & 186 & $0.000 e+00$ & $1.414 \mathrm{e}+05$ & 32 & 187 & $0.000 e+00$ & $5.953 e+03$ & 32 & 189 & $6.261 \mathrm{e}-04$ & $1.310 \mathrm{e}+07$ \\
\hline 32 & 190 & $4.607 e-03$ & $9.816 e+07$ & 32 & 191 & $2.084 \mathrm{e}-01$ & $7.997 e+09$ & 32 & 192 & $2.559 \mathrm{e}-01$ & $5.457 e+09$ \\
\hline 32 & 193 & $2.251 \mathrm{e}+00$ & $6.173 e+10$ & 32 & 196 & $7.656 \mathrm{e}-04$ & $2.984 e+07$ & 32 & 197 & $5.568 e-01$ & $1.598 e+10$ \\
\hline 32 & 199 & $7.716 \mathrm{e}+00$ & $1.725 \mathrm{e}+11$ & 32 & 201 & $1.596 \mathrm{e}-02$ & $4.618 e+08$ & 32 & 202 & $2.304 \mathrm{e}-01$ & $9.333 e+09$ \\
\hline 32 & 206 & $8.048 e-04$ & $2.381 e+07$ & 32 & 210 & $0.000 e+00$ & $1.397 e+05$ & 32 & 211 & $0.000 \mathrm{e}+00$ & $2.096 \mathrm{e}+05$ \\
\hline 32 & 212 & $0.000 e+00$ & $9.088 e+04$ & 32 & 216 & $1.109 \mathrm{e}-01$ & $6.976 e+09$ & 32 & 217 & $2.337 e-02$ & $1.051 \mathrm{e}+09$ \\
\hline 32 & 218 & $1.257 e-02$ & $7.940 e+08$ & 32 & 219 & $5.253 e-02$ & $2.372 \mathrm{e}+09$ & 32 & 220 & $5.874 e-03$ & $2.066 e+08$ \\
\hline 32 & 221 & $1.751 \mathrm{e}-04$ & $1.118 e+07$ & 32 & 227 & $0.000 e+00$ & $2.186 e+05$ & 32 & 228 & $0.000 e+00$ & $3.402 e+05$ \\
\hline 32 & 229 & $0.000 e+00$ & $1.518 e+05$ & 32 & 230 & $0.000 e+00$ & $1.632 \mathrm{e}+05$ & 32 & 231 & $0.000 e+00$ & $5.211 \mathrm{e}+05$ \\
\hline 32 & 232 & $0.000 e+00$ & $7.483 e+04$ & 32 & 235 & $0.000 e+00$ & $3.270 \mathrm{e}+04$ & 32 & 236 & $0.000 e+00$ & $5.166 e+04$ \\
\hline 32 & 237 & $0.000 e+00$ & $2.006 e+04$ & 32 & 238 & $0.000 e+00$ & $1.487 e+04$ & 32 & 240 & $0.000 e+00$ & $8.273 e+05$ \\
\hline 32 & 241 & $0.000 e+00$ & $2.264 \mathrm{e}+05$ & 32 & 243 & $8.251 \mathrm{e}-04$ & $3.351 e+07$ & 32 & 244 & $4.142 \mathrm{e}-04$ & $1.686 \mathrm{e}+07$ \\
\hline 32 & 247 & $4.477 e-02$ & $3.289 e+09$ & 32 & 248 & $4.861 \mathrm{e}-01$ & $2.551 e+10$ & 32 & 249 & $5.939 e-02$ & $2.425 e+09$ \\
\hline 32 & 250 & $1.533 \mathrm{e}-04$ & $1.134 \mathrm{e}+07$ & 32 & 251 & $0.000 e+00$ & $1.933 e+05$ & 32 & 252 & $1.529 \mathrm{e}-01$ & $8.136 \mathrm{e}+09$ \\
\hline 32 & 253 & $0.000 \mathrm{e}+00$ & $2.655 e+05$ & 32 & 254 & $2.130 \mathrm{e}+00$ & $8.819 e+10$ & 32 & 255 & $0.000 e+00$ & $8.327 \mathrm{e}+06$ \\
\hline 32 & 258 & $0.000 e+00$ & $9.342 \mathrm{e}+05$ & 32 & 259 & $0.000 e+00$ & $4.296 e+06$ & 32 & 260 & $0.000 e+00$ & $4.070 e+04$ \\
\hline 32 & 261 & $0.000 e+00$ & $1.699 \mathrm{e}+04$ & 32 & 263 & $7.372 \mathrm{e}-02$ & $5.519 e+09$ & 32 & 264 & $6.369 \mathrm{e}-03$ & $3.406 e+08$ \\
\hline 32 & 266 & $0.000 \mathrm{e}+00$ & $1.631 \mathrm{e}+04$ & 32 & 267 & $0.000 e+00$ & $3.695 e+05$ & 32 & 268 & $0.000 e+00$ & $8.404 e+05$ \\
\hline 32 & 269 & $0.000 e+00$ & $1.241 \mathrm{e}+05$ & 32 & 272 & $1.039 \mathrm{e}-03$ & $7.801 \mathrm{e}+07$ & 32 & 275 & $5.064 e-04$ & $2.721 \mathrm{e}+07$ \\
\hline 32 & 277 & $0.000 e+00$ & $2.114 \mathrm{e}+04$ & 32 & 278 & $0.000 e+00$ & $8.453 e+04$ & 32 & 279 & $0.000 e+00$ & $4.822 e+04$ \\
\hline 33 & 42 & $2.829 e-02$ & $4.600 e+07$ & 33 & 43 & $4.278 e-01$ & $4.186 e+08$ & 33 & 44 & $2.424 e+00$ & $1.702 e+09$ \\
\hline 33 & 51 & $0.000 e+00$ & $1.496 e+04$ & 33 & 55 & $4.927 e-02$ & $3.272 \mathrm{e}+08$ & 33 & 56 & $4.200 e-01$ & $1.698 \mathrm{e}+09$ \\
\hline
\end{tabular}


Table III. Ca IX Oscillator Strengths and Radiative Decay Rates for transitions involving levels higher than 4.

\begin{tabular}{|c|c|c|c|c|c|c|c|c|c|c|c|}
\hline $\begin{array}{l}\text { Low. } \\
\text { Lev. }\end{array}$ & $\begin{array}{l}\text { Upp } \\
\text { Lev }\end{array}$ & $\begin{array}{l}\text { Osc. } \\
\text { Str. }\end{array}$ & $\begin{array}{l}\text { Rad. } \\
\text { Rate }\end{array}$ & $\begin{array}{l}\text { Low. } \\
\text { Lev. }\end{array}$ & $\begin{array}{l}\text { Upp. } \\
\text { Lev. }\end{array}$ & $\begin{array}{l}\text { Osc. } \\
\text { Str. }\end{array}$ & $\begin{array}{l}\text { Rad. } \\
\text { Rate }\end{array}$ & $\begin{array}{l}\text { Low. } \\
\text { Lev. }\end{array}$ & $\begin{array}{l}\text { Upp } \\
\text { Lev }\end{array}$ & $\begin{array}{l}\text { Osc. } \\
\text { Str. }\end{array}$ & $\begin{array}{l}\text { Rad. } \\
\text { Rate }\end{array}$ \\
\hline$i$ & $j$ & $g f$ & $(1 / s)$ & $i$ & $j$ & $g f$ & $(1 / s)$ & $i$ & $j$ & $g f$ & $(1 / s)$ \\
\hline 33 & 57 & $1.854 \mathrm{e}+00$ & $5.559 e+09$ & 33 & 59 & $5.102 e-01$ & $3.767 e+09$ & 33 & 60 & $6.304 \mathrm{e}-02$ & $4.783 e+08$ \\
\hline 33 & 61 & $6.301 \mathrm{e}-01$ & $2.873 e+09$ & 33 & 62 & $1.993 e-02$ & $1.025 e+08$ & 33 & 64 & $6.543 e-01$ & $8.119 \mathrm{e}+09$ \\
\hline 33 & 70 & $0.000 e+00$ & $2.078 e+05$ & 33 & 71 & $0.000 e+00$ & $3.706 e+05$ & 33 & 72 & $0.000 e+00$ & $1.124 \mathrm{e}+05$ \\
\hline 33 & 73 & $0.000 e+00$ & $5.769 e+04$ & 33 & 76 & $0.000 e+00$ & $5.038 e+03$ & 33 & 78 & $0.000 e+00$ & $7.149 e+04$ \\
\hline 33 & 79 & $0.000 e+00$ & $1.316 \mathrm{e}+05$ & 33 & 80 & $0.000 e+00$ & $1.670 \mathrm{e}+05$ & 33 & 87 & $3.258 \mathrm{e}-03$ & $2.776 e+07$ \\
\hline 33 & 89 & $2.022 \mathrm{e}-01$ & $1.789 e+09$ & 33 & 90 & $4.148 e-02$ & $5.156 e+08$ & 33 & 91 & $3.116 \mathrm{e}-03$ & $6.471 \mathrm{e}+07$ \\
\hline 33 & 92 & $5.779 e-04$ & $7.318 e+06$ & 33 & 94 & $2.422 \mathrm{e}-01$ & $2.284 e+09$ & 33 & 95 & $3.860 \mathrm{e}-02$ & $5.116 e+08$ \\
\hline 33 & 96 & $2.345 e-03$ & $5.198 e+07$ & 33 & 98 & $0.000 e+00$ & $8.892 e+04$ & 33 & 99 & $0.000 e+00$ & $4.444 e+05$ \\
\hline 33 & 100 & $0.000 e+00$ & $1.333 e+06$ & 33 & 110 & $0.000 e+00$ & $3.362 e+04$ & 33 & 111 & $0.000 e+00$ & $1.885 e+04$ \\
\hline 33 & 113 & $0.000 e+00$ & $1.085 e+04$ & 33 & 114 & $0.000 e+00$ & $1.525 e+04$ & 33 & 115 & $0.000 e+00$ & $7.119 e+04$ \\
\hline 33 & 116 & $0.000 e+00$ & $1.597 e+05$ & 33 & 117 & $0.000 e+00$ & $1.501 e+05$ & 33 & 118 & $0.000 e+00$ & $5.427 e+04$ \\
\hline 33 & 119 & $0.000 e+00$ & $1.848 e+05$ & 33 & 120 & $0.000 e+00$ & $3.341 e+05$ & 33 & 121 & $0.000 e+00$ & $3.287 e+05$ \\
\hline 33 & 122 & $0.000 e+00$ & $2.501 e+05$ & 33 & 123 & $0.000 e+00$ & $1.182 \mathrm{e}+05$ & 33 & 131 & $7.677 e-04$ & $1.468 \mathrm{e}+07$ \\
\hline 33 & 133 & $2.385 e-04$ & $1.078 \mathrm{e}+07$ & 33 & 142 & $0.000 e+00$ & $1.889 e+03$ & 33 & 172 & $7.454 e-04$ & $1.840 e+07$ \\
\hline 33 & 175 & $0.000 e+00$ & $2.053 e+04$ & 33 & 176 & $0.000 e+00$ & $3.995 e+03$ & 33 & 184 & $9.846 e-05$ & $6.069 e+06$ \\
\hline 33 & 186 & $2.390 e-04$ & $8.842 e+06$ & 33 & 223 & $0.000 e+00$ & $5.024 e+03$ & 33 & 229 & $1.373 e-04$ & $6.877 e+06$ \\
\hline 33 & 234 & $1.781 \mathrm{e}-04$ & $2.110 \mathrm{e}+07$ & 34 & 69 & $4.443 e-02$ & $2.884 e+08$ & 34 & 75 & $5.324 e-03$ & $3.610 e+07$ \\
\hline 34 & 76 & $1.209 \mathrm{e}-03$ & $6.501 e+06$ & 34 & 107 & $0.000 e+00$ & $9.047 e+04$ & 34 & 108 & $0.000 e+00$ & $3.232 e+05$ \\
\hline 34 & 117 & $7.428 \mathrm{e}-01$ & $1.245 e+10$ & 34 & 119 & $1.496 \mathrm{e}-01$ & $2.531 e+09$ & 34 & 120 & $5.284 \mathrm{e}-01$ & $6.981 \mathrm{e}+09$ \\
\hline 34 & 132 & $0.000 e+00$ & $2.937 e+04$ & 34 & 141 & $2.469 e-03$ & $5.646 e+07$ & 34 & 142 & $1.331 \mathrm{e}-02$ & $2.377 e+08$ \\
\hline 34 & 149 & $0.000 e+00$ & $1.595 e+05$ & 34 & 150 & $0.000 e+00$ & $5.715 e+05$ & 34 & 151 & $0.000 e+00$ & $8.577 e+03$ \\
\hline 34 & 152 & $0.000 e+00$ & $9.643 e+04$ & 34 & 153 & $0.000 e+00$ & $5.560 e+05$ & 34 & 157 & $3.057 e-03$ & $5.706 e+07$ \\
\hline 34 & 160 & $1.429 \mathrm{e}-01$ & $2.186 e+09$ & 34 & 161 & $0.000 e+00$ & $1.649 \mathrm{e}+04$ & 34 & 162 & $8.518 \mathrm{e}-04$ & $1.602 \mathrm{e}+07$ \\
\hline 34 & 167 & $8.323 e-03$ & $1.582 \mathrm{e}+08$ & 34 & 168 & $3.046 \mathrm{e}-01$ & $4.739 e+09$ & 34 & 169 & $0.000 e+00$ & $5.399 e+04$ \\
\hline 34 & 170 & $1.322 \mathrm{e}-01$ & $2.064 \mathrm{e}+09$ & 34 & 175 & $2.534 \mathrm{e}-01$ & $4.900 e+09$ & 34 & 176 & $9.643 e-03$ & $2.400 e+08$ \\
\hline 34 & 178 & $7.600 e-03$ & $1.905 \mathrm{e}+08$ & 34 & 181 & $0.000 e+00$ & $2.033 e+04$ & 34 & 182 & $0.000 \mathrm{e}+00$ & $6.646 \mathrm{e}+04$ \\
\hline 34 & 186 & $0.000 \mathrm{e}+00$ & $5.540 e+05$ & 34 & 187 & $0.000 e+00$ & $2.003 e+04$ & 34 & 189 & $3.080 \mathrm{e}-03$ & $6.435 \mathrm{e}+07$ \\
\hline 34 & 190 & $2.048 \mathrm{e}-01$ & $4.358 e+09$ & 34 & 192 & $3.061 \mathrm{e}+00$ & $6.521 e+10$ & 34 & 193 & $2.146 \mathrm{e}-01$ & $5.881 \mathrm{e}+09$ \\
\hline 34 & 194 & $3.588 \mathrm{e}-03$ & $6.264 \mathrm{e}+07$ & 34 & 197 & $4.924 \mathrm{e}-03$ & $1.412 \mathrm{e}+08$ & 34 & 198 & $1.008 \mathrm{e}+01$ & $1.842 \mathrm{e}+11$ \\
\hline 34 & 199 & $5.378 e-01$ & $1.201 \mathrm{e}+10$ & 34 & 201 & $3.326 \mathrm{e}-01$ & $9.612 e+09$ & 34 & 211 & $0.000 e+00$ & $8.905 e+04$ \\
\hline 34 & 212 & $0.000 e+00$ & $3.212 \mathrm{e}+05$ & 34 & 217 & $1.591 \mathrm{e}-01$ & $7.151 e+09$ & 34 & 219 & $1.670 \mathrm{e}-02$ & $7.534 \mathrm{e}+08$ \\
\hline 34 & 220 & $8.752 \mathrm{e}-02$ & $3.075 e+09$ & 34 & 228 & $0.000 e+00$ & $1.385 e+05$ & 34 & 229 & $0.000 e+00$ & $5.076 e+05$ \\
\hline 34 & 230 & $0.000 e+00$ & $1.038 \mathrm{e}+04$ & 34 & 231 & $0.000 e+00$ & $1.327 e+05$ & 34 & 232 & $0.000 e+00$ & $6.679 \mathrm{e}+05$ \\
\hline 34 & 236 & $0.000 e+00$ & $2.527 e+04$ & 34 & 237 & $0.000 e+00$ & $7.952 e+04$ & 34 & 238 & $0.000 e+00$ & $7.130 \mathrm{e}+04$ \\
\hline 34 & 241 & $0.000 e+00$ & $8.799 e+05$ & 34 & 243 & $1.716 \mathrm{e}-03$ & $6.963 e+07$ & 34 & 245 & $4.152 \mathrm{e}-04$ & $1.382 \mathrm{e}+07$ \\
\hline 34 & 248 & $4.439 e-02$ & $2.328 \mathrm{e}+09$ & 34 & 249 & $6.935 \mathrm{e}-01$ & $2.829 e+10$ & 34 & 251 & $0.000 e+00$ & $1.252 \mathrm{e}+04$ \\
\hline 34 & 252 & $2.401 \mathrm{e}-03$ & $1.276 \mathrm{e}+08$ & 34 & 254 & $1.550 \mathrm{e}-01$ & $6.412 \mathrm{e}+09$ & 34 & 255 & $0.000 e+00$ & $8.679 e+04$ \\
\hline 34 & 256 & $2.793 e+00$ & $9.455 e+10$ & 34 & 257 & $0.000 \mathrm{e}+00$ & $8.982 e+06$ & 34 & 258 & $0.000 e+00$ & $3.772 \mathrm{e}+04$ \\
\hline 34 & 259 & $0.000 e+00$ & $7.398 e+05$ & 34 & 260 & $0.000 e+00$ & $5.100 e+06$ & 34 & 264 & $1.078 e-01$ & $5.762 e+09$ \\
\hline 34 & 265 & $0.000 e+00$ & $2.287 e+05$ & 34 & 267 & $0.000 e+00$ & $1.656 \mathrm{e}+04$ & 34 & 268 & $0.000 e+00$ & $2.862 e+05$ \\
\hline 34 & 269 & $0.000 e+00$ & $9.595 e+05$ & 34 & 270 & $0.000 e+00$ & $1.716 e+04$ & 34 & 277 & $0.000 e+00$ & $1.238 \mathrm{e}+05$ \\
\hline 34 & 278 & $0.000 e+00$ & $3.241 \mathrm{e}+04$ & 35 & 41 & $1.748 \mathrm{e}-03$ & $6.825 e+06$ & 35 & 42 & $4.425 e-02$ & $7.016 e+07$ \\
\hline 35 & 43 & $1.195 \mathrm{e}-01$ & $1.140 \mathrm{e}+08$ & 35 & 45 & $1.557 \mathrm{e}+00$ & $1.550 \mathrm{e}+09$ & 35 & 53 & $0.000 e+00$ & $1.946 \mathrm{e}+04$ \\
\hline 35 & 54 & $5.479 \mathrm{e}-01$ & $3.349 e+09$ & 35 & 55 & $2.087 e-01$ & $1.369 e+09$ & 35 & 56 & $1.217 \mathrm{e}-01$ & $4.860 e+08$ \\
\hline
\end{tabular}


Table III. Ca IX Oscillator Strengths and Radiative Decay Rates for transitions involving levels higher than 4.

\begin{tabular}{|c|c|c|c|c|c|c|c|c|c|c|c|}
\hline $\begin{array}{l}\text { Low. } \\
\text { Lev. }\end{array}$ & $\begin{array}{l}\text { Upp } \\
\text { Lev }\end{array}$ & $\begin{array}{l}\text { Osc. } \\
\text { Str. }\end{array}$ & $\begin{array}{l}\text { Rad. } \\
\text { Rate }\end{array}$ & $\begin{array}{l}\text { Low. } \\
\text { Lev. }\end{array}$ & $\begin{array}{l}\text { Upp } \\
\text { Lev }\end{array}$ & $\begin{array}{l}\text { Osc. } \\
\text { Str. }\end{array}$ & $\begin{array}{l}\text { Rad. } \\
\text { Rate }\end{array}$ & $\begin{array}{l}\text { Low. } \\
\text { Lev. }\end{array}$ & $\begin{array}{l}\text { Upp } \\
\text { Lev }\end{array}$ & $\begin{array}{l}\text { Osc. } \\
\text { Str. }\end{array}$ & $\begin{array}{l}\text { Rad. } \\
\text { Rate }\end{array}$ \\
\hline$i$ & $j$ & $g f$ & $(1 / s)$ & $i$ & $\mathrm{j}$ & $g f$ & $(1 / s)$ & $i$ & $j$ & $g f$ & $(1 / s)$ \\
\hline 35 & 58 & $1.154 \mathrm{e}-02$ & $2.507 e+08$ & 35 & 59 & $8.425 e-03$ & $6.146 e+07$ & 35 & 60 & $5.928 e-02$ & $4.444 e+08$ \\
\hline 35 & 61 & $7.442 e-03$ & $3.354 \mathrm{e}+07$ & 35 & 62 & $7.642 \mathrm{e}-01$ & $3.888 e+09$ & 35 & 63 & $2.602 \mathrm{e}-01$ & $7.703 e+09$ \\
\hline 35 & 64 & $3.948 e-02$ & $4.855 e+08$ & 35 & 65 & $2.031 \mathrm{e}-01$ & $8.199 e+09$ & 35 & 66 & $0.000 e+00$ & $6.653 e+03$ \\
\hline 35 & 68 & $0.000 e+00$ & $5.553 e+03$ & 35 & 70 & $0.000 e+00$ & $7.782 e+04$ & 35 & 72 & $0.000 e+00$ & $1.885 e+04$ \\
\hline 35 & 73 & $0.000 e+00$ & $1.374 \mathrm{e}+05$ & 35 & 75 & $0.000 e+00$ & $1.238 e+04$ & 35 & 77 & $0.000 e+00$ & $2.611 \mathrm{e}+04$ \\
\hline 35 & 78 & $0.000 e+00$ & $8.320 e+03$ & 35 & 79 & $0.000 e+00$ & $2.554 e+04$ & 35 & 81 & $0.000 e+00$ & $3.053 e+05$ \\
\hline 35 & 90 & $7.672 \mathrm{e}-03$ & $9.469 \mathrm{e}+07$ & 35 & 91 & $4.941 \mathrm{e}-03$ & $1.019 e+08$ & 35 & 92 & $2.107 e-01$ & $2.649 \mathrm{e}+09$ \\
\hline 35 & 95 & $5.353 e-03$ & $7.045 e+07$ & 35 & 96 & $3.619 \mathrm{e}-03$ & $7.967 e+07$ & 35 & 97 & $6.550 \mathrm{e}-02$ & $9.116 e+08$ \\
\hline 35 & 98 & $0.000 e+00$ & $6.329 e+04$ & 35 & 99 & $0.000 e+00$ & $8.904 e+04$ & 35 & 101 & $0.000 e+00$ & $1.080 e+06$ \\
\hline 35 & 109 & $3.300 e-02$ & $5.861 e+08$ & 35 & 112 & $0.000 e+00$ & $1.943 e+05$ & 35 & 113 & $0.000 e+00$ & $7.570 e+04$ \\
\hline 35 & 114 & $0.000 e+00$ & $4.145 e+04$ & 35 & 115 & $0.000 e+00$ & $3.207 e+04$ & 35 & 117 & $0.000 e+00$ & $1.384 e+04$ \\
\hline 35 & 118 & $0.000 e+00$ & $3.567 e+04$ & 35 & 119 & $0.000 e+00$ & $1.248 e+04$ & 35 & 123 & $0.000 e+00$ & $1.780 e+04$ \\
\hline 35 & 124 & $0.000 e+00$ & $2.585 e+05$ & 35 & 125 & $6.609 e-04$ & $2.845 e+07$ & 35 & 126 & $1.075 e-04$ & $4.687 e+06$ \\
\hline 35 & 127 & $0.000 e+00$ & $1.568 \mathrm{e}+05$ & 35 & 129 & $2.812 \mathrm{e}-05$ & $3.712 \mathrm{e}+06$ & 35 & 134 & $5.255 e-04$ & $1.449 \mathrm{e}+07$ \\
\hline 35 & 135 & $2.150 e-04$ & $3.036 \mathrm{e}+07$ & 35 & 136 & $0.000 e+00$ & $6.622 \mathrm{e}+03$ & 35 & 147 & $0.000 e+00$ & $3.024 e+04$ \\
\hline 35 & 154 & $7.366 e-04$ & $4.080 e+07$ & 35 & 156 & $0.000 e+00$ & $2.435 e+04$ & 35 & 176 & $0.000 e+00$ & $5.489 e+03$ \\
\hline 35 & 178 & $0.000 e+00$ & $2.402 e+04$ & 35 & 183 & $4.981 e-04$ & $1.758 e+07$ & 35 & 187 & $6.366 e-04$ & $2.353 e+07$ \\
\hline 35 & 196 & $0.000 e+00$ & $2.130 e+04$ & 35 & 207 & $1.529 \mathrm{e}-04$ & $3.165 e+07$ & 35 & 208 & $0.000 e+00$ & $3.187 e+04$ \\
\hline 35 & 213 & $1.389 e-04$ & $8.047 e+06$ & 35 & 225 & $0.000 e+00$ & $1.218 e+04$ & 35 & 233 & $1.492 \mathrm{e}-03$ & $1.744 \mathrm{e}+08$ \\
\hline 35 & 238 & $1.111 \mathrm{e}-04$ & $8.021 \mathrm{e}+06$ & 35 & 250 & $0.000 e+00$ & $2.544 e+04$ & 35 & 281 & $3.117 \mathrm{e}-05$ & $1.181 \mathrm{e}+07$ \\
\hline 35 & 283 & $0.000 e+00$ & $1.176 \mathrm{e}+04$ & 36 & 62 & $0.000 e+00$ & $4.145 e+03$ & 36 & 69 & $1.220 \mathrm{e}-02$ & $6.759 e+07$ \\
\hline 36 & 75 & $5.705 e-02$ & $3.313 e+08$ & 36 & 77 & $1.767 e-01$ & $1.051 \mathrm{e}+09$ & 36 & 101 & $2.032 \mathrm{e}-02$ & $1.880 \mathrm{e}+08$ \\
\hline 36 & 109 & $0.000 e+00$ & $3.943 e+05$ & 36 & 117 & $1.620 \mathrm{e}-03$ & $2.463 e+07$ & 36 & 119 & $8.542 \mathrm{e}-04$ & $1.312 \mathrm{e}+07$ \\
\hline 36 & 124 & $1.190 \mathrm{e}+00$ & $1.950 \mathrm{e}+10$ & 36 & 134 & $0.000 e+00$ & $5.415 e+04$ & 36 & 144 & $0.000 e+00$ & $6.935 e+05$ \\
\hline 36 & 147 & $7.166 \mathrm{e}-04$ & $1.549 e+07$ & 36 & 151 & $0.000 e+00$ & $3.329 e+03$ & 36 & 155 & $0.000 e+00$ & $2.181 e+04$ \\
\hline 36 & 157 & $2.959 \mathrm{e}-02$ & $5.094 \mathrm{e}+08$ & 36 & 160 & $3.286 \mathrm{e}-01$ & $4.637 e+09$ & 36 & 162 & $8.483 e-02$ & $1.472 \mathrm{e}+09$ \\
\hline 36 & 163 & $2.186 \mathrm{e}-04$ & $4.881 e+06$ & 36 & 167 & $8.971 \mathrm{e}-02$ & $1.574 \mathrm{e}+09$ & 36 & 168 & $7.072 \mathrm{e}-02$ & $1.016 e+09$ \\
\hline 36 & 170 & $1.177 \mathrm{e}+00$ & $1.697 \mathrm{e}+10$ & 36 & 175 & $1.843 e-02$ & $3.291 e+08$ & 36 & 176 & $1.562 \mathrm{e}-03$ & $3.591 e+07$ \\
\hline 36 & 178 & $2.898 \mathrm{e}-03$ & $6.713 e+07$ & 36 & 179 & $0.000 e+00$ & $1.117 e+04$ & 36 & 186 & $0.000 e+00$ & $9.230 e+03$ \\
\hline 36 & 187 & $0.000 e+00$ & $2.648 \mathrm{e}+05$ & 36 & 188 & $0.000 e+00$ & $1.094 \mathrm{e}+06$ & 36 & 189 & $2.192 \mathrm{e}+00$ & $4.244 e+10$ \\
\hline 36 & 190 & $1.118 \mathrm{e}-02$ & $2.207 e+08$ & 36 & 194 & $2.792 \mathrm{e}-03$ & $4.520 e+07$ & 36 & 206 & $1.263 \mathrm{e}-01$ & $3.472 \mathrm{e}+09$ \\
\hline 36 & 209 & $9.464 \mathrm{e}+00$ & $1.753 e+11$ & 36 & 213 & $0.000 e+00$ & $4.110 \mathrm{e}+05$ & 36 & 217 & $2.422 \mathrm{e}-04$ & $1.026 \mathrm{e}+07$ \\
\hline 36 & 224 & $2.625 \mathrm{e}-01$ & $1.138 \mathrm{e}+10$ & 36 & 226 & $0.000 e+00$ & $6.303 e+05$ & 36 & 238 & $0.000 e+00$ & $6.157 e+05$ \\
\hline 36 & 241 & $0.000 e+00$ & $4.946 \mathrm{e}+04$ & 36 & 242 & $0.000 e+00$ & $1.308 e+06$ & 36 & 243 & $4.168 \mathrm{e}-01$ & $1.601 \mathrm{e}+10$ \\
\hline 36 & 244 & $4.577 e-03$ & $1.763 e+08$ & 36 & 245 & $2.362 \mathrm{e}-04$ & $7.447 e+06$ & 36 & 249 & $1.480 \mathrm{e}-03$ & $5.718 e+07$ \\
\hline 36 & 251 & $0.000 e+00$ & $2.971 \mathrm{e}+06$ & 36 & 255 & $0.000 e+00$ & $6.973 e+04$ & 36 & 260 & $0.000 e+00$ & $1.686 e+04$ \\
\hline 36 & 261 & $0.000 e+00$ & $4.532 e+04$ & 36 & 265 & $0.000 e+00$ & $6.083 e+05$ & 36 & 266 & $0.000 e+00$ & $4.578 e+03$ \\
\hline 36 & 269 & $0.000 \mathrm{e}+00$ & $1.183 e+05$ & 36 & 270 & $0.000 e+00$ & $3.655 e+04$ & 36 & 275 & $6.099 e-02$ & $3.104 e+09$ \\
\hline 36 & 276 & $0.000 \mathrm{e}+00$ & $1.133 e+07$ & 36 & 280 & $0.000 \mathrm{e}+00$ & $1.832 \mathrm{e}+04$ & 36 & 282 & $3.774 \mathrm{e}+00$ & $1.246 \mathrm{e}+11$ \\
\hline 37 & 52 & $7.243 e-03$ & $1.774 \mathrm{e}+07$ & 37 & 53 & $2.189 e-02$ & $2.708 e+07$ & 37 & 65 & $0.000 e+00$ & $6.839 e+03$ \\
\hline 37 & 69 & $1.895 e-03$ & $1.048 \mathrm{e}+07$ & 37 & 75 & $6.798 e-03$ & $3.942 e+07$ & 37 & 77 & $1.965 e-02$ & $1.168 \mathrm{e}+08$ \\
\hline 37 & 79 & $3.784 \mathrm{e}-04$ & $5.668 e+06$ & 37 & 81 & $1.816 \mathrm{e}-02$ & $2.846 e+08$ & 37 & 92 & $0.000 e+00$ & $6.639 e+03$ \\
\hline 37 & 101 & $2.338 \mathrm{e}-02$ & $2.160 \mathrm{e}+08$ & 37 & 105 & $0.000 e+00$ & $3.278 e+04$ & 37 & 109 & $0.000 \mathrm{e}+00$ & $2.423 e+05$ \\
\hline 37 & 111 & $9.336 e-03$ & $3.183 e+08$ & 37 & 112 & $3.871 \mathrm{e}-01$ & $8.051 e+09$ & 37 & 113 & $7.833 e-02$ & $1.635 \mathrm{e}+09$ \\
\hline
\end{tabular}


Table III. Ca IX Oscillator Strengths and Radiative Decay Rates for transitions involving levels higher than 4.

\begin{tabular}{|c|c|c|c|c|c|c|c|c|c|c|c|}
\hline $\begin{array}{l}\text { Low. } \\
\text { Lev . }\end{array}$ & $\begin{array}{l}\text { Upp } \\
\text { Lev }\end{array}$ & $\begin{array}{l}\text { Osc. } \\
\text { Str. }\end{array}$ & $\begin{array}{l}\text { Rad. } \\
\text { Rate }\end{array}$ & $\begin{array}{l}\text { Low. } \\
\text { Lev. }\end{array}$ & $\begin{array}{l}\text { Upp. } \\
\text { Lev. }\end{array}$ & $\begin{array}{l}\text { Osc. } \\
\text { Str. }\end{array}$ & $\begin{array}{l}\text { Rad. } \\
\text { Rate }\end{array}$ & $\begin{array}{l}\text { Low. } \\
\text { Lev. }\end{array}$ & $\begin{array}{l}\text { - Upp } \\
\text {. Lev }\end{array}$ & $\begin{array}{l}\text { Osc. } \\
\text { Str. }\end{array}$ & $\begin{array}{l}\text { Rad. } \\
\text { Rate }\end{array}$ \\
\hline$i$ & $j$ & $g f$ & $(1 / s)$ & $i$ & $j$ & $g f$ & $(1 / s)$ & i & j & $g f$ & $(1 / s)$ \\
\hline 37 & 114 & $3.559 e-02$ & $1.246 \mathrm{e}+09$ & 37 & 115 & $2.730 e-04$ & $9.647 e+06$ & 37 & 117 & $2.310 e-03$ & $3.509 e+07$ \\
\hline 37 & 118 & $5.902 \mathrm{e}-03$ & $1.264 \mathrm{e}+08$ & 37 & 122 & $4.281 e-03$ & $1.611 \mathrm{e}+08$ & 37 & 123 & $8.982 \mathrm{e}-04$ & $2.030 e+07$ \\
\hline 37 & 124 & $1.435 e-01$ & $2.351 e+09$ & 37 & 127 & $2.603 e-01$ & $1.041 e+10$ & 37 & 129 & $0.000 e+00$ & $5.791 e+03$ \\
\hline 37 & 134 & $0.000 e+00$ & $8.338 e+03$ & 37 & 135 & $0.000 e+00$ & $2.285 e+05$ & 37 & 136 & $1.297 \mathrm{e}-04$ & $3.733 e+06$ \\
\hline 37 & 140 & $1.321 \mathrm{e}-04$ & $3.878 e+06$ & 37 & 144 & $0.000 e+00$ & $4.485 e+05$ & 37 & 147 & $7.621 \mathrm{e}-04$ & $1.646 e+07$ \\
\hline 37 & 151 & $0.000 e+00$ & $3.166 \mathrm{e}+03$ & 37 & 154 & $0.000 e+00$ & $4.428 e+05$ & 37 & 155 & $0.000 e+00$ & $1.978 e+04$ \\
\hline 37 & 163 & $1.965 e-02$ & $4.386 e+08$ & 37 & 176 & $1.398 \mathrm{e}-01$ & $3.212 \mathrm{e}+09$ & 37 & 178 & $2.315 e-01$ & $5.359 e+09$ \\
\hline 37 & 179 & $0.000 e+00$ & $2.750 e+04$ & 37 & 182 & $0.000 e+00$ & $4.654 e+03$ & 37 & 183 & $0.000 e+00$ & $2.067 e+04$ \\
\hline 37 & 188 & $0.000 e+00$ & $2.457 e+05$ & 37 & 191 & $7.986 e-03$ & $2.837 e+08$ & 37 & 196 & $2.871 \mathrm{e}+00$ & $1.037 e+11$ \\
\hline 37 & 197 & $5.664 \mathrm{e}-03$ & $1.508 \mathrm{e}+08$ & 37 & 201 & $2.996 \mathrm{e}-02$ & $8.040 e+08$ & 37 & 202 & $3.902 \mathrm{e}-03$ & $1.466 e+08$ \\
\hline 37 & 203 & $1.028 \mathrm{e}-02$ & $3.905 e+08$ & 37 & 204 & $4.522 \mathrm{e}-03$ & $2.865 e+08$ & 37 & 206 & $5.145 \mathrm{e}+00$ & $1.413 e+11$ \\
\hline 37 & 207 & $0.000 e+00$ & $1.773 e+06$ & 37 & 208 & $2.002 \mathrm{e}-01$ & $1.342 e+10$ & 37 & 213 & $0.000 e+00$ & $2.703 e+05$ \\
\hline 37 & 214 & $6.969 \mathrm{e}-02$ & $4.110 e+09$ & 37 & 217 & $4.469 \mathrm{e}-04$ & $1.893 e+07$ & 37 & 218 & $1.014 \mathrm{e}-03$ & $6.029 e+07$ \\
\hline 37 & 221 & $1.187 e-04$ & $7.140 e+06$ & 37 & 222 & $7.561 \mathrm{e}-04$ & $7.583 e+07$ & 37 & 224 & $3.602 \mathrm{e}-02$ & $1.561 \mathrm{e}+09$ \\
\hline 37 & 225 & $7.615 e-02$ & $7.773 e+09$ & 37 & 226 & $0.000 e+00$ & $4.194 e+05$ & 37 & 227 & $0.000 e+00$ & $6.568 e+03$ \\
\hline 37 & 233 & $0.000 e+00$ & $3.967 e+05$ & 37 & 238 & $0.000 e+00$ & $2.633 e+04$ & 37 & 239 & $0.000 e+00$ & $6.288 e+03$ \\
\hline 37 & 242 & $0.000 e+00$ & $6.575 e+04$ & 37 & 247 & $1.503 e-03$ & $1.045 e+08$ & 37 & 250 & $5.923 e-01$ & $4.145 e+10$ \\
\hline 37 & 252 & $1.077 \mathrm{e}-03$ & $5.425 e+07$ & 37 & 253 & $0.000 e+00$ & $1.615 e+04$ & 37 & 258 & $0.000 e+00$ & $5.894 e+04$ \\
\hline 37 & 259 & $0.000 e+00$ & $2.430 \mathrm{e}+04$ & 37 & 261 & $0.000 e+00$ & $5.305 e+06$ & 37 & 263 & $8.067 e-04$ & $5.716 e+07$ \\
\hline 37 & 264 & $2.441 \mathrm{e}-02$ & $1.235 \mathrm{e}+09$ & 37 & 265 & $0.000 e+00$ & $5.856 e+06$ & 37 & 269 & $0.000 e+00$ & $7.463 e+05$ \\
\hline 37 & 272 & $1.880 e-03$ & $1.336 \mathrm{e}+08$ & 37 & 273 & $1.293 e-03$ & $1.532 \mathrm{e}+08$ & 37 & 275 & $1.551 \mathrm{e}+00$ & $7.890 e+10$ \\
\hline 37 & 277 & $0.000 e+00$ & $5.693 e+03$ & 37 & 278 & $0.000 e+00$ & $5.259 e+04$ & 37 & 279 & $0.000 e+00$ & $7.579 e+03$ \\
\hline 37 & 280 & $0.000 e+00$ & $1.426 \mathrm{e}+06$ & 37 & 281 & $0.000 e+00$ & $4.516 e+06$ & 37 & 283 & $8.928 e-02$ & $1.082 \mathrm{e}+10$ \\
\hline 38 & 67 & $8.212 \mathrm{e}-04$ & $6.145 \mathrm{e}+06$ & 38 & 68 & $5.132 \mathrm{e}-04$ & $3.872 \mathrm{e}+06$ & 38 & 106 & $0.000 e+00$ & $9.266 e+04$ \\
\hline 38 & 107 & $0.000 e+00$ & $1.031 \mathrm{e}+04$ & 38 & 108 & $0.000 e+00$ & $4.125 e+04$ & 38 & 110 & $1.296 \mathrm{e}-02$ & $1.303 e+09$ \\
\hline 38 & 111 & $7.259 \mathrm{e}-03$ & $2.438 \mathrm{e}+08$ & 38 & 112 & $9.914 \mathrm{e}-03$ & $2.032 \mathrm{e}+08$ & 38 & 113 & $2.752 \mathrm{e}-02$ & $5.660 e+08$ \\
\hline 38 & 114 & $6.069 \mathrm{e}-03$ & $2.094 \mathrm{e}+08$ & 38 & 115 & $3.187 e-02$ & $1.110 \mathrm{e}+09$ & 38 & 116 & $1.088 \mathrm{e}-01$ & $2.276 e+09$ \\
\hline 38 & 118 & $4.680 \mathrm{e}-03$ & $9.884 \mathrm{e}+07$ & 38 & 121 & $1.361 \mathrm{e}-01$ & $1.515 e+10$ & 38 & 122 & $1.039 \mathrm{e}-01$ & $3.853 e+09$ \\
\hline 38 & 123 & $1.483 e-01$ & $3.303 e+09$ & 38 & 136 & $9.180 e-05$ & $2.610 \mathrm{e}+06$ & 38 & 137 & $5.590 e-04$ & $1.596 e+07$ \\
\hline 38 & 140 & $2.553 e-04$ & $7.402 e+06$ & 38 & 143 & $1.467 e-03$ & $4.320 e+07$ & 38 & 145 & $1.883 e-03$ & $9.279 e+07$ \\
\hline 38 & 146 & $2.261 \mathrm{e}-03$ & $3.351 \mathrm{e}+08$ & 38 & 148 & $0.000 e+00$ & $2.005 e+05$ & 38 & 149 & $0.000 e+00$ & $2.301 e+04$ \\
\hline 38 & 150 & $0.000 e+00$ & $8.844 \mathrm{e}+04$ & 38 & 180 & $0.000 e+00$ & $1.210 \mathrm{e}+05$ & 38 & 181 & $0.000 e+00$ & $1.824 \mathrm{e}+05$ \\
\hline 38 & 184 & $0.000 e+00$ & $1.614 \mathrm{e}+05$ & 38 & 186 & $0.000 e+00$ & $2.803 e+05$ & 38 & 187 & $0.000 e+00$ & $1.033 e+04$ \\
\hline 38 & 200 & $7.908 e-01$ & $4.896 e+10$ & 38 & 202 & $2.755 e+00$ & $1.024 e+11$ & 38 & 203 & $6.980 e-01$ & $2.623 e+10$ \\
\hline 38 & 204 & $5.615 e-01$ & $3.519 \mathrm{e}+10$ & 38 & 205 & $6.071 \mathrm{e}-01$ & $1.142 \mathrm{e}+11$ & 38 & 210 & $0.000 e+00$ & $1.035 e+05$ \\
\hline 38 & 211 & $0.000 e+00$ & $1.154 e+04$ & 38 & 212 & $0.000 e+00$ & $4.647 e+04$ & 38 & 215 & $6.044 e-03$ & $5.913 e+08$ \\
\hline 38 & 216 & $2.228 \mathrm{e}-02$ & $1.308 e+09$ & 38 & 218 & $2.241 \mathrm{e}-04$ & $1.321 \mathrm{e}+07$ & 38 & 221 & $3.052 e-02$ & $1.820 e+09$ \\
\hline 38 & 222 & $2.022 e-02$ & $2.011 \mathrm{e}+09$ & 38 & 223 & $2.603 e-02$ & $7.766 e+09$ & 38 & 227 & $0.000 e+00$ & $2.318 e+05$ \\
\hline 38 & 228 & $0.000 e+00$ & $2.869 \mathrm{e}+04$ & 38 & 229 & $0.000 e+00$ & $9.982 e+04$ & 38 & 235 & $0.000 e+00$ & $1.448 e+05$ \\
\hline 38 & 236 & $0.000 e+00$ & $2.129 \mathrm{e}+05$ & 38 & 238 & $0.000 e+00$ & $2.977 e+04$ & 38 & 240 & $0.000 e+00$ & $2.201 \mathrm{e}+05$ \\
\hline 38 & 241 & $0.000 e+00$ & $3.704 \mathrm{e}+05$ & 38 & 247 & $1.444 \mathrm{e}-04$ & $9.953 e+06$ & 38 & 258 & $0.000 e+00$ & $1.520 \mathrm{e}+04$ \\
\hline 38 & 262 & $1.995 e-01$ & $2.338 \mathrm{e}+10$ & 38 & 263 & $7.698 \mathrm{e}-01$ & $5.411 e+10$ & 38 & 267 & $0.000 e+00$ & $2.250 \mathrm{e}+06$ \\
\hline 38 & 268 & $0.000 e+00$ & $3.482 \mathrm{e}+06$ & 38 & 272 & $1.160 \mathrm{e}-01$ & $8.175 e+09$ & 38 & 273 & $1.477 \mathrm{e}-01$ & $1.736 e+10$ \\
\hline 38 & 274 & $1.556 \mathrm{e}-01$ & $5.487 e+10$ & 38 & 277 & $0.000 e+00$ & $1.643 e+06$ & 38 & 278 & $0.000 e+00$ & $5.284 e+05$ \\
\hline
\end{tabular}


Table III. Ca IX Oscillator Strengths and Radiative Decay Rates for transitions involving levels higher than 4.

\begin{tabular}{|c|c|c|c|c|c|c|c|c|c|c|c|}
\hline $\begin{array}{l}\text { Low. } \\
\text { Lev. }\end{array}$ & $\begin{array}{l}\text { - Upp } \\
\text {. Lev }\end{array}$ & $\begin{array}{l}\text { Osc. } \\
\text { Str. }\end{array}$ & $\begin{array}{l}\text { Rad. } \\
\text { Rate }\end{array}$ & $\begin{array}{l}\text { Low. } \\
\text { Lev. }\end{array}$ & $\begin{array}{l}\text { - Upp } \\
\text { - Lev }\end{array}$ & $\begin{array}{l}\text { Osc. } \\
\text { Str. }\end{array}$ & $\begin{array}{l}\text { Rad. } \\
\text { Rate }\end{array}$ & $\begin{array}{l}\text { Low. } \\
\text { Lev }\end{array}$ & $\begin{array}{l}\text { Upp } \\
\text { Lev }\end{array}$ & $\begin{array}{l}\text { Osc. } \\
\text { Str. }\end{array}$ & $\begin{array}{l}\text { Rad. } \\
\text { Rate }\end{array}$ \\
\hline$i$ & $j$ & $g f$ & $(1 / s)$ & i & $j$ & $g f$ & $(1 / s)$ & $i$ & j & $g f$ & $(1 / s)$ \\
\hline 38 & 279 & $0.000 e+00$ & $4.080 e+06$ & 39 & 66 & $5.814 \mathrm{e}-04$ & $7.250 \mathrm{e}+06$ & 39 & 107 & $0.000 e+00$ & $4.118 e+04$ \\
\hline 39 & 111 & $1.362 \mathrm{e}-02$ & $4.573 e+08$ & 39 & 114 & $1.503 e-03$ & $5.187 e+07$ & 39 & 115 & $5.607 e-02$ & $1.953 e+09$ \\
\hline 39 & 122 & $1.279 \mathrm{e}-01$ & $4.745 e+09$ & 39 & 127 & $3.914 \mathrm{e}-04$ & $1.543 e+07$ & 39 & 138 & $3.692 \mathrm{e}-04$ & $1.761 \mathrm{e}+07$ \\
\hline 39 & 145 & $1.688 \mathrm{e}-03$ & $8.318 e+07$ & 39 & 149 & $0.000 e+00$ & $8.926 e+04$ & 39 & 180 & $0.000 e+00$ & $1.223 e+05$ \\
\hline 39 & 186 & $0.000 e+00$ & $1.247 \mathrm{e}+05$ & 39 & 187 & $0.000 e+00$ & $4.957 e+03$ & 39 & 200 & $1.330 e+00$ & $8.231 \mathrm{e}+10$ \\
\hline 39 & 204 & $4.732 \mathrm{e}-01$ & $2.965 e+10$ & 39 & 211 & $0.000 e+00$ & $4.625 e+04$ & 39 & 215 & $1.001 \mathrm{e}-02$ & $9.793 e+08$ \\
\hline 39 & 222 & $2.508 \mathrm{e}-02$ & $2.494 \mathrm{e}+09$ & 39 & 225 & $9.515 e-05$ & $9.631 e+06$ & 39 & 228 & $0.000 e+00$ & $1.029 e+05$ \\
\hline 39 & 235 & $0.000 e+00$ & $1.487 e+05$ & 39 & 238 & $0.000 e+00$ & $1.107 e+04$ & 39 & 241 & $0.000 e+00$ & $1.658 e+05$ \\
\hline 39 & 262 & $3.460 \mathrm{e}-01$ & $4.053 e+10$ & 39 & 267 & $0.000 \mathrm{e}+00$ & $2.466 e+06$ & 39 & 273 & $1.168 \mathrm{e}-01$ & $1.373 e+10$ \\
\hline 39 & 278 & $0.000 e+00$ & $1.652 \mathrm{e}+06$ & 39 & 280 & $0.000 e+00$ & $2.119 e+04$ & 40 & 69 & $1.944 \mathrm{e}-03$ & $1.049 e+07$ \\
\hline 40 & 106 & $0.000 e+00$ & $3.041 \mathrm{e}+04$ & 40 & 107 & $0.000 e+00$ & $7.139 e+04$ & 40 & 108 & $0.000 e+00$ & $8.193 e+04$ \\
\hline 40 & 111 & $1.233 \mathrm{e}-02$ & $4.140 \mathrm{e}+08$ & 40 & 112 & $9.032 \mathrm{e}-03$ & $1.850 e+08$ & 40 & 113 & $6.773 e-02$ & $1.392 e+09$ \\
\hline 40 & 114 & $8.496 e-03$ & $2.929 \mathrm{e}+08$ & 40 & 115 & $9.315 e-04$ & $3.242 \mathrm{e}+07$ & 40 & 116 & $3.332 \mathrm{e}-02$ & $6.967 e+08$ \\
\hline 40 & 117 & $1.949 e-01$ & $2.916 \mathrm{e}+09$ & 40 & 118 & $9.886 e-04$ & $2.086 e+07$ & 40 & 119 & $1.046 e-02$ & $1.581 \mathrm{e}+08$ \\
\hline 40 & 122 & $1.642 \mathrm{e}-01$ & $6.088 e+09$ & 40 & 123 & $4.845 e-01$ & $1.079 e+10$ & 40 & 124 & $1.794 \mathrm{e}-03$ & $2.895 e+07$ \\
\hline 40 & 127 & $3.003 e-03$ & $1.183 e+08$ & 40 & 136 & $1.432 \mathrm{e}-04$ & $4.068 e+06$ & 40 & 139 & $3.749 e-04$ & $7.666 e+06$ \\
\hline 40 & 141 & $5.137 e-04$ & $1.066 \mathrm{e}+07$ & 40 & 143 & $7.752 \mathrm{e}-03$ & $2.282 \mathrm{e}+08$ & 40 & 145 & $2.709 \mathrm{e}-03$ & $1.334 e+08$ \\
\hline 40 & 148 & $0.000 e+00$ & $6.432 e+04$ & 40 & 149 & $0.000 e+00$ & $1.540 \mathrm{e}+05$ & 40 & 150 & $0.000 e+00$ & $1.795 e+05$ \\
\hline 40 & 165 & $0.000 e+00$ & $1.235 e+04$ & 40 & 176 & $7.945 e-04$ & $1.803 e+07$ & 40 & 178 & $1.184 \mathrm{e}-03$ & $2.706 e+07$ \\
\hline 40 & 179 & $0.000 e+00$ & $9.722 \mathrm{e}+03$ & 40 & 180 & $0.000 e+00$ & $1.651 \mathrm{e}+04$ & 40 & 181 & $0.000 e+00$ & $8.841 e+04$ \\
\hline 40 & 182 & $0.000 e+00$ & $2.618 \mathrm{e}+05$ & 40 & 184 & $0.000 e+00$ & $4.834 e+05$ & 40 & 185 & $0.000 e+00$ & $6.462 \mathrm{e}+05$ \\
\hline 40 & 186 & $0.000 e+00$ & $2.184 \mathrm{e}+05$ & 40 & 187 & $0.000 e+00$ & $9.073 e+03$ & 40 & 191 & $2.548 e-04$ & $8.943 e+06$ \\
\hline 40 & 196 & $1.303 e-02$ & $4.649 \mathrm{e}+08$ & 40 & 197 & $1.025 \mathrm{e}-02$ & $2.697 e+08$ & 40 & 200 & $2.973 e-02$ & $1.839 e+09$ \\
\hline 40 & 201 & $4.983 e+00$ & $1.322 \mathrm{e}+11$ & 40 & 202 & $8.301 \mathrm{e}-01$ & $3.084 \mathrm{e}+10$ & 40 & 203 & $2.330 e+00$ & $8.752 e+10$ \\
\hline 40 & 204 & $7.842 \mathrm{e}-01$ & $4.911 \mathrm{e}+10$ & 40 & 206 & $3.174 \mathrm{e}-02$ & $8.621 \mathrm{e}+08$ & 40 & 207 & $0.000 e+00$ & $1.043 e+04$ \\
\hline 40 & 208 & $1.331 \mathrm{e}-03$ & $8.821 \mathrm{e}+07$ & 40 & 210 & $0.000 e+00$ & $3.382 e+04$ & 40 & 211 & $0.000 e+00$ & $7.917 e+04$ \\
\hline 40 & 212 & $0.000 e+00$ & $9.237 e+04$ & 40 & 215 & $2.521 \mathrm{e}-04$ & $2.465 e+07$ & 40 & 216 & $4.716 \mathrm{e}-03$ & $2.768 e+08$ \\
\hline 40 & 217 & $3.670 \mathrm{e}-02$ & $1.540 \mathrm{e}+09$ & 40 & 219 & $6.649 \mathrm{e}-04$ & $2.802 \mathrm{e}+07$ & 40 & 221 & $9.956 e-02$ & $5.937 e+09$ \\
\hline 40 & 222 & $3.209 \mathrm{e}-02$ & $3.190 \mathrm{e}+09$ & 40 & 224 & $4.029 \mathrm{e}-04$ & $1.730 \mathrm{e}+07$ & 40 & 225 & $8.170 e-04$ & $8.266 e+07$ \\
\hline 40 & 227 & $0.000 e+00$ & $7.167 e+04$ & 40 & 228 & $0.000 e+00$ & $1.775 e+05$ & 40 & 229 & $0.000 e+00$ & $2.123 e+05$ \\
\hline 40 & 235 & $0.000 e+00$ & $1.913 e+04$ & 40 & 236 & $0.000 e+00$ & $9.997 e+04$ & 40 & 237 & $0.000 e+00$ & $3.099 e+05$ \\
\hline 40 & 238 & $0.000 e+00$ & $1.919 \mathrm{e}+04$ & 40 & 239 & $0.000 e+00$ & $8.852 e+05$ & 40 & 240 & $0.000 e+00$ & $6.659 \mathrm{e}+05$ \\
\hline 40 & 241 & $0.000 e+00$ & $2.963 e+05$ & 40 & 248 & $3.231 \mathrm{e}-04$ & $1.591 \mathrm{e}+07$ & 40 & 250 & $1.610 \mathrm{e}-03$ & $1.117 \mathrm{e}+08$ \\
\hline 40 & 258 & $0.000 e+00$ & $5.969 e+03$ & 40 & 259 & $0.000 e+00$ & $2.460 e+04$ & 40 & 261 & $0.000 e+00$ & $7.181 e+03$ \\
\hline 40 & 262 & $6.848 e-03$ & $8.020 e+08$ & 40 & 263 & $1.494 \mathrm{e}-01$ & $1.050 e+10$ & 40 & 264 & $1.267 e+00$ & $6.358 e+10$ \\
\hline 40 & 265 & $0.000 e+00$ & $4.879 e+05$ & 40 & 267 & $0.000 e+00$ & $2.631 e+05$ & 40 & 268 & $0.000 e+00$ & $1.485 e+06$ \\
\hline 40 & 269 & $0.000 e+00$ & $4.481 e+06$ & 40 & 272 & $6.611 \mathrm{e}-01$ & $4.659 e+10$ & 40 & 273 & $2.014 \mathrm{e}-01$ & $2.366 e+10$ \\
\hline 40 & 275 & $2.478 \mathrm{e}-02$ & $1.250 \mathrm{e}+09$ & 40 & 277 & $0.000 e+00$ & $3.797 e+06$ & 40 & 278 & $0.000 e+00$ & $3.181 \mathrm{e}+06$ \\
\hline 40 & 279 & $0.000 e+00$ & $1.352 \mathrm{e}+06$ & 40 & 280 & $0.000 e+00$ & $4.993 e+04$ & 40 & 281 & $0.000 e+00$ & $2.548 e+04$ \\
\hline 40 & 283 & $6.474 \mathrm{e}-04$ & $7.780 \mathrm{e}+07$ & 41 & 70 & $5.924 \mathrm{e}-04$ & $4.603 e+06$ & 41 & 73 & $2.537 e-03$ & $1.992 \mathrm{e}+07$ \\
\hline 41 & 79 & $2.798 e-04$ & $2.539 \mathrm{e}+06$ & 41 & 81 & $1.314 \mathrm{e}-02$ & $1.263 e+08$ & 41 & 97 & $0.000 e+00$ & $1.094 \mathrm{e}+04$ \\
\hline 41 & 109 & $0.000 e+00$ & $2.171 \mathrm{e}+04$ & 41 & 111 & $8.929 e-03$ & $2.215 e+08$ & 41 & 114 & $4.293 e-02$ & $1.099 \mathrm{e}+09$ \\
\hline 41 & 122 & $1.073 e-03$ & $2.986 e+07$ & 41 & 127 & $2.306 \mathrm{e}-01$ & $6.886 e+09$ & 41 & 138 & $3.746 e-04$ & $1.390 \mathrm{e}+07$ \\
\hline 41 & 156 & $4.104 e-03$ & $1.639 \mathrm{e}+08$ & 41 & 186 & $0.000 e+00$ & $1.007 e+04$ & 41 & 187 & $0.000 e+00$ & $2.695 e+05$ \\
\hline
\end{tabular}


Table III. Ca IX Oscillator Strengths and Radiative Decay Rates for transitions involving levels higher than 4.

\begin{tabular}{|c|c|c|c|c|c|c|c|c|c|c|c|}
\hline $\begin{array}{l}\text { Low. } \\
\text { Lev. }\end{array}$ & $\begin{array}{l}\text { Upp } \\
\text { Lev }\end{array}$ & $\begin{array}{l}\text { Osc. } \\
\text { Str. }\end{array}$ & $\begin{array}{l}\text { Rad. } \\
\text { Rate }\end{array}$ & $\begin{array}{l}\text { Low. } \\
\text { Lev. }\end{array}$ & $\begin{array}{l}\text { - Upp } \\
\text {. Lev }\end{array}$ & $\begin{array}{l}\text { Osc. } \\
\text { Str. }\end{array}$ & $\begin{array}{l}\text { Rad. } \\
\text { Rate }\end{array}$ & $\begin{array}{l}\text { Low. } \\
\text { Lev. }\end{array}$ & $\begin{array}{l}\text { Upp } \\
\text { Lev }\end{array}$ & $\begin{array}{l}\text { Osc. } \\
\text { Str. }\end{array}$ & $\begin{array}{l}\text { Rad. } \\
\text { Rate }\end{array}$ \\
\hline$i$ & $j$ & $g f$ & $(1 / s)$ & $i$ & $j$ & $g f$ & $(1 / s)$ & $i$ & j & $g f$ & $(1 / s)$ \\
\hline 41 & 208 & $1.829 \mathrm{e}+00$ & $9.825 e+10$ & 41 & 213 & $0.000 e+00$ & $3.140 \mathrm{e}+04$ & 41 & 215 & $6.419 \mathrm{e}-05$ & $5.287 e+06$ \\
\hline 41 & 222 & $1.383 e-04$ & $1.160 \mathrm{e}+07$ & 41 & 225 & $5.943 e-02$ & $5.081 e+09$ & 41 & 238 & $0.000 e+00$ & $2.040 e+05$ \\
\hline 41 & 241 & $0.000 e+00$ & $1.540 \mathrm{e}+04$ & 41 & 278 & $0.000 e+00$ & $2.432 \mathrm{e}+04$ & 41 & 280 & $0.000 e+00$ & $3.732 \mathrm{e}+06$ \\
\hline 41 & 283 & $5.566 e-01$ & $5.734 e+10$ & 42 & 49 & $5.750 e-01$ & $1.101 e+08$ & 42 & 64 & $0.000 e+00$ & $3.108 e+04$ \\
\hline 42 & 66 & $3.521 \mathrm{e}-01$ & $2.377 e+09$ & 42 & 67 & $7.000 e-02$ & $2.836 e+08$ & 42 & 68 & $2.560 \mathrm{e}-03$ & $1.049 \mathrm{e}+07$ \\
\hline 42 & 70 & $4.647 e-01$ & $3.311 \mathrm{e}+09$ & 42 & 71 & $4.319 e-01$ & $9.240 \mathrm{e}+09$ & 42 & 72 & $5.683 e-02$ & $2.439 e+08$ \\
\hline 42 & 73 & $2.793 e-02$ & $2.011 \mathrm{e}+08$ & 42 & 74 & $3.834 \mathrm{e}-01$ & $1.673 e+09$ & 42 & 78 & $2.134 \mathrm{e}-03$ & $1.065 e+07$ \\
\hline 42 & 79 & $1.609 \mathrm{e}-02$ & $1.348 \mathrm{e}+08$ & 42 & 80 & $1.105 e-02$ & $2.794 e+08$ & 42 & 82 & $0.000 e+00$ & $4.764 e+04$ \\
\hline 42 & 84 & $0.000 e+00$ & $1.957 e+04$ & 42 & 90 & $0.000 e+00$ & $3.156 e+04$ & 42 & 91 & $0.000 e+00$ & $3.440 e+04$ \\
\hline 42 & 95 & $0.000 e+00$ & $4.276 e+04$ & 42 & 96 & $0.000 e+00$ & $3.999 e+04$ & 42 & 98 & $1.880 e+00$ & $1.466 e+10$ \\
\hline 42 & 102 & $0.000 e+00$ & $7.286 e+05$ & 42 & 110 & $3.400 e-03$ & $2.407 e+08$ & 42 & 111 & $2.211 \mathrm{e}-03$ & $5.229 e+07$ \\
\hline 42 & 113 & $3.978 e-04$ & $5.782 e+06$ & 42 & 115 & $1.362 \mathrm{e}-03$ & $3.362 e+07$ & 42 & 116 & $1.552 \mathrm{e}-03$ & $2.302 \mathrm{e}+07$ \\
\hline 42 & 118 & $1.051 \mathrm{e}-02$ & $1.577 e+08$ & 42 & 121 & $1.195 \mathrm{e}-04$ & $9.535 e+06$ & 42 & 122 & $1.532 \mathrm{e}-04$ & $4.075 e+06$ \\
\hline 42 & 130 & $0.000 e+00$ & $5.459 e+03$ & 42 & 137 & $3.232 \mathrm{e}-04$ & $6.902 e+06$ & 42 & 138 & $2.714 \mathrm{e}-04$ & $9.683 e+06$ \\
\hline 42 & 145 & $9.526 e-04$ & $3.529 e+07$ & 42 & 146 & $1.464 \mathrm{e}-03$ & $1.632 \mathrm{e}+08$ & 42 & 148 & $0.000 e+00$ & $1.257 e+05$ \\
\hline 42 & 149 & $0.000 e+00$ & $1.252 \mathrm{e}+05$ & 42 & 150 & $0.000 e+00$ & $1.386 e+04$ & 42 & 151 & $0.000 e+00$ & $3.664 e+05$ \\
\hline 42 & 155 & $0.000 e+00$ & $1.858 e+04$ & 42 & 171 & $0.000 e+00$ & $7.070 e+04$ & 42 & 173 & $0.000 e+00$ & $1.152 \mathrm{e}+04$ \\
\hline 42 & 177 & $2.443 e-03$ & $5.945 e+07$ & 42 & 180 & $0.000 e+00$ & $1.441 \mathrm{e}+05$ & 42 & 181 & $0.000 e+00$ & $5.501 e+04$ \\
\hline 42 & 183 & $0.000 e+00$ & $1.137 e+04$ & 42 & 184 & $0.000 e+00$ & $1.433 e+05$ & 42 & 186 & $0.000 e+00$ & $2.789 e+04$ \\
\hline 42 & 191 & $7.751 \mathrm{e}-04$ & $2.099 \mathrm{e}+07$ & 42 & 215 & $1.338 \mathrm{e}-04$ & $1.074 \mathrm{e}+07$ & 42 & 230 & $0.000 e+00$ & $6.806 e+03$ \\
\hline 42 & 273 & $9.354 e-05$ & $9.183 e+06$ & 42 & 274 & $1.058 \mathrm{e}-04$ & $3.117 e+07$ & 43 & 49 & $1.061 \mathrm{e}-01$ & $2.018 e+07$ \\
\hline 43 & 50 & $8.507 e-01$ & $1.157 \mathrm{e}+08$ & 43 & 64 & $0.000 e+00$ & $5.169 e+04$ & 43 & 66 & $3.301 \mathrm{e}-01$ & $2.226 e+09$ \\
\hline 43 & 67 & $2.100 e-01$ & $8.497 e+08$ & 43 & 68 & $4.327 e-01$ & $1.770 \mathrm{e}+09$ & 43 & 69 & $4.137 e-02$ & $1.212 \mathrm{e}+08$ \\
\hline 43 & 70 & $5.824 \mathrm{e}-01$ & $4.143 e+09$ & 43 & 72 & $5.433 e-01$ & $2.328 e+09$ & 43 & 73 & $2.391 \mathrm{e}-01$ & $1.720 \mathrm{e}+09$ \\
\hline 43 & 75 & $4.949 e-01$ & $1.547 e+09$ & 43 & 77 & $1.308 \mathrm{e}-01$ & $4.228 e+08$ & 43 & 78 & $1.437 e-02$ & $7.163 e+07$ \\
\hline 43 & 79 & $9.583 e-03$ & $8.018 e+07$ & 43 & 81 & $3.459 \mathrm{e}-03$ & $3.072 e+07$ & 43 & 83 & $0.000 e+00$ & $7.579 e+04$ \\
\hline 43 & 89 & $0.000 \mathrm{e}+00$ & $2.313 e+04$ & 43 & 90 & $0.000 e+00$ & $2.058 e+04$ & 43 & 91 & $0.000 \mathrm{e}+00$ & $5.744 e+04$ \\
\hline 43 & 94 & $0.000 \mathrm{e}+00$ & $3.858 e+04$ & 43 & 95 & $0.000 e+00$ & $3.170 e+04$ & 43 & 96 & $0.000 e+00$ & $6.646 e+04$ \\
\hline 43 & 98 & $3.479 \mathrm{e}-01$ & $2.710 \mathrm{e}+09$ & 43 & 99 & $2.796 e+00$ & $1.556 e+10$ & 43 & 102 & $0.000 e+00$ & $2.023 e+05$ \\
\hline 43 & 103 & $0.000 e+00$ & $7.792 e+05$ & 43 & 111 & $5.630 e-03$ & $1.330 e+08$ & 43 & 112 & $6.311 \mathrm{e}-04$ & $9.127 e+06$ \\
\hline 43 & 113 & $1.728 \mathrm{e}-03$ & $2.509 \mathrm{e}+07$ & 43 & 114 & $1.558 \mathrm{e}-03$ & $3.801 e+07$ & 43 & 115 & $4.388 e-04$ & $1.083 e+07$ \\
\hline 43 & 116 & $8.999 e-04$ & $1.334 \mathrm{e}+07$ & 43 & 117 & $1.949 \mathrm{e}-03$ & $2.069 e+07$ & 43 & 118 & $2.835 e-03$ & $4.249 e+07$ \\
\hline 43 & 119 & $1.421 \mathrm{e}-02$ & $1.526 \mathrm{e}+08$ & 43 & 122 & $3.349 \mathrm{e}-04$ & $8.901 e+06$ & 43 & 123 & $2.715 e-04$ & $4.336 e+06$ \\
\hline 43 & 129 & $0.000 e+00$ & $1.306 \mathrm{e}+04$ & 43 & 132 & $0.000 e+00$ & $3.759 e+03$ & 43 & 136 & $1.776 e-04$ & $3.772 \mathrm{e}+06$ \\
\hline 43 & 139 & $3.890 e-04$ & $5.950 e+06$ & 43 & 140 & $1.918 \mathrm{e}-04$ & $4.166 e+06$ & 43 & 143 & $8.181 \mathrm{e}-04$ & $1.809 e+07$ \\
\hline 43 & 145 & $3.242 \mathrm{e}-03$ & $1.200 \mathrm{e}+08$ & 43 & 148 & $0.000 e+00$ & $2.099 e+05$ & 43 & 149 & $0.000 e+00$ & $9.029 e+04$ \\
\hline 43 & 150 & $0.000 e+00$ & $9.606 \mathrm{e}+04$ & 43 & 151 & $0.000 e+00$ & $1.075 e+05$ & 43 & 152 & $0.000 e+00$ & $4.159 e+05$ \\
\hline 43 & 163 & $2.331 \mathrm{e}-04$ & $3.888 \mathrm{e}+06$ & 43 & 171 & $0.000 e+00$ & $1.210 e+05$ & 43 & 172 & $0.000 e+00$ & $8.368 e+03$ \\
\hline 43 & 174 & $0.000 e+00$ & $8.675 e+03$ & 43 & 176 & $1.874 \mathrm{e}-03$ & $3.233 e+07$ & 43 & 177 & $4.486 e-04$ & $1.091 \mathrm{e}+07$ \\
\hline 43 & 178 & $1.615 \mathrm{e}-03$ & $2.810 \mathrm{e}+07$ & 43 & 180 & $0.000 e+00$ & $1.100 \mathrm{e}+05$ & 43 & 181 & $0.000 e+00$ & $1.411 \mathrm{e}+05$ \\
\hline 43 & 182 & $0.000 e+00$ & $4.703 e+04$ & 43 & 184 & $0.000 e+00$ & $2.656 e+04$ & 43 & 185 & $0.000 e+00$ & $3.145 e+05$ \\
\hline 43 & 186 & $0.000 e+00$ & $1.078 \mathrm{e}+05$ & 43 & 193 & $1.085 e-03$ & $2.100 e+07$ & 43 & 216 & $2.038 e-04$ & $9.815 e+06$ \\
\hline 43 & 227 & $0.000 e+00$ & $5.852 e+03$ & 43 & 231 & $0.000 e+00$ & $7.247 e+03$ & 43 & 234 & $0.000 e+00$ & $8.153 e+03$ \\
\hline 43 & 273 & $2.247 e-04$ & $2.205 e+07$ & 44 & 50 & $1.057 \mathrm{e}-01$ & $1.424 e+07$ & 44 & 51 & $1.228 \mathrm{e}+00$ & $1.289 \mathrm{e}+08$ \\
\hline
\end{tabular}


Table III. Ca IX Oscillator Strengths and Radiative Decay Rates for transitions involving levels higher than 4.

\begin{tabular}{|c|c|c|c|c|c|c|c|c|c|c|c|}
\hline $\begin{array}{l}\text { Low. } \\
\text { Lev. }\end{array}$ & $\begin{array}{l}\text { Upp } \\
\text { Lev }\end{array}$ & $\begin{array}{l}\text { Osc. } \\
\text { Str. }\end{array}$ & $\begin{array}{l}\text { Rad. } \\
\text { Rate }\end{array}$ & $\begin{array}{l}\text { Low. } \\
\text { Lev. }\end{array}$ & $\begin{array}{l}\text { - Upp } \\
\text {. Lev }\end{array}$ & $\begin{array}{l}\text { Osc. } \\
\text { Str. }\end{array}$ & $\begin{array}{l}\text { Rad. } \\
\text { Rate }\end{array}$ & $\begin{array}{l}\text { Low. } \\
\text { Lev. }\end{array}$ & $\begin{array}{l}\text { - Upp } \\
\text {. Lev }\end{array}$ & $\begin{array}{l}\text { Osc. } \\
\text { Str. }\end{array}$ & $\begin{array}{l}\text { Rad. } \\
\text { Rate }\end{array}$ \\
\hline$i$ & $j$ & $g f$ & $(1 / s)$ & $i$ & $\mathrm{j}$ & $g f$ & $(1 / s)$ & $i$ & j & $g f$ & $(1 / s)$ \\
\hline 44 & 64 & $0.000 e+00$ & $7.217 e+04$ & 44 & 67 & $4.558 e-01$ & $1.840 \mathrm{e}+09$ & 44 & 68 & $9.036 \mathrm{e}-02$ & $3.688 e+08$ \\
\hline 44 & 69 & $1.408 \mathrm{e}+00$ & $4.118 e+09$ & 44 & 72 & $1.460 \mathrm{e}+00$ & $6.243 e+09$ & 44 & 74 & $6.592 \mathrm{e}-03$ & $2.866 e+07$ \\
\hline 44 & 75 & $6.161 \mathrm{e}-02$ & $1.922 \mathrm{e}+08$ & 44 & 76 & $7.243 e-01$ & $1.808 e+09$ & 44 & 77 & $3.367 e-02$ & $1.086 e+08$ \\
\hline 44 & 78 & $4.942 \mathrm{e}-03$ & $2.459 \mathrm{e}+07$ & 44 & 83 & $0.000 e+00$ & $1.282 e+04$ & 44 & 85 & $0.000 e+00$ & $1.098 e+05$ \\
\hline 44 & 89 & $0.000 e+00$ & $4.841 \mathrm{e}+04$ & 44 & 90 & $0.000 e+00$ & $3.409 e+04$ & 44 & 94 & $0.000 e+00$ & $9.526 e+04$ \\
\hline 44 & 95 & $0.000 e+00$ & $4.962 \mathrm{e}+04$ & 44 & 98 & $9.913 e-03$ & $7.709 e+07$ & 44 & 99 & $3.494 \mathrm{e}-01$ & $1.941 \mathrm{e}+09$ \\
\hline 44 & 100 & $4.065 e+00$ & $1.757 e+10$ & 44 & 102 & $0.000 e+00$ & $1.347 e+04$ & 44 & 103 & $0.000 e+00$ & $1.560 e+05$ \\
\hline 44 & 104 & $0.000 e+00$ & $9.299 e+05$ & 44 & 112 & $1.967 e-03$ & $2.841 \mathrm{e}+07$ & 44 & 113 & $9.110 e-03$ & $1.321 e+08$ \\
\hline 44 & 116 & $7.961 \mathrm{e}-04$ & $1.179 e+07$ & 44 & 117 & $1.875 e-03$ & $1.987 e+07$ & 44 & 119 & $2.910 e-03$ & $3.123 e+07$ \\
\hline 44 & 120 & $1.966 \mathrm{e}-02$ & $1.649 \mathrm{e}+08$ & 44 & 123 & $1.052 \mathrm{e}-03$ & $1.679 \mathrm{e}+07$ & 44 & 132 & $0.000 \mathrm{e}+00$ & $6.766 e+03$ \\
\hline 44 & 133 & $0.000 e+00$ & $7.943 e+03$ & 44 & 136 & $2.512 \mathrm{e}-04$ & $5.330 e+06$ & 44 & 137 & $3.162 \mathrm{e}-04$ & $6.741 \mathrm{e}+06$ \\
\hline 44 & 141 & $2.089 e-04$ & $3.250 e+06$ & 44 & 142 & $6.505 e-04$ & $7.911 e+06$ & 44 & 143 & $5.745 e-03$ & $1.269 \mathrm{e}+08$ \\
\hline 44 & 148 & $0.000 e+00$ & $2.428 \mathrm{e}+04$ & 44 & 149 & $0.000 e+00$ & $1.451 e+05$ & 44 & 150 & $0.000 e+00$ & $2.497 e+05$ \\
\hline 44 & 151 & $0.000 e+00$ & $4.367 e+03$ & 44 & 152 & $0.000 e+00$ & $8.241 e+04$ & 44 & 153 & $0.000 e+00$ & $4.977 e+05$ \\
\hline 44 & 162 & $7.634 \mathrm{e}-04$ & $9.887 e+06$ & 44 & 171 & $0.000 e+00$ & $1.701 e+05$ & 44 & 175 & $4.776 e-03$ & $6.393 e+07$ \\
\hline 44 & 176 & $2.402 e-04$ & $4.139 \mathrm{e}+06$ & 44 & 180 & $0.000 e+00$ & $1.266 e+04$ & 44 & 181 & $0.000 e+00$ & $8.803 e+04$ \\
\hline 44 & 182 & $0.000 e+00$ & $2.336 e+05$ & 44 & 184 & $0.000 e+00$ & $1.450 e+05$ & 44 & 186 & $0.000 e+00$ & $1.692 \mathrm{e}+05$ \\
\hline 44 & 187 & $0.000 e+00$ & $6.811 \mathrm{e}+03$ & 44 & 192 & $1.397 e-03$ & $2.099 e+07$ & 44 & 217 & $3.716 e-04$ & $1.278 \mathrm{e}+07$ \\
\hline 44 & 229 & $0.000 e+00$ & $6.725 e+03$ & 44 & 232 & $0.000 e+00$ & $8.515 e+03$ & 44 & 234 & $0.000 e+00$ & $1.147 e+04$ \\
\hline 44 & 272 & $4.417 e-04$ & $2.598 e+07$ & 45 & 53 & $1.199 \mathrm{e}+00$ & $2.477 e+08$ & 45 & 63 & $0.000 e+00$ & $6.509 e+03$ \\
\hline 45 & 65 & $0.000 e+00$ & $1.771 \mathrm{e}+05$ & 45 & 66 & $6.665 e-02$ & $4.400 e+08$ & 45 & 67 & $4.958 e-01$ & $1.964 \mathrm{e}+09$ \\
\hline 45 & 68 & $5.262 \mathrm{e}-01$ & $2.108 e+09$ & 45 & 69 & $2.203 e-02$ & $6.322 \mathrm{e}+07$ & 45 & 70 & $2.279 \mathrm{e}-01$ & $1.588 e+09$ \\
\hline 45 & 72 & $3.828 \mathrm{e}-02$ & $1.607 e+08$ & 45 & 73 & $1.058 \mathrm{e}+00$ & $7.460 \mathrm{e}+09$ & 45 & 74 & $1.135 \mathrm{e}-01$ & $4.843 e+08$ \\
\hline 45 & 75 & $1.214 \mathrm{e}-01$ & $3.718 e+08$ & 45 & 77 & $3.669 \mathrm{e}-01$ & $1.162 \mathrm{e}+09$ & 45 & 78 & $6.008 e-03$ & $2.939 e+07$ \\
\hline 45 & 81 & $1.359 \mathrm{e}-02$ & $1.185 \mathrm{e}+08$ & 45 & 92 & $0.000 e+00$ & $1.645 e+05$ & 45 & 93 & $0.000 e+00$ & $3.330 e+05$ \\
\hline 45 & 97 & $0.000 \mathrm{e}+00$ & $3.243 e+04$ & 45 & 101 & $3.020 e+00$ & $1.713 e+10$ & 45 & 105 & $0.000 \mathrm{e}+00$ & $6.638 e+05$ \\
\hline 45 & 109 & $0.000 \mathrm{e}+00$ & $2.395 \mathrm{e}+04$ & 45 & 111 & $3.088 e-03$ & $7.216 \mathrm{e}+07$ & 45 & 112 & $6.262 \mathrm{e}-03$ & $8.957 e+07$ \\
\hline 45 & 113 & $1.462 \mathrm{e}-03$ & $2.099 \mathrm{e}+07$ & 45 & 114 & $9.871 \mathrm{e}-03$ & $2.381 e+08$ & 45 & 124 & $3.828 \mathrm{e}-02$ & $4.397 e+08$ \\
\hline 45 & 125 & $0.000 e+00$ & $8.514 \mathrm{e}+03$ & 45 & 127 & $6.673 e-04$ & $1.887 e+07$ & 45 & 135 & $0.000 e+00$ & $6.261 e+03$ \\
\hline 45 & 144 & $0.000 e+00$ & $3.168 \mathrm{e}+05$ & 45 & 147 & $5.750 e-04$ & $9.151 e+06$ & 45 & 151 & $0.000 e+00$ & $2.603 e+03$ \\
\hline 45 & 152 & $0.000 e+00$ & $1.202 \mathrm{e}+03$ & 45 & 154 & $0.000 e+00$ & $4.563 e+05$ & 45 & 155 & $0.000 e+00$ & $1.704 e+04$ \\
\hline 45 & 156 & $6.773 e-03$ & $2.580 \mathrm{e}+08$ & 45 & 179 & $0.000 e+00$ & $2.143 e+05$ & 45 & 182 & $0.000 e+00$ & $4.538 e+03$ \\
\hline 45 & 183 & $0.000 e+00$ & $5.007 e+04$ & 45 & 186 & $0.000 e+00$ & $7.544 e+03$ & 45 & 187 & $0.000 e+00$ & $2.164 e+05$ \\
\hline 45 & 188 & $0.000 e+00$ & $1.724 \mathrm{e}+05$ & 45 & 196 & $1.679 \mathrm{e}-02$ & $4.592 e+08$ & 45 & 206 & $3.895 e-02$ & $8.180 e+08$ \\
\hline 45 & 207 & $0.000 e+00$ & $1.763 e+05$ & 45 & 208 & $1.470 \mathrm{e}-03$ & $7.580 e+07$ & 45 & 224 & $5.208 e-03$ & $1.828 e+08$ \\
\hline 45 & 225 & $2.610 \mathrm{e}-04$ & $2.161 \mathrm{e}+07$ & 45 & 233 & $0.000 e+00$ & $4.460 e+04$ & 45 & 250 & $4.729 \mathrm{e}-03$ & $2.723 e+08$ \\
\hline 45 & 261 & $0.000 e+00$ & $2.595 \mathrm{e}+04$ & 45 & 265 & $0.000 e+00$ & $1.151 \mathrm{e}+04$ & 45 & 275 & $4.931 \mathrm{e}-03$ & $2.067 e+08$ \\
\hline 45 & 280 & $0.000 e+00$ & $1.390 \mathrm{e}+04$ & 45 & 281 & $0.000 e+00$ & $5.045 e+03$ & 46 & 54 & $2.448 e-02$ & $1.737 e+07$ \\
\hline 46 & 55 & $4.417 e-01$ & $3.827 e+08$ & 46 & 59 & $3.007 e-01$ & $3.449 e+08$ & 46 & 67 & $0.000 e+00$ & $9.541 e+03$ \\
\hline 46 & 68 & $0.000 e+00$ & $1.528 \mathrm{e}+04$ & 46 & 74 & $0.000 e+00$ & $9.949 e+03$ & 46 & 78 & $0.000 e+00$ & $5.757 e+03$ \\
\hline 46 & 91 & $3.968 e-03$ & $3.363 e+07$ & 46 & 106 & $5.071 \mathrm{e}-01$ & $6.885 e+09$ & 46 & 125 & $1.396 \mathrm{e}-02$ & $3.396 e+08$ \\
\hline 46 & 126 & $9.457 e-02$ & $2.339 \mathrm{e}+09$ & 46 & 130 & $1.426 \mathrm{e}-02$ & $3.605 e+08$ & 46 & 133 & $8.953 e-04$ & $2.308 e+07$ \\
\hline 46 & 136 & $0.000 e+00$ & $1.404 \mathrm{e}+04$ & 46 & 137 & $0.000 e+00$ & $4.212 e+05$ & 46 & 140 & $0.000 e+00$ & $8.366 e+03$ \\
\hline 46 & 143 & $0.000 e+00$ & $2.704 \mathrm{e}+04$ & 46 & 148 & $2.443 e-04$ & $8.149 e+06$ & 46 & 171 & $5.972 \mathrm{e}-04$ & $2.107 e+07$ \\
\hline
\end{tabular}


Table III. Ca IX Oscillator Strengths and Radiative Decay Rates for transitions involving levels higher than 4.

\begin{tabular}{|c|c|c|c|c|c|c|c|c|c|c|c|}
\hline $\begin{array}{l}\text { Low. } \\
\text { Lev. }\end{array}$ & $\begin{array}{l}\text { Upp } \\
\text { Lev }\end{array}$ & $\begin{array}{l}\text { Osc. } \\
\text { Str. }\end{array}$ & $\begin{array}{l}\text { Rad. } \\
\text { Rate }\end{array}$ & $\begin{array}{l}\text { Low. } \\
\text { Lev. }\end{array}$ & $\begin{array}{l}\text { - Upp } \\
\text {. Lev }\end{array}$ & $\begin{array}{l}\text { Osc. } \\
\text { Str. }\end{array}$ & $\begin{array}{l}\text { Rad. } \\
\text { Rate }\end{array}$ & $\begin{array}{l}\text { Low. } \\
\text { Lev. }\end{array}$ & $\begin{array}{l}\text { Upp } \\
\text { Lev }\end{array}$ & $\begin{array}{l}\text { Osc. } \\
\text { Str. }\end{array}$ & $\begin{array}{l}\text { Rad. } \\
\text { Rate }\end{array}$ \\
\hline$i$ & $j$ & $g f$ & $(1 / s)$ & $i$ & $j$ & $g f$ & $(1 / s)$ & $i$ & j & $g f$ & $(1 / s)$ \\
\hline 46 & 174 & $4.486 \mathrm{e}-04$ & $1.599 \mathrm{e}+07$ & 46 & 210 & $1.964 \mathrm{e}-04$ & $1.305 e+07$ & 47 & 54 & $1.207 e-01$ & $8.197 e+07$ \\
\hline 47 & 55 & $1.544 \mathrm{e}-01$ & $1.285 e+08$ & 47 & 56 & $9.981 \mathrm{e}-01$ & $5.197 e+08$ & 47 & 58 & $2.597 e-01$ & $8.462 \mathrm{e}+08$ \\
\hline 47 & 59 & $4.684 \mathrm{e}-01$ & $5.190 e+08$ & 47 & 60 & $4.547 e-02$ & $5.400 e+07$ & 47 & 61 & $2.428 \mathrm{e}-01$ & $1.738 e+08$ \\
\hline 47 & 62 & $7.930 e-03$ & $7.597 e+06$ & 47 & 63 & $3.924 \mathrm{e}-03$ & $2.623 e+07$ & 47 & 66 & $0.000 e+00$ & $3.156 e+04$ \\
\hline 47 & 67 & $0.000 e+00$ & $8.798 e+03$ & 47 & 69 & $0.000 e+00$ & $2.364 e+04$ & 47 & 72 & $0.000 e+00$ & $3.213 e+03$ \\
\hline 47 & 74 & $0.000 e+00$ & $1.707 e+04$ & 47 & 75 & $0.000 e+00$ & $1.042 \mathrm{e}+04$ & 47 & 78 & $0.000 e+00$ & $1.424 \mathrm{e}+04$ \\
\hline 47 & 79 & $0.000 e+00$ & $7.426 \mathrm{e}+03$ & 47 & 90 & $8.425 e-03$ & $4.213 e+07$ & 47 & 91 & $2.894 \mathrm{e}-03$ & $2.422 \mathrm{e}+07$ \\
\hline 47 & 92 & $3.467 e-03$ & $1.785 e+07$ & 47 & 95 & $2.188 e-03$ & $1.209 e+07$ & 47 & 97 & $3.646 \mathrm{e}-03$ & $2.195 e+07$ \\
\hline 47 & 106 & $3.685 e-01$ & $4.953 e+09$ & 47 & 107 & $1.116 \mathrm{e}+00$ & $9.012 e+09$ & 47 & 109 & $3.585 e-02$ & $3.093 e+08$ \\
\hline 47 & 115 & $0.000 e+00$ & $8.619 e+03$ & 47 & 125 & $5.179 e-02$ & $1.250 e+09$ & 47 & 126 & $3.043 e-04$ & $7.469 e+06$ \\
\hline 47 & 127 & $0.000 e+00$ & $5.159 e+03$ & 47 & 128 & $1.890 e-01$ & $2.795 e+09$ & 47 & 129 & $4.490 \mathrm{e}-02$ & $3.347 e+09$ \\
\hline 47 & 130 & $6.348 e-02$ & $1.593 e+09$ & 47 & 132 & $1.034 \mathrm{e}-02$ & $1.573 e+08$ & 47 & 133 & $1.001 \mathrm{e}-02$ & $2.561 e+08$ \\
\hline 47 & 134 & $8.341 e-04$ & $1.318 \mathrm{e}+07$ & 47 & 136 & $0.000 e+00$ & $1.947 e+05$ & 47 & 138 & $0.000 e+00$ & $4.298 e+05$ \\
\hline 47 & 139 & $0.000 e+00$ & $4.468 e+05$ & 47 & 140 & $0.000 e+00$ & $1.589 e+05$ & 47 & 141 & $0.000 e+00$ & $2.514 e+04$ \\
\hline 47 & 143 & $0.000 e+00$ & $1.032 \mathrm{e}+05$ & 47 & 145 & $0.000 e+00$ & $3.852 \mathrm{e}+04$ & 47 & 148 & $2.117 e-04$ & $7.019 e+06$ \\
\hline 47 & 149 & $5.714 e-04$ & $1.137 \mathrm{e}+07$ & 47 & 171 & $1.831 \mathrm{e}-03$ & $6.422 \mathrm{e}+07$ & 47 & 173 & $9.055 e-04$ & $1.915 e+07$ \\
\hline 47 & 174 & $3.266 e-04$ & $1.157 \mathrm{e}+07$ & 47 & 207 & $8.614 \mathrm{e}-05$ & $1.145 e+07$ & 47 & 210 & $1.454 \mathrm{e}-04$ & $9.613 e+06$ \\
\hline 47 & 211 & $4.486 e-04$ & $1.781 \mathrm{e}+07$ & 48 & 55 & $1.423 e-02$ & $1.051 e+07$ & 48 & 56 & $2.009 e-01$ & $9.300 e+07$ \\
\hline 48 & 57 & $1.697 e+00$ & $6.258 e+08$ & 48 & 59 & $1.392 \mathrm{e}-02$ & $1.390 e+07$ & 48 & 60 & $8.158 e-01$ & $8.765 e+08$ \\
\hline 48 & 61 & $1.066 e+00$ & $6.905 e+08$ & 48 & 62 & $1.024 \mathrm{e}-02$ & $9.000 e+06$ & 48 & 64 & $1.760 e-03$ & $5.734 \mathrm{e}+06$ \\
\hline 48 & 67 & $0.000 e+00$ & $5.233 e+03$ & 48 & 68 & $0.000 e+00$ & $4.741 e+03$ & 48 & 69 & $0.000 e+00$ & $1.223 e+04$ \\
\hline 48 & 70 & $0.000 e+00$ & $1.145 \mathrm{e}+04$ & 48 & 71 & $0.000 e+00$ & $1.124 \mathrm{e}+04$ & 48 & 72 & $0.000 e+00$ & $9.576 e+03$ \\
\hline 48 & 74 & $0.000 e+00$ & $6.779 \mathrm{e}+03$ & 48 & 75 & $0.000 e+00$ & $2.234 \mathrm{e}+04$ & 48 & 76 & $0.000 e+00$ & $4.076 e+04$ \\
\hline 48 & 78 & $0.000 e+00$ & $1.529 \mathrm{e}+04$ & 48 & 79 & $0.000 e+00$ & $3.051 e+04$ & 48 & 80 & $0.000 e+00$ & $3.984 e+04$ \\
\hline 48 & 89 & $1.345 e-02$ & $4.604 \mathrm{e}+07$ & 48 & 90 & $2.713 e-03$ & $1.307 e+07$ & 48 & 94 & $3.971 \mathrm{e}-03$ & $1.505 e+07$ \\
\hline 48 & 106 & $2.499 \mathrm{e}-02$ & $3.262 \mathrm{e}+08$ & 48 & 107 & $3.750 e-01$ & $2.940 e+09$ & 48 & 108 & $2.106 \mathrm{e}+00$ & $1.181 \mathrm{e}+10$ \\
\hline 48 & 116 & $0.000 e+00$ & $6.810 \mathrm{e}+03$ & 48 & 117 & $0.000 e+00$ & $7.685 e+03$ & 48 & 128 & $9.237 e-03$ & $1.337 \mathrm{e}+08$ \\
\hline 48 & 130 & $1.964 \mathrm{e}-02$ & $4.822 \mathrm{e}+08$ & 48 & 131 & $2.789 e-01$ & $2.944 e+09$ & 48 & 132 & $2.072 \mathrm{e}-01$ & $3.088 e+09$ \\
\hline 48 & 133 & $1.083 e-01$ & $2.714 \mathrm{e}+09$ & 48 & 134 & $1.070 \mathrm{e}-03$ & $1.656 \mathrm{e}+07$ & 48 & 136 & $0.000 e+00$ & $4.786 e+03$ \\
\hline 48 & 137 & $0.000 e+00$ & $5.038 e+04$ & 48 & 138 & $0.000 e+00$ & $5.101 e+04$ & 48 & 139 & $0.000 e+00$ & $2.897 e+04$ \\
\hline 48 & 140 & $0.000 e+00$ & $1.397 \mathrm{e}+05$ & 48 & 141 & $0.000 e+00$ & $4.538 e+05$ & 48 & 142 & $0.000 e+00$ & $4.735 e+05$ \\
\hline 48 & 143 & $0.000 e+00$ & $2.721 \mathrm{e}+05$ & 48 & 145 & $0.000 e+00$ & $3.933 e+05$ & 48 & 146 & $0.000 \mathrm{e}+00$ & $4.309 e+05$ \\
\hline 48 & 149 & $3.036 e-04$ & $5.928 e+06$ & 48 & 150 & $1.414 \mathrm{e}-03$ & $1.974 \mathrm{e}+07$ & 48 & 171 & $3.612 \mathrm{e}-03$ & $1.244 \mathrm{e}+08$ \\
\hline 48 & 172 & $1.665 e-03$ & $2.460 \mathrm{e}+07$ & 48 & 205 & $0.000 e+00$ & $1.277 e+04$ & 48 & 211 & $1.562 \mathrm{e}-04$ & $6.120 \mathrm{e}+06$ \\
\hline 48 & 212 & $8.765 e-04$ & $2.455 e+07$ & 49 & 70 & $0.000 e+00$ & $4.694 e+03$ & 49 & 71 & $0.000 e+00$ & $1.918 e+04$ \\
\hline 49 & 79 & $0.000 e+00$ & $3.775 e+03$ & 49 & 80 & $0.000 e+00$ & $1.073 e+04$ & 49 & 82 & $1.064 \mathrm{e}-02$ & $2.880 e+07$ \\
\hline 49 & 86 & $6.544 \mathrm{e}-01$ & $2.659 \mathrm{e}+09$ & 49 & 87 & $8.172 \mathrm{e}-02$ & $2.402 \mathrm{e}+08$ & 49 & 89 & $1.594 \mathrm{e}-02$ & $4.992 e+07$ \\
\hline 49 & 90 & $2.544 \mathrm{e}-01$ & $1.122 \mathrm{e}+09$ & 49 & 91 & $6.485 e-01$ & $4.787 e+09$ & 49 & 95 & $3.714 \mathrm{e}-02$ & $1.822 \mathrm{e}+08$ \\
\hline 49 & 96 & $1.125 \mathrm{e}-01$ & $9.254 \mathrm{e}+08$ & 49 & 98 & $0.000 e+00$ & $6.032 e+04$ & 49 & 99 & $0.000 e+00$ & $1.885 e+04$ \\
\hline 49 & 102 & $7.520 \mathrm{e}+00$ & $3.221 \mathrm{e}+10$ & 49 & 125 & $8.534 \mathrm{e}-04$ & $1.915 e+07$ & 49 & 126 & $1.332 \mathrm{e}-03$ & $3.042 \mathrm{e}+07$ \\
\hline 49 & 128 & $3.884 \mathrm{e}-04$ & $5.343 e+06$ & 49 & 130 & $2.351 \mathrm{e}-04$ & $5.493 e+06$ & 49 & 145 & $0.000 e+00$ & $7.767 e+03$ \\
\hline 49 & 146 & $0.000 e+00$ & $2.240 \mathrm{e}+04$ & 49 & 155 & $2.235 \mathrm{e}-03$ & $3.039 e+07$ & 49 & 157 & $0.000 e+00$ & $7.298 e+04$ \\
\hline 49 & 158 & $2.489 e-03$ & $3.399 e+07$ & 49 & 164 & $3.693 e-03$ & $5.133 e+07$ & 49 & 167 & $0.000 e+00$ & $3.583 e+04$ \\
\hline 49 & 173 & $3.260 e-03$ & $6.493 e+07$ & 49 & 174 & $1.975 e-02$ & $6.587 e+08$ & 49 & 176 & $0.000 e+00$ & $1.180 \mathrm{e}+04$ \\
\hline
\end{tabular}


Table III. Ca IX Oscillator Strengths and Radiative Decay Rates for transitions involving levels higher than 4.

\begin{tabular}{|c|c|c|c|c|c|c|c|c|c|c|c|}
\hline $\begin{array}{l}\text { Low. } \\
\text { Lev . }\end{array}$ & $\begin{array}{l}\text { Upp } \\
\text { Lev }\end{array}$ & $\begin{array}{l}\text { Osc. } \\
\text { Str. }\end{array}$ & $\begin{array}{l}\text { Rad. } \\
\text { Rate }\end{array}$ & $\begin{array}{l}\text { Low. } \\
\text { Lev. }\end{array}$ & $\begin{array}{l}\text { Upp. } \\
\text { Lev. }\end{array}$ & $\begin{array}{l}\text { Osc. } \\
\text { Str. }\end{array}$ & $\begin{array}{l}\text { Rad. } \\
\text { Rate }\end{array}$ & $\begin{array}{l}\text { Low. } \\
\text { Lev. }\end{array}$ & $\begin{array}{l}\text { Upp } \\
\text { Lev }\end{array}$ & $\begin{array}{l}\text { Osc. } \\
\text { Str. }\end{array}$ & $\begin{array}{l}\text { Rad. } \\
\text { Rate }\end{array}$ \\
\hline$i$ & $j$ & $g f$ & $(1 / s)$ & $i$ & $j$ & $g f$ & $(1 / s)$ & $i$ & $j$ & $g f$ & $(1 / s)$ \\
\hline 49 & 177 & $0.000 e+00$ & $3.451 e+04$ & 49 & 178 & $0.000 e+00$ & $4.073 e+03$ & 49 & 180 & $2.741 \mathrm{e}-04$ & $5.583 e+06$ \\
\hline 49 & 190 & $0.000 e+00$ & $8.024 e+04$ & 49 & 191 & $0.000 e+00$ & $1.571 \mathrm{e}+05$ & 49 & 192 & $0.000 e+00$ & $1.306 e+04$ \\
\hline 49 & 193 & $0.000 \mathrm{e}+00$ & $4.675 e+04$ & 49 & 197 & $0.000 e+00$ & $2.917 e+05$ & 49 & 199 & $0.000 e+00$ & $4.775 e+04$ \\
\hline 49 & 200 & $0.000 e+00$ & $2.418 e+05$ & 49 & 202 & $0.000 e+00$ & $1.196 e+05$ & 49 & 203 & $0.000 e+00$ & $1.379 \mathrm{e}+04$ \\
\hline 49 & 204 & $0.000 e+00$ & $1.657 \mathrm{e}+05$ & 49 & 205 & $0.000 e+00$ & $4.014 e+05$ & 49 & 230 & $5.501 \mathrm{e}-04$ & $1.902 \mathrm{e}+07$ \\
\hline 49 & 244 & $0.000 e+00$ & $2.674 \mathrm{e}+03$ & 49 & 258 & $1.159 \mathrm{e}-04$ & $4.301 e+06$ & 49 & 279 & $2.647 \mathrm{e}-04$ & $2.331 \mathrm{e}+07$ \\
\hline 50 & 70 & $0.000 e+00$ & $9.555 e+03$ & 50 & 72 & $0.000 e+00$ & $3.292 e+03$ & 50 & 78 & $0.000 e+00$ & $2.628 e+03$ \\
\hline 50 & 79 & $0.000 e+00$ & $7.461 e+03$ & 50 & 82 & $3.001 \mathrm{e}-02$ & $8.115 e+07$ & 50 & 84 & $7.772 \mathrm{e}-02$ & $2.175 e+08$ \\
\hline 50 & 86 & $2.457 e-01$ & $9.982 e+08$ & 50 & 87 & $7.604 \mathrm{e}-01$ & $2.234 \mathrm{e}+09$ & 50 & 88 & $9.523 e-02$ & $2.194 \mathrm{e}+08$ \\
\hline 50 & 89 & $3.296 \mathrm{e}-01$ & $1.032 \mathrm{e}+09$ & 50 & 90 & $8.473 e-01$ & $3.736 e+09$ & 50 & 92 & $8.883 e-03$ & $4.041 \mathrm{e}+07$ \\
\hline 50 & 93 & $4.388 e-03$ & $1.136 \mathrm{e}+07$ & 50 & 94 & $3.181 \mathrm{e}-02$ & $1.108 e+08$ & 50 & 95 & $1.045 e-01$ & $5.127 e+08$ \\
\hline 50 & 97 & $9.870 e-03$ & $5.300 e+07$ & 50 & 98 & $0.000 e+00$ & $2.638 e+04$ & 50 & 99 & $0.000 e+00$ & $4.946 e+04$ \\
\hline 50 & 100 & $0.000 e+00$ & $1.526 e+04$ & 50 & 102 & $6.331 \mathrm{e}-01$ & $2.711 e+09$ & 50 & 103 & $9.877 e+00$ & $3.295 e+10$ \\
\hline 50 & 105 & $1.889 e-02$ & $6.787 e+07$ & 50 & 128 & $3.067 e-03$ & $4.218 e+07$ & 50 & 131 & $4.018 e-04$ & $4.033 e+06$ \\
\hline 50 & 132 & $3.094 e-04$ & $4.386 e+06$ & 50 & 143 & $0.000 e+00$ & $4.597 e+03$ & 50 & 145 & $0.000 e+00$ & $1.345 e+04$ \\
\hline 50 & 155 & $7.505 e-04$ & $1.020 \mathrm{e}+07$ & 50 & 157 & $0.000 e+00$ & $1.650 e+04$ & 50 & 159 & $1.583 e-03$ & $3.031 e+07$ \\
\hline 50 & 160 & $0.000 e+00$ & $4.696 e+04$ & 50 & 161 & $4.320 e-03$ & $4.599 e+07$ & 50 & 163 & $0.000 e+00$ & $5.942 e+03$ \\
\hline 50 & 165 & $5.953 e-03$ & $6.453 e+07$ & 50 & 168 & $0.000 e+00$ & $6.468 e+04$ & 50 & 170 & $0.000 e+00$ & $4.012 e+03$ \\
\hline 50 & 172 & $3.145 e-03$ & $4.453 e+07$ & 50 & 173 & $2.640 \mathrm{e}-02$ & $5.257 e+08$ & 50 & 175 & $0.000 e+00$ & $1.224 \mathrm{e}+04$ \\
\hline 50 & 176 & $0.000 e+00$ & $9.018 e+03$ & 50 & 177 & $0.000 e+00$ & $1.525 e+04$ & 50 & 178 & $0.000 e+00$ & $1.282 e+04$ \\
\hline 50 & 180 & $3.427 e-04$ & $6.981 e+06$ & 50 & 183 & $9.203 e-04$ & $1.886 e+07$ & 50 & 190 & $0.000 e+00$ & $2.849 e+04$ \\
\hline 50 & 191 & $0.000 e+00$ & $7.370 e+04$ & 50 & 192 & $0.000 e+00$ & $2.612 \mathrm{e}+04$ & 50 & 193 & $0.000 e+00$ & $1.372 e+05$ \\
\hline 50 & 194 & $0.000 e+00$ & $9.322 e+04$ & 50 & 197 & $0.000 e+00$ & $9.039 e+04$ & 50 & 198 & $0.000 e+00$ & $3.971 \mathrm{e}+04$ \\
\hline 50 & 199 & $0.000 e+00$ & $2.720 e+05$ & 50 & 200 & $0.000 e+00$ & $1.673 e+05$ & 50 & 201 & $0.000 e+00$ & $8.102 e+04$ \\
\hline 50 & 202 & $0.000 e+00$ & $1.959 e+05$ & 50 & 203 & $0.000 e+00$ & $8.453 e+04$ & 50 & 204 & $0.000 e+00$ & $2.344 e+05$ \\
\hline 50 & 231 & $7.321 \mathrm{e}-04$ & $1.970 \mathrm{e}+07$ & 50 & 245 & $0.000 e+00$ & $2.806 e+03$ & 50 & 278 & $3.623 e-04$ & $1.914 \mathrm{e}+07$ \\
\hline 51 & 57 & $1.435 \mathrm{e}-02$ & $3.977 e+06$ & 51 & 72 & $0.000 e+00$ & $1.283 e+04$ & 51 & 78 & $0.000 \mathrm{e}+00$ & $1.000 e+04$ \\
\hline 51 & 83 & $1.485 \mathrm{e}-01$ & $3.204 \mathrm{e}+08$ & 51 & 84 & $8.386 e-02$ & $2.346 e+08$ & 51 & 85 & $1.336 \mathrm{e}-02$ & $2.424 e+07$ \\
\hline 51 & 87 & $2.843 e-01$ & $8.349 e+08$ & 51 & 88 & $1.394 \mathrm{e}+00$ & $3.210 e+09$ & 51 & 89 & $1.238 \mathrm{e}+00$ & $3.874 e+09$ \\
\hline 51 & 93 & $1.120 \mathrm{e}-02$ & $2.898 e+07$ & 51 & 94 & $9.883 e-02$ & $3.441 e+08$ & 51 & 99 & $0.000 e+00$ & $1.962 e+04$ \\
\hline 51 & 100 & $0.000 e+00$ & $7.192 \mathrm{e}+04$ & 51 & 102 & $9.626 \mathrm{e}-03$ & $4.121 e+07$ & 51 & 103 & $6.255 \mathrm{e}-01$ & $2.086 e+09$ \\
\hline 51 & 104 & $1.291 \mathrm{e}+01$ & $3.530 e+10$ & 51 & 105 & $3.561 \mathrm{e}-03$ & $1.279 \mathrm{e}+07$ & 51 & 131 & $4.651 \mathrm{e}-03$ & $4.669 e+07$ \\
\hline 51 & 143 & $0.000 e+00$ & $1.453 e+04$ & 51 & 153 & $3.800 e-04$ & $3.263 e+06$ & 51 & 155 & $1.730 \mathrm{e}-03$ & $2.352 e+07$ \\
\hline 51 & 158 & $3.729 \mathrm{e}-03$ & $5.089 e+07$ & 51 & 160 & $0.000 e+00$ & $1.510 e+04$ & 51 & 162 & $0.000 e+00$ & $7.865 e+03$ \\
\hline 51 & 166 & $0.000 e+00$ & $1.308 e+05$ & 51 & 169 & $1.285 e-02$ & $1.145 e+08$ & 51 & 170 & $0.000 e+00$ & $5.691 \mathrm{e}+03$ \\
\hline 51 & 172 & $3.682 \mathrm{e}-02$ & $5.212 \mathrm{e}+08$ & 51 & 175 & $0.000 e+00$ & $2.772 \mathrm{e}+04$ & 51 & 176 & $0.000 e+00$ & $4.594 e+03$ \\
\hline 51 & 178 & $0.000 e+00$ & $4.996 e+03$ & 51 & 190 & $0.000 e+00$ & $6.680 e+03$ & 51 & 192 & $0.000 e+00$ & $1.946 \mathrm{e}+05$ \\
\hline 51 & 193 & $0.000 e+00$ & $5.484 e+04$ & 51 & 194 & $0.000 e+00$ & $1.282 \mathrm{e}+04$ & 51 & 195 & $0.000 e+00$ & $1.006 e+05$ \\
\hline 51 & 198 & $0.000 e+00$ & $3.461 \mathrm{e}+05$ & 51 & 199 & $0.000 e+00$ & $6.639 e+04$ & 51 & 201 & $0.000 e+00$ & $3.149 \mathrm{e}+05$ \\
\hline 51 & 202 & $0.000 \mathrm{e}+00$ & $9.438 \mathrm{e}+04$ & 51 & 203 & $0.000 e+00$ & $3.002 e+05$ & 51 & 232 & $9.686 \mathrm{e}-04$ & $2.134 \mathrm{e}+07$ \\
\hline 51 & 246 & $0.000 e+00$ & $3.107 e+03$ & 51 & 260 & $1.750 e-04$ & $4.134 e+06$ & 51 & 277 & $4.999 e-04$ & $1.885 e+07$ \\
\hline 52 & 54 & $3.217 e-01$ & $1.253 e+08$ & 52 & 55 & $8.172 \mathrm{e}-02$ & $4.150 e+07$ & 52 & 59 & $3.751 \mathrm{e}-02$ & $2.728 e+07$ \\
\hline 52 & 60 & $6.384 e-03$ & $5.058 e+06$ & 52 & 62 & $1.463 e+00$ & $9.931 e+08$ & 52 & 63 & $4.163 e-01$ & $2.088 e+09$ \\
\hline 52 & 65 & $2.617 \mathrm{e}-03$ & $2.600 \mathrm{e}+07$ & 52 & 67 & $0.000 e+00$ & $8.204 e+03$ & 52 & 68 & $0.000 e+00$ & $1.101 \mathrm{e}+04$ \\
\hline
\end{tabular}


Table III. Ca IX Oscillator Strengths and Radiative Decay Rates for transitions involving levels higher than 4.

\begin{tabular}{|c|c|c|c|c|c|c|c|c|c|c|c|}
\hline $\begin{array}{l}\text { Low. } \\
\text { Lev. }\end{array}$ & $\begin{array}{l}\text { Upp } \\
\text { Lev }\end{array}$ & $\begin{array}{l}\text { Osc. } \\
\text { Str. }\end{array}$ & $\begin{array}{l}\text { Rad. } \\
\text { Rate }\end{array}$ & $\begin{array}{l}\text { Low. } \\
\text { Lev. }\end{array}$ & $\begin{array}{l}\text { Upp. } \\
\text { Lev. }\end{array}$ & $\begin{array}{l}\text { Osc. } \\
\text { Str. }\end{array}$ & $\begin{array}{l}\text { Rad. } \\
\text { Rate }\end{array}$ & $\begin{array}{l}\text { Low. } \\
\text { Lev. }\end{array}$ & $\begin{array}{l}\text { Upp } \\
\text { Lev }\end{array}$ & $\begin{array}{l}\text { Osc. } \\
\text { Str. }\end{array}$ & $\begin{array}{l}\text { Rad. } \\
\text { Rate }\end{array}$ \\
\hline$i$ & $j$ & $g f$ & $(1 / s)$ & $i$ & $j$ & $g f$ & $(1 / s)$ & $i$ & j & $g f$ & $(1 / s)$ \\
\hline 52 & 70 & $0.000 e+00$ & $3.682 \mathrm{e}+03$ & 52 & 73 & $0.000 e+00$ & $1.894 \mathrm{e}+04$ & 52 & 74 & $0.000 e+00$ & $5.248 e+03$ \\
\hline 52 & 75 & $0.000 e+00$ & $3.746 e+03$ & 52 & 77 & $0.000 e+00$ & $2.172 \mathrm{e}+04$ & 52 & 81 & $0.000 e+00$ & $1.309 e+04$ \\
\hline 52 & 92 & $1.537 e-01$ & $6.873 e+08$ & 52 & 97 & $1.121 \mathrm{e}-01$ & $5.923 e+08$ & 52 & 101 & $0.000 e+00$ & $3.805 e+04$ \\
\hline 52 & 106 & $9.932 \mathrm{e}-03$ & $1.194 \mathrm{e}+08$ & 52 & 107 & $2.116 \mathrm{e}-02$ & $1.528 e+08$ & 52 & 109 & $1.585 e+00$ & $1.227 e+10$ \\
\hline 52 & 112 & $0.000 e+00$ & $9.354 e+03$ & 52 & 124 & $0.000 e+00$ & $1.032 \mathrm{e}+05$ & 52 & 125 & $8.146 \mathrm{e}-02$ & $1.809 e+09$ \\
\hline 52 & 126 & $3.387 e-02$ & $7.658 \mathrm{e}+08$ & 52 & 127 & $0.000 e+00$ & $1.266 \mathrm{e}+05$ & 52 & 130 & $3.657 e-02$ & $8.459 e+08$ \\
\hline 52 & 132 & $1.159 \mathrm{e}-03$ & $1.627 e+07$ & 52 & 133 & $3.899 e-03$ & $9.207 e+07$ & 52 & 134 & $1.589 \mathrm{e}-01$ & $2.318 e+09$ \\
\hline 52 & 135 & $3.467 e-02$ & $2.613 e+09$ & 52 & 136 & $0.000 e+00$ & $3.015 e+05$ & 52 & 137 & $0.000 e+00$ & $8.076 e+03$ \\
\hline 52 & 140 & $0.000 e+00$ & $1.856 e+05$ & 52 & 143 & $0.000 e+00$ & $3.589 e+04$ & 52 & 147 & $0.000 e+00$ & $3.854 e+05$ \\
\hline 52 & 156 & $0.000 e+00$ & $3.290 e+05$ & 52 & 163 & $0.000 e+00$ & $5.334 e+03$ & 52 & 176 & $0.000 e+00$ & $1.788 e+04$ \\
\hline 52 & 178 & $0.000 e+00$ & $2.293 e+04$ & 52 & 187 & $6.677 e-04$ & $1.442 \mathrm{e}+07$ & 52 & 196 & $0.000 e+00$ & $1.858 e+04$ \\
\hline 52 & 206 & $0.000 e+00$ & $3.169 e+04$ & 52 & 207 & $3.580 e-03$ & $4.479 e+08$ & 52 & 208 & $0.000 e+00$ & $3.499 e+04$ \\
\hline 52 & 213 & $6.792 \mathrm{e}-04$ & $2.594 e+07$ & 52 & 224 & $0.000 e+00$ & $1.169 e+04$ & 52 & 233 & $4.201 \mathrm{e}-04$ & $3.376 e+07$ \\
\hline 52 & 238 & $1.045 e-04$ & $5.221 e+06$ & 52 & 281 & $1.665 e-05$ & $4.404 e+06$ & 53 & 62 & $3.209 \mathrm{e}-02$ & $1.664 \mathrm{e}+07$ \\
\hline 53 & 73 & $0.000 e+00$ & $1.296 \mathrm{e}+04$ & 53 & 81 & $0.000 e+00$ & $1.352 \mathrm{e}+04$ & 53 & 82 & $3.285 e-01$ & $7.802 e+08$ \\
\hline 53 & 84 & $7.054 e-01$ & $1.738 \mathrm{e}+09$ & 53 & 86 & $1.280 e-02$ & $4.587 e+07$ & 53 & 87 & $5.986 e-02$ & $1.553 e+08$ \\
\hline 53 & 89 & $1.111 \mathrm{e}-02$ & $3.084 e+07$ & 53 & 90 & $8.100 e-03$ & $3.168 e+07$ & 53 & 92 & $8.192 \mathrm{e}-01$ & $3.311 \mathrm{e}+09$ \\
\hline 53 & 93 & $5.124 \mathrm{e}-01$ & $1.180 \mathrm{e}+09$ & 53 & 94 & $2.935 e-03$ & $9.122 \mathrm{e}+06$ & 53 & 97 & $5.385 e-01$ & $2.595 e+09$ \\
\hline 53 & 101 & $0.000 e+00$ & $9.472 \mathrm{e}+04$ & 53 & 103 & $2.291 \mathrm{e}-02$ & $6.900 \mathrm{e}+07$ & 53 & 105 & $1.078 \mathrm{e}+01$ & $3.511 \mathrm{e}+10$ \\
\hline 53 & 109 & $1.351 \mathrm{e}-02$ & $9.692 e+07$ & 53 & 134 & $5.843 e-03$ & $8.069 e+07$ & 53 & 144 & $1.881 \mathrm{e}-02$ & $2.319 e+08$ \\
\hline 53 & 152 & $3.219 \mathrm{e}-04$ & $3.182 \mathrm{e}+06$ & 53 & 155 & $1.104 \mathrm{e}-03$ & $1.418 e+07$ & 53 & 156 & $0.000 e+00$ & $2.588 e+04$ \\
\hline 53 & 157 & $0.000 e+00$ & $2.519 \mathrm{e}+03$ & 53 & 159 & $1.440 \mathrm{e}-03$ & $2.606 e+07$ & 53 & 160 & $0.000 \mathrm{e}+00$ & $1.571 \mathrm{e}+04$ \\
\hline 53 & 161 & $3.505 e-03$ & $3.527 e+07$ & 53 & 162 & $0.000 e+00$ & $7.344 e+03$ & 53 & 167 & $0.000 e+00$ & $7.909 e+03$ \\
\hline 53 & 168 & $0.000 e+00$ & $3.380 e+03$ & 53 & 170 & $0.000 e+00$ & $5.432 e+04$ & 53 & 173 & $1.310 e-03$ & $2.469 e+07$ \\
\hline 53 & 176 & $0.000 e+00$ & $1.692 \mathrm{e}+04$ & 53 & 178 & $0.000 e+00$ & $2.793 e+04$ & 53 & 179 & $8.440 \mathrm{e}-02$ & $8.987 e+08$ \\
\hline 53 & 180 & $2.555 e-03$ & $4.925 e+07$ & 53 & 182 & $1.580 \mathrm{e}-03$ & $1.696 \mathrm{e}+07$ & 53 & 183 & $4.276 \mathrm{e}-02$ & $8.295 e+08$ \\
\hline 53 & 187 & $1.901 \mathrm{e}-03$ & $3.924 \mathrm{e}+07$ & 53 & 188 & $3.694 \mathrm{e}-03$ & $4.284 e+07$ & 53 & 189 & $0.000 \mathrm{e}+00$ & $1.325 e+05$ \\
\hline 53 & 196 & $0.000 \mathrm{e}+00$ & $3.013 e+05$ & 53 & 206 & $0.000 e+00$ & $3.554 e+05$ & 53 & 208 & $0.000 e+00$ & $4.206 e+05$ \\
\hline 53 & 209 & $0.000 e+00$ & $4.431 \mathrm{e}+05$ & 53 & 213 & $3.051 \mathrm{e}-03$ & $1.126 \mathrm{e}+08$ & 53 & 214 & $0.000 e+00$ & $1.674 \mathrm{e}+04$ \\
\hline 53 & 226 & $6.915 e-03$ & $2.287 e+08$ & 53 & 238 & $6.396 e-04$ & $3.101 e+07$ & 53 & 242 & $7.608 e-03$ & $2.059 e+08$ \\
\hline 53 & 243 & $0.000 e+00$ & $4.240 \mathrm{e}+04$ & 53 & 261 & $1.287 e-04$ & $4.633 e+06$ & 53 & 265 & $2.740 \mathrm{e}-04$ & $7.679 e+06$ \\
\hline 53 & 280 & $5.840 e-04$ & $2.995 e+07$ & 53 & 282 & $0.000 e+00$ & $1.770 e+04$ & 54 & 66 & $1.045 \mathrm{e}-01$ & $2.005 e+08$ \\
\hline 54 & 67 & $6.561 \mathrm{e}-01$ & $7.555 e+08$ & 54 & 68 & $1.114 \mathrm{e}+00$ & $1.310 \mathrm{e}+09$ & 54 & 70 & $5.931 \mathrm{e}-02$ & $1.257 e+08$ \\
\hline 54 & 71 & $1.789 e-03$ & $1.140 \mathrm{e}+07$ & 54 & 72 & $1.955 \mathrm{e}-02$ & $2.504 e+07$ & 54 & 73 & $1.056 \mathrm{e}-01$ & $2.283 e+08$ \\
\hline 54 & 74 & $4.003 e-03$ & $5.282 \mathrm{e}+06$ & 54 & 79 & $2.633 e-02$ & $7.443 e+07$ & 54 & 81 & $3.306 \mathrm{e}-01$ & $1.034 \mathrm{e}+09$ \\
\hline 54 & 97 & $0.000 e+00$ & $1.617 \mathrm{e}+04$ & 54 & 98 & $2.016 \mathrm{e}-02$ & $6.919 \mathrm{e}+07$ & 54 & 110 & $9.705 e-03$ & $3.866 e+08$ \\
\hline 54 & 111 & $2.682 \mathrm{e}-01$ & $3.572 \mathrm{e}+09$ & 54 & 112 & $6.946 \mathrm{e}-01$ & $5.700 e+09$ & 54 & 113 & $1.525 e-01$ & $1.258 \mathrm{e}+09$ \\
\hline 54 & 114 & $3.246 \mathrm{e}-01$ & $4.511 \mathrm{e}+09$ & 54 & 116 & $2.805 e-02$ & $2.378 e+08$ & 54 & 118 & $4.918 e-02$ & $4.230 e+08$ \\
\hline 54 & 121 & $4.077 e-03$ & $1.903 e+08$ & 54 & 122 & $2.031 \mathrm{e}-03$ & $3.159 e+07$ & 54 & 123 & $9.854 \mathrm{e}-04$ & $9.214 e+06$ \\
\hline 54 & 125 & $0.000 e+00$ & $7.573 e+04$ & 54 & 126 & $0.000 e+00$ & $6.706 e+04$ & 54 & 127 & $4.670 \mathrm{e}-03$ & $7.979 e+07$ \\
\hline 54 & 128 & $0.000 e+00$ & $4.506 \mathrm{e}+04$ & 54 & 132 & $0.000 e+00$ & $8.135 e+03$ & 54 & 134 & $0.000 e+00$ & $1.969 e+05$ \\
\hline 54 & 136 & $2.438 \mathrm{e}-01$ & $3.283 e+09$ & 54 & 137 & $1.398 \mathrm{e}-03$ & $1.895 e+07$ & 54 & 138 & $1.546 \mathrm{e}-02$ & $3.501 e+08$ \\
\hline 54 & 140 & $1.549 \mathrm{e}-02$ & $2.146 e+08$ & 54 & 143 & $1.353 \mathrm{e}-03$ & $1.918 e+07$ & 54 & 144 & $0.000 e+00$ & $1.108 e+04$ \\
\hline 54 & 145 & $2.508 e-03$ & $5.956 e+07$ & 54 & 151 & $0.000 e+00$ & $1.001 e+05$ & 54 & 155 & $0.000 e+00$ & $7.143 e+05$ \\
\hline
\end{tabular}


Table III. Ca IX Oscillator Strengths and Radiative Decay Rates for transitions involving levels higher than 4.

\begin{tabular}{|c|c|c|c|c|c|c|c|c|c|c|c|}
\hline $\begin{array}{l}\text { Low. } \\
\text { Lev. }\end{array}$ & $\begin{array}{l}\text { Upp } \\
\text { Lev }\end{array}$ & $\begin{array}{l}\text { Osc. } \\
\text { Str. }\end{array}$ & $\begin{array}{l}\text { Rad. } \\
\text { Rate }\end{array}$ & $\begin{array}{l}\text { Low. } \\
\text { Lev. }\end{array}$ & $\begin{array}{l}\text { Upp. } \\
\text { Lev }\end{array}$ & $\begin{array}{l}\text { Osc. } \\
\text { Str. }\end{array}$ & $\begin{array}{l}\text { Rad. } \\
\text { Rate }\end{array}$ & $\begin{array}{l}\text { Low. } \\
\text { Lev. }\end{array}$ & $\begin{array}{l}\text { - Upp } \\
\text {. Lev }\end{array}$ & $\begin{array}{l}\text { Osc. } \\
\text { Str. }\end{array}$ & $\begin{array}{l}\text { Rad. } \\
\text { Rate }\end{array}$ \\
\hline$i$ & $j$ & $g f$ & $(1 / s)$ & $i$ & $j$ & $g f$ & $(1 / s)$ & $i$ & j & $g f$ & $(1 / s)$ \\
\hline 54 & 156 & $6.307 e-02$ & $1.569 \mathrm{e}+09$ & 54 & 159 & $0.000 e+00$ & $1.062 \mathrm{e}+05$ & 54 & 164 & $0.000 e+00$ & $6.087 e+04$ \\
\hline 54 & 172 & $0.000 e+00$ & $8.433 e+03$ & 54 & 173 & $0.000 e+00$ & $3.233 e+04$ & 54 & 177 & $2.480 e-04$ & $3.956 e+06$ \\
\hline 54 & 180 & $0.000 e+00$ & $2.120 e+04$ & 54 & 183 & $0.000 e+00$ & $3.388 e+05$ & 54 & 186 & $0.000 e+00$ & $5.645 e+03$ \\
\hline 54 & 187 & $0.000 e+00$ & $5.854 e+04$ & 54 & 214 & $1.795 e-04$ & $6.427 e+06$ & 54 & 225 & $2.660 e-04$ & $1.666 \mathrm{e}+07$ \\
\hline 54 & 250 & $3.632 \mathrm{e}-04$ & $1.616 \mathrm{e}+07$ & 54 & 283 & $2.920 e-04$ & $2.271 \mathrm{e}+07$ & 55 & 66 & $1.004 \mathrm{e}-01$ & $1.688 e+08$ \\
\hline 55 & 67 & $2.654 \mathrm{e}-01$ & $2.678 \mathrm{e}+08$ & 55 & 68 & $2.061 \mathrm{e}-01$ & $2.127 e+08$ & 55 & 71 & $3.303 e-02$ & $1.856 e+08$ \\
\hline 55 & 73 & $5.887 e-02$ & $1.125 e+08$ & 55 & 74 & $1.717 e+00$ & $2.003 e+09$ & 55 & 78 & $3.625 e-03$ & $5.449 e+06$ \\
\hline 55 & 79 & $1.043 e-03$ & $2.647 \mathrm{e}+06$ & 55 & 80 & $1.306 \mathrm{e}-02$ & $1.004 e+08$ & 55 & 81 & $5.798 e-02$ & $1.637 e+08$ \\
\hline 55 & 98 & $1.481 \mathrm{e}-01$ & $4.713 e+08$ & 55 & 102 & $0.000 e+00$ & $1.818 e+04$ & 55 & 110 & $2.547 e-01$ & $9.659 e+09$ \\
\hline 55 & 111 & $2.606 \mathrm{e}-02$ & $3.304 e+08$ & 55 & 112 & $5.364 e-02$ & $4.194 e+08$ & 55 & 114 & $9.684 \mathrm{e}-02$ & $1.282 \mathrm{e}+09$ \\
\hline 55 & 115 & $1.716 \mathrm{e}-01$ & $2.307 e+09$ & 55 & 116 & $7.663 e-02$ & $6.194 e+08$ & 55 & 118 & $8.947 e-01$ & $7.341 \mathrm{e}+09$ \\
\hline 55 & 121 & $2.683 e-03$ & $1.197 e+08$ & 55 & 122 & $2.331 \mathrm{e}-02$ & $3.465 e+08$ & 55 & 123 & $7.361 \mathrm{e}-04$ & $6.579 e+06$ \\
\hline 55 & 125 & $0.000 e+00$ & $3.362 e+04$ & 55 & 126 & $0.000 e+00$ & $1.582 e+05$ & 55 & 127 & $4.906 e-04$ & $8.028 e+06$ \\
\hline 55 & 128 & $0.000 e+00$ & $3.822 \mathrm{e}+04$ & 55 & 130 & $0.000 e+00$ & $1.343 e+05$ & 55 & 132 & $0.000 e+00$ & $3.817 e+03$ \\
\hline 55 & 133 & $0.000 e+00$ & $1.554 \mathrm{e}+04$ & 55 & 134 & $0.000 e+00$ & $4.544 e+04$ & 55 & 136 & $4.448 e-03$ & $5.769 e+07$ \\
\hline 55 & 137 & $1.826 \mathrm{e}-01$ & $2.384 \mathrm{e}+09$ & 55 & 138 & $2.813 e-02$ & $6.138 e+08$ & 55 & 140 & $1.159 \mathrm{e}-01$ & $1.548 e+09$ \\
\hline 55 & 145 & $2.938 e-04$ & $6.726 e+06$ & 55 & 146 & $1.978 e-03$ & $1.364 \mathrm{e}+08$ & 55 & 151 & $0.000 e+00$ & $1.463 e+05$ \\
\hline 55 & 156 & $1.332 \mathrm{e}-02$ & $3.197 e+08$ & 55 & 158 & $0.000 e+00$ & $5.998 e+05$ & 55 & 159 & $0.000 e+00$ & $1.458 e+05$ \\
\hline 55 & 164 & $0.000 e+00$ & $3.185 e+05$ & 55 & 174 & $0.000 e+00$ & $1.866 e+04$ & 55 & 177 & $2.015 e-03$ & $3.104 \mathrm{e}+07$ \\
\hline 55 & 180 & $0.000 e+00$ & $4.692 \mathrm{e}+03$ & 55 & 183 & $0.000 e+00$ & $8.369 e+04$ & 55 & 184 & $0.000 e+00$ & $2.108 e+04$ \\
\hline 55 & 187 & $0.000 e+00$ & $1.150 \mathrm{e}+04$ & 55 & 191 & $5.602 e-04$ & $9.860 e+06$ & 55 & 204 & $5.845 e-04$ & $1.887 e+07$ \\
\hline 55 & 205 & $2.536 \mathrm{e}-04$ & $2.459 e+07$ & 55 & 218 & $6.771 \mathrm{e}-04$ & $2.395 e+07$ & 55 & 223 & $3.655 e-05$ & $6.563 e+06$ \\
\hline 55 & 247 & $3.383 e-04$ & $1.461 \mathrm{e}+07$ & 55 & 274 & $7.567 e-05$ & $1.682 \mathrm{e}+07$ & 56 & 66 & $3.189 \mathrm{e}-02$ & $5.205 e+07$ \\
\hline 56 & 67 & $2.013 e-01$ & $1.972 \mathrm{e}+08$ & 56 & 69 & $7.749 e-01$ & $5.576 e+08$ & 56 & 70 & $7.890 e-02$ & $1.434 \mathrm{e}+08$ \\
\hline 56 & 72 & $6.990 e-03$ & $7.684 \mathrm{e}+06$ & 56 & 73 & $1.219 \mathrm{e}-02$ & $2.265 e+07$ & 56 & 74 & $4.674 \mathrm{e}-01$ & $5.305 e+08$ \\
\hline 56 & 75 & $1.717 \mathrm{e}+00$ & $1.405 \mathrm{e}+09$ & 56 & 77 & $7.548 e-01$ & $6.589 e+08$ & 56 & 78 & $1.360 e-02$ & $1.994 \mathrm{e}+07$ \\
\hline 56 & 79 & $2.533 e-02$ & $6.274 \mathrm{e}+07$ & 56 & 98 & $3.020 \mathrm{e}-02$ & $9.454 e+07$ & 56 & 99 & $2.425 \mathrm{e}-01$ & $5.422 \mathrm{e}+08$ \\
\hline 56 & 102 & $0.000 \mathrm{e}+00$ & $5.536 e+03$ & 56 & 103 & $0.000 e+00$ & $2.146 e+04$ & 56 & 111 & $4.605 e-01$ & $5.777 e+09$ \\
\hline 56 & 112 & $2.030 \mathrm{e}-02$ & $1.570 \mathrm{e}+08$ & 56 & 113 & $8.383 e-02$ & $6.521 e+08$ & 56 & 114 & $9.128 \mathrm{e}-02$ & $1.196 \mathrm{e}+09$ \\
\hline 56 & 115 & $4.587 e-02$ & $6.104 \mathrm{e}+08$ & 56 & 116 & $1.313 \mathrm{e}-01$ & $1.050 e+09$ & 56 & 117 & $1.172 \mathrm{e}-01$ & $6.718 e+08$ \\
\hline 56 & 118 & $2.757 e-01$ & $2.239 \mathrm{e}+09$ & 56 & 119 & $1.394 \mathrm{e}+00$ & $8.124 e+09$ & 56 & 122 & $1.784 \mathrm{e}-02$ & $2.626 e+08$ \\
\hline 56 & 123 & $2.683 e-02$ & $2.374 \mathrm{e}+08$ & 56 & 124 & $2.187 e-02$ & $1.415 e+08$ & 56 & 125 & $0.000 e+00$ & $8.816 e+04$ \\
\hline 56 & 128 & $0.000 e+00$ & $1.073 e+05$ & 56 & 129 & $0.000 e+00$ & $4.182 e+05$ & 56 & 130 & $0.000 e+00$ & $1.409 e+05$ \\
\hline 56 & 131 & $0.000 e+00$ & $3.073 e+04$ & 56 & 132 & $0.000 e+00$ & $6.397 e+04$ & 56 & 133 & $0.000 e+00$ & $7.123 e+04$ \\
\hline 56 & 134 & $0.000 e+00$ & $4.248 e+03$ & 56 & 135 & $0.000 e+00$ & $5.138 e+03$ & 56 & 136 & $3.003 e-02$ & $3.863 e+08$ \\
\hline 56 & 137 & $8.969 e-03$ & $1.161 \mathrm{e}+08$ & 56 & 138 & $1.090 \mathrm{e}-02$ & $2.359 e+08$ & 56 & 139 & $2.975 e-01$ & $2.764 e+09$ \\
\hline 56 & 140 & $7.829 e-02$ & $1.037 e+09$ & 56 & 141 & $1.333 e-01$ & $1.267 e+09$ & 56 & 145 & $6.485 \mathrm{e}-03$ & $1.473 e+08$ \\
\hline 56 & 147 & $1.476 \mathrm{e}-02$ & $1.459 \mathrm{e}+08$ & 56 & 151 & $0.000 e+00$ & $3.357 e+04$ & 56 & 152 & $0.000 e+00$ & $1.322 \mathrm{e}+05$ \\
\hline 56 & 155 & $0.000 e+00$ & $1.019 \mathrm{e}+05$ & 56 & 158 & $0.000 e+00$ & $3.010 e+04$ & 56 & 159 & $0.000 e+00$ & $1.338 e+05$ \\
\hline 56 & 161 & $0.000 e+00$ & $7.122 \mathrm{e}+05$ & 56 & 163 & $3.669 \mathrm{e}-04$ & $3.805 e+06$ & 56 & 164 & $0.000 e+00$ & $3.097 e+05$ \\
\hline 56 & 165 & $0.000 e+00$ & $2.736 \mathrm{e}+05$ & 56 & 173 & $0.000 e+00$ & $3.081 e+04$ & 56 & 174 & $0.000 e+00$ & $2.965 e+04$ \\
\hline 56 & 176 & $2.148 \mathrm{e}-03$ & $2.324 \mathrm{e}+07$ & 56 & 177 & $4.044 \mathrm{e}-04$ & $6.184 e+06$ & 56 & 178 & $1.144 \mathrm{e}-03$ & $1.252 \mathrm{e}+07$ \\
\hline 56 & 179 & $0.000 e+00$ & $2.356 e+04$ & 56 & 184 & $0.000 e+00$ & $4.848 e+03$ & 56 & 185 & $0.000 e+00$ & $5.259 e+04$ \\
\hline 56 & 186 & $0.000 e+00$ & $1.618 \mathrm{e}+04$ & 56 & 193 & $9.790 e-04$ & $1.224 e+07$ & 56 & 203 & $9.246 e-04$ & $1.778 e+07$ \\
\hline
\end{tabular}


Table III. Ca IX Oscillator Strengths and Radiative Decay Rates for transitions involving levels higher than 4.

\begin{tabular}{|c|c|c|c|c|c|c|c|c|c|c|c|}
\hline $\begin{array}{l}\text { Low. } \\
\text { Lev }\end{array}$ & $\begin{array}{l}\text { Upp } \\
\text {. Lev }\end{array}$ & $\begin{array}{l}\text { Osc. } \\
\text { Str. }\end{array}$ & $\begin{array}{l}\text { Rad. } \\
\text { Rate }\end{array}$ & $\begin{array}{l}\text { Low. } \\
\text { Lev. }\end{array}$ & $\begin{array}{l}\text { Upp. } \\
\text { Lev }\end{array}$ & $\begin{array}{l}\text { Osc. } \\
\text { Str. }\end{array}$ & $\begin{array}{l}\text { Rad. } \\
\text { Rate }\end{array}$ & $\begin{array}{l}\text { Low. } \\
\text { Lev. }\end{array}$ & $\begin{array}{l}\text { - Upp } \\
\text {. Lev }\end{array}$ & $\begin{array}{l}\text { Osc. } \\
\text { Str. }\end{array}$ & $\begin{array}{l}\text { Rad. } \\
\text { Rate }\end{array}$ \\
\hline$i$ & $\mathrm{j}$ & $g f$ & $(1 / s)$ & $i$ & $j$ & $g f$ & $(1 / s)$ & $i$ & $j$ & $g f$ & $(1 / s)$ \\
\hline 56 & 204 & $7.823 e-04$ & $2.508 e+07$ & 56 & 218 & $1.181 \mathrm{e}-04$ & $4.157 e+06$ & 56 & 219 & $1.073 e-03$ & $2.702 e+07$ \\
\hline 56 & 222 & $8.612 \mathrm{e}-05$ & $5.129 \mathrm{e}+06$ & 56 & 248 & $5.835 e-04$ & $1.793 e+07$ & 56 & 273 & $1.450 e-04$ & $1.069 \mathrm{e}+07$ \\
\hline 57 & 68 & $1.283 e-02$ & $1.189 \mathrm{e}+07$ & 57 & 69 & $2.458 \mathrm{e}-01$ & $1.637 e+08$ & 57 & 72 & $1.867 \mathrm{e}-01$ & $1.906 e+08$ \\
\hline 57 & 74 & $2.391 \mathrm{e}-02$ & $2.523 e+07$ & 57 & 75 & $5.781 \mathrm{e}-01$ & $4.398 e+08$ & 57 & 76 & $4.486 e+00$ & $2.812 \mathrm{e}+09$ \\
\hline 57 & 77 & $9.045 e-02$ & $7.360 \mathrm{e}+07$ & 57 & 78 & $6.039 \mathrm{e}-02$ & $8.310 e+07$ & 57 & 99 & $2.968 \mathrm{e}-02$ & $6.353 e+07$ \\
\hline 57 & 100 & $3.450 e-01$ & $5.746 e+08$ & 57 & 104 & $0.000 \mathrm{e}+00$ & $2.422 \mathrm{e}+04$ & 57 & 112 & $1.455 \mathrm{e}-01$ & $1.095 e+09$ \\
\hline 57 & 113 & $6.830 e-01$ & $5.169 \mathrm{e}+09$ & 57 & 116 & $7.056 e-02$ & $5.493 e+08$ & 57 & 117 & $2.231 \mathrm{e}-01$ & $1.245 e+09$ \\
\hline 57 & 118 & $1.405 \mathrm{e}-02$ & $1.110 \mathrm{e}+08$ & 57 & 119 & $3.073 e-01$ & $1.744 e+09$ & 57 & 120 & $2.195 \mathrm{e}+00$ & $9.754 e+09$ \\
\hline 57 & 123 & $7.944 e-02$ & $6.850 e+08$ & 57 & 128 & $0.000 e+00$ & $1.146 e+04$ & 57 & 130 & $0.000 e+00$ & $6.061 e+04$ \\
\hline 57 & 131 & $0.000 e+00$ & $1.120 \mathrm{e}+05$ & 57 & 132 & $0.000 e+00$ & $2.665 e+05$ & 57 & 133 & $0.000 e+00$ & $3.406 e+05$ \\
\hline 57 & 137 & $2.794 \mathrm{e}-03$ & $3.539 \mathrm{e}+07$ & 57 & 139 & $1.508 \mathrm{e}-02$ & $1.372 \mathrm{e}+08$ & 57 & 140 & $7.402 \mathrm{e}-03$ & $9.597 e+07$ \\
\hline 57 & 141 & $1.688 \mathrm{e}-01$ & $1.570 \mathrm{e}+09$ & 57 & 142 & $6.202 \mathrm{e}-01$ & $4.518 e+09$ & 57 & 143 & $1.831 \mathrm{e}-02$ & $2.430 e+08$ \\
\hline 57 & 152 & $0.000 e+00$ & $3.996 e+03$ & 57 & 153 & $0.000 e+00$ & $4.908 e+04$ & 57 & 158 & $0.000 e+00$ & $2.675 e+04$ \\
\hline 57 & 161 & $0.000 e+00$ & $4.038 e+04$ & 57 & 162 & $8.681 \mathrm{e}-04$ & $6.857 e+06$ & 57 & 164 & $0.000 e+00$ & $7.205 e+04$ \\
\hline 57 & 165 & $0.000 e+00$ & $5.289 e+05$ & 57 & 169 & $0.000 e+00$ & $1.177 e+06$ & 57 & 171 & $0.000 e+00$ & $4.393 e+03$ \\
\hline 57 & 172 & $0.000 e+00$ & $1.010 e+05$ & 57 & 173 & $0.000 e+00$ & $3.961 e+04$ & 57 & 175 & $4.669 e-03$ & $3.847 e+07$ \\
\hline 57 & 184 & $0.000 e+00$ & $2.310 \mathrm{e}+04$ & 57 & 186 & $0.000 e+00$ & $2.664 \mathrm{e}+04$ & 57 & 192 & $1.658 \mathrm{e}-03$ & $1.581 \mathrm{e}+07$ \\
\hline 57 & 203 & $2.517 e-03$ & $4.755 e+07$ & 57 & 220 & $1.586 e-03$ & $3.071 \mathrm{e}+07$ & 57 & 221 & $1.748 e-04$ & $6.167 e+06$ \\
\hline 57 & 237 & $0.000 e+00$ & $5.105 e+03$ & 57 & 249 & $8.650 e-04$ & $2.043 e+07$ & 57 & 272 & $2.093 e-04$ & $9.152 \mathrm{e}+06$ \\
\hline 58 & 66 & $4.960 e-01$ & $6.753 e+08$ & 58 & 73 & $1.464 \mathrm{e}-02$ & $2.297 e+07$ & 58 & 79 & $9.944 \mathrm{e}-02$ & $2.129 \mathrm{e}+08$ \\
\hline 58 & 81 & $2.637 e-03$ & $6.341 \mathrm{e}+06$ & 58 & 111 & $2.592 \mathrm{e}-01$ & $3.052 \mathrm{e}+09$ & 58 & 114 & $8.229 e-03$ & $1.013 e+08$ \\
\hline 58 & 115 & $4.676 \mathrm{e}-01$ & $5.851 \mathrm{e}+09$ & 58 & 122 & $2.857 e-02$ & $3.967 e+08$ & 58 & 127 & $1.209 \mathrm{e}-03$ & $1.854 \mathrm{e}+07$ \\
\hline 58 & 128 & $0.000 e+00$ & $1.104 \mathrm{e}+05$ & 58 & 132 & $0.000 e+00$ & $3.952 \mathrm{e}+03$ & 58 & 138 & $7.895 e-02$ & $1.628 \mathrm{e}+09$ \\
\hline 58 & 145 & $7.147 e-03$ & $1.549 \mathrm{e}+08$ & 58 & 156 & $6.464 \mathrm{e}-04$ & $1.471 \mathrm{e}+07$ & 58 & 159 & $0.000 e+00$ & $5.254 e+05$ \\
\hline 58 & 173 & $0.000 e+00$ & $8.142 \mathrm{e}+04$ & 58 & 180 & $0.000 e+00$ & $5.380 e+03$ & 58 & 183 & $0.000 e+00$ & $1.345 \mathrm{e}+04$ \\
\hline 58 & 200 & $4.217 \mathrm{e}-03$ & $1.278 e+08$ & 58 & 204 & $2.180 \mathrm{e}-03$ & $6.719 e+07$ & 58 & 208 & $6.690 e-04$ & $2.234 \mathrm{e}+07$ \\
\hline 58 & 262 & $2.158 \mathrm{e}-04$ & $1.544 \mathrm{e}+07$ & 58 & 267 & $0.000 e+00$ & $1.218 e+04$ & 58 & 278 & $0.000 e+00$ & $7.670 \mathrm{e}+03$ \\
\hline 59 & 66 & $2.600 e-01$ & $3.477 \mathrm{e}+08$ & 59 & 67 & $5.318 \mathrm{e}-01$ & $4.269 e+08$ & 59 & 68 & $3.346 \mathrm{e}-01$ & $2.753 e+08$ \\
\hline 59 & 72 & $1.981 \mathrm{e}-01$ & $1.806 \mathrm{e}+08$ & 59 & 73 & $3.576 \mathrm{e}-02$ & $5.515 e+07$ & 59 & 74 & $1.281 \mathrm{e}-01$ & $1.209 \mathrm{e}+08$ \\
\hline 59 & 78 & $5.262 \mathrm{e}-01$ & $6.571 \mathrm{e}+08$ & 59 & 79 & $4.852 \mathrm{e}-03$ & $1.024 \mathrm{e}+07$ & 59 & 80 & $9.893 e-03$ & $6.334 e+07$ \\
\hline 59 & 81 & $1.550 \mathrm{e}-02$ & $3.676 e+07$ & 59 & 110 & $3.260 \mathrm{e}-01$ & $1.141 \mathrm{e}+10$ & 59 & 111 & $3.428 \mathrm{e}-01$ & $4.011 \mathrm{e}+09$ \\
\hline 59 & 112 & $2.325 e-02$ & $1.679 \mathrm{e}+08$ & 59 & 113 & $4.373 e-03$ & $3.177 e+07$ & 59 & 114 & $2.205 e-01$ & $2.700 \mathrm{e}+09$ \\
\hline 59 & 115 & $1.871 \mathrm{e}-01$ & $2.328 e+09$ & 59 & 116 & $7.504 \mathrm{e}-01$ & $5.611 e+09$ & 59 & 118 & $7.477 e-03$ & $5.678 e+07$ \\
\hline 59 & 121 & $9.468 \mathrm{e}-02$ & $3.921 \mathrm{e}+09$ & 59 & 122 & $1.264 \mathrm{e}-01$ & $1.746 e+09$ & 59 & 123 & $2.187 e-02$ & $1.815 \mathrm{e}+08$ \\
\hline 59 & 125 & $0.000 e+00$ & $6.339 \mathrm{e}+04$ & 59 & 126 & $0.000 e+00$ & $1.958 e+05$ & 59 & 128 & $0.000 e+00$ & $1.079 e+05$ \\
\hline 59 & 130 & $0.000 e+00$ & $7.998 e+03$ & 59 & 131 & $0.000 e+00$ & $1.231 \mathrm{e}+04$ & 59 & 132 & $0.000 e+00$ & $1.197 e+04$ \\
\hline 59 & 134 & $0.000 e+00$ & $1.824 \mathrm{e}+04$ & 59 & 136 & $7.894 \mathrm{e}-03$ & $9.630 e+07$ & 59 & 137 & $1.055 \mathrm{e}-01$ & $1.295 \mathrm{e}+09$ \\
\hline 59 & 138 & $4.690 \mathrm{e}-02$ & $9.628 \mathrm{e}+08$ & 59 & 140 & $6.635 \mathrm{e}-02$ & $8.339 e+08$ & 59 & 143 & $7.905 e-02$ & $1.017 e+09$ \\
\hline 59 & 145 & $2.080 e-03$ & $4.488 e+07$ & 59 & 146 & $1.385 e-04$ & $8.995 e+06$ & 59 & 148 & $0.000 e+00$ & $5.423 e+03$ \\
\hline 59 & 151 & $0.000 e+00$ & $2.790 \mathrm{e}+03$ & 59 & 155 & $0.000 e+00$ & $2.449 e+04$ & 59 & 156 & $4.385 e-03$ & $9.931 e+07$ \\
\hline 59 & 158 & $0.000 e+00$ & $4.190 \mathrm{e}+05$ & 59 & 159 & $0.000 e+00$ & $3.511 \mathrm{e}+05$ & 59 & 164 & $0.000 e+00$ & $2.291 \mathrm{e}+05$ \\
\hline 59 & 172 & $0.000 e+00$ & $3.334 e+05$ & 59 & 174 & $0.000 e+00$ & $3.129 e+04$ & 59 & 181 & $0.000 e+00$ & $9.246 e+03$ \\
\hline 59 & 183 & $0.000 e+00$ & $4.602 \mathrm{e}+04$ & 59 & 200 & $1.657 \mathrm{e}-03$ & $5.001 e+07$ & 59 & 202 & $6.266 \mathrm{e}-03$ & $1.136 \mathrm{e}+08$ \\
\hline 59 & 203 & $2.854 \mathrm{e}-03$ & $5.255 e+07$ & 59 & 204 & $1.184 \mathrm{e}-03$ & $3.636 e+07$ & 59 & 205 & $1.840 e-03$ & $1.697 \mathrm{e}+08$ \\
\hline
\end{tabular}


Table III. Ca IX Oscillator Strengths and Radiative Decay Rates for transitions involving levels higher than 4.

\begin{tabular}{|c|c|c|c|c|c|c|c|c|c|c|c|}
\hline $\begin{array}{l}\text { Low. } \\
\text { Lev. }\end{array}$ & $\begin{array}{l}\text { Upp } \\
\text { Lev }\end{array}$ & $\begin{array}{l}\text { Osc. } \\
\text { Str. }\end{array}$ & $\begin{array}{l}\text { Rad. } \\
\text { Rate }\end{array}$ & $\begin{array}{l}\text { Low. } \\
\text { Lev. }\end{array}$ & $\begin{array}{l}\text { Upp. } \\
\text { Lev }\end{array}$ & $\begin{array}{l}\text { Osc. } \\
\text { Str. }\end{array}$ & $\begin{array}{l}\text { Rad. } \\
\text { Rate }\end{array}$ & $\begin{array}{l}\text { Low. } \\
\text { Lev. }\end{array}$ & $\begin{array}{l}\text { Upp } \\
\text { Lev }\end{array}$ & $\begin{array}{l}\text { Osc. } \\
\text { Str. }\end{array}$ & $\begin{array}{l}\text { Rad. } \\
\text { Rate }\end{array}$ \\
\hline$i$ & $j$ & $g f$ & $(1 / s)$ & $i$ & $j$ & $g f$ & $(1 / s)$ & $i$ & j & $g f$ & $(1 / s)$ \\
\hline 59 & 263 & $2.815 e-04$ & $1.206 \mathrm{e}+07$ & 59 & 267 & $0.000 e+00$ & $8.809 e+03$ & 59 & 268 & $0.000 e+00$ & $1.205 e+04$ \\
\hline 59 & 273 & $4.145 e-04$ & $2.971 e+07$ & 59 & 274 & $2.081 \mathrm{e}-04$ & $4.475 e+07$ & 59 & 277 & $0.000 e+00$ & $1.713 e+04$ \\
\hline 60 & 66 & $2.509 e-02$ & $3.143 e+07$ & 60 & 67 & $4.546 e-02$ & $3.419 e+07$ & 60 & 68 & $6.423 e-02$ & $4.955 e+07$ \\
\hline 60 & 70 & $3.462 \mathrm{e}-01$ & $4.903 e+08$ & 60 & 71 & $1.565 e-01$ & $6.663 e+08$ & 60 & 72 & $4.183 e-01$ & $3.586 e+08$ \\
\hline 60 & 73 & $6.271 \mathrm{e}-02$ & $9.102 \mathrm{e}+07$ & 60 & 74 & $1.518 e-02$ & $1.349 e+07$ & 60 & 78 & $3.256 \mathrm{e}-01$ & $3.859 \mathrm{e}+08$ \\
\hline 60 & 79 & $5.938 e-01$ & $1.190 e+09$ & 60 & 80 & $3.047 e-01$ & $1.853 e+09$ & 60 & 81 & $1.977 \mathrm{e}-02$ & $4.466 e+07$ \\
\hline 60 & 110 & $1.437 e-03$ & $4.919 e+07$ & 60 & 111 & $5.763 e-02$ & $6.598 e+08$ & 60 & 112 & $1.840 \mathrm{e}-01$ & $1.300 \mathrm{e}+09$ \\
\hline 60 & 113 & $9.340 e-01$ & $6.641 \mathrm{e}+09$ & 60 & 114 & $2.120 \mathrm{e}-02$ & $2.540 e+08$ & 60 & 115 & $1.057 \mathrm{e}-01$ & $1.287 e+09$ \\
\hline 60 & 116 & $2.313 e-01$ & $1.694 \mathrm{e}+09$ & 60 & 118 & $9.099 e-03$ & $6.767 e+07$ & 60 & 121 & $9.853 e-03$ & $3.999 e+08$ \\
\hline 60 & 122 & $6.164 e-02$ & $8.341 e+08$ & 60 & 123 & $2.196 \mathrm{e}-01$ & $1.786 e+09$ & 60 & 127 & $4.358 e-04$ & $6.520 e+06$ \\
\hline 60 & 128 & $0.000 e+00$ & $1.069 e+04$ & 60 & 130 & $0.000 e+00$ & $6.633 e+03$ & 60 & 131 & $0.000 e+00$ & $1.601 \mathrm{e}+05$ \\
\hline 60 & 132 & $0.000 e+00$ & $3.190 e+04$ & 60 & 137 & $5.575 e-03$ & $6.733 e+07$ & 60 & 138 & $2.079 \mathrm{e}-03$ & $4.197 e+07$ \\
\hline 60 & 140 & $5.211 \mathrm{e}-03$ & $6.443 e+07$ & 60 & 143 & $1.358 \mathrm{e}-01$ & $1.720 e+09$ & 60 & 145 & $1.542 \mathrm{e}-01$ & $3.273 e+09$ \\
\hline 60 & 146 & $6.627 e-02$ & $4.237 e+09$ & 60 & 151 & $0.000 e+00$ & $1.148 e+03$ & 60 & 156 & $2.356 e-03$ & $5.253 e+07$ \\
\hline 60 & 158 & $0.000 e+00$ & $3.953 e+04$ & 60 & 159 & $0.000 e+00$ & $2.778 e+04$ & 60 & 164 & $0.000 e+00$ & $2.874 e+04$ \\
\hline 60 & 172 & $0.000 e+00$ & $2.592 e+05$ & 60 & 173 & $0.000 e+00$ & $6.508 e+05$ & 60 & 174 & $0.000 e+00$ & $1.031 \mathrm{e}+06$ \\
\hline 60 & 180 & $0.000 e+00$ & $2.929 e+04$ & 60 & 181 & $0.000 e+00$ & $1.441 e+04$ & 60 & 183 & $0.000 e+00$ & $1.302 e+04$ \\
\hline 60 & 200 & $6.039 e-04$ & $1.798 e+07$ & 60 & 202 & $2.320 e-03$ & $4.148 e+07$ & 60 & 204 & $1.227 e-03$ & $3.715 e+07$ \\
\hline 60 & 205 & $1.111 \mathrm{e}-03$ & $1.011 \mathrm{e}+08$ & 60 & 210 & $0.000 e+00$ & $5.855 e+03$ & 60 & 211 & $0.000 e+00$ & $6.236 e+03$ \\
\hline 60 & 212 & $0.000 e+00$ & $7.075 e+03$ & 60 & 268 & $0.000 e+00$ & $4.188 e+03$ & 60 & 272 & $8.753 e-04$ & $3.730 \mathrm{e}+07$ \\
\hline 60 & 273 & $2.320 e-04$ & $1.648 \mathrm{e}+07$ & 60 & 274 & $5.117 \mathrm{e}-05$ & $1.091 \mathrm{e}+07$ & 60 & 278 & $0.000 e+00$ & $1.472 \mathrm{e}+04$ \\
\hline 60 & 279 & $0.000 e+00$ & $2.798 e+04$ & 61 & 66 & $6.559 \mathrm{e}-03$ & $8.180 e+06$ & 61 & 67 & $4.569 e-02$ & $3.421 \mathrm{e}+07$ \\
\hline 61 & 68 & $1.551 \mathrm{e}-01$ & $1.192 \mathrm{e}+08$ & 61 & 69 & $1.455 \mathrm{e}+00$ & $8.032 e+08$ & 61 & 70 & $8.265 e-02$ & $1.166 \mathrm{e}+08$ \\
\hline 61 & 72 & $2.666 \mathrm{e}-01$ & $2.276 \mathrm{e}+08$ & 61 & 74 & $4.927 e-02$ & $4.362 e+07$ & 61 & 75 & $2.754 \mathrm{e}-01$ & $1.759 \mathrm{e}+08$ \\
\hline 61 & 77 & $4.100 e-02$ & $2.818 e+07$ & 61 & 78 & $4.921 \mathrm{e}-01$ & $5.811 e+08$ & 61 & 79 & $1.826 \mathrm{e}-01$ & $3.646 e+08$ \\
\hline 61 & 111 & $2.317 \mathrm{e}-01$ & $2.649 \mathrm{e}+09$ & 61 & 112 & $2.667 e-01$ & $1.883 e+09$ & 61 & 113 & $7.252 \mathrm{e}-01$ & $5.149 \mathrm{e}+09$ \\
\hline 61 & 114 & $2.459 \mathrm{e}-02$ & $2.943 e+08$ & 61 & 115 & $1.612 \mathrm{e}-02$ & $1.960 \mathrm{e}+08$ & 61 & 116 & $3.642 \mathrm{e}-01$ & $2.662 \mathrm{e}+09$ \\
\hline 61 & 117 & $1.964 \mathrm{e}+00$ & $1.029 \mathrm{e}+10$ & 61 & 118 & $6.512 \mathrm{e}-03$ & $4.836 e+07$ & 61 & 119 & $3.660 \mathrm{e}-02$ & $1.952 \mathrm{e}+08$ \\
\hline 61 & 122 & $5.103 e-02$ & $6.896 \mathrm{e}+08$ & 61 & 123 & $8.580 e-02$ & $6.970 e+08$ & 61 & 124 & $2.152 \mathrm{e}-02$ & $1.280 \mathrm{e}+08$ \\
\hline 61 & 125 & $0.000 e+00$ & $4.185 \mathrm{e}+04$ & 61 & 127 & $7.980 e-04$ & $1.192 \mathrm{e}+07$ & 61 & 128 & $0.000 e+00$ & $8.016 e+04$ \\
\hline 61 & 129 & $0.000 e+00$ & $9.397 e+04$ & 61 & 130 & $0.000 e+00$ & $7.539 e+04$ & 61 & 131 & $0.000 e+00$ & $1.926 \mathrm{e}+05$ \\
\hline 61 & 132 & $0.000 e+00$ & $1.191 \mathrm{e}+05$ & 61 & 133 & $0.000 e+00$ & $2.542 \mathrm{e}+04$ & 61 & 136 & $2.089 e-03$ & $2.504 e+07$ \\
\hline 61 & 137 & $1.219 \mathrm{e}-02$ & $1.471 \mathrm{e}+08$ & 61 & 138 & $1.054 \mathrm{e}-03$ & $2.127 e+07$ & 61 & 139 & $1.273 \mathrm{e}-01$ & $1.102 \mathrm{e}+09$ \\
\hline 61 & 140 & $2.066 \mathrm{e}-02$ & $2.552 \mathrm{e}+08$ & 61 & 141 & $1.508 e-01$ & $1.337 e+09$ & 61 & 143 & $1.036 \mathrm{e}-01$ & $1.311 \mathrm{e}+09$ \\
\hline 61 & 145 & $3.207 e-02$ & $6.802 \mathrm{e}+08$ & 61 & 150 & $0.000 e+00$ & $5.853 e+03$ & 61 & 151 & $0.000 e+00$ & $4.096 \mathrm{e}+03$ \\
\hline 61 & 152 & $0.000 e+00$ & $8.506 e+03$ & 61 & 155 & $0.000 e+00$ & $1.400 e+04$ & 61 & 158 & $0.000 e+00$ & $6.010 e+04$ \\
\hline 61 & 159 & $0.000 e+00$ & $1.617 \mathrm{e}+04$ & 61 & 161 & $0.000 e+00$ & $4.044 e+05$ & 61 & 164 & $0.000 e+00$ & $1.159 \mathrm{e}+05$ \\
\hline 61 & 165 & $0.000 e+00$ & $4.028 e+05$ & 61 & 172 & $0.000 e+00$ & $5.080 e+05$ & 61 & 173 & $0.000 e+00$ & $4.128 \mathrm{e}+05$ \\
\hline 61 & 174 & $0.000 e+00$ & $1.643 e+05$ & 61 & 180 & $0.000 e+00$ & $1.327 e+04$ & 61 & 181 & $0.000 e+00$ & $2.837 e+04$ \\
\hline 61 & 182 & $0.000 \mathrm{e}+00$ & $4.099 \mathrm{e}+04$ & 61 & 201 & $1.542 \mathrm{e}-02$ & $1.967 e+08$ & 61 & 202 & $2.337 e-03$ & $4.175 e+07$ \\
\hline 61 & 203 & $9.647 e-03$ & $1.751 \mathrm{e}+08$ & 61 & 204 & $3.880 e-03$ & $1.174 \mathrm{e}+08$ & 61 & 264 & $7.402 \mathrm{e}-04$ & $2.244 \mathrm{e}+07$ \\
\hline 61 & 265 & $0.000 e+00$ & $6.867 e+03$ & 61 & 268 & $0.000 e+00$ & $7.602 e+03$ & 61 & 269 & $0.000 e+00$ & $1.646 \mathrm{e}+04$ \\
\hline 61 & 272 & $3.384 \mathrm{e}-04$ & $1.441 \mathrm{e}+07$ & 61 & 277 & $0.000 e+00$ & $1.512 \mathrm{e}+04$ & 61 & 278 & $0.000 e+00$ & $1.437 \mathrm{e}+04$ \\
\hline 61 & 279 & $0.000 e+00$ & $6.286 e+03$ & 62 & 66 & $7.620 \mathrm{e}-03$ & $6.812 \mathrm{e}+06$ & 62 & 67 & $1.059 \mathrm{e}-01$ & $5.684 e+07$ \\
\hline
\end{tabular}


Table III. Ca IX Oscillator Strengths and Radiative Decay Rates for transitions involving levels higher than 4.

\begin{tabular}{|c|c|c|c|c|c|c|c|c|c|c|c|}
\hline $\begin{array}{l}\text { Low. } \\
\text { Lev. }\end{array}$ & $\begin{array}{l}\text { Upp } \\
\text { Lev }\end{array}$ & $\begin{array}{l}\text { Osc. } \\
\text { Str. }\end{array}$ & $\begin{array}{l}\text { Rad. } \\
\text { Rate }\end{array}$ & $\begin{array}{l}\text { Low. } \\
\text { Lev. }\end{array}$ & $\begin{array}{l}\text { Upp. } \\
\text { Lev. }\end{array}$ & $\begin{array}{l}\text { Osc. } \\
\text { Str. }\end{array}$ & $\begin{array}{l}\text { Rad. } \\
\text { Rate }\end{array}$ & $\begin{array}{l}\text { Low. } \\
\text { Lev. }\end{array}$ & $\begin{array}{l}\text { Upp } \\
\text { Lev }\end{array}$ & $\begin{array}{l}\text { Osc. } \\
\text { Str. }\end{array}$ & $\begin{array}{l}\text { Rad. } \\
\text { Rate }\end{array}$ \\
\hline$i$ & $j$ & $g f$ & $(1 / s)$ & $i$ & $j$ & $g f$ & $(1 / s)$ & $i$ & $j$ & $g f$ & $(1 / s)$ \\
\hline 62 & 68 & $9.219 \mathrm{e}-02$ & $5.100 e+07$ & 62 & 69 & $2.517 \mathrm{e}-02$ & $1.002 \mathrm{e}+07$ & 62 & 70 & $1.364 \mathrm{e}-02$ & $1.409 e+07$ \\
\hline 62 & 72 & $2.308 e-02$ & $1.445 e+07$ & 62 & 73 & $7.931 \mathrm{e}-02$ & $8.430 e+07$ & 62 & 74 & $3.059 \mathrm{e}-02$ & $1.999 \mathrm{e}+07$ \\
\hline 62 & 75 & $5.490 e-01$ & $2.593 e+08$ & 62 & 77 & $1.869 e+00$ & $9.609 e+08$ & 62 & 78 & $1.549 \mathrm{e}-02$ & $1.410 \mathrm{e}+07$ \\
\hline 62 & 81 & $8.866 e-02$ & $1.566 \mathrm{e}+08$ & 62 & 101 & $2.308 e-01$ & $4.012 e+08$ & 62 & 105 & $0.000 e+00$ & $3.273 e+04$ \\
\hline 62 & 111 & $8.289 e-02$ & $8.540 e+08$ & 62 & 112 & $3.612 \mathrm{e}-01$ & $2.301 e+09$ & 62 & 113 & $1.649 \mathrm{e}-01$ & $1.057 e+09$ \\
\hline 62 & 114 & $6.228 \mathrm{e}-01$ & $6.733 e+09$ & 62 & 115 & $6.120 e-03$ & $6.730 \mathrm{e}+07$ & 62 & 116 & $1.002 \mathrm{e}-02$ & $6.625 e+07$ \\
\hline 62 & 117 & $6.099 \mathrm{e}-03$ & $2.890 e+07$ & 62 & 118 & $1.254 \mathrm{e}-03$ & $8.425 e+06$ & 62 & 119 & $1.701 \mathrm{e}-02$ & $8.212 e+07$ \\
\hline 62 & 123 & $4.385 e-03$ & $3.238 e+07$ & 62 & 124 & $2.644 \mathrm{e}+00$ & $1.431 e+10$ & 62 & 125 & $0.000 e+00$ & $1.947 e+05$ \\
\hline 62 & 126 & $0.000 e+00$ & $8.056 e+04$ & 62 & 127 & $6.653 e-02$ & $9.078 e+08$ & 62 & 130 & $0.000 e+00$ & $8.067 e+04$ \\
\hline 62 & 132 & $0.000 e+00$ & $5.410 e+03$ & 62 & 133 & $0.000 e+00$ & $5.441 e+03$ & 62 & 134 & $0.000 e+00$ & $1.585 e+05$ \\
\hline 62 & 135 & $0.000 e+00$ & $4.100 e+05$ & 62 & 136 & $6.222 \mathrm{e}-02$ & $6.897 e+08$ & 62 & 137 & $2.519 \mathrm{e}-03$ & $2.811 e+07$ \\
\hline 62 & 138 & $4.025 e-04$ & $7.512 \mathrm{e}+06$ & 62 & 140 & $2.279 \mathrm{e}-02$ & $2.606 e+08$ & 62 & 141 & $4.239 e-03$ & $3.479 e+07$ \\
\hline 62 & 143 & $9.485 e-03$ & $1.112 \mathrm{e}+08$ & 62 & 144 & $0.000 e+00$ & $4.267 e+04$ & 62 & 147 & $2.482 \mathrm{e}-01$ & $2.126 e+09$ \\
\hline 62 & 151 & $0.000 e+00$ & $1.025 e+04$ & 62 & 154 & $0.000 e+00$ & $6.835 e+04$ & 62 & 155 & $0.000 e+00$ & $2.383 e+05$ \\
\hline 62 & 156 & $1.521 \mathrm{e}-02$ & $3.147 e+08$ & 62 & 158 & $0.000 e+00$ & $2.184 e+04$ & 62 & 164 & $0.000 e+00$ & $1.026 e+05$ \\
\hline 62 & 165 & $0.000 e+00$ & $1.213 e+04$ & 62 & 172 & $0.000 e+00$ & $5.236 e+04$ & 62 & 179 & $0.000 e+00$ & $1.049 e+06$ \\
\hline 62 & 180 & $0.000 e+00$ & $5.942 e+03$ & 62 & 182 & $0.000 e+00$ & $2.797 e+04$ & 62 & 183 & $0.000 e+00$ & $7.855 e+04$ \\
\hline 62 & 188 & $0.000 e+00$ & $9.456 e+04$ & 62 & 196 & $1.740 \mathrm{e}-02$ & $2.745 e+08$ & 62 & 206 & $4.278 e-02$ & $5.300 e+08$ \\
\hline 62 & 207 & $0.000 e+00$ & $9.027 e+04$ & 62 & 208 & $3.968 e-03$ & $1.226 \mathrm{e}+08$ & 62 & 214 & $5.108 e-04$ & $1.627 e+07$ \\
\hline 62 & 224 & $8.237 e-04$ & $1.947 e+07$ & 62 & 226 & $0.000 e+00$ & $7.137 e+03$ & 62 & 242 & $0.000 e+00$ & $8.495 e+03$ \\
\hline 62 & 250 & $1.745 e-03$ & $6.993 e+07$ & 62 & 261 & $0.000 e+00$ & $2.810 e+04$ & 62 & 265 & $0.000 e+00$ & $3.305 e+04$ \\
\hline 62 & 269 & $0.000 e+00$ & $9.900 e+03$ & 62 & 275 & $2.762 \mathrm{e}-03$ & $8.087 e+07$ & 62 & 280 & $0.000 e+00$ & $1.744 \mathrm{e}+04$ \\
\hline 62 & 281 & $0.000 e+00$ & $2.550 e+04$ & 63 & 66 & $1.696 \mathrm{e}-02$ & $8.698 e+06$ & 63 & 70 & $9.808 e-02$ & $6.075 e+07$ \\
\hline 63 & 73 & $4.649 \mathrm{e}-01$ & $2.988 e+08$ & 63 & 81 & $9.232 \mathrm{e}-02$ & $1.117 e+08$ & 63 & 92 & $0.000 e+00$ & $5.826 e+03$ \\
\hline 63 & 111 & $1.212 \mathrm{e}-01$ & $1.077 \mathrm{e}+09$ & 63 & 114 & $6.621 \mathrm{e}-01$ & $6.194 e+09$ & 63 & 115 & $2.332 \mathrm{e}-02$ & $2.222 \mathrm{e}+08$ \\
\hline 63 & 122 & $1.587 \mathrm{e}-03$ & $1.703 e+07$ & 63 & 127 & $1.922 \mathrm{e}-01$ & $2.307 e+09$ & 63 & 134 & $0.000 \mathrm{e}+00$ & $7.544 \mathrm{e}+04$ \\
\hline 63 & 145 & $2.381 \mathrm{e}-04$ & $4.209 \mathrm{e}+06$ & 63 & 156 & $7.389 e-02$ & $1.378 e+09$ & 63 & 173 & $0.000 \mathrm{e}+00$ & $8.703 e+03$ \\
\hline 63 & 180 & $0.000 \mathrm{e}+00$ & $2.539 \mathrm{e}+04$ & 63 & 183 & $0.000 e+00$ & $4.880 e+05$ & 63 & 208 & $6.099 \mathrm{e}-02$ & $1.731 \mathrm{e}+09$ \\
\hline 63 & 213 & $0.000 e+00$ & $9.652 \mathrm{e}+04$ & 63 & 225 & $5.310 e-04$ & $2.788 e+07$ & 63 & 238 & $0.000 e+00$ & $1.001 \mathrm{e}+04$ \\
\hline 63 & 280 & $0.000 e+00$ & $1.379 \mathrm{e}+05$ & 63 & 283 & $6.719 \mathrm{e}-03$ & $4.463 e+08$ & 64 & 70 & $4.690 \mathrm{e}-01$ & $8.342 e+07$ \\
\hline 64 & 71 & $2.106 \mathrm{e}-01$ & $1.130 \mathrm{e}+08$ & 64 & 72 & $9.199 \mathrm{e}-01$ & $1.006 e+08$ & 64 & 73 & $1.365 \mathrm{e}-01$ & $2.600 \mathrm{e}+07$ \\
\hline 64 & 78 & $7.871 \mathrm{e}-01$ & $1.917 \mathrm{e}+08$ & 64 & 79 & $4.063 e-01$ & $1.702 e+08$ & 64 & 80 & $1.295 \mathrm{e}-01$ & $1.668 \mathrm{e}+08$ \\
\hline 64 & 81 & $7.734 \mathrm{e}-03$ & $4.174 \mathrm{e}+06$ & 64 & 110 & $1.354 \mathrm{e}-01$ & $2.767 e+09$ & 64 & 111 & $3.197 e-01$ & $2.186 e+09$ \\
\hline 64 & 112 & $1.105 \mathrm{e}-01$ & $4.705 e+08$ & 64 & 113 & $5.052 e-01$ & $2.168 e+09$ & 64 & 114 & $7.693 e-02$ & $5.581 e+08$ \\
\hline 64 & 116 & $6.419 e-03$ & $2.859 \mathrm{e}+07$ & 64 & 121 & $2.668 \mathrm{e}-02$ & $6.779 e+08$ & 64 & 122 & $9.190 e-02$ & $7.784 e+08$ \\
\hline 64 & 123 & $2.045 e-01$ & $1.042 \mathrm{e}+09$ & 64 & 127 & $9.037 e-04$ & $8.681 e+06$ & 64 & 143 & $1.822 \mathrm{e}-03$ & $1.600 \mathrm{e}+07$ \\
\hline 64 & 145 & $1.300 \mathrm{e}-03$ & $1.916 \mathrm{e}+07$ & 64 & 146 & $4.955 e-04$ & $2.201 \mathrm{e}+07$ & 64 & 172 & $0.000 e+00$ & $8.648 e+03$ \\
\hline 64 & 173 & $0.000 e+00$ & $9.271 \mathrm{e}+03$ & 64 & 174 & $0.000 e+00$ & $1.056 \mathrm{e}+04$ & 64 & 210 & $0.000 e+00$ & $3.022 \mathrm{e}+05$ \\
\hline 64 & 211 & $0.000 \mathrm{e}+00$ & $3.019 \mathrm{e}+05$ & 64 & 212 & $0.000 \mathrm{e}+00$ & $3.041 e+05$ & 65 & 73 & $1.884 \mathrm{e}-01$ & $1.423 e+07$ \\
\hline 65 & 79 & $1.114 \mathrm{e}-02$ & $2.626 \mathrm{e}+06$ & 65 & 81 & $4.881 \mathrm{e}-01$ & $1.603 e+08$ & 65 & 111 & $3.541 e-02$ & $2.132 \mathrm{e}+08$ \\
\hline 65 & 114 & $1.128 \mathrm{e}-01$ & $7.234 \mathrm{e}+08$ & 65 & 115 & $2.736 \mathrm{e}-03$ & $1.793 e+07$ & 65 & 127 & $3.630 e-01$ & $3.133 e+09$ \\
\hline 65 & 134 & $0.000 e+00$ & $5.343 e+03$ & 65 & 156 & $1.232 \mathrm{e}-03$ & $1.772 \mathrm{e}+07$ & 65 & 180 & $0.000 e+00$ & $4.868 e+03$ \\
\hline 65 & 183 & $0.000 e+00$ & $8.637 e+04$ & 65 & 208 & $1.301 \mathrm{e}-02$ & $2.998 e+08$ & 65 & 213 & $0.000 e+00$ & $3.269 \mathrm{e}+05$ \\
\hline 65 & 238 & $0.000 e+00$ & $6.468 e+03$ & 65 & 280 & $0.000 e+00$ & $1.932 \mathrm{e}+04$ & 65 & 283 & $1.329 \mathrm{e}-03$ & $7.720 e+07$ \\
\hline
\end{tabular}


Table III. Ca IX Oscillator Strengths and Radiative Decay Rates for transitions involving levels higher than 4.

\begin{tabular}{|c|c|c|c|c|c|c|c|c|c|c|c|}
\hline $\begin{array}{l}\text { Low. } \\
\text { Lev. }\end{array}$ & $\begin{array}{l}\text { - Upp } \\
\text { Lev }\end{array}$ & $\begin{array}{l}\text { Osc. } \\
\text { Str. }\end{array}$ & $\begin{array}{l}\text { Rad. } \\
\text { Rate }\end{array}$ & $\begin{array}{l}\text { Low. } \\
\text { Lev. }\end{array}$ & $\begin{array}{l}\text { Upp. } \\
\text { Lev. }\end{array}$ & $\begin{array}{l}\text { Osc. } \\
\text { Str. }\end{array}$ & $\begin{array}{l}\text { Rad. } \\
\text { Rate }\end{array}$ & $\begin{array}{l}\text { Low. } \\
\text { Lev. }\end{array}$ & $\begin{array}{l}\text { Upp } \\
\text { Lev }\end{array}$ & $\begin{array}{l}\text { Osc. } \\
\text { Str. }\end{array}$ & $\begin{array}{l}\text { Rad. } \\
\text { Rate }\end{array}$ \\
\hline$i$ & $j$ & $g f$ & $(1 / s)$ & $i$ & $j$ & $g f$ & $(1 / s)$ & $i$ & j & $g f$ & $(1 / s)$ \\
\hline 66 & 86 & $5.949 e-01$ & $1.156 \mathrm{e}+08$ & 66 & 90 & $3.380 e-02$ & $9.321 e+06$ & 66 & 92 & $5.356 e-02$ & $1.668 \mathrm{e}+07$ \\
\hline 66 & 96 & $4.566 \mathrm{e}-02$ & $3.181 \mathrm{e}+07$ & 66 & 106 & $3.714 \mathrm{e}-03$ & $7.887 e+06$ & 66 & 107 & $7.156 \mathrm{e}-03$ & $9.144 \mathrm{e}+06$ \\
\hline 66 & 111 & $0.000 e+00$ & $2.392 \mathrm{e}+04$ & 66 & 122 & $0.000 e+00$ & $4.787 e+03$ & 66 & 125 & $2.093 e-01$ & $1.530 e+09$ \\
\hline 66 & 126 & $4.450 e-03$ & $3.354 \mathrm{e}+07$ & 66 & 128 & $1.512 \mathrm{e}-02$ & $6.887 e+07$ & 66 & 129 & $3.025 e-01$ & $6.993 e+09$ \\
\hline 66 & 130 & $1.208 \mathrm{e}-01$ & $9.471 \mathrm{e}+08$ & 66 & 134 & $2.779 \mathrm{e}-02$ & $1.424 e+08$ & 66 & 135 & $7.254 \mathrm{e}-03$ & $1.962 \mathrm{e}+08$ \\
\hline 66 & 136 & $0.000 e+00$ & $2.894 \mathrm{e}+04$ & 66 & 138 & $0.000 e+00$ & $3.857 e+04$ & 66 & 140 & $0.000 e+00$ & $3.012 \mathrm{e}+04$ \\
\hline 66 & 141 & $0.000 e+00$ & $6.978 e+03$ & 66 & 143 & $0.000 e+00$ & $5.246 e+03$ & 66 & 145 & $0.000 e+00$ & $2.551 e+04$ \\
\hline 66 & 148 & $4.681 \mathrm{e}-01$ & $5.894 e+09$ & 66 & 149 & $7.246 e-02$ & $5.477 e+08$ & 66 & 154 & $1.237 \mathrm{e}-02$ & $1.589 e+08$ \\
\hline 66 & 159 & $1.301 \mathrm{e}+00$ & $1.021 e+10$ & 66 & 163 & $0.000 e+00$ & $7.135 e+05$ & 66 & 171 & $4.137 e-03$ & $5.703 e+07$ \\
\hline 66 & 173 & $4.296 \mathrm{e}-02$ & $3.583 e+08$ & 66 & 174 & $6.706 e-02$ & $9.391 e+08$ & 66 & 177 & $0.000 e+00$ & $7.213 e+04$ \\
\hline 66 & 178 & $0.000 \mathrm{e}+00$ & $1.415 \mathrm{e}+04$ & 66 & 180 & $7.665 e-01$ & $6.618 e+09$ & 66 & 183 & $4.296 \mathrm{e}-02$ & $3.744 e+08$ \\
\hline 66 & 184 & $1.389 \mathrm{e}-02$ & $2.198 e+08$ & 66 & 185 & $5.848 e-02$ & $2.776 e+09$ & 66 & 186 & $1.107 e-03$ & $1.051 \mathrm{e}+07$ \\
\hline 66 & 187 & $5.572 \mathrm{e}-03$ & $5.324 e+07$ & 66 & 197 & $0.000 e+00$ & $2.110 e+04$ & 66 & 207 & $1.657 \mathrm{e}-03$ & $9.839 e+07$ \\
\hline 66 & 210 & $6.774 \mathrm{e}-04$ & $2.376 e+07$ & 66 & 214 & $0.000 e+00$ & $8.062 e+03$ & 66 & 224 & $0.000 e+00$ & $6.030 e+03$ \\
\hline 66 & 225 & $0.000 e+00$ & $1.093 e+04$ & 66 & 227 & $5.654 \mathrm{e}-04$ & $2.719 e+07$ & 66 & 239 & $1.214 \mathrm{e}-03$ & $1.846 e+08$ \\
\hline 66 & 240 & $5.890 e-04$ & $2.985 e+07$ & 66 & 267 & $5.774 \mathrm{e}-04$ & $1.845 e+07$ & 66 & 278 & $2.138 \mathrm{e}-04$ & $6.926 e+06$ \\
\hline 66 & 279 & $1.017 e-04$ & $5.496 e+06$ & 66 & 281 & $5.536 e-05$ & $9.047 e+06$ & 67 & 84 & $5.081 e-01$ & $5.935 e+07$ \\
\hline 67 & 87 & $2.926 \mathrm{e}-01$ & $4.293 e+07$ & 67 & 89 & $7.597 e-02$ & $1.459 e+07$ & 67 & 95 & $3.860 \mathrm{e}-02$ & $1.580 e+07$ \\
\hline 67 & 97 & $8.000 e-02$ & $4.409 \mathrm{e}+07$ & 67 & 108 & $1.304 \mathrm{e}-02$ & $1.195 e+07$ & 67 & 110 & $0.000 e+00$ & $3.661 \mathrm{e}+04$ \\
\hline 67 & 111 & $0.000 e+00$ & $1.853 e+04$ & 67 & 114 & $0.000 e+00$ & $7.153 e+03$ & 67 & 121 & $0.000 e+00$ & $6.296 e+03$ \\
\hline 67 & 125 & $1.796 \mathrm{e}-01$ & $1.312 \mathrm{e}+09$ & 67 & 126 & $4.815 e-01$ & $3.628 e+09$ & 67 & 127 & $0.000 e+00$ & $1.986 e+04$ \\
\hline 67 & 128 & $2.293 e-01$ & $1.044 \mathrm{e}+09$ & 67 & 130 & $1.310 \mathrm{e}-01$ & $1.027 e+09$ & 67 & 131 & $6.773 e-03$ & $2.286 e+07$ \\
\hline 67 & 132 & $9.390 e-03$ & $4.504 e+07$ & 67 & 133 & $1.718 \mathrm{e}-03$ & $1.395 e+07$ & 67 & 134 & $8.918 e-02$ & $4.569 e+08$ \\
\hline 67 & 136 & $0.000 e+00$ & $1.523 e+04$ & 67 & 137 & $0.000 e+00$ & $4.741 e+04$ & 67 & 138 & $0.000 e+00$ & $2.902 e+04$ \\
\hline 67 & 139 & $0.000 e+00$ & $8.777 e+03$ & 67 & 140 & $0.000 e+00$ & $5.016 e+03$ & 67 & 141 & $0.000 e+00$ & $1.908 e+04$ \\
\hline 67 & 142 & $0.000 e+00$ & $2.517 e+03$ & 67 & 143 & $0.000 e+00$ & $1.234 \mathrm{e}+04$ & 67 & 144 & $3.706 \mathrm{e}-01$ & $1.932 \mathrm{e}+09$ \\
\hline 67 & 146 & $0.000 e+00$ & $2.702 \mathrm{e}+04$ & 67 & 147 & $0.000 e+00$ & $9.023 e+03$ & 67 & 148 & $6.968 \mathrm{e}-02$ & $8.772 \mathrm{e}+08$ \\
\hline 67 & 149 & $4.397 e-01$ & $3.323 e+09$ & 67 & 150 & $4.007 e-03$ & $2.167 e+07$ & 67 & 151 & $1.643 e-02$ & $8.950 e+07$ \\
\hline 67 & 154 & $8.940 e-02$ & $1.149 \mathrm{e}+09$ & 67 & 155 & $1.340 \mathrm{e}+00$ & $7.438 e+09$ & 67 & 156 & $0.000 e+00$ & $2.431 \mathrm{e}+04$ \\
\hline 67 & 157 & $0.000 e+00$ & $6.434 \mathrm{e}+03$ & 67 & 158 & $1.228 \mathrm{e}+00$ & $6.860 e+09$ & 67 & 159 & $1.321 \mathrm{e}-01$ & $1.037 e+09$ \\
\hline 67 & 162 & $0.000 e+00$ & $8.108 e+05$ & 67 & 163 & $0.000 e+00$ & $1.221 \mathrm{e}+05$ & 67 & 171 & $1.893 \mathrm{e}-02$ & $2.609 e+08$ \\
\hline 67 & 172 & $4.031 \mathrm{e}-03$ & $2.384 e+07$ & 67 & 173 & $2.340 e-02$ & $1.951 e+08$ & 67 & 174 & $1.209 \mathrm{e}-02$ & $1.693 e+08$ \\
\hline 67 & 177 & $0.000 e+00$ & $2.833 e+04$ & 67 & 178 & $0.000 e+00$ & $9.715 e+04$ & 67 & 180 & $1.427 \mathrm{e}-01$ & $1.232 \mathrm{e}+09$ \\
\hline 67 & 181 & $6.592 \mathrm{e}-01$ & $4.069 e+09$ & 67 & 183 & $1.150 \mathrm{e}-01$ & $1.002 e+09$ & 67 & 184 & $9.521 \mathrm{e}-02$ & $1.506 e+09$ \\
\hline 67 & 186 & $1.833 e-02$ & $1.741 \mathrm{e}+08$ & 67 & 187 & $1.724 \mathrm{e}-01$ & $1.647 e+09$ & 67 & 189 & $0.000 e+00$ & $4.021 e+04$ \\
\hline 67 & 211 & $7.233 e-04$ & $1.523 e+07$ & 67 & 213 & $7.742 \mathrm{e}-04$ & $1.655 e+07$ & 67 & 216 & $0.000 e+00$ & $5.731 e+03$ \\
\hline 67 & 217 & $0.000 e+00$ & $5.954 e+03$ & 67 & 220 & $0.000 e+00$ & $1.160 e+04$ & 67 & 221 & $0.000 e+00$ & $5.999 e+03$ \\
\hline 67 & 222 & $0.000 e+00$ & $5.542 e+03$ & 67 & 223 & $0.000 e+00$ & $4.777 e+03$ & 67 & 228 & $4.887 e-04$ & $1.410 e+07$ \\
\hline 67 & 233 & $5.865 e-04$ & $2.837 e+07$ & 67 & 238 & $1.254 \mathrm{e}-03$ & $3.808 e+07$ & 67 & 240 & $1.684 \mathrm{e}-03$ & $8.536 e+07$ \\
\hline 67 & 241 & $3.516 \mathrm{e}-04$ & $1.070 \mathrm{e}+07$ & 67 & 261 & $5.859 e-04$ & $1.335 e+07$ & 67 & 268 & $4.926 e-04$ & $1.124 \mathrm{e}+07$ \\
\hline 67 & 277 & $3.772 \mathrm{e}-04$ & $8.725 e+06$ & 67 & 280 & $4.131 e-04$ & $1.346 e+07$ & 68 & 82 & $4.303 e-01$ & $3.976 e+07$ \\
\hline 68 & 87 & $3.888 e-01$ & $5.428 e+07$ & 68 & 95 & $4.402 \mathrm{e}-02$ & $1.740 \mathrm{e}+07$ & 68 & 97 & $7.708 e-02$ & $4.122 \mathrm{e}+07$ \\
\hline 68 & 102 & $2.369 \mathrm{e}-02$ & $1.259 \mathrm{e}+07$ & 68 & 107 & $5.955 e-03$ & $7.457 e+06$ & 68 & 110 & $0.000 e+00$ & $5.660 e+04$ \\
\hline 68 & 111 & $0.000 e+00$ & $5.972 \mathrm{e}+03$ & 68 & 114 & $0.000 e+00$ & $1.547 e+04$ & 68 & 121 & $0.000 e+00$ & $1.058 e+04$ \\
\hline
\end{tabular}


Table III. Ca IX Oscillator Strengths and Radiative Decay Rates for transitions involving levels higher than 4.

\begin{tabular}{|c|c|c|c|c|c|c|c|c|c|c|c|}
\hline $\begin{array}{l}\text { Low. } \\
\text { Lev . }\end{array}$ & $\begin{array}{l}\text { Upp } \\
\text { Lev }\end{array}$ & $\begin{array}{l}\text { Osc. } \\
\text { Str. }\end{array}$ & $\begin{array}{l}\text { Rad. } \\
\text { Rate }\end{array}$ & $\begin{array}{l}\text { Low. } \\
\text { Lev. }\end{array}$ & $\begin{array}{l}\text { Upp. } \\
\text { Lev. }\end{array}$ & $\begin{array}{l}\text { Osc. } \\
\text { Str. }\end{array}$ & $\begin{array}{l}\text { Rad. } \\
\text { Rate }\end{array}$ & $\begin{array}{l}\text { Low. } \\
\text { Lev. }\end{array}$ & $\begin{array}{l}\text { - Upp } \\
\text {. Lev }\end{array}$ & $\begin{array}{l}\text { Osc. } \\
\text { Str. }\end{array}$ & $\begin{array}{l}\text { Rad. } \\
\text { Rate }\end{array}$ \\
\hline$i$ & $j$ & $g f$ & $(1 / s)$ & $i$ & $j$ & $g f$ & $(1 / s)$ & i & j & $g f$ & $(1 / s)$ \\
\hline 68 & 125 & $4.559 \mathrm{e}-01$ & $3.297 e+09$ & 68 & 126 & $8.311 \mathrm{e}-02$ & $6.197 e+08$ & 68 & 127 & $0.000 e+00$ & $2.238 e+04$ \\
\hline 68 & 130 & $1.729 \mathrm{e}-01$ & $1.342 \mathrm{e}+09$ & 68 & 131 & $1.078 \mathrm{e}-02$ & $3.603 e+07$ & 68 & 132 & $1.013 e-01$ & $4.809 e+08$ \\
\hline 68 & 133 & $6.214 \mathrm{e}-03$ & $4.996 e+07$ & 68 & 134 & $1.305 e-01$ & $6.619 e+08$ & 68 & 136 & $0.000 e+00$ & $3.668 e+04$ \\
\hline 68 & 137 & $0.000 e+00$ & $5.609 e+04$ & 68 & 139 & $0.000 e+00$ & $4.954 e+03$ & 68 & 141 & $0.000 e+00$ & $6.128 e+03$ \\
\hline 68 & 142 & $0.000 e+00$ & $2.422 \mathrm{e}+03$ & 68 & 143 & $0.000 e+00$ & $1.360 e+04$ & 68 & 144 & $4.328 \mathrm{e}-01$ & $2.237 e+09$ \\
\hline 68 & 145 & $0.000 e+00$ & $9.078 e+03$ & 68 & 146 & $0.000 e+00$ & $2.830 \mathrm{e}+04$ & 68 & 147 & $0.000 e+00$ & $1.705 e+04$ \\
\hline 68 & 148 & $1.099 \mathrm{e}-01$ & $1.372 \mathrm{e}+09$ & 68 & 149 & $2.292 \mathrm{e}-01$ & $1.718 e+09$ & 68 & 150 & $4.662 \mathrm{e}-02$ & $2.500 e+08$ \\
\hline 68 & 151 & $5.903 e-02$ & $3.189 \mathrm{e}+08$ & 68 & 154 & $7.004 e-02$ & $8.928 e+08$ & 68 & 155 & $1.740 e+00$ & $9.584 e+09$ \\
\hline 68 & 156 & $0.000 e+00$ & $3.010 e+04$ & 68 & 157 & $0.000 e+00$ & $4.953 e+05$ & 68 & 158 & $9.304 e-01$ & $5.156 e+09$ \\
\hline 68 & 159 & $8.481 \mathrm{e}-03$ & $6.603 e+07$ & 68 & 162 & $0.000 e+00$ & $5.580 e+03$ & 68 & 164 & $2.885 e-01$ & $1.644 \mathrm{e}+09$ \\
\hline 68 & 167 & $0.000 \mathrm{e}+00$ & $3.390 \mathrm{e}+05$ & 68 & 172 & $3.139 \mathrm{e}-02$ & $1.842 \mathrm{e}+08$ & 68 & 173 & $9.843 e-02$ & $8.144 \mathrm{e}+08$ \\
\hline 68 & 174 & $1.578 \mathrm{e}-02$ & $2.192 \mathrm{e}+08$ & 68 & 175 & $0.000 e+00$ & $1.035 e+04$ & 68 & 176 & $0.000 e+00$ & $1.734 \mathrm{e}+05$ \\
\hline 68 & 177 & $0.000 e+00$ & $2.514 e+04$ & 68 & 178 & $0.000 e+00$ & $1.154 \mathrm{e}+04$ & 68 & 180 & $1.468 \mathrm{e}-01$ & $1.258 \mathrm{e}+09$ \\
\hline 68 & 181 & $4.335 e-01$ & $2.656 e+09$ & 68 & 183 & $2.633 e-01$ & $2.277 e+09$ & 68 & 184 & $3.543 e-02$ & $5.564 e+08$ \\
\hline 68 & 186 & $3.380 e-02$ & $3.187 e+08$ & 68 & 187 & $1.736 \mathrm{e}-01$ & $1.647 e+09$ & 68 & 189 & $0.000 e+00$ & $5.142 e+04$ \\
\hline 68 & 190 & $0.000 e+00$ & $1.877 e+04$ & 68 & 206 & $0.000 e+00$ & $1.870 e+04$ & 68 & 210 & $4.933 e-04$ & $1.722 e+07$ \\
\hline 68 & 213 & $9.629 \mathrm{e}-04$ & $2.048 e+07$ & 68 & 227 & $4.285 e-04$ & $2.052 e+07$ & 68 & 233 & $4.662 \mathrm{e}-04$ & $2.246 e+07$ \\
\hline 68 & 238 & $1.932 \mathrm{e}-03$ & $5.842 e+07$ & 68 & 240 & $9.618 e-04$ & $4.854 e+07$ & 68 & 244 & $0.000 e+00$ & $3.817 e+03$ \\
\hline 68 & 258 & $3.693 e-04$ & $8.335 e+06$ & 68 & 261 & $5.859 e-04$ & $1.329 e+07$ & 68 & 268 & $3.530 \mathrm{e}-04$ & $8.024 e+06$ \\
\hline 68 & 278 & $1.865 e-04$ & $6.019 e+06$ & 68 & 280 & $3.970 e-04$ & $1.288 e+07$ & 69 & 83 & $3.369 e-01$ & $2.735 e+07$ \\
\hline 69 & 88 & $8.590 e-01$ & $9.560 \mathrm{e}+07$ & 69 & 89 & $1.096 \mathrm{e}-01$ & $1.993 e+07$ & 69 & 94 & $9.486 e-02$ & $2.599 \mathrm{e}+07$ \\
\hline 69 & 103 & $1.887 e-02$ & $7.782 \mathrm{e}+06$ & 69 & 105 & $3.995 e-02$ & $2.021 e+07$ & 69 & 108 & $1.980 \mathrm{e}-02$ & $1.770 \mathrm{e}+07$ \\
\hline 69 & 111 & $0.000 e+00$ & $4.054 e+04$ & 69 & 113 & $0.000 e+00$ & $1.145 e+04$ & 69 & 122 & $0.000 e+00$ & $8.263 e+03$ \\
\hline 69 & 128 & $5.881 \mathrm{e}-01$ & $2.642 \mathrm{e}+09$ & 69 & 131 & $2.348 e-01$ & $7.825 e+08$ & 69 & 132 & $4.775 e-01$ & $2.261 \mathrm{e}+09$ \\
\hline 69 & 136 & $0.000 e+00$ & $2.205 \mathrm{e}+04$ & 69 & 139 & $0.000 e+00$ & $9.285 e+04$ & 69 & 142 & $0.000 e+00$ & $2.722 e+04$ \\
\hline 69 & 143 & $0.000 e+00$ & $5.576 e+04$ & 69 & 144 & $8.043 e-02$ & $4.149 e+08$ & 69 & 145 & $0.000 e+00$ & $3.546 e+04$ \\
\hline 69 & 149 & $1.812 \mathrm{e}-01$ & $1.356 \mathrm{e}+09$ & 69 & 150 & $9.468 e-01$ & $5.068 e+09$ & 69 & 151 & $3.100 \mathrm{e}-03$ & $1.672 \mathrm{e}+07$ \\
\hline 69 & 152 & $7.890 e-04$ & $3.319 e+06$ & 69 & 155 & $9.933 e-02$ & $5.460 e+08$ & 69 & 157 & $0.000 e+00$ & $3.888 e+04$ \\
\hline 69 & 158 & $5.973 e-03$ & $3.303 e+07$ & 69 & 159 & $1.504 \mathrm{e}-03$ & $1.169 \mathrm{e}+07$ & 69 & 160 & $0.000 e+00$ & $5.610 e+05$ \\
\hline 69 & 161 & $2.862 \mathrm{e}+00$ & $1.237 e+10$ & 69 & 165 & $1.860 \mathrm{e}-01$ & $8.265 e+08$ & 69 & 167 & $0.000 e+00$ & $2.312 e+04$ \\
\hline 69 & 168 & $0.000 e+00$ & $2.782 e+05$ & 69 & 170 & $0.000 e+00$ & $8.667 e+04$ & 69 & 172 & $2.928 e-01$ & $1.715 e+09$ \\
\hline 69 & 173 & $3.246 \mathrm{e}-02$ & $2.681 e+08$ & 69 & 175 & $0.000 e+00$ & $2.036 e+05$ & 69 & 176 & $0.000 e+00$ & $3.667 e+04$ \\
\hline 69 & 177 & $0.000 e+00$ & $5.578 e+03$ & 69 & 178 & $0.000 e+00$ & $1.759 e+04$ & 69 & 179 & $3.670 \mathrm{e}-02$ & $1.728 \mathrm{e}+08$ \\
\hline 69 & 180 & $1.978 \mathrm{e}-02$ & $1.692 \mathrm{e}+08$ & 69 & 181 & $3.530 e-01$ & $2.159 e+09$ & 69 & 182 & $1.566 e+00$ & $7.464 e+09$ \\
\hline 69 & 186 & $1.372 \mathrm{e}-01$ & $1.291 \mathrm{e}+09$ & 69 & 187 & $1.860 e-03$ & $1.761 e+07$ & 69 & 188 & $9.857 e-02$ & $5.271 \mathrm{e}+08$ \\
\hline 69 & 190 & $0.000 e+00$ & $4.529 e+03$ & 69 & 194 & $0.000 e+00$ & $1.552 \mathrm{e}+04$ & 69 & 198 & $0.000 e+00$ & $2.924 e+04$ \\
\hline 69 & 211 & $7.283 e-04$ & $1.524 \mathrm{e}+07$ & 69 & 212 & $1.342 \mathrm{e}-03$ & $2.008 e+07$ & 69 & 228 & $4.944 e-04$ & $1.419 \mathrm{e}+07$ \\
\hline 69 & 229 & $1.109 \mathrm{e}-03$ & $2.275 \mathrm{e}+07$ & 69 & 238 & $8.775 e-04$ & $2.651 e+07$ & 69 & 241 & $3.209 \mathrm{e}-03$ & $9.714 e+07$ \\
\hline 69 & 245 & $0.000 e+00$ & $2.564 e+03$ & 69 & 256 & $0.000 e+00$ & $1.237 e+04$ & 69 & 265 & $3.932 \mathrm{e}-04$ & $6.937 e+06$ \\
\hline 69 & 269 & $7.830 \mathrm{e}-04$ & $1.383 e+07$ & 69 & 277 & $5.428 \mathrm{e}-04$ & $1.249 e+07$ & 70 & 90 & $1.062 \mathrm{e}+00$ & $2.349 e+08$ \\
\hline 70 & 91 & $3.982 e-01$ & $1.495 e+08$ & 70 & 92 & $2.672 \mathrm{e}-01$ & $6.766 e+07$ & 70 & 95 & $3.612 \mathrm{e}-01$ & $1.237 e+08$ \\
\hline 70 & 96 & $6.070 e-02$ & $3.542 \mathrm{e}+07$ & 70 & 106 & $1.536 \mathrm{e}-02$ & $2.952 e+07$ & 70 & 107 & $2.729 \mathrm{e}-02$ & $3.157 e+07$ \\
\hline 70 & 109 & $1.426 \mathrm{e}-02$ & $1.958 e+07$ & 70 & 125 & $2.980 e-01$ & $2.065 e+09$ & 70 & 126 & $1.547 e-02$ & $1.107 e+08$ \\
\hline 70 & 128 & $2.437 e-01$ & $1.053 e+09$ & 70 & 129 & $1.431 \mathrm{e}-01$ & $3.143 e+09$ & 70 & 130 & $7.981 e-02$ & $5.945 e+08$ \\
\hline
\end{tabular}


Table III. Ca IX Oscillator Strengths and Radiative Decay Rates for transitions involving levels higher than 4.

\begin{tabular}{|c|c|c|c|c|c|c|c|c|c|c|c|}
\hline $\begin{array}{l}\text { Low. } \\
\text { Lev. }\end{array}$ & $\begin{array}{l}\text { - Upp } \\
\text {. Lev }\end{array}$ & $\begin{array}{l}\text { Osc. } \\
\text { Str. }\end{array}$ & $\begin{array}{l}\text { Rad. } \\
\text { Rate }\end{array}$ & $\begin{array}{l}\text { Low. } \\
\text { Lev. }\end{array}$ & $\begin{array}{l}\text { - Upp } \\
\text { Lev }\end{array}$ & $\begin{array}{l}\text { Osc. } \\
\text { Str. }\end{array}$ & $\begin{array}{l}\text { Rad. } \\
\text { Rate }\end{array}$ & $\begin{array}{l}\text { Low. } \\
\text { Lev. }\end{array}$ & $\begin{array}{l}\text {. Upp } \\
\text {. Lev }\end{array}$ & $\begin{array}{l}\text { Osc. } \\
\text { Str. }\end{array}$ & $\begin{array}{l}\text { Rad. } \\
\text { Rate }\end{array}$ \\
\hline$i$ & $j$ & $g f$ & $(1 / s)$ & i & $j$ & $g f$ & $(1 / s)$ & $i$ & $j$ & $g f$ & $(1 / s)$ \\
\hline 70 & 132 & $6.197 e-01$ & $2.827 e+09$ & 70 & 133 & $4.564 \mathrm{e}-01$ & $3.526 e+09$ & 70 & 134 & $1.435 \mathrm{e}-01$ & $7.003 e+08$ \\
\hline 70 & 135 & $4.759 e-02$ & $1.227 \mathrm{e}+09$ & 70 & 136 & $0.000 \mathrm{e}+00$ & $3.071 e+03$ & 70 & 140 & $0.000 e+00$ & $7.798 e+03$ \\
\hline 70 & 141 & $0.000 e+00$ & $7.157 e+03$ & 70 & 143 & $0.000 \mathrm{e}+00$ & $4.393 e+03$ & 70 & 145 & $0.000 e+00$ & $1.627 e+04$ \\
\hline 70 & 148 & $5.076 e-04$ & $6.139 \mathrm{e}+06$ & 70 & 149 & $9.739 \mathrm{e}-02$ & $7.070 e+08$ & 70 & 154 & $3.323 e-02$ & $4.103 e+08$ \\
\hline 70 & 156 & $0.000 e+00$ & $5.527 e+03$ & 70 & 159 & $1.176 \mathrm{e}-02$ & $8.872 \mathrm{e}+07$ & 70 & 163 & $0.000 e+00$ & $5.803 e+03$ \\
\hline 70 & 171 & $2.457 e-02$ & $3.259 \mathrm{e}+08$ & 70 & 173 & $3.074 \mathrm{e}-01$ & $2.467 e+09$ & 70 & 174 & $1.615 \mathrm{e}-01$ & $2.176 \mathrm{e}+09$ \\
\hline 70 & 176 & $0.000 e+00$ & $1.454 \mathrm{e}+05$ & 70 & 177 & $0.000 e+00$ & $1.265 e+05$ & 70 & 180 & $5.057 e-02$ & $4.205 e+08$ \\
\hline 70 & 183 & $7.768 e-02$ & $6.521 \mathrm{e}+08$ & 70 & 184 & $2.823 e-02$ & $4.308 e+08$ & 70 & 185 & $9.509 e-03$ & $4.354 e+08$ \\
\hline 70 & 186 & $3.689 e-02$ & $3.380 e+08$ & 70 & 187 & $6.567 e-03$ & $6.055 e+07$ & 70 & 191 & $0.000 e+00$ & $1.052 \mathrm{e}+04$ \\
\hline 70 & 207 & $1.195 \mathrm{e}-03$ & $6.867 e+07$ & 70 & 211 & $3.383 e-04$ & $6.955 e+06$ & 70 & 214 & $0.000 e+00$ & $5.852 e+04$ \\
\hline 70 & 215 & $0.000 e+00$ & $1.574 \mathrm{e}+05$ & 70 & 216 & $0.000 e+00$ & $1.341 e+04$ & 70 & 217 & $0.000 e+00$ & $9.449 e+04$ \\
\hline 70 & 218 & $0.000 e+00$ & $8.727 e+04$ & 70 & 219 & $0.000 e+00$ & $1.057 e+05$ & 70 & 221 & $0.000 e+00$ & $7.912 e+04$ \\
\hline 70 & 222 & $0.000 e+00$ & $5.240 \mathrm{e}+04$ & 70 & 224 & $0.000 e+00$ & $2.832 \mathrm{e}+04$ & 70 & 225 & $0.000 e+00$ & $4.848 e+04$ \\
\hline 70 & 240 & $3.284 e-04$ & $1.631 \mathrm{e}+07$ & 70 & 241 & $2.921 \mathrm{e}-04$ & $8.710 e+06$ & 70 & 278 & $3.911 \mathrm{e}-04$ & $\mathrm{Be}+07$ \\
\hline 70 & 279 & $2.436 \mathrm{e}-04$ & $1.291 \mathrm{e}+07$ & 70 & 280 & $1.384 \mathrm{e}-04$ & $4.424 e+06$ & 71 & 91 & $6.061 \mathrm{e}-01$ & $2.267 e+08$ \\
\hline 71 & 96 & $1.635 \mathrm{e}-01$ & $9.506 \mathrm{e}+07$ & 71 & 106 & $1.795 e-02$ & $3.444 e+07$ & 71 & 125 & $8.623 e-02$ & $5.972 e+08$ \\
\hline 71 & 126 & $7.315 e-02$ & $5.228 e+08$ & 71 & 130 & $2.389 e-01$ & $1.778 e+09$ & 71 & 133 & $3.154 \mathrm{e}-01$ & $2.434 e+09$ \\
\hline 71 & 143 & $0.000 e+00$ & $6.997 e+03$ & 71 & 148 & $2.553 e-02$ & $3.085 e+08$ & 71 & 171 & $9.746 e-03$ & $1.292 \mathrm{e}+08$ \\
\hline 71 & 174 & $1.552 \mathrm{e}-01$ & $2.090 e+09$ & 71 & 177 & $0.000 e+00$ & $8.504 e+04$ & 71 & 184 & $2.509 \mathrm{e}-02$ & $3.826 e+08$ \\
\hline 71 & 216 & $0.000 e+00$ & $1.159 \mathrm{e}+05$ & 71 & 218 & $0.000 e+00$ & $9.924 e+04$ & 71 & 221 & $0.000 e+00$ & $4.742 e+04$ \\
\hline 71 & 240 & $1.931 \mathrm{e}-04$ & $9.589 e+06$ & 71 & 279 & $2.303 e-04$ & $1.220 \mathrm{e}+07$ & 72 & 89 & $2.046 e+00$ & $3.092 e+08$ \\
\hline 72 & 90 & $4.510 e-01$ & $9.802 e+07$ & 72 & 91 & $3.491 \mathrm{e}-02$ & $1.288 \mathrm{e}+07$ & 72 & 94 & $8.280 e-01$ & $1.953 e+08$ \\
\hline 72 & 95 & $8.634 \mathrm{e}-02$ & $2.915 \mathrm{e}+07$ & 72 & 107 & $1.820 \mathrm{e}-02$ & $2.089 e+07$ & 72 & 108 & $6.350 e-02$ & $5.232 e+07$ \\
\hline 72 & 126 & $1.257 \mathrm{e}-01$ & $8.956 e+08$ & 72 & 128 & $3.576 \mathrm{e}-01$ & $1.540 \mathrm{e}+09$ & 72 & 130 & $3.701 \mathrm{e}-01$ & $2.746 e+09$ \\
\hline 72 & 131 & $1.201 \mathrm{e}+00$ & $3.837 e+09$ & 72 & 132 & $7.660 \mathrm{e}-01$ & $3.480 e+09$ & 72 & 133 & $4.176 \mathrm{e}-01$ & $3.213 e+09$ \\
\hline 72 & 134 & $1.029 \mathrm{e}-01$ & $5.001 e+08$ & 72 & 139 & $0.000 e+00$ & $5.553 e+03$ & 72 & 140 & $0.000 e+00$ & $6.216 e+03$ \\
\hline 72 & 141 & $0.000 e+00$ & $1.134 \mathrm{e}+04$ & 72 & 142 & $0.000 e+00$ & $2.647 e+03$ & 72 & 144 & $2.526 \mathrm{e}-02$ & $1.260 \mathrm{e}+08$ \\
\hline 72 & 145 & $0.000 e+00$ & $2.009 e+04$ & 72 & 146 & $0.000 e+00$ & $5.247 e+04$ & 72 & 148 & $1.075 e-03$ & $1.296 \mathrm{e}+07$ \\
\hline 72 & 150 & $1.923 e-01$ & $9.960 e+08$ & 72 & 151 & $9.722 \mathrm{e}-04$ & $5.072 \mathrm{e}+06$ & 72 & 154 & $4.691 \mathrm{e}-03$ & $5.775 e+07$ \\
\hline 72 & 155 & $1.533 e-02$ & $8.159 \mathrm{e}+07$ & 72 & 158 & $2.353 e-03$ & $1.260 \mathrm{e}+07$ & 72 & 162 & $0.000 e+00$ & $5.664 e+03$ \\
\hline 72 & 171 & $6.338 e-02$ & $8.382 e+08$ & 72 & 172 & $7.675 e-01$ & $4.355 e+09$ & 72 & 173 & $1.919 \mathrm{e}-01$ & $1.536 \mathrm{e}+09$ \\
\hline 72 & 174 & $1.920 \mathrm{e}-02$ & $2.580 e+08$ & 72 & 175 & $0.000 e+00$ & $2.138 e+05$ & 72 & 176 & $0.000 e+00$ & $4.796 e+04$ \\
\hline 72 & 177 & $0.000 e+00$ & $3.336 \mathrm{e}+04$ & 72 & 178 & $0.000 e+00$ & $7.756 e+04$ & 72 & 180 & $2.549 \mathrm{e}-02$ & $2.113 e+08$ \\
\hline 72 & 181 & $9.664 \mathrm{e}-02$ & $5.730 e+08$ & 72 & 183 & $2.387 e-02$ & $1.998 e+08$ & 72 & 184 & $1.671 \mathrm{e}-02$ & $2.543 e+08$ \\
\hline 72 & 186 & $1.148 \mathrm{e}-01$ & $1.049 \mathrm{e}+09$ & 72 & 187 & $3.055 e-02$ & $2.809 e+08$ & 72 & 192 & $0.000 e+00$ & $1.363 e+04$ \\
\hline 72 & 193 & $0.000 e+00$ & $8.792 \mathrm{e}+03$ & 72 & 212 & $5.542 e-04$ & $8.133 e+06$ & 72 & 215 & $0.000 e+00$ & $5.023 e+04$ \\
\hline 72 & 216 & $0.000 e+00$ & $1.126 \mathrm{e}+05$ & 72 & 217 & $0.000 e+00$ & $1.230 \mathrm{e}+05$ & 72 & 218 & $0.000 e+00$ & $2.761 e+04$ \\
\hline 72 & 219 & $0.000 e+00$ & $1.090 \mathrm{e}+05$ & 72 & 220 & $0.000 e+00$ & $2.275 e+05$ & 72 & 221 & $0.000 e+00$ & $8.548 e+04$ \\
\hline 72 & 222 & $0.000 e+00$ & $1.803 e+05$ & 72 & 223 & $0.000 e+00$ & $2.363 e+05$ & 72 & 234 & $3.518 e-04$ & $1.691 \mathrm{e}+07$ \\
\hline 72 & 241 & $1.262 \mathrm{e}-03$ & $3.757 e+07$ & 72 & 277 & $9.817 e-04$ & $2.224 \mathrm{e}+07$ & 72 & 278 & $3.027 e-04$ & $9.605 e+06$ \\
\hline 73 & 90 & $1.638 \mathrm{e}-01$ & $3.449 \mathrm{e}+07$ & 73 & 91 & $8.085 e-02$ & $2.892 \mathrm{e}+07$ & 73 & 92 & $1.799 e+00$ & $4.351 e+08$ \\
\hline 73 & 95 & $6.505 e-02$ & $2.142 \mathrm{e}+07$ & 73 & 96 & $3.412 \mathrm{e}-02$ & $1.915 e+07$ & 73 & 107 & $8.397 e-03$ & $9.509 e+06$ \\
\hline 73 & 109 & $6.669 e-02$ & $8.976 \mathrm{e}+07$ & 73 & 114 & $0.000 e+00$ & $9.759 e+03$ & 73 & 125 & $2.405 e-01$ & $1.649 \mathrm{e}+09$ \\
\hline 73 & 126 & $3.372 \mathrm{e}-01$ & $2.386 \mathrm{e}+09$ & 73 & 128 & $1.500 e-01$ & $6.414 e+08$ & 73 & 129 & $3.548 e-02$ & $7.706 e+08$ \\
\hline
\end{tabular}


Table III. Ca IX Oscillator Strengths and Radiative Decay Rates for transitions involving levels higher than 4.

\begin{tabular}{|c|c|c|c|c|c|c|c|c|c|c|c|}
\hline $\begin{array}{l}\text { Low. } \\
\text { Lev . }\end{array}$ & $\begin{array}{l}\text { Upp } \\
\text { Lev }\end{array}$ & $\begin{array}{l}\text { Osc. } \\
\text { Str. }\end{array}$ & $\begin{array}{l}\text { Rad. } \\
\text { Rate }\end{array}$ & $\begin{array}{l}\text { Low. } \\
\text { Lev. }\end{array}$ & $\begin{array}{l}\text { Upp. } \\
\text { Lev. }\end{array}$ & $\begin{array}{l}\text { Osc. } \\
\text { Str. }\end{array}$ & $\begin{array}{l}\text { Rad. } \\
\text { Rate }\end{array}$ & $\begin{array}{l}\text { Low. } \\
\text { Lev. }\end{array}$ & $\begin{array}{l}\text { - Upp } \\
\text {. Lev }\end{array}$ & $\begin{array}{l}\text { Osc. } \\
\text { Str. }\end{array}$ & $\begin{array}{l}\text { Rad. } \\
\text { Rate }\end{array}$ \\
\hline$i$ & $j$ & $g f$ & $(1 / s)$ & $i$ & $j$ & $g f$ & $(1 / s)$ & i & j & $g f$ & $(1 / s)$ \\
\hline 73 & 130 & $1.347 e-01$ & $9.923 e+08$ & 73 & 132 & $6.319 e-02$ & $2.851 e+08$ & 73 & 133 & $1.947 e-01$ & $1.488 e+09$ \\
\hline 73 & 134 & $5.895 e-01$ & $2.846 e+09$ & 73 & 135 & $4.061 e-01$ & $1.037 e+10$ & 73 & 136 & $0.000 e+00$ & $1.177 e+04$ \\
\hline 73 & 140 & $0.000 e+00$ & $1.118 e+04$ & 73 & 143 & $0.000 e+00$ & $7.064 e+03$ & 73 & 148 & $1.384 \mathrm{e}-02$ & $1.660 \mathrm{e}+08$ \\
\hline 73 & 149 & $3.878 e-03$ & $2.791 e+07$ & 73 & 154 & $1.420 \mathrm{e}-01$ & $1.739 e+09$ & 73 & 156 & $0.000 e+00$ & $2.185 e+04$ \\
\hline 73 & 159 & $9.872 \mathrm{e}-03$ & $7.386 e+07$ & 73 & 171 & $7.048 e-03$ & $9.273 e+07$ & 73 & 173 & $2.408 \mathrm{e}-02$ & $1.917 e+08$ \\
\hline 73 & 174 & $1.173 e-02$ & $1.569 \mathrm{e}+08$ & 73 & 176 & $0.000 e+00$ & $2.620 \mathrm{e}+04$ & 73 & 177 & $0.000 e+00$ & $7.441 \mathrm{e}+03$ \\
\hline 73 & 178 & $0.000 e+00$ & $2.013 e+05$ & 73 & 180 & $3.912 \mathrm{e}-03$ & $3.227 e+07$ & 73 & 183 & $4.369 \mathrm{e}-01$ & $3.639 e+09$ \\
\hline 73 & 184 & $1.697 e-03$ & $2.571 \mathrm{e}+07$ & 73 & 185 & $8.978 e-03$ & $4.080 e+08$ & 73 & 187 & $6.857 e-02$ & $6.274 e+08$ \\
\hline 73 & 207 & $4.705 e-03$ & $2.686 \mathrm{e}+08$ & 73 & 214 & $0.000 e+00$ & $1.548 e+05$ & 73 & 215 & $0.000 e+00$ & $3.558 e+04$ \\
\hline 73 & 217 & $0.000 e+00$ & $2.115 e+04$ & 73 & 218 & $0.000 e+00$ & $4.389 e+04$ & 73 & 219 & $0.000 e+00$ & $2.821 e+04$ \\
\hline 73 & 221 & $0.000 e+00$ & $3.131 \mathrm{e}+04$ & 73 & 222 & $0.000 e+00$ & $6.561 e+03$ & 73 & 224 & $0.000 e+00$ & $1.566 e+05$ \\
\hline 73 & 225 & $0.000 e+00$ & $2.843 e+05$ & 73 & 233 & $4.656 \mathrm{e}-04$ & $2.197 e+07$ & 73 & 238 & $2.715 e-04$ & $8.048 e+06$ \\
\hline 73 & 239 & $1.076 \mathrm{e}-04$ & $1.596 \mathrm{e}+07$ & 73 & 280 & $8.632 \mathrm{e}-04$ & $2.748 e+07$ & 73 & 281 & $4.337 e-05$ & $6.925 e+06$ \\
\hline 74 & 82 & $2.022 \mathrm{e}-01$ & $1.258 e+07$ & 74 & 84 & $2.821 \mathrm{e}-01$ & $2.177 e+07$ & 74 & 87 & $1.605 e-01$ & $1.634 \mathrm{e}+07$ \\
\hline 74 & 96 & $3.772 \mathrm{e}-02$ & $2.047 e+07$ & 74 & 97 & $2.250 e-02$ & $1.000 e+07$ & 74 & 102 & $1.569 \mathrm{e}-01$ & $7.135 e+07$ \\
\hline 74 & 106 & $5.075 e-03$ & $9.377 e+06$ & 74 & 110 & $0.000 e+00$ & $2.691 \mathrm{e}+04$ & 74 & 111 & $0.000 e+00$ & $9.217 \mathrm{e}+03$ \\
\hline 74 & 114 & $0.000 e+00$ & $1.495 e+04$ & 74 & 121 & $0.000 e+00$ & $6.665 e+03$ & 74 & 122 & $0.000 e+00$ & $4.805 e+03$ \\
\hline 74 & 125 & $5.652 \mathrm{e}-02$ & $3.838 e+08$ & 74 & 126 & $3.394 e-01$ & $2.378 e+09$ & 74 & 127 & $0.000 e+00$ & $5.000 e+03$ \\
\hline 74 & 128 & $1.397 e-01$ & $5.918 e+08$ & 74 & 130 & $2.255 \mathrm{e}-01$ & $1.647 e+09$ & 74 & 131 & $9.424 \mathrm{e}-03$ & $2.964 e+07$ \\
\hline 74 & 132 & $5.978 e-02$ & $2.673 e+08$ & 74 & 133 & $1.517 \mathrm{e}-03$ & $1.149 e+07$ & 74 & 134 & $4.786 e-02$ & $2.291 \mathrm{e}+08$ \\
\hline 74 & 136 & $0.000 e+00$ & $2.832 \mathrm{e}+03$ & 74 & 137 & $0.000 e+00$ & $5.035 e+04$ & 74 & 138 & $0.000 e+00$ & $6.225 e+04$ \\
\hline 74 & 139 & $0.000 e+00$ & $1.450 \mathrm{e}+04$ & 74 & 140 & $0.000 e+00$ & $2.115 e+04$ & 74 & 141 & $0.000 e+00$ & $1.888 e+04$ \\
\hline 74 & 142 & $0.000 e+00$ & $3.051 \mathrm{e}+03$ & 74 & 143 & $0.000 e+00$ & $5.598 e+03$ & 74 & 144 & $9.489 e-02$ & $4.673 e+08$ \\
\hline 74 & 146 & $0.000 e+00$ & $2.860 \mathrm{e}+04$ & 74 & 147 & $0.000 e+00$ & $1.243 e+04$ & 74 & 148 & $4.349 e-03$ & $5.177 e+07$ \\
\hline 74 & 149 & $7.224 \mathrm{e}-02$ & $5.163 e+08$ & 74 & 150 & $1.116 \mathrm{e}-02$ & $5.707 e+07$ & 74 & 151 & $1.479 \mathrm{e}-01$ & $7.622 \mathrm{e}+08$ \\
\hline 74 & 154 & $1.779 \mathrm{e}-02$ & $2.163 e+08$ & 74 & 155 & $4.316 \mathrm{e}-01$ & $2.268 e+09$ & 74 & 156 & $0.000 e+00$ & $1.331 \mathrm{e}+04$ \\
\hline 74 & 157 & $0.000 e+00$ & $2.940 \mathrm{e}+05$ & 74 & 158 & $1.013 e+00$ & $5.354 e+09$ & 74 & 159 & $3.981 \mathrm{e}-01$ & $2.958 e+09$ \\
\hline 74 & 162 & $0.000 e+00$ & $2.725 e+04$ & 74 & 163 & $0.000 e+00$ & $1.254 \mathrm{e}+05$ & 74 & 164 & $2.430 \mathrm{e}+00$ & $1.323 e+10$ \\
\hline 74 & 167 & $0.000 e+00$ & $3.457 e+05$ & 74 & 172 & $2.494 \mathrm{e}-02$ & $1.398 e+08$ & 74 & 173 & $1.183 e-01$ & $9.353 e+08$ \\
\hline 74 & 174 & $4.731 \mathrm{e}-03$ & $6.283 e+07$ & 74 & 175 & $0.000 e+00$ & $5.665 e+03$ & 74 & 176 & $0.000 e+00$ & $7.448 e+04$ \\
\hline 74 & 177 & $0.000 e+00$ & $2.376 \mathrm{e}+04$ & 74 & 178 & $0.000 e+00$ & $1.195 e+04$ & 74 & 180 & $1.721 \mathrm{e}-01$ & $1.410 \mathrm{e}+09$ \\
\hline 74 & 181 & $2.544 \mathrm{e}-01$ & $1.491 \mathrm{e}+09$ & 74 & 183 & $2.967 e-02$ & $2.454 e+08$ & 74 & 184 & $4.938 e-03$ & $7.431 \mathrm{e}+07$ \\
\hline 74 & 186 & $5.450 e-03$ & $4.924 \mathrm{e}+07$ & 74 & 187 & $3.434 \mathrm{e}-02$ & $3.122 \mathrm{e}+08$ & 74 & 190 & $0.000 e+00$ & $1.950 e+05$ \\
\hline 74 & 192 & $0.000 e+00$ & $9.139 e+03$ & 74 & 210 & $7.165 e-04$ & $2.431 e+07$ & 74 & 211 & $4.496 e-04$ & $9.159 e+06$ \\
\hline 74 & 227 & $3.307 e-04$ & $1.546 \mathrm{e}+07$ & 74 & 228 & $2.731 \mathrm{e}-04$ & $7.661 e+06$ & 74 & 233 & $1.658 \mathrm{e}-04$ & $7.796 e+06$ \\
\hline 74 & 238 & $4.034 \mathrm{e}-04$ & $1.192 \mathrm{e}+07$ & 74 & 240 & $1.482 \mathrm{e}-04$ & $7.304 e+06$ & 74 & 244 & $0.000 e+00$ & $2.480 e+04$ \\
\hline 74 & 247 & $0.000 e+00$ & $5.987 e+03$ & 74 & 258 & $1.216 \mathrm{e}-03$ & $2.681 \mathrm{e}+07$ & 74 & 261 & $2.581 \mathrm{e}-04$ & $5.723 e+06$ \\
\hline 74 & 267 & $1.880 \mathrm{e}-04$ & $5.846 e+06$ & 75 & 83 & $4.414 \mathrm{e}-01$ & $2.445 e+07$ & 75 & 88 & $2.415 e-01$ & $1.948 e+07$ \\
\hline 75 & 93 & $2.186 \mathrm{e}-01$ & $3.072 \mathrm{e}+07$ & 75 & 95 & $4.329 e-02$ & $1.354 \mathrm{e}+07$ & 75 & 103 & $1.690 \mathrm{e}-01$ & $5.935 e+07$ \\
\hline 75 & 105 & $2.083 e-01$ & $9.115 \mathrm{e}+07$ & 75 & 107 & $6.902 \mathrm{e}-03$ & $7.604 e+06$ & 75 & 111 & $0.000 e+00$ & $1.867 e+04$ \\
\hline 75 & 113 & $0.000 e+00$ & $1.726 \mathrm{e}+04$ & 75 & 123 & $0.000 e+00$ & $6.140 e+03$ & 75 & 128 & $4.711 \mathrm{e}-01$ & $1.986 e+09$ \\
\hline 75 & 131 & $2.701 \mathrm{e}-01$ & $8.457 e+08$ & 75 & 132 & $2.436 \mathrm{e}-01$ & $1.084 e+09$ & 75 & 134 & $2.041 \mathrm{e}-01$ & $9.724 e+08$ \\
\hline 75 & 136 & $0.000 e+00$ & $4.016 \mathrm{e}+04$ & 75 & 137 & $0.000 e+00$ & $9.304 e+03$ & 75 & 138 & $0.000 e+00$ & $1.766 e+04$ \\
\hline 75 & 139 & $0.000 e+00$ & $3.240 \mathrm{e}+04$ & 75 & 140 & $0.000 e+00$ & $1.978 e+04$ & 75 & 141 & $0.000 e+00$ & $1.349 e+04$ \\
\hline
\end{tabular}


Table III. Ca IX Oscillator Strengths and Radiative Decay Rates for transitions involving levels higher than 4.

\begin{tabular}{|c|c|c|c|c|c|c|c|c|c|c|c|}
\hline $\begin{array}{l}\text { Low. } \\
\text { Lev. }\end{array}$ & $\begin{array}{l}\text { - Upp } \\
\text {. Lev }\end{array}$ & $\begin{array}{l}\text { Osc. } \\
\text { Str. }\end{array}$ & $\begin{array}{l}\text { Rad. } \\
\text { Rate }\end{array}$ & $\begin{array}{l}\text { Low. } \\
\text { Lev. }\end{array}$ & $\begin{array}{l}\text { - Upp } \\
\text { Lev }\end{array}$ & $\begin{array}{l}\text { Osc. } \\
\text { Str. }\end{array}$ & $\begin{array}{l}\text { Rad. } \\
\text { Rate }\end{array}$ & $\begin{array}{l}\text { Low. } \\
\text { Lev. }\end{array}$ & $\begin{array}{l}\text { - Upp } \\
\text { - Lev }\end{array}$ & $\begin{array}{l}\text { Osc. } \\
\text { Str. }\end{array}$ & $\begin{array}{l}\text { Rad. } \\
\text { Rate }\end{array}$ \\
\hline$i$ & $j$ & $g f$ & $(1 / s)$ & i & $j$ & $g f$ & $(1 / s)$ & $i$ & j & $g f$ & $(1 / s)$ \\
\hline 75 & 142 & $0.000 e+00$ & $4.272 \mathrm{e}+04$ & 75 & 143 & $0.000 e+00$ & $2.040 \mathrm{e}+04$ & 75 & 144 & $2.539 \mathrm{e}-01$ & $1.246 \mathrm{e}+09$ \\
\hline 75 & 145 & $0.000 e+00$ & $2.833 e+04$ & 75 & 147 & $0.000 \mathrm{e}+00$ & $1.913 e+04$ & 75 & 149 & $1.258 \mathrm{e}-03$ & $8.955 e+06$ \\
\hline 75 & 150 & $2.260 \mathrm{e}-01$ & $1.151 \mathrm{e}+09$ & 75 & 151 & $1.765 e-02$ & $9.060 e+07$ & 75 & 152 & $2.998 e-01$ & $1.201 \mathrm{e}+09$ \\
\hline 75 & 155 & $2.529 \mathrm{e}-01$ & $1.324 \mathrm{e}+09$ & 75 & 156 & $0.000 e+00$ & $5.593 e+03$ & 75 & 157 & $0.000 e+00$ & $2.985 e+04$ \\
\hline 75 & 158 & $1.077 e-01$ & $5.676 e+08$ & 75 & 159 & $3.258 e-02$ & $2.412 \mathrm{e}+08$ & 75 & 160 & $0.000 e+00$ & $3.386 e+05$ \\
\hline 75 & 161 & $1.128 \mathrm{e}+00$ & $4.643 e+09$ & 75 & 162 & $0.000 e+00$ & $1.168 \mathrm{e}+05$ & 75 & 163 & $0.000 e+00$ & $2.250 \mathrm{e}+04$ \\
\hline 75 & 164 & $3.451 \mathrm{e}-01$ & $1.872 \mathrm{e}+09$ & 75 & 165 & $2.670 \mathrm{e}+00$ & $1.131 e+10$ & 75 & 167 & $0.000 e+00$ & $6.071 e+04$ \\
\hline 75 & 168 & $0.000 e+00$ & $2.602 \mathrm{e}+05$ & 75 & 170 & $0.000 e+00$ & $1.255 e+05$ & 75 & 172 & $2.666 \mathrm{e}-01$ & $1.489 e+09$ \\
\hline 75 & 173 & $2.538 \mathrm{e}-02$ & $2.000 e+08$ & 75 & 175 & $0.000 \mathrm{e}+00$ & $7.858 e+04$ & 75 & 176 & $0.000 e+00$ & $2.544 e+04$ \\
\hline 75 & 177 & $0.000 e+00$ & $6.649 e+03$ & 75 & 178 & $0.000 e+00$ & $1.643 e+04$ & 75 & 179 & $8.390 e-02$ & $3.773 e+08$ \\
\hline 75 & 180 & $1.208 \mathrm{e}-02$ & $9.862 \mathrm{e}+07$ & 75 & 181 & $7.978 \mathrm{e}-02$ & $4.659 e+08$ & 75 & 182 & $6.020 e-01$ & $2.739 e+09$ \\
\hline 75 & 186 & $1.633 e-02$ & $1.471 \mathrm{e}+08$ & 75 & 187 & $7.672 \mathrm{e}-03$ & $6.953 e+07$ & 75 & 188 & $5.576 e-01$ & $2.855 e+09$ \\
\hline 75 & 190 & $0.000 e+00$ & $1.673 e+04$ & 75 & 194 & $0.000 e+00$ & $1.521 e+05$ & 75 & 211 & $8.258 e-04$ & $1.679 \mathrm{e}+07$ \\
\hline 75 & 212 & $8.304 \mathrm{e}-04$ & $1.207 e+07$ & 75 & 213 & $1.515 \mathrm{e}-03$ & $3.126 e+07$ & 75 & 228 & $3.650 e-04$ & $2 e+07$ \\
\hline 75 & 229 & $5.252 e-04$ & $1.051 \mathrm{e}+07$ & 75 & 238 & $4.841 \mathrm{e}-04$ & $1.427 \mathrm{e}+07$ & 75 & 241 & $1.024 \mathrm{e}-03$ & $3.024 e+07$ \\
\hline 75 & 245 & $0.000 e+00$ & $1.893 e+04$ & 75 & 259 & $1.240 \mathrm{e}-03$ & $2.124 \mathrm{e}+07$ & 75 & 268 & $2.534 \mathrm{e}-04$ & $5.620 e+06$ \\
\hline 75 & 269 & $3.245 e-04$ & $5.598 e+06$ & 75 & 282 & $0.000 e+00$ & $2.417 e+04$ & 76 & 85 & $1.075 e+00$ & $4.772 e+07$ \\
\hline 76 & 94 & $6.964 \mathrm{e}-02$ & $1.359 \mathrm{e}+07$ & 76 & 103 & $1.724 \mathrm{e}-02$ & $5.603 e+06$ & 76 & 104 & $3.335 e-01$ & $8.930 e+07$ \\
\hline 76 & 108 & $8.733 e-03$ & $6.516 e+06$ & 76 & 112 & $0.000 e+00$ & $7.036 e+03$ & 76 & 113 & $0.000 e+00$ & $3.256 e+04$ \\
\hline 76 & 123 & $0.000 e+00$ & $1.425 \mathrm{e}+04$ & 76 & 131 & $1.534 \mathrm{e}+00$ & $4.665 e+09$ & 76 & 139 & $0.000 e+00$ & $6.065 e+03$ \\
\hline 76 & 140 & $0.000 e+00$ & $9.039 e+03$ & 76 & 141 & $0.000 \mathrm{e}+00$ & $9.016 e+04$ & 76 & 142 & $0.000 e+00$ & $1.132 \mathrm{e}+05$ \\
\hline 76 & 143 & $0.000 e+00$ & $1.475 \mathrm{e}+04$ & 76 & 150 & $6.637 \mathrm{e}-02$ & $3.306 e+08$ & 76 & 151 & $8.907 e-04$ & $4.470 e+06$ \\
\hline 76 & 152 & $8.223 e-02$ & $3.220 \mathrm{e}+08$ & 76 & 153 & $1.187 e+00$ & $3.820 e+09$ & 76 & 155 & $3.452 \mathrm{e}-03$ & $1.767 e+07$ \\
\hline 76 & 158 & $1.752 \mathrm{e}-02$ & $9.029 \mathrm{e}+07$ & 76 & 161 & $7.386 \mathrm{e}-02$ & $2.974 \mathrm{e}+08$ & 76 & 162 & $0.000 e+00$ & $6.107 e+03$ \\
\hline 76 & 164 & $3.172 \mathrm{e}-02$ & $1.683 e+08$ & 76 & 165 & $1.111 \mathrm{e}+00$ & $4.607 e+09$ & 76 & 166 & $0.000 e+00$ & $7.710 e+05$ \\
\hline 76 & 167 & $0.000 e+00$ & $1.728 e+04$ & 76 & 168 & $0.000 e+00$ & $2.123 e+05$ & 76 & 169 & $6.425 e+00$ & $2.195 \mathrm{e}+10$ \\
\hline 76 & 170 & $0.000 e+00$ & $1.452 \mathrm{e}+04$ & 76 & 172 & $1.124 \mathrm{e}-02$ & $6.144 \mathrm{e}+07$ & 76 & 175 & $0.000 e+00$ & $1.890 \mathrm{e}+04$ \\
\hline 76 & 179 & $9.610 e-03$ & $4.230 \mathrm{e}+07$ & 76 & 181 & $3.016 \mathrm{e}-02$ & $1.724 \mathrm{e}+08$ & 76 & 182 & $4.450 e-01$ & $1.983 e+09$ \\
\hline 76 & 194 & $0.000 e+00$ & $2.944 \mathrm{e}+04$ & 76 & 195 & $0.000 e+00$ & $2.948 e+05$ & 76 & 212 & $2.441 \mathrm{e}-03$ & $3.500 e+07$ \\
\hline 76 & 229 & $1.337 e-03$ & $2.645 \mathrm{e}+07$ & 76 & 245 & $0.000 e+00$ & $3.254 \mathrm{e}+03$ & 76 & 246 & $0.000 e+00$ & $3.313 e+04$ \\
\hline 76 & 249 & $0.000 e+00$ & $7.445 e+03$ & 76 & 260 & $2.562 \mathrm{e}-03$ & $3.552 \mathrm{e}+07$ & 76 & 269 & $2.706 \mathrm{e}-04$ & $4.617 \mathrm{e}+06$ \\
\hline 77 & 93 & $8.094 \mathrm{e}-01$ & $9.838 e+07$ & 77 & 97 & $2.628 \mathrm{e}-02$ & $1.032 \mathrm{e}+07$ & 77 & 103 & $5.805 e-02$ & $1.862 \mathrm{e}+07$ \\
\hline 77 & 105 & $7.470 \mathrm{e}-01$ & $3.015 \mathrm{e}+08$ & 77 & 109 & $9.427 e-03$ & $1.162 \mathrm{e}+07$ & 77 & 114 & $0.000 e+00$ & $4.462 e+04$ \\
\hline 77 & 127 & $0.000 e+00$ & $1.440 \mathrm{e}+04$ & 77 & 128 & $1.449 \mathrm{e}-01$ & $5.901 e+08$ & 77 & 131 & $2.824 \mathrm{e}-02$ & $8.545 e+07$ \\
\hline 77 & 132 & $6.189 e-02$ & $2.664 \mathrm{e}+08$ & 77 & 134 & $9.015 e-01$ & $4.158 e+09$ & 77 & 136 & $0.000 e+00$ & $1.695 \mathrm{e}+04$ \\
\hline 77 & 139 & $0.000 e+00$ & $7.432 \mathrm{e}+03$ & 77 & 140 & $0.000 e+00$ & $6.387 e+04$ & 77 & 142 & $0.000 e+00$ & $5.355 e+03$ \\
\hline 77 & 144 & $7.991 \mathrm{e}-01$ & $3.816 e+09$ & 77 & 145 & $0.000 e+00$ & $4.148 e+03$ & 77 & 147 & $0.000 e+00$ & $1.221 \mathrm{e}+05$ \\
\hline 77 & 149 & $1.615 \mathrm{e}-02$ & $1.120 \mathrm{e}+08$ & 77 & 150 & $1.715 e-03$ & $8.512 \mathrm{e}+06$ & 77 & 151 & $2.364 \mathrm{e}-02$ & $1.182 \mathrm{e}+08$ \\
\hline 77 & 152 & $1.632 \mathrm{e}-01$ & $6.365 e+08$ & 77 & 155 & $2.427 \mathrm{e}-02$ & $1.238 \mathrm{e}+08$ & 77 & 156 & $0.000 \mathrm{e}+00$ & $1.988 e+04$ \\
\hline 77 & 159 & $1.284 \mathrm{e}-02$ & $9.263 e+07$ & 77 & 161 & $4.953 e-01$ & $1.987 e+09$ & 77 & 162 & $0.000 e+00$ & $1.144 \mathrm{e}+04$ \\
\hline 77 & 163 & $0.000 e+00$ & $5.604 e+03$ & 77 & 164 & $4.552 \mathrm{e}-01$ & $2.406 e+09$ & 77 & 165 & $4.863 e-01$ & $2.008 e+09$ \\
\hline 77 & 167 & $0.000 e+00$ & $1.579 \mathrm{e}+05$ & 77 & 168 & $0.000 \mathrm{e}+00$ & $1.561 e+05$ & 77 & 170 & $0.000 e+00$ & $7.020 e+05$ \\
\hline 77 & 173 & $1.680 \mathrm{e}-03$ & $1.291 \mathrm{e}+07$ & 77 & 175 & $0.000 e+00$ & $4.665 e+03$ & 77 & 176 & $0.000 e+00$ & $9.361 e+03$ \\
\hline 77 & 179 & $1.785 e+00$ & $7.829 \mathrm{e}+09$ & 77 & 180 & $9.116 \mathrm{e}-03$ & $7.262 \mathrm{e}+07$ & 77 & 181 & $1.018 \mathrm{e}-01$ & $5.801 e+08$ \\
\hline
\end{tabular}


Table III. Ca IX Oscillator Strengths and Radiative Decay Rates for transitions involving levels higher than 4.

\begin{tabular}{|c|c|c|c|c|c|c|c|c|c|c|c|}
\hline $\begin{array}{l}\text { Low. } \\
\text { Lev. }\end{array}$ & $\begin{array}{l}\text { Upp } \\
\text { Lev }\end{array}$ & $\begin{array}{l}\text { Osc. } \\
\text { Str. }\end{array}$ & $\begin{array}{l}\text { Rad. } \\
\text { Rate }\end{array}$ & $\begin{array}{l}\text { Low. } \\
\text { Lev. }\end{array}$ & $\begin{array}{l}\text { Upp } \\
\text {. Lev }\end{array}$ & $\begin{array}{l}\text { Osc. } \\
\text { Str. }\end{array}$ & $\begin{array}{l}\text { Rad. } \\
\text { Rate }\end{array}$ & $\begin{array}{l}\text { Low. } \\
\text { Lev. }\end{array}$ & $\begin{array}{l}\text { - Upp } \\
\text {. Lev }\end{array}$ & $\begin{array}{l}\text { Osc. } \\
\text { Str. }\end{array}$ & $\begin{array}{l}\text { Rad. } \\
\text { Rate }\end{array}$ \\
\hline$i$ & $j$ & $g f$ & $(1 / s)$ & $i$ & $j$ & $g f$ & $(1 / s)$ & $i$ & j & $g f$ & $(1 / s)$ \\
\hline 77 & 182 & $7.850 e-03$ & $3.485 e+07$ & 77 & 187 & $9.988 e-03$ & $8.841 e+07$ & 77 & 188 & $1.859 e+00$ & $9.302 e+09$ \\
\hline 77 & 190 & $0.000 e+00$ & $1.105 e+04$ & 77 & 194 & $0.000 e+00$ & $7.645 e+04$ & 77 & 209 & $0.000 e+00$ & $6.276 e+04$ \\
\hline 77 & 211 & $3.207 e-04$ & $6.417 e+06$ & 77 & 213 & $5.455 e-03$ & $1.108 e+08$ & 77 & 228 & $2.598 e-04$ & $7.176 e+06$ \\
\hline 77 & 238 & $2.438 e-03$ & $7.096 \mathrm{e}+07$ & 77 & 241 & $2.741 \mathrm{e}-04$ & $7.993 e+06$ & 77 & 245 & $0.000 e+00$ & $8.287 e+03$ \\
\hline 77 & 259 & $4.948 e-04$ & $8.368 e+06$ & 77 & 265 & $8.276 e-04$ & $1.408 e+07$ & 77 & 275 & $0.000 e+00$ & $1.274 \mathrm{e}+04$ \\
\hline 77 & 282 & $0.000 e+00$ & $7.921 \mathrm{e}+04$ & 78 & 94 & $1.581 \mathrm{e}+00$ & $1.915 e+08$ & 78 & 95 & $2.968 e-01$ & $5.193 e+07$ \\
\hline 78 & 110 & $0.000 e+00$ & $8.102 e+03$ & 78 & 125 & $2.595 e-03$ & $1.517 e+07$ & 78 & 128 & $5.204 e-02$ & $1.903 e+08$ \\
\hline 78 & 130 & $1.279 \mathrm{e}-02$ & $8.079 e+07$ & 78 & 131 & $2.320 \mathrm{e}-01$ & $6.318 e+08$ & 78 & 132 & $1.118 e-02$ & $4.335 e+07$ \\
\hline 78 & 133 & $1.451 \mathrm{e}-01$ & $9.541 e+08$ & 78 & 137 & $0.000 e+00$ & $2.088 e+04$ & 78 & 138 & $0.000 e+00$ & $1.431 \mathrm{e}+04$ \\
\hline 78 & 139 & $0.000 e+00$ & $2.058 e+04$ & 78 & 140 & $0.000 e+00$ & $6.340 e+03$ & 78 & 141 & $0.000 e+00$ & $6.023 e+03$ \\
\hline 78 & 142 & $0.000 e+00$ & $1.145 \mathrm{e}+04$ & 78 & 143 & $0.000 e+00$ & $7.402 e+03$ & 78 & 144 & $2.804 e-03$ & $1.232 e+07$ \\
\hline 78 & 145 & $0.000 e+00$ & $3.882 e+04$ & 78 & 146 & $0.000 e+00$ & $7.501 e+04$ & 78 & 148 & $3.605 e-03$ & $3.836 e+07$ \\
\hline 78 & 149 & $7.631 \mathrm{e}-02$ & $4.875 e+08$ & 78 & 150 & $7.167 e-01$ & $3.276 e+09$ & 78 & 151 & $5.935 e-04$ & $2.735 e+06$ \\
\hline 78 & 155 & $2.621 \mathrm{e}-02$ & $1.233 e+08$ & 78 & 158 & $6.844 \mathrm{e}-02$ & $3.241 e+08$ & 78 & 159 & $9.220 \mathrm{e}-03$ & $6.137 e+07$ \\
\hline 78 & 162 & $0.000 e+00$ & $2.353 e+04$ & 78 & 164 & $8.069 e-03$ & $3.940 e+07$ & 78 & 171 & $3.306 e-01$ & $3.881 e+09$ \\
\hline 78 & 172 & $1.711 \mathrm{e}+00$ & $8.619 e+09$ & 78 & 173 & $3.425 e-01$ & $2.435 e+09$ & 78 & 174 & $2.793 e-02$ & $3.336 e+08$ \\
\hline 78 & 175 & $0.000 e+00$ & $4.330 e+05$ & 78 & 176 & $0.000 e+00$ & $8.605 e+04$ & 78 & 177 & $0.000 e+00$ & $4.038 e+04$ \\
\hline 78 & 178 & $0.000 e+00$ & $9.143 e+04$ & 78 & 180 & $1.441 \mathrm{e}-02$ & $1.064 \mathrm{e}+08$ & 78 & 181 & $3.398 e-02$ & $1.794 \mathrm{e}+08$ \\
\hline 78 & 183 & $1.893 e-02$ & $1.412 \mathrm{e}+08$ & 78 & 184 & $1.360 \mathrm{e}-01$ & $1.852 \mathrm{e}+09$ & 78 & 186 & $4.550 e-01$ & $3.722 \mathrm{e}+09$ \\
\hline 78 & 187 & $2.958 \mathrm{e}-02$ & $2.436 \mathrm{e}+08$ & 78 & 192 & $0.000 e+00$ & $4.146 e+04$ & 78 & 193 & $0.000 e+00$ & $1.833 e+04$ \\
\hline 78 & 215 & $0.000 e+00$ & $2.034 \mathrm{e}+04$ & 78 & 216 & $0.000 e+00$ & $4.519 e+04$ & 78 & 217 & $0.000 e+00$ & $4.843 e+04$ \\
\hline 78 & 218 & $0.000 e+00$ & $1.149 \mathrm{e}+04$ & 78 & 219 & $0.000 e+00$ & $4.754 e+04$ & 78 & 220 & $0.000 e+00$ & $1.092 \mathrm{e}+05$ \\
\hline 78 & 221 & $0.000 e+00$ & $5.080 e+04$ & 78 & 222 & $0.000 e+00$ & $1.003 e+05$ & 78 & 223 & $0.000 e+00$ & $1.281 \mathrm{e}+05$ \\
\hline 78 & 234 & $2.058 e-03$ & $9.306 e+07$ & 78 & 240 & $1.591 \mathrm{e}-04$ & $7.428 e+06$ & 78 & 241 & $1.102 \mathrm{e}-03$ & $3.088 e+07$ \\
\hline 78 & 249 & $0.000 e+00$ & $6.374 \mathrm{e}+03$ & 78 & 274 & $0.000 e+00$ & $9.103 e+03$ & 78 & 277 & $3.191 \mathrm{e}-03$ & $6.817 \mathrm{e}+07$ \\
\hline 78 & 278 & $6.508 e-04$ & $1.948 \mathrm{e}+07$ & 79 & 95 & $7.837 e-01$ & $1.321 \mathrm{e}+08$ & 79 & 96 & $2.703 e-01$ & $7.836 e+07$ \\
\hline 79 & 125 & $1.087 e-02$ & $6.303 e+07$ & 79 & 126 & $3.623 e-02$ & $2.173 e+08$ & 79 & 128 & $8.186 e-02$ & $2.969 \mathrm{e}+08$ \\
\hline 79 & 132 & $4.236 \mathrm{e}-03$ & $1.629 \mathrm{e}+07$ & 79 & 133 & $1.125 \mathrm{e}-01$ & $7.335 e+08$ & 79 & 134 & $1.017 e-02$ & $4.208 e+07$ \\
\hline 79 & 135 & $1.212 \mathrm{e}-03$ & $2.663 e+07$ & 79 & 136 & $0.000 e+00$ & $3.793 e+03$ & 79 & 138 & $0.000 e+00$ & $1.723 e+04$ \\
\hline 79 & 141 & $0.000 e+00$ & $1.933 e+04$ & 79 & 143 & $0.000 e+00$ & $2.867 e+04$ & 79 & 145 & $0.000 e+00$ & $2.850 e+04$ \\
\hline 79 & 148 & $9.321 \mathrm{e}-02$ & $9.858 e+08$ & 79 & 149 & $3.898 e-01$ & $2.475 e+09$ & 79 & 154 & $9.840 e-03$ & $1.064 \mathrm{e}+08$ \\
\hline 79 & 159 & $3.153 e-02$ & $2.086 e+08$ & 79 & 163 & $0.000 e+00$ & $1.049 e+04$ & 79 & 171 & $2.001 \mathrm{e}-01$ & $2.336 e+09$ \\
\hline 79 & 173 & $9.993 e-01$ & $7.062 e+09$ & 79 & 174 & $3.872 \mathrm{e}-01$ & $4.597 e+09$ & 79 & 176 & $0.000 e+00$ & $2.205 e+05$ \\
\hline 79 & 177 & $0.000 e+00$ & $2.553 e+05$ & 79 & 178 & $0.000 e+00$ & $9.246 e+04$ & 79 & 180 & $2.732 \mathrm{e}-02$ & $2.005 e+08$ \\
\hline 79 & 184 & $1.043 e-01$ & $1.414 \mathrm{e}+09$ & 79 & 185 & $1.195 \mathrm{e}-01$ & $4.858 e+09$ & 79 & 186 & $1.713 e-01$ & $1.394 \mathrm{e}+09$ \\
\hline 79 & 191 & $0.000 e+00$ & $2.860 \mathrm{e}+04$ & 79 & 193 & $0.000 e+00$ & $3.301 e+04$ & 79 & 207 & $1.459 e-03$ & $7.545 e+07$ \\
\hline 79 & 214 & $0.000 e+00$ & $7.106 e+03$ & 79 & 215 & $0.000 e+00$ & $5.990 e+04$ & 79 & 217 & $0.000 e+00$ & $3.641 \mathrm{e}+04$ \\
\hline 79 & 218 & $0.000 e+00$ & $4.038 e+04$ & 79 & 219 & $0.000 e+00$ & $4.970 \mathrm{e}+04$ & 79 & 221 & $0.000 e+00$ & $4.764 e+04$ \\
\hline 79 & 222 & $0.000 \mathrm{e}+00$ & $2.536 \mathrm{e}+04$ & 79 & 234 & $1.240 \mathrm{e}-03$ & $5.589 e+07$ & 79 & 239 & $2.004 \mathrm{e}-04$ & $2.799 \mathrm{e}+07$ \\
\hline 79 & 240 & $2.936 \mathrm{e}-04$ & $1.367 \mathrm{e}+07$ & 79 & 241 & $3.225 e-04$ & $9.011 e+06$ & 79 & 278 & $1.825 \mathrm{e}-03$ & $5.445 e+07$ \\
\hline 79 & 279 & $6.714 \mathrm{e}-04$ & $3.341 \mathrm{e}+07$ & 79 & 281 & $1.324 \mathrm{e}-04$ & $1.994 e+07$ & 80 & 96 & $3.376 e-01$ & $9.492 \mathrm{e}+07$ \\
\hline 80 & 125 & $2.007 e-02$ & $1.156 e+08$ & 80 & 126 & $1.474 \mathrm{e}-02$ & $8.783 e+07$ & 80 & 130 & $7.783 e-03$ & $4.848 e+07$ \\
\hline 80 & 133 & $4.120 e-02$ & $2.670 \mathrm{e}+08$ & 80 & 140 & $0.000 e+00$ & $9.821 e+03$ & 80 & 143 & $0.000 e+00$ & $2.079 e+04$ \\
\hline 80 & 148 & $1.641 \mathrm{e}-01$ & $1.727 e+09$ & 80 & 154 & $1.177 \mathrm{e}-03$ & $1.266 \mathrm{e}+07$ & 80 & 171 & $6.932 \mathrm{e}-02$ & $8.053 e+08$ \\
\hline
\end{tabular}


Table III. Ca IX Oscillator Strengths and Radiative Decay Rates for transitions involving levels higher than 4.

\begin{tabular}{|c|c|c|c|c|c|c|c|c|c|c|c|}
\hline $\begin{array}{l}\text { Low. } \\
\text { Lev. }\end{array}$ & $\begin{array}{l}\text { Upp } \\
\text { Lev }\end{array}$ & $\begin{array}{l}\text { Osc. } \\
\text { Str. }\end{array}$ & $\begin{array}{l}\text { Rad. } \\
\text { Rate }\end{array}$ & $\begin{array}{l}\text { Low. } \\
\text { Lev. }\end{array}$ & $\begin{array}{l}\text { - Upp } \\
\text { Lev }\end{array}$ & $\begin{array}{l}\text { Osc. } \\
\text { Str. }\end{array}$ & $\begin{array}{l}\text { Rad. } \\
\text { Rate }\end{array}$ & $\begin{array}{l}\text { Low. } \\
\text { Lev. }\end{array}$ & $\begin{array}{l}\text { - Upp } \\
\text { - Lev }\end{array}$ & $\begin{array}{l}\text { Osc. } \\
\text { Str. }\end{array}$ & $\begin{array}{l}\text { Rad. } \\
\text { Rate }\end{array}$ \\
\hline$i$ & $j$ & $g f$ & $(1 / s)$ & $i$ & $j$ & $g f$ & $(1 / s)$ & $i$ & j & $g f$ & $(1 / s)$ \\
\hline 80 & 174 & $5.017 e-01$ & $5.929 e+09$ & 80 & 177 & $0.000 \mathrm{e}+00$ & $2.443 e+05$ & 80 & 184 & $1.363 e-01$ & $1.839 e+09$ \\
\hline 80 & 191 & $0.000 e+00$ & $2.797 e+04$ & 80 & 216 & $0.000 \mathrm{e}+00$ & $3.162 \mathrm{e}+04$ & 80 & 218 & $0.000 e+00$ & $3.165 e+04$ \\
\hline 80 & 221 & $0.000 e+00$ & $1.886 e+04$ & 80 & 234 & $4.357 e-04$ & $1.959 \mathrm{e}+07$ & 80 & 240 & $3.139 e-04$ & $1.458 \mathrm{e}+07$ \\
\hline 80 & 279 & $8.817 e-04$ & $4.377 \mathrm{e}+07$ & 81 & 92 & $2.048 e-01$ & $1.394 \mathrm{e}+07$ & 81 & 97 & $8.368 e-01$ & $1.660 e+08$ \\
\hline 81 & 109 & $2.768 \mathrm{e}-02$ & $2.390 \mathrm{e}+07$ & 81 & 114 & $0.000 \mathrm{e}+00$ & $8.956 e+03$ & 81 & 125 & $8.832 e-03$ & $4.753 e+07$ \\
\hline 81 & 126 & $4.487 e-03$ & $2.502 \mathrm{e}+07$ & 81 & 128 & $6.941 \mathrm{e}-03$ & $2.342 \mathrm{e}+07$ & 81 & 129 & $6.001 \mathrm{e}-04$ & $1.030 e+07$ \\
\hline 81 & 133 & $1.251 \mathrm{e}-03$ & $7.609 \mathrm{e}+06$ & 81 & 134 & $4.701 \mathrm{e}-02$ & $1.818 e+08$ & 81 & 135 & $1.185 e-01$ & $2.438 e+09$ \\
\hline 81 & 136 & $0.000 e+00$ & $6.548 e+03$ & 81 & 140 & $0.000 \mathrm{e}+00$ & $5.176 \mathrm{e}+03$ & 81 & 147 & $0.000 e+00$ & $4.996 e+04$ \\
\hline 81 & 148 & $7.966 e-04$ & $7.978 e+06$ & 81 & 149 & $4.575 e-03$ & $2.751 \mathrm{e}+07$ & 81 & 154 & $7.273 e-01$ & $7.453 e+09$ \\
\hline 81 & 156 & $0.000 e+00$ & $9.568 e+04$ & 81 & 159 & $5.983 e-03$ & $3.753 e+07$ & 81 & 163 & $0.000 e+00$ & $8.926 e+03$ \\
\hline 81 & 171 & $7.339 \mathrm{e}-03$ & $8.134 \mathrm{e}+07$ & 81 & 174 & $5.133 e-03$ & $5.789 e+07$ & 81 & 176 & $0.000 e+00$ & $1.086 e+05$ \\
\hline 81 & 178 & $0.000 e+00$ & $3.021 \mathrm{e}+05$ & 81 & 180 & $4.380 e-02$ & $3.055 e+08$ & 81 & 183 & $9.886 e-01$ & $6.969 e+09$ \\
\hline 81 & 184 & $1.405 \mathrm{e}-03$ & $1.815 e+07$ & 81 & 185 & $3.972 \mathrm{e}-03$ & $1.539 \mathrm{e}+08$ & 81 & 186 & $5.995 e-03$ & $4.648 e+07$ \\
\hline 81 & 187 & $4.716 e-01$ & $3.681 \mathrm{e}+09$ & 81 & 206 & $0.000 e+00$ & $2.468 e+04$ & 81 & 207 & $8.603 e-02$ & $4.264 e+09$ \\
\hline 81 & 208 & $0.000 e+00$ & $3.816 e+04$ & 81 & 213 & $4.758 e-04$ & $8.904 e+06$ & 81 & 214 & $0.000 e+00$ & $8.224 e+04$ \\
\hline 81 & 224 & $0.000 e+00$ & $9.049 e+04$ & 81 & 225 & $0.000 e+00$ & $1.357 \mathrm{e}+05$ & 81 & 233 & $5.148 e-04$ & $2.223 e+07$ \\
\hline 81 & 238 & $2.379 \mathrm{e}-04$ & $6.466 \mathrm{e}+06$ & 81 & 275 & $0.000 e+00$ & $4.217 e+04$ & 81 & 280 & $1.442 \mathrm{e}-03$ & $4.223 e+07$ \\
\hline 81 & 281 & $7.580 e-03$ & $1.113 e+09$ & 81 & 283 & $0.000 e+00$ & $1.452 \mathrm{e}+04$ & 82 & 98 & $1.146 \mathrm{e}-01$ & $1.914 \mathrm{e}+07$ \\
\hline 82 & 124 & $4.750 e-03$ & $9.227 e+06$ & 82 & 125 & $0.000 e+00$ & $1.624 \mathrm{e}+04$ & 82 & 136 & $1.373 e-01$ & $6.802 e+08$ \\
\hline 82 & 137 & $2.975 e-02$ & $1.489 e+08$ & 82 & 139 & $9.097 e-03$ & $3.277 e+07$ & 82 & 140 & $8.353 e-03$ & $4.336 e+07$ \\
\hline 82 & 141 & $1.507 e-03$ & $5.628 \mathrm{e}+06$ & 82 & 151 & $0.000 e+00$ & $1.616 \mathrm{e}+04$ & 82 & 152 & $0.000 e+00$ & $1.073 e+03$ \\
\hline 82 & 155 & $0.000 e+00$ & $2.921 \mathrm{e}+04$ & 82 & 157 & $3.804 e+00$ & $1.243 e+10$ & 82 & 158 & $0.000 e+00$ & $1.234 \mathrm{e}+04$ \\
\hline 82 & 159 & $0.000 e+00$ & $1.115 e+04$ & 82 & 162 & $2.230 \mathrm{e}-01$ & $7.424 e+08$ & 82 & 163 & $8.507 e-01$ & $3.647 e+09$ \\
\hline 82 & 167 & $2.457 e-01$ & $8.385 e+08$ & 82 & 175 & $3.772 \mathrm{e}-03$ & $1.340 \mathrm{e}+07$ & 82 & 176 & $5.521 \mathrm{e}-03$ & $2.528 e+07$ \\
\hline 82 & 177 & $7.607 e-02$ & $4.945 e+08$ & 82 & 178 & $1.071 \mathrm{e}-01$ & $4.986 e+08$ & 82 & 189 & $1.331 \mathrm{e}+00$ & $5.638 e+09$ \\
\hline 82 & 190 & $2.774 \mathrm{e}+00$ & $1.224 \mathrm{e}+10$ & 82 & 191 & $7.702 \mathrm{e}-02$ & $6.125 e+08$ & 82 & 192 & $1.370 e-01$ & $6.059 e+08$ \\
\hline 82 & 196 & $1.286 \mathrm{e}-01$ & $1.059 \mathrm{e}+09$ & 82 & 197 & $3.374 e-01$ & $2.119 \mathrm{e}+09$ & 82 & 201 & $2.565 e-03$ & $1.638 \mathrm{e}+07$ \\
\hline 82 & 202 & $3.095 e-03$ & $2.768 e+07$ & 82 & 203 & $1.920 \mathrm{e}-03$ & $1.756 \mathrm{e}+07$ & 82 & 206 & $2.330 e-01$ & $1.560 \mathrm{e}+09$ \\
\hline 82 & 214 & $2.302 \mathrm{e}-04$ & $4.744 \mathrm{e}+06$ & 82 & 218 & $1.279 \mathrm{e}-03$ & $2.674 \mathrm{e}+07$ & 82 & 227 & $0.000 e+00$ & $1.103 e+04$ \\
\hline 82 & 247 & $3.992 \mathrm{e}-03$ & $1.078 e+08$ & 82 & 248 & $3.773 e-04$ & $7.280 e+06$ & 82 & 250 & $3.256 \mathrm{e}-03$ & $8.893 e+07$ \\
\hline 82 & 251 & $0.000 e+00$ & $8.925 e+03$ & 82 & 252 & $1.347 e-03$ & $2.659 \mathrm{e}+07$ & 82 & 253 & $0.000 e+00$ & $1.059 e+04$ \\
\hline 82 & 258 & $0.000 e+00$ & $4.240 e+04$ & 82 & 259 & $0.000 \mathrm{e}+00$ & $1.173 e+04$ & 82 & 266 & $0.000 \mathrm{e}+00$ & $1.248 \mathrm{e}+05$ \\
\hline 82 & 267 & $0.000 e+00$ & $1.372 \mathrm{e}+04$ & 82 & 268 & $0.000 \mathrm{e}+00$ & $4.341 e+03$ & 82 & 275 & $2.293 e-03$ & $4.598 e+07$ \\
\hline 82 & 278 & $0.000 e+00$ & $7.246 e+03$ & 82 & 279 & $0.000 e+00$ & $2.760 e+04$ & 83 & 99 & $2.053 e-01$ & $2.155 e+07$ \\
\hline 83 & 128 & $0.000 e+00$ & $1.788 e+04$ & 83 & 139 & $1.686 \mathrm{e}-01$ & $5.937 e+08$ & 83 & 141 & $5.720 e-02$ & $2.089 e+08$ \\
\hline 83 & 142 & $1.807 e-03$ & $5.188 e+06$ & 83 & 147 & $2.316 \mathrm{e}-03$ & $9.008 e+06$ & 83 & 151 & $0.000 e+00$ & $2.081 e+03$ \\
\hline 83 & 152 & $0.000 \mathrm{e}+00$ & $8.951 \mathrm{e}+03$ & 83 & 157 & $8.183 e-03$ & $2.618 \mathrm{e}+07$ & 83 & 160 & $3.808 e+00$ & $1.001 \mathrm{e}+10$ \\
\hline 83 & 161 & $0.000 e+00$ & $4.290 \mathrm{e}+04$ & 83 & 162 & $6.375 e-01$ & $2.079 e+09$ & 83 & 163 & $9.606 e-02$ & $4.033 e+08$ \\
\hline 83 & 164 & $0.000 e+00$ & $1.185 \mathrm{e}+04$ & 83 & 165 & $0.000 e+00$ & $1.078 e+04$ & 83 & 167 & $4.817 e-01$ & $1.611 \mathrm{e}+09$ \\
\hline 83 & 168 & $8.406 e-02$ & $2.301 \mathrm{e}+08$ & 83 & 170 & $5.062 \mathrm{e}-01$ & $1.397 e+09$ & 83 & 175 & $1.176 \mathrm{e}-02$ & $4.093 e+07$ \\
\hline 83 & 176 & $2.945 e-02$ & $1.321 \mathrm{e}+08$ & 83 & 178 & $9.992 e-02$ & $4.561 \mathrm{e}+08$ & 83 & 190 & $1.928 e-01$ & $8.356 e+08$ \\
\hline 83 & 192 & $9.333 e-02$ & $4.052 e+08$ & 83 & 193 & $1.393 e-01$ & $7.783 e+08$ & 83 & 194 & $5.332 \mathrm{e}+00$ & $1.901 e+10$ \\
\hline 83 & 197 & $2.176 \mathrm{e}-02$ & $1.343 e+08$ & 83 & 198 & $1.676 \mathrm{e}-01$ & $6.602 \mathrm{e}+08$ & 83 & 199 & $5.741 \mathrm{e}-01$ & $2.765 e+09$ \\
\hline 83 & 01 & $5.751 \mathrm{e}-02$ & $.610 e+08$ & 83 & 209 & $5.420 \mathrm{e}-02$ & $.550 e+08$ & 83 & 219 & $2.518 e-03$ & $3.725 e+07$ \\
\hline
\end{tabular}


Table III. Ca IX Oscillator Strengths and Radiative Decay Rates for transitions involving levels higher than 4.

\begin{tabular}{|c|c|c|c|c|c|c|c|c|c|c|c|}
\hline $\begin{array}{l}\text { Low. } \\
\text { Lev . }\end{array}$ & $\begin{array}{l}\text { Upp } \\
\text { Lev }\end{array}$ & $\begin{array}{l}\text { Osc. } \\
\text { Str. }\end{array}$ & $\begin{array}{l}\text { Rad. } \\
\text { Rate }\end{array}$ & $\begin{array}{l}\text { Low. } \\
\text { Lev. }\end{array}$ & $\begin{array}{l}\text { Upp. } \\
\text { Lev. }\end{array}$ & $\begin{array}{l}\text { Osc. } \\
\text { Str. }\end{array}$ & $\begin{array}{l}\text { Rad. } \\
\text { Rate }\end{array}$ & $\begin{array}{l}\text { Low } \\
\text { Lev }\end{array}$ & $\begin{array}{l}\text { - Upp } \\
\text {. Lev }\end{array}$ & $\begin{array}{l}\text { Osc. } \\
\text { Str. }\end{array}$ & $\begin{array}{l}\text { Rad. } \\
\text { Rate }\end{array}$ \\
\hline i & $j$ & $g f$ & $(1 / s)$ & $i$ & $j$ & $g f$ & $(1 / s)$ & $i$ & j & $g f$ & $(1 / s)$ \\
\hline 83 & 228 & $0.000 e+00$ & $1.156 \mathrm{e}+04$ & 83 & 248 & $6.169 \mathrm{e}-03$ & $1.179 e+08$ & 83 & 249 & $5.906 e-04$ & $8.780 e+06$ \\
\hline 83 & 253 & $0.000 e+00$ & $1.470 e+04$ & 83 & 254 & $2.419 e-03$ & $3.679 e+07$ & 83 & 255 & $0.000 e+00$ & $1.575 e+04$ \\
\hline 83 & 256 & $1.274 \mathrm{e}-03$ & $1.586 e+07$ & 83 & 258 & $0.000 e+00$ & $1.460 e+04$ & 83 & 259 & $0.000 e+00$ & $4.331 \mathrm{e}+04$ \\
\hline 83 & 260 & $0.000 e+00$ & $1.388 e+04$ & 83 & 264 & $2.455 e-03$ & $4.839 e+07$ & 83 & 266 & $0.000 e+00$ & $1.329 \mathrm{e}+04$ \\
\hline 83 & 267 & $0.000 e+00$ & $5.182 \mathrm{e}+03$ & 83 & 268 & $0.000 e+00$ & $1.676 e+04$ & 83 & 269 & $0.000 e+00$ & $4.805 e+03$ \\
\hline 83 & 270 & $0.000 e+00$ & $1.764 \mathrm{e}+05$ & 83 & 277 & $0.000 e+00$ & $8.032 \mathrm{e}+03$ & 83 & 278 & $0.000 e+00$ & $3.102 \mathrm{e}+04$ \\
\hline 84 & 112 & $2.771 \mathrm{e}-03$ & $5.331 e+06$ & 84 & 116 & $4.217 \mathrm{e}-03$ & $8.672 e+06$ & 84 & 124 & $9.670 \mathrm{e}-03$ & $1.803 e+07$ \\
\hline 84 & 126 & $0.000 e+00$ & $1.823 e+04$ & 84 & 134 & $0.000 e+00$ & $4.852 e+03$ & 84 & 136 & $5.707 e-02$ & $2.743 e+08$ \\
\hline 84 & 137 & $1.080 e-01$ & $5.245 e+08$ & 84 & 139 & $4.334 \mathrm{e}-03$ & $1.515 e+07$ & 84 & 140 & $5.239 \mathrm{e}-02$ & $2.640 e+08$ \\
\hline 84 & 142 & $5.357 e-03$ & $1.527 e+07$ & 84 & 147 & $6.568 e-03$ & $2.537 e+07$ & 84 & 151 & $0.000 e+00$ & $1.666 \mathrm{e}+03$ \\
\hline 84 & 155 & $0.000 e+00$ & $1.083 e+04$ & 84 & 157 & $4.030 e-02$ & $1.281 e+08$ & 84 & 158 & $0.000 e+00$ & $3.124 \mathrm{e}+04$ \\
\hline 84 & 162 & $4.566 \mathrm{e}+00$ & $1.479 \mathrm{e}+10$ & 84 & 163 & $2.120 e-02$ & $8.840 e+07$ & 84 & 164 & $0.000 e+00$ & $1.609 \mathrm{e}+04$ \\
\hline 84 & 167 & $9.804 \mathrm{e}-01$ & $3.256 e+09$ & 84 & 175 & $5.044 \mathrm{e}-02$ & $1.744 e+08$ & 84 & 176 & $2.598 e-01$ & $1.158 \mathrm{e}+09$ \\
\hline 84 & 177 & $4.839 e-02$ & $3.064 e+08$ & 84 & 178 & $4.691 \mathrm{e}-01$ & $2.127 e+09$ & 84 & 179 & $0.000 e+00$ & $1.001 \mathrm{e}+04$ \\
\hline 84 & 183 & $0.000 e+00$ & $1.305 e+04$ & 84 & 189 & $2.037 e+00$ & $8.421 e+09$ & 84 & 190 & $1.322 \mathrm{e}+00$ & $5.697 e+09$ \\
\hline 84 & 191 & $3.336 e-02$ & $2.590 e+08$ & 84 & 192 & $1.391 \mathrm{e}-01$ & $6.005 e+08$ & 84 & 193 & $5.937 e-02$ & $3.298 e+08$ \\
\hline 84 & 196 & $3.056 e-01$ & $2.457 e+09$ & 84 & 197 & $1.599 \mathrm{e}-01$ & $9.818 e+08$ & 84 & 199 & $2.108 \mathrm{e}-01$ & $1.010 e+09$ \\
\hline 84 & 201 & $1.102 \mathrm{e}-02$ & $6.876 e+07$ & 84 & 202 & $7.270 e-02$ & $6.357 e+08$ & 84 & 203 & $2.138 \mathrm{e}-02$ & $1.912 e+08$ \\
\hline 84 & 206 & $5.366 e-01$ & $3.513 e+09$ & 84 & 218 & $6.832 e-04$ & $1.407 e+07$ & 84 & 224 & $4.945 e-04$ & $7.526 e+06$ \\
\hline 84 & 233 & $0.000 e+00$ & $1.417 e+04$ & 84 & 247 & $8.926 e-04$ & $2.379 e+07$ & 84 & 250 & $8.163 e-03$ & $2.201 \mathrm{e}+08$ \\
\hline 84 & 251 & $0.000 e+00$ & $1.168 e+04$ & 84 & 252 & $7.076 e-04$ & $1.379 e+07$ & 84 & 253 & $0.000 e+00$ & $5.967 e+03$ \\
\hline 84 & 254 & $1.426 \mathrm{e}-03$ & $2.163 e+07$ & 84 & 258 & $0.000 e+00$ & $1.690 \mathrm{e}+04$ & 84 & 261 & $0.000 e+00$ & $5.725 e+03$ \\
\hline 84 & 263 & $2.666 \mathrm{e}-03$ & $7.333 e+07$ & 84 & 266 & $0.000 e+00$ & $4.879 e+04$ & 84 & 267 & $0.000 e+00$ & $7.503 e+03$ \\
\hline 84 & 275 & $5.409 e-03$ & $1.071 \mathrm{e}+08$ & 84 & 279 & $0.000 e+00$ & $1.090 e+04$ & 85 & 100 & $3.090 e-01$ & $2.184 \mathrm{e}+07$ \\
\hline 85 & 131 & $0.000 e+00$ & $1.591 \mathrm{e}+04$ & 85 & 142 & $2.640 \mathrm{e}-01$ & $7.401 e+08$ & 85 & 153 & $0.000 e+00$ & $3.484 e+03$ \\
\hline 85 & 157 & $2.207 e-03$ & $6.907 e+06$ & 85 & 160 & $2.107 e-01$ & $5.419 e+08$ & 85 & 162 & $1.079 \mathrm{e}-01$ & $3.441 \mathrm{e}+08$ \\
\hline 85 & 165 & $0.000 e+00$ & $2.252 \mathrm{e}+04$ & 85 & 166 & $3.666 \mathrm{e}+00$ & $8.270 e+09$ & 85 & 167 & $4.468 e-02$ & $1.461 \mathrm{e}+08$ \\
\hline 85 & 168 & $1.549 \mathrm{e}+00$ & $4.147 e+09$ & 85 & 169 & $0.000 e+00$ & $5.676 e+04$ & 85 & 170 & $3.544 e-01$ & $9.571 \mathrm{e}+08$ \\
\hline 85 & 175 & $9.201 \mathrm{e}-02$ & $3.134 \mathrm{e}+08$ & 85 & 190 & $1.066 \mathrm{e}-03$ & $4.530 e+06$ & 85 & 192 & $1.343 e-01$ & $5.719 e+08$ \\
\hline 85 & 194 & $2.823 e-01$ & $9.875 e+08$ & 85 & 195 & $7.394 e+00$ & $2.193 e+10$ & 85 & 198 & $5.308 e-01$ & $2.053 e+09$ \\
\hline 85 & 199 & $2.250 e-02$ & $1.064 \mathrm{e}+08$ & 85 & 220 & $3.180 e-03$ & $3.624 e+07$ & 85 & 229 & $0.000 e+00$ & $9.746 e+03$ \\
\hline 85 & 249 & $8.769 e-03$ & $1.290 \mathrm{e}+08$ & 85 & 255 & $0.000 e+00$ & $1.942 \mathrm{e}+04$ & 85 & 256 & $2.069 \mathrm{e}-03$ & $2.550 e+07$ \\
\hline 85 & 257 & $0.000 e+00$ & $3.388 e+04$ & 85 & 259 & $0.000 e+00$ & $1.280 e+04$ & 85 & 260 & $0.000 e+00$ & $5.755 e+04$ \\
\hline 85 & 268 & $0.000 e+00$ & $4.744 e+03$ & 85 & 269 & $0.000 e+00$ & $2.141 e+04$ & 85 & 270 & $0.000 e+00$ & $1.224 \mathrm{e}+04$ \\
\hline 85 & 271 & $0.000 e+00$ & $2.129 \mathrm{e}+05$ & 85 & 277 & $0.000 e+00$ & $4.433 e+04$ & 86 & 115 & $2.569 \mathrm{e}-02$ & $8.338 e+07$ \\
\hline 86 & 127 & $1.973 e-03$ & $9.373 e+06$ & 86 & 129 & $0.000 e+00$ & $2.441 e+04$ & 86 & 130 & $0.000 e+00$ & $7.443 e+03$ \\
\hline 86 & 136 & $3.029 e-02$ & $1.408 e+08$ & 86 & 138 & $1.737 e-01$ & $1.367 e+09$ & 86 & 140 & $2.312 \mathrm{e}-02$ & $1.128 \mathrm{e}+08$ \\
\hline 86 & 145 & $1.022 \mathrm{e}-03$ & $8.710 e+06$ & 86 & 147 & $1.426 \mathrm{e}-02$ & $5.337 e+07$ & 86 & 151 & $0.000 e+00$ & $1.593 e+03$ \\
\hline 86 & 159 & $0.000 e+00$ & $4.355 e+04$ & 86 & 163 & $4.977 e+00$ & $2.014 e+10$ & 86 & 164 & $0.000 e+00$ & $1.279 \mathrm{e}+04$ \\
\hline 86 & 174 & $0.000 \mathrm{e}+00$ & $8.996 e+03$ & 86 & 176 & $2.314 \mathrm{e}-02$ & $1.002 e+08$ & 86 & 177 & $4.104 \mathrm{e}-01$ & $2.524 \mathrm{e}+09$ \\
\hline 86 & 178 & $1.136 \mathrm{e}-01$ & $5.004 e+08$ & 86 & 191 & $4.396 e-01$ & $3.326 e+09$ & 86 & 197 & $2.409 e+00$ & $1.442 e+10$ \\
\hline 86 & 200 & $3.774 \mathrm{e}-01$ & $5.358 e+09$ & 86 & 202 & $1.274 \mathrm{e}-02$ & $1.087 e+08$ & 86 & 203 & $4.220 e-03$ & $3.683 e+07$ \\
\hline 86 & 204 & $5.825 e-03$ & $8.481 e+07$ & 86 & 206 & $3.054 \mathrm{e}-03$ & $1.952 \mathrm{e}+07$ & 86 & 208 & $6.296 \mathrm{e}-03$ & $1.029 e+08$ \\
\hline 86 & 215 & $4.280 e-04$ & $1.435 \mathrm{e}+07$ & 86 & 218 & $3.542 \mathrm{e}-04$ & $7.180 e+06$ & 86 & 239 & $0.000 e+00$ & $1.868 \mathrm{e}+04$ \\
\hline
\end{tabular}


Table III. Ca IX Oscillator Strengths and Radiative Decay Rates for transitions involving levels higher than 4.

\begin{tabular}{|c|c|c|c|c|c|c|c|c|c|c|c|}
\hline $\begin{array}{l}\text { Low. } \\
\text { Lev. }\end{array}$ & $\begin{array}{l}\text { Upp } \\
\text { Lev }\end{array}$ & $\begin{array}{l}\text { Osc. } \\
\text { Str. }\end{array}$ & $\begin{array}{l}\text { Rad. } \\
\text { Rate }\end{array}$ & $\begin{array}{l}\text { Low. } \\
\text { Lev. }\end{array}$ & $\begin{array}{l}\text { Upp. } \\
\text { Lev }\end{array}$ & $\begin{array}{l}\text { Osc. } \\
\text { Str. }\end{array}$ & $\begin{array}{l}\text { Rad. } \\
\text { Rate }\end{array}$ & $\begin{array}{l}\text { Low. } \\
\text { Lev. }\end{array}$ & $\begin{array}{l}\text {. Upp } \\
\text {. Lev }\end{array}$ & $\begin{array}{l}\text { Osc. } \\
\text { Str. }\end{array}$ & $\begin{array}{l}\text { Rad. } \\
\text { Rate }\end{array}$ \\
\hline$i$ & $j$ & $g f$ & $(1 / s)$ & $i$ & $j$ & $g f$ & $(1 / s)$ & $i$ & j & $g f$ & $(1 / s)$ \\
\hline 86 & 240 & $0.000 e+00$ & $9.236 \mathrm{e}+03$ & 86 & 252 & $1.343 e-02$ & $2.580 e+08$ & 86 & 258 & $0.000 e+00$ & $4.688 e+03$ \\
\hline 86 & 262 & $1.524 \mathrm{e}-02$ & $6.887 e+08$ & 86 & 263 & $1.684 \mathrm{e}-03$ & $4.569 e+07$ & 86 & 283 & $3.364 e-04$ & $1.585 e+07$ \\
\hline 87 & 116 & $3.220 e-02$ & $6.179 \mathrm{e}+07$ & 87 & 130 & $0.000 e+00$ & $1.248 e+04$ & 87 & 132 & $0.000 e+00$ & $4.539 e+03$ \\
\hline 87 & 137 & $1.333 e-01$ & $6.190 e+08$ & 87 & 139 & $1.857 \mathrm{e}-02$ & $6.207 e+07$ & 87 & 140 & $1.089 \mathrm{e}-01$ & $5.252 e+08$ \\
\hline 87 & 141 & $3.993 e-02$ & $1.386 \mathrm{e}+08$ & 87 & 142 & $3.716 \mathrm{e}-03$ & $1.014 \mathrm{e}+07$ & 87 & 143 & $2.000 e-03$ & $1.002 \mathrm{e}+07$ \\
\hline 87 & 147 & $8.083 e-03$ & $2.992 \mathrm{e}+07$ & 87 & 157 & $2.637 e-01$ & $8.042 e+08$ & 87 & 158 & $0.000 e+00$ & $1.712 \mathrm{e}+04$ \\
\hline 87 & 159 & $0.000 e+00$ & $1.377 e+04$ & 87 & 162 & $1.877 e+00$ & $5.835 e+09$ & 87 & 163 & $3.900 e-01$ & $1.561 \mathrm{e}+09$ \\
\hline 87 & 164 & $0.000 e+00$ & $1.257 e+04$ & 87 & 165 & $0.000 e+00$ & $1.640 e+04$ & 87 & 167 & $3.768 e+00$ & $1.202 \mathrm{e}+10$ \\
\hline 87 & 173 & $0.000 e+00$ & $1.169 \mathrm{e}+04$ & 87 & 175 & $3.372 \mathrm{e}-02$ & $1.121 e+08$ & 87 & 176 & $9.089 e-01$ & $3.895 e+09$ \\
\hline 87 & 177 & $1.931 \mathrm{e}-02$ & $1.175 \mathrm{e}+08$ & 87 & 178 & $7.080 e-02$ & $3.088 e+08$ & 87 & 189 & $1.639 \mathrm{e}-01$ & $6.537 e+08$ \\
\hline 87 & 190 & $3.752 \mathrm{e}-01$ & $1.561 \mathrm{e}+09$ & 87 & 191 & $2.430 e-02$ & $1.821 e+08$ & 87 & 192 & $1.216 \mathrm{e}-02$ & $5.068 e+07$ \\
\hline 87 & 193 & $5.423 e-01$ & $2.908 e+09$ & 87 & 196 & $2.094 \mathrm{e}-02$ & $1.625 e+08$ & 87 & 197 & $7.873 e-02$ & $4.673 e+08$ \\
\hline 87 & 199 & $2.933 e+00$ & $1.359 \mathrm{e}+10$ & 87 & 202 & $4.699 \mathrm{e}-01$ & $3.974 e+09$ & 87 & 203 & $2.782 \mathrm{e}-02$ & $2.407 e+08$ \\
\hline 87 & 206 & $3.734 e-02$ & $2.367 e+08$ & 87 & 216 & $5.722 \mathrm{e}-04$ & $1.146 e+07$ & 87 & 219 & $5.836 e-04$ & $8.417 e+06$ \\
\hline 87 & 240 & $0.000 e+00$ & $1.034 \mathrm{e}+04$ & 87 & 241 & $0.000 e+00$ & $5.712 e+03$ & 87 & 247 & $3.804 e-04$ & $9.950 e+06$ \\
\hline 87 & 250 & $6.877 e-04$ & $1.819 \mathrm{e}+07$ & 87 & 254 & $1.602 \mathrm{e}-02$ & $2.384 e+08$ & 87 & 255 & $0.000 e+00$ & $3.749 e+03$ \\
\hline 87 & 259 & $0.000 e+00$ & $8.858 e+03$ & 87 & 263 & $2.000 e-02$ & $5.399 e+08$ & 87 & 264 & $1.116 \mathrm{e}-03$ & $2.151 \mathrm{e}+07$ \\
\hline 87 & 266 & $0.000 e+00$ & $1.564 \mathrm{e}+04$ & 87 & 279 & $0.000 e+00$ & $7.380 e+03$ & 88 & 117 & $4.224 e-02$ & $5.758 e+07$ \\
\hline 88 & 131 & $0.000 e+00$ & $6.649 \mathrm{e}+03$ & 88 & 132 & $0.000 e+00$ & $1.290 \mathrm{e}+04$ & 88 & 139 & $1.169 \mathrm{e}-01$ & $3.877 e+08$ \\
\hline 88 & 141 & $1.515 \mathrm{e}-01$ & $5.218 e+08$ & 88 & 142 & $7.292 \mathrm{e}-02$ & $1.975 e+08$ & 88 & 157 & $6.310 e-02$ & $1.911 \mathrm{e}+08$ \\
\hline 88 & 160 & $1.171 \mathrm{e}+00$ & $2.914 \mathrm{e}+09$ & 88 & 161 & $0.000 e+00$ & $1.570 \mathrm{e}+04$ & 88 & 162 & $6.261 \mathrm{e}-02$ & $1.933 e+08$ \\
\hline 88 & 163 & $1.651 \mathrm{e}-02$ & $6.565 e+07$ & 88 & 165 & $0.000 e+00$ & $1.392 \mathrm{e}+04$ & 88 & 167 & $4.542 \mathrm{e}-01$ & $1.439 \mathrm{e}+09$ \\
\hline 88 & 168 & $5.009 e+00$ & $1.299 e+10$ & 88 & 169 & $0.000 e+00$ & $1.873 e+04$ & 88 & 170 & $1.077 \mathrm{e}+00$ & $2.817 e+09$ \\
\hline 88 & 172 & $0.000 e+00$ & $2.411 \mathrm{e}+04$ & 88 & 175 & $1.516 \mathrm{e}+00$ & $5.006 e+09$ & 88 & 176 & $2.014 \mathrm{e}-02$ & $8.572 \mathrm{e}+07$ \\
\hline 88 & 178 & $1.331 \mathrm{e}-02$ & $5.764 e+07$ & 88 & 189 & $4.475 e-03$ & $1.773 e+07$ & 88 & 190 & $1.219 \mathrm{e}-01$ & $5.037 e+08$ \\
\hline 88 & 192 & $5.894 \mathrm{e}-01$ & $2.441 \mathrm{e}+09$ & 88 & 193 & $1.538 \mathrm{e}-02$ & $8.199 e+07$ & 88 & 194 & $5.125 e-01$ & $1.743 e+09$ \\
\hline 88 & 198 & $4.165 \mathrm{e}+00$ & $1.569 \mathrm{e}+10$ & 88 & 199 & $7.065 e-02$ & $3.254 e+08$ & 88 & 201 & $5.959 e-01$ & $3.577 e+09$ \\
\hline 88 & 209 & $2.230 \mathrm{e}-02$ & $1.007 e+08$ & 88 & 217 & $1.000 \mathrm{e}-03$ & $1.427 e+07$ & 88 & 220 & $7.930 \mathrm{e}-04$ & $8.886 e+06$ \\
\hline 88 & 229 & $0.000 e+00$ & $8.744 \mathrm{e}+03$ & 88 & 241 & $0.000 e+00$ & $1.560 \mathrm{e}+04$ & 88 & 248 & $6.334 \mathrm{e}-04$ & $1.180 \mathrm{e}+07$ \\
\hline 88 & 256 & $2.263 e-02$ & $2.749 \mathrm{e}+08$ & 88 & 257 & $0.000 e+00$ & $4.788 e+03$ & 88 & 260 & $0.000 e+00$ & $9.833 e+03$ \\
\hline 88 & 264 & $2.820 \mathrm{e}-02$ & $5.419 e+08$ & 88 & 270 & $0.000 e+00$ & $1.845 e+04$ & 88 & 278 & $0.000 e+00$ & $7.979 e+03$ \\
\hline 89 & 100 & $1.227 e+00$ & $4.652 e+07$ & 89 & 112 & $3.526 e-03$ & $5.713 e+06$ & 89 & 113 & $1.510 \mathrm{e}-02$ & $2.476 e+07$ \\
\hline 89 & 117 & $7.631 \mathrm{e}-02$ & $9.561 \mathrm{e}+07$ & 89 & 120 & $5.670 e-02$ & $5.807 e+07$ & 89 & 123 & $3.774 \mathrm{e}-02$ & $8.114 e+07$ \\
\hline 89 & 132 & $0.000 e+00$ & $4.689 e+03$ & 89 & 133 & $0.000 e+00$ & $4.549 e+03$ & 89 & 136 & $3.060 \mathrm{e}-02$ & $1.321 e+08$ \\
\hline 89 & 137 & $1.874 \mathrm{e}-01$ & $8.182 \mathrm{e}+08$ & 89 & 139 & $6.301 \mathrm{e}-01$ & $1.981 e+09$ & 89 & 140 & $3.421 \mathrm{e}-02$ & $1.553 e+08$ \\
\hline 89 & 141 & $2.114 \mathrm{e}-01$ & $6.908 e+08$ & 89 & 142 & $7.284 \mathrm{e}-01$ & $1.872 \mathrm{e}+09$ & 89 & 143 & $7.563 e-01$ & $3.571 \mathrm{e}+09$ \\
\hline 89 & 147 & $2.682 \mathrm{e}-02$ & $9.367 e+07$ & 89 & 157 & $5.048 e-03$ & $1.455 e+07$ & 89 & 162 & $2.187 e-02$ & $6.428 e+07$ \\
\hline 89 & 167 & $1.462 \mathrm{e}-02$ & $4.412 \mathrm{e}+07$ & 89 & 172 & $0.000 \mathrm{e}+00$ & $1.594 \mathrm{e}+04$ & 89 & 173 & $0.000 e+00$ & $1.749 e+04$ \\
\hline 89 & 175 & $3.666 \mathrm{e}+00$ & $1.154 \mathrm{e}+10$ & 89 & 176 & $2.809 e-01$ & $1.140 \mathrm{e}+09$ & 89 & 177 & $2.166 \mathrm{e}-02$ & $1.250 \mathrm{e}+08$ \\
\hline 89 & 178 & $3.117 \mathrm{e}-01$ & $1.288 \mathrm{e}+09$ & 89 & 182 & $0.000 e+00$ & $5.312 e+03$ & 89 & 189 & $3.982 \mathrm{e}-02$ & $1.511 \mathrm{e}+08$ \\
\hline 89 & 190 & $5.433 e-02$ & $2.153 e+08$ & 89 & 192 & $9.392 \mathrm{e}-01$ & $3.728 e+09$ & 89 & 193 & $1.983 e-02$ & $1.013 e+08$ \\
\hline 89 & 196 & $3.454 \mathrm{e}-03$ & $2.557 e+07$ & 89 & 197 & $1.757 e-02$ & $9.963 e+07$ & 89 & 199 & $1.733 \mathrm{e}-01$ & $7.666 e+08$ \\
\hline 89 & 201 & $5.876 e-01$ & $3.390 \mathrm{e}+09$ & 89 & 202 & $1.641 \mathrm{e}-02$ & $1.326 \mathrm{e}+08$ & 89 & 203 & $4.630 \mathrm{e}-01$ & $3.831 e+09$ \\
\hline 89 & 206 & $7.807 e-03$ & $4.733 e+07$ & 89 & 220 & $3.137 \mathrm{e}-03$ & $3.426 e+07$ & 89 & 221 & $5.970 e-04$ & $1.193 e+07$ \\
\hline
\end{tabular}


Table III. Ca IX Oscillator Strengths and Radiative Decay Rates for transitions involving levels higher than 4.

\begin{tabular}{|c|c|c|c|c|c|c|c|c|c|c|c|}
\hline $\begin{array}{l}\text { Low. } \\
\text { Lev . }\end{array}$ & $\begin{array}{l}\text { Upp } \\
\text { Lev }\end{array}$ & $\begin{array}{l}\text { Osc. } \\
\text { Str. }\end{array}$ & $\begin{array}{l}\text { Rad. } \\
\text { Rate }\end{array}$ & $\begin{array}{l}\text { Low. } \\
\text { Lev. }\end{array}$ & $\begin{array}{l}\text { Upp. } \\
\text { Lev. }\end{array}$ & $\begin{array}{l}\text { Osc. } \\
\text { Str. }\end{array}$ & $\begin{array}{l}\text { Rad. } \\
\text { Rate }\end{array}$ & $\begin{array}{l}\text { Low. } \\
\text { Lev. }\end{array}$ & $\begin{array}{l}\text { - Upp } \\
\text {. Lev }\end{array}$ & $\begin{array}{l}\text { Osc. } \\
\text { Str. }\end{array}$ & $\begin{array}{l}\text { Rad. } \\
\text { Rate }\end{array}$ \\
\hline$i$ & $j$ & $g f$ & $(1 / s)$ & $i$ & $j$ & $g f$ & $(1 / s)$ & i & j & $g f$ & $(1 / s)$ \\
\hline 89 & 227 & $0.000 e+00$ & $8.177 e+03$ & 89 & 228 & $0.000 e+00$ & $4.603 e+04$ & 89 & 229 & $0.000 e+00$ & $6.268 e+04$ \\
\hline 89 & 231 & $0.000 e+00$ & $1.771 \mathrm{e}+04$ & 89 & 232 & $0.000 e+00$ & $9.595 e+04$ & 89 & 234 & $0.000 e+00$ & $2.116 e+04$ \\
\hline 89 & 235 & $0.000 e+00$ & $6.845 e+03$ & 89 & 236 & $0.000 e+00$ & $4.989 e+04$ & 89 & 237 & $0.000 e+00$ & $1.298 \mathrm{e}+05$ \\
\hline 89 & 240 & $0.000 e+00$ & $5.962 e+04$ & 89 & 241 & $0.000 e+00$ & $4.600 e+04$ & 89 & 264 & $5.065 e-03$ & $9.523 e+07$ \\
\hline 89 & 272 & $1.317 e-02$ & $3.482 e+08$ & 89 & 277 & $0.000 e+00$ & $5.702 e+03$ & 89 & 278 & $0.000 e+00$ & $4.467 e+03$ \\
\hline 90 & 98 & $1.167 e-01$ & $7.558 e+06$ & 90 & 99 & $9.464 \mathrm{e}-01$ & $4.384 e+07$ & 90 & 111 & $7.072 \mathrm{e}-03$ & $1.780 e+07$ \\
\hline 90 & 115 & $5.219 \mathrm{e}-03$ & $1.495 e+07$ & 90 & 116 & $4.673 e-02$ & $8.064 e+07$ & 90 & 119 & $4.751 e-02$ & $6.112 \mathrm{e}+07$ \\
\hline 90 & 122 & $2.230 \mathrm{e}-02$ & $7.893 e+07$ & 90 & 123 & $8.031 \mathrm{e}-03$ & $1.712 \mathrm{e}+07$ & 90 & 127 & $1.407 e-03$ & $6.033 e+06$ \\
\hline 90 & 133 & $0.000 e+00$ & $5.593 e+03$ & 90 & 136 & $3.017 \mathrm{e}-02$ & $1.295 e+08$ & 90 & 137 & $3.175 e-01$ & $1.378 e+09$ \\
\hline 90 & 138 & $1.605 e-01$ & $1.167 \mathrm{e}+09$ & 90 & 139 & $4.179 \mathrm{e}-02$ & $1.306 e+08$ & 90 & 140 & $5.229 e-02$ & $2.360 \mathrm{e}+08$ \\
\hline 90 & 141 & $5.875 e-01$ & $1.908 e+09$ & 90 & 143 & $3.536 \mathrm{e}-01$ & $1.660 \mathrm{e}+09$ & 90 & 145 & $4.498 e-01$ & $3.553 e+09$ \\
\hline 90 & 147 & $3.745 e-02$ & $1.300 e+08$ & 90 & 156 & $2.267 e-03$ & $1.939 e+07$ & 90 & 172 & $0.000 e+00$ & $1.051 \mathrm{e}+04$ \\
\hline 90 & 174 & $0.000 e+00$ & $2.409 \mathrm{e}+04$ & 90 & 176 & $1.550 e+00$ & $6.257 e+09$ & 90 & 177 & $4.378 e-01$ & $2.513 e+09$ \\
\hline 90 & 178 & $7.099 \mathrm{e}-01$ & $2.919 \mathrm{e}+09$ & 90 & 191 & $3.433 e-02$ & $2.440 e+08$ & 90 & 193 & $6.318 e-01$ & $3.213 e+09$ \\
\hline 90 & 197 & $8.091 \mathrm{e}-02$ & $4.567 e+08$ & 90 & 200 & $3.250 e-02$ & $4.351 e+08$ & 90 & 201 & $6.990 \mathrm{e}-02$ & $4.014 e+08$ \\
\hline 90 & 202 & $2.950 e-01$ & $2.374 \mathrm{e}+09$ & 90 & 203 & $7.855 e-02$ & $6.470 e+08$ & 90 & 204 & $2.075 e-01$ & $2.851 \mathrm{e}+09$ \\
\hline 90 & 206 & $4.347 e-03$ & $2.624 \mathrm{e}+07$ & 90 & 208 & $1.612 \mathrm{e}-03$ & $2.495 e+07$ & 90 & 219 & $2.582 \mathrm{e}-03$ & $3.607 e+07$ \\
\hline 90 & 222 & $3.381 \mathrm{e}-04$ & $1.123 e+07$ & 90 & 227 & $0.000 e+00$ & $7.574 e+04$ & 90 & 228 & $0.000 e+00$ & $2.641 \mathrm{e}+04$ \\
\hline 90 & 229 & $0.000 e+00$ & $2.947 e+04$ & 90 & 230 & $0.000 e+00$ & $2.580 e+04$ & 90 & 231 & $0.000 e+00$ & $9.112 \mathrm{e}+04$ \\
\hline 90 & 234 & $0.000 e+00$ & $2.021 \mathrm{e}+04$ & 90 & 235 & $0.000 e+00$ & $7.648 e+04$ & 90 & 236 & $0.000 e+00$ & $9.054 e+04$ \\
\hline 90 & 237 & $0.000 e+00$ & $3.203 e+04$ & 90 & 238 & $0.000 e+00$ & $8.250 e+03$ & 90 & 239 & $0.000 e+00$ & $1.489 e+05$ \\
\hline 90 & 240 & $0.000 e+00$ & $7.903 e+03$ & 90 & 241 & $0.000 e+00$ & $3.700 e+04$ & 90 & 263 & $2.181 \mathrm{e}-03$ & $5.726 e+07$ \\
\hline 90 & 272 & $2.577 \mathrm{e}-03$ & $6.796 e+07$ & 90 & 273 & $6.089 \mathrm{e}-03$ & $2.678 e+08$ & 90 & 279 & $0.000 e+00$ & $6.093 e+03$ \\
\hline 91 & 98 & $7.032 \mathrm{e}-01$ & $4.400 e+07$ & 91 & 110 & $3.648 \mathrm{e}-03$ & $2.716 e+07$ & 91 & 115 & $2.436 \mathrm{e}-02$ & $6.933 e+07$ \\
\hline 91 & 116 & $3.142 \mathrm{e}-03$ & $5.385 e+06$ & 91 & 118 & $3.443 e-02$ & $6.095 e+07$ & 91 & 121 & $1.045 e-02$ & $1.103 e+08$ \\
\hline 91 & 122 & $8.421 \mathrm{e}-03$ & $2.963 e+07$ & 91 & 133 & $0.000 e+00$ & $4.382 e+03$ & 91 & 136 & $9.843 e-02$ & $4.207 e+08$ \\
\hline 91 & 137 & $9.227 e-03$ & $3.987 e+07$ & 91 & 138 & $2.270 e-01$ & $1.643 e+09$ & 91 & 140 & $3.602 \mathrm{e}-01$ & $1.619 \mathrm{e}+09$ \\
\hline 91 & 143 & $4.573 e-02$ & $2.138 \mathrm{e}+08$ & 91 & 145 & $3.061 \mathrm{e}-01$ & $2.408 e+09$ & 91 & 146 & $2.578 e-01$ & $6.123 e+09$ \\
\hline 91 & 173 & $0.000 e+00$ & $1.189 \mathrm{e}+04$ & 91 & 174 & $0.000 e+00$ & $9.980 e+03$ & 91 & 177 & $1.416 \mathrm{e}+00$ & $8.094 e+09$ \\
\hline 91 & 191 & $3.809 e-01$ & $2.698 \mathrm{e}+09$ & 91 & 200 & $1.396 \mathrm{e}-01$ & $1.864 e+09$ & 91 & 202 & $5.419 e-02$ & $4.347 e+08$ \\
\hline 91 & 203 & $5.513 e-03$ & $4.527 e+07$ & 91 & 204 & $8.381 \mathrm{e}-02$ & $1.148 e+09$ & 91 & 205 & $8.937 e-02$ & $3.679 e+09$ \\
\hline 91 & 218 & $1.938 \mathrm{e}-03$ & $3.774 \mathrm{e}+07$ & 91 & 223 & $1.575 \mathrm{e}-04$ & $1.566 \mathrm{e}+07$ & 91 & 227 & $0.000 e+00$ & $4.598 e+04$ \\
\hline 91 & 228 & $0.000 e+00$ & $4.509 e+04$ & 91 & 230 & $0.000 e+00$ & $9.559 e+04$ & 91 & 234 & $0.000 e+00$ & $1.477 e+04$ \\
\hline 91 & 235 & $0.000 e+00$ & $1.174 \mathrm{e}+05$ & 91 & 236 & $0.000 e+00$ & $4.320 e+04$ & 91 & 240 & $0.000 e+00$ & $7.061 e+04$ \\
\hline 91 & 241 & $0.000 e+00$ & $1.268 \mathrm{e}+04$ & 91 & 262 & $9.130 \mathrm{e}-04$ & $3.988 e+07$ & 91 & 273 & $2.110 \mathrm{e}-03$ & $9.265 e+07$ \\
\hline 91 & 274 & $2.478 e-03$ & $3.265 e+08$ & 92 & 101 & $1.891 \mathrm{e}+00$ & $9.826 e+07$ & 92 & 112 & $5.480 e-02$ & $8.341 e+07$ \\
\hline 92 & 113 & $1.411 \mathrm{e}-02$ & $2.175 e+07$ & 92 & 114 & $6.774 \mathrm{e}-03$ & $1.782 \mathrm{e}+07$ & 92 & 124 & $3.754 e-01$ & $5.731 e+08$ \\
\hline 92 & 127 & $1.513 \mathrm{e}-01$ & $6.222 \mathrm{e}+08$ & 92 & 135 & $0.000 e+00$ & $7.059 e+03$ & 92 & 136 & $6.896 \mathrm{e}-01$ & $2.867 e+09$ \\
\hline 92 & 137 & $3.784 \mathrm{e}-02$ & $1.590 \mathrm{e}+08$ & 92 & 138 & $1.137 \mathrm{e}-03$ & $8.007 e+06$ & 92 & 139 & $3.678 \mathrm{e}-02$ & $1.113 e+08$ \\
\hline 92 & 140 & $2.466 \mathrm{e}-01$ & $1.079 \mathrm{e}+09$ & 92 & 141 & $2.094 \mathrm{e}-02$ & $6.593 e+07$ & 92 & 143 & $6.135 e-02$ & $2.793 e+08$ \\
\hline 92 & 147 & $6.607 e-01$ & $2.226 \mathrm{e}+09$ & 92 & 156 & $6.930 \mathrm{e}-01$ & $5.757 e+09$ & 92 & 176 & $1.469 \mathrm{e}-01$ & $5.767 e+08$ \\
\hline 92 & 178 & $3.462 \mathrm{e}-01$ & $1.384 \mathrm{e}+09$ & 92 & 179 & $0.000 \mathrm{e}+00$ & $1.378 e+04$ & 92 & 187 & $0.000 e+00$ & $4.434 e+03$ \\
\hline 92 & 188 & $0.000 e+00$ & $5.675 e+03$ & 92 & 191 & $1.341 \mathrm{e}-03$ & $9.295 e+06$ & 92 & 196 & $2.869 e-02$ & $2.064 \mathrm{e}+08$ \\
\hline 92 & 197 & $6.922 \mathrm{e}-03$ & $3.816 \mathrm{e}+07$ & 92 & 200 & $1.638 \mathrm{e}-03$ & $2.142 \mathrm{e}+07$ & 92 & 206 & $7.814 \mathrm{e}-02$ & $4.611 \mathrm{e}+08$ \\
\hline
\end{tabular}


Table III. Ca IX Oscillator Strengths and Radiative Decay Rates for transitions involving levels higher than 4.

\begin{tabular}{|c|c|c|c|c|c|c|c|c|c|c|c|}
\hline $\begin{array}{l}\text { Low. } \\
\text { Lev. }\end{array}$ & $\begin{array}{l}\text { - Upp } \\
\text {. Lev }\end{array}$ & $\begin{array}{l}\text { Osc. } \\
\text { Str. }\end{array}$ & $\begin{array}{l}\text { Rad. } \\
\text { Rate }\end{array}$ & $\begin{array}{l}\text { Low. } \\
\text { Lev. }\end{array}$ & $\begin{array}{l}\text { - Upp } \\
\text { Lev }\end{array}$ & $\begin{array}{l}\text { Osc. } \\
\text { Str. }\end{array}$ & $\begin{array}{l}\text { Rad. } \\
\text { Rate }\end{array}$ & $\begin{array}{l}\text { Low. } \\
\text { Lev. }\end{array}$ & $\begin{array}{l}\text {. Upp } \\
\text {. Lev }\end{array}$ & $\begin{array}{l}\text { Osc. } \\
\text { Str. }\end{array}$ & $\begin{array}{l}\text { Rad. } \\
\text { Rate }\end{array}$ \\
\hline$i$ & $j$ & $g f$ & $(1 / s)$ & i & $j$ & $g f$ & $(1 / s)$ & $i$ & j & $g f$ & $(1 / s)$ \\
\hline 92 & 208 & $4.398 \mathrm{e}-02$ & $6.659 e+08$ & 92 & 214 & $4.477 e-03$ & $8.483 e+07$ & 92 & 225 & $7.053 e-03$ & $2.380 e+08$ \\
\hline 92 & 226 & $0.000 e+00$ & $1.934 \mathrm{e}+05$ & 92 & 233 & $0.000 \mathrm{e}+00$ & $1.883 e+05$ & 92 & 238 & $0.000 e+00$ & $3.916 e+05$ \\
\hline 92 & 241 & $0.000 e+00$ & $4.136 \mathrm{e}+04$ & 92 & 242 & $0.000 e+00$ & $3.056 e+05$ & 92 & 275 & $9.578 e-04$ & $1.788 e+07$ \\
\hline 92 & 280 & $0.000 e+00$ & $9.809 e+03$ & 92 & 281 & $0.000 e+00$ & $5.490 e+05$ & 92 & 283 & $3.017 e-03$ & $1.360 e+08$ \\
\hline 93 & 101 & $5.215 e-01$ & $2.220 \mathrm{e}+07$ & 93 & 124 & $1.242 \mathrm{e}-01$ & $1.831 e+08$ & 93 & 134 & $0.000 e+00$ & $1.391 e+04$ \\
\hline 93 & 139 & $1.831 \mathrm{e}-03$ & $5.404 \mathrm{e}+06$ & 93 & 141 & $1.773 e-03$ & $5.446 e+06$ & 93 & 147 & $2.043 e-01$ & $6.722 \mathrm{e}+08$ \\
\hline 93 & 157 & $3.407 e-01$ & $9.267 e+08$ & 93 & 160 & $2.296 \mathrm{e}-01$ & $5.135 e+08$ & 93 & 162 & $8.112 \mathrm{e}-01$ & $2.252 \mathrm{e}+09$ \\
\hline 93 & 163 & $4.942 \mathrm{e}-02$ & $1.767 e+08$ & 93 & 167 & $9.390 \mathrm{e}-01$ & $2.679 e+09$ & 93 & 168 & $3.290 e-02$ & $7.682 \mathrm{e}+07$ \\
\hline 93 & 170 & $1.890 e+00$ & $4.455 e+09$ & 93 & 175 & $1.633 e-01$ & $4.865 e+08$ & 93 & 176 & $2.264 \mathrm{e}-01$ & $8.698 e+08$ \\
\hline 93 & 178 & $2.417 e-01$ & $9.458 e+08$ & 93 & 179 & $0.000 e+00$ & $5.413 e+04$ & 93 & 188 & $0.000 e+00$ & $1.098 e+04$ \\
\hline 93 & 189 & $4.216 \mathrm{e}-02$ & $1.522 \mathrm{e}+08$ & 93 & 190 & $1.183 e-03$ & $4.465 e+06$ & 93 & 192 & $4.787 e-03$ & $1.809 \mathrm{e}+07$ \\
\hline 93 & 193 & $2.085 e-03$ & $1.014 \mathrm{e}+07$ & 93 & 194 & $2.873 e-02$ & $8.920 e+07$ & 93 & 198 & $3.039 e-02$ & $1.050 e+08$ \\
\hline 93 & 199 & $6.593 e-03$ & $2.784 e+07$ & 93 & 201 & $4.962 \mathrm{e}-03$ & $2.733 e+07$ & 93 & 206 & $8.111 \mathrm{e}-02$ & $4.701 e+08$ \\
\hline 93 & 209 & $4.537 e+00$ & $1.894 \mathrm{e}+10$ & 93 & 224 & $1.434 \mathrm{e}-03$ & $2.017 e+07$ & 93 & 238 & $0.000 e+00$ & $2.259 e+04$ \\
\hline 93 & 243 & $8.309 e-03$ & $1.137 \mathrm{e}+08$ & 93 & 251 & $0.000 e+00$ & $1.869 e+05$ & 93 & 255 & $0.000 e+00$ & $7.707 e+03$ \\
\hline 93 & 261 & $0.000 e+00$ & $1.118 e+05$ & 93 & 265 & $0.000 e+00$ & $1.425 e+05$ & 93 & 269 & $0.000 e+00$ & $2.405 e+04$ \\
\hline 93 & 275 & $1.988 \mathrm{e}-02$ & $3.675 e+08$ & 93 & 276 & $0.000 e+00$ & $1.495 e+05$ & 93 & 280 & $0.000 e+00$ & $1.312 e+05$ \\
\hline 93 & 282 & $6.337 e-02$ & $7.689 \mathrm{e}+08$ & 94 & 100 & $8.370 e-01$ & $9.980 e+06$ & 94 & 113 & $7.380 e-03$ & $1.003 e+07$ \\
\hline 94 & 120 & $5.379 e-03$ & $4.618 e+06$ & 94 & 123 & $3.896 \mathrm{e}-03$ & $7.117 e+06$ & 94 & 133 & $0.000 e+00$ & $5.008 e+03$ \\
\hline 94 & 136 & $9.090 e-03$ & $3.504 e+07$ & 94 & 137 & $1.028 \mathrm{e}-01$ & $4.008 e+08$ & 94 & 139 & $4.125 \mathrm{e}-01$ & $1.159 \mathrm{e}+09$ \\
\hline 94 & 140 & $1.809 e-02$ & $7.352 e+07$ & 94 & 141 & $9.192 \mathrm{e}-02$ & $2.690 \mathrm{e}+08$ & 94 & 142 & $4.336 \mathrm{e}-01$ & $9.988 e+08$ \\
\hline 94 & 143 & $3.916 \mathrm{e}-02$ & $1.659 \mathrm{e}+08$ & 94 & 147 & $1.333 \mathrm{e}-02$ & $4.184 e+07$ & 94 & 162 & $1.897 \mathrm{e}-01$ & $5.034 e+08$ \\
\hline 94 & 163 & $7.553 e-03$ & $2.581 e+07$ & 94 & 169 & $0.000 e+00$ & $1.051 e+04$ & 94 & 172 & $0.000 e+00$ & $1.412 \mathrm{e}+04$ \\
\hline 94 & 173 & $0.000 e+00$ & $1.080 e+04$ & 94 & 175 & $2.891 \mathrm{e}+00$ & $8.247 e+09$ & 94 & 176 & $1.656 \mathrm{e}-01$ & $6.090 e+08$ \\
\hline 94 & 177 & $1.115 \mathrm{e}-02$ & $5.836 e+07$ & 94 & 178 & $1.681 \mathrm{e}-01$ & $6.301 \mathrm{e}+08$ & 94 & 189 & $1.072 \mathrm{e}-02$ & $3.720 \mathrm{e}+07$ \\
\hline 94 & 190 & $6.724 \mathrm{e}-02$ & $2.441 \mathrm{e}+08$ & 94 & 191 & $1.383 e-03$ & $9.044 e+06$ & 94 & 192 & $1.004 \mathrm{e}+00$ & $3.652 \mathrm{e}+09$ \\
\hline 94 & 193 & $6.603 e-02$ & $3.091 \mathrm{e}+08$ & 94 & 197 & $5.631 \mathrm{e}-03$ & $2.938 e+07$ & 94 & 199 & $2.392 \mathrm{e}-02$ & $9.742 \mathrm{e}+07$ \\
\hline 94 & 201 & $5.998 e-01$ & $3.187 e+09$ & 94 & 202 & $5.614 \mathrm{e}-02$ & $4.178 e+08$ & 94 & 203 & $5.927 e-01$ & $4.520 e+09$ \\
\hline 94 & 220 & $8.477 e-04$ & $8.786 e+06$ & 94 & 227 & $0.000 e+00$ & $1.588 e+04$ & 94 & 228 & $0.000 e+00$ & $9.825 e+04$ \\
\hline 94 & 229 & $0.000 e+00$ & $1.747 e+05$ & 94 & 231 & $0.000 e+00$ & $2.840 e+04$ & 94 & 232 & $0.000 e+00$ & $1.634 \mathrm{e}+05$ \\
\hline 94 & 234 & $0.000 e+00$ & $2.187 e+05$ & 94 & 235 & $0.000 e+00$ & $6.637 e+03$ & 94 & 236 & $0.000 e+00$ & $5.221 \mathrm{e}+04$ \\
\hline 94 & 237 & $0.000 e+00$ & $1.565 e+05$ & 94 & 238 & $0.000 e+00$ & $1.202 \mathrm{e}+04$ & 94 & 240 & $0.000 e+00$ & $1.413 e+05$ \\
\hline 94 & 241 & $0.000 e+00$ & $1.758 \mathrm{e}+05$ & 94 & 249 & $8.695 \mathrm{e}-04$ & $1.177 e+07$ & 94 & 260 & $0.000 e+00$ & $1.336 e+04$ \\
\hline 94 & 263 & $4.684 \mathrm{e}-04$ & $1.178 \mathrm{e}+07$ & 94 & 264 & $2.577 \mathrm{e}-03$ & $4.630 e+07$ & 94 & 272 & $1.885 e-02$ & $4.765 e+08$ \\
\hline 94 & 277 & $0.000 e+00$ & $5.402 \mathrm{e}+03$ & 95 & 111 & $3.488 e-03$ & $7.215 e+06$ & 95 & 122 & $2.630 \mathrm{e}-03$ & $7.900 e+06$ \\
\hline 95 & 133 & $0.000 e+00$ & $5.279 \mathrm{e}+03$ & 95 & 136 & $7.784 e-03$ & $2.981 e+07$ & 95 & 137 & $1.912 \mathrm{e}-01$ & $7.405 e+08$ \\
\hline 95 & 138 & $7.569 e-02$ & $4.915 e+08$ & 95 & 139 & $1.967 e-02$ & $5.489 e+07$ & 95 & 140 & $1.626 \mathrm{e}-02$ & $6.566 e+07$ \\
\hline 95 & 141 & $2.683 e-01$ & $7.801 \mathrm{e}+08$ & 95 & 143 & $4.869 e-02$ & $2.050 e+08$ & 95 & 145 & $1.308 e-02$ & $9.267 e+07$ \\
\hline 95 & 147 & $7.692 \mathrm{e}-03$ & $2.400 e+07$ & 95 & 151 & $0.000 e+00$ & $7.329 e+02$ & 95 & 152 & $0.000 \mathrm{e}+00$ & $1.037 e+03$ \\
\hline 95 & 156 & $2.053 e-03$ & $1.583 e+07$ & 95 & 163 & $7.567 e-02$ & $2.571 \mathrm{e}+08$ & 95 & 172 & $0.000 e+00$ & $9.014 e+03$ \\
\hline 95 & 174 & $0.000 e+00$ & $2.026 \mathrm{e}+04$ & 95 & 176 & $1.599 \mathrm{e}+00$ & $5.848 e+09$ & 95 & 177 & $3.633 e-01$ & $1.890 e+09$ \\
\hline 95 & 178 & $7.452 \mathrm{e}-01$ & $2.778 \mathrm{e}+09$ & 95 & 191 & $8.848 e-02$ & $5.759 e+08$ & 95 & 193 & $8.527 e-01$ & $3.971 \mathrm{e}+09$ \\
\hline 95 & 196 & $2.469 e-03$ & $1.669 \mathrm{e}+07$ & 95 & 197 & $1.553 e-02$ & $8.067 e+07$ & 95 & 200 & $8.242 e-02$ & $1.016 e+09$ \\
\hline 95 & 201 & $8.955 e-02$ & $4.735 e+08$ & 95 & 202 & $3.695 \mathrm{e}-01$ & $2.737 e+09$ & 95 & 203 & $1.288 \mathrm{e}-01$ & $9.775 e+08$ \\
\hline
\end{tabular}


Table III. Ca IX Oscillator Strengths and Radiative Decay Rates for transitions involving levels higher than 4.

\begin{tabular}{|c|c|c|c|c|c|c|c|c|c|c|c|}
\hline $\begin{array}{l}\text { Low. } \\
\text { Lev. }\end{array}$ & $\begin{array}{l}\text { - Upp } \\
\text {. Lev }\end{array}$ & $\begin{array}{l}\text { Osc. } \\
\text { Str. }\end{array}$ & $\begin{array}{l}\text { Rad. } \\
\text { Rate }\end{array}$ & $\begin{array}{l}\text { Low. } \\
\text { Lev. }\end{array}$ & $\begin{array}{l}\text { - Upp } \\
\text { Lev }\end{array}$ & $\begin{array}{l}\text { Osc. } \\
\text { Str. }\end{array}$ & $\begin{array}{l}\text { Rad. } \\
\text { Rate }\end{array}$ & $\begin{array}{l}\text { Low. } \\
\text { Lev }\end{array}$ & $\begin{array}{l}\text { Upp } \\
\text { Lev }\end{array}$ & $\begin{array}{l}\text { Osc. } \\
\text { Str. }\end{array}$ & $\begin{array}{l}\text { Rad. } \\
\text { Rate }\end{array}$ \\
\hline$i$ & $j$ & $g f$ & $(1 / s)$ & i & $j$ & $g f$ & $(1 / s)$ & $i$ & $j$ & $g f$ & $(1 / s)$ \\
\hline 95 & 204 & $3.330 \mathrm{e}-01$ & $4.216 \mathrm{e}+09$ & 95 & 206 & $1.181 \mathrm{e}-02$ & $6.576 \mathrm{e}+07$ & 95 & 208 & $3.859 e-03$ & $5.531 e+07$ \\
\hline 95 & 219 & $4.988 e-04$ & $6.609 \mathrm{e}+06$ & 95 & 227 & $0.000 \mathrm{e}+00$ & $1.294 \mathrm{e}+05$ & 95 & 228 & $0.000 e+00$ & $5.800 e+04$ \\
\hline 95 & 229 & $0.000 e+00$ & $6.069 \mathrm{e}+04$ & 95 & 230 & $0.000 \mathrm{e}+00$ & $3.271 e+04$ & 95 & 231 & $0.000 e+00$ & $1.198 \mathrm{e}+05$ \\
\hline 95 & 234 & $0.000 e+00$ & $1.426 \mathrm{e}+05$ & 95 & 235 & $0.000 e+00$ & $6.286 e+04$ & 95 & 236 & $0.000 e+00$ & $8.402 e+04$ \\
\hline 95 & 237 & $0.000 e+00$ & $2.913 e+04$ & 95 & 238 & $0.000 e+00$ & $1.831 e+04$ & 95 & 239 & $0.000 e+00$ & $2.924 \mathrm{e}+05$ \\
\hline 95 & 240 & $0.000 e+00$ & $2.880 e+04$ & 95 & 241 & $0.000 \mathrm{e}+00$ & $9.337 e+04$ & 95 & 248 & $5.612 \mathrm{e}-04$ & $9.738 e+06$ \\
\hline 95 & 259 & $0.000 e+00$ & $1.160 \mathrm{e}+04$ & 95 & 262 & $3.437 e-04$ & $1.437 e+07$ & 95 & 263 & $1.214 \mathrm{e}-03$ & $3.047 e+07$ \\
\hline 95 & 272 & $4.443 e-03$ & $1.120 \mathrm{e}+08$ & 95 & 273 & $1.128 \mathrm{e}-02$ & $4.743 e+08$ & 95 & 279 & $0.000 e+00$ & $5.965 e+03$ \\
\hline 95 & 281 & $0.000 e+00$ & $5.090 e+03$ & 96 & 110 & $1.624 \mathrm{e}-03$ & $9.883 e+06$ & 96 & 121 & $1.510 e-03$ & $1.347 e+07$ \\
\hline 96 & 136 & $4.032 \mathrm{e}-02$ & $1.534 \mathrm{e}+08$ & 96 & 137 & $1.934 \mathrm{e}-03$ & $7.443 e+06$ & 96 & 138 & $1.086 \mathrm{e}-01$ & $7.002 e+08$ \\
\hline 96 & 140 & $1.361 \mathrm{e}-01$ & $5.461 e+08$ & 96 & 143 & $7.011 \mathrm{e}-03$ & $2.932 \mathrm{e}+07$ & 96 & 145 & $2.260 \mathrm{e}-02$ & $1.591 \mathrm{e}+08$ \\
\hline 96 & 146 & $7.669 \mathrm{e}-03$ & $1.631 \mathrm{e}+08$ & 96 & 151 & $0.000 \mathrm{e}+00$ & $1.603 e+03$ & 96 & 156 & $1.058 e-03$ & $8.108 e+06$ \\
\hline 96 & 173 & $0.000 e+00$ & $1.289 \mathrm{e}+04$ & 96 & 174 & $0.000 \mathrm{e}+00$ & $1.194 \mathrm{e}+04$ & 96 & 177 & $1.814 \mathrm{e}+00$ & $9.384 e+09$ \\
\hline 96 & 191 & $6.417 e-01$ & $4.155 e+09$ & 96 & 196 & $2.112 \mathrm{e}-03$ & $1.420 \mathrm{e}+07$ & 96 & 200 & $2.292 \mathrm{e}-01$ & $2.812 \mathrm{e}+09$ \\
\hline 96 & 202 & $9.095 e-02$ & $6.704 e+08$ & 96 & 203 & $1.099 \mathrm{e}-02$ & $8.302 e+07$ & 96 & 204 & $1.633 e-01$ & $2.057 e+09$ \\
\hline 96 & 205 & $1.758 e-01$ & $6.656 \mathrm{e}+09$ & 96 & 218 & $3.114 \mathrm{e}-04$ & $5.748 e+06$ & 96 & 227 & $0.000 e+00$ & $7.148 e+04$ \\
\hline 96 & 228 & $0.000 e+00$ & $7.100 e+04$ & 96 & 229 & $0.000 e+00$ & $6.336 e+03$ & 96 & 230 & $0.000 e+00$ & $1.008 e+05$ \\
\hline 96 & 234 & $0.000 e+00$ & $8.062 e+04$ & 96 & 235 & $0.000 e+00$ & $8.356 e+04$ & 96 & 236 & $0.000 e+00$ & $3.087 e+04$ \\
\hline 96 & 240 & $0.000 e+00$ & $1.327 \mathrm{e}+05$ & 96 & 241 & $0.000 e+00$ & $2.386 e+04$ & 96 & 247 & $3.896 e-04$ & $9.436 e+06$ \\
\hline 96 & 258 & $0.000 e+00$ & $1.135 \mathrm{e}+04$ & 96 & 262 & $7.468 e-04$ & $3.114 \mathrm{e}+07$ & 96 & 272 & $3.644 e-04$ & $9.163 e+06$ \\
\hline 96 & 273 & $4.635 e-03$ & $1.944 e+08$ & 96 & 274 & $5.687 e-03$ & $7.156 e+08$ & 97 & 112 & $1.268 \mathrm{e}-02$ & $1.396 e+07$ \\
\hline 97 & 124 & $6.125 \mathrm{e}-02$ & $7.140 e+07$ & 97 & 127 & $3.758 \mathrm{e}-02$ & $1.204 \mathrm{e}+08$ & 97 & 135 & $0.000 e+00$ & $2.800 e+04$ \\
\hline 97 & 136 & $2.250 \mathrm{e}-03$ & $7.736 \mathrm{e}+06$ & 97 & 147 & $9.954 \mathrm{e}-03$ & $2.808 e+07$ & 97 & 156 & $7.701 \mathrm{e}-02$ & $5.384 e+08$ \\
\hline 97 & 163 & $1.678 \mathrm{e}-01$ & $5.177 e+08$ & 97 & 176 & $1.591 \mathrm{e}+00$ & $5.303 e+09$ & 97 & 178 & $3.436 \mathrm{e}+00$ & $1.168 \mathrm{e}+10$ \\
\hline 97 & 183 & $0.000 e+00$ & $5.422 e+04$ & 97 & 193 & $1.732 \mathrm{e}-02$ & $7.430 \mathrm{e}+07$ & 97 & 196 & $6.124 \mathrm{e}-01$ & $3.819 e+09$ \\
\hline 97 & 200 & $4.045 e-03$ & $4.617 e+07$ & 97 & 202 & $3.691 \mathrm{e}-03$ & $2.531 e+07$ & 97 & 203 & $2.378 e-03$ & $1.673 e+07$ \\
\hline 97 & 204 & $3.879 e-03$ & $4.552 \mathrm{e}+07$ & 97 & 206 & $1.964 \mathrm{e}+00$ & $1.015 e+10$ & 97 & 207 & $0.000 e+00$ & $1.514 \mathrm{e}+04$ \\
\hline 97 & 208 & $7.925 e-01$ & $1.058 \mathrm{e}+10$ & 97 & 214 & $1.432 \mathrm{e}-03$ & $2.489 e+07$ & 97 & 224 & $2.046 \mathrm{e}-03$ & $2.675 e+07$ \\
\hline 97 & 225 & $6.479 \mathrm{e}-03$ & $2.011 \mathrm{e}+08$ & 97 & 226 & $0.000 e+00$ & $1.381 \mathrm{e}+04$ & 97 & 233 & $0.000 e+00$ & $9.042 e+04$ \\
\hline 97 & 238 & $0.000 e+00$ & $1.028 \mathrm{e}+05$ & 97 & 241 & $0.000 e+00$ & $1.148 e+04$ & 97 & 242 & $0.000 e+00$ & $4.472 \mathrm{e}+04$ \\
\hline 97 & 250 & $1.639 \mathrm{e}-03$ & $3.863 e+07$ & 97 & 261 & $0.000 e+00$ & $1.997 e+04$ & 97 & 275 & $2.273 e-02$ & $3.943 e+08$ \\
\hline 97 & 280 & $0.000 e+00$ & $5.383 e+03$ & 97 & 281 & $0.000 e+00$ & $3.241 e+05$ & 97 & 283 & $4.310 e-02$ & $1.807 e+09$ \\
\hline 98 & 125 & $1.560 \mathrm{e}-03$ & $4.492 \mathrm{e}+06$ & 98 & 126 & $2.440 \mathrm{e}-03$ & $7.377 e+06$ & 98 & 151 & $8.984 e-02$ & $2.521 e+08$ \\
\hline 98 & 155 & $2.177 \mathrm{e}-01$ & $6.273 e+08$ & 98 & 158 & $5.906 e-02$ & $1.716 e+08$ & 98 & 159 & $5.747 e-01$ & $2.350 e+09$ \\
\hline 98 & 164 & $7.385 e-01$ & $2.231 \mathrm{e}+09$ & 98 & 172 & $2.206 \mathrm{e}-02$ & $6.935 e+07$ & 98 & 173 & $3.065 e-01$ & $1.362 \mathrm{e}+09$ \\
\hline 98 & 174 & $7.080 \mathrm{e}-01$ & $5.298 e+09$ & 98 & 180 & $5.345 e-02$ & $2.491 e+08$ & 98 & 181 & $1.363 e-02$ & $4.545 e+07$ \\
\hline 98 & 183 & $9.198 \mathrm{e}-03$ & $4.341 \mathrm{e}+07$ & 98 & 230 & $2.770 \mathrm{e}-03$ & $4.192 \mathrm{e}+07$ & 98 & 244 & $0.000 e+00$ & $2.420 e+05$ \\
\hline 98 & 247 & $0.000 e+00$ & $2.311 \mathrm{e}+05$ & 98 & 248 & $0.000 e+00$ & $6.876 e+04$ & 98 & 252 & $0.000 e+00$ & $2.557 e+05$ \\
\hline 98 & 254 & $0.000 e+00$ & $4.201 \mathrm{e}+04$ & 98 & 258 & $1.598 \mathrm{e}-03$ & $2.688 e+07$ & 98 & 262 & $0.000 e+00$ & $2.191 \mathrm{e}+05$ \\
\hline 98 & 263 & $0.000 e+00$ & $1.042 \mathrm{e}+05$ & 98 & 272 & $0.000 \mathrm{e}+00$ & $2.138 \mathrm{e}+04$ & 98 & 273 & $0.000 e+00$ & $1.691 \mathrm{e}+05$ \\
\hline 98 & 274 & $0.000 e+00$ & $4.022 \mathrm{e}+05$ & 99 & 128 & $5.549 e-03$ & $1.018 \mathrm{e}+07$ & 99 & 151 & $4.915 e-02$ & $1.379 \mathrm{e}+08$ \\
\hline 99 & 152 & $3.671 \mathrm{e}-02$ & $8.044 \mathrm{e}+07$ & 99 & 155 & $1.582 \mathrm{e}-02$ & $4.557 e+07$ & 99 & 158 & $6.818 \mathrm{e}-01$ & $1.981 \mathrm{e}+09$ \\
\hline 99 & 159 & $2.773 e-01$ & $1.133 e+09$ & 99 & 161 & $2.075 e-01$ & $4.718 e+08$ & 99 & 164 & $9.220 \mathrm{e}-02$ & $2.785 e+08$ \\
\hline 99 & 165 & $1.191 e+00$ & $2.814 \mathrm{e}+09$ & 99 & 172 & $3.756 \mathrm{e}-01$ & $1.181 \mathrm{e}+09$ & 99 & 173 & $8.024 e-01$ & $3.565 e+09$ \\
\hline
\end{tabular}


Table III. Ca IX Oscillator Strengths and Radiative Decay Rates for transitions involving levels higher than 4.

\begin{tabular}{|c|c|c|c|c|c|c|c|c|c|c|c|}
\hline $\begin{array}{l}\text { Low. } \\
\text { Lev. }\end{array}$ & $\begin{array}{l}\text { Upp } \\
\text { Lev }\end{array}$ & $\begin{array}{l}\text { Osc. } \\
\text { Str. }\end{array}$ & $\begin{array}{l}\text { Rad. } \\
\text { Rate }\end{array}$ & $\begin{array}{l}\text { Low. } \\
\text { Lev. }\end{array}$ & $\begin{array}{l}\text { Upp. } \\
\text { Lev. }\end{array}$ & $\begin{array}{l}\text { Osc. } \\
\text { Str. }\end{array}$ & $\begin{array}{l}\text { Rad. } \\
\text { Rate }\end{array}$ & $\begin{array}{l}\text { Low. } \\
\text { Lev. }\end{array}$ & $\begin{array}{l}\text { Upp } \\
\text { Lev }\end{array}$ & $\begin{array}{l}\text { Osc. } \\
\text { Str. }\end{array}$ & $\begin{array}{l}\text { Rad. } \\
\text { Rate }\end{array}$ \\
\hline i & $j$ & $g f$ & $(1 / s)$ & $i$ & $j$ & $g f$ & $(1 / s)$ & $i$ & j & $g f$ & $(1 / s)$ \\
\hline 99 & 179 & $3.886 e-02$ & $9.941 \mathrm{e}+07$ & 99 & 180 & $4.532 e-03$ & $2.111 e+07$ & 99 & 181 & $7.732 \mathrm{e}-02$ & $2.578 e+08$ \\
\hline 99 & 182 & $1.095 \mathrm{e}-02$ & $2.846 \mathrm{e}+07$ & 99 & 183 & $5.369 e-02$ & $2.534 e+08$ & 99 & 231 & $3.537 e-03$ & $4.166 e+07$ \\
\hline 99 & 244 & $0.000 \mathrm{e}+00$ & $3.581 \mathrm{e}+04$ & 99 & 245 & $0.000 e+00$ & $2.526 e+05$ & 99 & 247 & $0.000 e+00$ & $1.076 e+05$ \\
\hline 99 & 248 & $0.000 e+00$ & $1.945 \mathrm{e}+05$ & 99 & 249 & $0.000 e+00$ & $5.600 e+04$ & 99 & 252 & $0.000 e+00$ & $6.995 e+04$ \\
\hline 99 & 254 & $0.000 e+00$ & $2.345 e+05$ & 99 & 256 & $0.000 e+00$ & $3.539 e+04$ & 99 & 259 & $1.978 \mathrm{e}-03$ & $2.589 e+07$ \\
\hline 99 & 262 & $0.000 e+00$ & $1.614 \mathrm{e}+05$ & 99 & 263 & $0.000 e+00$ & $1.562 \mathrm{e}+05$ & 99 & 264 & $0.000 e+00$ & $8.454 e+04$ \\
\hline 99 & 272 & $0.000 e+00$ & $1.102 \mathrm{e}+05$ & 99 & 273 & $0.000 e+00$ & $2.324 e+05$ & 100 & 131 & $8.417 e-03$ & $1.169 \mathrm{e}+07$ \\
\hline 100 & 151 & $1.430 e-03$ & $4.011 e+06$ & 100 & 152 & $4.652 \mathrm{e}-02$ & $1.019 e+08$ & 100 & 153 & $1.162 \mathrm{e}-02$ & $2.095 e+07$ \\
\hline 100 & 155 & $6.712 \mathrm{e}-02$ & $1.934 \mathrm{e}+08$ & 100 & 158 & $3.628 \mathrm{e}-01$ & $1.054 e+09$ & 100 & 161 & $1.159 \mathrm{e}+00$ & $2.635 e+09$ \\
\hline 100 & 164 & $4.482 e-02$ & $1.353 e+08$ & 100 & 165 & $2.897 e-01$ & $6.846 e+08$ & 100 & 169 & $1.817 \mathrm{e}+00$ & $3.546 e+09$ \\
\hline 100 & 172 & $1.114 \mathrm{e}+00$ & $3.502 \mathrm{e}+09$ & 100 & 179 & $2.295 \mathrm{e}-02$ & $5.870 e+07$ & 100 & 182 & $1.047 \mathrm{e}-01$ & $2.720 e+08$ \\
\hline 100 & 232 & $4.456 e-03$ & $4.299 e+07$ & 100 & 245 & $0.000 e+00$ & $3.042 e+04$ & 100 & 246 & $0.000 e+00$ & $2.834 e+05$ \\
\hline 100 & 248 & $0.000 e+00$ & $8.244 \mathrm{e}+04$ & 100 & 249 & $0.000 e+00$ & $2.867 e+05$ & 100 & 254 & $0.000 e+00$ & $5.302 e+04$ \\
\hline 100 & 256 & $0.000 e+00$ & $2.948 e+05$ & 100 & 260 & $2.510 e-03$ & $2.688 e+07$ & 100 & 263 & $0.000 e+00$ & $1.201 \mathrm{e}+05$ \\
\hline 100 & 264 & $0.000 e+00$ & $2.852 \mathrm{e}+05$ & 100 & 272 & $0.000 e+00$ & $2.687 e+05$ & 101 & 109 & $1.154 \mathrm{e}-01$ & $1.833 e+07$ \\
\hline 101 & 134 & $1.891 \mathrm{e}-02$ & $3.899 e+07$ & 101 & 151 & $4.766 e-02$ & $1.273 e+08$ & 101 & 152 & $4.073 e-03$ & $8.498 e+06$ \\
\hline 101 & 155 & $8.216 e-01$ & $2.256 \mathrm{e}+09$ & 101 & 156 & $0.000 e+00$ & $6.268 e+03$ & 101 & 158 & $5.235 e-02$ & $1.450 e+08$ \\
\hline 101 & 159 & $3.166 \mathrm{e}-02$ & $1.233 e+08$ & 101 & 161 & $4.070 e-02$ & $8.823 e+07$ & 101 & 164 & $3.154 \mathrm{e}-01$ & $9.088 e+08$ \\
\hline 101 & 165 & $4.829 e-03$ & $1.089 e+07$ & 101 & 172 & $5.976 e-02$ & $1.794 e+08$ & 101 & 173 & $3.150 \mathrm{e}-02$ & $1.337 e+08$ \\
\hline 101 & 179 & $1.097 e+00$ & $2.683 e+09$ & 101 & 180 & $6.443 e-02$ & $2.870 e+08$ & 101 & 182 & $2.152 \mathrm{e}-02$ & $5.347 e+07$ \\
\hline 101 & 183 & $1.098 e+00$ & $4.956 e+09$ & 101 & 186 & $1.399 \mathrm{e}-03$ & $7.108 e+06$ & 101 & 187 & $3.020 e-02$ & $1.547 e+08$ \\
\hline 101 & 188 & $1.104 \mathrm{e}-01$ & $3.214 \mathrm{e}+08$ & 101 & 213 & $9.439 e-04$ & $1.360 e+07$ & 101 & 226 & $1.921 \mathrm{e}-03$ & $2.809 e+07$ \\
\hline 101 & 242 & $3.918 \mathrm{e}-03$ & $4.806 e+07$ & 101 & 243 & $0.000 e+00$ & $3.250 e+05$ & 101 & 244 & $0.000 e+00$ & $3.645 e+03$ \\
\hline 01 & 250 & $0.000 e+00$ & $3.831 \mathrm{e}+05$ & 101 & 261 & $3.904 e-04$ & $6.478 e+06$ & 101 & 265 & $1.233 e-02$ & $1.593 e+08$ \\
\hline 01 & 269 & $2.268 \mathrm{e}-03$ & $2.934 \mathrm{e}+07$ & 101 & 275 & $0.000 e+00$ & $4.043 e+05$ & 101 & 282 & $0.000 e+00$ & $2.458 e+05$ \\
\hline 01 & 283 & $0.000 e+00$ & $3.816 \mathrm{e}+05$ & 102 & 136 & $6.327 e-03$ & $1.879 \mathrm{e}+07$ & 102 & 137 & $7.903 e-03$ & $2.377 e+07$ \\
\hline 02 & 140 & $1.546 \mathrm{e}-02$ & $4.875 e+07$ & 102 & 141 & $1.679 \mathrm{e}-03$ & $3.816 e+06$ & 102 & 157 & $1.794 \mathrm{e}+00$ & $3.684 e+09$ \\
\hline 02 & 163 & $4.216 \mathrm{e}-01$ & $1.142 \mathrm{e}+09$ & 102 & 167 & $8.868 e-01$ & $1.924 \mathrm{e}+09$ & 102 & 175 & $1.013 e-02$ & $2.309 e+07$ \\
\hline 02 & 176 & $2.165 \mathrm{e}-01$ & $6.367 e+08$ & 102 & 177 & $9.867 e-01$ & $4.134 e+09$ & 102 & 178 & $2.646 e-02$ & $7.948 e+07$ \\
\hline 102 & 190 & $1.204 \mathrm{e}+00$ & $3.586 e+09$ & 102 & 191 & $1.284 \mathrm{e}-01$ & $6.893 e+08$ & 102 & 192 & $8.534 e-02$ & $2.548 e+08$ \\
\hline 02 & 193 & $1.279 \mathrm{e}-02$ & $4.918 \mathrm{e}+07$ & 102 & 197 & $1.943 e-01$ & $8.414 e+08$ & 102 & 199 & $2.694 \mathrm{e}-02$ & $9.109 e+07$ \\
\hline 102 & 218 & $6.724 \mathrm{e}-04$ & $1.113 e+07$ & 102 & 244 & $2.779 e-03$ & $3.386 e+07$ & 102 & 247 & $1.928 \mathrm{e}-03$ & $4.248 e+07$ \\
\hline 102 & 253 & $0.000 e+00$ & $2.011 \mathrm{e}+05$ & 102 & 255 & $0.000 e+00$ & $1.467 e+04$ & 102 & 258 & $0.000 e+00$ & $1.989 e+05$ \\
\hline 102 & 259 & $0.000 e+00$ & $1.001 e+05$ & 102 & 260 & $0.000 e+00$ & $4.719 e+03$ & 102 & 265 & $0.000 e+00$ & $4.744 e+03$ \\
\hline 102 & 266 & $0.000 e+00$ & $1.342 e+05$ & 102 & 267 & $0.000 e+00$ & $2.741 e+05$ & 102 & 268 & $0.000 e+00$ & $7.338 e+04$ \\
\hline 102 & 278 & $0.000 e+00$ & $6.141 e+04$ & 102 & 279 & $0.000 e+00$ & $3.121 e+05$ & 103 & 139 & $1.556 \mathrm{e}-02$ & $3.368 e+07$ \\
\hline 103 & 141 & $2.454 \mathrm{e}-02$ & $5.565 e+07$ & 103 & 142 & $3.018 e-03$ & $5.396 e+06$ & 103 & 157 & $3.119 \mathrm{e}-01$ & $6.391 e+08$ \\
\hline 103 & 160 & $1.491 e+00$ & $2.514 \mathrm{e}+09$ & 103 & 162 & $2.692 \mathrm{e}-01$ & $5.647 e+08$ & 103 & 163 & $1.914 \mathrm{e}-01$ & $5.171 e+08$ \\
\hline 103 & 167 & $8.865 e-02$ & $1.919 \mathrm{e}+08$ & 103 & 168 & $1.813 e+00$ & $3.212 \mathrm{e}+09$ & 103 & 170 & $4.916 \mathrm{e}-02$ & $8.804 e+07$ \\
\hline 103 & 175 & $2.509 \mathrm{e}-01$ & $5.709 e+08$ & 103 & 176 & $5.019 \mathrm{e}-01$ & $1.473 e+09$ & 103 & 178 & $6.321 \mathrm{e}-01$ & $1.895 e+09$ \\
\hline .03 & 190 & $7.611 \mathrm{e}-02$ & $2.264 \mathrm{e}+08$ & 103 & 193 & $1.580 \mathrm{e}-01$ & $6.063 e+08$ & 103 & 194 & $1.728 e+00$ & $4.233 e+09$ \\
\hline 103 & 197 & $9.898 e-03$ & $4.280 e+07$ & 103 & 198 & $3.115 e-02$ & $8.599 e+07$ & 103 & 199 & $2.419 \mathrm{e}-01$ & $8.166 e+08$ \\
\hline 103 & 219 & $9.146 \mathrm{e}-04$ & $1.083 e+07$ & 103 & 245 & $3.441 \mathrm{e}-03$ & $3.430 e+07$ & 103 & 248 & $2.665 e-03$ & $4.193 e+07$ \\
\hline 103 & 251 & $0.000 e+00$ & $4.322 \mathrm{e}+03$ & 103 & 253 & $0.000 e+00$ & $9.793 e+04$ & 103 & 255 & $0.000 e+00$ & $1.802 e+05$ \\
\hline
\end{tabular}


Table III. Ca IX Oscillator Strengths and Radiative Decay Rates for transitions involving levels higher than 4.

\begin{tabular}{|c|c|c|c|c|c|c|c|c|c|c|c|}
\hline $\begin{array}{l}\text { Low. } \\
\text { Lev. }\end{array}$ & $\begin{array}{l}\text { - Upp } \\
\text {. Lev }\end{array}$ & $\begin{array}{l}\text { Osc. } \\
\text { Str. }\end{array}$ & $\begin{array}{l}\text { Rad. } \\
\text { Rate }\end{array}$ & $\begin{array}{l}\text { Low. } \\
\text { Lev. }\end{array}$ & $\begin{array}{l}\text { - Upp } \\
\text { Lev }\end{array}$ & $\begin{array}{l}\text { Osc. } \\
\text { Str. }\end{array}$ & $\begin{array}{l}\text { Rad. } \\
\text { Rate }\end{array}$ & $\begin{array}{l}\text { Low. } \\
\text { Lev }\end{array}$ & $\begin{array}{l}\text { Upp } \\
\text { Lev }\end{array}$ & $\begin{array}{l}\text { Osc. } \\
\text { Str. }\end{array}$ & $\begin{array}{l}\text { Rad. } \\
\text { Rate }\end{array}$ \\
\hline$i$ & $j$ & $g f$ & $(1 / s)$ & i & $j$ & $g f$ & $(1 / s)$ & $i$ & j & $g f$ & $(1 / s)$ \\
\hline 103 & 257 & $0.000 e+00$ & $1.622 \mathrm{e}+04$ & 103 & 258 & $0.000 e+00$ & $5.944 e+04$ & 103 & 259 & $0.000 e+00$ & $1.214 \mathrm{e}+05$ \\
\hline 103 & 260 & $0.000 e+00$ & $1.019 \mathrm{e}+05$ & 103 & 265 & $0.000 e+00$ & $5.385 e+03$ & 103 & 266 & $0.000 e+00$ & $6.479 e+03$ \\
\hline 103 & 267 & $0.000 e+00$ & $6.390 \mathrm{e}+04$ & 103 & 268 & $0.000 e+00$ & $2.161 \mathrm{e}+05$ & 103 & 269 & $0.000 e+00$ & $5.498 e+04$ \\
\hline 103 & 270 & $0.000 e+00$ & $1.328 \mathrm{e}+05$ & 103 & 277 & $0.000 e+00$ & $4.407 e+04$ & 103 & 278 & $0.000 e+00$ & $2.432 \mathrm{e}+05$ \\
\hline 103 & 280 & $0.000 e+00$ & $5.787 e+03$ & 104 & 142 & $5.821 \mathrm{e}-02$ & $1.038 e+08$ & 104 & 157 & $5.550 e-03$ & $1.134 \mathrm{e}+07$ \\
\hline 104 & 160 & $5.758 e-01$ & $9.683 e+08$ & 104 & 162 & $3.440 \mathrm{e}-01$ & $7.198 e+08$ & 104 & 166 & $4.080 e+00$ & $6.069 e+09$ \\
\hline 104 & 167 & $2.303 e-02$ & $4.971 \mathrm{e}+07$ & 104 & 168 & $1.809 \mathrm{e}-01$ & $3.198 e+08$ & 104 & 170 & $2.813 e-01$ & $5.024 e+08$ \\
\hline 104 & 175 & $1.318 e+00$ & $2.991 \mathrm{e}+09$ & 104 & 190 & $7.339 e-03$ & $2.178 \mathrm{e}+07$ & 104 & 192 & $1.879 \mathrm{e}-01$ & $5.588 e+08$ \\
\hline 104 & 194 & $6.851 \mathrm{e}-02$ & $1.674 \mathrm{e}+08$ & 104 & 195 & $2.403 e+00$ & $4.981 e+09$ & 104 & 198 & $3.335 e-01$ & $9.187 e+08$ \\
\hline 104 & 199 & $1.020 \mathrm{e}-02$ & $3.436 \mathrm{e}+07$ & 104 & 220 & $1.287 e-03$ & $1.187 e+07$ & 104 & 246 & $4.218 \mathrm{e}-03$ & $3.556 e+07$ \\
\hline 104 & 249 & $3.652 \mathrm{e}-03$ & $4.464 \mathrm{e}+07$ & 104 & 251 & $0.000 e+00$ & $5.418 e+03$ & 104 & 253 & $0.000 e+00$ & $8.274 e+03$ \\
\hline 104 & 255 & $0.000 e+00$ & $1.008 e+05$ & 104 & 257 & $0.000 e+00$ & $2.928 e+05$ & 104 & 259 & $0.000 e+00$ & $4.504 e+04$ \\
\hline 104 & 260 & $0.000 e+00$ & $1.574 \mathrm{e}+05$ & 104 & 265 & $0.000 e+00$ & $3.932 \mathrm{e}+04$ & 104 & 268 & $0.000 e+00$ & 4.70 \\
\hline 104 & 269 & $0.000 e+00$ & $2.297 e+05$ & 104 & 270 & $0.000 e+00$ & $4.823 e+03$ & 104 & 271 & $0.000 e+00$ & 1.32 \\
\hline 104 & 277 & $0.000 e+00$ & $2.579 \mathrm{e}+05$ & 105 & 124 & $1.192 \mathrm{e}-01$ & $9.424 e+07$ & 105 & 134 & $0.000 e+00$ & 5.29 \\
\hline 105 & 139 & $9.855 e-03$ & $1.913 e+07$ & 105 & 147 & $3.151 \mathrm{e}-01$ & $6.985 e+08$ & 105 & 157 & $3.924 \mathrm{e}-02$ & $7.287 e+07$ \\
\hline 105 & 160 & $1.301 \mathrm{e}+00$ & $1.988 e+09$ & 105 & 162 & $7.222 \mathrm{e}-02$ & $1.374 \mathrm{e}+08$ & 105 & 163 & $6.423 e-02$ & $1.575 e+08$ \\
\hline 105 & 167 & $6.840 e-02$ & $1.345 e+08$ & 105 & 168 & $3.641 \mathrm{e}-01$ & $5.861 e+08$ & 105 & 170 & $5.089 e+00$ & $8.284 e+09$ \\
\hline 105 & 175 & $3.217 e-03$ & $6.666 e+06$ & 105 & 176 & $3.825 e-01$ & $1.022 \mathrm{e}+09$ & 105 & 178 & $5.170 e-01$ & $1.413 e+09$ \\
\hline 105 & 179 & $0.000 e+00$ & $1.180 e+04$ & 105 & 188 & $0.000 e+00$ & $3.775 e+03$ & 105 & 189 & $3.341 \mathrm{e}-01$ & $8.694 e+08$ \\
\hline 105 & 190 & $1.429 \mathrm{e}-03$ & $3.917 e+06$ & 105 & 194 & $2.003 e-02$ & $4.523 e+07$ & 105 & 206 & $2.063 e-01$ & $8.948 e+08$ \\
\hline 105 & 209 & $3.939 e+00$ & $1.253 e+10$ & 105 & 224 & $4.547 e-03$ & $5.333 e+07$ & 105 & 243 & $8.731 \mathrm{e}-03$ & $1.017 e+08$ \\
\hline 105 & 251 & $0.000 e+00$ & $1.462 \mathrm{e}+05$ & 105 & 255 & $0.000 e+00$ & $5.586 e+03$ & 105 & 261 & $0.000 e+00$ & $2.467 e+05$ \\
\hline 05 & 265 & $0.000 e+00$ & $1.576 \mathrm{e}+05$ & 105 & 269 & $0.000 e+00$ & $2.590 e+04$ & 105 & 275 & $1.699 \mathrm{e}-02$ & $2.683 e+08$ \\
\hline 05 & 276 & $0.000 e+00$ & $1.206 \mathrm{e}+05$ & 105 & 280 & $0.000 e+00$ & $2.031 e+05$ & 105 & 282 & $6.745 e-02$ & $7.012 e+08$ \\
\hline 06 & 110 & $4.577 e-02$ & $8.823 e+07$ & 106 & 111 & $2.058 e-02$ & $1.340 \mathrm{e}+07$ & 106 & 114 & $3.367 e-02$ & $2.638 e+07$ \\
\hline .06 & 115 & $5.241 \mathrm{e}-01$ & $4.372 \mathrm{e}+08$ & 106 & 116 & $4.420 e-02$ & $2.227 e+07$ & 106 & 118 & $1.282 \mathrm{e}+00$ & $6.853 e+08$ \\
\hline 06 & 121 & $2.498 e-01$ & $9.097 e+08$ & 106 & 122 & $2.052 \mathrm{e}-01$ & $2.492 \mathrm{e}+08$ & 106 & 123 & $1.535 \mathrm{e}-02$ & $1.125 \mathrm{e}+07$ \\
\hline 06 & 136 & $9.271 \mathrm{e}-03$ & $1.998 \mathrm{e}+07$ & 106 & 137 & $2.906 \mathrm{e}-03$ & $6.359 e+06$ & 106 & 138 & $9.946 e-03$ & $3.655 e+07$ \\
\hline 06 & 140 & $2.835 e-02$ & $6.554 \mathrm{e}+07$ & 106 & 143 & $2.265 e-03$ & $5.532 e+06$ & 106 & 145 & $1.561 \mathrm{e}-02$ & $6.439 \mathrm{e}+07$ \\
\hline 06 & 146 & $1.377 e-02$ & $1.719 \mathrm{e}+08$ & 106 & 148 & $0.000 e+00$ & $9.366 e+03$ & 106 & 149 & $0.000 e+00$ & $9.467 e+03$ \\
\hline 106 & 151 & $0.000 e+00$ & $1.134 \mathrm{e}+04$ & 106 & 171 & $0.000 e+00$ & $6.301 e+03$ & 106 & 177 & $1.822 \mathrm{e}-02$ & $5.849 e+07$ \\
\hline 106 & 180 & $0.000 e+00$ & $2.439 \mathrm{e}+04$ & 106 & 181 & $0.000 e+00$ & $8.986 e+03$ & 106 & 184 & $0.000 e+00$ & $2.845 e+04$ \\
\hline 106 & 186 & $0.000 e+00$ & $5.498 e+03$ & 106 & 191 & $4.568 e-03$ & $1.943 e+07$ & 106 & 205 & $1.463 e-03$ & $3.768 e+07$ \\
\hline 106 & 214 & $3.452 \mathrm{e}-03$ & $4.939 e+07$ & 106 & 215 & $1.008 e-01$ & $2.423 e+09$ & 106 & 216 & $1.572 \mathrm{e}-02$ & $2.269 \mathrm{e}+08$ \\
\hline 106 & 218 & $2.954 \mathrm{e}-01$ & $4.298 e+09$ & 106 & 221 & $2.418 \mathrm{e}-03$ & $3.602 \mathrm{e}+07$ & 106 & 222 & $3.233 e-02$ & $8.027 e+08$ \\
\hline 106 & 223 & $3.941 e-02$ & $2.936 \mathrm{e}+09$ & 106 & 227 & $0.000 e+00$ & $1.987 e+05$ & 106 & 228 & $0.000 e+00$ & $2.053 e+05$ \\
\hline 106 & 229 & $0.000 e+00$ & $1.971 \mathrm{e}+04$ & 106 & 230 & $0.000 e+00$ & $4.347 e+05$ & 106 & 234 & $0.000 e+00$ & $1.062 \mathrm{e}+05$ \\
\hline 106 & 235 & $0.000 e+00$ & $2.437 \mathrm{e}+05$ & 106 & 236 & $0.000 e+00$ & $8.339 e+04$ & 106 & 240 & $0.000 \mathrm{e}+00$ & $1.685 e+05$ \\
\hline 106 & 241 & $0.000 e+00$ & $3.060 e+04$ & 107 & 111 & $1.012 \mathrm{e}-01$ & $6.560 \mathrm{e}+07$ & 107 & 112 & $1.701 \mathrm{e}-02$ & $7.442 e+06$ \\
\hline .07 & 113 & $1.208 \mathrm{e}-02$ & $5.409 \mathrm{e}+06$ & 107 & 114 & $1.347 \mathrm{e}-02$ & $1.051 e+07$ & 107 & 115 & $2.066 \mathrm{e}-01$ & $1.715 e+08$ \\
\hline 07 & 116 & $9.996 \mathrm{e}-01$ & $5.015 e+08$ & 107 & 117 & $2.129 \mathrm{e}-02$ & $7.728 e+06$ & 107 & 118 & $6.605 e-02$ & $3.517 e+07$ \\
\hline 107 & 119 & $1.893 e+00$ & $7.339 e+08$ & 107 & 122 & $5.207 e-01$ & $6.301 e+08$ & 107 & 123 & $2.021 \mathrm{e}-01$ & $1.476 e+08$ \\
\hline .07 & 136 & $2.724 e-03$ & $5.859 \mathrm{e}+06$ & 107 & 137 & $1.904 \mathrm{e}-02$ & $4.158 e+07$ & 107 & 138 & $1.119 \mathrm{e}-02$ & $4.104 e+07$ \\
\hline
\end{tabular}


Table III. Ca IX Oscillator Strengths and Radiative Decay Rates for transitions involving levels higher than 4.

\begin{tabular}{|c|c|c|c|c|c|c|c|c|c|c|c|}
\hline $\begin{array}{l}\text { Low. } \\
\text { Lev . }\end{array}$ & $\begin{array}{l}\text { Upp } \\
\text { Lev }\end{array}$ & $\begin{array}{l}\text { Osc. } \\
\text { Str. }\end{array}$ & $\begin{array}{l}\text { Rad. } \\
\text { Rate }\end{array}$ & $\begin{array}{l}\text { Low. } \\
\text { Lev. }\end{array}$ & $\begin{array}{l}\text { Upp. } \\
\text { Lev. }\end{array}$ & $\begin{array}{l}\text { Osc. } \\
\text { Str. }\end{array}$ & $\begin{array}{l}\text { Rad. } \\
\text { Rate }\end{array}$ & $\begin{array}{l}\text { Low. } \\
\text { Lev. }\end{array}$ & $\begin{array}{l}\text { Upp } \\
\text { Lev }\end{array}$ & $\begin{array}{l}\text { Osc. } \\
\text { Str. }\end{array}$ & $\begin{array}{l}\text { Rad. } \\
\text { Rate }\end{array}$ \\
\hline$i$ & $j$ & $g f$ & $(1 / s)$ & $i$ & $j$ & $g f$ & $(1 / s)$ & $i$ & j & $g f$ & $(1 / s)$ \\
\hline 107 & 139 & $8.782 e-03$ & $1.386 \mathrm{e}+07$ & 107 & 141 & $4.908 e-02$ & $8.176 e+07$ & 107 & 143 & $1.857 \mathrm{e}-02$ & $4.527 e+07$ \\
\hline 107 & 145 & $2.459 \mathrm{e}-02$ & $1.012 \mathrm{e}+08$ & 107 & 148 & $0.000 e+00$ & $1.542 \mathrm{e}+04$ & 107 & 149 & $0.000 e+00$ & $6.650 e+03$ \\
\hline 107 & 150 & $0.000 \mathrm{e}+00$ & $7.443 e+03$ & 107 & 151 & $0.000 e+00$ & $3.211 \mathrm{e}+03$ & 107 & 152 & $0.000 \mathrm{e}+00$ & $1.631 \mathrm{e}+04$ \\
\hline 107 & 171 & $0.000 e+00$ & $1.048 e+04$ & 107 & 176 & $1.292 \mathrm{e}-02$ & $2.899 e+07$ & 107 & 177 & $3.338 e-03$ & $1.070 e+07$ \\
\hline 107 & 178 & $1.258 \mathrm{e}-02$ & $2.893 e+07$ & 107 & 180 & $0.000 e+00$ & $1.825 e+04$ & 107 & 181 & $0.000 e+00$ & $2.360 e+04$ \\
\hline 107 & 182 & $0.000 e+00$ & $7.496 \mathrm{e}+03$ & 107 & 184 & $0.000 e+00$ & $5.248 e+03$ & 107 & 185 & $0.000 e+00$ & $6.366 e+04$ \\
\hline 107 & 186 & $0.000 e+00$ & $2.167 e+04$ & 107 & 193 & $6.321 \mathrm{e}-03$ & $1.922 \mathrm{e}+07$ & 107 & 204 & $2.959 \mathrm{e}-03$ & $2.533 e+07$ \\
\hline 107 & 214 & $3.135 e-03$ & $4.481 e+07$ & 107 & 215 & $3.975 e-02$ & $9.543 e+08$ & 107 & 216 & $1.725 e-01$ & $2.488 e+09$ \\
\hline 107 & 217 & $1.035 e-02$ & $1.068 e+08$ & 107 & 218 & $3.339 \mathrm{e}-02$ & $4.854 e+08$ & 107 & 219 & $4.333 e-01$ & $4.509 e+09$ \\
\hline 107 & 221 & $3.432 \mathrm{e}-02$ & $5.109 e+08$ & 107 & 222 & $8.646 e-02$ & $2.145 e+09$ & 107 & 227 & $0.000 e+00$ & $3.295 e+05$ \\
\hline 107 & 228 & $0.000 e+00$ & $1.405 e+05$ & 107 & 229 & $0.000 e+00$ & $1.615 e+05$ & 107 & 230 & $0.000 e+00$ & $1.195 e+05$ \\
\hline 107 & 231 & $0.000 e+00$ & $4.715 e+05$ & 107 & 234 & $0.000 e+00$ & $1.747 e+05$ & 107 & 235 & $0.000 e+00$ & $1.815 e+05$ \\
\hline 107 & 236 & $0.000 e+00$ & $2.200 \mathrm{e}+05$ & 107 & 237 & $0.000 e+00$ & $6.930 e+04$ & 107 & 238 & $0.000 e+00$ & $9.832 e+03$ \\
\hline 107 & 239 & $0.000 e+00$ & $3.893 e+05$ & 107 & 240 & $0.000 e+00$ & $3.132 e+04$ & 107 & 241 & $0.000 e+00$ & $1.242 \mathrm{e}+05$ \\
\hline 108 & 112 & $7.290 \mathrm{e}-02$ & $3.165 e+07$ & 108 & 113 & $2.528 \mathrm{e}-01$ & $1.123 e+08$ & 108 & 116 & $1.892 \mathrm{e}-01$ & $9.423 e+07$ \\
\hline 108 & 117 & $1.723 e+00$ & $6.210 e+08$ & 108 & 119 & $2.874 \mathrm{e}-02$ & $1.107 e+07$ & 108 & 120 & $2.511 \mathrm{e}+00$ & $7.716 e+08$ \\
\hline 108 & 123 & $9.044 e-01$ & $6.568 e+08$ & 108 & 136 & $2.852 \mathrm{e}-03$ & $6.114 \mathrm{e}+06$ & 108 & 137 & $1.665 \mathrm{e}-02$ & $3.623 e+07$ \\
\hline 108 & 139 & $4.143 e-02$ & $6.515 e+07$ & 108 & 140 & $2.100 e-03$ & $4.830 e+06$ & 108 & 141 & $5.047 e-03$ & $8.380 e+06$ \\
\hline 108 & 142 & $7.972 \mathrm{e}-02$ & $1.046 e+08$ & 108 & 143 & $4.198 e-02$ & $1.020 e+08$ & 108 & 149 & $0.000 e+00$ & $1.048 e+04$ \\
\hline 108 & 150 & $0.000 e+00$ & $1.825 \mathrm{e}+04$ & 108 & 152 & $0.000 e+00$ & $2.978 e+03$ & 108 & 153 & $0.000 e+00$ & $2.305 e+04$ \\
\hline 108 & 162 & $4.769 e-03$ & $7.533 e+06$ & 108 & 171 & $0.000 e+00$ & $1.413 e+04$ & 108 & 175 & $3.383 e-02$ & $5.865 e+07$ \\
\hline 108 & 181 & $0.000 e+00$ & $1.465 \mathrm{e}+04$ & 108 & 182 & $0.000 e+00$ & $3.904 e+04$ & 108 & 184 & $0.000 e+00$ & $2.983 e+04$ \\
\hline 108 & 186 & $0.000 e+00$ & $3.427 e+04$ & 108 & 192 & $7.899 \mathrm{e}-03$ & $1.860 \mathrm{e}+07$ & 108 & 201 & $5.408 e-03$ & $1.917 e+07$ \\
\hline 108 & 203 & $5.753 e-03$ & $2.944 e+07$ & 108 & 216 & $4.670 \mathrm{e}-02$ & $6.726 e+08$ & 108 & 217 & $3.191 \mathrm{e}-01$ & $3.288 e+09$ \\
\hline 08 & 218 & $3.790 e-04$ & $5.502 e+06$ & 108 & 219 & $2.478 \mathrm{e}-02$ & $2.575 e+08$ & 108 & 220 & $5.805 e-01$ & $4.706 e+09$ \\
\hline 08 & 221 & $1.632 \mathrm{e}-01$ & $2.426 \mathrm{e}+09$ & 108 & 227 & $0.000 e+00$ & $3.645 e+04$ & 108 & 228 & $0.000 e+00$ & $2.223 e+05$ \\
\hline .08 & 229 & $0.000 e+00$ & $3.830 \mathrm{e}+05$ & 108 & 230 & $0.000 e+00$ & $6.048 e+03$ & 108 & 231 & $0.000 e+00$ & $8.891 e+04$ \\
\hline 108 & 232 & $0.000 e+00$ & $5.598 e+05$ & 108 & 234 & $0.000 e+00$ & $2.331 e+05$ & 108 & 235 & $0.000 e+00$ & $1.946 e+04$ \\
\hline 108 & 236 & $0.000 e+00$ & $1.408 \mathrm{e}+05$ & 108 & 237 & $0.000 e+00$ & $3.750 e+05$ & 108 & 238 & $0.000 e+00$ & $1.672 \mathrm{e}+04$ \\
\hline 108 & 240 & $0.000 e+00$ & $1.896 \mathrm{e}+05$ & 108 & 241 & $0.000 e+00$ & $2.050 e+05$ & 109 & 111 & $5.371 \mathrm{e}-02$ & $2.492 \mathrm{e}+07$ \\
\hline 109 & 112 & $7.828 e-01$ & $2.503 e+08$ & 109 & 113 & $1.918 \mathrm{e}-01$ & $6.302 \mathrm{e}+07$ & 109 & 114 & $2.641 \mathrm{e}-01$ & $1.522 \mathrm{e}+08$ \\
\hline 109 & 118 & $1.307 e-02$ & $5.250 e+06$ & 109 & 124 & $2.153 e+00$ & $9.690 e+08$ & 109 & 127 & $4.819 e-01$ & $6.543 e+08$ \\
\hline 109 & 136 & $1.391 \mathrm{e}-01$ & $2.614 \mathrm{e}+08$ & 109 & 137 & $3.639 e-03$ & $6.948 e+06$ & 109 & 138 & $4.220 \mathrm{e}-03$ & $1.354 e+07$ \\
\hline 109 & 140 & $4.596 \mathrm{e}-02$ & $9.307 e+07$ & 109 & 143 & $6.759 e-03$ & $1.451 \mathrm{e}+07$ & 109 & 144 & $0.000 e+00$ & $1.519 \mathrm{e}+04$ \\
\hline 109 & 145 & $1.388 \mathrm{e}-03$ & $5.039 e+06$ & 109 & 147 & $1.739 e-02$ & $2.808 e+07$ & 109 & 154 & $0.000 e+00$ & $1.673 e+04$ \\
\hline 109 & 156 & $1.670 \mathrm{e}-01$ & $6.811 e+08$ & 109 & 176 & $7.961 \mathrm{e}-03$ & $1.598 \mathrm{e}+07$ & 109 & 178 & $1.167 \mathrm{e}-02$ & $2.402 \mathrm{e}+07$ \\
\hline 109 & 187 & $0.000 e+00$ & $5.111 e+04$ & 109 & 188 & $0.000 e+00$ & $6.857 e+04$ & 109 & 196 & $1.457 e-02$ & $5.908 e+07$ \\
\hline 109 & 207 & $0.000 e+00$ & $7.896 e+04$ & 109 & 213 & $0.000 e+00$ & $1.161 e+04$ & 109 & 214 & $2.266 \mathrm{e}-01$ & $3.077 e+09$ \\
\hline 109 & 216 & $1.018 \mathrm{e}-03$ & $1.394 \mathrm{e}+07$ & 109 & 217 & $1.036 \mathrm{e}-03$ & $1.015 e+07$ & 109 & 218 & $2.623 e-03$ & $3.624 \mathrm{e}+07$ \\
\hline 109 & 219 & $6.409 e-04$ & $6.338 e+06$ & 109 & 221 & $5.773 e-04$ & $8.170 e+06$ & 109 & 224 & $6.308 e-01$ & $6.486 e+09$ \\
\hline 109 & 225 & $9.996 e-02$ & $2.446 \mathrm{e}+09$ & 109 & 226 & $0.000 e+00$ & $6.726 e+05$ & 109 & 233 & $0.000 e+00$ & $6.613 e+05$ \\
\hline 109 & 238 & $0.000 \mathrm{e}+00$ & $2.873 e+05$ & 109 & 241 & $0.000 e+00$ & $2.407 e+04$ & 109 & 242 & $0.000 e+00$ & $3.241 \mathrm{e}+05$ \\
\hline 109 & 261 & $0.000 e+00$ & $1.205 \mathrm{e}+04$ & 109 & 265 & $0.000 e+00$ & $4.299 e+04$ & 109 & 269 & $0.000 e+00$ & $7.977 e+03$ \\
\hline 109 & 275 & $3.547 e-03$ & $5.003 e+07$ & 109 & 280 & $0.000 e+00$ & $1.764 e+04$ & 109 & 281 & $0.000 e+00$ & $2.039 e+05$ \\
\hline
\end{tabular}


Table III. Ca IX Oscillator Strengths and Radiative Decay Rates for transitions involving levels higher than 4.

\begin{tabular}{|c|c|c|c|c|c|c|c|c|c|c|c|}
\hline $\begin{array}{l}\text { Low. } \\
\text { Lev . }\end{array}$ & $\begin{array}{l}\text { Upp } \\
\text { Lev }\end{array}$ & $\begin{array}{l}\text { Osc. } \\
\text { Str. }\end{array}$ & $\begin{array}{l}\text { Rad. } \\
\text { Rate }\end{array}$ & $\begin{array}{l}\text { Low. } \\
\text { Lev. }\end{array}$ & $\begin{array}{l}\text { Upp } \\
\text {. Lev }\end{array}$ & $\begin{array}{l}\text { Osc. } \\
\text { Str. }\end{array}$ & $\begin{array}{l}\text { Rad. } \\
\text { Rate }\end{array}$ & $\begin{array}{l}\text { Low } \\
\text { Lev }\end{array}$ & $\begin{array}{l}\text { Upp } \\
. \mathrm{Lev}\end{array}$ & $\begin{array}{l}\text { Osc. } \\
\text { Str. }\end{array}$ & $\begin{array}{l}\text { Rad. } \\
\text { Rate }\end{array}$ \\
\hline$i$ & $j$ & $g f$ & $(1 / s)$ & $i$ & $j$ & $g f$ & $(1 / s)$ & $i$ & j & $g f$ & $(1 / s)$ \\
\hline 109 & 283 & $1.563 \mathrm{e}-03$ & $5.351 e+07$ & 110 & 125 & $9.623 e-02$ & $1.904 \mathrm{e}+07$ & 110 & 126 & $7.477 \mathrm{e}-01$ & $1.769 e+08$ \\
\hline 110 & 130 & $1.272 \mathrm{e}-01$ & $3.725 e+07$ & 110 & 137 & $0.000 e+00$ & $6.441 e+03$ & 110 & 148 & $7.393 e-03$ & $1.229 e+07$ \\
\hline 110 & 171 & $3.589 e-03$ & $7.588 e+06$ & 110 & 184 & $1.713 e-02$ & $5.061 e+07$ & 110 & 210 & $5.874 \mathrm{e}-01$ & $7.886 e+09$ \\
\hline 110 & 218 & $0.000 e+00$ & $1.064 \mathrm{e}+04$ & 110 & 227 & $5.775 e-03$ & $1.262 \mathrm{e}+08$ & 110 & 234 & $1.276 \mathrm{e}-03$ & $2.880 e+07$ \\
\hline 110 & 240 & $6.438 e-03$ & $1.521 \mathrm{e}+08$ & 110 & 247 & $0.000 e+00$ & $1.518 e+04$ & 110 & 263 & $0.000 e+00$ & $2.483 e+04$ \\
\hline 110 & 272 & $0.000 e+00$ & $1.524 \mathrm{e}+04$ & 111 & 125 & $5.428 e-01$ & $1.047 e+08$ & 111 & 128 & $1.392 \mathrm{e}+00$ & $2.009 e+08$ \\
\hline 111 & 129 & $3.370 e-01$ & $2.647 e+08$ & 111 & 130 & $3.100 \mathrm{e}-01$ & $8.895 e+07$ & 111 & 132 & $1.131 \mathrm{e}-01$ & $2.154 e+07$ \\
\hline 111 & 133 & $5.690 \mathrm{e}-02$ & $1.952 \mathrm{e}+07$ & 111 & 134 & $1.208 \mathrm{e}-01$ & $3.138 e+07$ & 111 & 135 & $4.317 e-02$ & $7.060 \mathrm{e}+07$ \\
\hline 111 & 136 & $0.000 \mathrm{e}+00$ & $4.350 e+03$ & 111 & 138 & $0.000 e+00$ & $6.093 e+03$ & 111 & 139 & $0.000 e+00$ & $6.418 e+03$ \\
\hline 111 & 148 & $1.162 \mathrm{e}-02$ & $1.916 \mathrm{e}+07$ & 111 & 149 & $1.184 \mathrm{e}-02$ & $1.173 e+07$ & 111 & 154 & $1.309 e-02$ & $2.283 e+07$ \\
\hline 111 & 171 & $1.013 \mathrm{e}-02$ & $2.124 \mathrm{e}+07$ & 111 & 184 & $9.127 e-03$ & $2.678 e+07$ & 111 & 185 & $2.058 e-02$ & $1.812 \mathrm{e}+08$ \\
\hline 111 & 186 & $2.272 \mathrm{e}-02$ & $4.005 e+07$ & 111 & 187 & $2.853 e-03$ & $5.101 e+06$ & 111 & 207 & $1.765 e-03$ & $2.525 e+07$ \\
\hline 111 & 210 & $3.268 e-01$ & $4.373 e+09$ & 111 & 211 & $1.157 e+00$ & $9.304 e+09$ & 111 & 213 & $3.023 e-01$ & $2.489 e+09$ \\
\hline 111 & 218 & $0.000 e+00$ & $4.898 e+03$ & 111 & 219 & $0.000 e+00$ & $1.498 e+04$ & 111 & 224 & $0.000 e+00$ & $1.301 \mathrm{e}+04$ \\
\hline 111 & 227 & $5.795 e-03$ & $1.264 \mathrm{e}+08$ & 111 & 228 & $1.030 e-02$ & $1.348 e+08$ & 111 & 233 & $4.032 e-03$ & $8.872 e+07$ \\
\hline 111 & 234 & $3.080 e-03$ & $6.937 e+07$ & 111 & 235 & $5.182 e-04$ & $7.038 e+06$ & 111 & 238 & $1.527 e-02$ & $2.154 \mathrm{e}+08$ \\
\hline 111 & 239 & $6.448 e-03$ & $4.558 e+08$ & 111 & 240 & $3.690 \mathrm{e}-03$ & $8.697 e+07$ & 111 & 241 & $2.701 \mathrm{e}-03$ & $3.821 e+07$ \\
\hline 111 & 247 & $0.000 e+00$ & $2.172 \mathrm{e}+04$ & 111 & 248 & $0.000 e+00$ & $1.504 e+04$ & 111 & 250 & $0.000 e+00$ & $1.720 e+04$ \\
\hline 111 & 262 & $0.000 e+00$ & $5.604 e+04$ & 111 & 264 & $0.000 e+00$ & $1.782 e+04$ & 111 & 272 & $0.000 e+00$ & $3.367 e+04$ \\
\hline 111 & 273 & $0.000 e+00$ & $1.971 \mathrm{e}+04$ & 111 & 281 & $1.222 \mathrm{e}-03$ & $9.598 e+07$ & 111 & 283 & $0.000 e+00$ & $2.397 e+04$ \\
\hline 112 & 130 & $2.691 \mathrm{e}-02$ & $6.377 e+06$ & 112 & 131 & $3.742 e-01$ & $3.909 e+07$ & 112 & 132 & $3.189 e-01$ & $5.068 e+07$ \\
\hline 112 & 133 & $1.544 \mathrm{e}-01$ & $4.450 e+07$ & 112 & 134 & $3.343 e-02$ & $7.442 \mathrm{e}+06$ & 112 & 142 & $0.000 e+00$ & $1.291 \mathrm{e}+03$ \\
\hline 112 & 144 & $1.514 \mathrm{e}+00$ & $8.952 e+08$ & 112 & 149 & $1.154 \mathrm{e}-02$ & $1.058 e+07$ & 112 & 151 & $7.132 \mathrm{e}-03$ & $4.791 e+06$ \\
\hline 112 & 154 & $2.706 \mathrm{e}-01$ & $4.376 e+08$ & 112 & 171 & $1.159 \mathrm{e}-02$ & $2.270 e+07$ & 112 & 181 & $8.790 e-03$ & $8.280 e+06$ \\
\hline 112 & 183 & $7.096 \mathrm{e}-02$ & $9.556 e+07$ & 112 & 184 & $2.027 e-02$ & $5.613 e+07$ & 112 & 186 & $1.387 e-01$ & $2.308 e+08$ \\
\hline 112 & 187 & $8.713 e-01$ & $1.471 \mathrm{e}+09$ & 112 & 211 & $1.145 \mathrm{e}-01$ & $8.963 e+08$ & 112 & 212 & $4.957 e-01$ & $2.776 e+09$ \\
\hline 112 & 213 & $6.728 e-01$ & $5.393 e+09$ & 112 & 214 & $0.000 e+00$ & $1.606 e+05$ & 112 & 218 & $0.000 e+00$ & $4.331 e+03$ \\
\hline 112 & 220 & $0.000 e+00$ & $9.253 e+03$ & 112 & 224 & $0.000 e+00$ & $1.868 e+05$ & 112 & 225 & $0.000 e+00$ & $5.979 e+04$ \\
\hline 112 & 226 & $2.884 \mathrm{e}-01$ & $2.611 \mathrm{e}+09$ & 112 & 227 & $2.770 e-04$ & $5.914 e+06$ & 112 & 228 & $2.989 e-03$ & $3.831 \mathrm{e}+07$ \\
\hline 112 & 229 & $2.982 \mathrm{e}-03$ & $2.733 e+07$ & 112 & 230 & $2.312 \mathrm{e}-03$ & $2.130 e+07$ & 112 & 233 & $3.820 e-02$ & $8.232 \mathrm{e}+08$ \\
\hline 112 & 234 & $2.132 \mathrm{e}-03$ & $4.703 e+07$ & 112 & 235 & $3.919 e-04$ & $5.213 e+06$ & 112 & 236 & $1.829 \mathrm{e}-03$ & $1.739 \mathrm{e}+07$ \\
\hline 112 & 238 & $9.536 \mathrm{e}-02$ & $1.319 \mathrm{e}+09$ & 112 & 240 & $4.184 \mathrm{e}-03$ & $9.663 e+07$ & 112 & 243 & $0.000 e+00$ & $6.172 \mathrm{e}+05$ \\
\hline 112 & 248 & $0.000 e+00$ & $1.178 \mathrm{e}+04$ & 112 & 250 & $0.000 e+00$ & $4.162 e+05$ & 112 & 261 & $1.683 e-03$ & $1.787 e+07$ \\
\hline 112 & 263 & $0.000 e+00$ & $1.995 e+04$ & 112 & 264 & $0.000 e+00$ & $2.759 e+04$ & 112 & 272 & $0.000 e+00$ & $1.355 e+04$ \\
\hline 112 & 273 & $0.000 e+00$ & $4.150 e+04$ & 112 & 274 & $0.000 e+00$ & $4.851 e+04$ & 112 & 275 & $0.000 e+00$ & $6.160 \mathrm{e}+05$ \\
\hline 112 & 280 & $7.756 e-04$ & $1.190 \mathrm{e}+07$ & 112 & 283 & $0.000 e+00$ & $1.574 \mathrm{e}+05$ & 113 & 128 & $5.249 e-02$ & $5.870 e+06$ \\
\hline 113 & 130 & $1.217 \mathrm{e}-01$ & $2.768 e+07$ & 113 & 131 & $1.659 e+00$ & $1.664 \mathrm{e}+08$ & 113 & 132 & $1.242 \mathrm{e}+00$ & $1.899 e+08$ \\
\hline 113 & 133 & $6.715 e-01$ & $1.864 \mathrm{e}+08$ & 113 & 134 & $4.752 \mathrm{e}-02$ & $1.024 e+07$ & 113 & 141 & $0.000 e+00$ & $5.248 e+03$ \\
\hline 113 & 142 & $0.000 e+00$ & $5.603 e+03$ & 113 & 144 & $3.458 e-01$ & $2.010 e+08$ & 113 & 145 & $0.000 e+00$ & $5.797 e+03$ \\
\hline 113 & 146 & $0.000 e+00$ & $6.711 \mathrm{e}+03$ & 113 & 148 & $7.088 e-03$ & $1.064 \mathrm{e}+07$ & 113 & 149 & $1.924 \mathrm{e}-02$ & $1.735 e+07$ \\
\hline 113 & 150 & $4.780 e-02$ & $3.095 e+07$ & 113 & 151 & $2.513 e-03$ & $1.662 \mathrm{e}+06$ & 113 & 154 & $5.902 e-02$ & $9.395 e+07$ \\
\hline 113 & 171 & $3.376 \mathrm{e}-02$ & $6.519 \mathrm{e}+07$ & 113 & 181 & $5.255 e-03$ & $4.883 e+06$ & 113 & 183 & $2.225 \mathrm{e}-02$ & $2.956 e+07$ \\
\hline 113 & 184 & $4.890 e-02$ & $1.338 \mathrm{e}+08$ & 113 & 186 & $3.972 \mathrm{e}-02$ & $6.530 e+07$ & 113 & 187 & $2.765 e-01$ & $4.613 e+08$ \\
\hline 113 & 210 & $3.034 \mathrm{e}-02$ & $3.931 \mathrm{e}+08$ & 113 & 211 & $2.631 \mathrm{e}-01$ & $2.047 e+09$ & 113 & 212 & $2.085 e+00$ & $1.161 \mathrm{e}+10$ \\
\hline
\end{tabular}


Table III. Ca IX Oscillator Strengths and Radiative Decay Rates for transitions involving levels higher than 4.

\begin{tabular}{|c|c|c|c|c|c|c|c|c|c|c|c|}
\hline $\begin{array}{l}\text { Low. } \\
\text { Lev . }\end{array}$ & $\begin{array}{l}\text { Upp } \\
\text { Lev }\end{array}$ & $\begin{array}{l}\text { Osc. } \\
\text { Str. }\end{array}$ & $\begin{array}{l}\text { Rad. } \\
\text { Rate }\end{array}$ & $\begin{array}{l}\text { Low. } \\
\text { Lev. }\end{array}$ & $\begin{array}{l}\text { Upp. } \\
\text { Lev. }\end{array}$ & $\begin{array}{l}\text { Osc. } \\
\text { Str. }\end{array}$ & $\begin{array}{l}\text { Rad. } \\
\text { Rate }\end{array}$ & $\begin{array}{l}\text { Low. } \\
\text { Lev. }\end{array}$ & $\begin{array}{l}\text { Upp } \\
\text { Lev }\end{array}$ & $\begin{array}{l}\text { Osc. } \\
\text { Str. }\end{array}$ & $\begin{array}{l}\text { Rad. } \\
\text { Rate }\end{array}$ \\
\hline$i$ & $j$ & $g f$ & $(1 / s)$ & $i$ & $j$ & $g f$ & $(1 / s)$ & $i$ & j & $g f$ & $(1 / s)$ \\
\hline 113 & 213 & $1.942 \mathrm{e}-01$ & $1.548 e+09$ & 113 & 214 & $0.000 e+00$ & $3.628 e+04$ & 113 & 217 & $0.000 e+00$ & $1.363 e+04$ \\
\hline 113 & 220 & $0.000 \mathrm{e}+00$ & $2.939 \mathrm{e}+04$ & 113 & 224 & $0.000 e+00$ & $4.198 e+04$ & 113 & 225 & $0.000 \mathrm{e}+00$ & $1.447 e+04$ \\
\hline 113 & 226 & $6.399 \mathrm{e}-02$ & $5.767 e+08$ & 113 & 227 & $1.888 \mathrm{e}-03$ & $4.013 e+07$ & 113 & 228 & $7.371 \mathrm{e}-03$ & $9.407 e+07$ \\
\hline 113 & 229 & $3.186 \mathrm{e}-02$ & $2.907 e+08$ & 113 & 230 & $8.199 \mathrm{e}-04$ & $7.520 e+06$ & 113 & 233 & $8.032 \mathrm{e}-03$ & $1.723 e+08$ \\
\hline 113 & 234 & $8.735 e-03$ & $1.919 e+08$ & 113 & 235 & $1.100 \mathrm{e}-03$ & $1.457 e+07$ & 113 & 236 & $1.127 e-03$ & $1.067 e+07$ \\
\hline 113 & 238 & $8.479 \mathrm{e}-03$ & $1.168 \mathrm{e}+08$ & 113 & 240 & $1.296 \mathrm{e}-02$ & $2.980 e+08$ & 113 & 241 & $4.051 \mathrm{e}-02$ & $5.594 e+08$ \\
\hline 113 & 243 & $0.000 e+00$ & $1.391 \mathrm{e}+05$ & 113 & 247 & $0.000 e+00$ & $1.469 e+04$ & 113 & 248 & $0.000 e+00$ & $1.979 e+04$ \\
\hline 113 & 249 & $0.000 e+00$ & $3.974 \mathrm{e}+04$ & 113 & 250 & $0.000 e+00$ & $9.124 e+04$ & 113 & 262 & $0.000 e+00$ & $2.573 e+04$ \\
\hline 113 & 263 & $0.000 e+00$ & $4.457 e+04$ & 113 & 264 & $0.000 e+00$ & $3.684 e+04$ & 113 & 272 & $0.000 e+00$ & $5.321 e+04$ \\
\hline 113 & 273 & $0.000 e+00$ & $1.087 e+05$ & 113 & 274 & $0.000 e+00$ & $1.525 e+05$ & 113 & 275 & $0.000 e+00$ & $1.564 \mathrm{e}+05$ \\
\hline 113 & 283 & $0.000 e+00$ & $3.788 e+04$ & 114 & 125 & $1.510 \mathrm{e}-01$ & $1.970 e+07$ & 114 & 126 & $1.543 \mathrm{e}-01$ & $2.505 e+07$ \\
\hline 114 & 128 & $1.008 e-01$ & $1.029 \mathrm{e}+07$ & 114 & 129 & $2.068 \mathrm{e}-02$ & $1.167 e+07$ & 114 & 130 & $4.130 e-01$ & $8.646 e+07$ \\
\hline 114 & 133 & $5.431 \mathrm{e}-02$ & $1.400 \mathrm{e}+07$ & 114 & 134 & $1.535 \mathrm{e}+00$ & $3.096 e+08$ & 114 & 135 & $4.217 e-01$ & $5.515 e+08$ \\
\hline 114 & 140 & $0.000 e+00$ & $3.283 e+03$ & 114 & 147 & $0.000 e+00$ & $7.205 e+03$ & 114 & 149 & $1.552 \mathrm{e}-02$ & $1.356 e+07$ \\
\hline 114 & 154 & $6.456 e-02$ & $9.966 e+07$ & 114 & 156 & $0.000 e+00$ & $9.955 e+03$ & 114 & 171 & $5.464 \mathrm{e}-03$ & $1.026 e+07$ \\
\hline 114 & 180 & $2.278 \mathrm{e}-02$ & $2.877 e+07$ & 114 & 184 & $1.198 \mathrm{e}-02$ & $3.203 e+07$ & 114 & 185 & $1.390 \mathrm{e}-03$ & $1.115 e+07$ \\
\hline 114 & 186 & $3.436 \mathrm{e}-03$ & $5.518 e+06$ & 114 & 187 & $4.898 e-02$ & $7.985 e+07$ & 114 & 207 & $1.305 e-02$ & $1.736 e+08$ \\
\hline 114 & 210 & $1.345 e-01$ & $1.724 \mathrm{e}+09$ & 114 & 211 & $1.701 \mathrm{e}-01$ & $1.309 e+09$ & 114 & 213 & $1.528 \mathrm{e}+00$ & $1.205 e+10$ \\
\hline 114 & 218 & $0.000 e+00$ & $1.021 e+04$ & 114 & 224 & $0.000 e+00$ & $5.664 e+04$ & 114 & 225 & $0.000 e+00$ & $1.480 e+04$ \\
\hline 114 & 228 & $6.445 e-03$ & $8.156 e+07$ & 114 & 233 & $2.377 \mathrm{e}-02$ & $5.059 e+08$ & 114 & 234 & $1.824 \mathrm{e}-03$ & $3.973 e+07$ \\
\hline 114 & 235 & $3.686 \mathrm{e}-03$ & $4.842 e+07$ & 114 & 238 & $4.350 e-02$ & $5.943 e+08$ & 114 & 239 & $1.067 e-03$ & $7.301 \mathrm{e}+07$ \\
\hline 114 & 240 & $2.605 \mathrm{e}-03$ & $5.943 e+07$ & 114 & 241 & $1.265 \mathrm{e}-02$ & $1.733 e+08$ & 114 & 248 & $0.000 e+00$ & $1.669 \mathrm{e}+04$ \\
\hline 114 & 250 & $0.000 e+00$ & $9.008 e+04$ & 114 & 263 & $0.000 e+00$ & $1.095 e+04$ & 114 & 272 & $0.000 e+00$ & $1.692 \mathrm{e}+04$ \\
\hline 14 & 275 & $0.000 e+00$ & $4.080 e+04$ & 114 & 280 & $8.403 e-04$ & $1.274 \mathrm{e}+07$ & 114 & 281 & $6.281 \mathrm{e}-03$ & $4.783 e+08$ \\
\hline 14 & 283 & $0.000 e+00$ & $1.293 e+05$ & 115 & 129 & $1.190 \mathrm{e}-02$ & $5.871 e+06$ & 115 & 134 & $2.328 \mathrm{e}-02$ & $4.252 \mathrm{e}+06$ \\
\hline 15 & 135 & $9.568 e-03$ & $1.147 e+07$ & 115 & 148 & $4.565 e-01$ & $6.332 \mathrm{e}+08$ & 115 & 149 & $1.281 \mathrm{e}-01$ & $1.067 e+08$ \\
\hline 15 & 159 & $1.123 \mathrm{e}-02$ & $1.047 e+07$ & 115 & 173 & $2.306 e-02$ & $2.550 e+07$ & 115 & 174 & $1.097 \mathrm{e}-02$ & $2.063 e+07$ \\
\hline 15 & 180 & $1.449 \mathrm{e}+00$ & $1.759 \mathrm{e}+09$ & 115 & 183 & $1.047 e-01$ & $1.303 e+08$ & 115 & 184 & $9.758 e-02$ & $2.519 e+08$ \\
\hline 115 & 185 & $1.699 \mathrm{e}-01$ & $1.316 \mathrm{e}+09$ & 115 & 186 & $3.141 \mathrm{e}-03$ & $4.873 e+06$ & 115 & 210 & $3.381 \mathrm{e}-01$ & $4.266 e+09$ \\
\hline 115 & 211 & $1.268 \mathrm{e}-01$ & $9.612 \mathrm{e}+08$ & 115 & 213 & $3.249 \mathrm{e}-02$ & $2.523 e+08$ & 115 & 215 & $0.000 e+00$ & $5.916 e+04$ \\
\hline 115 & 216 & $0.000 e+00$ & $4.671 \mathrm{e}+04$ & 115 & 218 & $0.000 e+00$ & $1.428 e+05$ & 115 & 219 & $0.000 e+00$ & $5.751 e+04$ \\
\hline 115 & 221 & $0.000 e+00$ & $9.027 e+03$ & 115 & 222 & $0.000 e+00$ & $3.834 e+04$ & 115 & 227 & $6.549 e-02$ & $1.364 e+09$ \\
\hline 115 & 228 & $2.005 e-02$ & $2.507 e+08$ & 115 & 233 & $1.910 \mathrm{e}-03$ & $4.015 e+07$ & 115 & 235 & $2.748 \mathrm{e}-01$ & $3.567 e+09$ \\
\hline 115 & 238 & $7.979 e-04$ & $1.077 e+07$ & 115 & 239 & $1.607 e-02$ & $1.087 e+09$ & 115 & 240 & $8.626 \mathrm{e}-03$ & $1.945 e+08$ \\
\hline 115 & 247 & $0.000 e+00$ & $4.561 e+05$ & 115 & 248 & $0.000 e+00$ & $1.506 e+05$ & 115 & 252 & $0.000 e+00$ & $5.133 e+05$ \\
\hline 115 & 262 & $0.000 e+00$ & $2.260 e+05$ & 115 & 263 & $0.000 e+00$ & $1.680 \mathrm{e}+05$ & 115 & 264 & $0.000 e+00$ & $1.034 \mathrm{e}+04$ \\
\hline 115 & 267 & $5.438 e-04$ & $7.920 e+06$ & 115 & 273 & $0.000 e+00$ & $6.657 e+04$ & 116 & 132 & $4.141 \mathrm{e}-02$ & $5.124 e+06$ \\
\hline 116 & 148 & $1.954 \mathrm{e}-01$ & $2.695 e+08$ & 116 & 149 & $6.164 \mathrm{e}-01$ & $5.110 e+08$ & 116 & 150 & $1.265 \mathrm{e}-01$ & $7.531 e+07$ \\
\hline 116 & 151 & $9.575 e-02$ & $5.825 e+07$ & 116 & 155 & $2.565 e-02$ & $1.652 \mathrm{e}+07$ & 116 & 172 & $1.560 \mathrm{e}-02$ & $1.203 e+07$ \\
\hline 116 & 180 & $4.853 e-01$ & $5.868 e+08$ & 116 & 181 & $2.071 e+00$ & $1.795 e+09$ & 116 & 183 & $2.196 \mathrm{e}-02$ & $2.723 e+07$ \\
\hline 16 & 184 & $3.403 e-01$ & $8.751 \mathrm{e}+08$ & 116 & 186 & $9.008 e-02$ & $1.392 \mathrm{e}+08$ & 116 & 187 & $5.991 \mathrm{e}-03$ & $9.399 e+06$ \\
\hline 16 & 210 & $3.946 e-02$ & $4.970 e+08$ & 116 & 211 & $7.047 e-01$ & $5.331 e+09$ & 116 & 212 & $5.745 e-02$ & $3.110 e+08$ \\
\hline 116 & 213 & $3.039 e-03$ & $2.356 \mathrm{e}+07$ & 116 & 215 & $0.000 e+00$ & $5.788 e+04$ & 116 & 216 & $0.000 e+00$ & $8.366 e+04$ \\
\hline 116 & 217 & $0.000 e+00$ & $3.677 \mathrm{e}+04$ & 116 & 218 & $0.000 e+00$ & $4.595 e+04$ & 116 & 219 & $0.000 e+00$ & $1.586 e+05$ \\
\hline
\end{tabular}


Table III. Ca IX Oscillator Strengths and Radiative Decay Rates for transitions involving levels higher than 4.

\begin{tabular}{|c|c|c|c|c|c|c|c|c|c|c|c|}
\hline $\begin{array}{l}\text { Low. } \\
\text { Lev . }\end{array}$ & $\begin{array}{l}\text { Upp } \\
\text { Lev }\end{array}$ & $\begin{array}{l}\text { Osc. } \\
\text { Str. }\end{array}$ & $\begin{array}{l}\text { Rad. } \\
\text { Rate }\end{array}$ & $\begin{array}{l}\text { Low. } \\
\text { Lev. }\end{array}$ & $\begin{array}{l}\text { Upp. } \\
\text { Lev. }\end{array}$ & $\begin{array}{l}\text { Osc. } \\
\text { Str. }\end{array}$ & $\begin{array}{l}\text { Rad. } \\
\text { Rate }\end{array}$ & $\begin{array}{l}\text { Low. } \\
\text { Lev. }\end{array}$ & $\begin{array}{l}\text { Upp } \\
\text { Lev }\end{array}$ & $\begin{array}{l}\text { Osc. } \\
\text { Str. }\end{array}$ & $\begin{array}{l}\text { Rad. } \\
\text { Rate }\end{array}$ \\
\hline$i$ & $j$ & $g f$ & $(1 / s)$ & $i$ & $j$ & $g f$ & $(1 / s)$ & $i$ & j & $g f$ & $(1 / s)$ \\
\hline 116 & 220 & $0.000 e+00$ & $4.115 e+04$ & 116 & 221 & $0.000 e+00$ & $3.932 \mathrm{e}+04$ & 116 & 222 & $0.000 e+00$ & $2.208 e+04$ \\
\hline 116 & 223 & $0.000 \mathrm{e}+00$ & $2.111 \mathrm{e}+04$ & 116 & 227 & $2.866 \mathrm{e}-02$ & $5.961 e+08$ & 116 & 228 & $8.660 \mathrm{e}-02$ & $1.081 \mathrm{e}+09$ \\
\hline 116 & 229 & $2.364 \mathrm{e}-02$ & $2.110 \mathrm{e}+08$ & 116 & 230 & $2.090 \mathrm{e}-02$ & $1.875 e+08$ & 116 & 235 & $8.175 e-02$ & $1.060 \mathrm{e}+09$ \\
\hline 116 & 236 & $3.688 e-01$ & $3.417 \mathrm{e}+09$ & 116 & 240 & $3.048 e-02$ & $6.864 e+08$ & 116 & 241 & $9.493 e-03$ & $1.283 e+08$ \\
\hline 116 & 244 & $0.000 e+00$ & $4.217 e+04$ & 116 & 247 & $0.000 e+00$ & $3.812 e+05$ & 116 & 248 & $0.000 e+00$ & $3.190 e+05$ \\
\hline 116 & 249 & $0.000 e+00$ & $1.244 \mathrm{e}+05$ & 116 & 252 & $0.000 e+00$ & $2.757 e+05$ & 116 & 254 & $0.000 e+00$ & $4.663 e+05$ \\
\hline 116 & 262 & $0.000 e+00$ & $3.152 \mathrm{e}+05$ & 116 & 263 & $0.000 e+00$ & $1.337 e+05$ & 116 & 264 & $0.000 e+00$ & $1.207 e+05$ \\
\hline 116 & 268 & $7.692 \mathrm{e}-04$ & $7.992 \mathrm{e}+06$ & 116 & 272 & $0.000 e+00$ & $3.795 e+04$ & 116 & 273 & $0.000 e+00$ & $2.766 e+04$ \\
\hline 116 & 274 & $0.000 e+00$ & $1.901 \mathrm{e}+05$ & 117 & 132 & $5.386 e-02$ & $6.492 e+06$ & 117 & 149 & $2.192 \mathrm{e}-01$ & $1.799 e+08$ \\
\hline 117 & 150 & $1.091 \mathrm{e}+00$ & $6.432 \mathrm{e}+08$ & 117 & 151 & $4.792 \mathrm{e}-03$ & $2.886 e+06$ & 117 & 152 & $1.709 \mathrm{e}-01$ & $8.084 e+07$ \\
\hline 117 & 172 & $1.254 \mathrm{e}-02$ & $9.585 e+06$ & 117 & 179 & $6.100 e-02$ & $3.970 e+07$ & 117 & 180 & $2.551 \mathrm{e}-02$ & $3.060 e+07$ \\
\hline 117 & 181 & $5.754 \mathrm{e}-01$ & $4.945 \mathrm{e}+08$ & 117 & 182 & $2.981 e+00$ & $2.002 e+09$ & 117 & 186 & $5.917 e-01$ & $9.075 e+08$ \\
\hline 117 & 187 & $1.617 \mathrm{e}-02$ & $2.519 \mathrm{e}+07$ & 117 & 188 & $6.981 \mathrm{e}-03$ & $6.289 e+06$ & 117 & 211 & $2.023 e-02$ & $1.526 e+08$ \\
\hline 117 & 212 & $1.093 e+00$ & $5.897 e+09$ & 117 & 216 & $0.000 e+00$ & $3.947 e+04$ & 117 & 217 & $0.000 e+00$ & $1.860 \mathrm{e}+05$ \\
\hline 117 & 219 & $0.000 e+00$ & $2.274 \mathrm{e}+04$ & 117 & 220 & $0.000 e+00$ & $2.187 e+05$ & 117 & 221 & $0.000 e+00$ & $7.056 e+04$ \\
\hline 117 & 222 & $0.000 e+00$ & $4.977 e+03$ & 117 & 226 & $8.858 e-04$ & $7.789 e+06$ & 117 & 228 & $3.295 e-02$ & $4.103 e+08$ \\
\hline 117 & 229 & $1.591 \mathrm{e}-01$ & $1.417 \mathrm{e}+09$ & 117 & 230 & $1.639 \mathrm{e}-03$ & $1.467 e+07$ & 117 & 231 & $3.188 \mathrm{e}-02$ & $2.221 \mathrm{e}+08$ \\
\hline 117 & 235 & $4.020 e-03$ & $5.198 e+07$ & 117 & 236 & $9.323 e-02$ & $8.616 e+08$ & 117 & 237 & $5.395 e-01$ & $3.882 e+09$ \\
\hline 117 & 238 & $5.282 e-03$ & $7.104 e+07$ & 117 & 241 & $5.179 e-02$ & $6.985 e+08$ & 117 & 242 & $1.327 e-03$ & $1.000 \mathrm{e}+07$ \\
\hline 117 & 244 & $0.000 e+00$ & $8.128 e+03$ & 117 & 245 & $0.000 e+00$ & $5.413 e+04$ & 117 & 247 & $0.000 e+00$ & $4.832 e+04$ \\
\hline 117 & 248 & $0.000 e+00$ & $2.868 e+05$ & 117 & 249 & $0.000 e+00$ & $5.341 e+05$ & 117 & 252 & $0.000 e+00$ & $3.367 e+04$ \\
\hline 117 & 254 & $0.000 e+00$ & $2.410 e+05$ & 117 & 256 & $0.000 e+00$ & $5.758 e+05$ & 117 & 262 & $0.000 e+00$ & $3.260 e+04$ \\
\hline 117 & 263 & $0.000 e+00$ & $1.954 e+05$ & 117 & 264 & $0.000 e+00$ & $3.217 e+05$ & 117 & 269 & $7.795 e-04$ & $6.285 e+06$ \\
\hline 117 & 272 & $0.000 e+00$ & $1.240 e+05$ & 117 & 273 & $0.000 e+00$ & $9.899 e+04$ & 118 & 144 & $1.280 e-02$ & $6.474 \mathrm{e}+06$ \\
\hline 18 & 148 & $5.788 e-02$ & $7.614 \mathrm{e}+07$ & 118 & 149 & $9.428 e-02$ & $7.454 e+07$ & 118 & 150 & $1.781 \mathrm{e}-02$ & $1.011 \mathrm{e}+07$ \\
\hline 18 & 151 & $1.793 e+00$ & $1.041 \mathrm{e}+09$ & 118 & 154 & $5.724 e-03$ & $8.018 e+06$ & 118 & 155 & $4.440 e-01$ & $2.733 e+08$ \\
\hline 118 & 158 & $2.572 \mathrm{e}-01$ & $1.612 \mathrm{e}+08$ & 118 & 159 & $1.507 e-02$ & $1.336 \mathrm{e}+07$ & 118 & 164 & $1.491 \mathrm{e}-01$ & $1.014 \mathrm{e}+08$ \\
\hline 18 & 173 & $1.033 \mathrm{e}-02$ & $1.091 \mathrm{e}+07$ & 118 & 180 & $4.749 e-01$ & $5.521 e+08$ & 118 & 181 & $3.357 e-01$ & $2.798 e+08$ \\
\hline 118 & 183 & $1.723 \mathrm{e}-02$ & $2.055 e+07$ & 118 & 184 & $1.388 \mathrm{e}-02$ & $3.448 e+07$ & 118 & 186 & $7.005 e-03$ & $1.046 e+07$ \\
\hline 118 & 187 & $1.091 \mathrm{e}-02$ & $1.653 e+07$ & 118 & 190 & $0.000 e+00$ & $6.799 e+03$ & 118 & 210 & $6.698 \mathrm{e}-01$ & $8.305 e+09$ \\
\hline 118 & 211 & $2.565 e-02$ & $1.910 \mathrm{e}+08$ & 118 & 213 & $1.201 \mathrm{e}-02$ & $9.169 e+07$ & 118 & 214 & $0.000 e+00$ & $5.485 e+03$ \\
\hline 118 & 215 & $0.000 e+00$ & $2.408 e+05$ & 118 & 216 & $0.000 e+00$ & $4.106 e+04$ & 118 & 218 & $0.000 e+00$ & $1.215 e+05$ \\
\hline 118 & 219 & $0.000 e+00$ & $7.245 e+03$ & 118 & 221 & $0.000 e+00$ & $7.226 e+03$ & 118 & 222 & $0.000 e+00$ & $1.338 e+05$ \\
\hline 118 & 223 & $0.000 e+00$ & $4.827 e+05$ & 118 & 226 & $2.069 \mathrm{e}-03$ & $1.802 e+07$ & 118 & 227 & $1.108 \mathrm{e}-02$ & $2.277 e+08$ \\
\hline 118 & 228 & $1.636 \mathrm{e}-02$ & $2.018 e+08$ & 118 & 229 & $2.574 \mathrm{e}-03$ & $2.269 e+07$ & 118 & 230 & $5.758 e-01$ & $5.103 e+09$ \\
\hline 118 & 233 & $1.229 \mathrm{e}-03$ & $2.550 e+07$ & 118 & 235 & $5.866 e-02$ & $7.514 e+08$ & 118 & 236 & $5.134 \mathrm{e}-02$ & $4.701 e+08$ \\
\hline 118 & 238 & $1.987 e-03$ & $2.648 e+07$ & 118 & 240 & $1.365 e-03$ & $3.038 e+07$ & 118 & 244 & $0.000 e+00$ & $1.112 \mathrm{e}+06$ \\
\hline 118 & 247 & $0.000 e+00$ & $6.773 e+04$ & 118 & 248 & $0.000 e+00$ & $1.208 e+05$ & 118 & 249 & $0.000 e+00$ & $1.286 e+04$ \\
\hline 118 & 250 & $0.000 e+00$ & $7.992 \mathrm{e}+03$ & 118 & 252 & $0.000 e+00$ & $3.287 e+05$ & 118 & 254 & $0.000 e+00$ & $1.577 e+05$ \\
\hline 118 & 258 & $8.071 \mathrm{e}-03$ & $8.203 e+07$ & 118 & 263 & $0.000 e+00$ & $3.621 e+04$ & 118 & 264 & $0.000 e+00$ & $1.229 \mathrm{e}+04$ \\
\hline 18 & 267 & $3.668 e-04$ & $5.276 \mathrm{e}+06$ & 118 & 279 & $3.509 e-04$ & $8.594 e+06$ & 119 & 149 & $9.281 \mathrm{e}-02$ & $7.221 e+07$ \\
\hline 19 & 150 & $1.396 \mathrm{e}-01$ & $7.799 e+07$ & 119 & 151 & $1.540 \mathrm{e}-01$ & $8.802 e+07$ & 119 & 152 & $2.827 e+00$ & $1.269 \mathrm{e}+09$ \\
\hline 119 & 155 & $3.158 e-02$ & $1.914 \mathrm{e}+07$ & 119 & 158 & $6.948 e-02$ & $4.288 e+07$ & 119 & 161 & $4.144 \mathrm{e}-01$ & $2.017 e+08$ \\
\hline 119 & 165 & $2.189 \mathrm{e}-01$ & $1.157 e+08$ & 119 & 172 & $1.366 \mathrm{e}-02$ & $9.963 e+06$ & 119 & 173 & $1.194 \mathrm{e}-02$ & $1.244 \mathrm{e}+07$ \\
\hline
\end{tabular}


Table III. Ca IX Oscillator Strengths and Radiative Decay Rates for transitions involving levels higher than 4.

\begin{tabular}{|c|c|c|c|c|c|c|c|c|c|c|c|}
\hline $\begin{array}{l}\text { Low } \\
\text { Lev }\end{array}$ & $\begin{array}{l}\text {. Upp } \\
\text {. Lev }\end{array}$ & $\begin{array}{l}\text { Osc. } \\
\text { Str. }\end{array}$ & $\begin{array}{l}\text { Rad. } \\
\text { Rate }\end{array}$ & $\begin{array}{l}\text { Low. } \\
\text { Lev. }\end{array}$ & $\begin{array}{l}\text { Upp. } \\
\text { Lev. }\end{array}$ & $\begin{array}{l}\text { Osc. } \\
\text { Str. }\end{array}$ & $\begin{array}{l}\text { Rad. } \\
\text { Rate }\end{array}$ & $\begin{array}{l}\text { Low. } \\
\text { Lev. }\end{array}$ & $\begin{array}{l}\text { Upp } \\
\text { Lev }\end{array}$ & $\begin{array}{l}\text { Osc. } \\
\text { Str. }\end{array}$ & $\begin{array}{l}\text { Rad. } \\
\text { Rate }\end{array}$ \\
\hline$i$ & $j$ & $g f$ & $(1 / s)$ & $i$ & $j$ & $g f$ & $(1 / s)$ & $i$ & j & $g f$ & $(1 / s)$ \\
\hline 119 & 180 & $6.269 e-02$ & $7.193 e+07$ & 119 & 181 & $6.598 \mathrm{e}-01$ & $5.426 e+08$ & 119 & 182 & $4.600 e-01$ & $2.957 e+08$ \\
\hline 119 & 186 & $2.848 \mathrm{e}-02$ & $4.201 \mathrm{e}+07$ & 119 & 188 & $5.866 e-03$ & $5.088 e+06$ & 119 & 194 & $0.000 e+00$ & $7.806 e+03$ \\
\hline 119 & 211 & $9.798 e-01$ & $7.260 \mathrm{e}+09$ & 119 & 212 & $9.431 \mathrm{e}-03$ & $5.001 e+07$ & 119 & 213 & $5.661 \mathrm{e}-03$ & $4.300 e+07$ \\
\hline 119 & 215 & $0.000 e+00$ & $1.216 \mathrm{e}+05$ & 119 & 216 & $0.000 e+00$ & $1.982 e+05$ & 119 & 217 & $0.000 e+00$ & $1.550 e+04$ \\
\hline 119 & 218 & $0.000 e+00$ & $2.259 \mathrm{e}+04$ & 119 & 219 & $0.000 e+00$ & $1.039 e+05$ & 119 & 221 & $0.000 e+00$ & $6.575 e+04$ \\
\hline 119 & 222 & $0.000 e+00$ & $3.040 \mathrm{e}+05$ & 119 & 228 & $1.802 \mathrm{e}-02$ & $2.214 \mathrm{e}+08$ & 119 & 229 & $2.489 e-02$ & $2.185 e+08$ \\
\hline 119 & 230 & $4.993 e-02$ & $4.407 e+08$ & 119 & 231 & $7.600 \mathrm{e}-01$ & $5.223 e+09$ & 119 & 235 & $9.359 e-03$ & $1.194 \mathrm{e}+08$ \\
\hline 119 & 236 & $8.204 \mathrm{e}-02$ & $7.481 e+08$ & 119 & 237 & $7.332 \mathrm{e}-02$ & $5.206 e+08$ & 119 & 241 & $3.021 \mathrm{e}-03$ & $4.021 e+07$ \\
\hline 119 & 242 & $9.292 \mathrm{e}-04$ & $6.913 e+06$ & 119 & 244 & $0.000 e+00$ & $1.451 e+05$ & 119 & 245 & $0.000 e+00$ & $1.136 e+06$ \\
\hline 119 & 247 & $0.000 e+00$ & $4.286 e+04$ & 119 & 248 & $0.000 e+00$ & $5.275 e+04$ & 119 & 249 & $0.000 e+00$ & $1.425 e+05$ \\
\hline 119 & 252 & $0.000 e+00$ & $1.156 \mathrm{e}+05$ & 119 & 253 & $8.305 e-04$ & $6.528 e+06$ & 119 & 254 & $0.000 e+00$ & $3.096 e+05$ \\
\hline 119 & 256 & $0.000 e+00$ & $1.679 e+05$ & 119 & 258 & $6.615 \mathrm{e}-04$ & $6.698 e+06$ & 119 & 259 & $1.012 \mathrm{e}-02$ & $7.978 e+07$ \\
\hline 119 & 264 & $0.000 e+00$ & $4.983 e+04$ & 119 & 268 & $5.752 \mathrm{e}-04$ & $5.887 e+06$ & 119 & 272 & $0.000 e+00$ & $1.037 e+04$ \\
\hline 119 & 278 & $5.752 e-04$ & $8.415 e+06$ & 120 & 150 & $2.305 e-01$ & $1.260 e+08$ & 120 & 152 & $1.802 \mathrm{e}-01$ & $7.918 e+07$ \\
\hline 120 & 153 & $4.313 e+00$ & $1.571 \mathrm{e}+09$ & 120 & 161 & $8.831 \mathrm{e}-02$ & $4.211 e+07$ & 120 & 169 & $3.811 \mathrm{e}-01$ & $1.649 e+08$ \\
\hline 120 & 172 & $1.199 \mathrm{e}-02$ & $8.578 e+06$ & 120 & 179 & $3.597 e-02$ & $2.199 e+07$ & 120 & 181 & $8.505 e-02$ & $6.871 e+07$ \\
\hline 120 & 182 & $1.284 \mathrm{e}+00$ & $8.111 \mathrm{e}+08$ & 120 & 195 & $0.000 e+00$ & $8.668 e+03$ & 120 & 212 & $1.278 \mathrm{e}+00$ & $6.731 \mathrm{e}+09$ \\
\hline 120 & 216 & $0.000 e+00$ & $7.804 e+04$ & 120 & 217 & $0.000 e+00$ & $2.428 e+05$ & 120 & 219 & $0.000 e+00$ & $8.666 e+03$ \\
\hline 120 & 220 & $0.000 e+00$ & $9.033 e+04$ & 120 & 221 & $0.000 e+00$ & $3.164 e+05$ & 120 & 229 & $4.348 e-02$ & $3.798 e+08$ \\
\hline 120 & 230 & $6.474 \mathrm{e}-04$ & $5.684 e+06$ & 120 & 231 & $5.118 e-02$ & $3.498 e+08$ & 120 & 232 & $1.042 \mathrm{e}+00$ & $5.839 e+09$ \\
\hline 120 & 236 & $1.253 e-02$ & $1.136 \mathrm{e}+08$ & 120 & 237 & $1.791 \mathrm{e}-01$ & $1.265 e+09$ & 120 & 244 & $0.000 e+00$ & $5.905 e+03$ \\
\hline 120 & 245 & $0.000 e+00$ & $1.248 \mathrm{e}+05$ & 120 & 246 & $0.000 e+00$ & $1.312 \mathrm{e}+06$ & 120 & 248 & $0.000 e+00$ & $4.568 e+04$ \\
\hline 120 & 249 & $0.000 e+00$ & $1.697 e+05$ & 120 & 254 & $0.000 e+00$ & $1.118 \mathrm{e}+05$ & 120 & 255 & $1.331 \mathrm{e}-03$ & $8.523 e+06$ \\
\hline 120 & 256 & $0.000 e+00$ & $5.459 e+05$ & 120 & 259 & $6.373 e-04$ & $4.997 e+06$ & 120 & 260 & $1.384 \mathrm{e}-02$ & $8.885 e+07$ \\
\hline 20 & 264 & $0.000 e+00$ & $3.884 \mathrm{e}+04$ & 120 & 269 & $8.347 e-04$ & $6.613 e+06$ & 120 & 277 & $9.600 e-04$ & $9.976 e+06$ \\
\hline 21 & 148 & $2.187 e-01$ & $2.141 \mathrm{e}+08$ & 121 & 171 & $1.413 \mathrm{e}-01$ & $1.882 \mathrm{e}+08$ & 121 & 174 & $9.092 e-03$ & $1.273 e+07$ \\
\hline 21 & 184 & $3.977 \mathrm{e}-01$ & $8.001 \mathrm{e}+08$ & 121 & 210 & $2.525 e-02$ & $2.858 e+08$ & 121 & 216 & $0.000 \mathrm{e}+00$ & $6.924 e+03$ \\
\hline 21 & 218 & $0.000 e+00$ & $7.341 \mathrm{e}+04$ & 121 & 227 & $4.996 \mathrm{e}-02$ & $9.562 e+08$ & 121 & 233 & $8.384 e-04$ & $1.621 \mathrm{e}+07$ \\
\hline 21 & 234 & $2.335 \mathrm{e}-02$ & $4.627 e+08$ & 121 & 240 & $4.611 \mathrm{e}-02$ & $9.587 e+08$ & 121 & 247 & $0.000 e+00$ & $1.465 e+05$ \\
\hline 121 & 263 & $0.000 e+00$ & $2.645 e+05$ & 121 & 272 & $0.000 e+00$ & $1.514 \mathrm{e}+05$ & 121 & 279 & $1.083 e-03$ & $2.487 e+07$ \\
\hline 122 & 148 & $1.457 \mathrm{e}-01$ & $1.426 \mathrm{e}+08$ & 122 & 149 & $5.045 e-01$ & $2.966 e+08$ & 122 & 171 & $4.185 e-01$ & $5.569 e+08$ \\
\hline 122 & 173 & $1.855 e-02$ & $1.520 \mathrm{e}+07$ & 122 & 174 & $7.016 e-03$ & $9.816 e+06$ & 122 & 184 & $3.046 e-01$ & $6.126 e+08$ \\
\hline 122 & 185 & $3.803 e-01$ & $2.295 \mathrm{e}+09$ & 122 & 186 & $4.952 e-01$ & $5.984 e+08$ & 122 & 187 & $4.905 e-03$ & $6.031 e+06$ \\
\hline 122 & 207 & $1.705 e-03$ & $1.825 e+07$ & 122 & 210 & $1.930 \mathrm{e}-02$ & $2.183 e+08$ & 122 & 211 & $3.706 e-02$ & $2.518 e+08$ \\
\hline 122 & 213 & $1.358 \mathrm{e}-03$ & $9.473 e+06$ & 122 & 215 & $0.000 e+00$ & $2.996 e+04$ & 122 & 216 & $0.000 e+00$ & $5.414 e+03$ \\
\hline 122 & 218 & $0.000 e+00$ & $6.761 e+04$ & 122 & 219 & $0.000 e+00$ & $9.781 e+04$ & 122 & 227 & $3.233 e-02$ & $6.187 e+08$ \\
\hline 122 & 228 & $1.144 \mathrm{e}-01$ & $1.314 \mathrm{e}+09$ & 122 & 234 & $6.725 e-02$ & $1.332 \mathrm{e}+09$ & 122 & 238 & $8.880 e-03$ & $1.105 e+08$ \\
\hline 122 & 239 & $4.440 e-02$ & $2.769 e+09$ & 122 & 240 & $3.486 e-02$ & $7.248 e+08$ & 122 & 241 & $4.896 e-02$ & $6.112 \mathrm{e}+08$ \\
\hline 122 & 247 & $0.000 e+00$ & $1.227 e+05$ & 122 & 248 & $0.000 e+00$ & $2.173 e+05$ & 122 & 262 & $0.000 e+00$ & $5.285 e+05$ \\
\hline 122 & 263 & $0.000 e+00$ & $1.075 e+05$ & 122 & 264 & $0.000 e+00$ & $2.142 \mathrm{e}+05$ & 122 & 272 & $0.000 e+00$ & $3.970 e+05$ \\
\hline 122 & 273 & $0.000 e+00$ & $1.759 \mathrm{e}+05$ & 122 & 278 & $2.337 e-03$ & $3.217 e+07$ & 122 & 279 & $7.844 e-04$ & $1.801 e+07$ \\
\hline 122 & 281 & $1.895 e-04$ & $1.322 \mathrm{e}+07$ & 123 & 131 & $2.036 \mathrm{e}-01$ & $5.201 e+06$ & 123 & 132 & $9.070 \mathrm{e}-02$ & $3.826 e+06$ \\
\hline 123 & 133 & $7.478 e-02$ & $6.176 \mathrm{e}+06$ & 123 & 148 & $8.016 e-03$ & $7.786 e+06$ & 123 & 149 & $1.330 \mathrm{e}-01$ & $7.765 e+07$ \\
\hline 123 & 150 & $9.131 \mathrm{e}-01$ & $3.832 \mathrm{e}+08$ & 123 & 171 & $6.685 \mathrm{e}-01$ & $8.842 \mathrm{e}+08$ & 123 & 172 & $2.952 \mathrm{e}-02$ & $1.680 \mathrm{e}+07$ \\
\hline
\end{tabular}


Table III. Ca IX Oscillator Strengths and Radiative Decay Rates for transitions involving levels higher than 4.

\begin{tabular}{|c|c|c|c|c|c|c|c|c|c|c|c|}
\hline $\begin{array}{l}\text { Low. } \\
\text { Lev. }\end{array}$ & $\begin{array}{l}\text { - Upp } \\
\text {. Lev }\end{array}$ & $\begin{array}{l}\text { Osc. } \\
\text { Str. }\end{array}$ & $\begin{array}{l}\text { Rad. } \\
\text { Rate }\end{array}$ & $\begin{array}{l}\text { Low. } \\
\text { Lev. }\end{array}$ & $\begin{array}{l}\text { - Upp } \\
\text { Lev }\end{array}$ & $\begin{array}{l}\text { Osc. } \\
\text { Str. }\end{array}$ & $\begin{array}{l}\text { Rad. } \\
\text { Rate }\end{array}$ & $\begin{array}{l}\text { Low. } \\
\text { Lev }\end{array}$ & $\begin{array}{l}\text { Upp } \\
\text { Lev }\end{array}$ & $\begin{array}{l}\text { Osc. } \\
\text { Str. }\end{array}$ & $\begin{array}{l}\text { Rad. } \\
\text { Rate }\end{array}$ \\
\hline$i$ & $j$ & $g f$ & $(1 / s)$ & i & $j$ & $g f$ & $(1 / s)$ & $i$ & j & $g f$ & $(1 / s)$ \\
\hline 123 & 184 & $4.561 \mathrm{e}-01$ & $9.127 e+08$ & 123 & 186 & $1.400 \mathrm{e}+00$ & $1.684 \mathrm{e}+09$ & 123 & 187 & $7.126 \mathrm{e}-02$ & $8.719 e+07$ \\
\hline 123 & 205 & $0.000 e+00$ & $1.289 \mathrm{e}+04$ & 123 & 210 & $1.069 \mathrm{e}-03$ & $1.207 e+07$ & 123 & 211 & $1.021 \mathrm{e}-02$ & $6.921 \mathrm{e}+07$ \\
\hline 123 & 212 & $2.356 \mathrm{e}-02$ & $1.143 e+08$ & 123 & 213 & $1.407 e-03$ & $9.793 e+06$ & 123 & 215 & $0.000 e+00$ & $1.098 e+04$ \\
\hline 123 & 216 & $0.000 e+00$ & $2.563 e+04$ & 123 & 217 & $0.000 e+00$ & $2.900 e+04$ & 123 & 218 & $0.000 e+00$ & $8.117 e+03$ \\
\hline 123 & 219 & $0.000 e+00$ & $4.178 \mathrm{e}+04$ & 123 & 220 & $0.000 e+00$ & $1.294 \mathrm{e}+05$ & 123 & 227 & $1.579 \mathrm{e}-03$ & $3.017 e+07$ \\
\hline 123 & 228 & $2.773 e-02$ & $3.181 \mathrm{e}+08$ & 123 & 229 & $1.977 \mathrm{e}-01$ & $1.621 \mathrm{e}+09$ & 123 & 234 & $1.027 e-01$ & $2.030 \mathrm{e}+09$ \\
\hline 123 & 238 & $9.432 \mathrm{e}-03$ & $1.172 \mathrm{e}+08$ & 123 & 240 & $5.253 e-02$ & $1.090 e+09$ & 123 & 241 & $1.565 e-01$ & $1.950 e+09$ \\
\hline 123 & 247 & $0.000 e+00$ & $1.149 \mathrm{e}+04$ & 123 & 248 & $0.000 e+00$ & $7.437 e+04$ & 123 & 249 & $0.000 e+00$ & $2.980 e+05$ \\
\hline 123 & 262 & $0.000 e+00$ & $8.728 e+04$ & 123 & 263 & $0.000 \mathrm{e}+00$ & $2.681 e+05$ & 123 & 264 & $0.000 e+00$ & $4.344 e+05$ \\
\hline 123 & 272 & $0.000 e+00$ & $4.141 \mathrm{e}+05$ & 123 & 273 & $0.000 e+00$ & $7.375 e+05$ & 123 & 274 & $0.000 e+00$ & $8.943 e+05$ \\
\hline 123 & 277 & $4.259 \mathrm{e}-03$ & $4.178 e+07$ & 123 & 278 & $7.512 \mathrm{e}-04$ & $1.032 \mathrm{e}+07$ & 124 & 134 & $1.245 \mathrm{e}-01$ & $7.383 e+06$ \\
\hline 124 & 144 & $4.974 \mathrm{e}-01$ & $1.650 \mathrm{e}+08$ & 124 & 151 & $6.258 e-03$ & $2.457 e+06$ & 124 & 155 & $8.321 \mathrm{e}-02$ & $3.50^{\circ}$ \\
\hline 124 & 164 & $2.525 \mathrm{e}-02$ & $1.201 \mathrm{e}+07$ & 124 & 179 & $9.007 e-02$ & $4.103 e+07$ & 124 & 186 & $5.779 e-03$ & e+06 \\
\hline 124 & 187 & $2.773 e-01$ & $3.182 \mathrm{e}+08$ & 124 & 188 & $6.122 \mathrm{e}+00$ & $4.089 e+09$ & 124 & 209 & $0.000 e+00$ & $3.966 e+04$ \\
\hline 124 & 211 & $5.718 e-03$ & $3.774 \mathrm{e}+07$ & 124 & 212 & $2.514 \mathrm{e}-03$ & $1.187 \mathrm{e}+07$ & 124 & 213 & $9.770 e-01$ & $6.620 \mathrm{e}+09$ \\
\hline 124 & 214 & $0.000 e+00$ & $2.609 \mathrm{e}+05$ & 124 & 224 & $0.000 e+00$ & $1.185 e+05$ & 124 & 225 & $0.000 e+00$ & $4.059 e+05$ \\
\hline 124 & 226 & $1.058 e-01$ & $8.392 e+08$ & 124 & 229 & $1.748 e-03$ & $1.404 e+07$ & 124 & 231 & $3.241 \mathrm{e}-03$ & $2.038 e+07$ \\
\hline 124 & 238 & $4.059 e-02$ & $4.944 e+08$ & 124 & 241 & $3.759 \mathrm{e}-03$ & $4.593 e+07$ & 124 & 242 & $1.343 e+00$ & $9.176 e+09$ \\
\hline 124 & 243 & $0.000 e+00$ & $5.097 e+05$ & 124 & 244 & $0.000 e+00$ & $4.018 e+03$ & 124 & 250 & $0.000 e+00$ & $3.452 e+04$ \\
\hline 124 & 265 & $3.173 e-02$ & $2.322 \mathrm{e}+08$ & 124 & 269 & $5.889 e-03$ & $4.318 e+07$ & 124 & 275 & $0.000 e+00$ & $2.033 e+05$ \\
\hline 124 & 280 & $2.741 \mathrm{e}-03$ & $3.729 \mathrm{e}+07$ & 124 & 282 & $0.000 e+00$ & $1.064 e+06$ & 125 & 136 & $2.230 \mathrm{e}+00$ & $5.636 e+08$ \\
\hline 125 & 137 & $1.278 \mathrm{e}-01$ & $3.375 \mathrm{e}+07$ & 125 & 138 & $2.515 \mathrm{e}-01$ & $1.131 \mathrm{e}+08$ & 125 & 145 & $2.833 e-02$ & $1.744 \mathrm{e}+07$ \\
\hline 125 & 156 & $3.421 \mathrm{e}-01$ & $2.765 e+08$ & 125 & 214 & $7.511 \mathrm{e}-01$ & $5.960 e+09$ & 125 & 215 & $5.191 \mathrm{e}-02$ & $6.937 e+08$ \\
\hline 125 & 216 & $6.137 e-02$ & $4.928 e+08$ & 125 & 218 & $2.168 \mathrm{e}-01$ & $1.760 e+09$ & 125 & 221 & $3.871 \mathrm{e}-03$ & $3.242 \mathrm{e}+07$ \\
\hline 25 & 222 & $3.730 e-02$ & $5.207 e+08$ & 125 & 223 & $1.489 \mathrm{e}-03$ & $6.238 e+07$ & 125 & 225 & $1.949 \mathrm{e}-01$ & $2.853 e+09$ \\
\hline 25 & 247 & $3.297 e-03$ & $3.978 e+07$ & 125 & 250 & $4.358 e-03$ & $5.347 e+07$ & 125 & 262 & $1.253 e-03$ & $2.639 \mathrm{e}+07$ \\
\hline 25 & 283 & $1.662 \mathrm{e}-03$ & $3.719 \mathrm{e}+07$ & 126 & 136 & $4.194 \mathrm{e}-02$ & $9.285 e+06$ & 126 & 137 & $2.162 \mathrm{e}+00$ & $5.015 e+08$ \\
\hline 26 & 140 & $5.200 e-01$ & $1.422 \mathrm{e}+08$ & 126 & 143 & $3.302 \mathrm{e}-02$ & $1.055 e+07$ & 126 & 156 & $1.549 \mathrm{e}-01$ & $1.139 \mathrm{e}+08$ \\
\hline 26 & 214 & $1.386 \mathrm{e}-01$ & $1.075 e+09$ & 126 & 215 & $2.893 e-01$ & $3.778 e+09$ & 126 & 216 & $1.296 \mathrm{e}-02$ & $1.017 e+08$ \\
\hline 126 & 218 & $6.715 e-01$ & $5.327 e+09$ & 126 & 222 & $7.089 e-02$ & $9.676 e+08$ & 126 & 223 & $1.021 \mathrm{e}-01$ & $4.183 e+09$ \\
\hline 126 & 225 & $7.165 e-02$ & $1.026 \mathrm{e}+09$ & 126 & 247 & $4.908 e-03$ & $5.812 \mathrm{e}+07$ & 126 & 250 & $2.105 e-03$ & $2.536 e+07$ \\
\hline 126 & 262 & $5.846 e-04$ & $1.209 \mathrm{e}+07$ & 126 & 263 & $2.998 \mathrm{e}-03$ & $3.720 \mathrm{e}+07$ & 126 & 272 & $1.046 \mathrm{e}-03$ & $1.307 e+07$ \\
\hline 126 & 283 & $6.353 e-04$ & $1.396 \mathrm{e}+07$ & 127 & 154 & $5.017 e-01$ & $3.510 e+08$ & 127 & 180 & $1.052 \mathrm{e}-02$ & $6.893 e+06$ \\
\hline 127 & 183 & $2.382 \mathrm{e}-01$ & $1.615 e+08$ & 127 & 185 & $1.354 \mathrm{e}-03$ & $6.138 e+06$ & 127 & 186 & $3.776 \mathrm{e}-02$ & $3.428 e+07$ \\
\hline 127 & 187 & $1.315 e+00$ & $1.218 \mathrm{e}+09$ & 127 & 207 & $5.867 e-01$ & $5.086 e+09$ & 127 & 210 & $6.058 e-04$ & $6.105 \mathrm{e}+06$ \\
\hline 127 & 213 & $7.173 e-03$ & $4.463 e+07$ & 127 & 214 & $0.000 e+00$ & $3.410 e+04$ & 127 & 224 & $0.000 e+00$ & $1.064 \mathrm{e}+05$ \\
\hline 127 & 233 & $6.406 e-02$ & $1.134 \mathrm{e}+09$ & 127 & 234 & $7.549 e-04$ & $1.371 e+07$ & 127 & 238 & $2.550 e-01$ & $2.916 e+09$ \\
\hline 127 & 241 & $2.133 e-02$ & $2.446 e+08$ & 127 & 250 & $0.000 e+00$ & $5.840 e+05$ & 127 & 275 & $0.000 e+00$ & $2.010 e+05$ \\
\hline 127 & 280 & $2.981 \mathrm{e}-03$ & $3.819 \mathrm{e}+07$ & 127 & 281 & $7.581 \mathrm{e}-02$ & $4.880 e+09$ & 127 & 283 & $0.000 e+00$ & $7.884 e+05$ \\
\hline 128 & 136 & $3.003 e-01$ & $6.440 \mathrm{e}+07$ & 128 & 137 & $2.069 \mathrm{e}-02$ & $4.652 \mathrm{e}+06$ & 128 & 138 & $6.298 e-02$ & $2.417 e+07$ \\
\hline 128 & 139 & $3.275 e+00$ & $5.450 \mathrm{e}+08$ & 128 & 140 & $4.382 \mathrm{e}-01$ & $1.164 \mathrm{e}+08$ & 128 & 141 & $7.416 \mathrm{e}-01$ & $1.452 \mathrm{e}+08$ \\
\hline 128 & 143 & $4.029 e-02$ & $1.253 \mathrm{e}+07$ & 128 & 145 & $1.150 \mathrm{e}-02$ & $6.185 e+06$ & 128 & 214 & $1.521 \mathrm{e}-02$ & $1.173 e+08$ \\
\hline 28 & 215 & $7.054 \mathrm{e}-02$ & $9.163 e+08$ & 128 & 216 & $3.639 \mathrm{e}-01$ & $2.841 e+09$ & 128 & 218 & $2.417 e-01$ & $1.907 e+09$ \\
\hline 28 & 219 & $1.342 \mathrm{e}+00$ & $7.585 e+09$ & 128 & 221 & $2.453 e-02$ & $1.999 e+08$ & 128 & 222 & $6.911 \mathrm{e}-02$ & $9.385 e+08$ \\
\hline
\end{tabular}


Table III. Ca IX Oscillator Strengths and Radiative Decay Rates for transitions involving levels higher than 4.

\begin{tabular}{|c|c|c|c|c|c|c|c|c|c|c|c|}
\hline $\begin{array}{l}\text { Low. } \\
\text { Lev . }\end{array}$ & $\begin{array}{l}\text { Upp } \\
\text { Lev }\end{array}$ & $\begin{array}{l}\text { Osc. } \\
\text { Str. }\end{array}$ & $\begin{array}{l}\text { Rad. } \\
\text { Rate }\end{array}$ & $\begin{array}{l}\text { Low. } \\
\text { Lev. }\end{array}$ & $\begin{array}{l}\text { Upp. } \\
\text { Lev. }\end{array}$ & $\begin{array}{l}\text { Osc. } \\
\text { Str. }\end{array}$ & $\begin{array}{l}\text { Rad. } \\
\text { Rate }\end{array}$ & $\begin{array}{l}\text { Low. } \\
\text { Lev. }\end{array}$ & $\begin{array}{l}\text { Upp } \\
\text { Lev }\end{array}$ & $\begin{array}{l}\text { Osc. } \\
\text { Str. }\end{array}$ & $\begin{array}{l}\text { Rad. } \\
\text { Rate }\end{array}$ \\
\hline$i$ & $j$ & $g f$ & $(1 / s)$ & $i$ & $j$ & $g f$ & $(1 / s)$ & $i$ & j & $g f$ & $(1 / s)$ \\
\hline 128 & 224 & $6.535 e-02$ & $3.889 e+08$ & 128 & 225 & $8.678 e-04$ & $1.236 e+07$ & 128 & 247 & $1.447 e-03$ & $1.706 e+07$ \\
\hline 128 & 248 & $1.254 \mathrm{e}-02$ & $1.057 e+08$ & 128 & 259 & $0.000 e+00$ & $4.866 e+03$ & 128 & 262 & $8.373 e-04$ & $1.724 \mathrm{e}+07$ \\
\hline 128 & 263 & $3.295 \mathrm{e}-03$ & $4.071 \mathrm{e}+07$ & 128 & 264 & $3.264 \mathrm{e}-03$ & $2.881 e+07$ & 129 & 138 & $7.799 \mathrm{e}-01$ & $2.791 e+08$ \\
\hline 129 & 145 & $9.385 e-02$ & $4.761 \mathrm{e}+07$ & 129 & 215 & $3.995 e-01$ & $5.128 e+09$ & 129 & 222 & $1.298 \mathrm{e}-01$ & $1.742 \mathrm{e}+09$ \\
\hline 129 & 225 & $1.064 \mathrm{e}-02$ & $1.499 e+08$ & 129 & 262 & $2.073 e-03$ & $4.228 e+07$ & 130 & 137 & $3.073 e-01$ & $5.928 e+07$ \\
\hline 130 & 138 & $4.779 \mathrm{e}-01$ & $1.576 \mathrm{e}+08$ & 130 & 140 & $1.334 \mathrm{e}+00$ & $3.080 e+08$ & 130 & 143 & $3.590 \mathrm{e}-01$ & $9.810 e+07$ \\
\hline 130 & 145 & $3.665 e-02$ & $1.736 \mathrm{e}+07$ & 130 & 146 & $7.433 e-02$ & $1.084 e+08$ & 130 & 156 & $1.531 \mathrm{e}-01$ & $9.865 e+07$ \\
\hline 130 & 214 & $9.693 e-02$ & $7.289 e+08$ & 130 & 215 & $8.660 e-02$ & $1.097 e+09$ & 130 & 216 & $7.986 e-01$ & $6.079 e+09$ \\
\hline 130 & 218 & $7.328 e-02$ & $5.640 \mathrm{e}+08$ & 130 & 221 & $1.642 \mathrm{e}-03$ & $1.305 e+07$ & 130 & 222 & $2.444 e-01$ & $3.238 e+09$ \\
\hline 130 & 223 & $1.571 \mathrm{e}-01$ & $6.245 e+09$ & 130 & 225 & $5.232 \mathrm{e}-02$ & $7.277 e+08$ & 130 & 247 & $8.764 e-04$ & $1.012 \mathrm{e}+07$ \\
\hline 130 & 250 & $1.272 \mathrm{e}-03$ & $1.495 \mathrm{e}+07$ & 130 & 262 & $2.791 \mathrm{e}-03$ & $5.632 e+07$ & 130 & 263 & $2.692 \mathrm{e}-03$ & $3.260 \mathrm{e}+07$ \\
\hline 130 & 272 & $2.982 \mathrm{e}-03$ & $3.636 e+07$ & 130 & 283 & $4.905 e-04$ & $1.053 e+07$ & 131 & 139 & $7.428 e-02$ & $1.039 e+07$ \\
\hline 131 & 140 & $5.408 e-02$ & $1.221 \mathrm{e}+07$ & 131 & 141 & $1.293 e+00$ & $2.157 e+08$ & 131 & 142 & $5.387 e+00$ & $7.348 e+08$ \\
\hline 131 & 143 & $1.509 \mathrm{e}-01$ & $4.041 \mathrm{e}+07$ & 131 & 192 & $1.747 e-02$ & $1.005 e+07$ & 131 & 216 & $5.533 e-02$ & $4.196 e+08$ \\
\hline 131 & 217 & $4.280 e-01$ & $2.324 \mathrm{e}+09$ & 131 & 218 & $1.005 e-02$ & $7.707 e+07$ & 131 & 219 & $2.727 e-01$ & $1.498 e+09$ \\
\hline 131 & 220 & $2.161 \mathrm{e}+00$ & $9.268 e+09$ & 131 & 221 & $4.050 e-02$ & $3.207 e+08$ & 131 & 237 & $0.000 e+00$ & $7.681 \mathrm{e}+03$ \\
\hline 131 & 248 & $1.564 \mathrm{e}-03$ & $1.287 e+07$ & 131 & 249 & $2.167 e-02$ & $1.388 e+08$ & 131 & 260 & $0.000 e+00$ & $6.173 e+03$ \\
\hline 131 & 263 & $1.392 \mathrm{e}-03$ & $1.681 \mathrm{e}+07$ & 131 & 264 & $1.089 e-02$ & $9.389 e+07$ & 132 & 137 & $1.211 \mathrm{e}-01$ & $2.112 \mathrm{e}+07$ \\
\hline 132 & 138 & $2.044 \mathrm{e}-02$ & $6.103 e+06$ & 132 & 139 & $5.350 e-01$ & $6.942 e+07$ & 132 & 140 & $3.442 \mathrm{e}-01$ & $7.250 e+07$ \\
\hline 132 & 141 & $1.927 e+00$ & $3.003 e+08$ & 132 & 143 & $1.049 \mathrm{e}+00$ & $2.635 \mathrm{e}+08$ & 132 & 145 & $3.874 \mathrm{e}-01$ & $1.690 \mathrm{e}+08$ \\
\hline 132 & 214 & $2.393 e-02$ & $1.772 \mathrm{e}+08$ & 132 & 215 & $1.758 e-03$ & $2.193 e+07$ & 132 & 216 & $1.015 \mathrm{e}-01$ & $7.609 e+08$ \\
\hline 132 & 217 & $1.761 \mathrm{e}+00$ & $9.451 e+09$ & 132 & 218 & $6.799 \mathrm{e}-03$ & $5.152 e+07$ & 132 & 219 & $4.431 e-02$ & $2.405 e+08$ \\
\hline 132 & 221 & $5.259 e-01$ & $4.117 e+09$ & 132 & 222 & $1.278 \mathrm{e}-01$ & $1.668 \mathrm{e}+09$ & 132 & 224 & $1.239 e-02$ & $7.089 e+07$ \\
\hline 32 & 248 & $1.372 \mathrm{e}-03$ & $1.118 e+07$ & 132 & 263 & $3.348 e-03$ & $4.005 e+07$ & 132 & 264 & $6.531 \mathrm{e}-03$ & $5.580 e+07$ \\
\hline 32 & 272 & $1.982 \mathrm{e}-03$ & $2.387 e+07$ & 132 & 273 & $6.410 e-04$ & $1.288 e+07$ & 133 & 143 & $1.215 e+00$ & $2.846 e+08$ \\
\hline 33 & 145 & $1.221 \mathrm{e}+00$ & $4.974 \mathrm{e}+08$ & 133 & 146 & $5.199 e-01$ & $6.530 e+08$ & 133 & 156 & $3.799 e-02$ & $2.152 \mathrm{e}+07$ \\
\hline 33 & 214 & $7.836 \mathrm{e}-03$ & $5.727 e+07$ & 133 & 215 & $4.391 e-02$ & $5.407 e+08$ & 133 & 216 & $1.544 \mathrm{e}-01$ & $1.143 e+09$ \\
\hline 33 & 218 & $1.153 e-03$ & $8.626 \mathrm{e}+06$ & 133 & 221 & $9.311 e-01$ & $7.199 e+09$ & 133 & 222 & $2.455 \mathrm{e}-01$ & $3.164 \mathrm{e}+09$ \\
\hline 33 & 223 & $4.973 e-02$ & $1.923 e+09$ & 133 & 225 & $1.073 e-02$ & $1.453 e+08$ & 133 & 272 & $1.629 \mathrm{e}-02$ & $1.942 \mathrm{e}+08$ \\
\hline 33 & 273 & $1.257 e-02$ & $2.500 e+08$ & 133 & 274 & $4.658 \mathrm{e}-03$ & $2.781 e+08$ & 133 & 277 & $0.000 e+00$ & $4.409 e+03$ \\
\hline 33 & 278 & $0.000 e+00$ & $8.163 e+03$ & 133 & 279 & $0.000 e+00$ & $1.174 \mathrm{e}+04$ & 134 & 136 & $2.331 \mathrm{e}-01$ & $2.590 \mathrm{e}+07$ \\
\hline 134 & 140 & $1.570 e-01$ & $2.336 \mathrm{e}+07$ & 134 & 143 & $8.371 \mathrm{e}-02$ & $1.533 e+07$ & 134 & 147 & $3.375 e+00$ & $5.242 e+08$ \\
\hline 134 & 156 & $1.593 e-01$ & $7.376 e+07$ & 134 & 176 & $2.329 e-02$ & $6.795 e+06$ & 134 & 178 & $2.582 \mathrm{e}-02$ & $8.052 e+06$ \\
\hline 134 & 213 & $0.000 e+00$ & $6.593 e+03$ & 134 & 214 & $6.300 e-01$ & $4.416 e+09$ & 134 & 215 & $3.682 \mathrm{e}-03$ & $4.349 e+07$ \\
\hline 134 & 216 & $3.207 e-02$ & $2.276 \mathrm{e}+08$ & 134 & 217 & $1.238 \mathrm{e}-02$ & $6.290 e+07$ & 134 & 219 & $4.051 e-02$ & $2.083 e+08$ \\
\hline 134 & 221 & $2.418 e-02$ & $1.795 e+08$ & 134 & 222 & $6.667 e-03$ & $8.250 e+07$ & 134 & 224 & $1.731 \mathrm{e}+00$ & $9.397 e+09$ \\
\hline 134 & 225 & $3.857 e-02$ & $5.018 e+08$ & 134 & 226 & $0.000 e+00$ & $3.232 e+04$ & 134 & 233 & $0.000 e+00$ & $1.226 e+04$ \\
\hline 134 & 242 & $0.000 e+00$ & $7.055 e+03$ & 134 & 250 & $1.365 e-02$ & $1.517 e+08$ & 134 & 261 & $0.000 e+00$ & $1.748 e+04$ \\
\hline 134 & 265 & $0.000 e+00$ & $1.690 \mathrm{e}+04$ & 134 & 269 & $0.000 e+00$ & $5.334 e+03$ & 134 & 275 & $3.220 \mathrm{e}-02$ & $2.669 e+08$ \\
\hline 134 & 280 & $0.000 e+00$ & $5.526 \mathrm{e}+03$ & 134 & 281 & $0.000 e+00$ & $2.312 e+04$ & 134 & 283 & $5.400 \mathrm{e}-04$ & $1.099 e+07$ \\
\hline 35 & 156 & $8.148 e-01$ & $2.934 \mathrm{e}+08$ & 135 & 215 & $6.141 \mathrm{e}-03$ & $6.918 e+07$ & 135 & 225 & $6.895 e-01$ & $8.577 e+09$ \\
\hline 35 & 238 & $0.000 \mathrm{e}+00$ & $5.757 e+03$ & 135 & 280 & $0.000 e+00$ & $1.962 e+04$ & 135 & 283 & $1.436 e-02$ & $2.819 \mathrm{e}+08$ \\
\hline 36 & 151 & $1.930 e-01$ & $3.731 \mathrm{e}+06$ & 136 & 155 & $1.209 \mathrm{e}+00$ & $3.161 \mathrm{e}+07$ & 136 & 183 & $1.338 \mathrm{e}-01$ & $1.688 e+07$ \\
\hline 36 & 187 & $2.627 e-02$ & $6.417 e+06$ & 136 & 226 & $9.891 \mathrm{e}-01$ & $5.362 e+09$ & 136 & 227 & $1.125 \mathrm{e}-02$ & $1.442 \mathrm{e}+08$ \\
\hline
\end{tabular}


Table III. Ca IX Oscillator Strengths and Radiative Decay Rates for transitions involving levels higher than 4.

\begin{tabular}{|c|c|c|c|c|c|c|c|c|c|c|c|}
\hline $\begin{array}{l}\text { Low } \\
\text { Lev }\end{array}$ & $\begin{array}{l}\text {. Upp } \\
\text {. Lev }\end{array}$ & $\begin{array}{l}\text { Osc. } \\
\text { Str. }\end{array}$ & $\begin{array}{l}\text { Rad. } \\
\text { Rate }\end{array}$ & $\begin{array}{l}\text { Low. } \\
\text { Lev. }\end{array}$ & $\begin{array}{l}\text { Upp. } \\
\text { Lev. }\end{array}$ & $\begin{array}{l}\text { Osc. } \\
\text { Str. }\end{array}$ & $\begin{array}{l}\text { Rad. } \\
\text { Rate }\end{array}$ & $\begin{array}{l}\text { Low. } \\
\text { Lev. }\end{array}$ & $\begin{array}{l}\text { Upp } \\
\text { Lev }\end{array}$ & $\begin{array}{l}\text { Osc. } \\
\text { Str. }\end{array}$ & $\begin{array}{l}\text { Rad. } \\
\text { Rate }\end{array}$ \\
\hline$i$ & $j$ & $g f$ & $(1 / s)$ & $i$ & $j$ & $g f$ & $(1 / s)$ & $i$ & j & $g f$ & $(1 / s)$ \\
\hline 136 & 228 & $5.905 e-02$ & $4.547 e+08$ & 136 & 229 & $3.996 \mathrm{e}-03$ & $2.200 \mathrm{e}+07$ & 136 & 230 & $3.354 e-01$ & $1.859 \mathrm{e}+09$ \\
\hline 136 & 233 & $1.782 \mathrm{e}-01$ & $2.312 \mathrm{e}+09$ & 136 & 234 & $2.378 e-02$ & $3.182 \mathrm{e}+08$ & 136 & 235 & $8.768 \mathrm{e}-02$ & $7.083 e+08$ \\
\hline 136 & 236 & $4.524 \mathrm{e}-02$ & $2.612 \mathrm{e}+08$ & 136 & 238 & $5.793 e-01$ & $4.917 e+09$ & 136 & 240 & $2.209 \mathrm{e}-02$ & $3.134 \mathrm{e}+08$ \\
\hline 136 & 241 & $2.634 \mathrm{e}-03$ & $2.244 \mathrm{e}+07$ & 136 & 258 & $6.181 \mathrm{e}-03$ & $4.068 e+07$ & 136 & 261 & $9.712 \mathrm{e}-03$ & $6.455 e+07$ \\
\hline 136 & 267 & $1.720 e-03$ & $1.606 \mathrm{e}+07$ & 136 & 277 & $1.168 \mathrm{e}-03$ & $7.988 e+06$ & 136 & 280 & $1.186 \mathrm{e}-02$ & $1.148 e+08$ \\
\hline 137 & 151 & $1.259 \mathrm{e}-01$ & $2.117 \mathrm{e}+06$ & 137 & 158 & $1.352 \mathrm{e}+00$ & $3.437 e+07$ & 137 & 210 & $1.851 \mathrm{e}-03$ & $1.209 \mathrm{e}+07$ \\
\hline 137 & 211 & $1.369 \mathrm{e}-02$ & $5.375 e+07$ & 137 & 212 & $9.101 \mathrm{e}-03$ & $2.558 e+07$ & 137 & 226 & $1.681 \mathrm{e}-02$ & $9.040 e+07$ \\
\hline 137 & 227 & $2.323 e-01$ & $2.956 \mathrm{e}+09$ & 137 & 228 & $5.278 e-01$ & $4.032 e+09$ & 137 & 229 & $4.666 e-03$ & $2.549 e+07$ \\
\hline 137 & 230 & $4.749 e-01$ & $2.612 \mathrm{e}+09$ & 137 & 233 & $1.565 e-02$ & $2.016 e+08$ & 137 & 234 & $4.474 e-02$ & $5.938 e+08$ \\
\hline 137 & 235 & $3.472 \mathrm{e}-01$ & $2.783 e+09$ & 137 & 236 & $3.217 \mathrm{e}-01$ & $1.843 e+09$ & 137 & 238 & $1.670 \mathrm{e}-02$ & $1.407 e+08$ \\
\hline 137 & 240 & $1.925 e-01$ & $2.709 e+09$ & 137 & 247 & $0.000 e+00$ & $6.359 e+03$ & 137 & 248 & $0.000 e+00$ & $6.676 e+03$ \\
\hline 137 & 258 & $6.118 e-03$ & $3.996 e+07$ & 137 & 268 & $5.438 e-03$ & $3.601 e+07$ & 137 & 277 & $3.694 \mathrm{e}-03$ & $2.507 e+07$ \\
\hline 137 & 278 & $5.240 e-04$ & $4.984 e+06$ & 138 & 159 & $8.767 e-01$ & $3.094 e+07$ & 138 & 185 & $6.442 \mathrm{e}-03$ & $7.036 e+06$ \\
\hline 138 & 207 & $3.364 \mathrm{e}-03$ & $1.164 \mathrm{e}+07$ & 138 & 210 & $1.285 \mathrm{e}-02$ & $8.346 e+07$ & 138 & 211 & 8.490 & $3.313 e+07$ \\
\hline 138 & 227 & $5.764 e-01$ & $7.304 e+09$ & 138 & 228 & $6.272 \mathrm{e}-02$ & $4.772 e+08$ & 138 & 233 & $6.141 \mathrm{e}-03$ & $7.876 e+07$ \\
\hline 138 & 234 & $1.479 \mathrm{e}-02$ & $1.955 e+08$ & 138 & 235 & $5.029 e-01$ & $4.015 e+09$ & 138 & 238 & $2.478 \mathrm{e}-03$ & $2.079 e+07$ \\
\hline 138 & 239 & $1.200 e-01$ & $5.048 e+09$ & 138 & 240 & $2.179 \mathrm{e}-02$ & $3.056 e+08$ & 138 & 241 & $8.047 e-04$ & $6.776 e+06$ \\
\hline 138 & 247 & $0.000 e+00$ & $1.234 \mathrm{e}+04$ & 138 & 252 & $0.000 e+00$ & $1.347 e+04$ & 138 & 267 & $4.369 e-03$ & $4.035 e+07$ \\
\hline 138 & 278 & $2.192 \mathrm{e}-03$ & $2.078 e+07$ & 138 & 279 & $6.404 \mathrm{e}-04$ & $1.012 \mathrm{e}+07$ & 138 & 281 & $4.066 e-03$ & $1.958 e+08$ \\
\hline 139 & 152 & $1.782 \mathrm{e}-01$ & $2.205 e+06$ & 139 & 161 & $1.750 e+00$ & $3.380 e+07$ & 139 & 211 & $1.824 \mathrm{e}-03$ & $7.096 e+06$ \\
\hline 139 & 212 & $2.694 \mathrm{e}-02$ & $7.504 e+07$ & 139 & 213 & $5.190 e-03$ & $2.090 e+07$ & 139 & 226 & $8.429 e-02$ & $4.504 e+08$ \\
\hline 139 & 228 & $2.680 \mathrm{e}-01$ & $2.035 e+09$ & 139 & 229 & $6.018 \mathrm{e}-01$ & $3.267 e+09$ & 139 & 230 & $5.848 e-02$ & $3.196 e+08$ \\
\hline 139 & 231 & $8.539 e-01$ & $3.634 \mathrm{e}+09$ & 139 & 235 & $3.205 e-02$ & $2.553 e+08$ & 139 & 236 & $4.802 \mathrm{e}-01$ & $2.734 \mathrm{e}+09$ \\
\hline 39 & 237 & $3.988 \mathrm{e}-01$ & $1.769 \mathrm{e}+09$ & 139 & 238 & $3.100 e-02$ & $2.596 e+08$ & 139 & 241 & $1.325 e-01$ & $1.113 e+09$ \\
\hline 39 & 242 & $3.897 e-02$ & $1.833 e+08$ & 139 & 249 & $0.000 e+00$ & $7.805 e+03$ & 139 & 253 & $7.500 e-04$ & $3.781 e+06$ \\
\hline 39 & 256 & $0.000 e+00$ & $1.275 \mathrm{e}+04$ & 139 & 259 & $1.123 \mathrm{e}-02$ & $5.676 e+07$ & 139 & 265 & $1.219 \mathrm{e}-03$ & $6.231 \mathrm{e}+06$ \\
\hline 39 & 268 & $1.224 \mathrm{e}-03$ & $8.055 e+06$ & 139 & 269 & $5.119 e-03$ & $2.622 \mathrm{e}+07$ & 139 & 277 & $2.903 e-03$ & $1.960 \mathrm{e}+07$ \\
\hline 40 & 164 & $1.237 e+00$ & $3.082 \mathrm{e}+07$ & 140 & 210 & $2.930 e-03$ & $1.835 e+07$ & 140 & 211 & $5.073 e-03$ & $1.909 e+07$ \\
\hline 40 & 212 & $2.228 \mathrm{e}-03$ & $6.004 \mathrm{e}+06$ & 140 & 226 & $2.859 \mathrm{e}-01$ & $1.491 \mathrm{e}+09$ & 140 & 227 & $2.994 \mathrm{e}-03$ & $3.696 e+07$ \\
\hline 140 & 228 & $3.061 \mathrm{e}-01$ & $2.269 \mathrm{e}+09$ & 140 & 229 & $1.645 e-02$ & $8.718 e+07$ & 140 & 230 & $7.667 e-01$ & $4.091 e+09$ \\
\hline 40 & 233 & $5.760 e-02$ & $7.198 e+08$ & 140 & 234 & $5.148 e-03$ & $6.635 e+07$ & 140 & 235 & $8.158 e-02$ & $6.349 e+08$ \\
\hline 140 & 236 & $4.102 \mathrm{e}-01$ & $2.282 \mathrm{e}+09$ & 140 & 238 & $1.991 \mathrm{e}-01$ & $1.630 \mathrm{e}+09$ & 140 & 240 & $6.003 e-02$ & $8.212 \mathrm{e}+08$ \\
\hline 140 & 241 & $3.711 \mathrm{e}-03$ & $3.049 e+07$ & 140 & 258 & $8.953 e-03$ & $5.690 e+07$ & 140 & 261 & $6.131 \mathrm{e}-03$ & $3.936 e+07$ \\
\hline 140 & 267 & $4.248 \mathrm{e}-03$ & $3.831 \mathrm{e}+07$ & 140 & 268 & $1.015 e-03$ & $6.538 e+06$ & 140 & 277 & $7.345 e-04$ & $4.853 e+06$ \\
\hline 140 & 278 & $6.578 e-04$ & $6.090 e+06$ & 140 & 279 & $5.152 \mathrm{e}-04$ & $7.956 e+06$ & 140 & 280 & $4.598 e-03$ & $4.303 e+07$ \\
\hline 141 & 165 & $1.597 e+00$ & $3.032 e+07$ & 141 & 211 & $4.854 \mathrm{e}-03$ & $1.811 \mathrm{e}+07$ & 141 & 212 & $1.689 \mathrm{e}-02$ & $4.512 \mathrm{e}+07$ \\
\hline 141 & 229 & $7.095 e-01$ & $3.738 e+09$ & 141 & 230 & $8.592 \mathrm{e}-02$ & $4.558 e+08$ & 141 & 231 & $1.123 e+00$ & $4.640 e+09$ \\
\hline 141 & 235 & $1.856 \mathrm{e}-02$ & $1.436 e+08$ & 141 & 236 & $1.293 \mathrm{e}-01$ & $7.154 e+08$ & 141 & 237 & $7.869 e-01$ & $3.390 e+09$ \\
\hline 141 & 238 & $4.257 e-03$ & $3.465 e+07$ & 141 & 241 & $1.248 \mathrm{e}-01$ & $1.019 e+09$ & 141 & 242 & $6.189 \mathrm{e}-03$ & $2.830 e+07$ \\
\hline 141 & 249 & $0.000 \mathrm{e}+00$ & $7.584 e+03$ & 141 & 253 & $1.224 \mathrm{e}-03$ & $6.002 e+06$ & 141 & 258 & $7.468 \mathrm{e}-04$ & $4.720 e+06$ \\
\hline 141 & 259 & $1.499 \mathrm{e}-02$ & $7.375 e+07$ & 141 & 267 & $5.053 e-04$ & $4.533 e+06$ & 141 & 268 & $7.519 e-03$ & $4.818 e+07$ \\
\hline 41 & 269 & $2.829 \mathrm{e}-03$ & $1.410 \mathrm{e}+07$ & 141 & 277 & $2.087 e-03$ & $1.372 \mathrm{e}+07$ & 141 & 278 & $1.041 \mathrm{e}-03$ & $9.589 e+06$ \\
\hline 42 & 169 & $2.316 \mathrm{e}+00$ & $3.510 \mathrm{e}+07$ & 142 & 229 & $1.621 \mathrm{e}-01$ & $8.463 e+08$ & 142 & 230 & $1.950 e-03$ & $1.025 e+07$ \\
\hline 42 & 231 & $1.451 \mathrm{e}-01$ & $5.941 \mathrm{e}+08$ & 142 & 232 & $2.681 \mathrm{e}+00$ & $8.999 e+09$ & 142 & 236 & $5.067 e-02$ & $2.778 e+08$ \\
\hline
\end{tabular}


Table III. Ca IX Oscillator Strengths and Radiative Decay Rates for transitions involving levels higher than 4.

\begin{tabular}{|c|c|c|c|c|c|c|c|c|c|c|c|}
\hline $\begin{array}{l}\text { Low } \\
\text { Lev }\end{array}$ & $\begin{array}{l}\text { Upp } \\
\text {. Lev }\end{array}$ & $\begin{array}{l}\text { Osc. } \\
\text { Str. }\end{array}$ & $\begin{array}{l}\text { Rad. } \\
\text { Rate }\end{array}$ & $\begin{array}{l}\text { Low. } \\
\text { Lev. }\end{array}$ & $\begin{array}{l}\text { Upp. } \\
\text { Lev. }\end{array}$ & $\begin{array}{l}\text { Osc. } \\
\text { Str. }\end{array}$ & $\begin{array}{l}\text { Rad. } \\
\text { Rate }\end{array}$ & $\begin{array}{l}\text { Low. } \\
\text { Lev. }\end{array}$ & $\begin{array}{l}\text { - Upp } \\
\text { - Lev }\end{array}$ & $\begin{array}{l}\text { Osc. } \\
\text { Str. }\end{array}$ & $\begin{array}{l}\text { Rad. } \\
\text { Rate }\end{array}$ \\
\hline$i$ & $j$ & $g f$ & $(1 / s)$ & $i$ & $j$ & $g f$ & $(1 / s)$ & i & j & $g f$ & $(1 / s)$ \\
\hline 142 & 237 & $7.947 e-01$ & $3.393 e+09$ & 142 & 246 & $0.000 e+00$ & $2.629 e+03$ & 142 & 255 & $3.707 e-03$ & $1.477 e+07$ \\
\hline 142 & 259 & $1.505 e-03$ & $7.343 e+06$ & 142 & 260 & $3.860 e-02$ & $1.542 \mathrm{e}+08$ & 142 & 265 & $1.794 \mathrm{e}-03$ & $8.851 e+06$ \\
\hline 142 & 268 & $6.547 e-04$ & $4.161 \mathrm{e}+06$ & 142 & 269 & $1.040 \mathrm{e}-02$ & $5.143 e+07$ & 142 & 277 & $7.590 e-04$ & $4.948 e+06$ \\
\hline 143 & 172 & $1.198 e+00$ & $2.960 \mathrm{e}+07$ & 143 & 173 & $3.080 e-01$ & $1.185 e+07$ & 143 & 210 & $1.137 \mathrm{e}-03$ & $6.814 e+06$ \\
\hline 143 & 211 & $8.652 \mathrm{e}-03$ & $3.115 e+07$ & 143 & 212 & $9.034 \mathrm{e}-03$ & $2.330 e+07$ & 143 & 226 & $2.343 e-02$ & $1.184 \mathrm{e}+08$ \\
\hline 143 & 228 & $1.152 \mathrm{e}-02$ & $8.275 e+07$ & 143 & 229 & $9.201 \mathrm{e}-01$ & $4.727 e+09$ & 143 & 233 & $4.632 \mathrm{e}-03$ & $5.611 e+07$ \\
\hline 143 & 234 & $5.580 e-01$ & $6.974 \mathrm{e}+09$ & 143 & 235 & $6.280 e-03$ & $4.740 e+07$ & 143 & 236 & $7.223 e-02$ & $3.898 e+08$ \\
\hline 143 & 238 & $4.840 e-03$ & $3.846 e+07$ & 143 & 240 & $1.140 \mathrm{e}-01$ & $1.514 e+09$ & 143 & 241 & $7.775 e-01$ & $6.200 e+09$ \\
\hline 143 & 277 & $3.090 e-02$ & $1.985 e+08$ & 143 & 278 & $8.280 e-03$ & $7.455 e+07$ & 143 & 279 & $7.702 \mathrm{e}-04$ & $1.157 e+07$ \\
\hline 143 & 280 & $1.181 \mathrm{e}-03$ & $1.074 \mathrm{e}+07$ & 144 & 189 & $9.917 e-01$ & $1.023 e+08$ & 144 & 196 & $6.552 \mathrm{e}-02$ & $1.899 e+07$ \\
\hline 144 & 206 & $4.915 e-01$ & $1.874 \mathrm{e}+08$ & 144 & 213 & $0.000 e+00$ & $6.438 e+04$ & 144 & 214 & $6.391 \mathrm{e}-01$ & $3.120 e+09$ \\
\hline 144 & 217 & $1.572 \mathrm{e}-03$ & $5.581 e+06$ & 144 & 218 & $5.011 \mathrm{e}-03$ & $2.518 e+07$ & 144 & 224 & $3.130 \mathrm{e}-01$ & $1.202 \mathrm{e}+09$ \\
\hline 144 & 226 & $0.000 \mathrm{e}+00$ & $7.746 e+03$ & 144 & 233 & $0.000 e+00$ & $7.151 e+04$ & 144 & 238 & $0.000 e+00$ & $8.632 e+04$ \\
\hline 144 & 241 & $0.000 e+00$ & $5.140 e+03$ & 144 & 242 & $0.000 e+00$ & $3.663 e+04$ & 144 & 243 & $2.686 e+00$ & $1.208 e+10$ \\
\hline 144 & 244 & $2.687 e-02$ & $1.217 \mathrm{e}+08$ & 144 & 250 & $3.603 e-02$ & $3.021 e+08$ & 144 & 251 & $0.000 e+00$ & $8.711 \mathrm{e}+05$ \\
\hline 144 & 252 & $4.451 \mathrm{e}-03$ & $2.722 \mathrm{e}+07$ & 144 & 255 & $0.000 e+00$ & $1.866 e+04$ & 144 & 261 & $0.000 e+00$ & $1.264 \mathrm{e}+05$ \\
\hline 144 & 264 & $1.084 e-02$ & $6.726 e+07$ & 144 & 265 & $0.000 e+00$ & $4.675 e+05$ & 144 & 269 & $0.000 e+00$ & $7.105 e+04$ \\
\hline 144 & 275 & $9.610 e-01$ & $6.049 e+09$ & 144 & 280 & $0.000 e+00$ & $9.664 \mathrm{e}+03$ & 145 & 173 & $6.443 e-01$ & $2.223 e+07$ \\
\hline 145 & 174 & $2.957 e-01$ & $1.904 \mathrm{e}+07$ & 145 & 210 & $5.822 e-03$ & $3.450 e+07$ & 145 & 211 & $5.198 e-03$ & $1.851 \mathrm{e}+07$ \\
\hline 145 & 227 & $5.295 e-02$ & $6.286 e+08$ & 145 & 228 & $5.037 e-01$ & $3.590 e+09$ & 145 & 233 & $8.629 e-03$ & $1.037 e+08$ \\
\hline 145 & 234 & $3.402 \mathrm{e}-01$ & $4.220 e+09$ & 145 & 235 & $2.580 e-02$ & $1.933 e+08$ & 145 & 238 & $3.072 \mathrm{e}-02$ & $2.423 e+08$ \\
\hline 145 & 239 & $1.398 \mathrm{e}-01$ & $5.526 \mathrm{e}+09$ & 145 & 240 & $1.835 \mathrm{e}-01$ & $2.419 e+09$ & 145 & 241 & $2.196 \mathrm{e}-01$ & $1.739 e+09$ \\
\hline 145 & 278 & $1.847 \mathrm{e}-02$ & $1.651 \mathrm{e}+08$ & 145 & 279 & $8.255 e-03$ & $1.231 \mathrm{e}+08$ & 145 & 281 & $1.719 \mathrm{e}-03$ & $7.811 \mathrm{e}+07$ \\
\hline 146 & 174 & $3.194 \mathrm{e}-01$ & $1.917 e+07$ & 146 & 210 & $3.434 \mathrm{e}-03$ & $2.020 e+07$ & 146 & 227 & $1.825 \mathrm{e}-01$ & $2.156 e+09$ \\
\hline 146 & 233 & $2.970 e-03$ & $3.552 \mathrm{e}+07$ & 146 & 234 & $1.179 \mathrm{e}-01$ & $1.455 e+09$ & 146 & 240 & $2.015 \mathrm{e}-01$ & $2.643 e+09$ \\
\hline 146 & 279 & $9.452 e-03$ & $1.403 e+08$ & 147 & 179 & $1.854 \mathrm{e}+00$ & $3.945 e+07$ & 147 & 187 & $4.675 \mathrm{e}-02$ & $6.101 \mathrm{e}+06$ \\
\hline 147 & 188 & $9.746 e-02$ & $8.097 e+06$ & 147 & 213 & $7.597 e-02$ & $2.723 e+08$ & 147 & 214 & $0.000 e+00$ & $1.820 \mathrm{e}+04$ \\
\hline 147 & 225 & $0.000 e+00$ & $6.854 \mathrm{e}+03$ & 147 & 226 & $1.031 \mathrm{e}+00$ & $5.061 e+09$ & 147 & 228 & $1.314 \mathrm{e}-02$ & $9.172 \mathrm{e}+07$ \\
\hline 147 & 229 & $2.987 e-02$ & $1.491 \mathrm{e}+08$ & 147 & 230 & $1.906 \mathrm{e}-03$ & $9.579 e+06$ & 147 & 231 & $8.387 e-02$ & $3.283 e+08$ \\
\hline 147 & 235 & $3.230 \mathrm{e}-03$ & $2.371 \mathrm{e}+07$ & 147 & 236 & $3.977 \mathrm{e}-02$ & $2.087 e+08$ & 147 & 237 & $3.288 e-03$ & $1.344 \mathrm{e}+07$ \\
\hline 147 & 238 & $9.094 \mathrm{e}-02$ & $7.031 \mathrm{e}+08$ & 147 & 241 & $2.106 \mathrm{e}-02$ & $1.634 \mathrm{e}+08$ & 147 & 242 & $9.292 \mathrm{e}-01$ & $4.037 e+09$ \\
\hline 147 & 243 & $0.000 e+00$ & $4.864 e+04$ & 147 & 258 & $1.573 e-03$ & $9.466 e+06$ & 147 & 259 & $1.126 \mathrm{e}-03$ & $5.273 e+06$ \\
\hline 147 & 265 & $2.354 \mathrm{e}-03$ & $1.115 e+07$ & 147 & 269 & $1.423 e-03$ & $6.754 e+06$ & 147 & 280 & $6.161 \mathrm{e}-03$ & $5.466 e+07$ \\
\hline 147 & 282 & $0.000 e+00$ & $1.403 e+05$ & 148 & 191 & $4.783 e-01$ & $9.370 e+07$ & 148 & 200 & $2.125 \mathrm{e}-01$ & $1.322 \mathrm{e}+08$ \\
\hline 148 & 202 & $6.415 e-02$ & $2.411 e+07$ & 148 & 205 & $2.465 e-02$ & $5.211 e+07$ & 148 & 210 & $0.000 e+00$ & $1.275 e+04$ \\
\hline 148 & 211 & $0.000 e+00$ & $1.272 \mathrm{e}+04$ & 148 & 214 & $1.047 e-03$ & $4.897 e+06$ & 148 & 215 & $1.529 \mathrm{e}-01$ & $1.208 e+09$ \\
\hline 148 & 216 & $5.255 e-02$ & $2.496 e+08$ & 148 & 218 & $4.487 e-03$ & $2.161 e+07$ & 148 & 221 & $3.221 \mathrm{e}-03$ & $1.616 e+07$ \\
\hline 148 & 222 & $5.782 e-02$ & $4.835 e+08$ & 148 & 223 & $8.463 e-02$ & $2.124 \mathrm{e}+09$ & 148 & 230 & $0.000 e+00$ & $1.979 e+04$ \\
\hline 148 & 234 & $0.000 e+00$ & $4.300 e+04$ & 148 & 235 & $0.000 e+00$ & $2.470 e+04$ & 148 & 236 & $0.000 e+00$ & $8.974 e+03$ \\
\hline 148 & 240 & $0.000 e+00$ & $3.334 e+04$ & 148 & 241 & $0.000 e+00$ & $6.147 e+03$ & 148 & 247 & $1.250 \mathrm{e}+00$ & $9.937 e+09$ \\
\hline 148 & 258 & $0.000 e+00$ & $5.254 \mathrm{e}+05$ & 148 & 262 & $3.502 \mathrm{e}-01$ & $4.909 e+09$ & 148 & 263 & $1.049 \mathrm{e}-01$ & $8.821 e+08$ \\
\hline 148 & 267 & $0.000 e+00$ & $3.250 e+05$ & 148 & 268 & $0.000 e+00$ & $8.409 e+04$ & 148 & 273 & $6.532 \mathrm{e}-03$ & $9.243 e+07$ \\
\hline 148 & 274 & $2.406 \mathrm{e}-02$ & $1.022 \mathrm{e}+09$ & 148 & 278 & $0.000 e+00$ & $5.285 e+04$ & 148 & 279 & $0.000 e+00$ & $6.160 \mathrm{e}+04$ \\
\hline 148 & 283 & $1.322 \mathrm{e}-03$ & $1.996 \mathrm{e}+07$ & 149 & 191 & $8.371 \mathrm{e}-02$ & $1.634 \mathrm{e}+07$ & 149 & 193 & $7.146 \mathrm{e}-01$ & $1.008 e+08$ \\
\hline
\end{tabular}


Table III. Ca IX Oscillator Strengths and Radiative Decay Rates for transitions involving levels higher than 4.

\begin{tabular}{|c|c|c|c|c|c|c|c|c|c|c|c|}
\hline $\begin{array}{l}\text { Low } \\
\text { Lev }\end{array}$ & $\begin{array}{l}\text {. Upp } \\
\text {. Lev }\end{array}$ & $\begin{array}{l}\text { Osc. } \\
\text { Str. }\end{array}$ & $\begin{array}{l}\text { Rad. } \\
\text { Rate }\end{array}$ & $\begin{array}{l}\text { Low. } \\
\text { Lev. }\end{array}$ & $\begin{array}{l}\text { Upp. } \\
\text { Lev. }\end{array}$ & $\begin{array}{l}\text { Osc. } \\
\text { Str. }\end{array}$ & $\begin{array}{l}\text { Rad. } \\
\text { Rate }\end{array}$ & $\begin{array}{l}\text { Low. } \\
\text { Lev. }\end{array}$ & $\begin{array}{l}\text { Upp } \\
\text { Lev }\end{array}$ & $\begin{array}{l}\text { Osc. } \\
\text { Str. }\end{array}$ & $\begin{array}{l}\text { Rad. } \\
\text { Rate }\end{array}$ \\
\hline$i$ & $j$ & $g f$ & $(1 / s)$ & $i$ & $j$ & $g f$ & $(1 / s)$ & $i$ & j & $g f$ & $(1 / s)$ \\
\hline 149 & 200 & $5.857 e-02$ & $3.635 e+07$ & 149 & 201 & $6.952 \mathrm{e}-02$ & $1.857 e+07$ & 149 & 202 & $3.248 \mathrm{e}-01$ & $1.218 e+08$ \\
\hline 149 & 204 & $6.515 e-02$ & $4.547 e+07$ & 149 & 210 & $0.000 e+00$ & $2.139 e+04$ & 149 & 211 & $0.000 \mathrm{e}+00$ & $9.075 e+03$ \\
\hline 149 & 212 & $0.000 \mathrm{e}+00$ & $1.035 \mathrm{e}+04$ & 149 & 215 & $4.288 e-02$ & $3.386 e+08$ & 149 & 216 & $2.271 \mathrm{e}-01$ & $1.078 e+09$ \\
\hline 149 & 217 & $5.438 e-02$ & $1.850 \mathrm{e}+08$ & 149 & 218 & $8.016 e-03$ & $3.858 e+07$ & 149 & 219 & $6.332 \mathrm{e}-03$ & $2.185 e+07$ \\
\hline 149 & 221 & $5.377 e-02$ & $2.696 \mathrm{e}+08$ & 149 & 222 & $1.966 \mathrm{e}-01$ & $1.643 e+09$ & 149 & 230 & $0.000 e+00$ & $5.916 e+03$ \\
\hline 149 & 231 & $0.000 e+00$ & $2.158 \mathrm{e}+04$ & 149 & 234 & $0.000 e+00$ & $7.107 e+04$ & 149 & 235 & $0.000 e+00$ & $1.795 e+04$ \\
\hline 149 & 236 & $0.000 e+00$ & $2.181 \mathrm{e}+04$ & 149 & 237 & $0.000 e+00$ & $7.560 e+03$ & 149 & 239 & $0.000 e+00$ & $7.679 e+04$ \\
\hline 149 & 240 & $0.000 e+00$ & $5.875 e+03$ & 149 & 241 & $0.000 e+00$ & $2.476 e+04$ & 149 & 247 & $2.213 \mathrm{e}-01$ & $1.758 e+09$ \\
\hline 149 & 248 & $1.855 e+00$ & $1.053 e+10$ & 149 & 253 & $0.000 e+00$ & $3.969 e+04$ & 149 & 258 & $0.000 e+00$ & $1.095 e+05$ \\
\hline 149 & 259 & $0.000 e+00$ & $5.269 e+05$ & 149 & 262 & $9.555 e-02$ & $1.339 \mathrm{e}+09$ & 149 & 263 & $5.495 e-01$ & $4.621 e+09$ \\
\hline 149 & 264 & $1.042 \mathrm{e}-01$ & $6.260 e+08$ & 149 & 265 & $0.000 e+00$ & $5.725 e+03$ & 149 & 267 & $0.000 e+00$ & $2.179 e+05$ \\
\hline 149 & 268 & $0.000 e+00$ & $3.159 e+05$ & 149 & 269 & $0.000 e+00$ & $5.904 e+04$ & 149 & 272 & $2.780 e-03$ & $2.357 e+07$ \\
\hline 149 & 273 & $6.977 e-02$ & $9.867 e+08$ & 149 & 277 & $0.000 e+00$ & $4.266 e+04$ & 149 & 278 & $0.000 e+00$ & $5.093 e+04$ \\
\hline 149 & 279 & $0.000 e+00$ & $1.024 \mathrm{e}+05$ & 150 & 190 & $5.305 e-02$ & $5.655 e+06$ & 150 & 192 & $9.783 e-01$ & $1.054 e+08$ \\
\hline 150 & 193 & $8.460 e-02$ & $1.180 \mathrm{e}+07$ & 150 & 201 & $5.684 e-01$ & $1.506 e+08$ & 150 & 202 & $6.763 e-02$ & $2.517 e+07$ \\
\hline 150 & 203 & $1.056 \mathrm{e}-01$ & $4.374 e+07$ & 150 & 211 & $0.000 e+00$ & $1.387 e+04$ & 150 & 212 & $0.000 e+00$ & $2.492 \mathrm{e}+04$ \\
\hline 150 & 216 & $3.762 \mathrm{e}-02$ & $1.782 \mathrm{e}+08$ & 150 & 217 & $3.799 \mathrm{e}-01$ & $1.289 e+09$ & 150 & 218 & $2.688 \mathrm{e}-03$ & $1.291 \mathrm{e}+07$ \\
\hline 150 & 219 & $1.269 \mathrm{e}-02$ & $4.370 e+07$ & 150 & 220 & $1.541 \mathrm{e}-02$ & $4.149 e+07$ & 150 & 221 & $3.645 \mathrm{e}-01$ & $1.823 e+09$ \\
\hline 150 & 231 & $0.000 e+00$ & $4.362 e+03$ & 150 & 232 & $0.000 e+00$ & $2.638 e+04$ & 150 & 234 & $0.000 e+00$ & $9.689 e+04$ \\
\hline 150 & 236 & $0.000 e+00$ & $1.414 \mathrm{e}+04$ & 150 & 237 & $0.000 e+00$ & $3.599 e+04$ & 150 & 240 & $0.000 e+00$ & $3.712 \mathrm{e}+04$ \\
\hline 150 & 241 & $0.000 e+00$ & $4.098 e+04$ & 150 & 243 & $2.227 e-03$ & $9.661 \mathrm{e}+06$ & 150 & 244 & $1.157 \mathrm{e}-03$ & $5.057 e+06$ \\
\hline 150 & 247 & $5.452 \mathrm{e}-03$ & $4.324 \mathrm{e}+07$ & 150 & 248 & $2.178 e-01$ & $1.234 \mathrm{e}+09$ & 150 & 249 & $2.670 \mathrm{e}+00$ & $1.178 \mathrm{e}+10$ \\
\hline 150 & 253 & $0.000 e+00$ & $4.521 \mathrm{e}+03$ & 150 & 255 & $0.000 e+00$ & $5.928 e+04$ & 150 & 258 & $0.000 e+00$ & $5.054 e+03$ \\
\hline 150 & 259 & $0.000 e+00$ & $6.940 e+04$ & 150 & 260 & $0.000 e+00$ & $5.791 e+05$ & 150 & 263 & $8.684 \mathrm{e}-02$ & $7.289 e+08$ \\
\hline 150 & 264 & $9.285 e-01$ & $5.568 e+09$ & 150 & 265 & $0.000 e+00$ & $7.179 e+04$ & 150 & 267 & $0.000 e+00$ & $2.001 \mathrm{e}+04$ \\
\hline 150 & 268 & $0.000 e+00$ & $1.622 \mathrm{e}+05$ & 150 & 269 & $0.000 e+00$ & $4.288 e+05$ & 150 & 272 & $1.354 \mathrm{e}-01$ & $1.146 \mathrm{e}+09$ \\
\hline 150 & 275 & $1.114 \mathrm{e}-02$ & $6.780 e+07$ & 150 & 277 & $0.000 e+00$ & $1.310 e+05$ & 150 & 278 & $0.000 e+00$ & $7.240 \mathrm{e}+04$ \\
\hline 150 & 279 & $0.000 e+00$ & $1.177 \mathrm{e}+04$ & 151 & 190 & $4.026 \mathrm{e}-01$ & $4.098 e+07$ & 151 & 197 & $1.326 \mathrm{e}-01$ & $3.129 e+07$ \\
\hline 51 & 210 & $0.000 e+00$ & $4.173 e+04$ & 151 & 211 & $0.000 e+00$ & $6.712 \mathrm{e}+03$ & 151 & 214 & $3.850 e-03$ & $1.779 e+07$ \\
\hline 151 & 216 & $6.854 \mathrm{e}-03$ & $3.217 e+07$ & 151 & 218 & $6.448 e-01$ & $3.069 e+09$ & 151 & 219 & $4.928 e-02$ & $1.681 \mathrm{e}+08$ \\
\hline 51 & 227 & $0.000 e+00$ & $1.538 \mathrm{e}+04$ & 151 & 230 & $0.000 e+00$ & $6.873 e+04$ & 151 & 231 & $0.000 e+00$ & $1.059 \mathrm{e}+04$ \\
\hline 151 & 235 & $0.000 e+00$ & $6.731 \mathrm{e}+04$ & 151 & 236 & $0.000 e+00$ & $1.283 e+04$ & 151 & 243 & $4.092 \mathrm{e}-02$ & $1.763 e+08$ \\
\hline 151 & 244 & $1.079 e+00$ & $4.683 e+09$ & 151 & 247 & $1.090 \mathrm{e}-02$ & $8.579 e+07$ & 151 & 248 & $2.348 \mathrm{e}-02$ & $1.321 e+08$ \\
\hline 151 & 250 & $2.212 \mathrm{e}-02$ & $1.778 e+08$ & 151 & 252 & $1.012 \mathrm{e}+00$ & $5.936 e+09$ & 151 & 253 & $0.000 e+00$ & $1.869 e+05$ \\
\hline 151 & 254 & $2.084 \mathrm{e}-02$ & $9.523 e+07$ & 151 & 255 & $0.000 e+00$ & $3.243 e+04$ & 151 & 258 & $0.000 e+00$ & $2.554 e+04$ \\
\hline 151 & 260 & $0.000 e+00$ & $4.807 e+03$ & 151 & 263 & $3.861 \mathrm{e}-03$ & $3.219 \mathrm{e}+07$ & 151 & 264 & $4.969 e-03$ & $2.959 e+07$ \\
\hline 151 & 266 & $0.000 e+00$ & $8.100 e+05$ & 151 & 275 & $1.862 \mathrm{e}-02$ & $1.125 e+08$ & 152 & 190 & $3.239 \mathrm{e}-02$ & $3.230 e+06$ \\
\hline 152 & 194 & $7.793 e-01$ & $6.589 e+07$ & 152 & 199 & $2.094 \mathrm{e}-01$ & $3.846 e+07$ & 152 & 211 & $0.000 e+00$ & $4.225 e+04$ \\
\hline 152 & 217 & $1.442 \mathrm{e}-02$ & $4.829 e+07$ & 152 & 219 & $1.003 e+00$ & $3.410 e+09$ & 152 & 220 & $5.484 e-02$ & $1.457 e+08$ \\
\hline 152 & 228 & $0.000 e+00$ & $1.665 e+04$ & 152 & 230 & $0.000 e+00$ & $1.661 \mathrm{e}+04$ & 152 & 231 & $0.000 e+00$ & $7.196 e+04$ \\
\hline 152 & 232 & $0.000 e+00$ & $9.671 \mathrm{e}+03$ & 152 & 235 & $0.000 e+00$ & $1.588 e+04$ & 152 & 236 & $0.000 e+00$ & $6.559 e+04$ \\
\hline 152 & 237 & $0.000 e+00$ & $1.243 e+04$ & 152 & 243 & $1.555 \mathrm{e}-02$ & $6.679 e+07$ & 152 & 244 & $7.751 \mathrm{e}-02$ & $3.354 e+08$ \\
\hline 152 & 245 & $2.195 e+00$ & $7.781 e+09$ & 152 & 248 & $1.439 \mathrm{e}-03$ & $8.074 e+06$ & 152 & 249 & $4.210 e-02$ & $1.838 e+08$ \\
\hline 152 & 251 & $0.000 e+00$ & $7.340 e+03$ & 152 & 252 & $5.694 \mathrm{e}-02$ & $3.331 e+08$ & 152 & 253 & $0.000 e+00$ & $1.836 e+04$ \\
\hline
\end{tabular}


Table III. Ca IX Oscillator Strengths and Radiative Decay Rates for transitions involving levels higher than 4.

\begin{tabular}{|c|c|c|c|c|c|c|c|c|c|c|c|}
\hline $\begin{array}{l}\text { Low. } \\
\text { Lev. }\end{array}$ & $\begin{array}{l}\text { - Upp } \\
\text {. Lev }\end{array}$ & $\begin{array}{l}\text { Osc. } \\
\text { Str. }\end{array}$ & $\begin{array}{l}\text { Rad. } \\
\text { Rate }\end{array}$ & $\begin{array}{l}\text { Low. } \\
\text { Lev. }\end{array}$ & $\begin{array}{l}\text { - Upp } \\
\text { Lev }\end{array}$ & $\begin{array}{l}\text { Osc. } \\
\text { Str. }\end{array}$ & $\begin{array}{l}\text { Rad. } \\
\text { Rate }\end{array}$ & $\begin{array}{l}\text { Low. } \\
\text { Lev }\end{array}$ & $\begin{array}{l}\text { Upp } \\
\text { Lev }\end{array}$ & $\begin{array}{l}\text { Osc. } \\
\text { Str. }\end{array}$ & $\begin{array}{l}\text { Rad. } \\
\text { Rate }\end{array}$ \\
\hline$i$ & $j$ & $g f$ & $(1 / s)$ & i & $j$ & $g f$ & $(1 / s)$ & $i$ & j & $g f$ & $(1 / s)$ \\
\hline 152 & 254 & $1.103 e+00$ & $5.025 e+09$ & 152 & 255 & $0.000 e+00$ & $1.860 \mathrm{e}+05$ & 152 & 256 & $1.118 \mathrm{e}-02$ & $4.171 \mathrm{e}+07$ \\
\hline 152 & 257 & $0.000 e+00$ & $3.999 \mathrm{e}+04$ & 152 & 258 & $0.000 e+00$ & $6.675 e+03$ & 152 & 259 & $0.000 e+00$ & $4.523 e+04$ \\
\hline 152 & 264 & $1.125 \mathrm{e}-02$ & $6.681 \mathrm{e}+07$ & 152 & 266 & $0.000 e+00$ & $9.337 e+04$ & 152 & 270 & $0.000 e+00$ & $9.550 e+05$ \\
\hline 152 & 282 & $5.155 \mathrm{e}-03$ & $2.087 e+07$ & 153 & 194 & $3.809 \mathrm{e}-02$ & $3.135 e+06$ & 153 & 195 & $1.214 \mathrm{e}+00$ & $8.572 \mathrm{e}+07$ \\
\hline 153 & 198 & $3.021 e-01$ & $4.438 e+07$ & 153 & 212 & $0.000 e+00$ & $4.799 e+04$ & 153 & 220 & $1.438 \mathrm{e}+00$ & $3.799 e+09$ \\
\hline 153 & 229 & $0.000 e+00$ & $2.187 e+04$ & 153 & 231 & $0.000 \mathrm{e}+00$ & $1.387 e+04$ & 153 & 232 & $0.000 e+00$ & $9.294 e+04$ \\
\hline 153 & 236 & $0.000 e+00$ & $1.277 \mathrm{e}+04$ & 153 & 237 & $0.000 e+00$ & $8.671 e+04$ & 153 & 244 & $1.120 \mathrm{e}-03$ & $4.827 e+06$ \\
\hline 153 & 245 & $1.193 e-01$ & $4.210 \mathrm{e}+08$ & 153 & 246 & $3.796 \mathrm{e}+00$ & $1.135 e+10$ & 153 & 249 & $7.259 e-03$ & $3.156 e+07$ \\
\hline 153 & 254 & $4.983 e-02$ & $2.261 \mathrm{e}+08$ & 153 & 255 & $0.000 e+00$ & $1.149 \mathrm{e}+04$ & 153 & 256 & $1.184 \mathrm{e}+00$ & $4.400 \mathrm{e}+09$ \\
\hline 153 & 257 & $0.000 e+00$ & $2.460 \mathrm{e}+05$ & 153 & 259 & $0.000 e+00$ & $7.014 e+03$ & 153 & 260 & $0.000 e+00$ & $6.130 \mathrm{e}+04$ \\
\hline 153 & 270 & $0.000 e+00$ & $8.746 e+04$ & 153 & 271 & $0.000 e+00$ & $1.125 \mathrm{e}+06$ & 154 & 196 & $5.072 \mathrm{e}-01$ & $1.088 e+08$ \\
\hline 154 & 208 & $4.497 e-01$ & $4.742 \mathrm{e}+08$ & 154 & 213 & $0.000 e+00$ & $1.734 \mathrm{e}+04$ & 154 & 214 & $5.584 \mathrm{e}-02$ & $4 e+08$ \\
\hline 154 & 223 & $6.239 e-04$ & $1.526 \mathrm{e}+07$ & 154 & 225 & $2.572 \mathrm{e}-01$ & $2.230 \mathrm{e}+09$ & 154 & 226 & $0.000 e+00$ & 3.33 \\
\hline 154 & 233 & $0.000 e+00$ & $6.822 e+04$ & 154 & 238 & $0.000 e+00$ & $1.682 \mathrm{e}+04$ & 154 & 250 & $1.186 e+00$ & 9.43 \\
\hline 154 & 261 & $0.000 e+00$ & $4.924 e+05$ & 154 & 262 & $7.615 e-04$ & $1.047 \mathrm{e}+07$ & 154 & 274 & $3.598 e-04$ & $1.499 \mathrm{e}+07$ \\
\hline 154 & 280 & $0.000 e+00$ & $6.281 \mathrm{e}+05$ & 154 & 283 & $4.535 e-01$ & $6.718 e+09$ & 155 & 189 & $1.872 \mathrm{e}-01$ & $1.232 \mathrm{e}+07$ \\
\hline 155 & 190 & $3.698 e-01$ & $3.309 \mathrm{e}+07$ & 155 & 210 & $0.000 e+00$ & $1.040 \mathrm{e}+04$ & 155 & 214 & $9.642 \mathrm{e}-03$ & $4.345 e+07$ \\
\hline 155 & 216 & $1.170 \mathrm{e}-03$ & $5.357 e+06$ & 155 & 218 & $1.411 \mathrm{e}-01$ & $6.549 e+08$ & 155 & 219 & $9.361 \mathrm{e}-03$ & $3.116 e+07$ \\
\hline 155 & 224 & $9.385 e-03$ & $3.340 e+07$ & 155 & 227 & $0.000 e+00$ & $6.008 e+03$ & 155 & 230 & $0.000 e+00$ & $1.412 \mathrm{e}+04$ \\
\hline 155 & 235 & $0.000 e+00$ & $1.084 e+04$ & 155 & 243 & $1.491 \mathrm{e}+00$ & $6.300 e+09$ & 155 & 244 & $1.433 e+00$ & $6.099 e+09$ \\
\hline 155 & 247 & $4.942 e-02$ & $3.818 e+08$ & 155 & 248 & $4.672 \mathrm{e}-02$ & $2.580 e+08$ & 155 & 249 & $2.651 \mathrm{e}-03$ & $1.139 \mathrm{e}+07$ \\
\hline 55 & 250 & $3.683 e-01$ & $2.906 \mathrm{e}+09$ & 155 & 251 & $0.000 e+00$ & $3.949 e+03$ & 155 & 252 & $9.029 e-03$ & $5.200 \mathrm{e}+07$ \\
\hline 155 & 253 & $0.000 e+00$ & $3.396 \mathrm{e}+04$ & 155 & 254 & $6.604 \mathrm{e}-03$ & $2.961 \mathrm{e}+07$ & 155 & 255 & $0.000 e+00$ & $1.057 e+04$ \\
\hline 55 & 258 & $0.000 e+00$ & $6.359 \mathrm{e}+03$ & 155 & 263 & $2.968 \mathrm{e}-02$ & $2.429 e+08$ & 155 & 264 & $7.487 e-02$ & $4.377 e+08$ \\
\hline 55 & 266 & $0.000 e+00$ & $1.402 \mathrm{e}+05$ & 155 & 272 & $4.597 e-02$ & $3.793 e+08$ & 155 & 275 & $6.469 \mathrm{e}-01$ & $3.838 e+09$ \\
\hline 56 & 183 & $6.797 e-01$ & $1.773 e+07$ & 156 & 207 & $1.164 \mathrm{e}-01$ & $2.496 e+08$ & 156 & 213 & $2.813 e-02$ & $9.446 e+07$ \\
\hline 56 & 214 & $0.000 e+00$ & $4.482 e+03$ & 156 & 224 & $0.000 e+00$ & $7.965 e+03$ & 156 & 225 & $0.000 e+00$ & $7.235 \mathrm{e}+03$ \\
\hline 56 & 228 & $1.068 \mathrm{e}-03$ & $7.119 \mathrm{e}+06$ & 156 & 233 & $1.135 \mathrm{e}+00$ & $1.276 \mathrm{e}+10$ & 156 & 234 & $1.137 \mathrm{e}-02$ & $1.321 \mathrm{e}+08$ \\
\hline 56 & 235 & $5.265 e-03$ & $3.693 e+07$ & 156 & 238 & $1.687 \mathrm{e}-01$ & $1.248 e+09$ & 156 & 239 & $5.665 e-03$ & $2.101 \mathrm{e}+08$ \\
\hline 56 & 241 & $2.705 e-02$ & $2.008 \mathrm{e}+08$ & 156 & 250 & $0.000 e+00$ & $7.903 e+04$ & 156 & 275 & $0.000 e+00$ & $2.638 e+04$ \\
\hline 56 & 280 & $1.556 e-02$ & $1.325 \mathrm{e}+08$ & 156 & 281 & $2.559 \mathrm{e}-01$ & $1.096 e+10$ & 156 & 283 & $0.000 e+00$ & $6.723 e+04$ \\
\hline 157 & 226 & $1.474 \mathrm{e}-03$ & $6.874 \mathrm{e}+06$ & 157 & 230 & $1.317 \mathrm{e}-02$ & $6.294 e+07$ & 157 & 242 & $5.534 \mathrm{e}-03$ & $2.292 \mathrm{e}+07$ \\
\hline 157 & 244 & $0.000 e+00$ & $1.239 \mathrm{e}+04$ & 157 & 251 & $3.564 \mathrm{e}-02$ & $1.293 e+08$ & 157 & 253 & $1.527 e+00$ & $6.804 e+09$ \\
\hline 157 & 258 & $2.347 e-01$ & $1.348 \mathrm{e}+09$ & 157 & 260 & $4.505 e-03$ & $1.649 \mathrm{e}+07$ & 157 & 261 & $8.099 e-02$ & $4.702 \mathrm{e}+08$ \\
\hline 157 & 265 & $2.847 e-01$ & $1.288 \mathrm{e}+09$ & 157 & 266 & $1.582 \mathrm{e}-02$ & $5.863 e+07$ & 157 & 268 & $4.119 e-02$ & $2.400 \mathrm{e}+08$ \\
\hline 157 & 269 & $1.570 \mathrm{e}-02$ & $7.118 e+07$ & 158 & 190 & $2.891 \mathrm{e}-01$ & $2.479 e+07$ & 158 & 216 & $1.082 \mathrm{e}-02$ & $4.917 e+07$ \\
\hline 158 & 217 & $5.258 e-03$ & $1.711 \mathrm{e}+07$ & 158 & 218 & $5.754 \mathrm{e}-02$ & $2.651 e+08$ & 158 & 219 & $4.135 e-02$ & $1.366 e+08$ \\
\hline 158 & 220 & $1.941 \mathrm{e}-03$ & $5.014 \mathrm{e}+06$ & 158 & 221 & $1.256 \mathrm{e}-03$ & $6.032 \mathrm{e}+06$ & 158 & 227 & $0.000 e+00$ & $1.095 e+04$ \\
\hline 158 & 231 & $0.000 e+00$ & $5.689 e+03$ & 158 & 235 & $0.000 e+00$ & $6.600 e+03$ & 158 & 243 & $2.487 e-02$ & $1.045 e+08$ \\
\hline 158 & 244 & $1.425 e+00$ & $6.030 \mathrm{e}+09$ & 158 & 247 & $2.693 e-01$ & $2.068 \mathrm{e}+09$ & 158 & 248 & $8.775 e-01$ & $4.815 e+09$ \\
\hline 158 & 249 & $6.615 \mathrm{e}-03$ & $2.825 \mathrm{e}+07$ & 158 & 250 & $3.602 \mathrm{e}-02$ & $2.825 e+08$ & 158 & 252 & $8.477 e-02$ & $4.853 e+08$ \\
\hline 58 & 253 & $0.000 e+00$ & $2.696 \mathrm{e}+04$ & 158 & 254 & $2.706 \mathrm{e}-01$ & $1.206 e+09$ & 158 & 255 & $0.000 e+00$ & $1.295 e+04$ \\
\hline 58 & 258 & $0.000 e+00$ & $1.289 \mathrm{e}+04$ & 158 & 259 & $0.000 e+00$ & $1.495 e+04$ & 158 & 263 & $4.260 e-01$ & $3.465 e+09$ \\
\hline 58 & 264 & $2.240 \mathrm{e}-02$ & $1.302 \mathrm{e}+08$ & 158 & 266 & $0.000 e+00$ & $8.044 e+04$ & 158 & 272 & $6.697 e-02$ & $5.493 e+08$ \\
\hline
\end{tabular}


Table III. Ca IX Oscillator Strengths and Radiative Decay Rates for transitions involving levels higher than 4.

\begin{tabular}{|c|c|c|c|c|c|c|c|c|c|c|c|}
\hline $\begin{array}{l}\text { Low. } \\
\text { Lev . }\end{array}$ & $\begin{array}{l}\text { Upp } \\
\text { Lev }\end{array}$ & $\begin{array}{l}\text { Osc. } \\
\text { Str. }\end{array}$ & $\begin{array}{l}\text { Rad. } \\
\text { Rate }\end{array}$ & $\begin{array}{l}\text { Low. } \\
\text { Lev. }\end{array}$ & $\begin{array}{l}\text { Upp. } \\
\text { Lev. }\end{array}$ & $\begin{array}{l}\text { Osc. } \\
\text { Str. }\end{array}$ & $\begin{array}{l}\text { Rad. } \\
\text { Rate }\end{array}$ & $\begin{array}{l}\text { Low. } \\
\text { Lev. }\end{array}$ & $\begin{array}{l}\text { Upp } \\
\text { Lev }\end{array}$ & $\begin{array}{l}\text { Osc. } \\
\text { Str. }\end{array}$ & $\begin{array}{l}\text { Rad. } \\
\text { Rate }\end{array}$ \\
\hline$i$ & $j$ & $g f$ & $(1 / s)$ & $i$ & $j$ & $g f$ & $(1 / s)$ & $i$ & j & $g f$ & $(1 / s)$ \\
\hline 158 & 275 & $3.927 e-02$ & $2.316 e+08$ & 159 & 214 & $1.036 \mathrm{e}-03$ & $4.609 e+06$ & 159 & 215 & $7.151 e-03$ & $5.378 e+07$ \\
\hline 159 & 216 & $3.918 e-03$ & $1.771 \mathrm{e}+07$ & 159 & 218 & $1.685 \mathrm{e}-02$ & $7.726 e+07$ & 159 & 219 & $1.896 \mathrm{e}-03$ & $6.234 \mathrm{e}+06$ \\
\hline 159 & 227 & $0.000 \mathrm{e}+00$ & $6.552 \mathrm{e}+03$ & 159 & 247 & $1.120 \mathrm{e}+00$ & $8.572 e+09$ & 159 & 248 & $4.102 \mathrm{e}-02$ & $2.243 e+08$ \\
\hline 159 & 250 & $1.601 \mathrm{e}-02$ & $1.251 \mathrm{e}+08$ & 159 & 252 & $3.714 \mathrm{e}-01$ & $2.119 e+09$ & 159 & 253 & $0.000 e+00$ & $4.265 e+04$ \\
\hline 159 & 258 & $0.000 e+00$ & $1.866 \mathrm{e}+04$ & 159 & 262 & $3.836 \mathrm{e}-01$ & $5.182 e+09$ & 159 & 263 & $2.608 e-03$ & $2.115 e+07$ \\
\hline 159 & 264 & $2.750 e-03$ & $1.593 e+07$ & 159 & 272 & $1.117 \mathrm{e}-02$ & $9.129 e+07$ & 159 & 273 & $2.114 \mathrm{e}-02$ & $2.883 e+08$ \\
\hline 159 & 275 & $3.797 e-03$ & $2.231 \mathrm{e}+07$ & 159 & 283 & $2.518 \mathrm{e}-02$ & $3.668 e+08$ & 160 & 231 & $1.143 e-02$ & $4.234 e+07$ \\
\hline 160 & 245 & $0.000 e+00$ & $8.716 e+03$ & 160 & 251 & $7.439 e-01$ & $2.689 e+09$ & 160 & 253 & $9.141 \mathrm{e}-02$ & $4.058 e+08$ \\
\hline 160 & 255 & $1.266 \mathrm{e}+00$ & $4.603 e+09$ & 160 & 257 & $3.479 \mathrm{e}-02$ & $1.071 e+08$ & 160 & 259 & $2.099 \mathrm{e}-01$ & $9.349 e+08$ \\
\hline 160 & 260 & $1.448 \mathrm{e}-01$ & $5.282 e+08$ & 160 & 265 & $1.521 \mathrm{e}-01$ & $6.854 e+08$ & 160 & 269 & $1.098 \mathrm{e}-01$ & $4.957 e+08$ \\
\hline 160 & 270 & $1.591 \mathrm{e}-02$ & $4.978 e+07$ & 160 & 276 & $8.979 \mathrm{e}-02$ & $2.865 e+08$ & 161 & 194 & $5.563 e-01$ & $3.927 e+07$ \\
\hline 161 & 217 & $2.055 e-02$ & $6.648 e+07$ & 161 & 219 & $9.570 e-02$ & $3.142 e+08$ & 161 & 220 & $5.656 e-02$ & $1.452 \mathrm{e}+08$ \\
\hline 161 & 228 & $0.000 e+00$ & $9.463 e+03$ & 161 & 231 & $0.000 e+00$ & $4.911 e+03$ & 161 & 232 & $0.000 e+00$ & $5.965 e+03$ \\
\hline 161 & 236 & $0.000 e+00$ & $6.535 e+03$ & 161 & 243 & $5.812 \mathrm{e}-02$ & $2.429 e+08$ & 161 & 244 & $1.217 \mathrm{e}-01$ & $5.125 e+08$ \\
\hline 161 & 245 & $2.425 \mathrm{e}+00$ & $8.368 e+09$ & 161 & 248 & $3.675 e-01$ & $2.008 e+09$ & 161 & 249 & $9.617 \mathrm{e}-01$ & $4.088 e+09$ \\
\hline 161 & 252 & $4.262 e-03$ & $2.429 \mathrm{e}+07$ & 161 & 254 & $1.588 e-01$ & $7.045 e+08$ & 161 & 255 & $0.000 e+00$ & $2.416 e+04$ \\
\hline 161 & 256 & $2.870 e-01$ & $1.043 e+09$ & 161 & 257 & $0.000 e+00$ & $1.179 e+04$ & 161 & 259 & $0.000 e+00$ & $1.527 e+04$ \\
\hline 161 & 260 & $0.000 e+00$ & $1.930 \mathrm{e}+04$ & 161 & 264 & $3.311 \mathrm{e}-01$ & $1.915 e+09$ & 161 & 266 & $0.000 e+00$ & $9.581 e+03$ \\
\hline 161 & 270 & $0.000 e+00$ & $9.215 e+04$ & 161 & 275 & $4.135 e-03$ & $2.428 e+07$ & 161 & 276 & $0.000 e+00$ & $5.336 e+03$ \\
\hline 161 & 282 & $3.573 e-02$ & $1.411 \mathrm{e}+08$ & 162 & 226 & $3.625 e-03$ & $1.661 \mathrm{e}+07$ & 162 & 236 & $1.420 \mathrm{e}-03$ & $6.970 e+06$ \\
\hline 162 & 242 & $1.361 \mathrm{e}-02$ & $5.543 e+07$ & 162 & 251 & $7.900 e-02$ & $2.820 e+08$ & 162 & 253 & $1.355 \mathrm{e}-01$ & $5.943 e+08$ \\
\hline 162 & 255 & $8.443 e-02$ & $3.033 e+08$ & 162 & 258 & $5.683 e-02$ & $3.212 \mathrm{e}+08$ & 162 & 259 & $9.509 e-01$ & $4.184 e+09$ \\
\hline 162 & 260 & $6.796 \mathrm{e}-02$ & $2.448 \mathrm{e}+08$ & 162 & 261 & $3.017 \mathrm{e}-01$ & $1.724 e+09$ & 162 & 265 & $1.021 \mathrm{e}+00$ & $4.546 e+09$ \\
\hline 62 & 268 & $4.994 e-01$ & $2.864 \mathrm{e}+09$ & 162 & 269 & $1.012 \mathrm{e}-02$ & $4.514 e+07$ & 162 & 277 & $1.986 \mathrm{e}-01$ & $1.170 \mathrm{e}+09$ \\
\hline 63 & 226 & $3.697 e-03$ & $1.692 \mathrm{e}+07$ & 163 & 235 & $2.379 e-03$ & $1.631 \mathrm{e}+07$ & 163 & 253 & $1.729 \mathrm{e}-01$ & $7.575 e+08$ \\
\hline 63 & 258 & $1.554 \mathrm{e}+00$ & $8.775 e+09$ & 163 & 259 & $1.518 \mathrm{e}-01$ & $6.671 \mathrm{e}+08$ & 163 & 261 & $4.674 \mathrm{e}-02$ & $2.667 e+08$ \\
\hline 63 & 267 & $6.117 \mathrm{e}-01$ & $4.905 e+09$ & 163 & 269 & $1.153 e-03$ & $5.139 e+06$ & 163 & 277 & $7.708 e-03$ & $4.535 e+07$ \\
\hline 63 & 278 & $7.185 \mathrm{e}-02$ & $5.924 e+08$ & 163 & 280 & $6.941 \mathrm{e}-02$ & $5.788 e+08$ & 164 & 190 & $3.712 \mathrm{e}-01$ & $2.576 e+07$ \\
\hline 64 & 216 & $4.208 e-03$ & $1.841 \mathrm{e}+07$ & 164 & 217 & $1.884 \mathrm{e}-03$ & $5.908 e+06$ & 164 & 218 & $5.215 \mathrm{e}-02$ & $2.315 e+08$ \\
\hline 64 & 219 & $2.523 e-03$ & $8.031 \mathrm{e}+06$ & 164 & 224 & $2.710 \mathrm{e}-03$ & $9.236 e+06$ & 164 & 230 & $0.000 e+00$ & $5.039 e+03$ \\
\hline 164 & 243 & $2.505 e-01$ & $1.022 \mathrm{e}+09$ & 164 & 244 & $1.747 e+00$ & $7.185 e+09$ & 164 & 247 & $4.508 e-03$ & $3.364 e+07$ \\
\hline 164 & 248 & $5.700 e-01$ & $3.040 e+09$ & 164 & 249 & $1.574 \mathrm{e}-02$ & $6.532 \mathrm{e}+07$ & 164 & 250 & $1.403 e-01$ & $1.070 e+09$ \\
\hline 164 & 252 & $1.294 \mathrm{e}-01$ & $7.204 e+08$ & 164 & 254 & $1.986 \mathrm{e}-01$ & $8.607 e+08$ & 164 & 255 & $0.000 e+00$ & $2.733 e+04$ \\
\hline 164 & 259 & $0.000 e+00$ & $9.862 \mathrm{e}+03$ & 164 & 263 & $1.282 \mathrm{e}-01$ & $1.014 \mathrm{e}+09$ & 164 & 264 & $9.265 e-03$ & $5.236 e+07$ \\
\hline 164 & 266 & $0.000 e+00$ & $2.006 \mathrm{e}+04$ & 164 & 272 & $1.490 \mathrm{e}-03$ & $1.189 e+07$ & 164 & 275 & $2.829 \mathrm{e}-01$ & $1.623 e+09$ \\
\hline 165 & 194 & $5.413 e-01$ & $3.097 e+07$ & 165 & 217 & $1.066 \mathrm{e}-02$ & $3.323 e+07$ & 165 & 219 & $7.810 e-02$ & $2.471 e+08$ \\
\hline 165 & 220 & $1.179 \mathrm{e}-02$ & $2.918 e+07$ & 165 & 229 & $0.000 e+00$ & $7.243 e+03$ & 165 & 231 & $0.000 e+00$ & $5.161 e+03$ \\
\hline 165 & 244 & $1.020 \mathrm{e}-01$ & $4.175 e+08$ & 165 & 245 & $2.347 e+00$ & $7.869 e+09$ & 165 & 248 & $1.546 \mathrm{e}-02$ & $8.207 e+07$ \\
\hline 165 & 249 & $1.116 \mathrm{e}+00$ & $4.612 \mathrm{e}+09$ & 165 & 252 & $1.383 e-02$ & $7.667 e+07$ & 165 & 254 & $2.324 \mathrm{e}-01$ & $1.003 e+09$ \\
\hline 165 & 256 & $3.083 e-01$ & $1.090 \mathrm{e}+09$ & 165 & 257 & $0.000 e+00$ & $4.319 e+04$ & 165 & 260 & $0.000 e+00$ & $1.739 e+04$ \\
\hline 165 & 264 & $2.211 \mathrm{e}-01$ & $1.244 \mathrm{e}+09$ & 165 & 270 & $0.000 e+00$ & $2.018 e+04$ & 165 & 275 & $4.139 \mathrm{e}-03$ & $2.364 \mathrm{e}+07$ \\
\hline 166 & 220 & $0.000 e+00$ & $4.810 \mathrm{e}+03$ & 166 & 232 & $3.412 \mathrm{e}-02$ & $1.002 e+08$ & 166 & 246 & $0.000 e+00$ & $1.890 \mathrm{e}+04$ \\
\hline 166 & 251 & $6.581 \mathrm{e}-03$ & $2.306 \mathrm{e}+07$ & 166 & 255 & $1.446 \mathrm{e}-01$ & $5.098 e+08$ & 166 & 257 & $2.462 \mathrm{e}+00$ & $7.353 e+09$ \\
\hline 166 & 260 & $1.194 \mathrm{e}-01$ & $4.222 \mathrm{e}+08$ & 166 & 270 & $6.295 e-03$ & $1.910 e+07$ & 166 & 271 & $3.360 e-03$ & $8.848 e+06$ \\
\hline
\end{tabular}


Table III. Ca IX Oscillator Strengths and Radiative Decay Rates for transitions involving levels higher than 4.

\begin{tabular}{|c|c|c|c|c|c|c|c|c|c|c|c|}
\hline $\begin{array}{l}\text { Low } \\
\text { Lev }\end{array}$ & $\begin{array}{l}\text { Upp } \\
\text { Lev }\end{array}$ & $\begin{array}{l}\text { Osc. } \\
\text { Str. }\end{array}$ & $\begin{array}{l}\text { Rad. } \\
\text { Rate }\end{array}$ & $\begin{array}{l}\text { Low. } \\
\text { Lev. }\end{array}$ & $\begin{array}{l}\text { Upp. } \\
\text { Lev. }\end{array}$ & $\begin{array}{l}\text { Osc. } \\
\text { Str. }\end{array}$ & $\begin{array}{l}\text { Rad. } \\
\text { Rate }\end{array}$ & $\begin{array}{l}\text { Low. } \\
\text { Lev. }\end{array}$ & $\begin{array}{l}\text { Upp } \\
\text { Lev }\end{array}$ & $\begin{array}{l}\text { Osc. } \\
\text { Str. }\end{array}$ & $\begin{array}{l}\text { Rad. } \\
\text { Rate }\end{array}$ \\
\hline$i$ & $j$ & $g f$ & $(1 / s)$ & $i$ & $j$ & $g f$ & $(1 / s)$ & $i$ & j & $g f$ & $(1 / s)$ \\
\hline 167 & 226 & $3.516 e-03$ & $1.572 \mathrm{e}+07$ & 167 & 230 & $5.460 e-03$ & $2.502 \mathrm{e}+07$ & 167 & 236 & $1.575 e-03$ & $7.550 e+06$ \\
\hline 167 & 242 & $1.185 e-02$ & $4.716 e+07$ & 167 & 244 & $0.000 e+00$ & $3.623 e+03$ & 167 & 251 & $2.014 \mathrm{e}-02$ & $7.033 e+07$ \\
\hline 167 & 253 & $6.081 \mathrm{e}-02$ & $2.609 \mathrm{e}+08$ & 167 & 255 & $1.782 \mathrm{e}-01$ & $6.263 e+08$ & 167 & 258 & $1.357 e-02$ & $7.506 e+07$ \\
\hline 167 & 259 & $9.516 \mathrm{e}-01$ & $4.097 e+09$ & 167 & 260 & $1.069 \mathrm{e}-01$ & $3.767 e+08$ & 167 & 261 & $2.463 e-01$ & $1.377 e+09$ \\
\hline 167 & 265 & $1.016 \mathrm{e}+00$ & $4.426 e+09$ & 167 & 268 & $2.526 \mathrm{e}-01$ & $1.417 e+09$ & 167 & 269 & $7.617 e-02$ & $3.325 e+08$ \\
\hline 167 & 277 & $2.424 \mathrm{e}-03$ & $1.398 \mathrm{e}+07$ & 168 & 231 & $1.162 \mathrm{e}-02$ & $4.145 e+07$ & 168 & 232 & $1.711 \mathrm{e}-03$ & $5.005 e+06$ \\
\hline 168 & 237 & $2.760 e-03$ & $1.030 \mathrm{e}+07$ & 168 & 245 & $0.000 e+00$ & $5.710 e+03$ & 168 & 246 & $0.000 e+00$ & $3.332 \mathrm{e}+03$ \\
\hline 168 & 251 & $9.613 e-02$ & $3.356 \mathrm{e}+08$ & 168 & 253 & $2.925 e-02$ & $1.255 e+08$ & 168 & 255 & $2.597 e-01$ & $9.125 e+08$ \\
\hline 168 & 257 & $3.886 e-01$ & $1.156 \mathrm{e}+09$ & 168 & 259 & $4.920 e-03$ & $2.117 e+07$ & 168 & 260 & $1.959 e+00$ & $6.900 e+09$ \\
\hline 168 & 265 & $1.185 e-01$ & $5.161 \mathrm{e}+08$ & 168 & 266 & $4.164 \mathrm{e}-03$ & $1.486 e+07$ & 168 & 269 & $3.286 e-01$ & $1.434 \mathrm{e}+09$ \\
\hline 168 & 276 & $1.268 \mathrm{e}-02$ & $3.913 e+07$ & 169 & 195 & $1.115 e+00$ & $5.191 e+07$ & 169 & 220 & $9.576 e-02$ & $2.347 e+08$ \\
\hline 169 & 232 & $0.000 e+00$ & $4.510 e+03$ & 169 & 244 & $2.131 \mathrm{e}-03$ & $8.659 e+06$ & 169 & 245 & $1.730 \mathrm{e}-01$ & $5.760 e+08$ \\
\hline 169 & 246 & $4.872 \mathrm{e}+00$ & $1.374 \mathrm{e}+10$ & 169 & 249 & $3.233 e-01$ & $1.326 e+09$ & 169 & 254 & 2.644 & $1.133 e+08$ \\
\hline 169 & 256 & $7.193 e-01$ & $2.524 \mathrm{e}+09$ & 169 & 257 & $0.000 e+00$ & $8.303 e+03$ & 169 & 271 & $0.000 e+00$ & $3.586 e+04$ \\
\hline 170 & 231 & $1.693 e-03$ & $5.989 e+06$ & 170 & 242 & $6.989 e-03$ & $2.760 e+07$ & 170 & 251 & $1.064 e+00$ & $3.686 e+09$ \\
\hline 170 & 253 & $2.481 \mathrm{e}-02$ & $1.056 e+08$ & 170 & 255 & $5.648 e-01$ & $1.969 e+09$ & 170 & 257 & $4.493 e-02$ & $1.327 e+08$ \\
\hline 170 & 259 & $5.285 e-02$ & $2.257 e+08$ & 170 & 260 & $4.602 \mathrm{e}-01$ & $1.609 e+09$ & 170 & 265 & $4.580 e-02$ & $1.979 e+08$ \\
\hline 170 & 266 & $4.317 e-03$ & $1.529 \mathrm{e}+07$ & 170 & 269 & $3.007 e-01$ & $1.303 e+09$ & 170 & 270 & $4.002 e-03$ & $1.201 \mathrm{e}+07$ \\
\hline 170 & 276 & $2.180 \mathrm{e}-01$ & $6.674 e+08$ & 170 & 282 & $0.000 e+00$ & $1.654 e+04$ & 171 & 203 & $5.352 e-01$ & $1.442 \mathrm{e}+08$ \\
\hline 171 & 204 & $3.172 \mathrm{e}-01$ & $1.432 \mathrm{e}+08$ & 171 & 205 & $1.062 \mathrm{e}-01$ & $1.451 e+08$ & 171 & 221 & $1.216 \mathrm{e}-01$ & $5.427 e+08$ \\
\hline 171 & 222 & $7.565 \mathrm{e}-02$ & $5.627 e+08$ & 171 & 223 & $2.537 e-02$ & $5.663 e+08$ & 171 & 227 & $0.000 e+00$ & $4.133 e+04$ \\
\hline 171 & 228 & $0.000 e+00$ & $4.168 \mathrm{e}+04$ & 171 & 229 & $0.000 e+00$ & $4.134 e+04$ & 171 & 262 & $5.659 e-03$ & $7.251 \mathrm{e}+07$ \\
\hline 171 & 263 & $1.460 \mathrm{e}-02$ & $1.123 e+08$ & 171 & 272 & $9.613 e-01$ & $7.455 e+09$ & 171 & 273 & $5.718 \mathrm{e}-01$ & $7.399 e+09$ \\
\hline 171 & 274 & $1.908 \mathrm{e}-01$ & $7.412 \mathrm{e}+09$ & 171 & 277 & $0.000 e+00$ & $3.951 e+05$ & 171 & 278 & $0.000 e+00$ & $3.874 e+05$ \\
\hline 71 & 279 & $0.000 e+00$ & $3.883 e+05$ & 171 & 280 & $0.000 e+00$ & $4.750 e+03$ & 172 & 216 & $1.345 \mathrm{e}-02$ & $5.654 \mathrm{e}+07$ \\
\hline 172 & 218 & $1.538 \mathrm{e}-03$ & $6.561 \mathrm{e}+06$ & 172 & 219 & $8.219 e-03$ & $2.514 \mathrm{e}+07$ & 172 & 220 & $2.215 \mathrm{e}-03$ & $5.299 e+06$ \\
\hline 172 & 221 & $6.392 \mathrm{e}-03$ & $2.848 \mathrm{e}+07$ & 172 & 240 & $0.000 e+00$ & $5.285 e+03$ & 172 & 243 & $5.001 \mathrm{e}-02$ & $1.979 \mathrm{e}+08$ \\
\hline 172 & 247 & $5.334 \mathrm{e}-03$ & $3.860 \mathrm{e}+07$ & 172 & 248 & $2.882 \mathrm{e}-02$ & $1.491 e+08$ & 172 & 249 & $7.125 \mathrm{e}-01$ & $2.868 e+09$ \\
\hline 172 & 250 & $1.982 \mathrm{e}-02$ & $1.466 \mathrm{e}+08$ & 172 & 254 & $5.413 e-03$ & $2.277 e+07$ & 172 & 255 & $0.000 e+00$ & $1.225 e+04$ \\
\hline 172 & 259 & $0.000 e+00$ & $7.863 e+03$ & 172 & 263 & $1.184 \mathrm{e}-01$ & $9.090 e+08$ & 172 & 264 & $1.188 \mathrm{e}+00$ & $6.519 e+09$ \\
\hline 172 & 272 & $1.152 \mathrm{e}+00$ & $8.926 e+09$ & 172 & 275 & $8.391 \mathrm{e}-02$ & $4.674 e+08$ & 173 & 215 & $1.200 \mathrm{e}-02$ & $8.308 e+07$ \\
\hline 173 & 218 & $6.543 e-03$ & $2.763 e+07$ & 173 & 219 & $2.404 \mathrm{e}-03$ & $7.280 e+06$ & 173 & 221 & $1.261 \mathrm{e}-03$ & $5.563 e+06$ \\
\hline 173 & 222 & $3.604 \mathrm{e}-03$ & $2.650 \mathrm{e}+07$ & 173 & 239 & $0.000 e+00$ & $1.154 e+04$ & 173 & 247 & $6.656 e-03$ & $4.780 e+07$ \\
\hline 173 & 248 & $5.027 e-01$ & $2.580 e+09$ & 173 & 250 & $3.998 e-02$ & $2.935 e+08$ & 173 & 253 & $0.000 e+00$ & $1.327 e+04$ \\
\hline 173 & 258 & $0.000 e+00$ & $7.354 e+03$ & 173 & 262 & $1.172 \mathrm{e}-01$ & $1.489 e+09$ & 173 & 263 & $6.098 \mathrm{e}-01$ & $4.649 e+09$ \\
\hline 173 & 264 & $1.601 \mathrm{e}-01$ & $8.717 e+08$ & 173 & 272 & $3.212 \mathrm{e}-01$ & $2.470 \mathrm{e}+09$ & 173 & 273 & $6.345 e-01$ & $8.140 \mathrm{e}+09$ \\
\hline 173 & 275 & $3.118 \mathrm{e}-03$ & $1.724 \mathrm{e}+07$ & 173 & 283 & $2.227 e-02$ & $3.058 e+08$ & 174 & 215 & $1.589 \mathrm{e}-03$ & $1.088 e+07$ \\
\hline 174 & 222 & $1.501 \mathrm{e}-03$ & $1.093 e+07$ & 174 & 223 & $1.932 \mathrm{e}-03$ & $4.220 e+07$ & 174 & 247 & $2.668 \mathrm{e}-01$ & $1.901 \mathrm{e}+09$ \\
\hline 174 & 250 & $1.040 \mathrm{e}-03$ & $7.575 e+06$ & 174 & 262 & $3.887 e-01$ & $4.899 e+09$ & 174 & 263 & $1.435 \mathrm{e}-01$ & $1.085 e+09$ \\
\hline 174 & 272 & $3.045 e-02$ & $2.323 e+08$ & 174 & 273 & $3.138 \mathrm{e}-01$ & $3.994 e+09$ & 174 & 274 & $3.336 e-01$ & $1.274 \mathrm{e}+10$ \\
\hline 175 & 229 & $3.327 e-03$ & $1.455 \mathrm{e}+07$ & 175 & 242 & $1.480 \mathrm{e}-03$ & $5.675 e+06$ & 175 & 253 & $7.178 e-03$ & $2.971 \mathrm{e}+07$ \\
\hline 175 & 255 & $5.599 e-03$ & $1.898 \mathrm{e}+07$ & 175 & 258 & $3.880 e-03$ & $2.069 e+07$ & 175 & 259 & $7.588 e-02$ & $3.151 \mathrm{e}+08$ \\
\hline 175 & 260 & $4.182 \mathrm{e}-02$ & $1.422 \mathrm{e}+08$ & 175 & 261 & $2.188 \mathrm{e}-02$ & $1.180 e+08$ & 175 & 265 & $7.147 e-02$ & $3.003 e+08$ \\
\hline 175 & 268 & $3.496 \mathrm{e}-02$ & $1.893 e+08$ & 175 & 269 & $2.299 \mathrm{e}+00$ & $9.682 e+09$ & 175 & 277 & $1.516 e+00$ & $8.435 e+09$ \\
\hline
\end{tabular}


Table III. Ca IX Oscillator Strengths and Radiative Decay Rates for transitions involving levels higher than 4.

\begin{tabular}{|c|c|c|c|c|c|c|c|c|c|c|c|}
\hline $\begin{array}{l}\text { Low } \\
\text { Lev }\end{array}$ & $\begin{array}{l}\text {. Upp } \\
\text {. Lev }\end{array}$ & $\begin{array}{l}\text { Osc. } \\
\text { Str. }\end{array}$ & $\begin{array}{l}\text { Rad. } \\
\text { Rate }\end{array}$ & $\begin{array}{l}\text { Low. } \\
\text { Lev. }\end{array}$ & $\begin{array}{l}\text { Upp. } \\
\text { Lev. }\end{array}$ & $\begin{array}{l}\text { Osc. } \\
\text { Str. }\end{array}$ & $\begin{array}{l}\text { Rad. } \\
\text { Rate }\end{array}$ & $\begin{array}{l}\text { Low. } \\
\text { Lev. }\end{array}$ & $\begin{array}{l}\text { Upp } \\
\text { Lev }\end{array}$ & $\begin{array}{l}\text { Osc. } \\
\text { Str. }\end{array}$ & $\begin{array}{l}\text { Rad. } \\
\text { Rate }\end{array}$ \\
\hline$i$ & $j$ & $g f$ & $(1 / s)$ & $i$ & $j$ & $g f$ & $(1 / s)$ & $i$ & j & $g f$ & $(1 / s)$ \\
\hline 176 & 213 & $2.535 e-03$ & $7.504 e+06$ & 176 & 226 & $8.378 e-03$ & $3.589 e+07$ & 176 & 228 & $1.469 \mathrm{e}-03$ & $8.953 e+06$ \\
\hline 176 & 253 & $9.438 e-03$ & $3.897 e+07$ & 176 & 258 & $7.936 e-02$ & $4.223 e+08$ & 176 & 259 & $2.977 \mathrm{e}-02$ & $1.233 e+08$ \\
\hline 176 & 261 & $8.031 \mathrm{e}-01$ & $4.321 \mathrm{e}+09$ & 176 & 265 & $4.443 e-02$ & $1.862 \mathrm{e}+08$ & 176 & 267 & $6.441 \mathrm{e}-03$ & $4.870 e+07$ \\
\hline 176 & 268 & $9.400 e-01$ & $5.078 e+09$ & 176 & 269 & $5.766 e-02$ & $2.423 e+08$ & 176 & 277 & $8.269 \mathrm{e}-02$ & $4.591 e+08$ \\
\hline 176 & 278 & $7.026 e-01$ & $5.467 e+09$ & 176 & 280 & $4.013 e-01$ & $3.159 e+09$ & 177 & 227 & $2.249 \mathrm{e}-03$ & $2.250 e+07$ \\
\hline 177 & 267 & $1.208 \mathrm{e}+00$ & $9.018 e+09$ & 177 & 268 & $1.551 \mathrm{e}-01$ & $8.269 e+08$ & 177 & 277 & $6.370 \mathrm{e}-03$ & $3.491 e+07$ \\
\hline 177 & 278 & $1.771 \mathrm{e}-01$ & $1.360 e+09$ & 177 & 279 & $7.926 e-01$ & $1.016 e+10$ & 178 & 213 & $3.467 e-03$ & $1.000 e+07$ \\
\hline 178 & 226 & $1.173 e-02$ & $4.938 e+07$ & 178 & 228 & $1.315 e-03$ & $7.879 e+06$ & 178 & 261 & $1.001 e+00$ & $5.300 e+09$ \\
\hline 178 & 267 & $8.205 e-02$ & $6.107 e+08$ & 178 & 268 & $7.208 e-01$ & $3.832 \mathrm{e}+09$ & 178 & 269 & $7.284 \mathrm{e}-02$ & $3.013 e+08$ \\
\hline 178 & 277 & $9.627 e-02$ & $5.263 e+08$ & 178 & 278 & $3.903 e-01$ & $2.990 \mathrm{e}+09$ & 178 & 280 & $8.730 \mathrm{e}-01$ & $6.767 e+09$ \\
\hline 179 & 217 & $2.105 e-02$ & $6.049 e+07$ & 179 & 220 & $1.742 \mathrm{e}-02$ & $3.976 e+07$ & 179 & 224 & $6.909 e-02$ & $2.166 e+08$ \\
\hline 179 & 226 & $0.000 e+00$ & $1.758 e+04$ & 179 & 242 & $0.000 \mathrm{e}+00$ & $1.830 e+04$ & 179 & 243 & $1.411 \mathrm{e}+00$ & $5.384 e+09$ \\
\hline 179 & 244 & $3.462 \mathrm{e}-03$ & $1.331 \mathrm{e}+07$ & 179 & 245 & $9.218 \mathrm{e}-02$ & $2.905 e+08$ & 179 & 248 & $2.393 e-02$ & $1.194 e+08$ \\
\hline 179 & 249 & $9.633 e-02$ & $3.740 e+08$ & 179 & 251 & $0.000 e+00$ & $3.475 e+04$ & 179 & 254 & $1.243 e-02$ & $5.049 e+07$ \\
\hline 179 & 256 & $7.676 \mathrm{e}-02$ & $2.553 e+08$ & 179 & 257 & $0.000 e+00$ & $1.534 \mathrm{e}+04$ & 179 & 260 & $0.000 e+00$ & $1.284 e+04$ \\
\hline 179 & 269 & $0.000 e+00$ & $4.323 e+03$ & 179 & 275 & $2.034 \mathrm{e}-01$ & $1.095 e+09$ & 179 & 276 & $0.000 e+00$ & $1.560 \mathrm{e}+05$ \\
\hline 179 & 282 & $4.333 e-01$ & $1.572 \mathrm{e}+09$ & 180 & 197 & $7.542 \mathrm{e}-01$ & $8.532 e+07$ & 180 & 210 & $0.000 e+00$ & $2.458 e+04$ \\
\hline 180 & 211 & $0.000 e+00$ & $1.058 e+04$ & 180 & 214 & $1.806 \mathrm{e}-03$ & $7.030 e+06$ & 180 & 215 & $4.712 \mathrm{e}-01$ & $3.102 e+09$ \\
\hline 180 & 216 & $1.306 \mathrm{e}-01$ & $5.170 e+08$ & 180 & 217 & $6.386 e-03$ & $1.812 \mathrm{e}+07$ & 180 & 218 & $2.562 \mathrm{e}-01$ & $1.029 e+09$ \\
\hline 180 & 219 & $3.230 \mathrm{e}-02$ & $9.308 e+07$ & 180 & 222 & $8.018 e-04$ & $5.617 e+06$ & 180 & 227 & $0.000 e+00$ & $4.872 \mathrm{e}+04$ \\
\hline 180 & 228 & $0.000 e+00$ & $2.143 e+04$ & 180 & 230 & $0.000 \mathrm{e}+00$ & $4.767 e+04$ & 180 & 231 & $0.000 e+00$ & $7.227 e+03$ \\
\hline 180 & 235 & $0.000 e+00$ & $5.504 e+03$ & 180 & 239 & $0.000 e+00$ & $7.117 e+04$ & 180 & 240 & $0.000 e+00$ & $2.610 \mathrm{e}+04$ \\
\hline 180 & 247 & $4.238 \mathrm{e}-01$ & $2.930 e+09$ & 180 & 248 & $2.860 \mathrm{e}-02$ & $1.413 e+08$ & 180 & 250 & $8.924 e-02$ & $6.309 e+08$ \\
\hline 180 & 252 & $2.937 e+00$ & $1.517 e+10$ & 180 & 253 & $0.000 e+00$ & $5.665 e+05$ & 180 & 258 & $0.000 e+00$ & $2.551 e+05$ \\
\hline 180 & 261 & $0.000 e+00$ & $7.903 e+03$ & 180 & 262 & $7.383 e-02$ & $9.040 e+08$ & 180 & 263 & $5.047 e-02$ & $3.708 e+08$ \\
\hline 180 & 264 & $7.113 e-03$ & $3.733 e+07$ & 180 & 265 & $0.000 e+00$ & $6.521 e+03$ & 180 & 267 & $0.000 e+00$ & $6.114 \mathrm{e}+04$ \\
\hline 180 & 268 & $0.000 e+00$ & $1.085 e+04$ & 180 & 272 & $8.847 e-03$ & $6.557 e+07$ & 180 & 273 & $1.846 e-02$ & $2.283 e+08$ \\
\hline 180 & 279 & $0.000 e+00$ & $7.115 e+03$ & 180 & 283 & $5.009 e-02$ & $6.637 e+08$ & 181 & 199 & $1.079 e+00$ & $9.628 e+07$ \\
\hline 181 & 210 & $0.000 e+00$ & $1.255 \mathrm{e}+04$ & 181 & 211 & $0.000 e+00$ & $1.944 \mathrm{e}+04$ & 181 & 212 & $0.000 e+00$ & $8.540 e+03$ \\
\hline 181 & 216 & $6.962 \mathrm{e}-01$ & $2.751 \mathrm{e}+09$ & 181 & 217 & $1.550 \mathrm{e}-01$ & $4.388 e+08$ & 181 & 218 & $7.785 e-02$ & $3.123 e+08$ \\
\hline 181 & 219 & $3.495 e-01$ & $1.005 e+09$ & 181 & 220 & $3.814 \mathrm{e}-02$ & $8.582 e+07$ & 181 & 221 & $1.305 e-03$ & $5.474 e+06$ \\
\hline 181 & 227 & $0.000 e+00$ & $2.561 \mathrm{e}+04$ & 181 & 228 & $0.000 \mathrm{e}+00$ & $3.861 e+04$ & 181 & 229 & $0.000 e+00$ & $1.692 \mathrm{e}+04$ \\
\hline 181 & 230 & $0.000 e+00$ & $1.389 e+04$ & 181 & 231 & $0.000 \mathrm{e}+00$ & $4.582 e+04$ & 181 & 232 & $0.000 e+00$ & $6.506 e+03$ \\
\hline 181 & 240 & $0.000 e+00$ & $5.334 e+04$ & 181 & 241 & $0.000 e+00$ & $1.568 e+04$ & 181 & 247 & $5.018 e-02$ & $3.464 e+08$ \\
\hline 181 & 248 & $5.024 \mathrm{e}-01$ & $2.479 e+09$ & 181 & 249 & $3.955 e-02$ & $1.519 e+08$ & 181 & 251 & $0.000 e+00$ & $1.291 e+04$ \\
\hline 181 & 252 & $2.757 e-01$ & $1.423 e+09$ & 181 & 253 & $0.000 e+00$ & $1.893 e+04$ & 181 & 254 & $4.247 e+00$ & $1.706 e+10$ \\
\hline 181 & 255 & $0.000 e+00$ & $5.885 e+05$ & 181 & 258 & $0.000 e+00$ & $6.820 e+04$ & 181 & 259 & $0.000 e+00$ & $3.106 e+05$ \\
\hline 181 & 263 & $1.644 \mathrm{e}-01$ & $1.206 \mathrm{e}+09$ & 181 & 264 & $4.301 \mathrm{e}-02$ & $2.254 \mathrm{e}+08$ & 181 & 267 & $0.000 e+00$ & $2.696 e+04$ \\
\hline 181 & 268 & $0.000 e+00$ & $6.143 e+04$ & 181 & 269 & $0.000 \mathrm{e}+00$ & $8.936 e+03$ & 181 & 272 & $1.600 \mathrm{e}-02$ & $1.184 \mathrm{e}+08$ \\
\hline 181 & 278 & $0.000 e+00$ & $6.340 e+03$ & 182 & 198 & $1.383 e+00$ & $9.930 e+07$ & 182 & 211 & $0.000 e+00$ & $8.003 e+03$ \\
\hline 182 & 212 & $0.000 e+00$ & $2.935 e+04$ & 182 & 217 & $9.891 \mathrm{e}-01$ & $2.793 e+09$ & 182 & 219 & $1.016 \mathrm{e}-01$ & $2.915 e+08$ \\
\hline 182 & 220 & $5.654 \mathrm{e}-01$ & $1.269 \mathrm{e}+09$ & 182 & 228 & $0.000 e+00$ & $1.588 e+04$ & 182 & 229 & $0.000 e+00$ & $5.596 e+04$ \\
\hline 182 & 231 & $0.000 e+00$ & $1.119 \mathrm{e}+04$ & 182 & 232 & $0.000 e+00$ & $5.746 e+04$ & 182 & 237 & $0.000 e+00$ & $7.155 e+03$ \\
\hline 182 & 238 & $0.000 e+00$ & $4.926 \mathrm{e}+03$ & 182 & 241 & $0.000 e+00$ & $5.713 e+04$ & 182 & 243 & $4.372 \mathrm{e}-02$ & $1.646 e+08$ \\
\hline
\end{tabular}


Table III. Ca IX Oscillator Strengths and Radiative Decay Rates for transitions involving levels higher than 4.

\begin{tabular}{|c|c|c|c|c|c|c|c|c|c|c|c|}
\hline $\begin{array}{l}\text { Low. } \\
\text { Lev . }\end{array}$ & $\begin{array}{l}\text { Upp } \\
\text { Lev }\end{array}$ & $\begin{array}{l}\text { Osc. } \\
\text { Str. }\end{array}$ & $\begin{array}{l}\text { Rad. } \\
\text { Rate }\end{array}$ & $\begin{array}{l}\text { Low. } \\
\text { Lev. }\end{array}$ & $\begin{array}{l}\text { Upp. } \\
\text { Lev. }\end{array}$ & $\begin{array}{l}\text { Osc. } \\
\text { Str. }\end{array}$ & $\begin{array}{l}\text { Rad. } \\
\text { Rate }\end{array}$ & $\begin{array}{l}\text { Low. } \\
\text { Lev. }\end{array}$ & $\begin{array}{l}\text { - Upp } \\
\text {. Lev }\end{array}$ & $\begin{array}{l}\text { Osc. } \\
\text { Str. }\end{array}$ & $\begin{array}{l}\text { Rad. } \\
\text { Rate }\end{array}$ \\
\hline$i$ & $j$ & $g f$ & $(1 / s)$ & $i$ & $j$ & $g f$ & $(1 / s)$ & i & j & $g f$ & $(1 / s)$ \\
\hline 182 & 248 & $4.393 e-02$ & $2.163 e+08$ & 182 & 249 & $6.345 e-01$ & $2.432 \mathrm{e}+09$ & 182 & 252 & $4.396 e-03$ & $2.264 e+07$ \\
\hline 182 & 254 & $2.878 \mathrm{e}-01$ & $1.154 \mathrm{e}+09$ & 182 & 255 & $0.000 e+00$ & $6.768 e+03$ & 182 & 256 & $5.492 e+00$ & $1.803 e+10$ \\
\hline 182 & 257 & $0.000 \mathrm{e}+00$ & $6.213 e+05$ & 182 & 259 & $0.000 e+00$ & $5.265 e+04$ & 182 & 260 & $0.000 e+00$ & $3.577 e+05$ \\
\hline 182 & 264 & $2.828 e-01$ & $1.479 \mathrm{e}+09$ & 182 & 265 & $0.000 e+00$ & $1.815 e+04$ & 182 & 268 & $0.000 e+00$ & $2.033 e+04$ \\
\hline 182 & 269 & $0.000 e+00$ & $6.630 \mathrm{e}+04$ & 182 & 275 & $7.642 \mathrm{e}-03$ & $4.061 e+07$ & 182 & 276 & $0.000 e+00$ & $2.932 \mathrm{e}+03$ \\
\hline 182 & 277 & $0.000 e+00$ & $8.740 \mathrm{e}+03$ & 182 & 282 & $5.602 \mathrm{e}-03$ & $2.007 e+07$ & 183 & 208 & $3.595 e-02$ & $2.304 e+07$ \\
\hline 183 & 214 & $2.640 \mathrm{e}-02$ & $1.013 e+08$ & 183 & 215 & $2.888 \mathrm{e}-02$ & $1.875 e+08$ & 183 & 216 & $4.975 e-03$ & $1.942 \mathrm{e}+07$ \\
\hline 183 & 218 & $8.979 e-03$ & $3.559 e+07$ & 183 & 219 & $2.152 \mathrm{e}-03$ & $6.118 e+06$ & 183 & 233 & $0.000 e+00$ & $1.454 e+04$ \\
\hline 183 & 239 & $0.000 e+00$ & $6.766 e+03$ & 183 & 247 & $3.544 \mathrm{e}-03$ & $2.424 e+07$ & 183 & 248 & $1.760 e-02$ & $8.607 e+07$ \\
\hline 183 & 250 & $1.283 e+00$ & $8.978 e+09$ & 183 & 252 & $2.510 \mathrm{e}-01$ & $1.283 e+09$ & 183 & 253 & $0.000 e+00$ & $3.141 \mathrm{e}+04$ \\
\hline 183 & 258 & $0.000 e+00$ & $1.772 e+04$ & 183 & 261 & $0.000 e+00$ & $2.700 e+04$ & 183 & 262 & $4.441 \mathrm{e}-02$ & $5.382 e+08$ \\
\hline 183 & 263 & $4.122 \mathrm{e}-03$ & $2.998 e+07$ & 183 & 265 & $0.000 e+00$ & $2.077 e+04$ & 183 & 269 & $0.000 e+00$ & $4.135 e+03$ \\
\hline 183 & 272 & $1.305 e-02$ & $9.572 e+07$ & 183 & 273 & $1.416 \mathrm{e}-02$ & $1.733 e+08$ & 183 & 275 & $9.997 e-02$ & $5.275 e+08$ \\
\hline 183 & 280 & $0.000 e+00$ & $6.036 e+03$ & 183 & 281 & $0.000 e+00$ & $7.345 e+03$ & 183 & 283 & $8.420 e-01$ & $1.105 e+10$ \\
\hline 184 & 202 & $2.255 e-01$ & $1.777 \mathrm{e}+07$ & 184 & 205 & $5.250 e-02$ & $2.653 e+07$ & 184 & 210 & $0.000 e+00$ & $8.700 e+03$ \\
\hline 184 & 215 & $2.166 \mathrm{e}-02$ & $1.229 \mathrm{e}+08$ & 184 & 216 & $8.587 e-02$ & $2.930 e+08$ & 184 & 221 & $1.864 \mathrm{e}-01$ & $6.780 e+08$ \\
\hline 184 & 222 & $1.194 \mathrm{e}-01$ & $7.241 e+08$ & 184 & 223 & $1.547 \mathrm{e}-01$ & $2.815 e+09$ & 184 & 227 & $0.000 e+00$ & $2.141 \mathrm{e}+04$ \\
\hline 184 & 229 & $0.000 e+00$ & $9.452 \mathrm{e}+03$ & 184 & 235 & $0.000 e+00$ & $1.806 e+04$ & 184 & 236 & $0.000 e+00$ & $2.663 e+04$ \\
\hline 184 & 240 & $0.000 e+00$ & $1.805 e+04$ & 184 & 241 & $0.000 e+00$ & $3.056 e+04$ & 184 & 262 & $3.383 e-01$ & $3.718 e+09$ \\
\hline 84 & 263 & $1.291 \mathrm{e}+00$ & $8.514 e+09$ & 184 & 267 & $0.000 e+00$ & $1.541 e+05$ & 184 & 268 & $0.000 e+00$ & $2.373 e+05$ \\
\hline 84 & 272 & $1.792 \mathrm{e}-01$ & $1.193 e+09$ & 184 & 273 & $2.423 e-01$ & $2.691 \mathrm{e}+09$ & 184 & 274 & $2.524 \mathrm{e}-01$ & $8.415 e+09$ \\
\hline 84 & 277 & $0.000 e+00$ & $1.231 \mathrm{e}+05$ & 184 & 278 & $0.000 e+00$ & $4.130 e+04$ & 184 & 279 & $0.000 e+00$ & $3.158 e+05$ \\
\hline 185 & 215 & $3.868 \mathrm{e}-02$ & $2.194 e+08$ & 185 & 222 & $1.512 \mathrm{e}-01$ & $9.167 e+08$ & 185 & 228 & $0.000 e+00$ & $9.547 e+03$ \\
\hline 85 & 235 & $0.000 e+00$ & $1.855 \mathrm{e}+04$ & 185 & 241 & $0.000 e+00$ & $1.355 e+04$ & 185 & 262 & $5.815 e-01$ & $6.390 e+09$ \\
\hline 85 & 267 & $0.000 e+00$ & $1.686 \mathrm{e}+05$ & 185 & 273 & $1.862 \mathrm{e}-01$ & $2.067 e+09$ & 185 & 278 & $0.000 e+00$ & $1.263 e+05$ \\
\hline 86 & 201 & $3.940 e-01$ & $2.190 e+07$ & 186 & 203 & $1.883 e-01$ & $1.849 \mathrm{e}+07$ & 186 & 211 & $0.000 e+00$ & $6.778 e+03$ \\
\hline 86 & 212 & $0.000 e+00$ & $7.595 e+03$ & 186 & 214 & $2.069 e-02$ & $6.924 \mathrm{e}+07$ & 186 & 216 & $1.329 \mathrm{e}-02$ & $4.531 e+07$ \\
\hline 86 & 217 & $1.331 \mathrm{e}-01$ & $3.253 e+08$ & 186 & 219 & $2.286 e-03$ & $5.682 e+06$ & 186 & 221 & $5.632 \mathrm{e}-01$ & $2.047 e+09$ \\
\hline 86 & 222 & $1.921 \mathrm{e}-01$ & $1.164 \mathrm{e}+09$ & 186 & 225 & $1.311 \mathrm{e}-02$ & $8.533 e+07$ & 186 & 227 & $0.000 e+00$ & $7.692 \mathrm{e}+03$ \\
\hline 86 & 228 & $0.000 e+00$ & $1.612 \mathrm{e}+04$ & 186 & 229 & $0.000 e+00$ & $1.838 e+04$ & 186 & 236 & $0.000 e+00$ & $1.194 \mathrm{e}+04$ \\
\hline 86 & 237 & $0.000 e+00$ & $3.803 e+04$ & 186 & 239 & $0.000 e+00$ & $7.050 e+04$ & 186 & 240 & $0.000 e+00$ & $5.305 e+04$ \\
\hline 186 & 241 & $0.000 e+00$ & $2.416 \mathrm{e}+04$ & 186 & 250 & $3.220 \mathrm{e}-02$ & $2.038 e+08$ & 186 & 261 & $0.000 e+00$ & $1.783 e+04$ \\
\hline 186 & 262 & $1.202 \mathrm{e}-02$ & $1.320 \mathrm{e}+08$ & 186 & 263 & $2.501 \mathrm{e}-01$ & $1.648 e+09$ & 186 & 264 & $2.135 \mathrm{e}+00$ & $1.005 e+10$ \\
\hline 186 & 265 & $0.000 e+00$ & $1.089 e+05$ & 186 & 267 & $0.000 e+00$ & $1.706 e+04$ & 186 & 268 & $0.000 e+00$ & $9.550 e+04$ \\
\hline 186 & 269 & $0.000 e+00$ & $2.282 e+05$ & 186 & 272 & $1.025 e+00$ & $6.817 e+09$ & 186 & 273 & $3.129 \mathrm{e}-01$ & $3.472 \mathrm{e}+09$ \\
\hline 186 & 275 & $5.942 \mathrm{e}-02$ & $2.844 e+08$ & 186 & 277 & $0.000 e+00$ & $2.732 \mathrm{e}+05$ & 186 & 278 & $0.000 e+00$ & $2.417 e+05$ \\
\hline 186 & 279 & $0.000 e+00$ & $1.003 e+05$ & 186 & 281 & $0.000 e+00$ & $1.097 e+04$ & 186 & 283 & $9.295 e-03$ & $1.110 e+08$ \\
\hline 187 & 206 & $6.394 \mathrm{e}-01$ & $5.313 e+07$ & 187 & 208 & $3.549 e-02$ & $1.369 e+07$ & 187 & 213 & $0.000 e+00$ & $2.060 e+04$ \\
\hline 187 & 214 & $2.706 \mathrm{e}-01$ & $8.962 \mathrm{e}+08$ & 187 & 216 & $2.159 \mathrm{e}-03$ & $7.283 e+06$ & 187 & 217 & $3.590 \mathrm{e}-03$ & $8.683 e+06$ \\
\hline 187 & 218 & $3.584 \mathrm{e}-03$ & $1.229 \mathrm{e}+07$ & 187 & 221 & $2.911 \mathrm{e}-02$ & $1.048 e+08$ & 187 & 222 & $2.529 \mathrm{e}-03$ & $1.517 \mathrm{e}+07$ \\
\hline 187 & 224 & $7.050 e-02$ & $1.875 \mathrm{e}+08$ & 187 & 225 & $4.700 e-01$ & $3.029 e+09$ & 187 & 226 & $0.000 e+00$ & $2.135 \mathrm{e}+04$ \\
\hline 187 & 233 & $0.000 e+00$ & $8.018 e+04$ & 187 & 242 & $0.000 e+00$ & $7.681 e+04$ & 187 & 247 & $2.802 \mathrm{e}-03$ & $1.719 \mathrm{e}+07$ \\
\hline 187 & 250 & $6.766 \mathrm{e}-01$ & $4.249 \mathrm{e}+09$ & 187 & 261 & $0.000 e+00$ & $3.147 e+05$ & 187 & 263 & $8.188 e-03$ & $5.357 e+07$ \\
\hline 187 & 264 & $3.688 e-02$ & $1.724 \mathrm{e}+08$ & 187 & 265 & $0.000 e+00$ & $2.463 e+05$ & 187 & 268 & $0.000 e+00$ & $6.104 e+03$ \\
\hline
\end{tabular}


Table III. Ca IX Oscillator Strengths and Radiative Decay Rates for transitions involving levels higher than 4.

\begin{tabular}{|c|c|c|c|c|c|c|c|c|c|c|c|}
\hline $\begin{array}{l}\text { Low. } \\
\text { Lev . }\end{array}$ & $\begin{array}{l}\text { Upp } \\
\text { Lev }\end{array}$ & $\begin{array}{l}\text { Osc. } \\
\text { Str. }\end{array}$ & $\begin{array}{l}\text { Rad. } \\
\text { Rate }\end{array}$ & $\begin{array}{l}\text { Low. } \\
\text { Lev. }\end{array}$ & $\begin{array}{l}\text { Upp. } \\
\text { Lev. }\end{array}$ & $\begin{array}{l}\text { Osc. } \\
\text { Str. }\end{array}$ & $\begin{array}{l}\text { Rad. } \\
\text { Rate }\end{array}$ & $\begin{array}{l}\text { Low. } \\
\text { Lev. }\end{array}$ & $\begin{array}{l}\text { Upp } \\
\text { Lev }\end{array}$ & $\begin{array}{l}\text { Osc. } \\
\text { Str. }\end{array}$ & $\begin{array}{l}\text { Rad. } \\
\text { Rate }\end{array}$ \\
\hline$i$ & $j$ & $g f$ & $(1 / s)$ & $i$ & $j$ & $g f$ & $(1 / s)$ & $i$ & j & $g f$ & $(1 / s)$ \\
\hline 187 & 269 & $0.000 e+00$ & $1.130 \mathrm{e}+05$ & 187 & 272 & $4.375 e-02$ & $2.889 e+08$ & 187 & 273 & $1.336 \mathrm{e}-02$ & $1.471 e+08$ \\
\hline 187 & 275 & $2.811 \mathrm{e}+00$ & $1.336 \mathrm{e}+10$ & 187 & 277 & $0.000 e+00$ & $1.443 e+04$ & 187 & 278 & $0.000 \mathrm{e}+00$ & $4.736 e+03$ \\
\hline 187 & 280 & $0.000 \mathrm{e}+00$ & $1.077 \mathrm{e}+05$ & 187 & 281 & $0.000 e+00$ & $2.892 e+05$ & 187 & 283 & $2.461 \mathrm{e}-01$ & $2.917 e+09$ \\
\hline 188 & 209 & $2.400 e+00$ & $2.697 \mathrm{e}+08$ & 188 & 213 & $0.000 e+00$ & $3.208 e+04$ & 188 & 224 & $1.686 \mathrm{e}+00$ & $4.365 e+09$ \\
\hline 188 & 226 & $0.000 e+00$ & $7.095 e+04$ & 188 & 238 & $0.000 e+00$ & $3.741 e+04$ & 188 & 242 & $0.000 e+00$ & $1.136 e+05$ \\
\hline 188 & 243 & $3.941 \mathrm{e}-01$ & $1.292 \mathrm{e}+09$ & 188 & 244 & $5.443 e-03$ & $1.800 \mathrm{e}+07$ & 188 & 251 & $0.000 e+00$ & $1.541 \mathrm{e}+05$ \\
\hline 188 & 255 & $0.000 e+00$ & $3.825 e+03$ & 188 & 265 & $0.000 e+00$ & $1.328 e+04$ & 188 & 275 & $1.442 \mathrm{e}-01$ & $6.718 e+08$ \\
\hline 188 & 276 & $0.000 e+00$ & $6.909 e+05$ & 188 & 282 & $7.192 e+00$ & $2.268 e+10$ & 189 & 214 & $0.000 e+00$ & $1.028 e+04$ \\
\hline 189 & 224 & $0.000 e+00$ & $7.907 e+03$ & 189 & 226 & $1.432 \mathrm{e}-01$ & $5.037 e+08$ & 189 & 243 & $0.000 e+00$ & $2.167 e+04$ \\
\hline 189 & 250 & $0.000 e+00$ & $1.026 \mathrm{e}+04$ & 189 & 251 & $1.069 \mathrm{e}+01$ & $3.012 \mathrm{e}+10$ & 189 & 253 & $1.113 \mathrm{e}-02$ & $3.852 \mathrm{e}+07$ \\
\hline 189 & 255 & $2.447 e-01$ & $6.941 \mathrm{e}+08$ & 189 & 258 & $7.604 \mathrm{e}-03$ & $3.394 e+07$ & 189 & 259 & $2.863 e-03$ & $9.949 e+06$ \\
\hline 189 & 260 & $2.189 \mathrm{e}-02$ & $6.227 e+07$ & 189 & 261 & $3.018 e-01$ & $1.364 e+09$ & 189 & 265 & $6.878 \mathrm{e}-01$ & $2.421 e+09$ \\
\hline 189 & 266 & $1.590 e-02$ & $4.587 e+07$ & 189 & 269 & $9.010 e-02$ & $3.180 e+08$ & 189 & 275 & $0.000 e+00$ & $2.955 e+04$ \\
\hline 189 & 282 & $0.000 e+00$ & $2.002 e+04$ & 190 & 218 & $0.000 e+00$ & $1.193 e+04$ & 190 & 229 & $7.968 e-03$ & $2.715 e+07$ \\
\hline 190 & 230 & $1.112 \mathrm{e}-01$ & $3.821 \mathrm{e}+08$ & 190 & 231 & $5.777 e-03$ & $1.546 e+07$ & 190 & 244 & $0.000 e+00$ & $3.410 e+04$ \\
\hline 190 & 245 & $0.000 e+00$ & $4.236 e+03$ & 190 & 251 & $5.793 e-03$ & $1.558 e+07$ & 190 & 252 & $0.000 e+00$ & $1.468 e+04$ \\
\hline 190 & 253 & $2.384 e-01$ & $7.881 \mathrm{e}+08$ & 190 & 258 & $1.342 \mathrm{e}-01$ & $5.718 e+08$ & 190 & 259 & $2.309 \mathrm{e}-02$ & $7.661 \mathrm{e}+07$ \\
\hline 190 & 260 & $6.448 e-01$ & $1.752 \mathrm{e}+09$ & 190 & 261 & $8.784 e-03$ & $3.790 e+07$ & 190 & 265 & $3.394 \mathrm{e}-03$ & $1.141 \mathrm{e}+07$ \\
\hline 190 & 266 & $1.153 e+01$ & $3.177 \mathrm{e}+10$ & 190 & 268 & $1.070 e-03$ & $4.635 e+06$ & 190 & 269 & $7.654 \mathrm{e}-02$ & $2.580 e+08$ \\
\hline 190 & 277 & $2.739 e-02$ & $1.224 \mathrm{e}+08$ & 191 & 215 & $0.000 e+00$ & $4.979 e+03$ & 191 & 223 & $0.000 e+00$ & $9.061 e+03$ \\
\hline 191 & 227 & $6.865 \mathrm{e}-02$ & $5.437 e+08$ & 191 & 228 & $1.160 \mathrm{e}-02$ & $5.516 e+07$ & 191 & 230 & $4.198 e-03$ & $1.440 \mathrm{e}+07$ \\
\hline 191 & 235 & $3.672 \mathrm{e}-03$ & $1.856 e+07$ & 191 & 244 & $0.000 e+00$ & $5.724 e+03$ & 191 & 247 & $0.000 e+00$ & $7.830 e+03$ \\
\hline 191 & 252 & $0.000 e+00$ & $1.402 \mathrm{e}+04$ & 191 & 258 & $5.624 \mathrm{e}+00$ & $2.394 e+10$ & 191 & 261 & $1.479 \mathrm{e}-02$ & $6.374 \mathrm{e}+07$ \\
\hline 91 & 262 & $0.000 e+00$ & $2.755 e+04$ & 191 & 263 & $0.000 e+00$ & $1.096 e+04$ & 191 & 267 & $5.605 e-01$ & $3.396 e+09$ \\
\hline 91 & 268 & $1.801 \mathrm{e}-02$ & $7.796 \mathrm{e}+07$ & 191 & 274 & $0.000 e+00$ & $1.509 e+04$ & 191 & 278 & $3.937 e-02$ & $2.463 e+08$ \\
\hline 91 & 279 & $2.169 \mathrm{e}-01$ & $2.264 \mathrm{e}+09$ & 192 & 221 & $0.000 e+00$ & $6.803 e+03$ & 192 & 229 & $1.369 \mathrm{e}-01$ & $4.654 \mathrm{e}+08$ \\
\hline 92 & 230 & $6.793 e-03$ & $2.329 \mathrm{e}+07$ & 192 & 232 & $5.999 e-03$ & $1.314 \mathrm{e}+07$ & 192 & 237 & $4.908 e-03$ & $1.381 \mathrm{e}+07$ \\
\hline 92 & 244 & $0.000 e+00$ & $2.956 \mathrm{e}+03$ & 192 & 246 & $0.000 e+00$ & $6.800 e+03$ & 192 & 249 & $0.000 e+00$ & $8.961 e+03$ \\
\hline 92 & 251 & $8.295 e-02$ & $2.226 \mathrm{e}+08$ & 192 & 253 & $3.043 e-03$ & $1.004 \mathrm{e}+07$ & 192 & 255 & $9.462 \mathrm{e}-01$ & $2.557 e+09$ \\
\hline 92 & 256 & $0.000 e+00$ & $1.453 e+04$ & 192 & 258 & $2.435 e-02$ & $1.036 e+08$ & 192 & 259 & $2.926 \mathrm{e}-01$ & $9.689 e+08$ \\
\hline 92 & 260 & $7.978 e+00$ & $2.163 e+10$ & 192 & 261 & $1.378 \mathrm{e}-03$ & $5.936 e+06$ & 192 & 264 & $0.000 e+00$ & $2.855 e+04$ \\
\hline 192 & 265 & $2.117 e-01$ & $7.104 e+08$ & 192 & 266 & $7.456 e-01$ & $2.051 e+09$ & 192 & 268 & $6.970 \mathrm{e}-02$ & $3.015 e+08$ \\
\hline 192 & 269 & $9.687 e-01$ & $3.259 \mathrm{e}+09$ & 192 & 272 & $0.000 e+00$ & $1.378 e+04$ & 192 & 277 & $4.181 \mathrm{e}-01$ & $1.865 e+09$ \\
\hline 193 & 222 & $0.000 e+00$ & $6.247 e+03$ & 193 & 228 & $1.013 e-01$ & $4.806 e+08$ & 193 & 229 & $1.151 \mathrm{e}-02$ & $3.909 e+07$ \\
\hline 193 & 231 & $5.119 e-03$ & $1.365 e+07$ & 193 & 236 & $4.214 \mathrm{e}-03$ & $1.519 e+07$ & 193 & 245 & $0.000 e+00$ & $5.999 e+03$ \\
\hline 193 & 248 & $0.000 e+00$ & $6.786 e+03$ & 193 & 253 & $5.886 e-01$ & $1.939 \mathrm{e}+09$ & 193 & 254 & $0.000 e+00$ & $1.243 e+04$ \\
\hline 193 & 258 & $3.622 \mathrm{e}-01$ & $1.539 e+09$ & 193 & 259 & $6.815 e+00$ & $2.254 e+10$ & 193 & 261 & $1.168 \mathrm{e}-02$ & $5.024 e+07$ \\
\hline 193 & 262 & $0.000 e+00$ & $1.037 e+04$ & 193 & 263 & $0.000 e+00$ & $2.134 \mathrm{e}+04$ & 193 & 265 & $3.624 \mathrm{e}-03$ & $1.215 e+07$ \\
\hline 93 & 267 & $6.319 \mathrm{e}-02$ & $3.821 \mathrm{e}+08$ & 193 & 268 & $8.563 e-01$ & $3.699 e+09$ & 193 & 269 & $1.343 e-02$ & $4.514 e+07$ \\
\hline 93 & 273 & $0.000 e+00$ & $1.269 \mathrm{e}+04$ & 193 & 277 & $2.979 \mathrm{e}-02$ & $1.327 e+08$ & 193 & 278 & $3.198 e-01$ & $1.997 \mathrm{e}+09$ \\
\hline 93 & 280 & $3.061 \mathrm{e}-03$ & $1.936 \mathrm{e}+07$ & 194 & 219 & $0.000 e+00$ & $1.163 e+04$ & 194 & 231 & $1.434 \mathrm{e}-01$ & $3.811 \mathrm{e}+08$ \\
\hline 94 & 232 & $5.263 e-03$ & $1.147 e+07$ & 194 & 244 & $0.000 e+00$ & $4.370 e+03$ & 194 & 245 & $0.000 e+00$ & $3.322 \mathrm{e}+04$ \\
\hline & 246 & $0.000 e+00$ & $2.953 e+03$ & 194 & 251 & $1.747 e-02$ & $4.669 e+07$ & 94 & 253 & $1.334 \mathrm{e}-03$ & $4.381 e+06$ \\
\hline 194 & 254 & $0.000 e+00$ & $1.314 \mathrm{e}+04$ & 194 & 255 & $1.994 \mathrm{e}-01$ & $5.366 e+08$ & 194 & 257 & $8.776 \mathrm{e}-02$ & $2.001 e+08$ \\
\hline
\end{tabular}


Table III. Ca IX Oscillator Strengths and Radiative Decay Rates for transitions involving levels higher than 4.

\begin{tabular}{|c|c|c|c|c|c|c|c|c|c|c|c|}
\hline $\begin{array}{l}\text { Low. } \\
\text { Lev . }\end{array}$ & $\begin{array}{l}\text { Upp } \\
\text { Lev }\end{array}$ & $\begin{array}{l}\text { Osc. } \\
\text { Str. }\end{array}$ & $\begin{array}{l}\text { Rad. } \\
\text { Rate }\end{array}$ & $\begin{array}{l}\text { Low. } \\
\text { Lev. }\end{array}$ & $\begin{array}{l}\text { Upp. } \\
\text { Lev. }\end{array}$ & $\begin{array}{l}\text { Osc. } \\
\text { Str. }\end{array}$ & $\begin{array}{l}\text { Rad. } \\
\text { Rate }\end{array}$ & $\begin{array}{l}\text { Low. } \\
\text { Lev. }\end{array}$ & $\begin{array}{l}\text { - Upp } \\
\text {. Lev }\end{array}$ & $\begin{array}{l}\text { Osc. } \\
\text { Str. }\end{array}$ & $\begin{array}{l}\text { Rad. } \\
\text { Rate }\end{array}$ \\
\hline$i$ & $j$ & $g f$ & $(1 / s)$ & $i$ & $j$ & $g f$ & $(1 / s)$ & i & j & $g f$ & $(1 / s)$ \\
\hline 194 & 259 & $2.110 \mathrm{e}-01$ & $6.956 \mathrm{e}+08$ & 194 & 265 & $2.089 \mathrm{e}-03$ & $6.980 e+06$ & 194 & 266 & $4.426 e-01$ & $1.212 \mathrm{e}+09$ \\
\hline 194 & 270 & $1.455 \mathrm{e}+01$ & $3.377 \mathrm{e}+10$ & 194 & 276 & $3.725 e-02$ & $8.849 e+07$ & 195 & 220 & $0.000 e+00$ & $1.171 \mathrm{e}+04$ \\
\hline 195 & 232 & $1.720 \mathrm{e}-01$ & $3.739 \mathrm{e}+08$ & 195 & 245 & $0.000 e+00$ & $3.574 \mathrm{e}+03$ & 195 & 246 & $0.000 e+00$ & $3.610 e+04$ \\
\hline 195 & 251 & $1.839 \mathrm{e}-03$ & $4.902 \mathrm{e}+06$ & 195 & 255 & $5.895 e-03$ & $1.583 e+07$ & 195 & 256 & $0.000 e+00$ & $1.469 \mathrm{e}+04$ \\
\hline 195 & 257 & $2.155 e-01$ & $4.900 e+08$ & 195 & 260 & $2.759 \mathrm{e}-01$ & $7.429 e+08$ & 195 & 266 & $3.561 \mathrm{e}-03$ & $9.731 e+06$ \\
\hline 195 & 270 & $4.454 \mathrm{e}-01$ & $1.031 \mathrm{e}+09$ & 195 & 271 & $1.741 \mathrm{e}+01$ & $3.498 e+10$ & 196 & 225 & $0.000 e+00$ & $1.423 e+04$ \\
\hline 196 & 226 & $4.907 e-03$ & $1.563 e+07$ & 196 & 233 & $7.948 e-02$ & $6.110 e+08$ & 196 & 243 & $0.000 e+00$ & $7.279 e+03$ \\
\hline 196 & 258 & $1.437 e-02$ & $5.874 \mathrm{e}+07$ & 196 & 261 & $5.441 \mathrm{e}+00$ & $2.253 e+10$ & 196 & 268 & $5.680 e-03$ & $2.362 \mathrm{e}+07$ \\
\hline 196 & 275 & $0.000 e+00$ & $2.388 e+04$ & 196 & 277 & $2.777 e-03$ & $1.192 \mathrm{e}+07$ & 196 & 278 & $7.535 e-03$ & $4.531 e+07$ \\
\hline 196 & 279 & $7.314 \mathrm{e}-04$ & $7.339 e+06$ & 196 & 280 & $1.142 \mathrm{e}+00$ & $6.958 e+09$ & 196 & 283 & $0.000 e+00$ & $5.447 e+04$ \\
\hline 197 & 215 & $0.000 e+00$ & $1.103 e+04$ & 197 & 218 & $0.000 e+00$ & $5.137 e+03$ & 197 & 230 & $4.812 \mathrm{e}-02$ & $1.439 e+08$ \\
\hline 197 & 231 & $3.408 e-03$ & $7.940 e+06$ & 197 & 235 & $2.135 \mathrm{e}-01$ & $9.440 e+08$ & 197 & 236 & $2.015 e-02$ & $6.372 \mathrm{e}+07$ \\
\hline 197 & 244 & $0.000 e+00$ & $1.634 \mathrm{e}+04$ & 197 & 247 & $0.000 \mathrm{e}+00$ & $2.733 e+04$ & 197 & 252 & $0.000 e+00$ & $1.935 e+04$ \\
\hline 197 & 253 & $8.472 \mathrm{e}+00$ & $2.474 \mathrm{e}+10$ & 197 & 258 & $8.240 e-01$ & $3.103 e+09$ & 197 & 259 & $3.815 e-01$ & $1.119 \mathrm{e}+09$ \\
\hline 197 & 261 & $2.053 e-03$ & $7.834 e+06$ & 197 & 262 & $0.000 e+00$ & $1.064 e+04$ & 197 & 265 & $3.282 \mathrm{e}-03$ & $9.759 e+06$ \\
\hline 197 & 267 & $1.724 \mathrm{e}-01$ & $9.252 e+08$ & 197 & 268 & $8.530 e-03$ & $3.269 e+07$ & 197 & 269 & $1.569 \mathrm{e}-02$ & $4.678 e+07$ \\
\hline 197 & 277 & $4.286 e-03$ & $1.697 \mathrm{e}+07$ & 198 & 217 & $0.000 e+00$ & $8.997 e+03$ & 198 & 220 & $0.000 e+00$ & $6.489 e+03$ \\
\hline 198 & 231 & $3.642 \mathrm{e}-03$ & $8.454 e+06$ & 198 & 232 & $7.952 \mathrm{e}-02$ & $1.514 \mathrm{e}+08$ & 198 & 237 & $3.655 e-01$ & $8.971 e+08$ \\
\hline 198 & 246 & $0.000 e+00$ & $1.875 e+04$ & 198 & 249 & $0.000 e+00$ & $2.644 e+04$ & 198 & 251 & $1.735 \mathrm{e}-03$ & $4.105 e+06$ \\
\hline 198 & 255 & $9.632 \mathrm{e}-02$ & $2.296 \mathrm{e}+08$ & 198 & 256 & $0.000 e+00$ & $2.135 e+04$ & 198 & 257 & $1.340 \mathrm{e}+01$ & $2.707 e+10$ \\
\hline 198 & 259 & $4.239 e-02$ & $1.238 \mathrm{e}+08$ & 198 & 260 & $1.615 \mathrm{e}+00$ & $3.864 e+09$ & 198 & 265 & $5.461 \mathrm{e}-02$ & $1.618 e+08$ \\
\hline 198 & 266 & $1.981 \mathrm{e}-03$ & $4.811 \mathrm{e}+06$ & 198 & 269 & $2.690 \mathrm{e}-01$ & $7.992 e+08$ & 198 & 270 & $4.674 e-02$ & $9.622 \mathrm{e}+07$ \\
\hline 198 & 276 & $2.229 \mathrm{e}-03$ & $4.702 \mathrm{e}+06$ & 199 & 216 & $0.000 e+00$ & $8.520 e+03$ & 199 & 219 & $0.000 e+00$ & $4.473 e+03$ \\
\hline 99 & 230 & $3.391 \mathrm{e}-03$ & $1.010 e+07$ & 199 & 231 & $6.042 e-02$ & $1.402 \mathrm{e}+08$ & 199 & 232 & $3.399 e-03$ & $6.468 e+06$ \\
\hline 99 & 236 & $2.802 \mathrm{e}-01$ & $8.822 \mathrm{e}+08$ & 199 & 237 & $1.989 \mathrm{e}-02$ & $4.878 e+07$ & 199 & 244 & $0.000 e+00$ & $2.707 e+03$ \\
\hline 99 & 245 & $0.000 e+00$ & $1.597 e+04$ & 199 & 248 & $0.000 e+00$ & $2.196 e+04$ & 199 & 251 & $1.843 e-01$ & $4.357 e+08$ \\
\hline 99 & 253 & $1.467 \mathrm{e}-01$ & $4.267 e+08$ & 199 & 254 & $0.000 e+00$ & $1.689 e+04$ & 199 & 255 & $1.006 \mathrm{e}+01$ & $2.398 e+10$ \\
\hline 99 & 258 & $4.127 e-02$ & $1.548 \mathrm{e}+08$ & 199 & 259 & $1.303 e+00$ & $3.805 e+09$ & 199 & 260 & $6.548 e-01$ & $1.566 \mathrm{e}+09$ \\
\hline 99 & 261 & $1.760 \mathrm{e}-03$ & $6.688 \mathrm{e}+06$ & 199 & 265 & $4.412 \mathrm{e}-03$ & $1.306 e+07$ & 199 & 266 & $4.282 \mathrm{e}-02$ & $1.040 e+08$ \\
\hline 99 & 268 & $2.450 e-01$ & $9.352 e+08$ & 199 & 269 & $5.618 e-03$ & $1.669 e+07$ & 199 & 277 & $1.220 \mathrm{e}-03$ & $4.812 \mathrm{e}+06$ \\
\hline 200 & 222 & $0.000 e+00$ & $6.286 e+03$ & 200 & 227 & $3.095 e-02$ & $2.087 e+08$ & 200 & 228 & $8.367 e-03$ & $3.389 e+07$ \\
\hline 200 & 235 & $7.209 e-03$ & $3.118 e+07$ & 200 & 239 & $2.878 e-02$ & $6.681 \mathrm{e}+08$ & 200 & 240 & $1.931 \mathrm{e}-02$ & $1.495 e+08$ \\
\hline 200 & 241 & $1.019 \mathrm{e}-03$ & $4.738 e+06$ & 200 & 247 & $0.000 \mathrm{e}+00$ & $1.652 e+04$ & 200 & 267 & $3.455 \mathrm{e}+00$ & $1.817 e+10$ \\
\hline 200 & 273 & $0.000 e+00$ & $1.947 e+04$ & 200 & 278 & $2.460 \mathrm{e}-01$ & $1.339 e+09$ & 200 & 279 & $4.183 e-01$ & $3.798 e+09$ \\
\hline 200 & 280 & $4.657 e-03$ & $2.570 e+07$ & 201 & 221 & $0.000 e+00$ & $7.625 e+03$ & 201 & 222 & $0.000 e+00$ & $6.135 e+03$ \\
\hline 201 & 228 & $1.055 \mathrm{e}-02$ & $4.269 e+07$ & 201 & 229 & $8.232 \mathrm{e}-02$ & $2.382 \mathrm{e}+08$ & 201 & 237 & $1.505 e-02$ & $3.622 \mathrm{e}+07$ \\
\hline 201 & 238 & $8.399 e-03$ & $3.881 e+07$ & 201 & 241 & $1.049 \mathrm{e}-01$ & $4.872 e+08$ & 201 & 248 & $0.000 e+00$ & $9.025 e+03$ \\
\hline 201 & 249 & $0.000 e+00$ & $2.294 e+04$ & 201 & 253 & $2.412 \mathrm{e}-02$ & $6.894 e+07$ & 201 & 259 & $2.632 \mathrm{e}-02$ & $7.552 \mathrm{e}+07$ \\
\hline 201 & 261 & $3.642 \mathrm{e}-03$ & $1.360 \mathrm{e}+07$ & 201 & 265 & $1.156 \mathrm{e}+00$ & $3.365 e+09$ & 201 & 267 & $1.951 \mathrm{e}-02$ & $1.025 e+08$ \\
\hline 201 & 268 & $5.616 \mathrm{e}-01$ & $2.107 e+09$ & 201 & 269 & $6.095 e+00$ & $1.779 e+10$ & 201 & 272 & $0.000 e+00$ & $2.983 e+04$ \\
\hline 01 & 273 & $0.000 e+00$ & $2.459 \mathrm{e}+04$ & 201 & 277 & $1.540 \mathrm{e}+00$ & $5.971 e+09$ & 201 & 278 & $1.909 \mathrm{e}-01$ & $1.038 e+09$ \\
\hline 01 & 280 & $8.953 e-04$ & $4.935 e+06$ & 202 & 223 & $0.000 e+00$ & $1.335 e+04$ & 202 & 227 & $9.602 e-03$ & $6.462 \mathrm{e}+07$ \\
\hline 02 & 228 & $4.714 \mathrm{e}-02$ & $1.905 \mathrm{e}+08$ & 202 & 229 & $9.387 e-03$ & $2.714 \mathrm{e}+07$ & 202 & 236 & $1.066 \mathrm{e}-02$ & $3.291 \mathrm{e}+07$ \\
\hline 02 & 238 & $1.379 \mathrm{e}-03$ & $6.366 \mathrm{e}+06$ & 202 & 240 & $6.209 \mathrm{e}-02$ & $4.796 e+08$ & 202 & 241 & $1.837 e-02$ & $8.524 e+07$ \\
\hline
\end{tabular}


Table III. Ca IX Oscillator Strengths and Radiative Decay Rates for transitions involving levels higher than 4.

\begin{tabular}{|c|c|c|c|c|c|c|c|c|c|c|c|}
\hline $\begin{array}{l}\text { Low. } \\
\text { Lev. }\end{array}$ & $\begin{array}{l}\text { Upp } \\
\text { Lev }\end{array}$ & $\begin{array}{l}\text { Osc. } \\
\text { Str. }\end{array}$ & $\begin{array}{l}\text { Rad. } \\
\text { Rate }\end{array}$ & $\begin{array}{l}\text { Low. } \\
\text { Lev. }\end{array}$ & $\begin{array}{l}\text { Upp. } \\
\text { Lev. }\end{array}$ & $\begin{array}{l}\text { Osc. } \\
\text { Str. }\end{array}$ & $\begin{array}{l}\text { Rad. } \\
\text { Rate }\end{array}$ & $\begin{array}{l}\text { Low. } \\
\text { Lev. }\end{array}$ & $\begin{array}{l}\text { Upp } \\
\text { Lev }\end{array}$ & $\begin{array}{l}\text { Osc. } \\
\text { Str. }\end{array}$ & $\begin{array}{l}\text { Rad. } \\
\text { Rate }\end{array}$ \\
\hline$i$ & $j$ & $g f$ & $(1 / s)$ & $i$ & $j$ & $g f$ & $(1 / s)$ & $i$ & j & $g f$ & $(1 / s)$ \\
\hline 202 & 247 & $0.000 e+00$ & $1.093 e+04$ & 202 & 248 & $0.000 e+00$ & $1.417 e+04$ & 202 & 258 & $1.561 \mathrm{e}-02$ & $5.750 e+07$ \\
\hline 202 & 261 & $1.497 \mathrm{e}-03$ & $5.587 e+06$ & 202 & 267 & $5.972 e-01$ & $3.135 e+09$ & 202 & 268 & $5.082 \mathrm{e}+00$ & $1.905 e+10$ \\
\hline 202 & 272 & $0.000 \mathrm{e}+00$ & $1.722 \mathrm{e}+04$ & 202 & 274 & $0.000 e+00$ & $5.033 e+04$ & 202 & 277 & $1.628 \mathrm{e}-01$ & $6.311 e+08$ \\
\hline 202 & 278 & $8.281 \mathrm{e}-01$ & $4.498 e+09$ & 202 & 279 & $1.775 e-01$ & $1.608 e+09$ & 202 & 280 & $3.100 e-03$ & $1.707 e+07$ \\
\hline 203 & 222 & $0.000 e+00$ & $6.327 e+03$ & 203 & 223 & $0.000 e+00$ & $8.228 e+03$ & 203 & 228 & $2.126 \mathrm{e}-03$ & $8.308 e+06$ \\
\hline 203 & 229 & $1.378 \mathrm{e}-02$ & $3.853 e+07$ & 203 & 234 & $8.346 e-02$ & $5.760 e+08$ & 203 & 240 & $2.877 e-03$ & $2.154 \mathrm{e}+07$ \\
\hline 203 & 241 & $1.132 \mathrm{e}-02$ & $5.089 e+07$ & 203 & 249 & $0.000 e+00$ & $7.858 e+03$ & 203 & 261 & $3.033 e-03$ & $1.099 e+07$ \\
\hline 203 & 263 & $0.000 e+00$ & $1.541 \mathrm{e}+04$ & 203 & 264 & $0.000 e+00$ & $1.473 e+04$ & 203 & 268 & $1.373 e-03$ & $4.999 e+06$ \\
\hline 203 & 273 & $0.000 e+00$ & $2.023 e+04$ & 203 & 274 & $0.000 e+00$ & $3.138 e+04$ & 203 & 277 & $5.736 e+00$ & $2.160 e+10$ \\
\hline 203 & 278 & $1.063 e+00$ & $5.611 e+09$ & 203 & 279 & $7.501 \mathrm{e}-02$ & $6.605 e+08$ & 203 & 280 & $8.839 e-03$ & $4.730 e+07$ \\
\hline 204 & 227 & $1.274 \mathrm{e}-03$ & $8.278 e+06$ & 204 & 228 & $8.197 e-03$ & $3.199 e+07$ & 204 & 234 & $4.863 e-02$ & $3.353 e+08$ \\
\hline 204 & 239 & $1.756 e-03$ & $3.939 e+07$ & 204 & 240 & $3.824 \mathrm{e}-03$ & $2.859 e+07$ & 204 & 241 & $3.752 \mathrm{e}-03$ & $1.685 e+07$ \\
\hline 204 & 262 & $0.000 e+00$ & $1.352 e+04$ & 204 & 267 & $7.859 \mathrm{e}-03$ & $4.001 e+07$ & 204 & 272 & $0.000 e+00$ & $1.294 e+04$ \\
\hline 204 & 273 & $0.000 e+00$ & $1.180 e+04$ & 204 & 278 & $2.958 e+00$ & $1.559 e+10$ & 204 & 279 & $1.149 e+00$ & $1.011 e+10$ \\
\hline 204 & 280 & $1.638 \mathrm{e}-02$ & $8.756 e+07$ & 205 & 227 & $3.023 e-03$ & $1.960 \mathrm{e}+07$ & 205 & 234 & $1.617 \mathrm{e}-02$ & $1.112 \mathrm{e}+08$ \\
\hline 205 & 240 & $3.233 e-03$ & $2.412 \mathrm{e}+07$ & 205 & 279 & $1.376 \mathrm{e}+00$ & $1.208 e+10$ & 206 & 214 & $0.000 e+00$ & $6.795 e+03$ \\
\hline 206 & 225 & $0.000 e+00$ & $9.936 e+03$ & 206 & 226 & $7.303 e-02$ & $1.922 \mathrm{e}+08$ & 206 & 238 & $2.189 \mathrm{e}-01$ & $9.454 e+08$ \\
\hline 206 & 241 & $1.679 \mathrm{e}-02$ & $7.286 e+07$ & 206 & 242 & $1.802 \mathrm{e}-01$ & $4.391 e+08$ & 206 & 243 & $0.000 e+00$ & $1.883 e+04$ \\
\hline 206 & 250 & $0.000 e+00$ & $3.935 e+04$ & 206 & 253 & $3.681 \mathrm{e}-03$ & $9.867 e+06$ & 206 & 258 & $8.298 e-03$ & $2.869 e+07$ \\
\hline 206 & 259 & $1.837 e-02$ & $4.944 e+07$ & 206 & 261 & $1.106 \mathrm{e}+00$ & $3.876 e+09$ & 206 & 265 & $6.864 \mathrm{e}+00$ & $1.875 e+10$ \\
\hline 206 & 269 & $1.314 \mathrm{e}+00$ & $3.600 e+09$ & 206 & 277 & $1.281 \mathrm{e}-03$ & $4.668 e+06$ & 206 & 278 & $1.326 \mathrm{e}-03$ & $6.773 e+06$ \\
\hline 206 & 280 & $2.917 e-01$ & $1.511 \mathrm{e}+09$ & 206 & 283 & $0.000 e+00$ & $1.487 e+04$ & 207 & 225 & $1.503 e-01$ & $6.512 \mathrm{e}+08$ \\
\hline 207 & 238 & $0.000 e+00$ & $4.789 e+04$ & 207 & 280 & $0.000 e+00$ & $1.694 \mathrm{e}+05$ & 207 & 283 & $9.625 e-01$ & $8.577 e+09$ \\
\hline 08 & 225 & $0.000 e+00$ & $9.099 e+03$ & 208 & 233 & $6.225 \mathrm{e}-02$ & $3.419 e+08$ & 208 & 238 & $6.617 \mathrm{e}-02$ & $2.486 e+08$ \\
\hline 08 & 241 & $5.042 \mathrm{e}-03$ & $1.904 \mathrm{e}+07$ & 208 & 250 & $0.000 e+00$ & $2.536 e+04$ & 208 & 267 & $1.378 e-03$ & $5.964 e+06$ \\
\hline 208 & 278 & $2.486 \mathrm{e}-02$ & $1.117 e+08$ & 208 & 280 & $4.355 e+00$ & $1.987 e+10$ & 208 & 281 & $3.501 \mathrm{e}-02$ & $8.053 e+08$ \\
\hline 08 & 283 & $0.000 e+00$ & $2.591 \mathrm{e}+04$ & 209 & 224 & $0.000 e+00$ & $1.481 e+04$ & 209 & 242 & $7.646 \mathrm{e}-01$ & $1.554 \mathrm{e}+09$ \\
\hline 09 & 243 & $0.000 e+00$ & $1.753 e+04$ & 209 & 251 & $5.812 \mathrm{e}-01$ & $1.065 e+09$ & 209 & 255 & $1.222 \mathrm{e}-02$ & $2.258 e+07$ \\
\hline 209 & 257 & $3.540 \mathrm{e}-03$ & $5.544 \mathrm{e}+06$ & 209 & 260 & $2.177 e-03$ & $4.039 e+06$ & 209 & 265 & $9.231 \mathrm{e}-02$ & $2.125 e+08$ \\
\hline 209 & 269 & $1.915 e-02$ & $4.425 e+07$ & 209 & 270 & $3.822 \mathrm{e}-02$ & $6.117 e+07$ & 209 & 276 & $1.650 \mathrm{e}+01$ & $2.715 e+10$ \\
\hline 209 & 282 & $0.000 e+00$ & $5.589 e+04$ & 210 & 215 & $7.382 \mathrm{e}-01$ & $1.406 e+08$ & 210 & 216 & $1.406 \mathrm{e}-01$ & $1.627 e+07$ \\
\hline 210 & 218 & $1.523 e+00$ & $1.923 e+08$ & 210 & 222 & $3.045 e-01$ & $8.146 e+07$ & 210 & 223 & $3.633 e-01$ & $2.921 e+08$ \\
\hline 210 & 240 & $0.000 e+00$ & $4.603 e+03$ & 211 & 215 & $2.805 e-01$ & $5.300 e+07$ & 211 & 216 & $1.227 e+00$ & $1.408 e+08$ \\
\hline 211 & 217 & $1.078 \mathrm{e}-01$ & $9.011 \mathrm{e}+06$ & 211 & 218 & $1.393 e-01$ & $1.745 e+07$ & 211 & 219 & $2.278 e+00$ & $2.085 e+08$ \\
\hline 211 & 221 & $3.192 \mathrm{e}-01$ & $5.085 e+07$ & 211 & 222 & $7.815 e-01$ & $2.076 e+08$ & 211 & 231 & $0.000 e+00$ & $4.451 e+03$ \\
\hline 11 & 239 & $0.000 e+00$ & $1.045 e+04$ & 212 & 216 & $3.208 e-01$ & $3.629 \mathrm{e}+07$ & 212 & 217 & $2.281 e+00$ & $1.879 e+08$ \\
\hline 212 & 219 & $9.726 e-02$ & $8.776 e+06$ & 212 & 220 & $3.098 e+00$ & $2.245 e+08$ & 212 & 221 & $1.448 \mathrm{e}+00$ & $2.278 e+08$ \\
\hline 12 & 232 & $0.000 e+00$ & $5.299 e+03$ & 212 & 237 & $0.000 e+00$ & $6.673 e+03$ & 212 & 240 & $0.000 e+00$ & $5.001 e+03$ \\
\hline 12 & 241 & $0.000 e+00$ & $5.468 e+03$ & 213 & 214 & $1.529 e+00$ & $1.268 \mathrm{e}+08$ & 213 & 224 & $2.512 \mathrm{e}+00$ & $2.833 e+08$ \\
\hline 13 & 225 & $1.047 e+00$ & $3.298 e+08$ & 213 & 238 & $0.000 e+00$ & $8.502 e+03$ & 213 & 242 & $0.000 e+00$ & $8.519 e+03$ \\
\hline 13 & 250 & $1.356 \mathrm{e}-02$ & $1.287 e+07$ & 213 & 275 & $5.069 e-02$ & $3.969 e+07$ & 213 & 281 & $0.000 e+00$ & $1.775 e+04$ \\
\hline 14 & 226 & $2.490 \mathrm{e}+00$ & $3.455 e+08$ & 214 & 230 & $2.795 e-02$ & $4.441 e+06$ & 214 & 233 & $3.935 \mathrm{e}-01$ & $1.490 \mathrm{e}+08$ \\
\hline 14 & 235 & $2.218 \mathrm{e}-02$ & $6.186 \mathrm{e}+06$ & 214 & 238 & $1.635 \mathrm{e}+00$ & $5.874 e+08$ & 214 & 241 & $7.542 e-02$ & $2.755 e+07$ \\
\hline 14 & 280 & $2.119 \mathrm{e}-02$ & $1.348 \mathrm{e}+07$ & 215 & 227 & $6.666 \mathrm{e}-01$ & $2.202 \mathrm{e}+08$ & 215 & 228 & $2.031 \mathrm{e}-01$ & $4.041 e+07$ \\
\hline
\end{tabular}


Table III. Ca IX Oscillator Strengths and Radiative Decay Rates for transitions involving levels higher than 4.

\begin{tabular}{|c|c|c|c|c|c|c|c|c|c|c|c|}
\hline $\begin{array}{l}\text { Low. } \\
\text { Lev. }\end{array}$ & $\begin{array}{l}\text { Upp } \\
\text { Lev }\end{array}$ & $\begin{array}{l}\text { Osc. } \\
\text { Str. }\end{array}$ & $\begin{array}{l}\text { Rad. } \\
\text { Rate }\end{array}$ & $\begin{array}{l}\text { Low. } \\
\text { Lev. }\end{array}$ & $\begin{array}{l}\text { - Upp. } \\
\text { Lev. }\end{array}$ & $\begin{array}{l}\text { Osc. } \\
\text { Str. }\end{array}$ & $\begin{array}{l}\text { Rad. } \\
\text { Rate }\end{array}$ & $\begin{array}{l}\text { Low. } \\
\text { Lev. }\end{array}$ & $\begin{array}{l}\text { Upp. } \\
\text { Lev. }\end{array}$ & $\begin{array}{l}\text { Osc. } \\
\text { Str. }\end{array}$ & $\begin{array}{l}\text { Rad. } \\
\text { Rate }\end{array}$ \\
\hline$i$ & $j$ & $g f$ & $(1 / s)$ & $i$ & $j$ & $g f$ & $(1 / s)$ & $i$ & j & $g f$ & $(1 / s)$ \\
\hline 215 & 235 & $1.725 \mathrm{e}+00$ & $4.547 e+08$ & 215 & 239 & $2.021 \mathrm{e}-01$ & $3.498 e+08$ & 215 & 240 & $1.195 \mathrm{e}-01$ & $6.900 \mathrm{e}+07$ \\
\hline 216 & 227 & $2.419 \mathrm{e}-01$ & $7.913 e+07$ & 216 & 228 & $9.733 e-01$ & $1.918 e+08$ & 216 & 229 & $1.885 e-01$ & $2.674 e+07$ \\
\hline 216 & 230 & $3.592 \mathrm{e}-02$ & $5.304 e+06$ & 216 & 235 & $4.568 e-01$ & $1.194 e+08$ & 216 & 236 & $2.416 e+00$ & $4.529 e+08$ \\
\hline 216 & 240 & $4.552 \mathrm{e}-01$ & $2.609 e+08$ & 216 & 241 & $9.374 \mathrm{e}-02$ & $3.236 e+07$ & 217 & 228 & $2.622 \mathrm{e}-01$ & $5.092 e+07$ \\
\hline 217 & 229 & $1.647 e+00$ & $2.302 e+08$ & 217 & 231 & $6.807 e-02$ & $7.761 e+06$ & 217 & 235 & $2.135 \mathrm{e}-02$ & $5.508 e+06$ \\
\hline 217 & 236 & $5.285 e-01$ & $9.781 e+07$ & 217 & 237 & $3.498 \mathrm{e}+00$ & $5.071 e+08$ & 217 & 238 & $6.549 e-02$ & $2.197 e+07$ \\
\hline 217 & 241 & $6.927 \mathrm{e}-01$ & $2.365 e+08$ & 218 & 227 & $7.874 \mathrm{e}-02$ & $2.404 \mathrm{e}+07$ & 218 & 228 & $6.864 \mathrm{e}-02$ & $1.263 \mathrm{e}+07$ \\
\hline 218 & 230 & $3.426 \mathrm{e}+00$ & $4.731 \mathrm{e}+08$ & 218 & 235 & $7.756 e-01$ & $1.909 e+08$ & 218 & 236 & $2.340 \mathrm{e}-01$ & $4.133 e+07$ \\
\hline 218 & 238 & $2.664 \mathrm{e}-02$ & $8.578 e+06$ & 219 & 228 & $1.211 \mathrm{e}-01$ & $2.186 e+07$ & 219 & 229 & $1.048 \mathrm{e}-01$ & $1.362 \mathrm{e}+07$ \\
\hline 219 & 230 & $2.892 \mathrm{e}-01$ & $3.921 e+07$ & 219 & 231 & $4.492 e+00$ & $4.770 e+08$ & 219 & 235 & $1.067 e-01$ & $2.584 e+07$ \\
\hline 219 & 236 & $1.039 \mathrm{e}+00$ & $1.804 \mathrm{e}+08$ & 219 & 237 & $3.146 \mathrm{e}-01$ & $4.283 e+07$ & 220 & 229 & $2.432 \mathrm{e}-01$ & $3.076 e+07$ \\
\hline 220 & 231 & $2.828 e-01$ & $2.924 \mathrm{e}+07$ & 220 & 232 & $5.893 e+00$ & $5.047 e+08$ & 220 & 236 & $1.204 \mathrm{e}-01$ & $2.044 e+07$ \\
\hline 220 & 237 & $1.747 \mathrm{e}+00$ & $2.324 \mathrm{e}+08$ & 221 & 228 & $1.757 e-01$ & $2.585 e+07$ & 221 & 229 & $1.269 \mathrm{e}+00$ & $1.346 \mathrm{e}+08$ \\
\hline 221 & 234 & $8.149 e-01$ & $2.650 e+08$ & 221 & 238 & $9.574 \mathrm{e}-02$ & $2.610 e+07$ & 221 & 240 & $6.032 \mathrm{e}-01$ & $2.783 e+08$ \\
\hline 221 & 241 & $1.815 \mathrm{e}+00$ & $5.045 e+08$ & 222 & 227 & $1.951 \mathrm{e}-01$ & $4.760 e+07$ & 222 & 228 & $6.856 e-01$ & $1.009 \mathrm{e}+08$ \\
\hline 222 & 234 & $5.128 e-01$ & $1.667 e+08$ & 222 & 238 & $6.567 e-02$ & $1.790 \mathrm{e}+07$ & 222 & 239 & $4.763 e-01$ & $6.585 e+08$ \\
\hline 222 & 240 & $3.736 e-01$ & $1.723 e+08$ & 222 & 241 & $5.490 e-01$ & $1.526 e+08$ & 222 & 281 & $1.610 \mathrm{e}-03$ & $4.275 e+06$ \\
\hline 223 & 227 & $2.904 \mathrm{e}-01$ & $7.073 e+07$ & 223 & 234 & $1.744 \mathrm{e}-01$ & $5.661 e+07$ & 223 & 240 & $4.813 e-01$ & $2.217 e+08$ \\
\hline 224 & 226 & $8.995 e-01$ & $7.052 e+07$ & 224 & 238 & $3.231 \mathrm{e}-01$ & $7.755 e+07$ & 224 & 241 & $3.428 \mathrm{e}-02$ & $8.399 e+06$ \\
\hline 224 & 242 & $5.341 \mathrm{e}+00$ & $7.599 e+08$ & 224 & 265 & $1.211 \mathrm{e}-01$ & $2.663 e+07$ & 224 & 269 & $2.217 e-02$ & $4.926 e+06$ \\
\hline 224 & 280 & $9.764 \mathrm{e}-03$ & $4.622 e+06$ & 225 & 233 & $8.382 e-01$ & $1.521 e+08$ & 225 & 238 & $1.036 \mathrm{e}+00$ & $2.123 e+08$ \\
\hline 225 & 241 & $1.004 \mathrm{e}-01$ & $2.104 \mathrm{e}+07$ & 225 & 280 & $1.489 e-02$ & $6.306 e+06$ & 225 & 281 & $8.209 e-01$ & $1.784 \mathrm{e}+09$ \\
\hline 226 & 243 & $1.857 \mathrm{e}+00$ & $3.896 e+07$ & 226 & 275 & $7.893 e-01$ & $5.714 e+07$ & 227 & 247 & $7.410 e-01$ & $2.866 e+07$ \\
\hline 227 & 262 & $3.140 \mathrm{e}-01$ & $4.037 e+07$ & 227 & 274 & $3.285 \mathrm{e}-02$ & $1.401 e+07$ & 228 & 248 & $1.105 e+00$ & $3.051 e+07$ \\
\hline 228 & 262 & $8.370 e-02$ & $1.069 e+07$ & 228 & 263 & $4.953 e-01$ & $3.802 e+07$ & 228 & 273 & $9.066 e-02$ & $1.273 e+07$ \\
\hline 229 & 249 & $1.578 e+00$ & $3.362 e+07$ & 229 & 264 & $8.422 e-01$ & $4.565 e+07$ & 229 & 272 & $1.685 e-01$ & $1.390 e+07$ \\
\hline 230 & 244 & $1.906 \mathrm{e}+00$ & $3.206 e+07$ & 230 & 252 & $3.120 \mathrm{e}-01$ & $1.339 e+07$ & 231 & 245 & $2.368 e+00$ & $3.269 e+07$ \\
\hline 231 & 254 & $3.935 \mathrm{e}-01$ & $1.313 e+07$ & 232 & 246 & $2.897 e+00$ & $3.352 e+07$ & 232 & 256 & $5.185 e-01$ & $1.399 e+07$ \\
\hline 233 & 250 & $7.603 e-01$ & $3.337 e+07$ & 233 & 283 & $6.588 e-01$ & $1.505 e+08$ & 234 & 272 & $7.111 \mathrm{e}-01$ & $3.785 e+07$ \\
\hline 234 & 273 & $4.195 e-01$ & $3.767 e+07$ & 234 & 274 & $1.395 \mathrm{e}-01$ & $3.785 e+07$ & 235 & 252 & $1.213 e+00$ & $3.081 \mathrm{e}+07$ \\
\hline 236 & 254 & $1.594 \mathrm{e}+00$ & $3.163 e+07$ & 237 & 256 & $2.084 e+00$ & $3.365 e+07$ & 238 & 275 & $8.087 e-01$ & $1.474 \mathrm{e}+07$ \\
\hline
\end{tabular}


Table IV. Ca IX Fractional Level Populations

\begin{tabular}{|c|c|c|c|c|c|c|c|}
\hline $\begin{array}{c}\text { og (Den.) } \\
\text { Key }\end{array}$ & 8 & 9 & 10 & $\begin{array}{c}11 \\
\text { Population }\end{array}$ & 12 & 13 & 14 \\
\hline 1 & $9.938-01$ & $9.853-01$ & $9.446-01$ & $9.073-01$ & $9.003-01$ & $8.994-01$ & $8.982-01$ \\
\hline 2 & $5.197-03$ & $5.977-03$ & $9.704-03$ & $1.311-02$ & $1.375-02$ & $1.384-02$ & $1.397-02$ \\
\hline 3 & $4.039-10$ & $4.134-09$ & $4.584-08$ & $4.996-07$ & $5.074-06$ & $5.081-05$ & $5.071-04$ \\
\hline 4 & $9.568-04$ & $8.703-03$ & $4.572-02$ & $7.956-02$ & $8.592-02$ & $8.667-02$ & $8.729-02$ \\
\hline 5 & $3.648-12$ & $3.617-11$ & $3.470-10$ & $3.335-09$ & $3.310-08$ & $3.307-07$ & $3.302-06$ \\
\hline 6 & 1. $252-12$ & $1.247-11$ & 1. $221-10$ & $1.198-09$ & $1.194-08$ & $1.193-07$ & $1.192-06$ \\
\hline 7 & $1.934-16$ & $2.258-15$ & $3.806-14$ & $5.223-13$ & $5.516-12$ & $5.817-11$ & $8.531-10$ \\
\hline 8 & $9.727-15$ & $1.450-13$ & $3.733-12$ & $5.820-11$ & $6.213-10$ & $6.262-09$ & $6.333-08$ \\
\hline 9 & $7.085-15$ & $1.682-13$ & $6.331-12$ & $1.058-10$ & $1.138-09$ & $1.148-08$ & $1.159-07$ \\
\hline 10 & $1.095-15$ & $1.099-14$ & $1.120-13$ & 1. $139-12$ & $1.143-11$ & $1.143-10$ & $1.144-09$ \\
\hline 11 & $1.034-14$ & $1.112-13$ & 1. $483-12$ & $1.821-11$ & $1.885-10$ & $1.894-09$ & $1.916-08$ \\
\hline 12 & $1.008-14$ & $1.119-13$ & 1. 649-12 & $2.134-11$ & $2.226-10$ & $2.239-09$ & $2.278-08$ \\
\hline 13 & $1.462-14$ & $2.061-13$ & $4.923-12$ & $7.539-11$ & $8.032-10$ & $8.089-09$ & $8.139-08$ \\
\hline 14 & $6.208-14$ & $6.157-13$ & $5.914-12$ & $5.691-11$ & $5.649-10$ & $5.644-09$ & $5.637-08$ \\
\hline 15 & $2.531-15$ & $2.872-14$ & $4.504-13$ & $5.996-12$ & $6.277-11$ & $6.318-10$ & $6.426-09$ \\
\hline 16 & 1. $322-15$ & $1.928-14$ & $4.826-13$ & $7.475-12$ & $7.974-11$ & $8.045-10$ & $8.216-09$ \\
\hline 17 & $1.668-15$ & $3.940-14$ & 1. $480-12$ & $2.472-11$ & $2.659-10$ & $2.681-09$ & $2.699-08$ \\
\hline 18 & $4.461-16$ & $4.660-15$ & $5.607-14$ & $6.474-13$ & $6.637-12$ & $6.657-11$ & $6.687-10$ \\
\hline 19 & $2.621-16$ & $2.937-15$ & $4.447-14$ & $5.828-13$ & $6.088-12$ & $6.126-11$ & $6.227-10$ \\
\hline 20 & $6.772-17$ & $7.984-16$ & $1.378-14$ & $1.907-13$ & $2.007-12$ & $2.023-11$ & $2.079-10$ \\
\hline 21 & $3.836-17$ & $6.065-16$ & $1.671-14$ & $2.645-13$ & $2.828-12$ & $2.850-11$ & $2.868-10$ \\
\hline 22 & $5.772-17$ & $1.011-15$ & $3.081-14$ & $4.974-13$ & $5.330-12$ & $5.373-11$ & $5.417-10$ \\
\hline 23 & $9.546-17$ & $1.708-15$ & $5.309-14$ & $8.600-13$ & $9.219-12$ & $9.293-11$ & $9.360-10$ \\
\hline 24 & $5.728-17$ & $1.352-15$ & $5.077-14$ & $8.481-13$ & $9.122-12$ & $9.200-11$ & $9.293-10$ \\
\hline 25 & 1. $159-15$ & $1.159-14$ & $1.161-13$ & 1. $162-12$ & $1.162-11$ & 1. $162-10$ & $1.162-09$ \\
\hline 26 & $4.519-16$ & $4.512-15$ & $4.476-14$ & $4.443-13$ & $4.436-12$ & $4.436-11$ & $4.434-10$ \\
\hline 27 & $1.836-16$ & $1.970-15$ & $2.610-14$ & $3.195-13$ & $3.305-12$ & $3.319-11$ & $3.337-10$ \\
\hline 28 & $9.603-15$ & $9.521-14$ & $9.129-13$ & $8.772-12$ & $8.704-11$ & $8.696-10$ & $8.684-09$ \\
\hline 29 & $2.685-16$ & $2.918-15$ & $4.031-14$ & $5.049-13$ & $5.241-12$ & $5.265-11$ & $5.302-10$ \\
\hline 30 & $6.635-16$ & $6.678-15$ & $6.879-14$ & $7.063-13$ & $7.098-12$ & $7.110-11$ & $7.192-10$ \\
\hline 31 & $7.375-19$ & $8.333-18$ & 1. $291-16$ & $1.710-15$ & $1.789-14$ & $1.799-13$ & $1.820-12$ \\
\hline 32 & $1.013-18$ & $1.229-17$ & $2.263-16$ & $3.209-15$ & $3.386-14$ & $3.408-13$ & $3.436-12$ \\
\hline 33 & $7.307-16$ & $9.366-15$ & $1.921-13$ & $2.821-12$ & $2.990-11$ & $3.010-10$ & $3.028-09$ \\
\hline 34 & $1.093-18$ & $1.373-17$ & $2.706-16$ & $3.926-15$ & $4.155-14$ & $4.183-13$ & $4.219-12$ \\
\hline 35 & 1. $168-15$ & $1.159-14$ & $1.119-13$ & $1.082-12$ & $1.075-11$ & $1.074-10$ & $1.073-09$ \\
\hline 36 & $9.097-18$ & $9.682-17$ & 1. $248-15$ & $1.503-14$ & $1.551-13$ & $1.557-12$ & $1.566-11$ \\
\hline 37 & 1. $250-16$ & 1. $243-15$ & $1.210-14$ & $1.180-13$ & $1.175-12$ & $1.174-11$ & $1.173-10$ \\
\hline 38 & $9.206-19$ & $1.076-17$ & $1.816-16$ & $2.493-15$ & $2.621-14$ & $2.636-13$ & $2.657-12$ \\
\hline 39 & $2.347-19$ & $2.357-18$ & $2.406-17$ & $2.451-16$ & $2.461-15$ & $2.468-14$ & $2.533-13$ \\
\hline 40 & $1.819-18$ & $2.168-17$ & $3.839-16$ & $5.366-15$ & $5.653-14$ & $5.687-13$ & $5.723-12$ \\
\hline 41 & $1.284-17$ & $1.274-16$ & $1.226-15$ & $1.182-14$ & $1.173-13$ & 1. $172-12$ & $1.171-11$ \\
\hline 42 & $1.064-16$ & $1.081-15$ & $1.164-14$ & $1.240-13$ & $1.254-12$ & $1.256-11$ & $1.260-10$ \\
\hline 43 & $1.670-16$ & $1.694-15$ & $1.804-14$ & $1.905-13$ & $1.924-12$ & $1.926-11$ & $1.932-10$ \\
\hline 44 & $2.284-16$ & $2.368-15$ & $2.773-14$ & $3.144-13$ & $3.213-12$ & $3.221-11$ & $3.229-10$ \\
\hline 45 & $1.992-15$ & $1.978-14$ & $1.909-13$ & $1.846-12$ & $1.834-11$ & $1.833-10$ & $1.831-09$ \\
\hline 46 & $1.816-17$ & $2.086-16$ & $3.376-15$ & $4.555-14$ & $4.777-13$ & $4.806-12$ & $4.853-11$ \\
\hline
\end{tabular}


Table IV. Ca IX Fractional Level Populations

\begin{tabular}{|c|c|c|c|c|c|c|c|}
\hline $\begin{array}{l}\text { og (Den.) } \\
\text { Key }\end{array}$ & 8 & 9 & 10 & $\begin{array}{c}11 \\
\text { Population }\end{array}$ & 12 & 13 & 14 \\
\hline 47 & $1.992-18$ & $2.138-17$ & $2.838-16$ & $3.480-15$ & $3.615-14$ & $3.784-13$ & $5.340-12$ \\
\hline 48 & $4.396-18$ & $3.149-16$ & $1.610-14$ & $2.794-13$ & $3.016-12$ & $3.042-11$ & $3.064-10$ \\
\hline 49 & $8.802-17$ & $8.941-16$ & $9.605-15$ & $1.021-13$ & $1.033-12$ & $1.034-11$ & $1.038-10$ \\
\hline 50 & $1.132-16$ & $1.153-15$ & 1. $255-14$ & $1.348-13$ & $1.366-12$ & $1.368-11$ & $1.374-10$ \\
\hline 51 & $1.461-16$ & $1.541-15$ & 1. $924-14$ & $2.274-13$ & $2.340-12$ & $2.347-11$ & $2.354-10$ \\
\hline 52 & $2.555-17$ & $2.550-16$ & $2.530-15$ & $2.511-14$ & $2.508-13$ & $2.507-12$ & $2.508-11$ \\
\hline 53 & $9.236-16$ & $9.165-15$ & $8.823-14$ & $8.510-13$ & $8.451-12$ & $8.444-11$ & $8.434-10$ \\
\hline 54 & $3.048-18$ & $3.320-17$ & $4.618-16$ & $5.804-15$ & $6.028-14$ & $6.058-13$ & $6.119-12$ \\
\hline 55 & $4.618-18$ & $5.114-17$ & $7.482-16$ & $9.648-15$ & $1.006-13$ & $1.011-12$ & $1.022-11$ \\
\hline 56 & $3.769-18$ & $4.296-17$ & $6.816-16$ & $9.120-15$ & $9.554-14$ & $9.619-13$ & $9.802-12$ \\
\hline 57 & $6.348-18$ & $9.540-17$ & $2.480-15$ & $3.874-14$ & $4.137-13$ & $4.168-12$ & $4.195-11$ \\
\hline 58 & $1.420-18$ & $1.448-17$ & $1.583-16$ & $1.706-15$ & $1.729-14$ & $1.737-13$ & $1.783-12$ \\
\hline 59 & $4.028-18$ & $4.516-17$ & $6.849-16$ & $8.983-15$ & $9.385-14$ & $9.442-13$ & $9.582-12$ \\
\hline 60 & $4.521-18$ & $5.959-17$ & $1.283-15$ & $1.911-14$ & $2.030-13$ & $2.044-12$ & $2.057-11$ \\
\hline 61 & $2.859-18$ & $4.759-17$ & $1.384-15$ & $2.214-14$ & $2.370-13$ & $2.389-12$ & $2.409-11$ \\
\hline 62 & $7.008-18$ & $7.333-17$ & $8.884-16$ & $1.030-14$ & $1.057-13$ & $1.060-12$ & $1.064-11$ \\
\hline 63 & $3.874-16$ & $3.843-15$ & $3.692-14$ & $3.554-13$ & $3.528-12$ & $3.525-11$ & $3.521-10$ \\
\hline 64 & $4.571-17$ & $4.637-16$ & $4.952-15$ & $5.240-14$ & $5.294-13$ & $5.301-12$ & $5.310-11$ \\
\hline 65 & $7.212-16$ & $7.151-15$ & $6.857-14$ & $6.589-13$ & $6.539-12$ & $6.533-11$ & $6.524-10$ \\
\hline 66 & $4.255-18$ & $4.299-17$ & $4.506-16$ & $4.695-15$ & $4.731-14$ & $4.739-13$ & $4.781-12$ \\
\hline 67 & $3.359-18$ & $3.845-17$ & $6.166-16$ & $8.287-15$ & $8.687-14$ & $8.739-13$ & $8.829-12$ \\
\hline 68 & $2.314-18$ & $2.734-17$ & $4.743-16$ & $6.580-15$ & $6.925-14$ & $6.970-13$ & $7.041-12$ \\
\hline 69 & $1.408-18$ & $1.839-17$ & $3.900-16$ & $5.783-15$ & $6.138-14$ & $6.186-13$ & $6.276-12$ \\
\hline 70 & $5.479-17$ & $5.547-16$ & $5.873-15$ & $6.172-14$ & $6.228-13$ & $6.238-12$ & $6.276-11$ \\
\hline 71 & $2.025-17$ & $2.165-16$ & $2.834-15$ & $3.446-14$ & $3.561-13$ & $3.575-12$ & $3.595-11$ \\
\hline 72 & $5.726-17$ & $6.612-16$ & $1.085-14$ & $1.472-13$ & $1.544-12$ & $1.553-11$ & $1.561-10$ \\
\hline 73 & $9.728-17$ & $9.686-16$ & $9.486-15$ & $9.304-14$ & $9.270-13$ & $9.266-12$ & $9.265-11$ \\
\hline 74 & $1.015-17$ & 1. $141-16$ & $1.745-15$ & $2.297-14$ & $2.401-13$ & $2.414-12$ & $2.439-11$ \\
\hline 75 & $7.390-18$ & $8.403-17$ & 1. $324-15$ & $1.767-14$ & $1.850-13$ & $1.860-12$ & $1.873-11$ \\
\hline 76 & $2.828-17$ & $3.643-16$ & $7.533-15$ & $1.109-13$ & $1.176-12$ & 1. $184-11$ & $1.191-10$ \\
\hline 77 & $6.621-18$ & $6.962-17$ & $8.594-16$ & $1.009-14$ & $1.037-13$ & $1.040-12$ & $1.044-11$ \\
\hline 78 & $2.739-17$ & $3.115-16$ & $4.909-15$ & $6.548-14$ & $6.857-13$ & $6.893-12$ & $6.924-11$ \\
\hline 79 & $1.545-17$ & $1.558-16$ & $1.618-15$ & $1.673-14$ & $1.684-13$ & $1.686-12$ & $1.704-11$ \\
\hline 80 & $5.731-18$ & $6.049-17$ & $7.571-16$ & $8.962-15$ & $9.224-14$ & $9.257-13$ & $9.308-12$ \\
\hline 81 & $4.752-17$ & $4.721-16$ & $4.572-15$ & $4.437-14$ & $4.411-13$ & $4.408-12$ & $4.404-11$ \\
\hline 82 & $3.356-18$ & $3.566-17$ & $4.566-16$ & $5.481-15$ & $5.653-14$ & $5.680-13$ & $5.764-12$ \\
\hline 83 & $4.295-18$ & $4.626-17$ & $6.209-16$ & $7.656-15$ & $7.929-14$ & $7.973-13$ & $8.112-12$ \\
\hline 84 & $1.920-18$ & $2.173-17$ & $3.385-16$ & $4.492-15$ & $4.701-14$ & $4.728-13$ & $4.773-12$ \\
\hline 85 & $5.492-18$ & $7.354-17$ & $1.625-15$ & $2.439-14$ & $2.592-13$ & $2.610-12$ & $2.625-11$ \\
\hline 86 & $8.023-19$ & $9.317-18$ & $1.550-16$ & $2.116-15$ & $2.222-14$ & $2.239-13$ & $2.292-12$ \\
\hline 87 & $1.079-18$ & $1.571-17$ & $3.921-16$ & $6.069-15$ & $6.473-14$ & $6.524-13$ & $6.592-12$ \\
\hline 88 & $8.625-19$ & $1.794-17$ & $6.243-16$ & $1.031-14$ & $1.107-13$ & $1.117-12$ & $1.125-11$ \\
\hline 89 & $1.493-17$ & $1.582-16$ & $2.004-15$ & $2.389-14$ & $2.462-13$ & $2.471-12$ & $2.482-11$ \\
\hline 90 & $1.344-17$ & $1.408-16$ & $1.713-15$ & $1.991-14$ & $2.043-13$ & $2.050-12$ & $2.058-11$ \\
\hline 91 & $9.037-18$ & $9.512-17$ & $1.178-15$ & $1.386-14$ & $1.425-13$ & $1.429-12$ & $1.434-11$ \\
\hline 92 & $2.121-16$ & $2.106-15$ & $2.034-14$ & 1. $969-13$ & $1.956-12$ & $1.955-11$ & $1.953-10$ \\
\hline
\end{tabular}


Table IV. Ca IX Fractional Level Populations

\begin{tabular}{|c|c|c|c|c|c|c|c|}
\hline og (Den.) & 8 & 9 & 10 & 11 & 12 & 13 & 14 \\
\hline Key & \multicolumn{7}{|c|}{ Population } \\
\hline 93 & $3.970-17$ & $3.979-16$ & $4.021-15$ & $4.060-14$ & $4.067-13$ & $4.068-12$ & $4.069-11$ \\
\hline 94 & $2.042-17$ & $2.084-16$ & $2.287-15$ & $2.473-14$ & $2.508-13$ & $2.512-12$ & $2.516-11$ \\
\hline 95 & $1.224-17$ & 1. $239-16$ & $1.310-15$ & $1.375-14$ & $1.387-13$ & $1.389-12$ & $1.392-11$ \\
\hline 96 & $6.179-18$ & $6.295-17$ & $6.848-16$ & $7.353-15$ & $7.448-14$ & $7.460-13$ & $7.476-12$ \\
\hline 97 & $3.217-18$ & $3.297-17$ & $3.677-16$ & $4.025-15$ & $4.090-14$ & $4.098-13$ & $4.108-12$ \\
\hline 98 & $3.720-17$ & $3.748-16$ & $3.886-15$ & $4.012-14$ & $4.036-13$ & $4.040-12$ & $4.049-11$ \\
\hline 99 & $4.916-17$ & $4.965-16$ & $5.202-15$ & $5.419-14$ & $5.460-13$ & $5.466-12$ & $5.480-11$ \\
\hline 100 & $6.391-17$ & $6.594-16$ & $7.565-15$ & $8.452-14$ & $8.619-13$ & $8.639-12$ & $8.654-11$ \\
\hline 101 & $2.609-16$ & $2.590-15$ & $2.500-14$ & $2.418-13$ & $2.402-12$ & $2.400-11$ & $2.398-10$ \\
\hline 102 & $1.660-17$ & $1.664-16$ & $1.680-15$ & $1.695-14$ & $1.698-13$ & $1.698-12$ & $1.699-11$ \\
\hline 103 & $2.054-17$ & $2.056-16$ & $2.068-15$ & $2.080-14$ & $2.082-13$ & $2.082-12$ & $2.083-11$ \\
\hline 104 & $2.391-17$ & $2.402-16$ & $2.454-15$ & $2.501-14$ & $2.510-13$ & $2.511-12$ & $2.512-11$ \\
\hline 105 & $3.313-17$ & $3.293-16$ & $3.199-15$ & $3.112-14$ & $3.096-13$ & $3.094-12$ & $3.091-11$ \\
\hline 106 & $1.934-19$ & $1.988-18$ & $2.246-17$ & $2.481-16$ & $2.526-15$ & $2.531-14$ & $2.539-13$ \\
\hline 107 & $3.145-19$ & $3.215-18$ & $3.551-17$ & $3.859-16$ & $3.917-15$ & $3.924-14$ & $3.937-13$ \\
\hline 108 & $4.347-19$ & $4.535-18$ & $5.432-17$ & $6.253-16$ & $6.407-15$ & $6.426-14$ & $6.446-13$ \\
\hline 109 & $5.769-18$ & $5.736-17$ & $5.579-16$ & $5.436-15$ & $5.409-14$ & $5.406-13$ & $5.401-12$ \\
\hline 110 & $1.799-18$ & $2.064-17$ & $3.332-16$ & $4.491-15$ & $4.709-14$ & $4.737-13$ & $4.785-12$ \\
\hline 111 & $2.174-19$ & $2.302-18$ & $2.914-17$ & $3.474-16$ & $3.592-15$ & $3.727-14$ & $4.962-13$ \\
\hline 112 & $1.046-19$ & $5.703-18$ & $2.796-16$ & $4.831-15$ & $5.213-14$ & $5.258-13$ & $5.296-12$ \\
\hline 113 & $3.577-19$ & $2.354-17$ & $1.190-15$ & $2.062-14$ & $2.226-13$ & $2.245-12$ & $2.261-11$ \\
\hline 114 & $2.190-19$ & $2.606-18$ & $4.597-17$ & $6.417-16$ & $6.761-15$ & $6.821-14$ & $7.056-13$ \\
\hline 115 & $2.852-20$ & $2.989-19$ & $3.641-18$ & $4.238-17$ & $4.350-16$ & $4.364-15$ & $4.387-14$ \\
\hline 116 & $2.954-20$ & $5.101-19$ & $1.536-17$ & $2.474-16$ & $2.650-15$ & $2.671-14$ & $2.690-13$ \\
\hline 117 & $3.088-20$ & $3.411-19$ & $4.957-18$ & $6.369-17$ & $6.635-16$ & $6.667-15$ & $6.705-14$ \\
\hline 118 & $1.585-19$ & $1.665-18$ & $2.044-17$ & $2.391-16$ & $2.456-15$ & $2.466-14$ & $2.494-13$ \\
\hline 119 & $1.475-19$ & 1. 544-18 & $1.876-17$ & $2.180-16$ & $2.237-15$ & $2.246-14$ & $2.270-13$ \\
\hline 120 & $1.713-19$ & $1.866-18$ & $2.600-17$ & $3.270-16$ & $3.396-15$ & $3.411-14$ & $3.425-13$ \\
\hline 121 & $2.270-19$ & $2.585-18$ & $4.092-17$ & $5.470-16$ & $5.730-15$ & $5.763-14$ & $5.819-13$ \\
\hline 122 & $6.838-20$ & $7.348-19$ & $9.786-18$ & $1.202-16$ & 1. $246-15$ & $1.273-14$ & $1.498-13$ \\
\hline 123 & $1.812-19$ & $7.443-18$ & $3.435-16$ & $5.895-15$ & $6.358-14$ & $6.412-13$ & $6.458-12$ \\
\hline 124 & $5.446-19$ & $5.424-18$ & $5.317-17$ & $5.220-16$ & $5.201-15$ & $5.199-14$ & $5.196-13$ \\
\hline 125 & $2.569-19$ & $2.784-18$ & $3.814-17$ & $4.756-16$ & $4.934-15$ & $4.963-14$ & $5.059-13$ \\
\hline 126 & $4.713-19$ & $5.282-18$ & $7.998-17$ & $1.048-15$ & $1.095-14$ & $1.101-13$ & $1.114-12$ \\
\hline 127 & $8.395-19$ & $8.423-18$ & $8.553-17$ & $8.673-16$ & $8.695-15$ & $8.698-14$ & $8.702-13$ \\
\hline 128 & $3.799-19$ & $4.236-18$ & $6.323-17$ & $8.232-16$ & $8.593-15$ & $8.658-14$ & $8.920-13$ \\
\hline 129 & 1. 266-19 & $1.338-18$ & $1.682-17$ & $1.996-16$ & $2.056-15$ & $2.068-14$ & $2.126-13$ \\
\hline 130 & $2.528-19$ & $3.204-18$ & $6.435-17$ & $9.389-16$ & $9.945-15$ & $1.002-13$ & $1.016-12$ \\
\hline 131 & $4.434-19$ & $8.217-18$ & $2.629-16$ & $4.282-15$ & $4.593-14$ & $4.629-13$ & $4.662-12$ \\
\hline 132 & $3.330-19$ & $5.979-18$ & $1.864-16$ & $3.021-15$ & $3.239-14$ & $3.265-13$ & $3.289-12$ \\
\hline 133 & $2.365-19$ & $3.553-18$ & $9.232-17$ & $1.442-15$ & $1.540-14$ & $1.552-13$ & $1.563-12$ \\
\hline 134 & $2.507-19$ & $3.250-18$ & $6.803-17$ & $1.005-15$ & $1.066-14$ & $1.074-13$ & $1.083-12$ \\
\hline 135 & $7.967-19$ & $8.048-18$ & $8.434-17$ & $8.788-16$ & $8.854-15$ & $8.862-14$ & $8.871-13$ \\
\hline 136 & $5.200-19$ & $5.666-18$ & $7.893-17$ & $9.929-16$ & $1.031-14$ & $1.038-13$ & $1.060-12$ \\
\hline 137 & $7.461-19$ & $8.470-18$ & $1.329-16$ & $1.770-15$ & $1.853-14$ & $1.864-13$ & $1.886-12$ \\
\hline 138 & $1.258-19$ & $1.472-18$ & $2.496-17$ & $3.433-16$ & $3.609-15$ & $3.639-14$ & $3.741-13$ \\
\hline
\end{tabular}


Table IV. Ca IX Fractional Level Populations

\begin{tabular}{|c|c|c|c|c|c|c|c|}
\hline $\operatorname{gg}($ Den. $)$ & 8 & 9 & 10 & 11 & 12 & 13 & 14 \\
\hline Key & & & & Population & & & \\
\hline 139 & $5.185-19$ & $5.758-18$ & $8.500-17$ & $1.101-15$ & $1.148-14$ & $1.156-13$ & $1.190-12$ \\
\hline 140 & $4.170-19$ & $5.942-18$ & $1.441-16$ & $2.215-15$ & $2.361-14$ & $2.379-13$ & $2.406-12$ \\
\hline 141 & $5.532-19$ & $9.506-18$ & $2.850-16$ & $4.586-15$ & $4.912-14$ & $4.951-13$ & $4.988-12$ \\
\hline 142 & $2.950-18$ & $4.600-17$ & 1. $249-15$ & $1.969-14$ & $2.105-13$ & $2.121-12$ & $2.135-11$ \\
\hline 143 & $3.606-19$ & $5.415-18$ & $1.406-16$ & $2.197-15$ & $2.346-14$ & $2.364-13$ & $2.386-12$ \\
\hline 144 & $2.415-20$ & $2.644-19$ & $3.742-18$ & $4.746-17$ & $4.935-16$ & $4.960-15$ & $5.003-14$ \\
\hline 145 & $2.089-19$ & $3.445-18$ & $9.929-17$ & $1.586-15$ & $1.697-14$ & $1.710-13$ & $1.724-12$ \\
\hline 146 & $6.909-20$ & 1. 203-18 & $3.650-17$ & $5.888-16$ & $6.309-15$ & $6.358-14$ & $6.403-13$ \\
\hline 147 & $3.067-19$ & $3.722-18$ & $6.852-17$ & $9.714-16$ & $1.025-14$ & $1.032-13$ & $1.040-12$ \\
\hline 148 & $1.290-20$ & $1.321-19$ & $1.466-18$ & $1.599-17$ & $1.624-16$ & $1.628-15$ & $1.634-14$ \\
\hline 149 & $2.062-20$ & $2.122-19$ & $2.408-18$ & $2.669-17$ & $2.718-16$ & $2.724-15$ & $2.736-14$ \\
\hline 150 & $2.909-20$ & $3.082-19$ & $3.908-18$ & $4.663-17$ & $4.805-16$ & $4.822-15$ & $4.839-14$ \\
\hline 151 & $2.341-18$ & $2.551-17$ & $3.552-16$ & $4.468-15$ & $4.641-14$ & $4.668-13$ & $4.749-12$ \\
\hline 152 & $7.402-19$ & $8.551-18$ & $1.404-16$ & $1.905-15$ & $2.000-14$ & $2.015-13$ & $2.062-12$ \\
\hline 153 & $2.452-19$ & $4.172-18$ & 1. $239-16$ & $1.990-15$ & $2.132-14$ & $2.148-13$ & $2.163-12$ \\
\hline 154 & $2.256-20$ & $2.260-19$ & $2.281-18$ & $2.300-17$ & $2.304-16$ & $2.305-15$ & $2.305-14$ \\
\hline 155 & $1.901-19$ & $2.096-18$ & $3.026-17$ & $3.877-16$ & $4.037-15$ & $4.063-14$ & $4.151-13$ \\
\hline 156 & $1.207-18$ & 1. $227-17$ & 1. $324-16$ & $1.412-15$ & $1.428-14$ & $1.430-13$ & $1.433-12$ \\
\hline 157 & $6.295-19$ & $6.822-18$ & $9.343-17$ & $1.165-15$ & $1.208-14$ & 1. $215-13$ & 1. $238-12$ \\
\hline 158 & $5.258-19$ & $6.022-18$ & $9.674-17$ & $1.301-15$ & $1.364-14$ & $1.372-13$ & $1.388-12$ \\
\hline 159 & $2.103-19$ & $2.427-18$ & $3.972-17$ & $5.385-16$ & $5.652-15$ & $5.695-14$ & $5.839-13$ \\
\hline 160 & $4.610-19$ & $4.833-18$ & $5.900-17$ & $6.876-16$ & $7.061-15$ & $7.102-14$ & $7.317-13$ \\
\hline 161 & $2.765-19$ & $3.101-18$ & $4.704-17$ & $6.170-16$ & $6.448-15$ & $6.498-14$ & $6.704-13$ \\
\hline 162 & $2.285-19$ & $2.746-18$ & $4.952-17$ & $6.968-16$ & $7.348-15$ & $7.400-14$ & $7.509-13$ \\
\hline 163 & $1.354-19$ & 1. $612-18$ & $2.846-17$ & $3.974-16$ & $4.187-15$ & $4.219-14$ & $4.315-13$ \\
\hline 164 & $1.951-19$ & $2.972-18$ & $7.847-17$ & 1. $230-15$ & $1.314-14$ & $1.325-13$ & $1.339-12$ \\
\hline 165 & $2.729-19$ & $4.899-18$ & $1.527-16$ & $2.475-15$ & $2.653-14$ & $2.674-13$ & $2.694-12$ \\
\hline 166 & $6.232-19$ & $1.090-17$ & $3.321-16$ & $5.360-15$ & $5.743-14$ & $5.789-13$ & $5.830-12$ \\
\hline 167 & $1.068-19$ & $2.160-18$ & $7.376-17$ & 1. 214-15 & $1.304-14$ & $1.315-13$ & 1. $327-12$ \\
\hline 168 & $2.004-19$ & $4.002-18$ & $1.355-16$ & $2.228-15$ & $2.392-14$ & $2.411-13$ & $2.429-12$ \\
\hline 169 & $4.334-19$ & $7.086-18$ & $2.024-16$ & $3.226-15$ & $3.452-14$ & $3.479-13$ & $3.503-12$ \\
\hline 170 & $2.902-19$ & $3.722-18$ & $7.638-17$ & $1.122-15$ & $1.189-14$ & $1.197-13$ & 1. 207-12 \\
\hline 171 & $3.650-20$ & $3.748-19$ & $4.218-18$ & $4.647-17$ & $4.728-16$ & $4.738-15$ & $4.752-14$ \\
\hline 172 & $4.977-19$ & $6.436-18$ & $1.341-16$ & $1.978-15$ & $2.098-14$ & $2.113-13$ & $2.133-12$ \\
\hline 173 & $3.926-19$ & $5.269-18$ & $1.169-16$ & $1.756-15$ & $1.866-14$ & $1.879-13$ & $1.893-12$ \\
\hline 174 & $2.069-19$ & $3.044-18$ & $7.705-17$ & $1.197-15$ & $1.277-14$ & 1. $286-13$ & 1. 295-12 \\
\hline 175 & $2.150-19$ & $2.981-18$ & $6.956-17$ & $1.059-15$ & $1.127-14$ & $1.136-13$ & $1.147-12$ \\
\hline 176 & $2.800-19$ & $3.441-18$ & $6.503-17$ & $9.302-16$ & $9.829-15$ & $9.892-14$ & $9.961-13$ \\
\hline 177 & $1.057-19$ & $1.717-18$ & $4.873-17$ & $7.757-16$ & $8.300-15$ & $8.364-14$ & $8.423-13$ \\
\hline 178 & $3.331-19$ & $3.865-18$ & $6.417-17$ & $8.751-16$ & $9.190-15$ & $9.243-14$ & $9.300-13$ \\
\hline 179 & $4.707-19$ & $5.494-18$ & $9.253-17$ & 1.269-15 & $1.334-14$ & $1.342-13$ & $1.351-12$ \\
\hline 180 & $2.348-19$ & $2.472-18$ & $3.069-17$ & $3.614-16$ & $3.717-15$ & $3.729-14$ & $3.743-13$ \\
\hline 181 & $3.844-20$ & $5.690-19$ & $1.451-17$ & $2.258-16$ & $2.410-15$ & $2.428-14$ & $2.445-13$ \\
\hline 182 & $5.623-20$ & $7.943-19$ & $1.903-17$ & $2.916-16$ & $3.107-15$ & $3.130-14$ & $3.155-13$ \\
\hline 183 & $1.887-18$ & $1.920-17$ & $2.082-16$ & $2.230-15$ & $2.258-14$ & $2.261-13$ & $2.265-12$ \\
\hline 184 & $3.872-20$ & $3.971-19$ & $4.442-18$ & $4.872-17$ & $4.953-16$ & $4.963-15$ & $4.979-14$ \\
\hline
\end{tabular}


Table IV. Ca IX Fractional Level Populations

\begin{tabular}{|c|c|c|c|c|c|c|c|}
\hline $\begin{array}{c}\text { og }(\text { Den. }) \\
\text { Key }\end{array}$ & 8 & 9 & 10 & $\begin{array}{c}11 \\
\text { Population }\end{array}$ & 12 & 13 & 14 \\
\hline 185 & $1.259-20$ & $1.260-19$ & $1.266-18$ & $1.272-17$ & 1. 273-16 & $1.273-15$ & $1.279-14$ \\
\hline 186 & $7.073-20$ & $7.307-19$ & $8.428-18$ & $9.452-17$ & $9.644-16$ & $9.668-15$ & $9.695-14$ \\
\hline 187 & $2.766-19$ & $2.801-18$ & $2.967-17$ & $3.120-16$ & $3.149-15$ & $3.152-14$ & $3.156-13$ \\
\hline 188 & $1.930-19$ & $2.114-18$ & $2.992-17$ & $3.795-16$ & $3.946-15$ & $3.964-14$ & $3.988-13$ \\
\hline 189 & 1. 328-20 & $1.436-19$ & $1.947-18$ & $2.415-17$ & $2.504-16$ & $2.514-15$ & $2.530-14$ \\
\hline 190 & $1.506-19$ & $1.556-18$ & $1.792-17$ & $2.008-16$ & $2.048-15$ & $2.054-14$ & $2.068-13$ \\
\hline 191 & $8.164-21$ & $9.439-20$ & $1.553-18$ & $2.110-17$ & $2.214-16$ & $2.228-15$ & $2.248-14$ \\
\hline 192 & $1.639-20$ & $1.942-19$ & $3.387-18$ & $4.707-17$ & $4.956-16$ & $4.986-15$ & $5.018-14$ \\
\hline 193 & $9.410-21$ & $1.103-19$ & $1.878-18$ & $2.586-17$ & $2.719-16$ & $2.736-15$ & $2.763-14$ \\
\hline 194 & $2.087-19$ & $2.215-18$ & $2.828-17$ & $3.388-16$ & $3.494-15$ & $3.507-14$ & $3.528-13$ \\
\hline 195 & $2.469-19$ & $2.764-18$ & $4.177-17$ & $5.468-16$ & $5.711-15$ & $5.739-14$ & $5.765-13$ \\
\hline 196 & $5.495-21$ & $5.640-20$ & $6.335-19$ & $6.970-18$ & $7.089-17$ & $7.104-16$ & $7.122-15$ \\
\hline 197 & $2.525-21$ & $2.661-20$ & $3.310-19$ & $3.903-18$ & $4.015-17$ & $4.029-16$ & $4.053-15$ \\
\hline 198 & $3.303-21$ & $3.615-20$ & $5.107-19$ & $6.470-18$ & $6.727-17$ & $6.758-16$ & $6.794-15$ \\
\hline 199 & $2.795-21$ & $3.045-20$ & $4.240-19$ & $5.333-18$ & $5.538-17$ & $5.563-16$ & $5.593-15$ \\
\hline 200 & $2.351-21$ & $2.368-20$ & $2.447-19$ & $2.520-18$ & $2.533-17$ & $2.535-16$ & $2.540-15$ \\
\hline 201 & $5.513-21$ & $5.600-20$ & $6.019-19$ & $6.402-18$ & $6.474-17$ & $6.483-16$ & $6.493-15$ \\
\hline 202 & $3.851-21$ & $3.911-20$ & $4.195-19$ & $4.454-18$ & $4.503-17$ & $4.509-16$ & $4.517-15$ \\
\hline 203 & $1.524-20$ & $1.552-19$ & $1.684-18$ & $1.805-17$ & $1.827-16$ & $1.830-15$ & $1.834-14$ \\
\hline 204 & $9.084-21$ & $9.198-20$ & $9.744-19$ & $1.024-17$ & $1.034-16$ & $1.035-15$ & $1.037-14$ \\
\hline 205 & $3.120-21$ & $3.183-20$ & $3.485-19$ & $3.762-18$ & $3.813-17$ & $3.820-16$ & $3.826-15$ \\
\hline 206 & $2.670-19$ & $2.652-18$ & $2.564-17$ & $2.484-16$ & $2.469-15$ & $2.467-14$ & $2.465-13$ \\
\hline 207 & $4.725-20$ & $4.765-19$ & $4.959-18$ & $5.137-17$ & $5.170-16$ & $5.174-15$ & $5.180-14$ \\
\hline 208 & $1.588-20$ & $1.583-19$ & $1.558-18$ & $1.535-17$ & $1.530-16$ & $1.530-15$ & $1.529-14$ \\
\hline 209 & $1.812-20$ & $1.837-19$ & $1.955-18$ & $2.062-17$ & $2.082-16$ & $2.085-15$ & $2.088-14$ \\
\hline 210 & $6.165-21$ & $6.199-20$ & $6.359-19$ & $6.505-18$ & $6.533-17$ & $6.536-16$ & $6.546-15$ \\
\hline 211 & $1.006-20$ & $1.010-19$ & $1.033-18$ & $1.053-17$ & $1.057-16$ & $1.058-15$ & $1.059-14$ \\
\hline 212 & $1.402-20$ & $1.426-19$ & $1.540-18$ & $1.644-17$ & $1.664-16$ & $1.666-15$ & $1.668-14$ \\
\hline 213 & $3.198-20$ & $3.192-19$ & $3.165-18$ & $3.139-17$ & $3.135-16$ & $3.134-15$ & $3.133-14$ \\
\hline 214 & $1.812-21$ & $1.901-20$ & $2.323-19$ & $2.709-18$ & $2.782-17$ & $2.791-16$ & $2.804-15$ \\
\hline 215 & $1.889-21$ & $1.925-20$ & $2.095-19$ & $2.250-18$ & $2.279-17$ & $2.283-16$ & $2.294-15$ \\
\hline 216 & $3.088-21$ & $3.197-20$ & $3.716-19$ & $4.191-18$ & $4.280-17$ & $4.291-16$ & $4.307-15$ \\
\hline 217 & $4.261-21$ & $4.446-20$ & $5.327-19$ & $6.133-18$ & $6.284-17$ & $6.303-16$ & $6.325-15$ \\
\hline 218 & $5.653-21$ & $5.706-20$ & $5.958-19$ & $6.189-18$ & $6.233-17$ & $6.240-16$ & $6.259-15$ \\
\hline 219 & $7.707-21$ & $7.831-20$ & $8.422-19$ & $8.962-18$ & $9.064-17$ & $9.077-16$ & $9.102-15$ \\
\hline 220 & $1.007-20$ & $1.043-19$ & $1.211-18$ & $1.365-17$ & 1. $394-16$ & $1.397-15$ & $1.400-14$ \\
\hline 221 & $7.434-21$ & $7.611-20$ & $8.457-19$ & $9.230-18$ & $9.375-17$ & $9.392-16$ & $9.409-15$ \\
\hline 222 & $4.577-21$ & $4.603-20$ & $4.728-19$ & $4.842-18$ & $4.864-17$ & $4.868-16$ & $4.881-15$ \\
\hline 223 & $1.585-21$ & $1.609-20$ & $1.726-19$ & $1.833-18$ & $1.853-17$ & $1.856-16$ & $1.860-15$ \\
\hline 224 & $1.616-20$ & $1.654-19$ & $1.836-18$ & $2.002-17$ & $2.034-16$ & $2.038-15$ & $2.043-14$ \\
\hline 225 & $3.660-20$ & $3.648-19$ & $3.593-18$ & $3.543-17$ & $3.533-16$ & $3.532-15$ & $3.531-14$ \\
\hline 226 & $3.138-21$ & $3.182-20$ & $3.391-19$ & $3.582-18$ & $3.618-17$ & $3.623-16$ & $3.630-15$ \\
\hline 227 & $2.851-21$ & $2.869-20$ & $2.953-19$ & $3.031-18$ & $3.045-17$ & $3.047-16$ & $3.052-15$ \\
\hline 228 & $4.659-21$ & $4.691-20$ & $4.843-19$ & $4.982-18$ & $5.009-17$ & $5.012-16$ & $5.020-15$ \\
\hline 229 & $6.521-21$ & $6.619-20$ & $7.087-19$ & $7.515-18$ & $7.595-17$ & $7.605-16$ & $7.614-15$ \\
\hline 230 & $8.326-21$ & $8.379-20$ & $8.632-19$ & $8.863-18$ & $8.906-17$ & $8.913-16$ & $8.937-15$ \\
\hline
\end{tabular}


Table IV. Ca IX Fractional Level Populations

\begin{tabular}{|c|c|c|c|c|c|c|c|}
\hline og (Den.) & 8 & 9 & 10 & 11 & 12 & 13 & 14 \\
\hline Key & \multicolumn{7}{|c|}{ Population } \\
\hline 231 & $1.060-20$ & $1.076-19$ & $1.151-18$ & $1.220-17$ & $1.233-16$ & $1.234-15$ & $1.237-14$ \\
\hline 232 & $1.280-20$ & $1.317-19$ & $1.497-18$ & $1.662-17$ & $1.693-16$ & $1.697-15$ & $1.700-14$ \\
\hline 233 & $3.731-21$ & $3.724-20$ & $3.692-19$ & $3.663-18$ & $3.658-17$ & $3.657-16$ & $3.656-15$ \\
\hline 234 & $6.961-21$ & $6.957-20$ & $6.938-19$ & $6.920-18$ & $6.916-17$ & $6.916-16$ & $6.916-15$ \\
\hline 235 & $2.708-21$ & $2.733-20$ & $2.852-19$ & $2.961-18$ & $2.981-17$ & $2.984-16$ & $2.990-15$ \\
\hline 236 & $3.782-21$ & $3.838-20$ & $4.102-19$ & $4.344-18$ & $4.389-17$ & $4.395-16$ & $4.403-15$ \\
\hline 237 & $4.868-21$ & $4.965-20$ & $5.429-19$ & $5.853-18$ & $5.933-17$ & $5.943-16$ & $5.953-15$ \\
\hline 238 & $1.884-20$ & $1.888-19$ & $1.906-18$ & $1.922-17$ & $1.925-16$ & $1.925-15$ & $1.926-14$ \\
\hline 239 & $2.774-21$ & $2.761-20$ & $2.699-19$ & $2.643-18$ & $2.633-17$ & $2.632-16$ & $2.638-15$ \\
\hline 240 & $8.250-21$ & $8.343-20$ & $8.786-19$ & $9.191-18$ & $9.267-17$ & $9.277-16$ & $9.292-15$ \\
\hline 241 & $1.388-20$ & $1.412-19$ & $1.526-18$ & $1.631-17$ & $1.651-16$ & $1.653-15$ & $1.655-14$ \\
\hline 242 & $7.385-21$ & $7.565-20$ & $8.423-19$ & $9.207-18$ & $9.355-17$ & $9.373-16$ & $9.398-15$ \\
\hline 243 & $2.509-21$ & $2.601-20$ & $3.041-19$ & $3.443-18$ & $3.519-17$ & $3.528-16$ & $3.544-15$ \\
\hline 244 & $1.123-20$ & $1.140-19$ & $1.220-18$ & $1.293-17$ & $1.306-16$ & $1.308-15$ & $1.314-14$ \\
\hline 245 & 1. 365-20 & $1.402-19$ & $1.574-18$ & $1.732-17$ & $1.762-16$ & $1.766-15$ & $1.772-14$ \\
\hline 246 & $1.611-20$ & $1.689-19$ & $2.059-18$ & $2.398-17$ & $2.462-16$ & $2.469-15$ & $2.476-14$ \\
\hline 247 & $1.605-21$ & $1.737-20$ & $2.369-19$ & $2.946-18$ & $3.055-17$ & $3.070-16$ & $3.104-15$ \\
\hline 248 & $1.698-21$ & $1.914-20$ & $2.949-19$ & $3.894-18$ & $4.072-17$ & $4.096-16$ & $4.143-15$ \\
\hline 249 & $2.183-21$ & $2.786-20$ & $5.666-19$ & $8.298-18$ & $8.794-17$ & $8.852-16$ & $8.907-15$ \\
\hline 250 & $2.569-21$ & $2.616-20$ & $2.837-19$ & $3.040-18$ & $3.078-17$ & $3.083-16$ & $3.089-15$ \\
\hline 251 & $6.173-22$ & $6.563-21$ & $8.429-20$ & $1.014-18$ & $1.046-17$ & $1.050-16$ & $1.056-15$ \\
\hline 252 & $8.225-22$ & $8.520-21$ & $9.932-20$ & $1.122-18$ & $1.146-17$ & $1.150-16$ & $1.156-15$ \\
\hline 253 & $6.374-22$ & $6.670-21$ & $8.086-20$ & $9.380-19$ & $9.624-18$ & $9.657-17$ & $9.723-16$ \\
\hline 254 & $9.955-22$ & $1.050-20$ & $1.310-19$ & $1.549-18$ & $1.593-17$ & $1.599-16$ & $1.606-15$ \\
\hline 255 & $7.609-22$ & $8.107-21$ & $1.049-19$ & 1.266-18 & $1.307-17$ & $1.313-16$ & $1.320-15$ \\
\hline 256 & $1.186-21$ & $1.262-20$ & $1.625-19$ & 1. $957-18$ & $2.019-17$ & $2.027-16$ & $2.036-15$ \\
\hline 257 & $7.643-22$ & $8.373-21$ & $1.186-19$ & $1.505-18$ & $1.565-17$ & $1.573-16$ & $1.583-15$ \\
\hline 258 & $1.040-21$ & $1.050-20$ & $1.099-19$ & $1.143-18$ & $1.151-17$ & $1.152-16$ & $1.154-15$ \\
\hline 259 & $1.319-21$ & $1.340-20$ & $1.443-19$ & $1.538-18$ & $1.555-17$ & $1.557-16$ & $1.560-15$ \\
\hline 260 & $1.530-21$ & $1.565-20$ & $1.734-19$ & $1.889-18$ & $1.918-17$ & $1.921-16$ & $1.925-15$ \\
\hline 261 & 1. 168-21 & $1.177-20$ & $1.220-19$ & 1. 259-18 & 1. $267-17$ & $1.267-16$ & $1.269-15$ \\
\hline 262 & 1. $377-21$ & $1.390-20$ & $1.452-19$ & $1.508-18$ & $1.519-17$ & $1.521-16$ & $1.527-15$ \\
\hline 263 & $2.434-21$ & $2.504-20$ & $2.838-19$ & $3.144-18$ & $3.202-17$ & $3.209-16$ & $3.219-15$ \\
\hline 264 & $3.298-21$ & $3.408-20$ & $3.934-19$ & $4.414-18$ & $4.505-17$ & $4.516-16$ & $4.530-15$ \\
\hline 265 & $6.164-21$ & $6.192-20$ & $6.329-19$ & $6.453-18$ & $6.477-17$ & $6.480-16$ & $6.484-15$ \\
\hline 266 & $2.472-21$ & $2.515-20$ & $2.720-19$ & $2.908-18$ & $2.943-17$ & $2.948-16$ & $2.961-15$ \\
\hline 267 & $9.467-22$ & $9.528-21$ & $9.817-20$ & $1.008-18$ & $1.013-17$ & $1.014-16$ & $1.015-15$ \\
\hline 268 & $1.315-21$ & $1.330-20$ & $1.404-19$ & $1.472-18$ & $1.485-17$ & $1.486-16$ & $1.488-15$ \\
\hline 269 & $2.511-21$ & $2.538-20$ & $2.664-19$ & $2.780-18$ & $2.802-17$ & $2.804-16$ & $2.807-15$ \\
\hline 270 & $2.880-21$ & $2.967-20$ & $3.380-19$ & $3.758-18$ & $3.829-17$ & $3.838-16$ & $3.852-15$ \\
\hline 271 & $3.274-21$ & $3.443-20$ & $4.250-19$ & $4.988-18$ & $5.126-17$ & $5.143-16$ & $5.157-15$ \\
\hline 272 & $7.071-21$ & $7.270-20$ & $8.218-19$ & $9.084-18$ & $9.247-17$ & $9.267-16$ & $9.292-15$ \\
\hline 273 & $4.216-21$ & $4.310-20$ & $4.758-19$ & $5.169-18$ & $5.246-17$ & $5.256-16$ & $5.271-15$ \\
\hline 274 & $1.448-21$ & $1.492-20$ & $1.704-19$ & $1.898-18$ & $1.934-17$ & $1.939-16$ & $1.942-15$ \\
\hline 275 & $3.035-20$ & $3.023-19$ & $2.962-18$ & $2.906-17$ & $2.895-16$ & $2.894-15$ & $2.892-14$ \\
\hline 276 & $2.276-21$ & $2.394-20$ & $2.958-19$ & $3.473-18$ & $3.570-17$ & $3.582-16$ & $3.597-15$ \\
\hline
\end{tabular}


Table IV. Ca IX Fractional Level Populations

\begin{tabular}{|c|c|c|c|c|c|c|c|}
\hline og (Den.) & 8 & 9 & 10 & 11 & 12 & 13 & 14 \\
\hline Key & \multicolumn{7}{|c|}{ Population } \\
\hline 277 & $4.495-21$ & $4.497-20$ & $4.506-19$ & $4.513-18$ & $4.515-17$ & $4.515-16$ & $4.516-1$ \\
\hline 278 & $3.291-21$ & $3.293-20$ & $3.306-19$ & $3.317-18$ & $3.319-17$ & $3.319-16$ & $9-15$ \\
\hline 279 & $1.930-21$ & $1.937-20$ & $1.971-19$ & $2.002-18$ & $2.007-17$ & $2.008-16$ & $2.008-15$ \\
\hline 280 & $1.194-20$ & $1.189-19$ & $1.163-18$ & $1.139-17$ & $1.134-16$ & $1.134-15$ & $1.133-14$ \\
\hline 281 & $2.132-20$ & $2.119-19$ & $2.058-18$ & $2.001-17$ & $1.991-16$ & $1.989-15$ & $1.988-1$ \\
\hline 282 & $1.476-21$ & $1.556-20$ & $1.938-19$ & $2.288-18$ & $2.354-17$ & $2.362-16$ & $2.374-1$ \\
\hline 283 & $1.090-20$ & $1.084-19$ & $1.053-18$ & $1.025-17$ & $1.020-16$ & $1.019-15$ & $1.019-1$ \\
\hline
\end{tabular}


Table V. Intensities of Selected Bright Ca IX Lines

\begin{tabular}{|c|c|c|c|c|c|c|c|c|c|}
\hline \multicolumn{2}{|c|}{$\log ($ Den. $)$} & & \multirow[t]{2}{*}{9} & \multirow[t]{2}{*}{10} & \multirow{2}{*}{\multicolumn{2}{|c|}{$\begin{array}{l}11 \\
\text { Intensity }\end{array}$}} & \multirow[t]{2}{*}{13} & \multirow[t]{2}{*}{14} \\
\hline j & $i$ & & & & & & & & \\
\hline 92 & 5 & 108.071 & $6.47-06$ & $6.43-05$ & $6.21-04$ & $6.01-03$ & $5.97-02$ & $5.96-01$ & $5.96+00$ \\
\hline 65 & 5 & 116.190 & $3.07-05$ & $3.04-04$ & $2.91-03$ & $2.80-02$ & $2.78-01$ & $2.78+00$ & $2.77+01$ \\
\hline 35 & 1 & 120.147 & $4.32-05$ & $4.29-04$ & $4.14-03$ & $4.00-02$ & $3.98-01$ & $3.97+00$ & $3.97+01$ \\
\hline 30 & 1 & 120.565 & $2.99-06$ & $3.01-05$ & $3.10-04$ & $3.18-03$ & $3.19-02$ & $3.20-01$ & $3.24+00$ \\
\hline 43 & 3 & 129.428 & $5.95-06$ & $6.04-05$ & $6.43-04$ & $6.79-03$ & $6.86-02$ & $6.87-01$ & $6.89+00$ \\
\hline 44 & 4 & 129.935 & $1.09-05$ & $1.13-04$ & $1.32-03$ & $1.50-02$ & $1.53-01$ & $1.53+00$ & $1.54+01$ \\
\hline 100 & 13 & 133.820 & $3.51-06$ & $3.62-05$ & $4.16-04$ & $4.64-03$ & $4.74-02$ & $4.75-01$ & $4.75+00$ \\
\hline 45 & 5 & 141.357 & $4.39-05$ & $4.35-04$ & $4.20-03$ & $4.07-02$ & $4.04-01$ & $4.04+00$ & $4.03+01$ \\
\hline 101 & 14 & 144.344 & $1.14-05$ & $1.13-04$ & $1.09-03$ & $1.06-02$ & $1.05-01$ & $1.05+00$ & $1.05+01$ \\
\hline 53 & 6 & 159.540 & $2.52-05$ & $2.50-04$ & $2.40-03$ & $2.32-02$ & $2.30-01$ & $2.30+00$ & $2.30+01$ \\
\hline 27 & 3 & 162.374 & $4.19-06$ & $4.50-05$ & $5.96-04$ & $7.30-03$ & $7.55-02$ & $7.58-01$ & $7.62+00$ \\
\hline 27 & 4 & 163.231 & $7.05-06$ & $7.57-05$ & $1.00-03$ & $1.23-02$ & $1.27-01$ & $1.27+00$ & $1.28+01$ \\
\hline 48 & 8 & 165.424 & $4.67-08$ & $3.34-06$ & $1.71-04$ & $2.97-03$ & $3.20-02$ & $3.23-01$ & $3.25+00$ \\
\hline 48 & 9 & 166.415 & $1.35-07$ & $9.70-06$ & $4.96-04$ & $8.60-03$ & $9.29-02$ & $9.37-01$ & $9.43+00$ \\
\hline 28 & 5 & 178.575 & $4.23-04$ & $4.19-03$ & $4.02-02$ & $3.86-01$ & $3.83+00$ & $3.83+01$ & $3.82+02$ \\
\hline 49 & 11 & 184.336 & $9.29-06$ & $9.43-05$ & $1.01-03$ & $1.08-02$ & $1.09-01$ & $1.09+00$ & $1.10+01$ \\
\hline 50 & 12 & 184.374 & $1.26-05$ & $1.29-04$ & $1.40-03$ & $1.51-02$ & $1.53-01$ & $1.53+00$ & $1.53+01$ \\
\hline 51 & 13 & 184.432 & $1.84-05$ & $1.94-04$ & $2.42-03$ & $2.86-02$ & $2.94-01$ & $2.95+00$ & $2.96+01$ \\
\hline 35 & 6 & 201.589 & $1.26-05$ & $1.25-04$ & $1.21-03$ & $1.17-02$ & $1.16-01$ & $1.16+00$ & $1.16+01$ \\
\hline 53 & 14 & 201.847 & $8.40-05$ & $8.33-04$ & $8.02-03$ & $7.74-02$ & $7.68-01$ & $7.68+00$ & $7.67+01$ \\
\hline 33 & 12 & 239.030 & $1.55-06$ & $1.99-05$ & $4.07-04$ & $5.98-03$ & $6.34-02$ & $6.38-01$ & $6.42+00$ \\
\hline 33 & 13 & 239.135 & $8.69-06$ & $1.11-04$ & $2.29-03$ & $3.36-02$ & $3.56-01$ & $3.58+00$ & $3.60+01$ \\
\hline 30 & 12 & 239.783 & $6.39-06$ & $6.43-05$ & $6.63-04$ & $6.81-03$ & $6.84-02$ & $6.85-01$ & $6.93+00$ \\
\hline 29 & 11 & 239.881 & $3.84-06$ & $4.18-05$ & $5.77-04$ & $7.23-03$ & $7.50-02$ & $7.53-01$ & $7.59+00$ \\
\hline 25 & 6 & 324.088 & $1.98-05$ & $1.98-04$ & $1.98-03$ & $1.99-02$ & $1.99-01$ & $1.99+00$ & $1.99+01$ \\
\hline 11 & 2 & 371.896 & $7.90-05$ & $8.49-04$ & $1.13-02$ & $1.39-01$ & $1.44+00$ & $1.45+01$ & $1.46+02$ \\
\hline 12 & 3 & 373.799 & $1.02-04$ & $1.14-03$ & $1.67-02$ & $2.17-01$ & $2.26+00$ & $2.27+01$ & $2.31+02$ \\
\hline 11 & 3 & 373.986 & $5.81-05$ & $6.25-04$ & $8.33-03$ & $1.02-01$ & $1.06+00$ & $1.07+01$ & $1.08+02$ \\
\hline 13 & 4 & 378.086 & $1.92-04$ & $2.70-03$ & $6.45-02$ & $9.88-01$ & $1.05+01$ & $1.06+02$ & $1.07+03$ \\
\hline 12 & 4 & 378.375 & $3.29-05$ & $3.65-04$ & $5.38-03$ & $6.96-02$ & $7.26-01$ & $7.30+00$ & $7.43+01$ \\
\hline 11 & 4 & 378.567 & $3.74-06$ & $4.02-05$ & $5.35-04$ & $6.58-03$ & $6.81-02$ & $6.84-01$ & $6.92+00$ \\
\hline 23 & 8 & 383.457 & $6.88-07$ & $1.23-05$ & $3.82-04$ & $6.20-03$ & $6.64-02$ & $6.69-01$ & $6.74+00$ \\
\hline 22 & 8 & 384.275 & $6.17-07$ & $1.08-05$ & $3.29-04$ & $5.32-03$ & $5.70-02$ & $5.74-01$ & $5.79+00$ \\
\hline 20 & 7 & 385.518 & $8.36-07$ & $9.86-06$ & $1.70-04$ & $2.36-03$ & $2.48-02$ & $2.50-01$ & $2.57+00$ \\
\hline 24 & 9 & 388.761 & $1.01-06$ & $2.39-05$ & $8.98-04$ & $1.50-02$ & $1.61-01$ & $1.63+00$ & $1.64+01$ \\
\hline 23 & 9 & 388.827 & $9.36-07$ & $1.67-05$ & $5.20-04$ & $8.43-03$ & $9.04-02$ & $9.11-01$ & $9 \cdot 17+00$ \\
\hline 19 & 8 & 389.934 & $2.51-06$ & $2.82-05$ & $4.26-04$ & $5.59-03$ & $5.84-02$ & $5.87-01$ & $5.97+00$ \\
\hline 26 & 10 & 391.737 & $6.93-06$ & $6.92-05$ & $6.87-04$ & $6.81-03$ & $6.81-02$ & $6.80-01$ & $6.80+00$ \\
\hline 14 & 5 & 395.022 & $1.52-03$ & $1.51-02$ & $1.45-01$ & $1.39+00$ & $1.38+01$ & $1.38+02$ & $1.38+03$ \\
\hline 18 & 6 & 424.366 & $4.37-06$ & $4.56-05$ & $5.49-04$ & $6.34-03$ & $6.50-02$ & $6.52-01$ & $6.55+00$ \\
\hline 15 & 6 & 439.747 & $8.89-07$ & $1.01-05$ & $1.58-04$ & $2.11-03$ & $2.20-02$ & $2.22-01$ & $2.26+00$ \\
\hline 5 & 1 & 466.240 & $4.15-02$ & $4.12-01$ & $3.95+00$ & $3.80+01$ & $3.77+02$ & $3.76+03$ & $3.76+04$ \\
\hline 9 & 3 & 498.011 & $1.72-05$ & $4.09-04$ & $1.54-02$ & $2.58-01$ & $2.77+00$ & $2.79+01$ & $2.82+02$ \\
\hline 8 & 2 & 503.272 & $3.22-05$ & $4.81-04$ & $1.24-02$ & $1.93-01$ & $2.06+00$ & $2.08+01$ & $2.10+02$ \\
\hline 9 & 4 & 506.168 & $4.99-05$ & $1.18-03$ & $4.46-02$ & $7.46-01$ & $8.02+00$ & $8.09+01$ & $8.16+02$ \\
\hline 8 & 3 & 507.108 & $2.36-05$ & $3.53-04$ & $9.08-03$ & $1.41-01$ & $1.51+00$ & $1.52+01$ & $1.54+02$ \\
\hline
\end{tabular}


Table V. Intensities of Selected Bright Ca IX Lines

\begin{tabular}{|c|c|c|c|c|c|c|c|c|c|}
\hline \multicolumn{3}{|c|}{$\log ($ Den.) } & 8 & 9 & 10 & 11 & 12 & 13 & 14 \\
\hline j & $i$ & \multicolumn{4}{|c|}{ Wavelength } & \multicolumn{4}{|l|}{ Intensity } \\
\hline 7 & 3 & 512.052 & $1.82-06$ & $2.12-05$ & $3.58-04$ & $4.91-03$ & $5.19-02$ & $5.47-01$ & $8.02+00$ \\
\hline 8 & 4 & 515.567 & $3.73-05$ & $5.57-04$ & $1.43-02$ & $2.23-01$ & $2.39+00$ & $2.40+01$ & $2.43+02$ \\
\hline 6 & 3 & 521.859 & $1.39-04$ & $1.38-03$ & $1.35-02$ & $1.33-01$ & $1.32+00$ & $1.32+01$ & $1.32+02$ \\
\hline 23 & 12 & 525.048 & $3.71-07$ & $6.64-06$ & $2.06-04$ & $3.34-03$ & $3.58-02$ & $3.61-01$ & $3.64+00$ \\
\hline 24 & 13 & 525.484 & $2.27-07$ & $5.36-06$ & $2.01-04$ & $3.36-03$ & $3.62-02$ & $3.65-01$ & $3.69+00$ \\
\hline 6 & 4 & 530.822 & $2.86-04$ & $2.85-03$ & $2.79-02$ & $2.74-01$ & $2.73+00$ & $2.73+01$ & $2.73+02$ \\
\hline 19 & 13 & 537.852 & $1.23-06$ & $1.38-05$ & $2.09-04$ & $2.74-03$ & $2.86-02$ & $2.88-01$ & $2.92+00$ \\
\hline 10 & 5 & 42.262 & $1.06-05$ & $1.06-04$ & $1.08-03$ & $1.10-02$ & $1.10-01$ & $1.11+00$ & $1.11+01$ \\
\hline 25 & 14 & 564.402 & $8.92-06$ & $8.93-05$ & $8.94-04$ & $8.95-03$ & $8.95-02$ & $8.95-01$ & $8.95+00$ \\
\hline 17 & 13 & 641.891 & $3.76-06$ & $8.88-05$ & $3.33-03$ & $5.57-02$ & $5.99-01$ & $6.04+00$ & $6.08+01$ \\
\hline 16 & 12 & 51.251 & $2.46-06$ & $3.58-05$ & $8.97-04$ & $1.39-02$ & $1.48-01$ & $1.50+00$ & $1.53+01$ \\
\hline 16 & 13 & 652.109 & $3.87-07$ & $5.64-06$ & $1.41-04$ & $2.19-03$ & $2.33-02$ & $2.35-01$ & $2.40+00$ \\
\hline 15 & 11 & 659.471 & $4.14-06$ & $4.69-05$ & $7.36-04$ & $9.80-03$ & $1.03-01$ & $1.03+00$ & $1.05+01$ \\
\hline 15 & 12 & 660.054 & $8.90-07$ & $1.01-05$ & $1.58-04$ & $2.11-03$ & $2.21-02$ & $2.22-01$ & $2.26+00$ \\
\hline 3 & 1 & 691.421 & $9.89-04$ & $1.01-02$ & $1.12-01$ & $1.22+00$ & $1.24+01$ & $1.24+02$ & $1.24+03$ \\
\hline 9 & 5 & 763.669 & $2.56-07$ & $6.09-06$ & $2.29-04$ & $3.83-03$ & $4.12-02$ & $4.16-01$ & $4.19+00$ \\
\hline 6 & 5 & 821.215 & $8.11-04$ & $8.07-03$ & $7.91-02$ & $7.76-01$ & $7.73+00$ & $7.73+01$ & $7.72+02$ \\
\hline
\end{tabular}

